NISTIR 7886

\title{
Finite-element Models of the National Fire Research Laboratory (NFRL) and Modular Support Structure
}

\author{
Dat Duthinh
}

http://dx.doi.org/10.6028/NIST.IR.7886

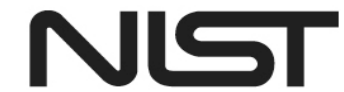

National Institute of Standards and Technology

U.S. Department of Commerce 


\section{Finite-element Models of the National Fire Research Laboratory (NFRL) and Modular Support Structure}

Dat Duthinh

Materials and Structural Systems Division

Engineering Laboratory

http://dx.doi.org/10.6028/NIST.IR.7886

October 2012

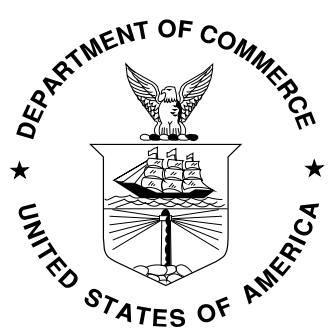

U.S. Department of Commerce Rebecca Blank, Acting Secretary

National Institute of Standards and Technology Patrick D. Gallagher, Under Secretary of Commerce for Standards and Technology and Director 


\section{Table of contents}

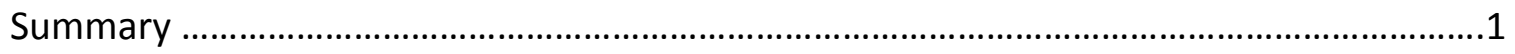

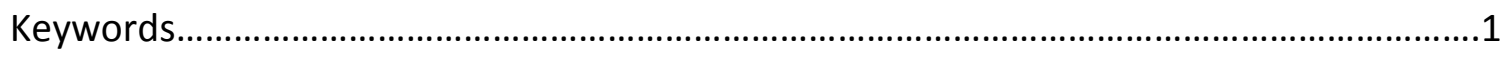

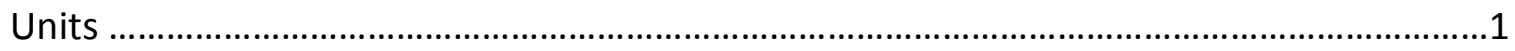

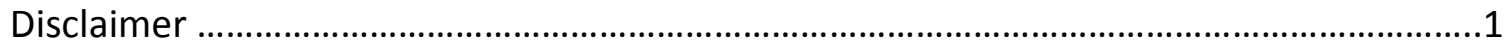

Part 1 Finite-Element Model of the National Fire Research Laboratory (NFRL).............. 2

Chapter 1 Finite-Element Model (FEM) of the National Fire Research Laboratory (NFRL)............. 2

Part 2 Finite-element model of the modular support structure .....................................54

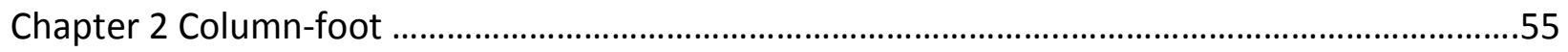

Chapter 3 Wrapped column-foot.................................................................................................

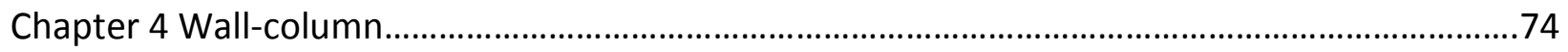

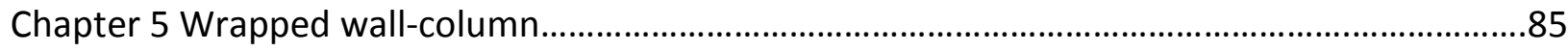

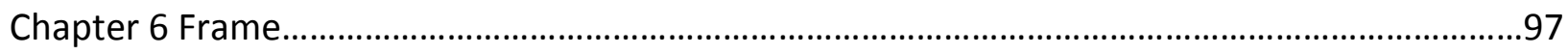

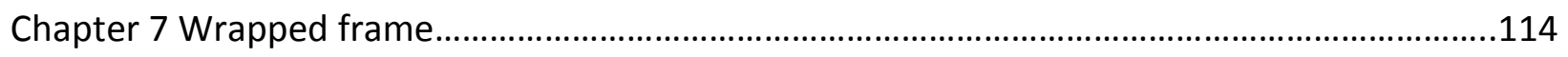

Chapter 8Web splice ........................................................................................................131

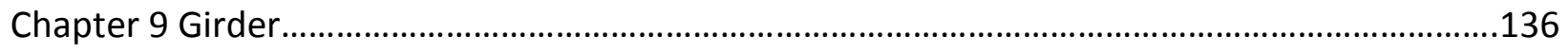

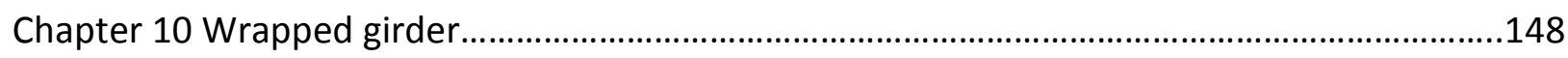

Chapter 11 Girder angle..........................................................................................................161

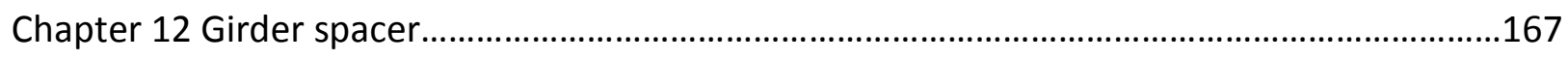

Chapter 13 Assembling the support structure..........................................................................174

Chapter 14 Assembling the support and the concrete structure s.............................................227

Appendix Modular Support Drawings.............................................................................294 


\section{Finite-element models of the}

\section{National Fire Research Laboratory (NFRL) and modular support structure}

Summary: This report documents the development of finite-element models (FEM) of the National Fire Research Laboratory (NFRL) and its modular support structure. The models enable a future user of the NFRL to add a potential test structure and perform thermal and structural simulations of the test. This report consists of two parts. Part 1 covers the NFRL proper, namely the strong floor, strong wall, basement walls and slab. It describes the model and provides results of structural analyses of all the load cases that were specified in the design documents. Part 2 covers the modular support structure, with the various components developed individually, then assembled in an example test case.

Keywords: finite-element model; fire research; modular structure; prestressed concrete; reinforced concrete; steel; structural analysis; thermal analysis.

Units: As the design of the NFRL was specified and performed in customary units, so were the present calculations. SI units are included in parenthesis in the text.

Disclaimer: Some commercial products are identified in this document for traceability of results. Such identification does not imply recommendation or endorsement by the National Institute of Standards and Technology, nor does it imply that the products identified are necessarily the best available for the purpose. 


\section{Part 1, Chapter 1}

\section{Finite-Element Model (FEM) of}

\section{the National Fire Research Laboratory (NFRL)}

This report documents the development of finite-element models (FEM) of the National Fire Research Laboratory (NFRL, Part 1) and its modular support structure (Part 2). The models enable a future user of the NFRL to add a potential test structure and perform thermal and structural simulations of the test. Part 1 covers the NFRL proper, namely the strong floor, strong wall, basement walls and slab. It describes the model and provides results of structural analyses of all the load cases that were specified in the design documents.

Elements: The model includes the strong wall, strong floor, basement walls and slab (Fig. 1.1), all represented by 59400 solid elements (ANSYS 8-node SOLID 185 for structural and thermal analyses). As mesh nodes are defined at all loading and anchorage points, typical element size is $2 \mathrm{ft}(0.61 \mathrm{~m})$, which is the distance between two adjacent loading points in the strong floor and strong wall. To accommodate various geometric constraints, however, many elements are smaller than that. Also, for proper modeling of structural behavior, the through-thickness element dimension is much smaller than $2 \mathrm{ft}(0.61 \mathrm{~m})$. The $4 \mathrm{ft}(1.22 \mathrm{~m})$ thick strong floor is modeled with 5 layers of elements (including one layer for the topmost 6 inch $(0.15 \mathrm{~m})$ sacrificial layer), the $4 \mathrm{ft}(1.22 \mathrm{~m})$ thick strong wall with 4 layers, and the 16 inch $(0.41 \mathrm{~m})$

Table 1.1 Properties

\begin{tabular}{|c|c|}
\hline Concrete & Starting numbers \\
\hline Keypoints & 0 \\
\hline Lines & 0 \\
\hline Areas & 0 \\
\hline Volumes & 0 \\
\hline Elements & 0 \\
\hline Nodes & 0 \\
\hline Coordinates system & $\begin{array}{l}\text { Global: } x \text { points North, y West, } z \text { up } \\
\text { Origin: Southeast corner, bottom of base slab, } \\
\text { beneath overhang of strong floor (Fig. 2). }\end{array}$ \\
\hline Element type & $\begin{array}{l}1 \text { BEAM188 ( } 1 \text { orientation }+2 \text { end nodes) } \\
2 \text { SURF154 ( } 4 \text { nodes loading surface) } \\
3 \text { SOLID185 ( } 8 \text { nodes) }\end{array}$ \\
\hline Sections & 1 rectangular solid (loading strip) \\
\hline $\begin{array}{l}\text { Concrete } \\
\text { Materials }\end{array}$ & $\begin{array}{l}1 f_{c}^{\prime}=3500 \text { psi }(24 \mathrm{MPa}) \text { slab on grade; } \\
2 f_{c}^{\prime}=4000 \text { psi }(28 \mathrm{MPa}) \text { basement North box walls; } \\
3 f_{c}^{\prime}=4000 \text { psi }(28 \mathrm{MPa}) \text { basement walls; } \\
4 f_{c}^{\prime}=5000 \mathrm{psi}(34 \mathrm{MPa}) \text { columns; } \\
5 f_{c}^{\prime}=6000 \mathrm{psi}(41 \mathrm{MPa}) \text { strong floor; } \\
6 f_{c}^{\prime}=6000 \mathrm{psi}(41 \mathrm{MPa}) \text { strong wall. }\end{array}$ \\
\hline
\end{tabular}


thick basement walls with 2 or 3 layers each (Figs. 1.2 - 1.4). The NFRL model also includes 5400 surface elements (ANSYS 4-node SURF 154) and 500 beam elements (ANSYS BEAM 188 with two end nodes and one orientation node) for applying surface and line loads. The coordinate system and other properties are listed in Table 1.1.

Materials: Since the NFRL is designed to operate well within the elastic range, the material properties are linearly elastic. The modulus of elasticity $E$ of normal weight concrete is ( $\mathrm{ACl} 318-05 \S 8.5 .1$ ):

$$
\frac{E}{p s i}=57000 \sqrt{\frac{f_{c}^{\prime}}{p s i}} \text { or } \frac{E}{M P a}=4700 \sqrt{\frac{f_{c}^{\prime}}{M P a}}
$$

where $f_{c}^{\prime}$ is the specified compressive (cylinder crushing) strength. For the strong floor and strong wall, $f^{\prime}{ }_{c}=6000 \mathrm{psi}(41 \mathrm{MPa})$ and $E=4400 \mathrm{ksi}(30 \mathrm{GPa})$; for the basement walls, $f_{c}^{\prime}=4000 \mathrm{psi}(28 \mathrm{MPa})$ and $E=3600 \mathrm{ksi}(25 \mathrm{GPa})$.

For normal weight concrete, the average splitting tensile strength $f_{c t}$ is ( $\left.\mathrm{ACl} 318-05 \S \mathrm{R} 11.2 .1 .1\right)$ :

$$
\frac{f_{c t}}{p s i}=6.7 \sqrt{\frac{f_{c}^{\prime}}{p s i}} \quad \text { or } \quad \frac{f_{c t}}{M P a}=0.56 \sqrt{\frac{f_{c}^{\prime}}{M P a}}
$$

For the strong floor and strong wall, $f_{c t}=520 \mathrm{psi}(3.6 \mathrm{MPa})$; and for the basement walls, $f_{c t}=420 \mathrm{psi}$ $(2.9 \mathrm{MPa})$. In the design of reinforced or post-tensioned concrete structures by finite-elements, the current practice is to calculate elastic stresses with plain concrete elements, and use the results to design the passive or tensioned reinforcements section by section. Since the present FEM is not intended to be used for structural design, which was already completed before this project was initiated, the second step is omitted.

Load steps: All the load steps that were specified in the design documents of the NFRL (Capital Improvements Group, 2010) were run for verification (Table 1.2). The results confirmed the soundness of the design and the validity of the elastic assumption. Only a few localized sections at corners and stress concentrations exceeded the concrete splitting strength. Considering that the design load cases are extremely demanding and unlikely to be reached ever, and the neglect of the tensile reinforcement in the model, these potential crack locations should not cause concern. Note that load step 7, (Figs. 1.16-1.17, is not part of the design basis.

The APDL (ANSYS Programming Design Language) commands for the model and the load steps are included at the end of the chapter. 
Table 1.2 Load steps

\begin{tabular}{|c|c|}
\hline $\begin{array}{l}\text { Load } \\
\text { steps }\end{array}$ & Description \\
\hline 3 & Gravity + Prestress (G + PS) \\
\hline 4 & $\mathrm{G}+\mathrm{PS}+\mathrm{FX} 10 \mathrm{k} / \mathrm{ft}(146 \mathrm{kN} / \mathrm{m})$ top of strong wall \\
\hline 5 & $\mathrm{G}+\mathrm{PS}-\mathrm{FX} 10 \mathrm{k} / \mathrm{ft}(146 \mathrm{kN} / \mathrm{m})$ top of strong wall \\
\hline 6 & $\mathrm{G}+\mathrm{PS}-\mathrm{FZ} 1.35 \mathrm{k} / \mathrm{ft}^{2}(64.6 \mathrm{kPa})$ entire floor \\
\hline 7 & G + PS + FX 50 k/load point (222 kN/lp) facing wall - FX reaction at wall (not in design basis) \\
\hline 8 & G + PS + FX 50 k/load point (222 kN/lp) entire floor \\
\hline 9 & G + PS - FX $50 \mathrm{k} / \mathrm{load}$ point ( $222 \mathrm{kN} / \mathrm{lp})$ entire floor \\
\hline 10 & G + PS + FY $50 \mathrm{k} /$ load point (222 kN/lp) entire floor \\
\hline 11 & $\mathrm{G}+\mathrm{PS}-\mathrm{FZ} 1.80 \mathrm{k} / \mathrm{ft}^{2}(86.2 \mathrm{kPa})$ over $20^{\prime} \times 20^{\prime}(6.1 \mathrm{~m} \times 6.1 \mathrm{~m})$ at floor center \\
\hline 12 & G + PS - FZ $1.80 \mathrm{k} / \mathrm{ft}^{2}(86.2 \mathrm{kPa})$ over $20^{\prime} \times 20^{\prime}(6.1 \mathrm{~m} \times 6.1 \mathrm{~m})$ at floor Southwest corner \\
\hline 13 & $\mathrm{G}+\mathrm{PS}-\mathrm{FZ} 1.80 \mathrm{k} / \mathrm{ft}^{2}(86.2 \mathrm{kPa})$ over $20^{\prime} \times 20^{\prime}(6.1 \mathrm{~m} \times 6.1 \mathrm{~m})$ at floor Northeast corner \\
\hline 14 & G + PS + FZ $100 \mathrm{k}(445 \mathrm{kN})$ every 4' $(1.2 \mathrm{~m})$ \\
\hline 15 & G + PS - FZ $100 \mathrm{k}(445 \mathrm{kN})$ every 4' $(1.2 \mathrm{~m})$ \\
\hline 16 & G + PS \pm FZ $100 \mathrm{k}(445 \mathrm{kN})$ every 4' (1.2 m) alternate \\
\hline 17 & G + PS + FZ $100 \mathrm{k}(445 \mathrm{kN})$ every 6' $(1.8 \mathrm{~m})-\mathrm{FZ} 100 \mathrm{k}(445 \mathrm{kN})$ every 2' $(0.61 \mathrm{~m})$ centered \\
\hline 18 & $\begin{array}{l}\text { G + PS + FX } 50 \mathrm{k} / \text { load point }(222 \mathrm{kN} / \mathrm{lp}) \text { entire floor + FZ } 100 \mathrm{k}(445 \mathrm{kN}) \text { every 6' }(1.8 \mathrm{~m})-\mathrm{FZ} 100 \mathrm{k} \\
(445 \mathrm{kN}) \text { every 2' }(0.61 \mathrm{~m}) \text { centered }\end{array}$ \\
\hline 19 & G + PS + FX 50 k/load point (222 kN/lp) entire floor + FZ 100 k (445 kN) every 4'(1.2 m) \\
\hline 20 & G + PS + FX 50 k/load point (222 kN/lp) entire floor - FZ 100 k (445 kN) every 4' (1.2 m) \\
\hline 21 & G + PS + FX $50 \mathrm{k} /$ load point (222 kN/lp) entire floor \pm FZ $100 \mathrm{k}(445 \mathrm{kN})$ every 4' $(1.2 \mathrm{~m})$ alternate \\
\hline
\end{tabular}

\section{References:}

American Concrete Institute International (2005) "Building Code Requirements for Structural Concrete," ACl 318-05, Farmington Hills, MI 48333

Capital Improvements Group (2010) "Building 205 Addition and Modification 100 \% Submission Design Drawings," National Institute of Standards and Technology, Gaithersburg, MD 20899 


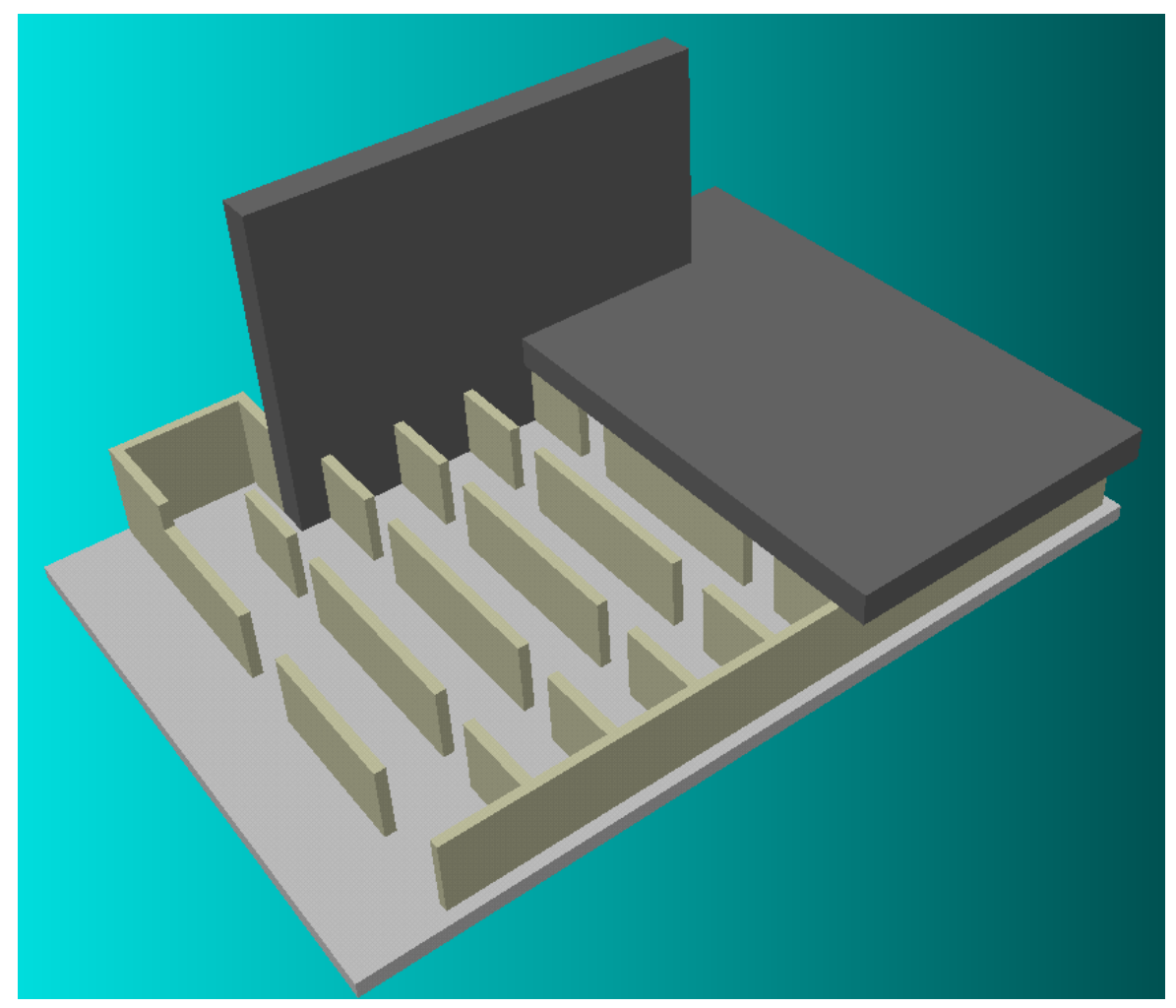

Fig. 1.1 View of NFRL with part of strong floor removed

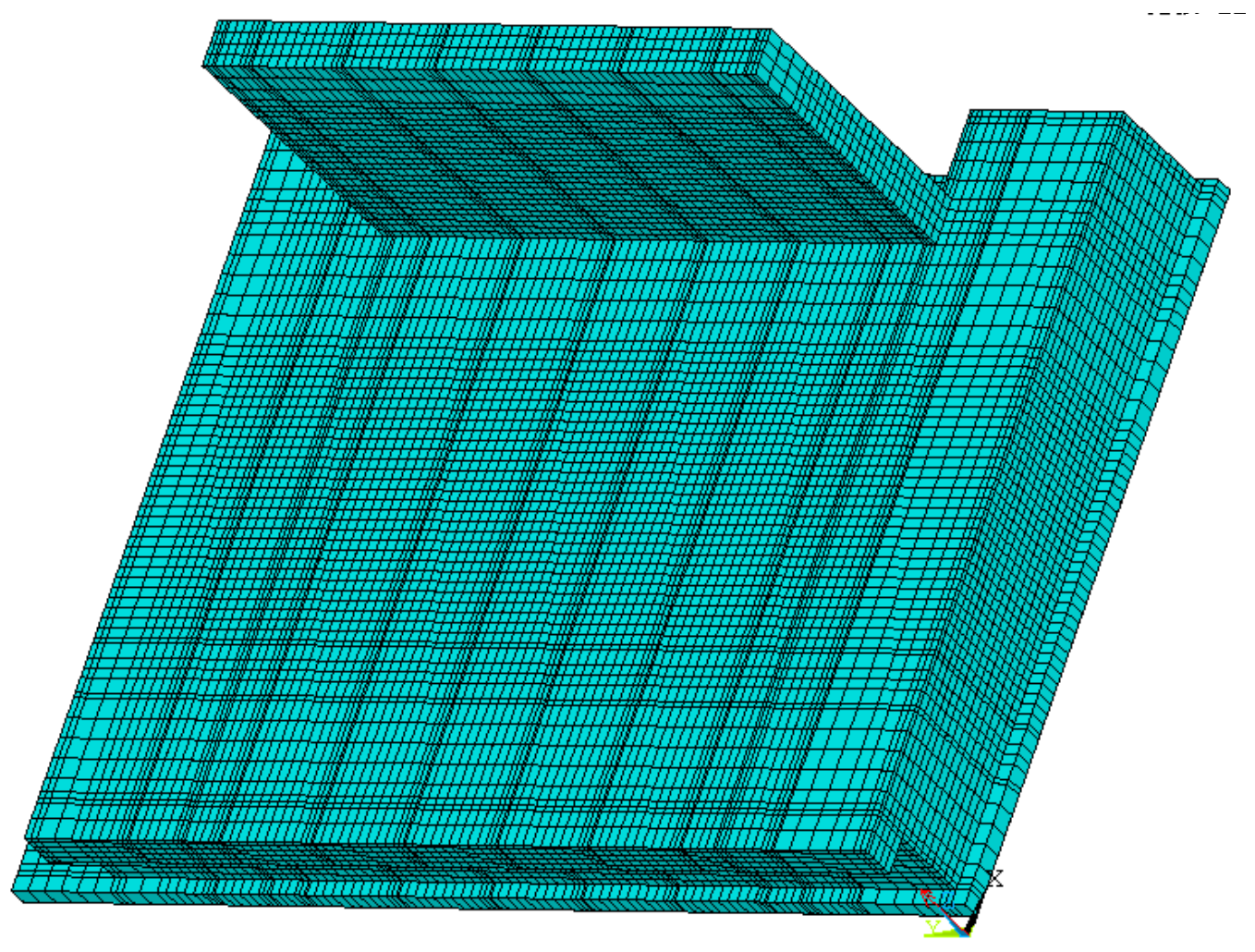

Fig. 1.2 Mesh of strong floor, strong wall and side wall 


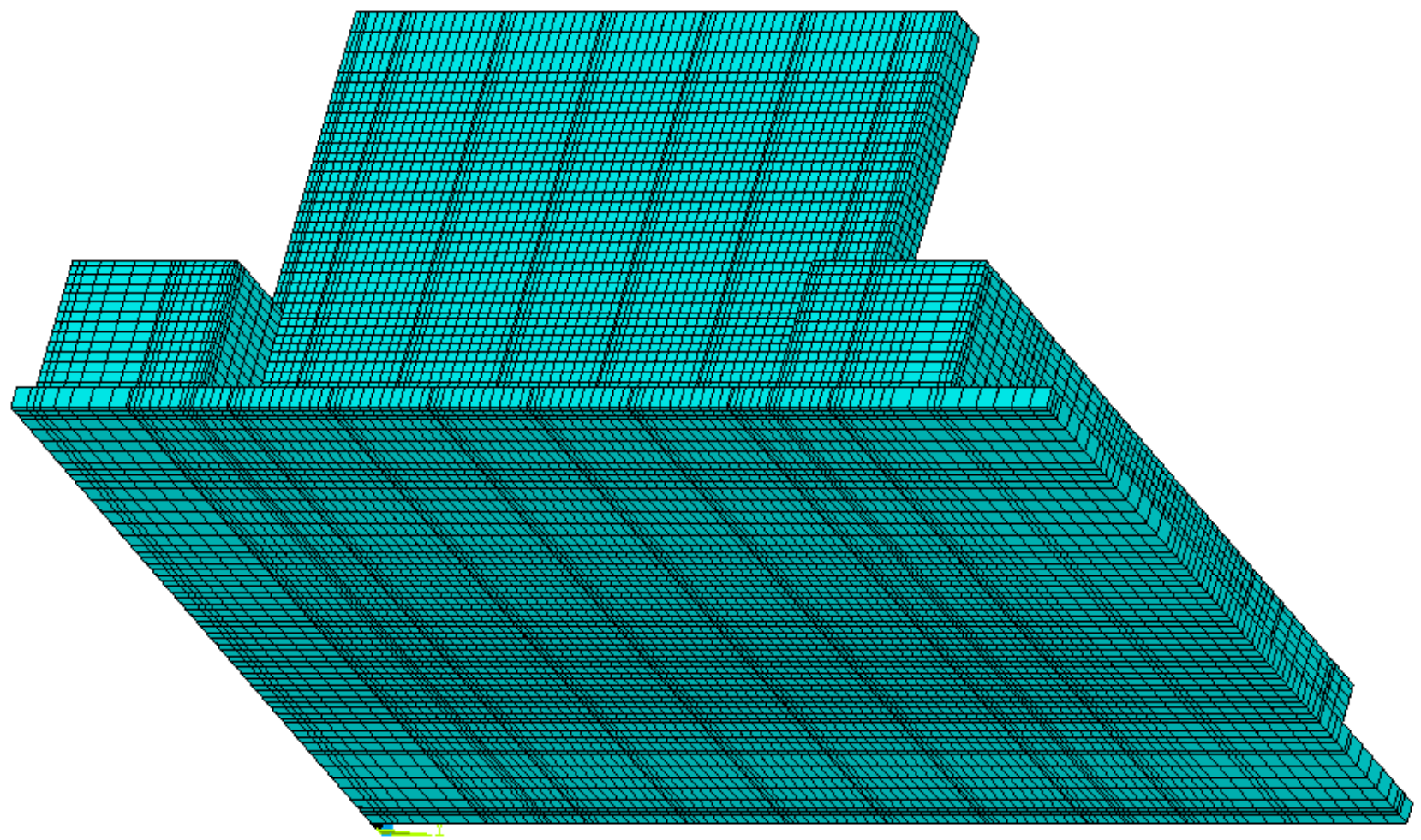

Fig. 1.3 Mesh of base slab, strong wall and back walls

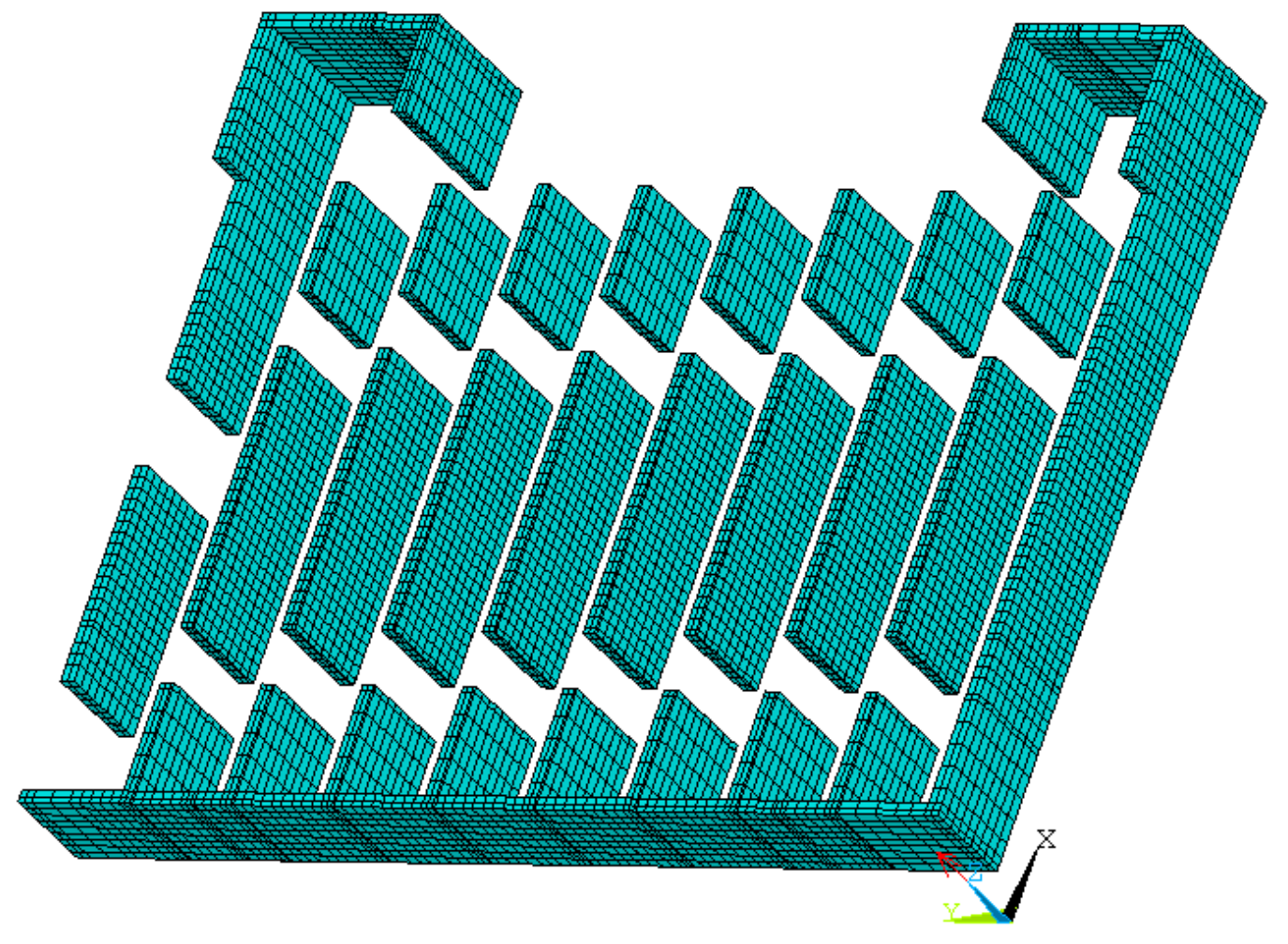

Fig. 1.4 Mesh of basement walls 


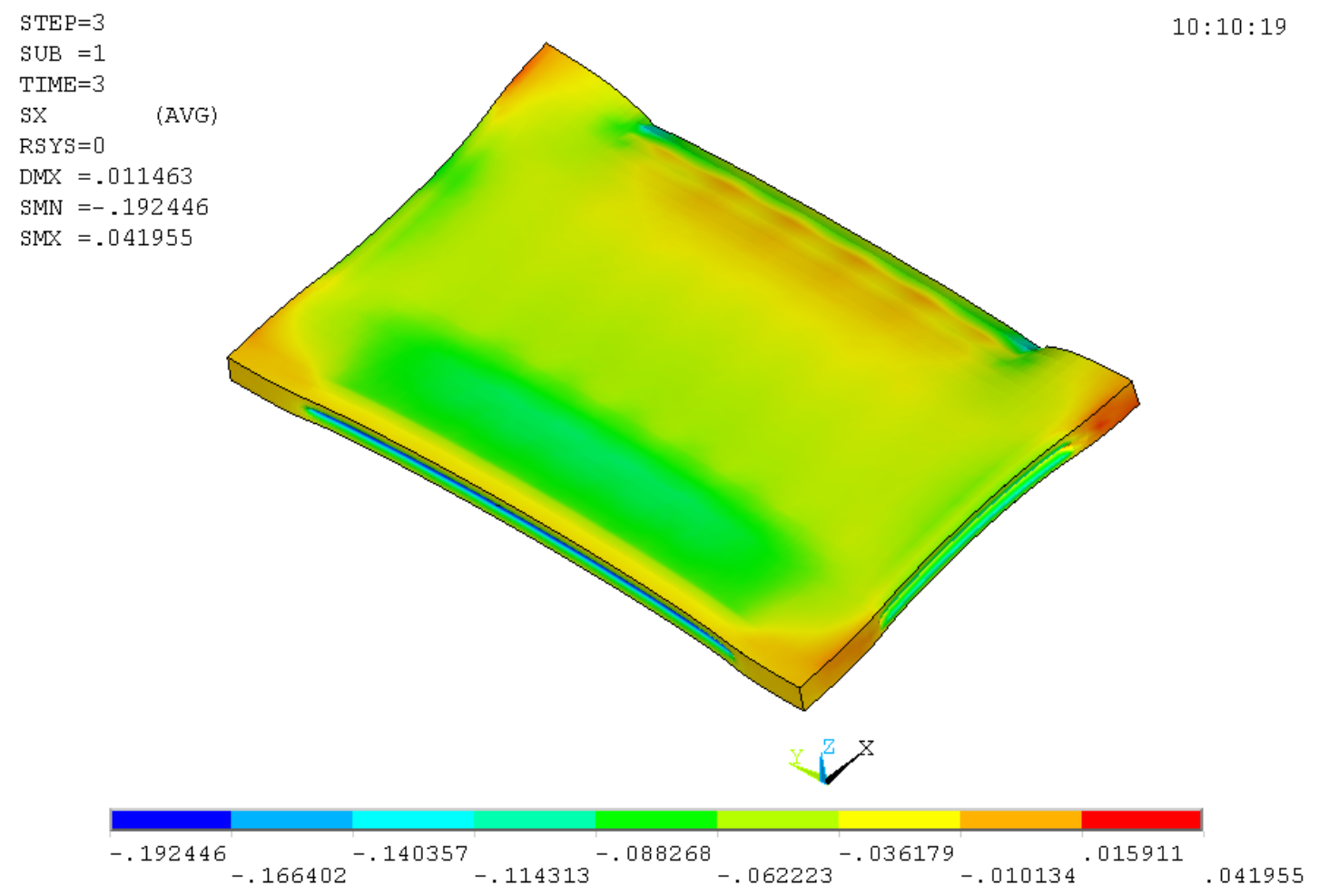

NERL STRONG FLOOR, STRONG WALL, BOX GIRDERS

Fig. 1.5 LS 3: Gravity and prestress (G+PS). $x$ - stre ss (1ksi=6.89 MPa), strong floor

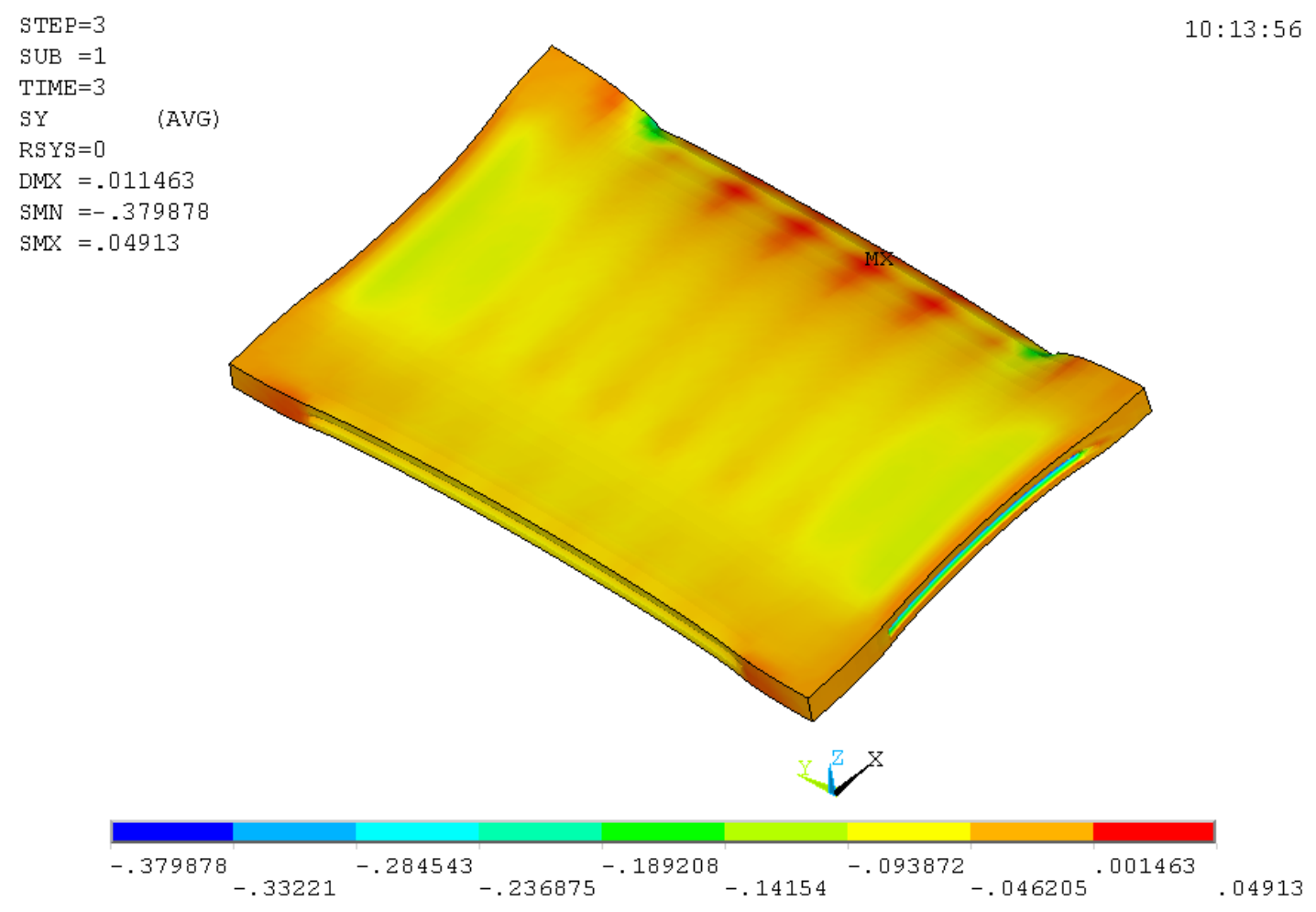

NFRL STRONG FLOOR. STRONG WALL, BOX GIRDERS

Fig. 1.6 LS 3: Gravity and prestress (G+PS). y - stress (1ksi=6.89 MPa), strong floor 


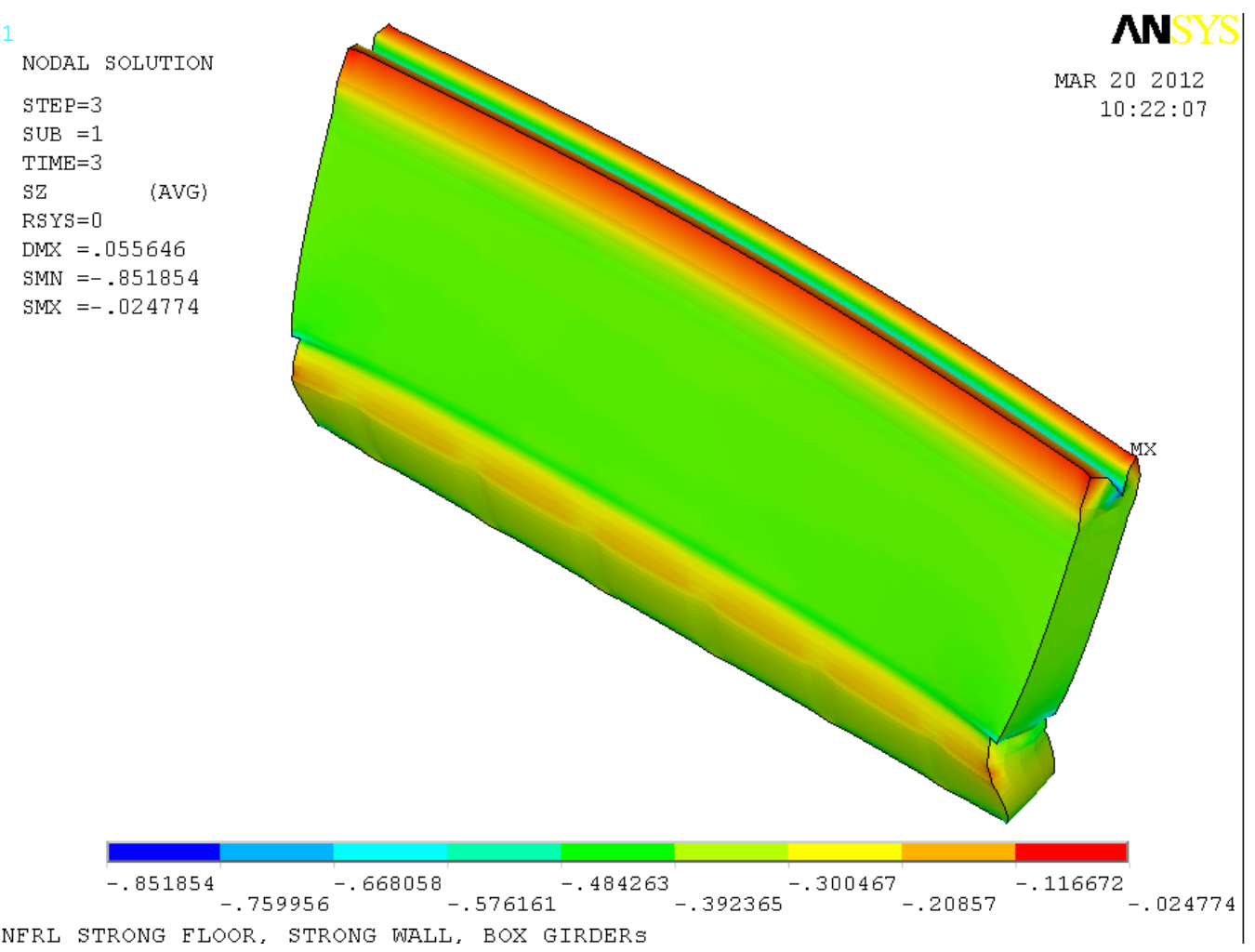

Fig. 1.7 LS 3: Gravity and prestress (G+PS). Vertical stress (1ksi=6.89 MPa), strong wall 


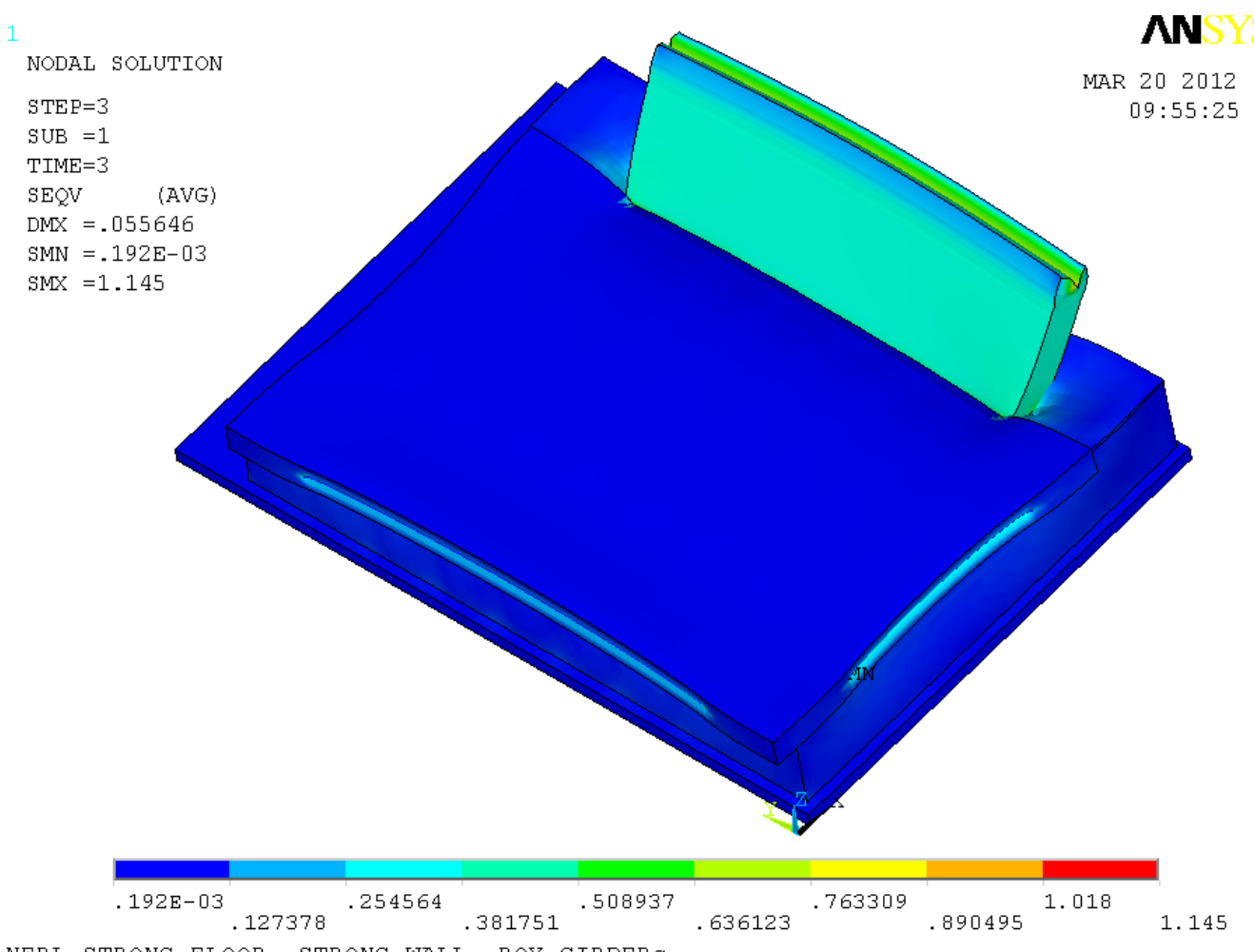

NERL STRONG FLOOR, STRONG WALL, BOX GIRDERS

Fig. 1.8 LS 3: Gravity and prestress (G+PS). Von Mises stress (1ksi=6.89 MPa), strong wall, floor

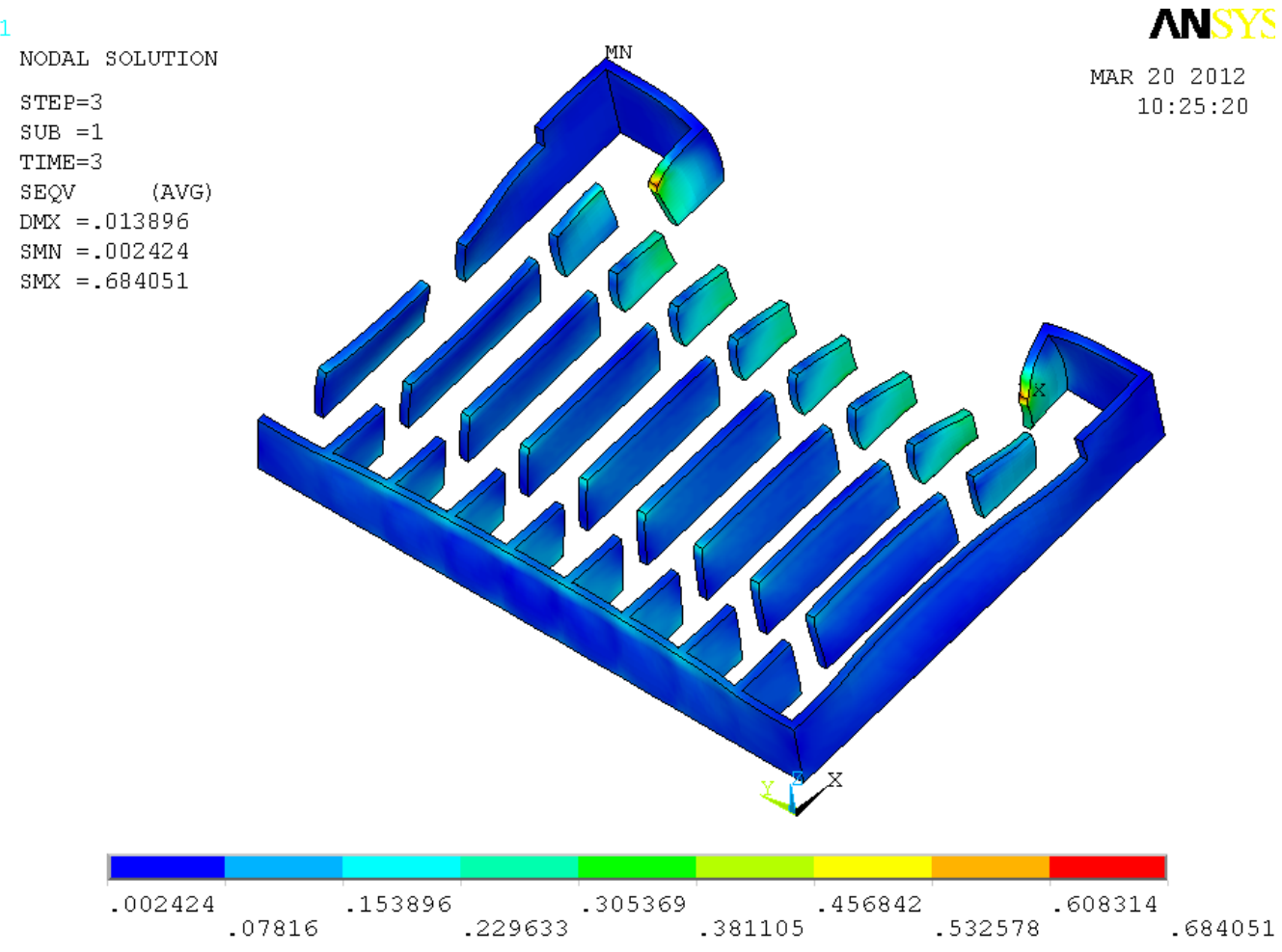

NERL STRONG FLOOR, STRONG WALL, BOX GIRDERS

Fig. 1.9 LS 3: Gravity and prestress (G+PS). Von Mises stress (1ksi=6.89 MPa), basement 
NODAL SOLUTION

$\mathrm{STEP}=4$

SUB $=1$

TIME $=4$

Sz

RSYS $=0$

$\mathrm{DMX}=.337257$

SMN $=-1.612$

$\mathrm{SMX}=.234327$

\section{NN}

MAR 202012

$10: 31: 05$

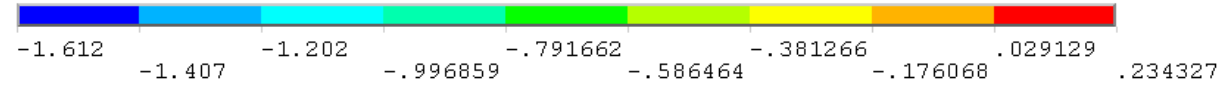

NFRL STRONG FLOOR, STRONG WALL, BOX GIRDERS

Fig. 1.10 LS 4: G+PS+FX $10 \mathrm{kip} / \mathrm{ft}(146 \mathrm{kN} / \mathrm{m})$ wall top. Vertical stress (1ksi=6.89 MPa), strong wall, floor

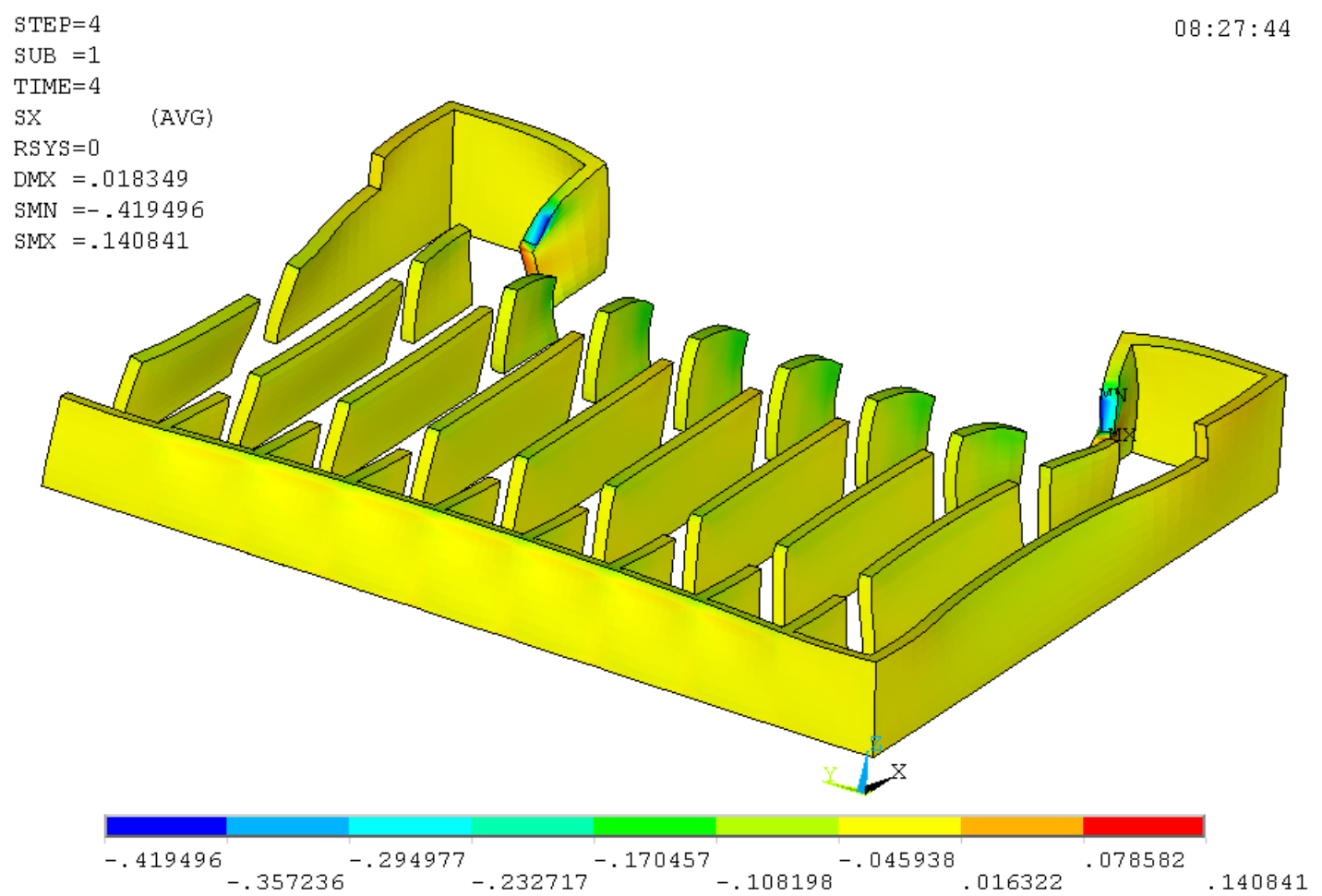

NFRL STRONG FLOOR, STRONG WALL, BOX GIRDERS

Fig. 1.11 LS 4: G+PS+FX $10 \mathrm{kip} / \mathrm{ft}(146 \mathrm{kN} / \mathrm{m})$ top of wall. $x$ stress (1ksi=6.89 MPa), basement walls 


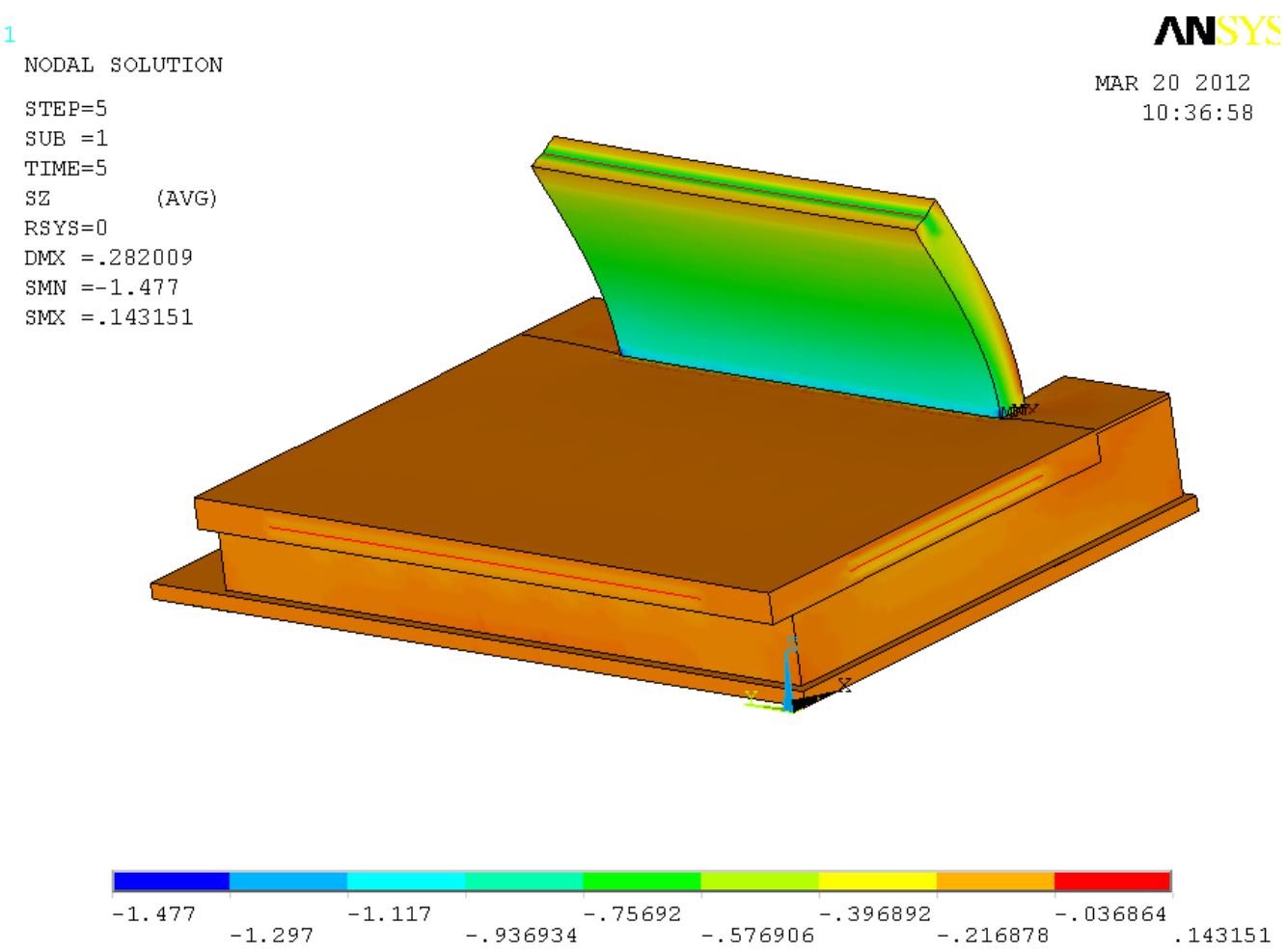

NFRL STRONG FLOOR, STRONG WALL, BOX GIRDERS

Fig. 1.12 LS 5: G+PS-FX $10 \mathrm{kip} / \mathrm{ft}(146 \mathrm{kN} / \mathrm{m})$ wall top. Vertical stress (1ksi=6.89 MPa), strong wall, floor

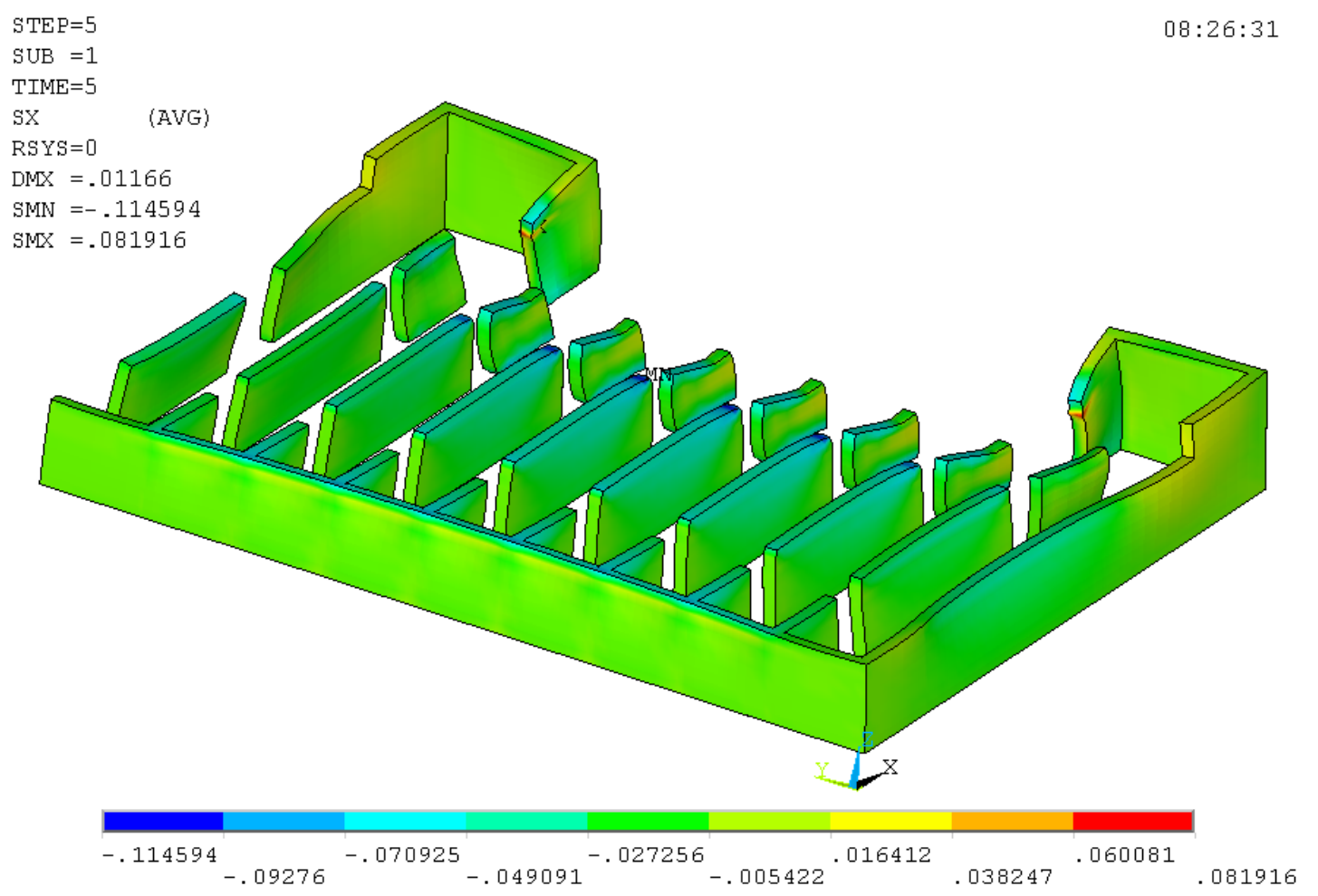

NERL STRONG FLOOR, STRONG WALL, BOX GIRDERS

Fig. 1.13 LS 5: G+PS-FX $10 \mathrm{kip} / \mathrm{ft}(146 \mathrm{kN} / \mathrm{m})$ top of wall. $x$ stress (1ksi=6.89 MPa), basement walls 
TIME $=6$

$\mathrm{SEQV} \quad$ (AVG

$\mathrm{DMX}=.053183$

SMN $=.181 \mathrm{E}-03$

$\operatorname{SMX}=1.104$
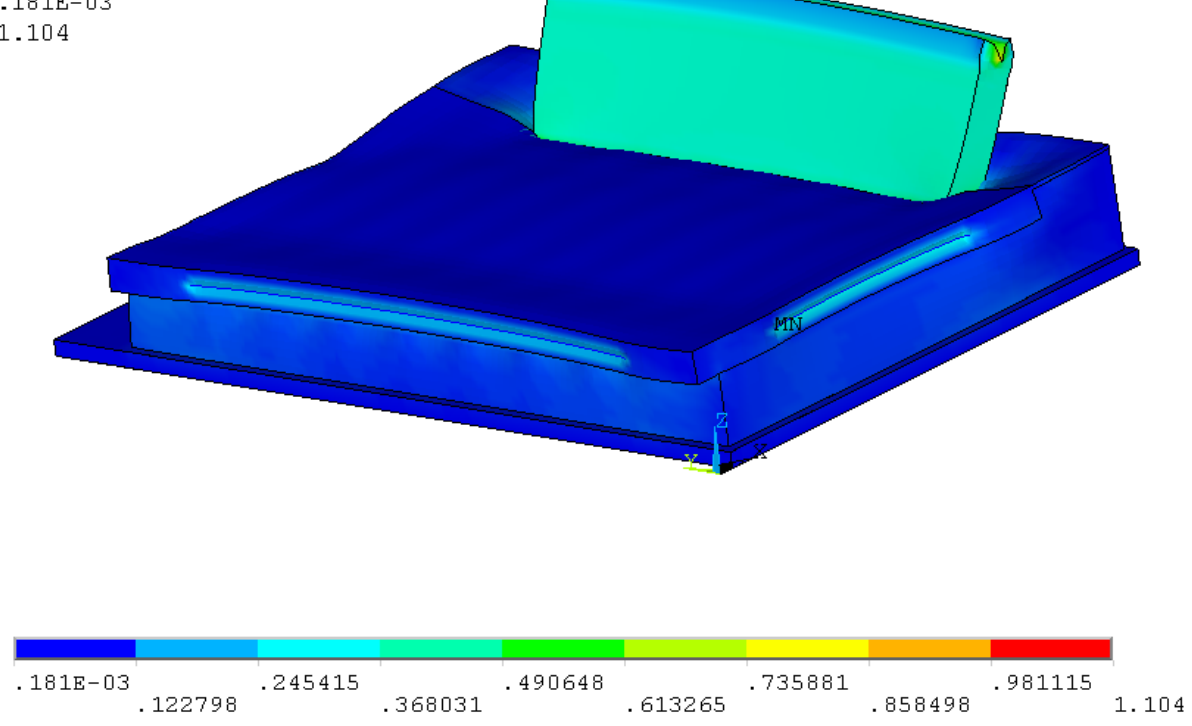

NFRL STRONG FLOOR, STRONG WALL, BOX GIRDERS

Fig.1.14 LS 6: G+PS-FZ 1.35 k/f² (64.6 kPa) on entire floor. Von Mises stress (1ksi=6.89 MPa), wall, floor

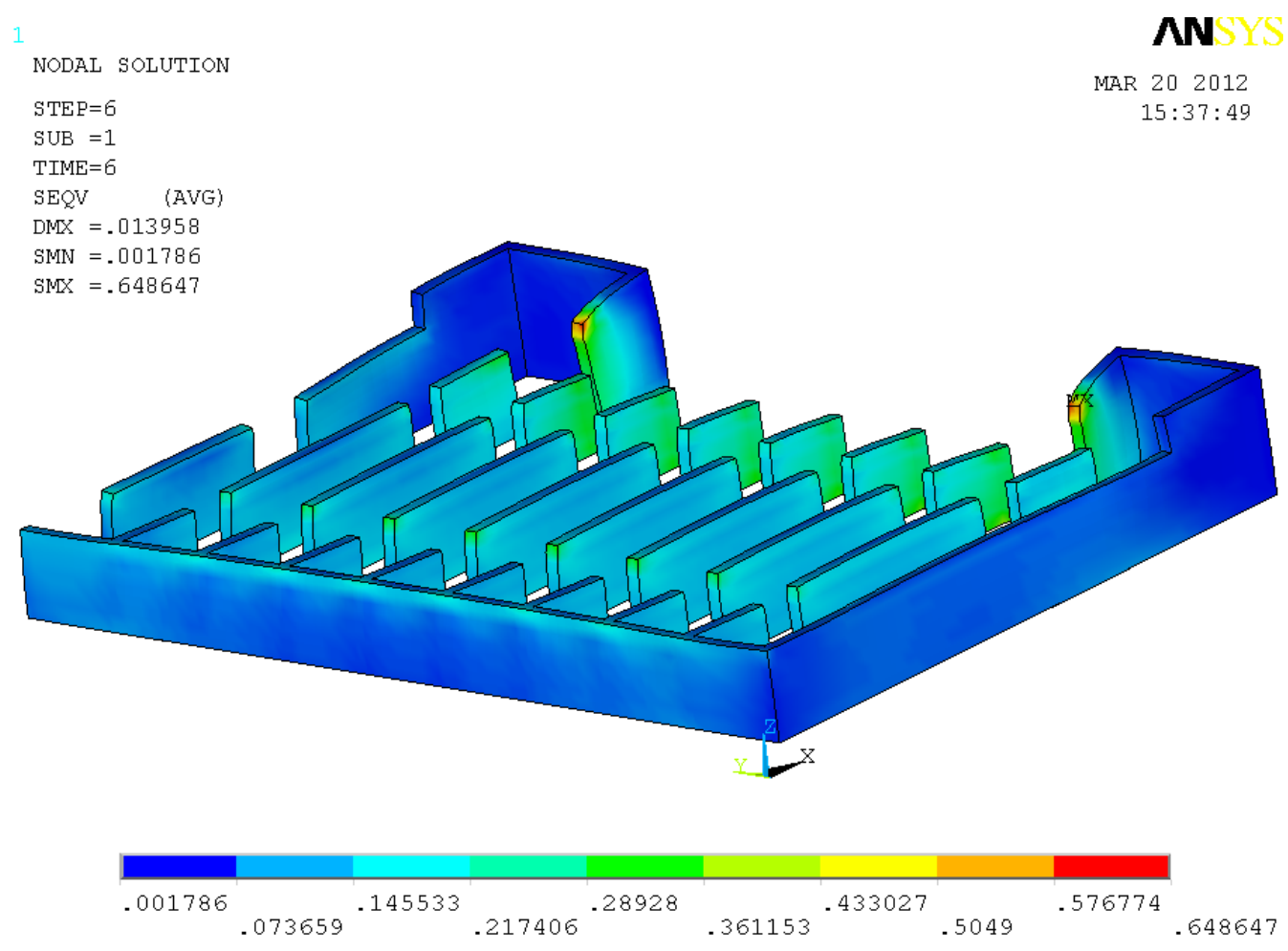

NERL STRONG FLOOR, STRONG WALL, BOX GIRDERS

Fig. 1.15 LS 6: G+PS-FZ $1.35 \mathrm{k} / \mathrm{f}^{2}(64.6 \mathrm{kPa})$ on entire floor. Von Mises stress (1ksi=6.89 MPa), basement 
RSYS $=0$

$\mathrm{DMX}=.36067$

$\mathrm{SMN}=-4.272$

SMX $=4.553$

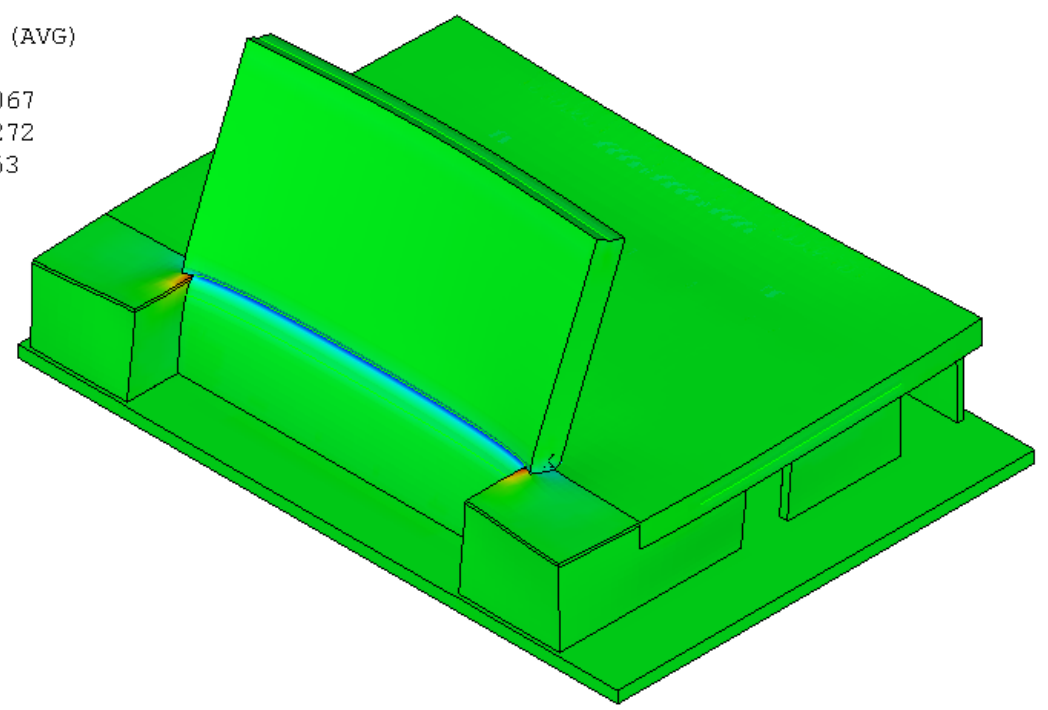

$08: 02: 05$

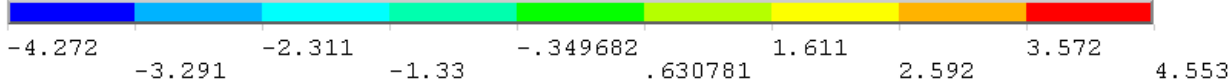

NERL STRONG FLOOR, STRONG WALL, BOX GIRDERS

Fig. 1.16 LS 7: G+PS+FX 50 k/floor load pt ( $222 \mathrm{kN} / \mathrm{lp}$ ) facing wall+ wall reaction. Deformations and horizontal $x$ stress $(1 \mathrm{ksi}=6.89 \mathrm{MPa})$, strong wall and floor

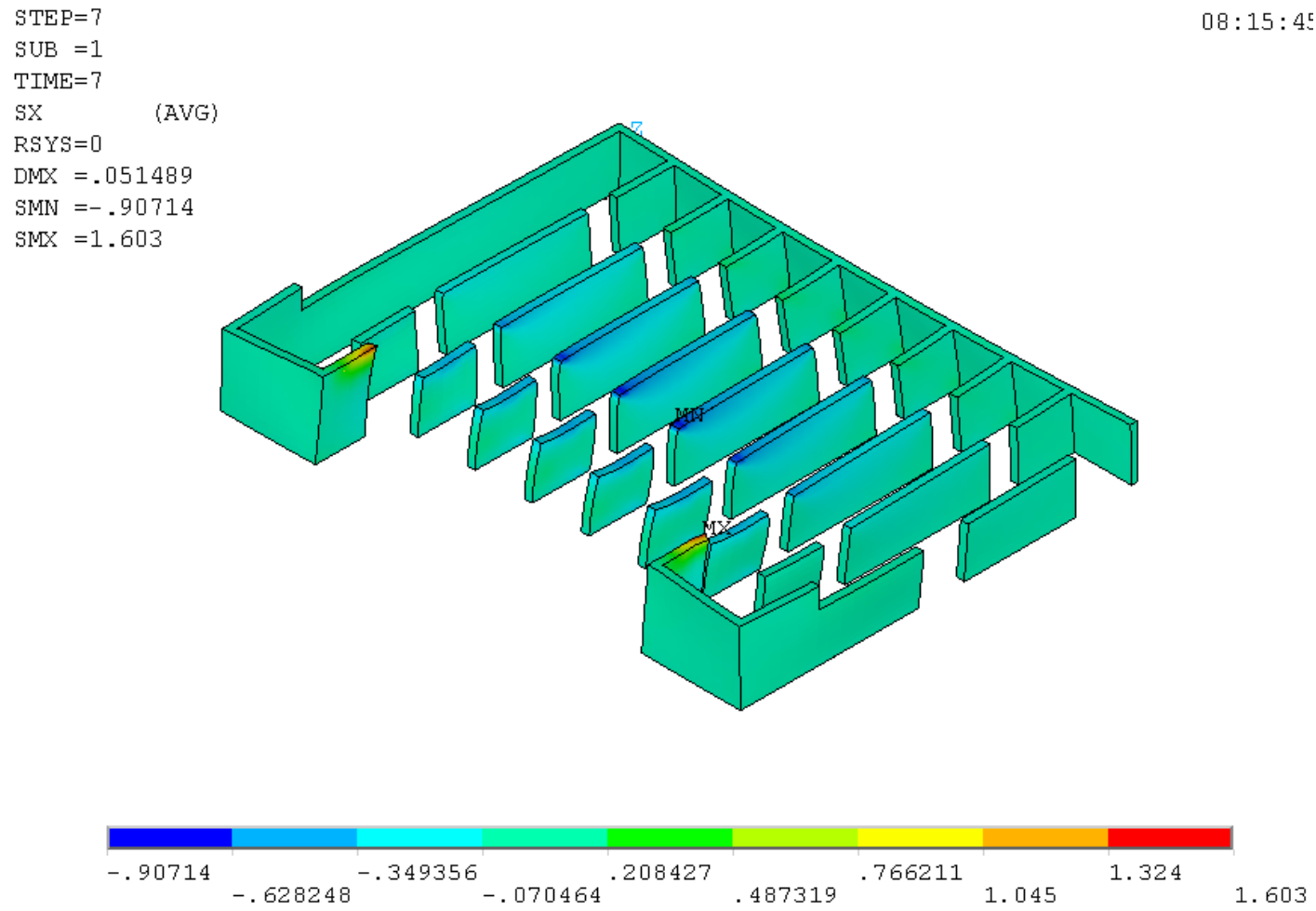

NERL STRONG FLOOR. STRONG WALL. BOX GIRDERS

Fig. 1.17 LS 7: G+PS+FX 50 k/floor load pt ( $222 \mathrm{kN} / \mathrm{lp}$ ) facing wall+ wall reaction. Deformations and $x$ stress ( $1 \mathrm{ksi}=6.89 \mathrm{MPa})$, basement walls 


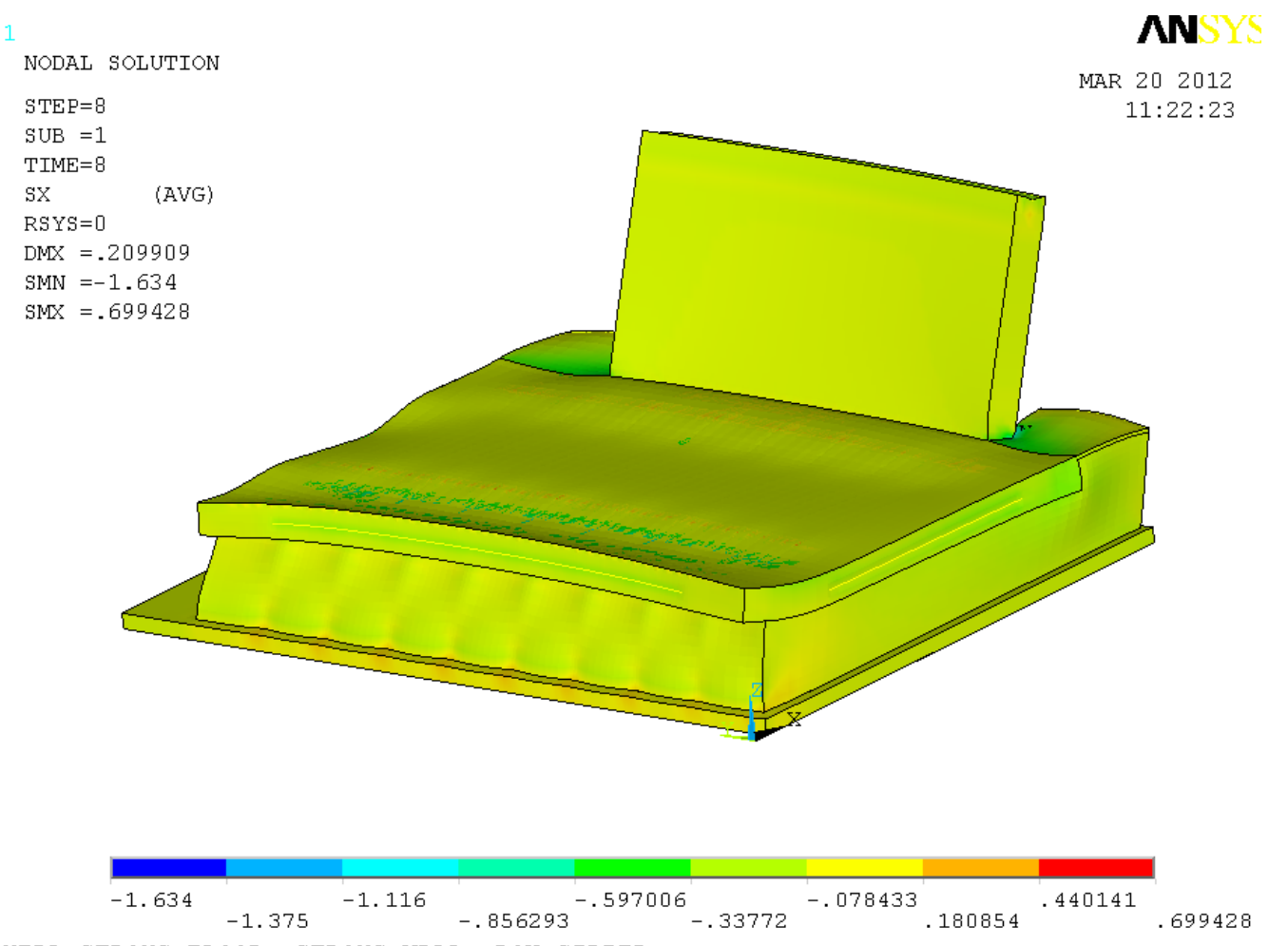

NERL STRONG FLOOR, STRONG WALL, BOX GIRDERS

Fig. 1.18 LS 8: G+PS+FX $50 \mathrm{k} / \mathrm{ld}$ pt (222 kN/lp) entire floor. Horizontal x stress (1ksi=6.89 MPa), wall, floor

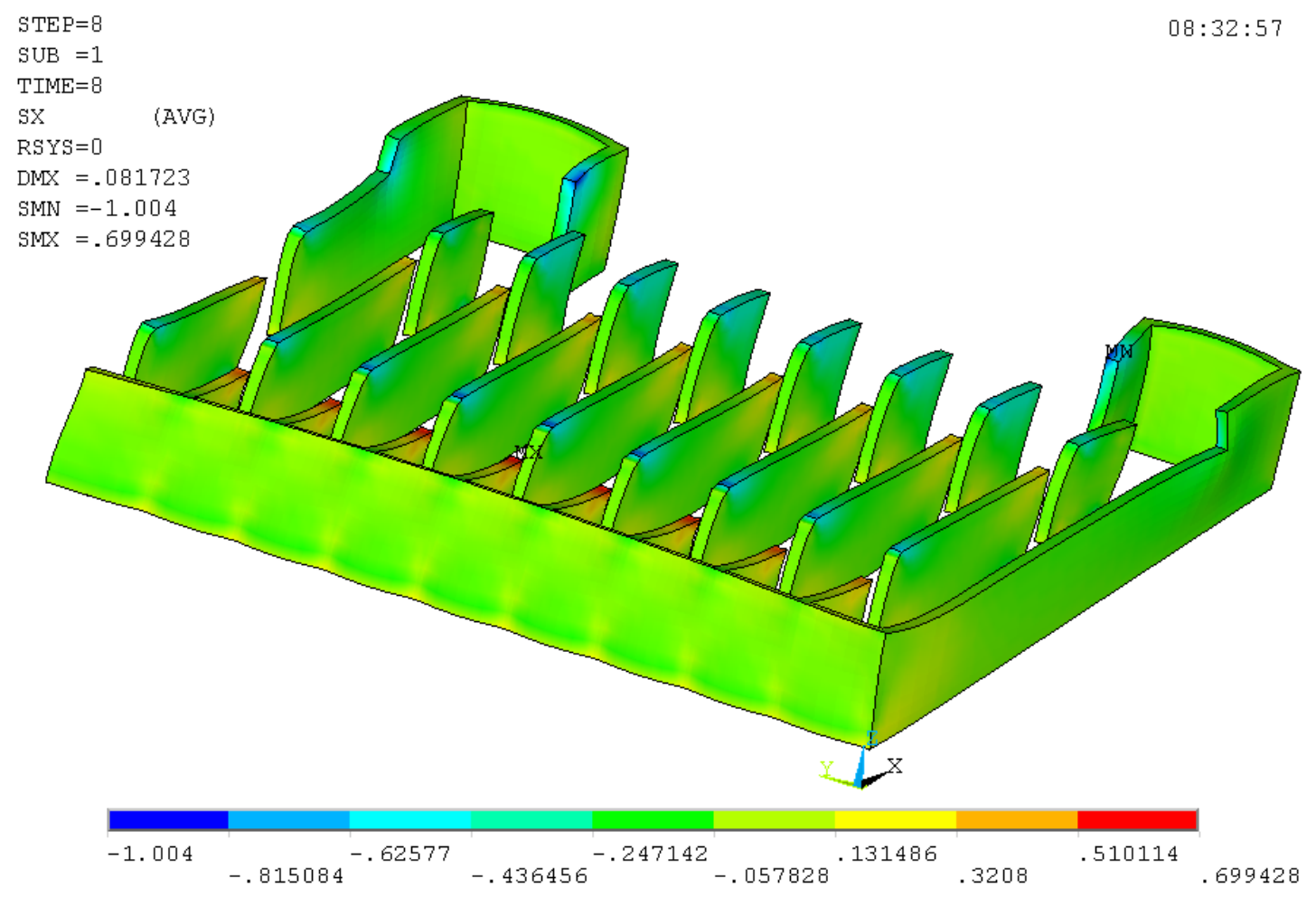

NFRL STRONG FLOOR, STRONG WALL, BOX GIRDERS

Fig. 1.19 LS 8: G+PS+FX $50 \mathrm{k} / \mathrm{ld}$ pt (222 kN/lp) entire floor. $x$ stress (1ksi=6.89 MPa), basement 


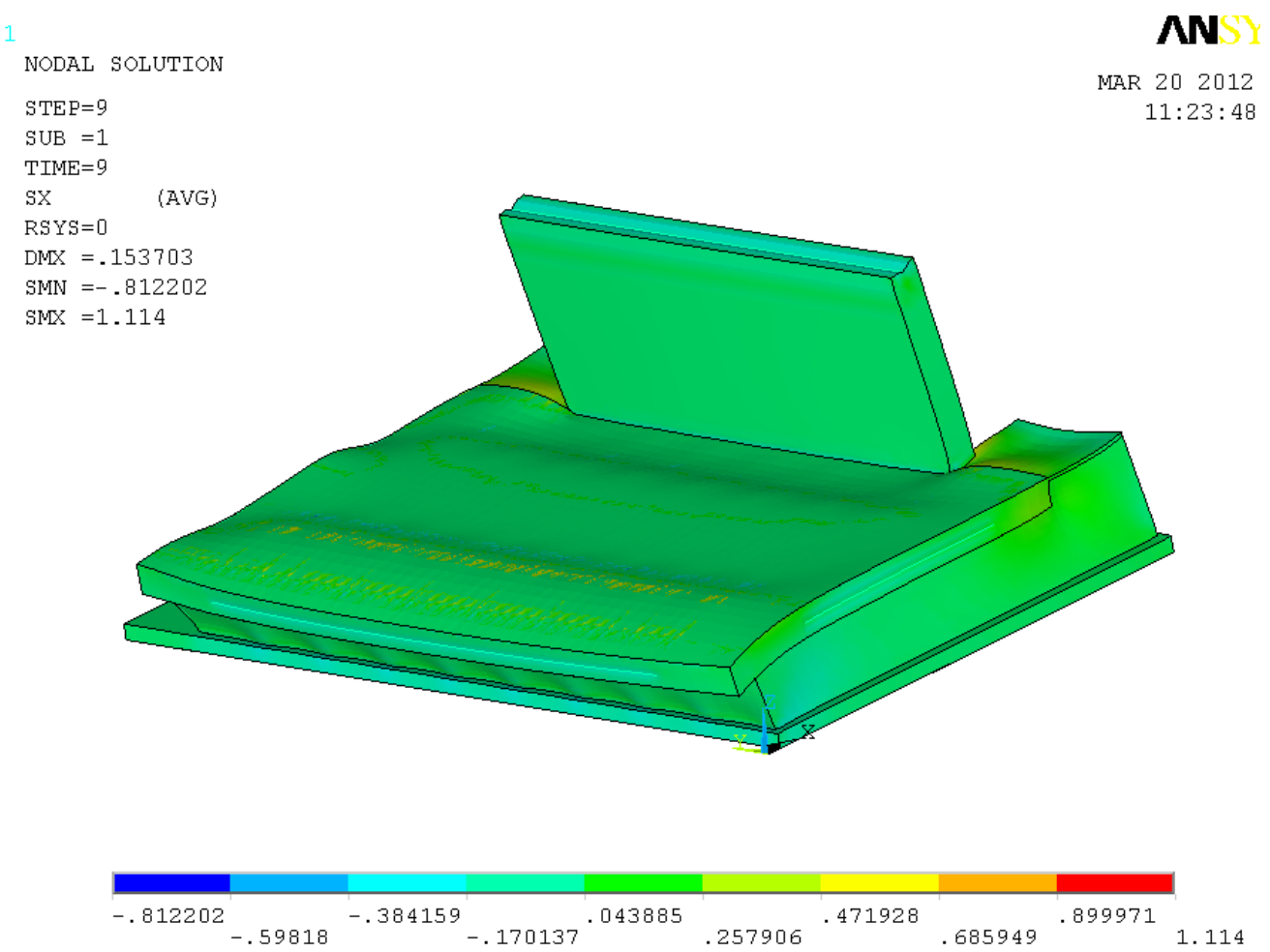

NFRL STRONG FLOOR, STRONG WALL, BOX GIRDERS

Fig. 1.20 LS 9: G+PS-FX 50 k/ld pt (222 kN/lp) entire floor. Horizontal x stress (1ksi=6.89 MPa), wall, floor

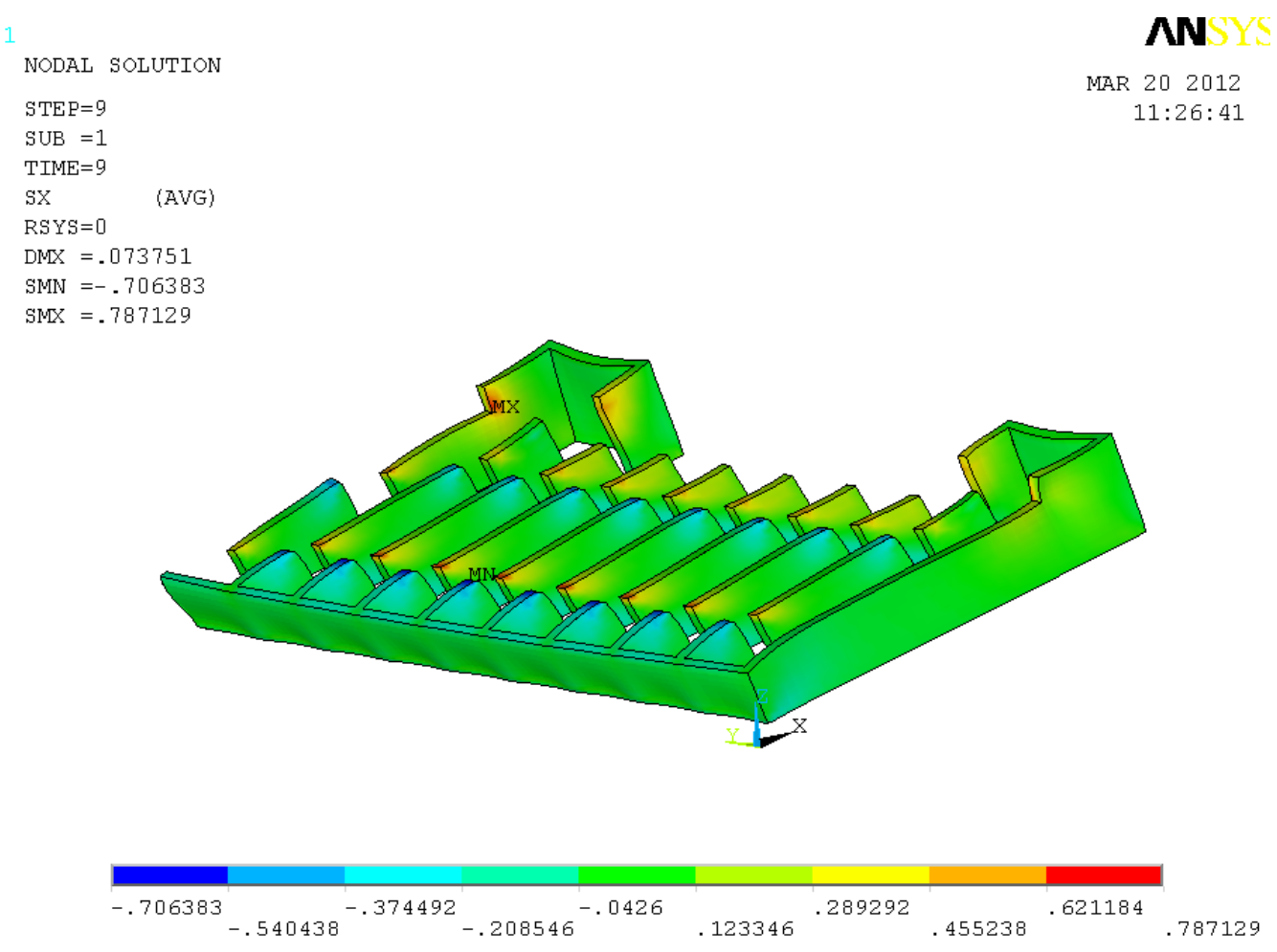

NFRL STRONG FLOOR, STRONG WALL, BOX GIRDERS

Fig. 1.21 LS 9: G+PS-FX 50 k/ld pt (222 kN/lp) entire floor. Horizontal x stress (1ksi=6.89 MPa), basement 


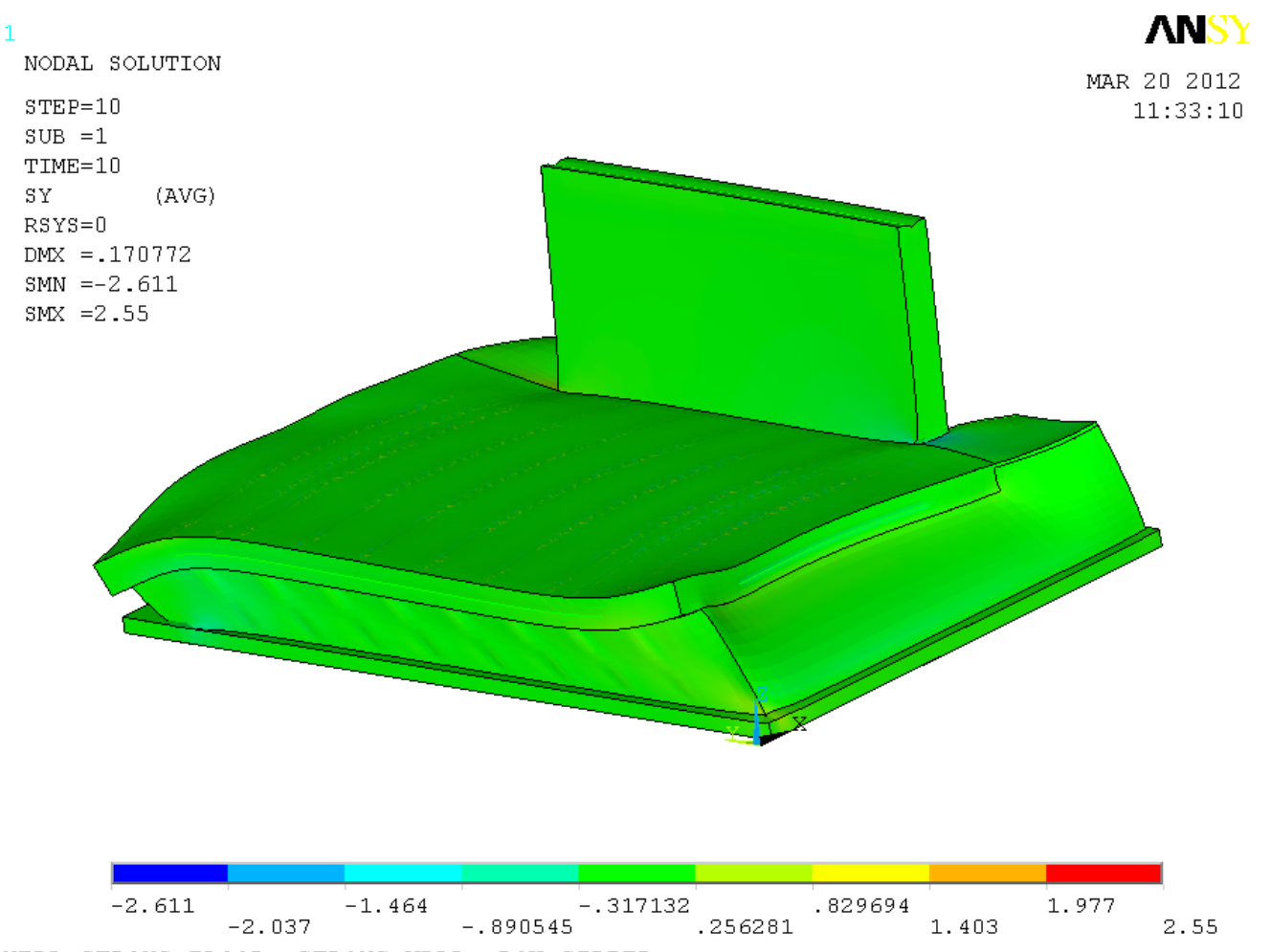

NFRL STRONG FLOOR, STRONG WALL, BOX GIRDERS

Fig.1.22 LS 10: G+PS+FY 50 k/Ip (222 kN/Ip) entire floor. Horizontal y stress (1ksi=6.89 MPa), wall, floor

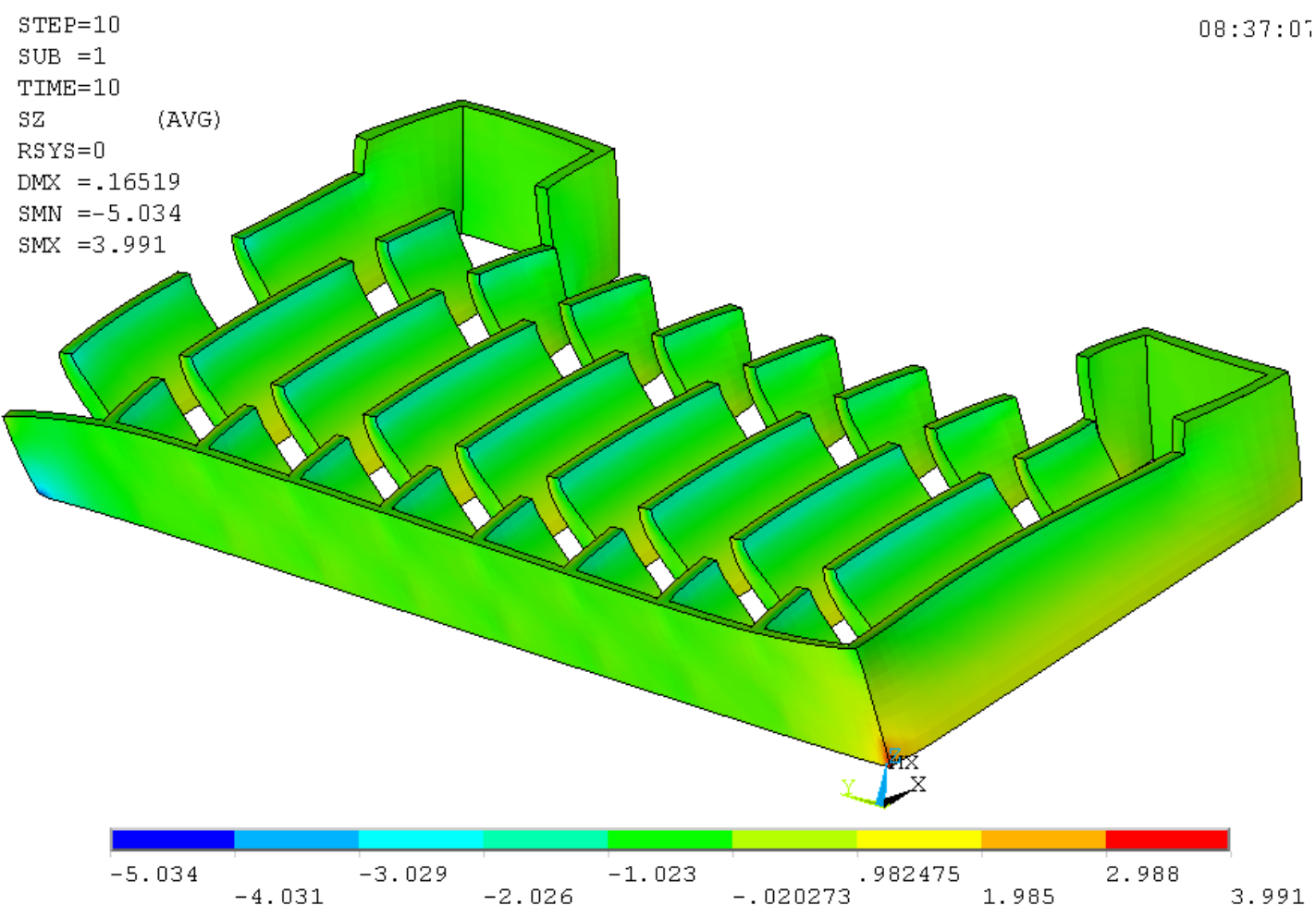

NERL STRONG FLOOR. STRONG WALL, BOX GIRDERS

Fig. 1.23 LS 10: G+PS+FY $50 \mathrm{k} / \mathrm{lp}(222 \mathrm{kN} / \mathrm{lp}$ ) entire floor. Vertical z stress (1ksi=6.89 MPa), basement 


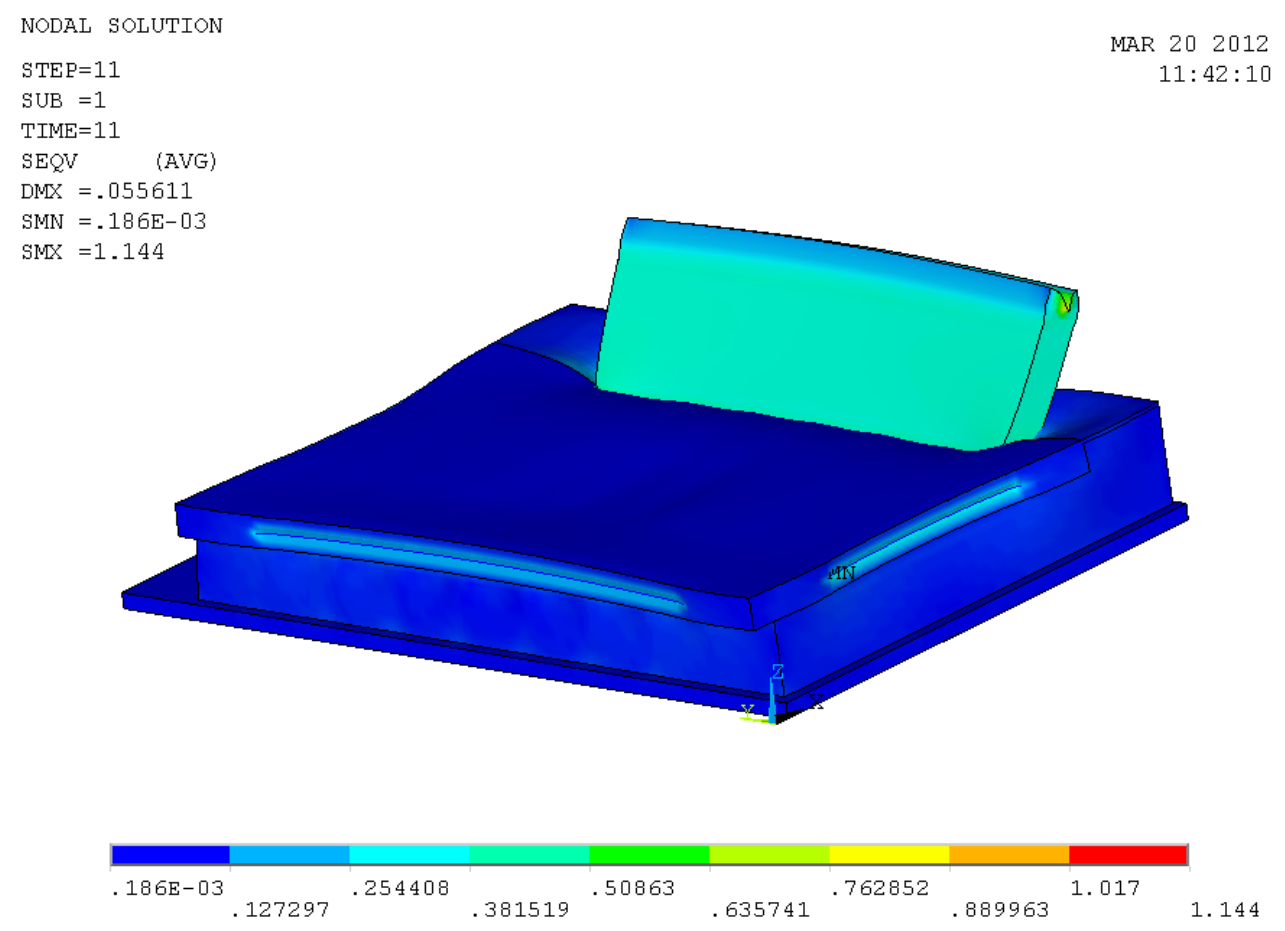

NFRL STRONG FLOOR, STRONG WALL, BOX GIRDERS

Fig. 1.24 LS 11: G+PS-FZ $1.8 \mathrm{k} / \mathrm{ft}^{2}(86.2 \mathrm{kPa})$ over $20^{\prime}$ by $20^{\prime}(6.1 \mathrm{~m} \times 6.1 \mathrm{~m})$ floor center. Deformations, Von Mises stress $(1 \mathrm{ksi}=6.89 \mathrm{MPa})$

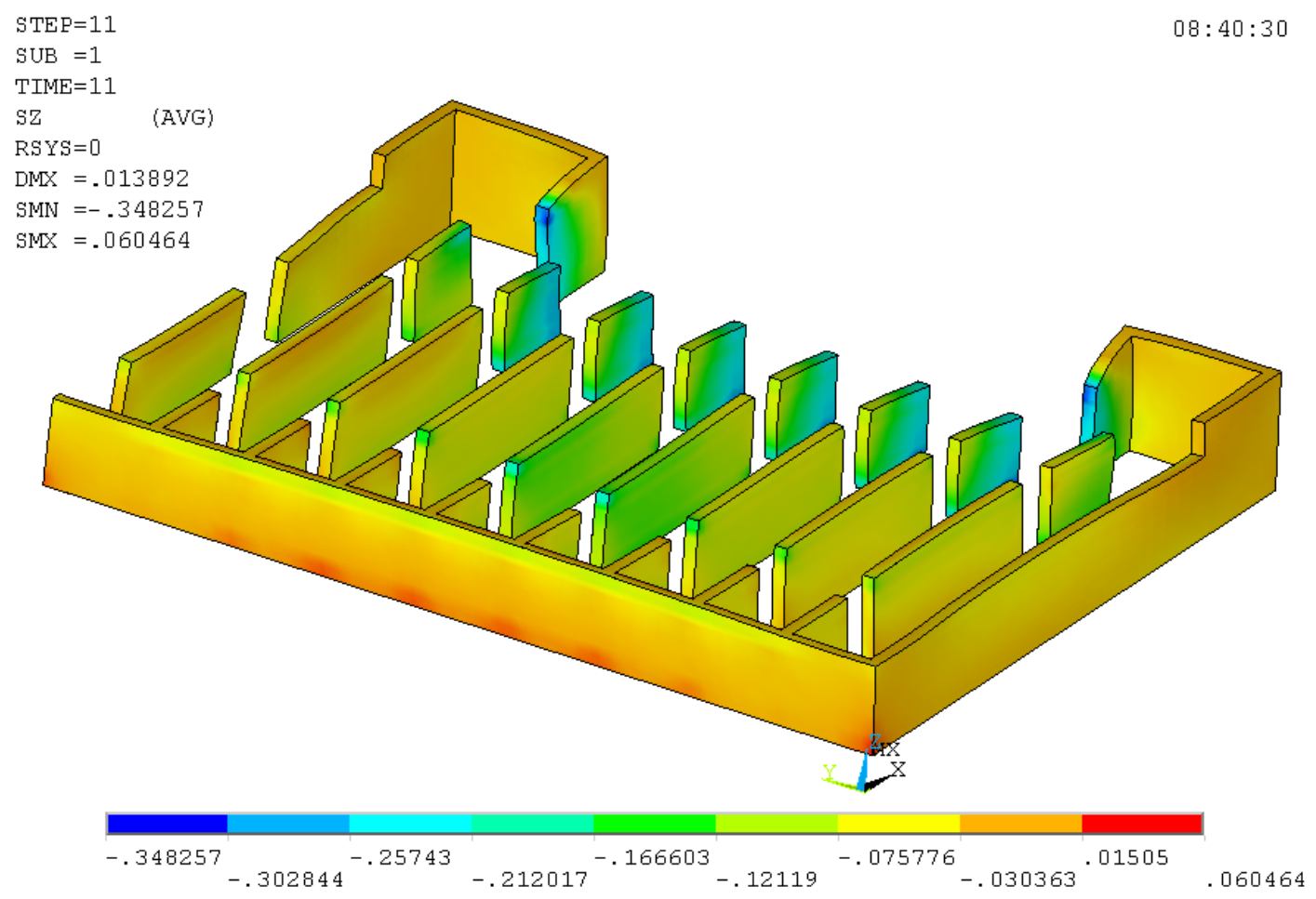

NERL STRONG FLOOR. STRONG WALL, BOX GIRDERS

Fig. 1.25 LS 11: G+PS-FZ 1.8 k/ft ${ }^{2}(86.2 \mathrm{kPa})$ over $20^{\prime}$ by $20^{\prime}(6.1 \mathrm{~m} \times 6.1 \mathrm{~m})$ floor center. Deformations, vertical z stress $(1 \mathrm{ksi}=6.89 \mathrm{MPa})$, basement 


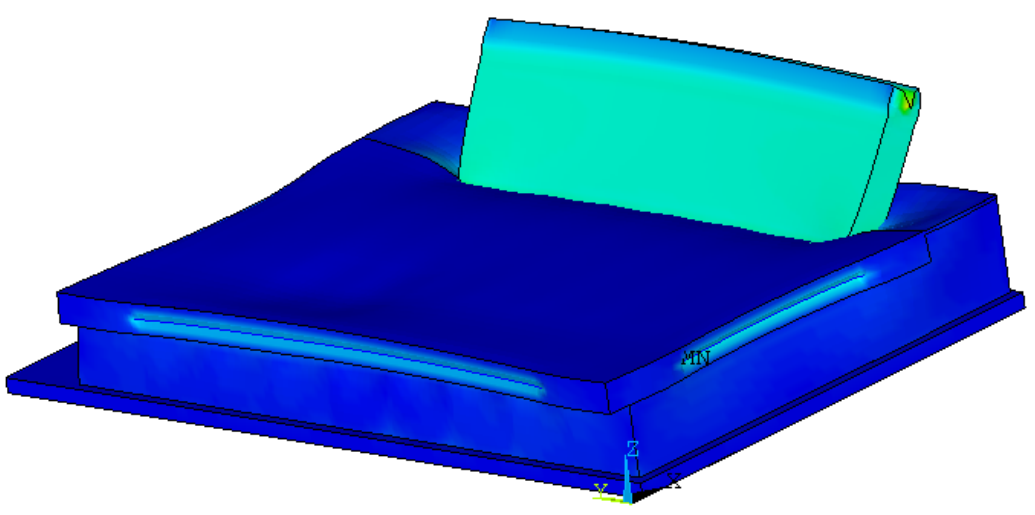

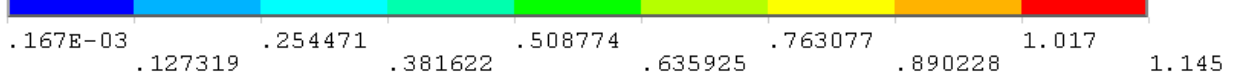

NERL STRONG FLOOR, STRONG WALL, BOX GIRDERS

Fig. 1.26 LS 12: G+PS-FZ 1.8 k/ft ${ }^{2}(86.2 \mathrm{kPa})$ over 20' by $20^{\prime}(6.1 \mathrm{~m} \times 6.1 \mathrm{~m})$ floor SW corner. Deformations, Von Mises stress ( $1 \mathrm{ksi}=6.89 \mathrm{MPa})$, strong floor and wall

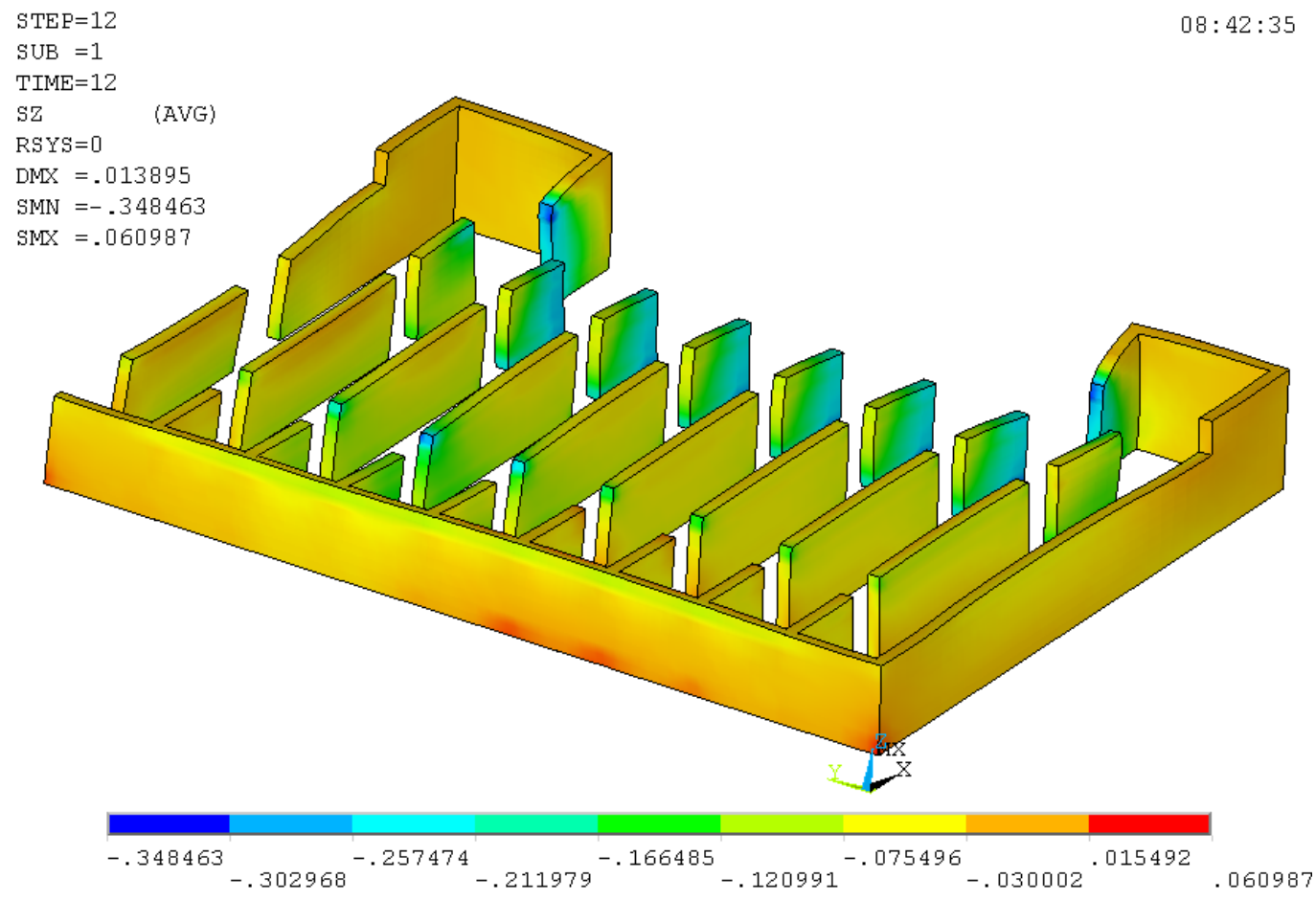

NERL STRONG FLOOR. STRONG WALL, BOX GIRDERS

Fig. 1.27 LS 12: G+PS-FZ $1.8 \mathrm{k} / \mathrm{ft}^{2}$ (86.2 kPa) over 20' by $20^{\prime}(6.1 \mathrm{~m} \times 6.1 \mathrm{~m}$ ) floor SW corner (x points North). Deformations, vertical z stress ( $1 \mathrm{ksi}=6.89 \mathrm{MPa})$ 


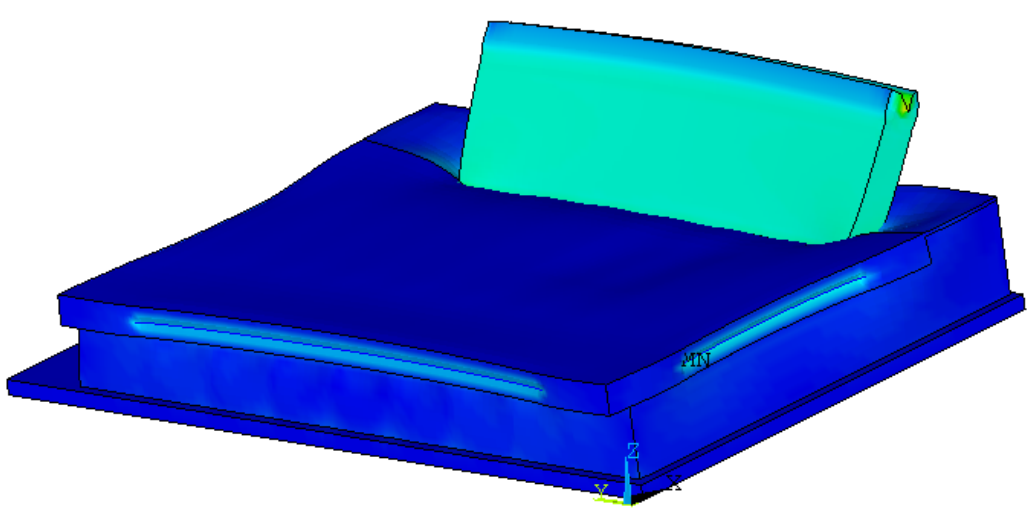

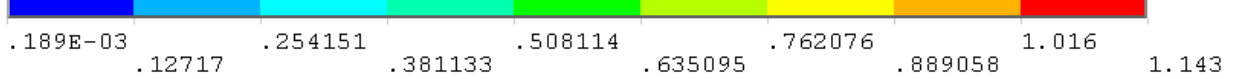

NFRL STRONG FLOOR, STRONG WALL, BOX GIRDERS

Fig. 1.28 LS 13: G+PS-FZ $1.8 \mathrm{k} / \mathrm{ft}^{2}(86.2 \mathrm{kPa})$ over 20' by 20' $(6.1 \mathrm{~m} \times 6.1 \mathrm{~m})$ floor NE corner. Deformations, Von Mises stress ( $1 \mathrm{ksi}=6.89 \mathrm{MPa})$, strong floor and wall

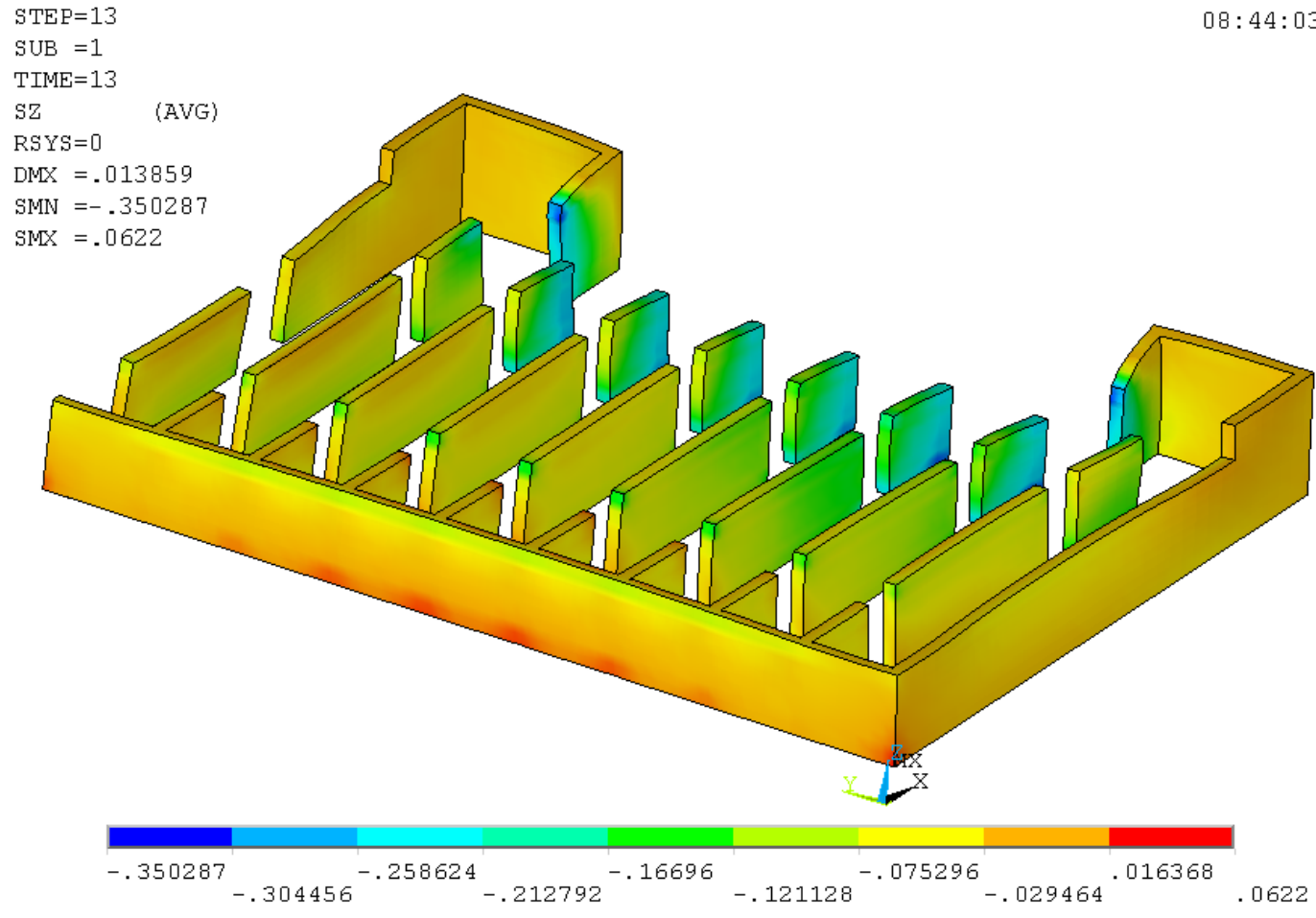

NERL STRONG FLOOR. STRONG WALL, BOX GIRDERS

Fig. 1.29 LS 13: G+PS-FZ $1.8 \mathrm{k} / \mathrm{ft}^{2}(86.2 \mathrm{kPa})$ over 20' by 20' $(6.1 \mathrm{~m} \times 6.1 \mathrm{~m})$ floor NE corner (x points North). Deformations, vertical z stress ( $1 \mathrm{ksi}=6.89 \mathrm{MPa})$, basement 


\section{NODAL SOLUTION}

$\mathrm{STEP}=14$

$\mathrm{SUB}=1$

$14: 11: 10$

TIME $=14$

SEQV (AVG)

$\mathrm{DMX}=.07513$

SMN $=.764 \mathrm{E}-04$

$\mathrm{SMX}=2.227$
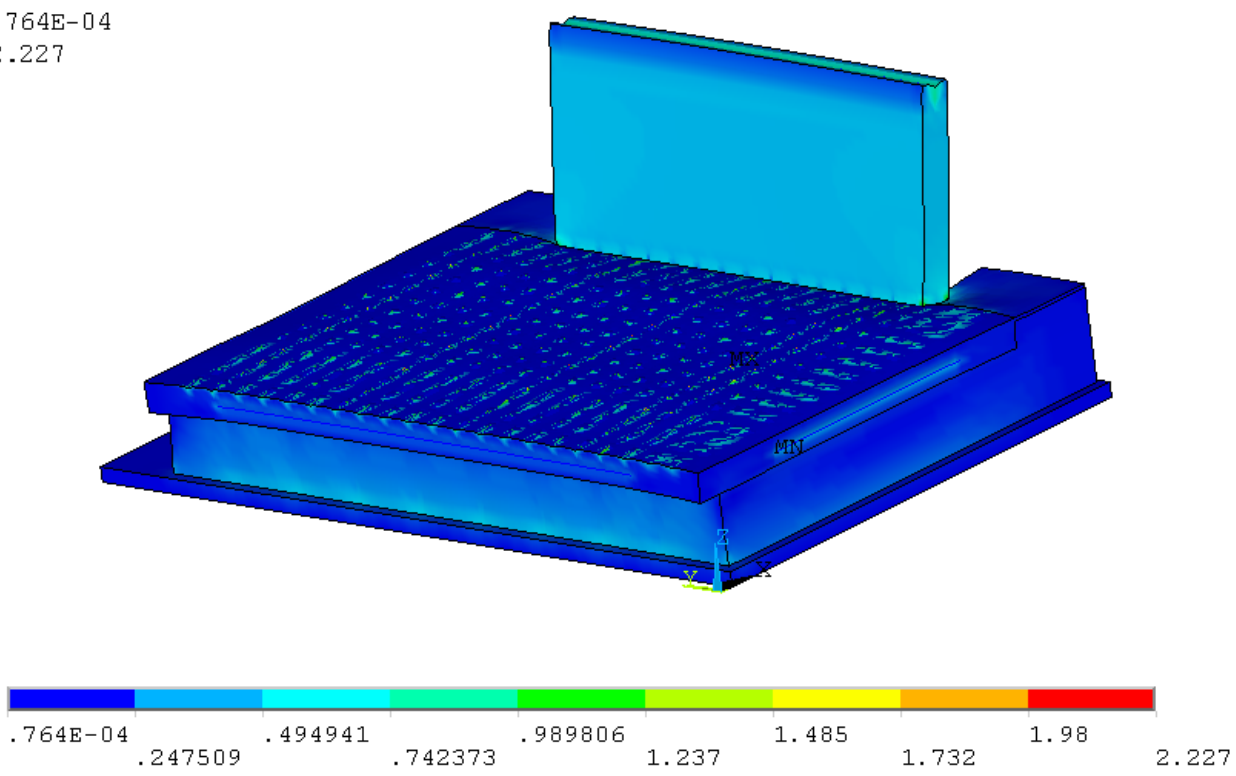

NFRL STRONG FLOOR, STRONG INALL, BOX GIRDERS

Fig. 1.30 LS 14: G+PS+FZ 100 k (445 kN) every 4' (1.2 m). Von Mises stress (1ksi=6.89 MPa), floor and wall

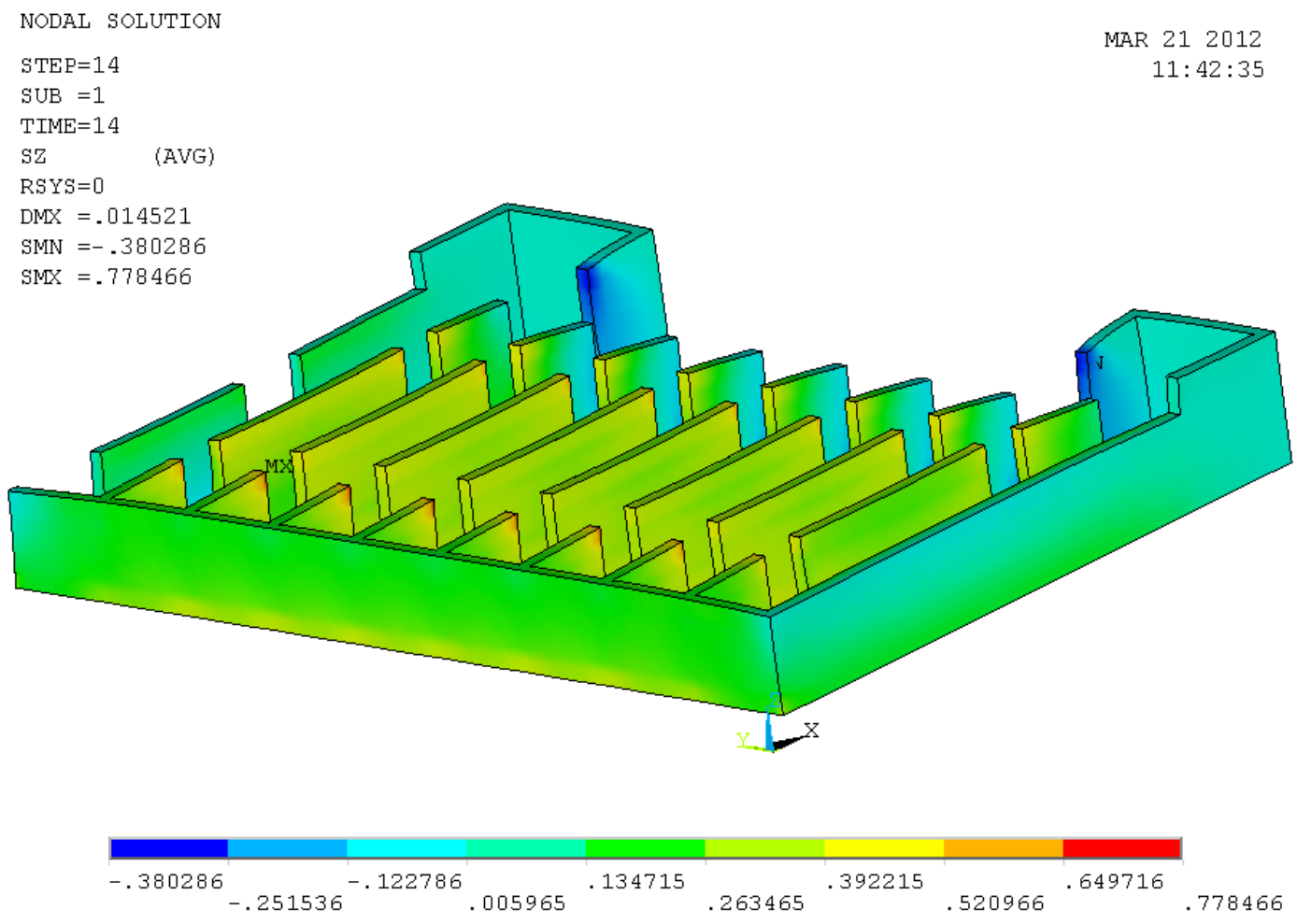

NERL STRONG FLOOR, STRONG WALL, BOX GIRDERS

Fig. 1.31 LS 14: G+PS+FZ 100 k (445 kN) every 4' (1.2 m). Vertical z stress (1ksi=6.89 MPa), basement 
NODAL SOLUTION

$\mathrm{STEP}=15$

MAR $20 \quad 2012$

$\mathrm{SUB}=1$

TIME $=15$

SEQV

(AVG)

$\mathrm{DMX}=.051502$

$\mathrm{SMN}=.403 \mathrm{E}-03$

$\mathrm{SMX}=2.157$
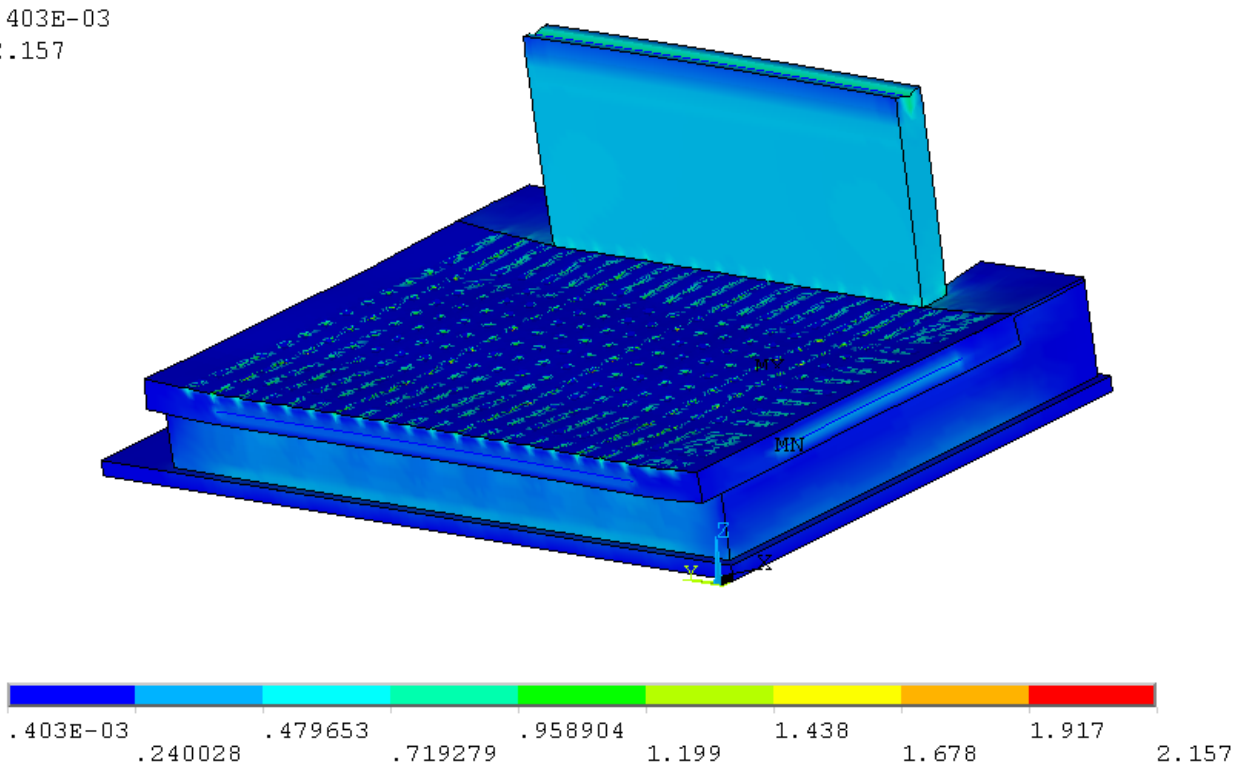

NERL STRONG FLOOR, STRONG WALL, BOX GIRDERS

Fig. 1.32 LS 15: G+PS-FZ 100 k (445 kN) every 4' (1.2 m). Von Mises stress (1ksi=6.89 MPa), floor and wall

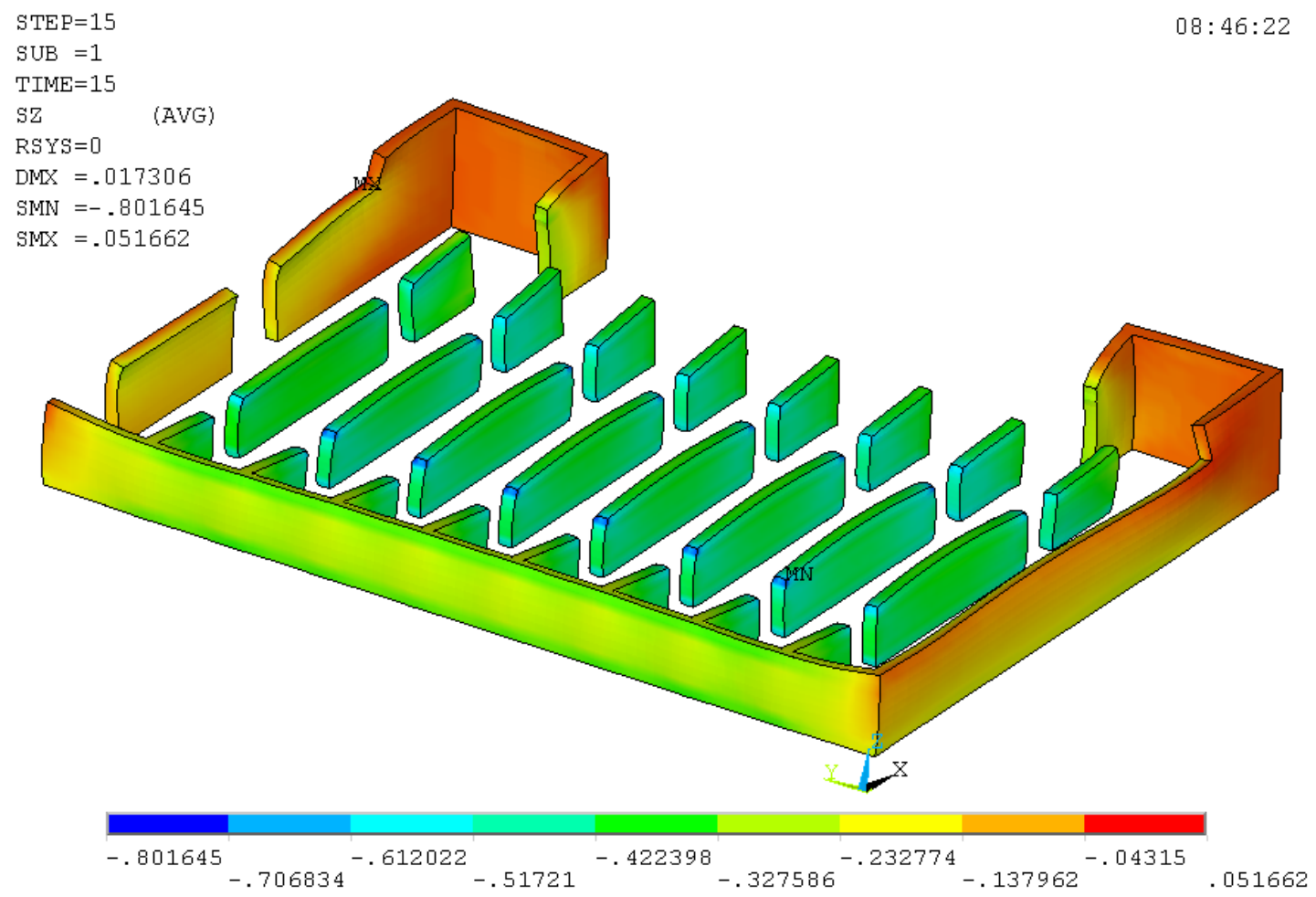

NFRL STRONG FLOOR. STRONG WALL. BOX GIRDERS

Fig. 1.33 LS 15: G+PS-FZ 100 k (445 kN) every 4' (1.2 m). Vertical z stress (1ksi=6.89 MPa), basement 
TIME $=16$

SEQV (AVG)

$\mathrm{DMX}=.054936$

$\mathrm{SMN}=.103 \mathrm{E}-03$

SMX $=2.101$
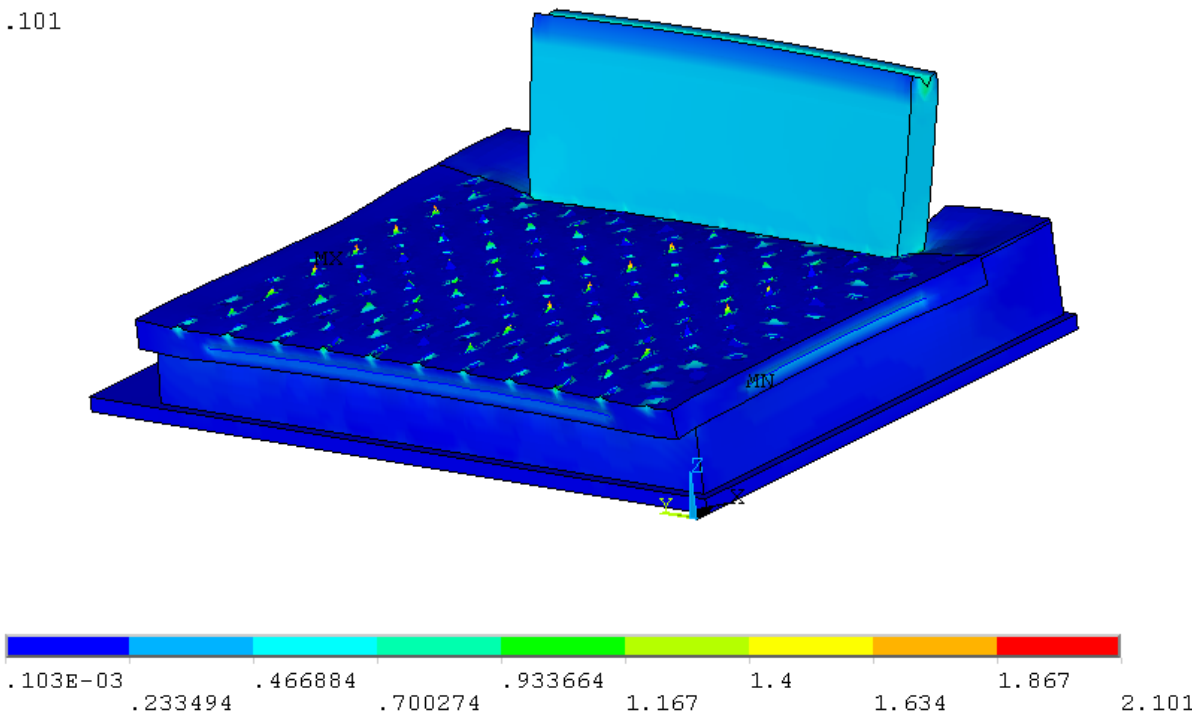

NERL STRONG FLOOR, STRONG WALL, BOX GIRDERS

Fig. 1.34 LS 16: G+PS+-FZ 100 k (445 kN) every 4' (1.2 m) alternate. Deformations, Von Mises stress (1ksi=6.89 MPa), strong floor and wall

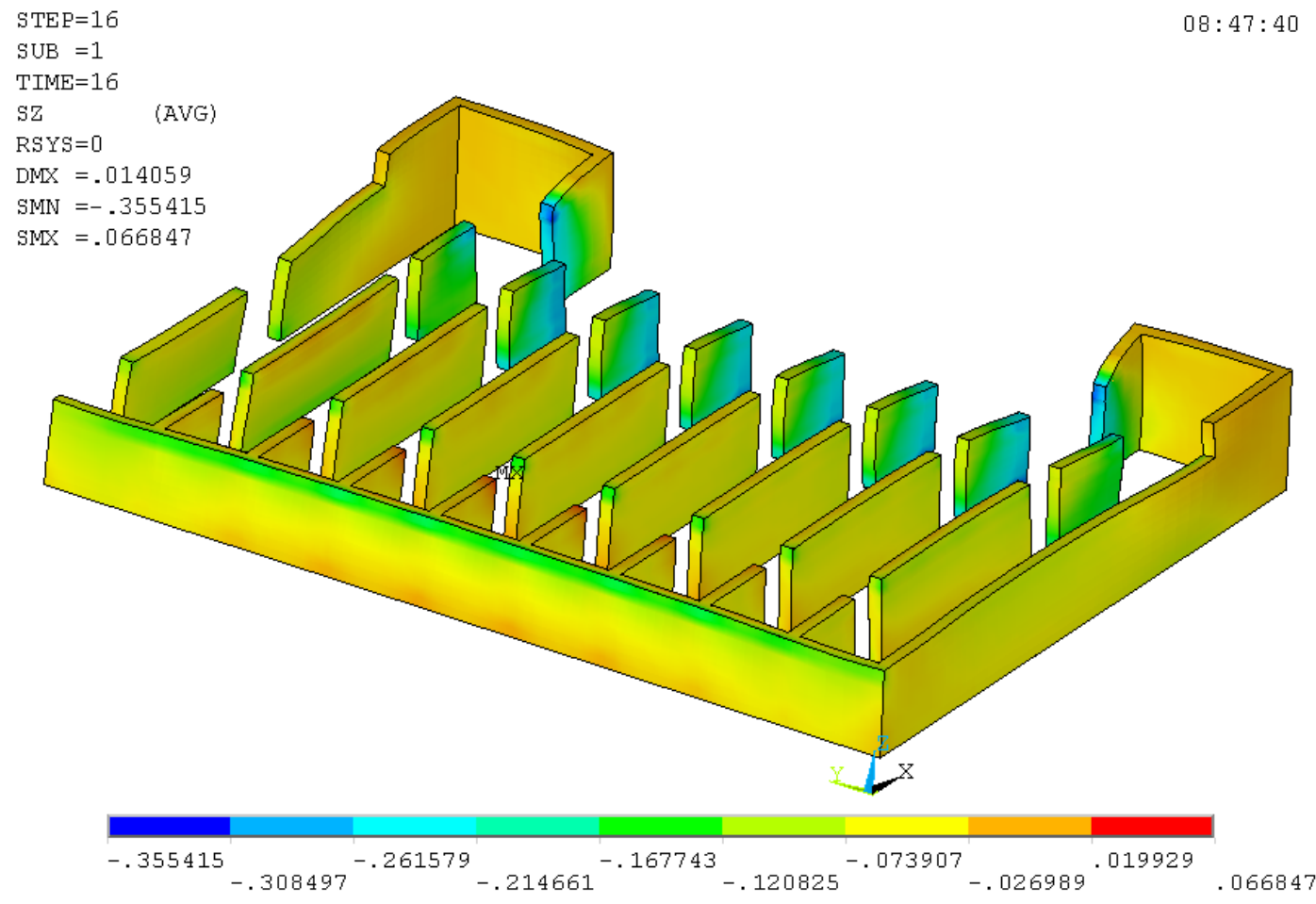

NERL STRONG FLOOR, STRONG WALL, BOX GIRDERS

Fig. 1.35 LS 16: G+PS+-FZ 100 k (445 kN) every 4' (1.2 m) alternate. Deformations, Vertical z stress $(1 \mathrm{ksi}=6.89 \mathrm{MPa})$, basement 


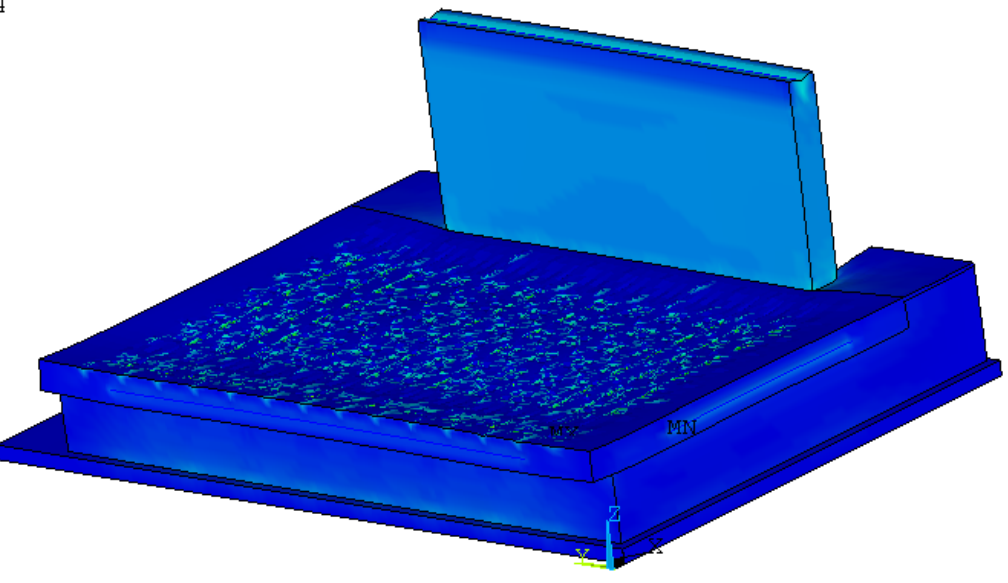

\begin{tabular}{llllllllll}
\hline $.909 \mathrm{E}-04$ & & .743067 & 1.486 & & 2.229 & & 2.972 & 3.343
\end{tabular}

NFRL STRONG FLOOR, STRONG WALL, BOX GIRDERS

Fig. 1.36 LS 17: G+PS +floor $100 \mathrm{k}(445 \mathrm{kN})$ up every 6' (1.8 m), down every 2' $(0.61 \mathrm{~m})$ centered. Deformations, Von Mises stress (1ksi=6.89 MPa), floor and wall

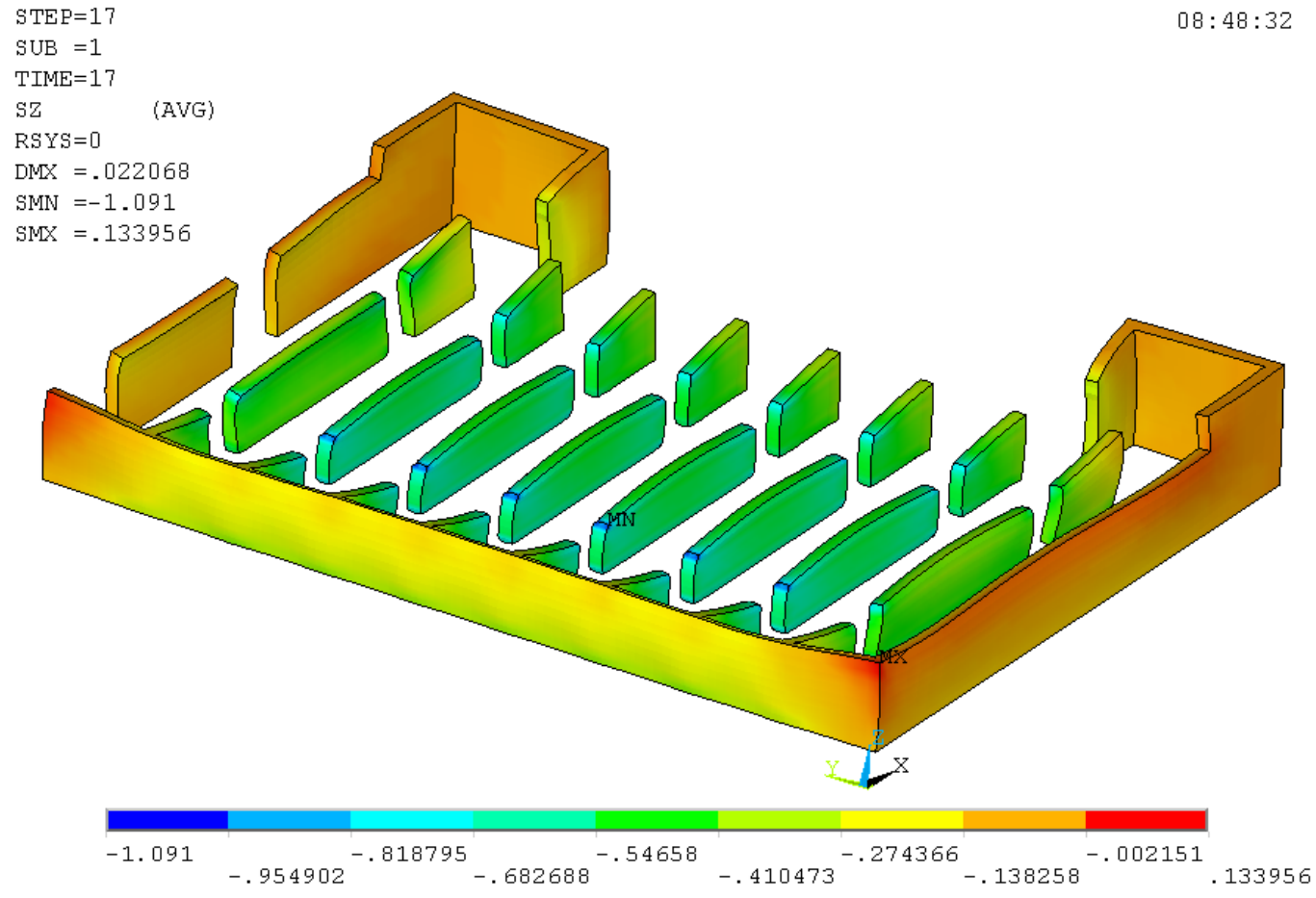

NERL STRONG FLOOR. STRONG WALL, BOX GIRDERS

Fig.1.37 LS 17: G+PS +floor 100 k (445 kN) up every 6' (1.8 m), down every 2' (0.61 m) centered. Deformations, Vertical z stress ( $1 \mathrm{ksi}=6.89 \mathrm{MPa})$, basement 
SUB $=1$

TIME $=18$

SEQV (AVG)

$\mathrm{DMX}=.184324$

SMN $=.644 \mathrm{E}-03$

SMX $=4.708$

$$
14: 52: 17
$$
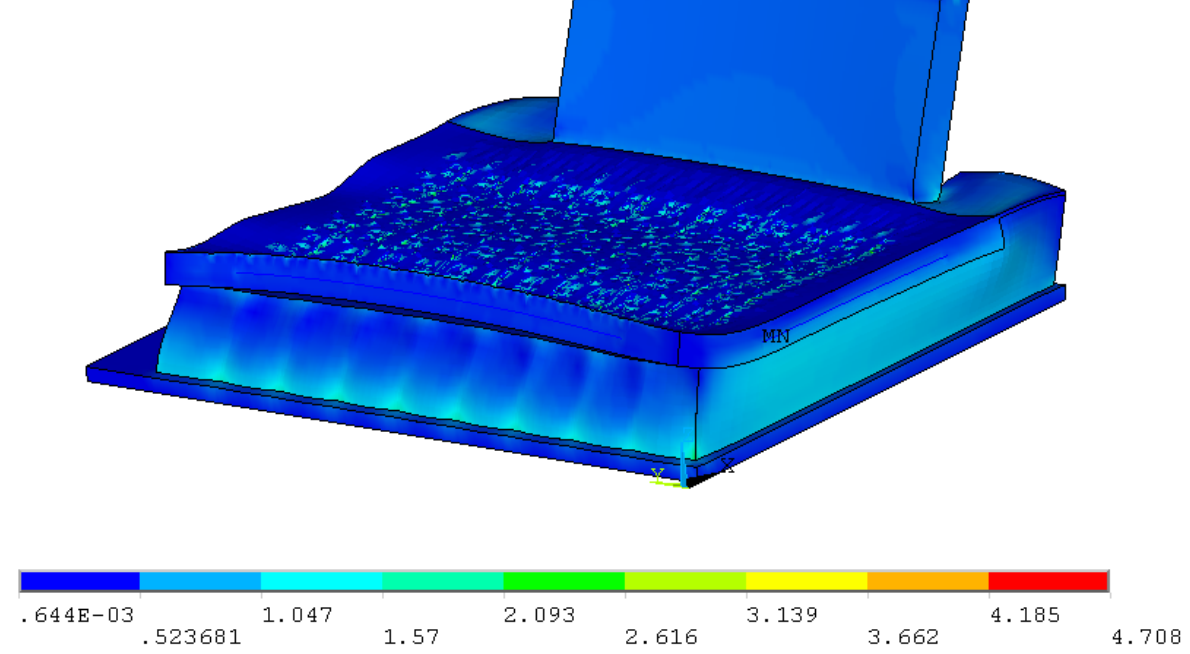

NERL STRONG FLOOR, STRONG WALL, BOX GIRDERS

Fig. 1.38 LS 18: G+PS +floor $100 \mathrm{k}(445 \mathrm{kN})$ up every 6' $(1.8 \mathrm{~m})$, down every 2' $(0.61 \mathrm{~m})$ centered + FX $50 \mathrm{k}$ every floor load point (222 kN/Ip). Deformations, Von Mises stress (1ksi=6.89 MPa), floor and wall

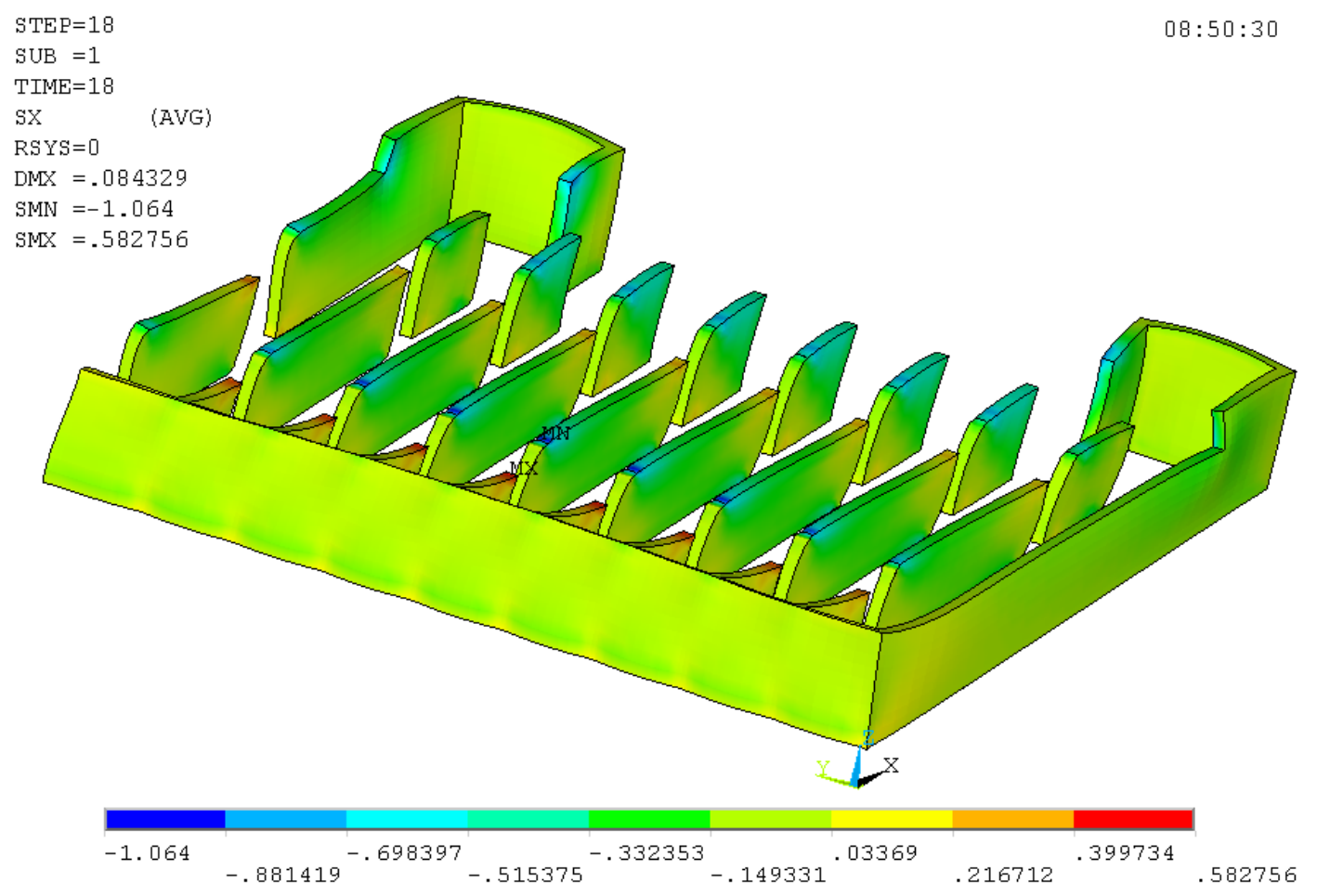

NERL STRONG FLOOR. STRONG WALL. BOX GIRDERS

Fig. 1.39 LS 18: G+PS +floor $100 \mathrm{k}(445 \mathrm{kN})$ up every 6' $(1.8 \mathrm{~m})$, down every 2' $(0.61 \mathrm{~m})$ centered + FX $50 \mathrm{k}$ every floor load point (222 kN/lp). Deformations, horizontal $x$ stress ( $1 \mathrm{ksi}=6.89 \mathrm{MPa})$, basement 


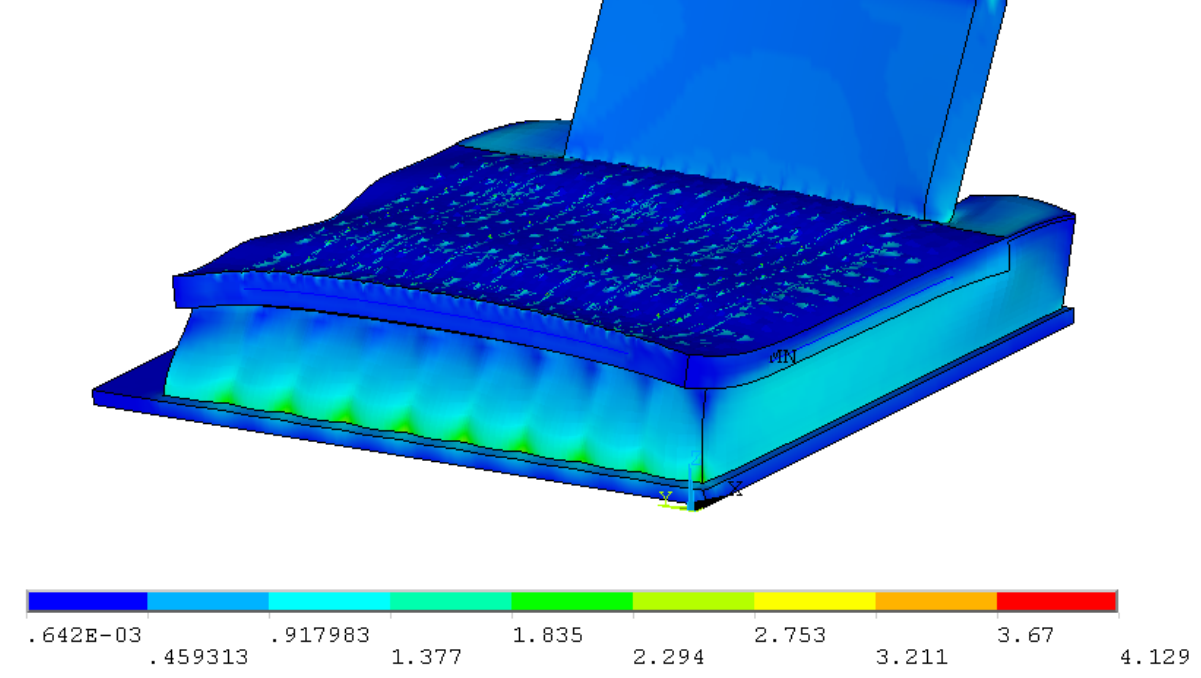

NERL STRONG FLOOR, STRONG WALL, BOX GIRDERS

Fig. 1.40 LS 19: G+PS +floor 100 k (445 kN) up every 4'(1.2 m) + FX 50 k every floor load point (222 $\mathrm{kN} / \mathrm{lp})$. Deformations, Von Mises stress ( $1 \mathrm{ksi}=6.89 \mathrm{MPa})$, strong floor and strong wall

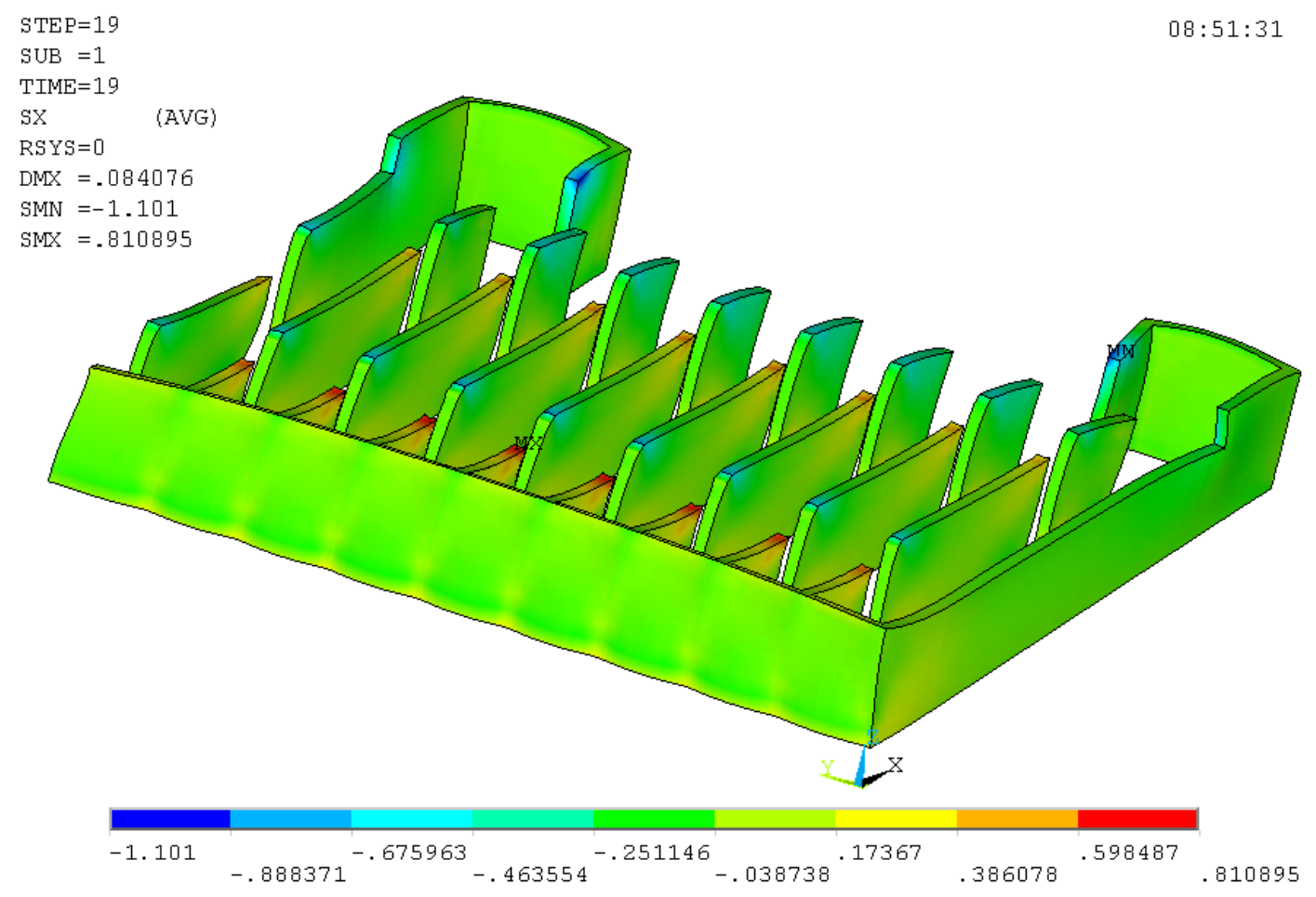

NERL STRONG FLOOR, STRONG WALL, BOX GIRDERS

Fig. 1.41 LS 19: G+PS +floor $100 \mathrm{k}(445 \mathrm{kN})$ up every 4' (1.2 m) + FX 50 k every floor load point (222 $\mathrm{kN} / \mathrm{lp})$. Deformations, horizontal $\mathrm{x}$ stress $(1 \mathrm{ksi}=6.89 \mathrm{MPa})$, basement 


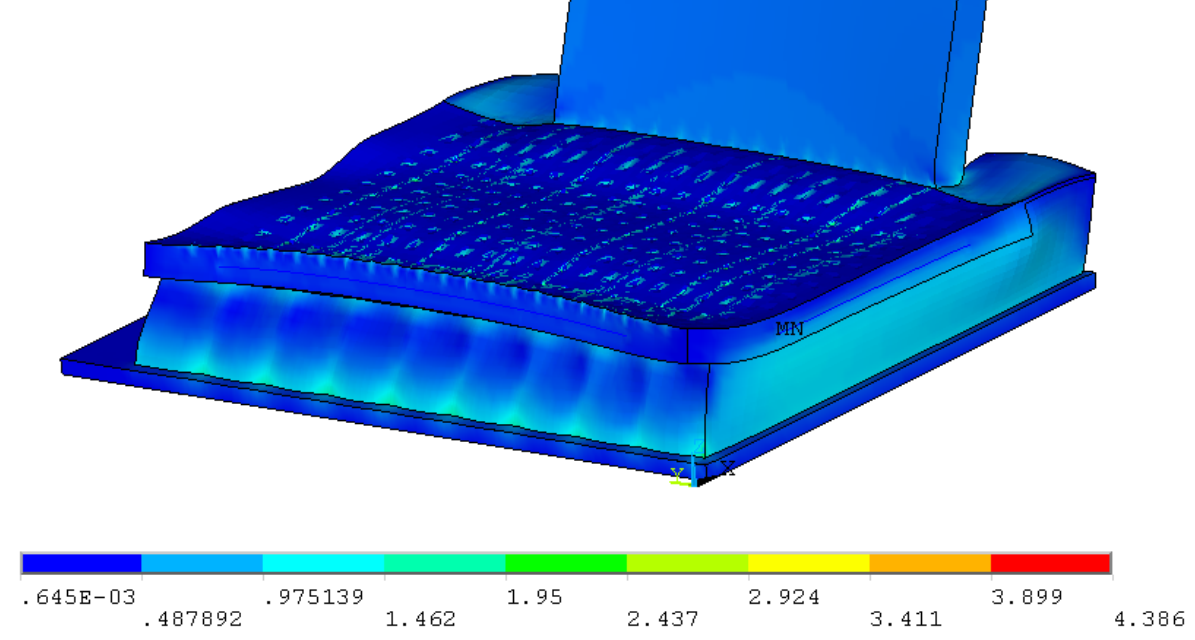

NERL STRONG FLOOR, STRONG WALL, BOX GIRDERS

Fig. 1.42 LS 20: G+PS -floor $100 \mathrm{k}(445 \mathrm{kN}$ ) down every 4' (1.2 m) + FX 50 k every floor load point (222 $\mathrm{kN} / \mathrm{lp})$. Deformations, Von Mises stress (1ksi=6.89 MPa), strong floor and strong wall

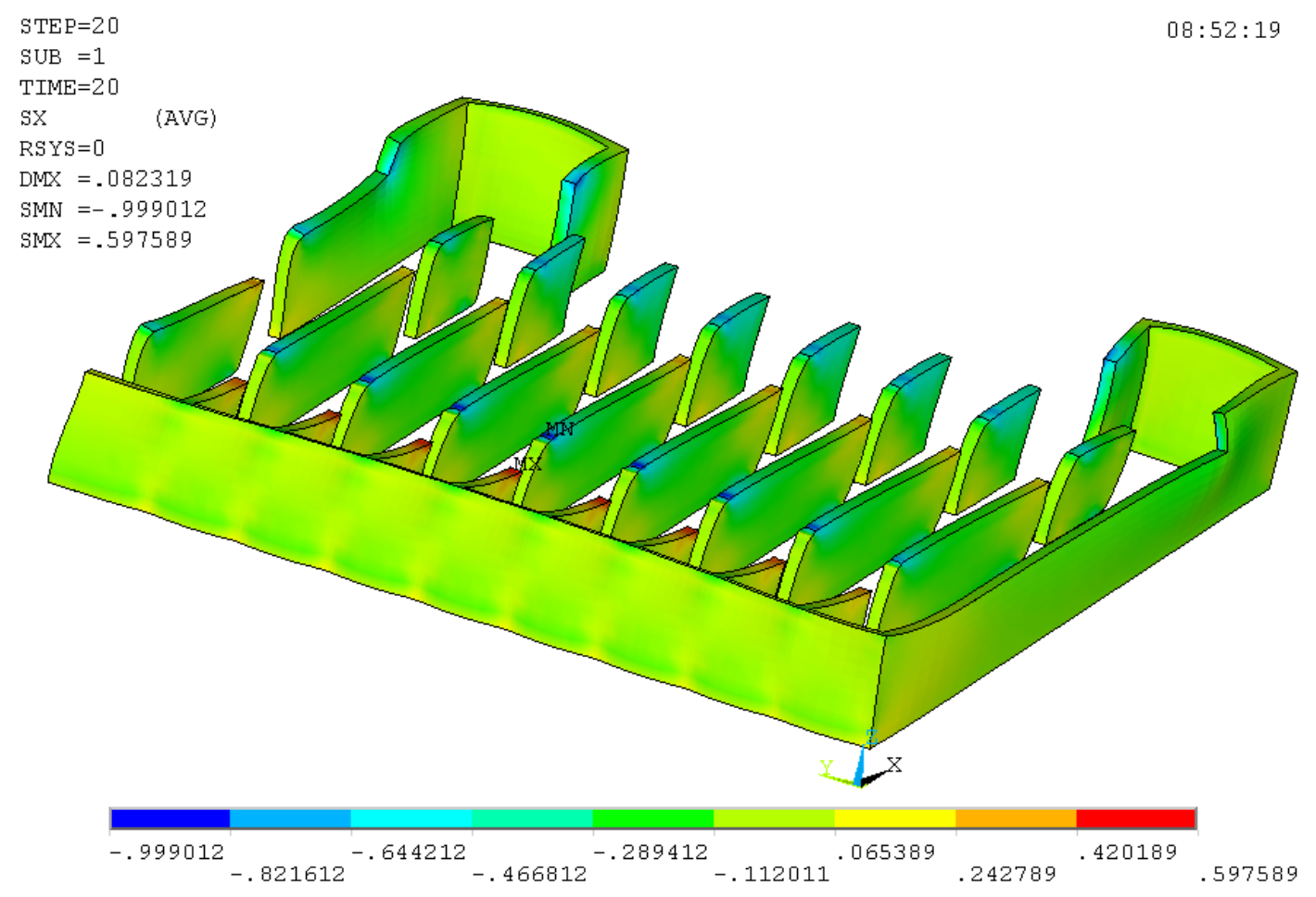

NFRL STRONG FLOOR. STRONG WALL, BOX GIRDERS

Fig. 1.43 LS 20: G+PS -floor $100 \mathrm{k}(445 \mathrm{kN})$ down every 4' (1.2 m) + FX 50 k every floor load point (222 $\mathrm{kN} / \mathrm{lp})$. Deformations, horizontal $\mathrm{x}$ stress $(1 \mathrm{ksi}=6.89 \mathrm{MPa})$, basement 


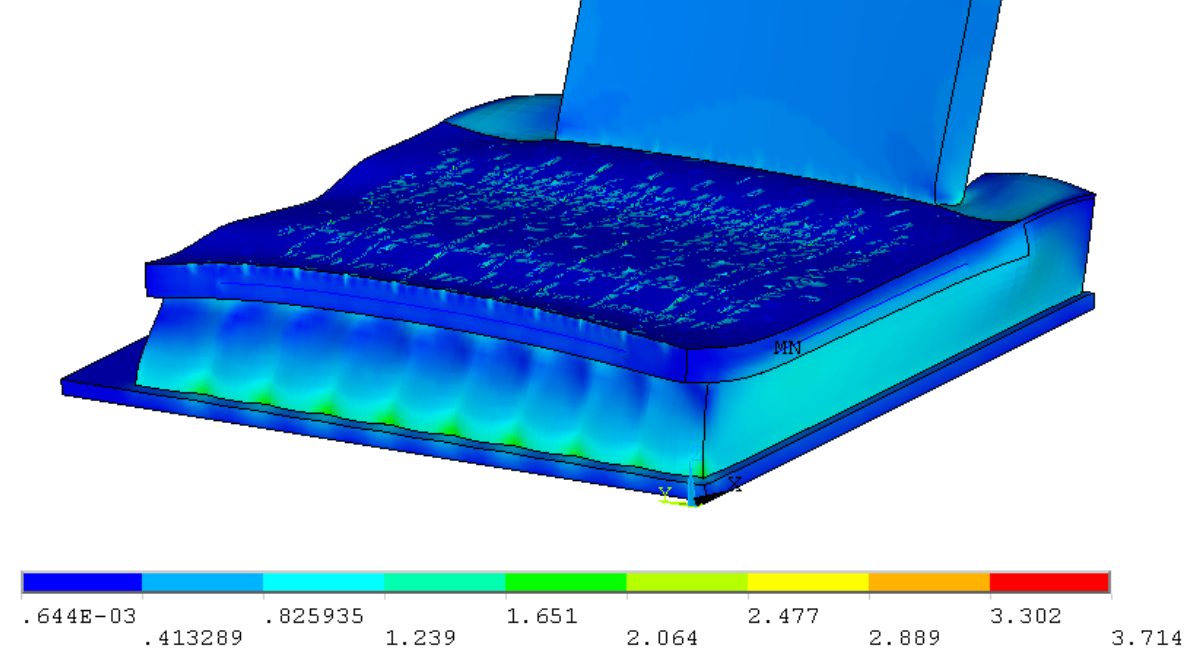

NFRL STRONG FLOOR, STRONG WALL, BOX GIRDERS

Fig. 1.44 LS 21: G+PS \pm floor $100 \mathrm{k}(445 \mathrm{kN}$ ) up down alternate every 4' (1.2 m) + FX 50 k every floor load point (222 kN/lp). Deformations, Von Mises stress (1ksi=6.89 MPa), strong floor and strong wall

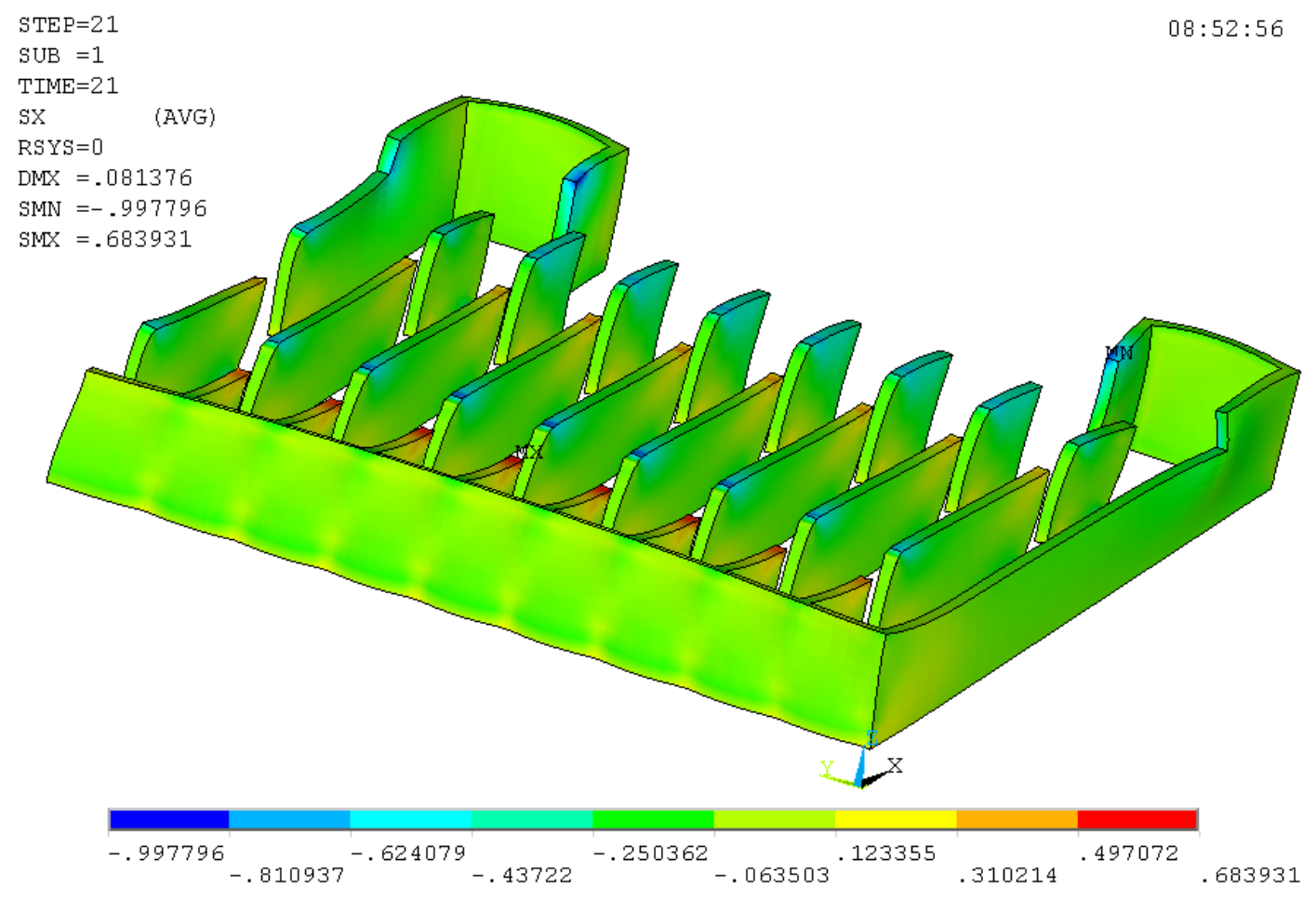

NERL STRONG FLOOR. STRONG WALL. BOX GIRDERS

Fig. 1.45 LS 21: G+PS \pm floor $100 \mathrm{k}(445 \mathrm{kN})$ up down alternate every 4' (1.2 m) + FX 50 k every floor load point $(222 \mathrm{kN} / \mathrm{lp})$. Deformations, horizontal $x$ stress $(1 \mathrm{ksi}=6.89 \mathrm{MPa})$, basement 


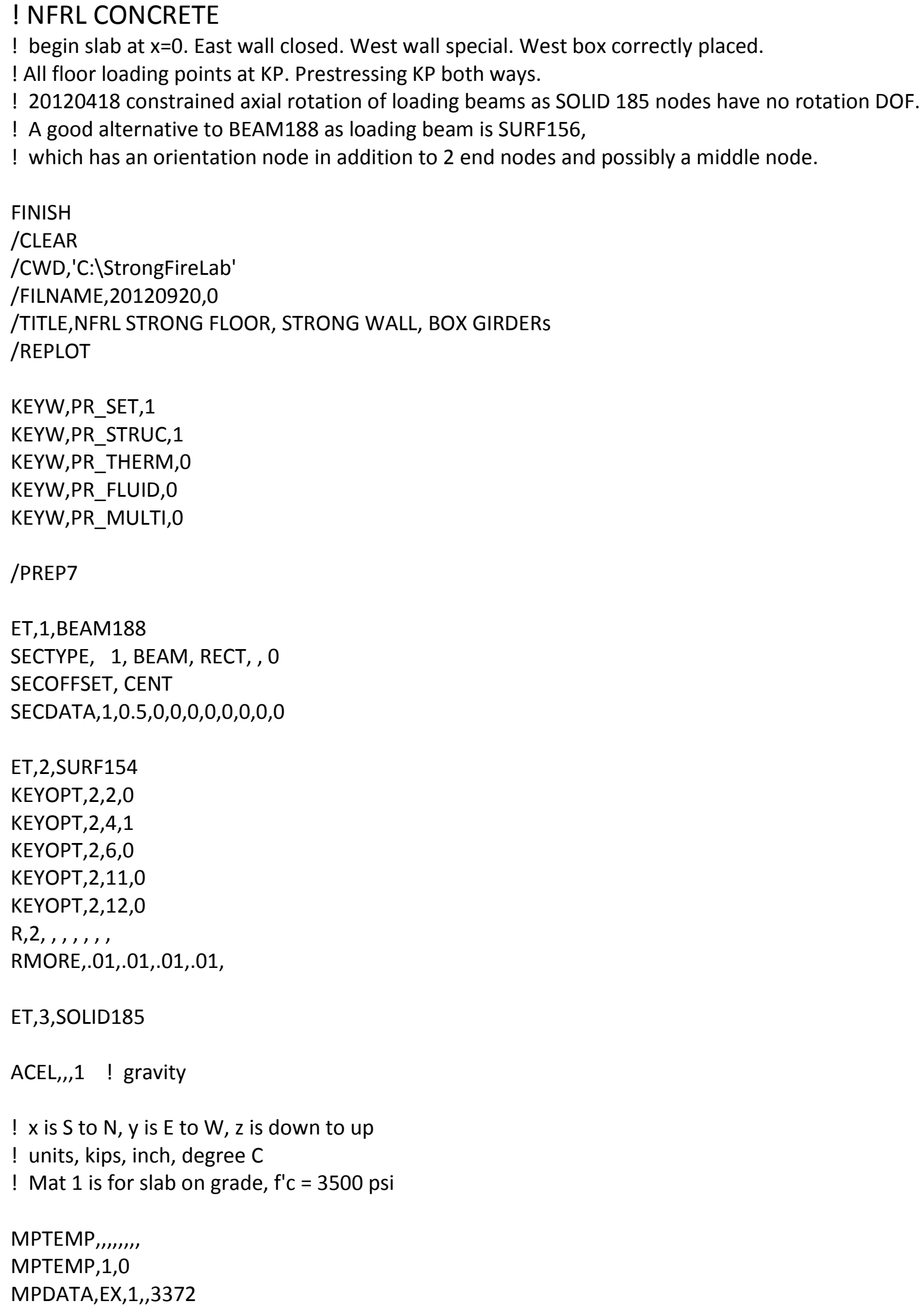


MPDATA,PRXY, $1,0.25$

MPTEMP $, \ldots, \ldots, \ldots$,

MPTEMP, 1,0

MPDATA,DENS,1,8.33E-5！144 LBF/FT^3

! Mat 2 is for foundation North box walls, f'c $=4000$ psi

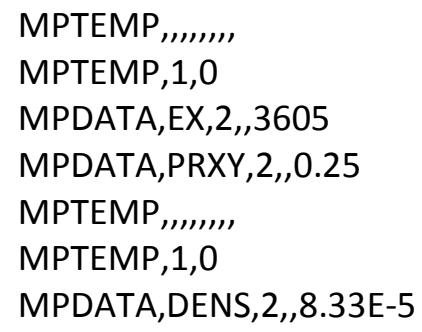

! Mat 3 is for foundation walls, $f^{\prime} c=4000$ psi

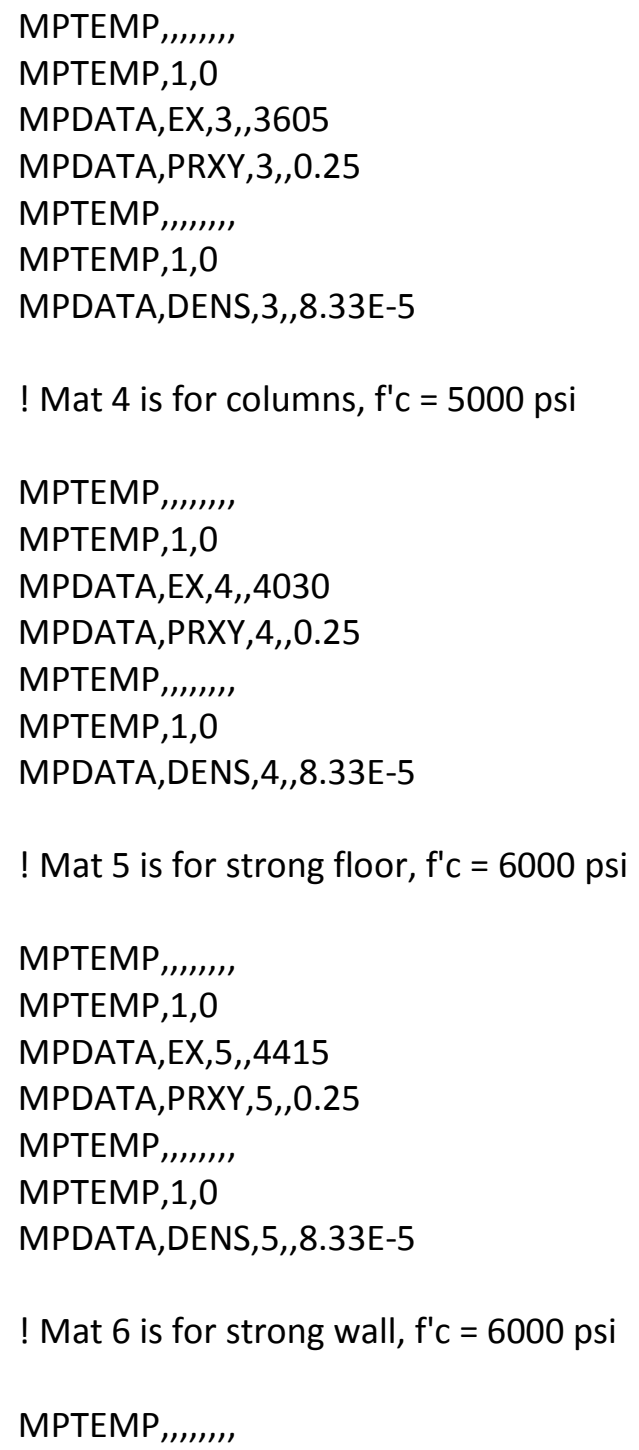




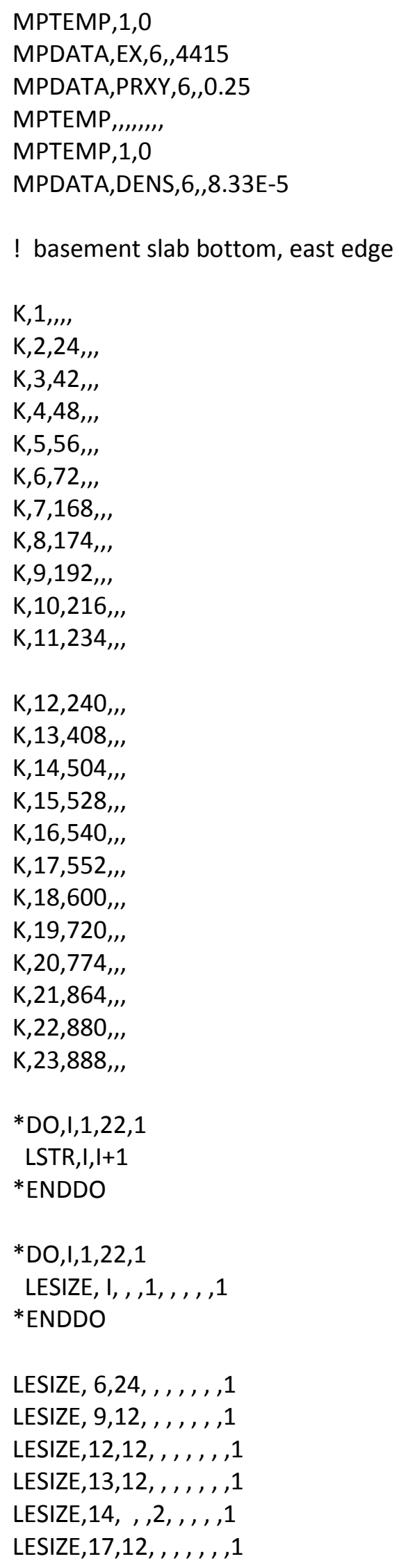




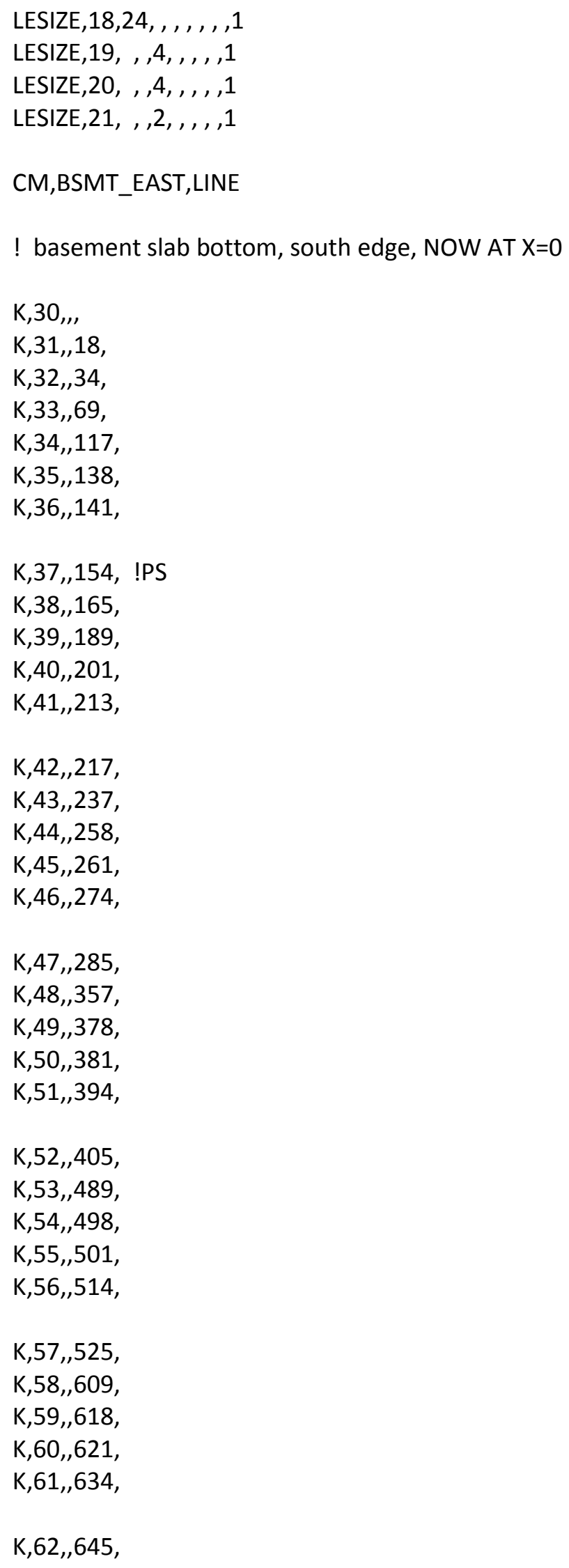


$\mathrm{K}, 63,729$,

$\mathrm{K}, 64,, 738$,

$\mathrm{K}, 65,, 741$,

$\mathrm{K}, 66,754$,

$\mathrm{K}, 67,765$,

$\mathrm{K}, 68,, 837$,

$\mathrm{K}, 69,, 858$,

$\mathrm{K}, 70,861$,

$\mathrm{K}, 71,874$,

$\mathrm{K}, 72,, 885$,

$\mathrm{K}, 73,, 905$,

$\mathrm{K}, 74,, 909$,

$\mathrm{K}, 75,, 921$,

$\mathrm{K}, 76,, 933$,

$\mathrm{K}, 77,, 957$,

$\mathrm{K}, 78,, 978$, !PS

$\mathrm{K}, 79,, 981$,

$\mathrm{K}, 80,994$,

$\mathrm{K}, 81,, 1005$,

$\mathrm{K}, 82,, 1053$,

$\mathrm{K}, 83,, 1098$,

$\mathrm{K}, 84,, 1114$,

$\mathrm{K}, 85,, 1242$,

*DO,I,30,84,1

LSTR,I,I+1

*ENDDO

*DO,I,23,77,1

LESIZE, I, , ,1, , , , , 1

*ENDDO

LESIZE, $24,,, 2,,,,, 1$

LESIZE, 25, , , 2, , , , , 1

LESIZE,26,24, , , , , , ,

LESIZE, 29, , , 2, , , , , 1

LESIZE,31,12, , , , , , , 1

LESIZE, 33, , , 2, , , , 1

LESIZE, 35, , , 2, , , , , 1

LESIZE,36, , , 2, , , , , 1

LESIZE, 38, , , 2, , , , , 1

LESIZE,40,12, , , , , , , 1

LESIZE,41, , , 2, , , , , 1

LESIZE,43, , , 2, , , , , 1 


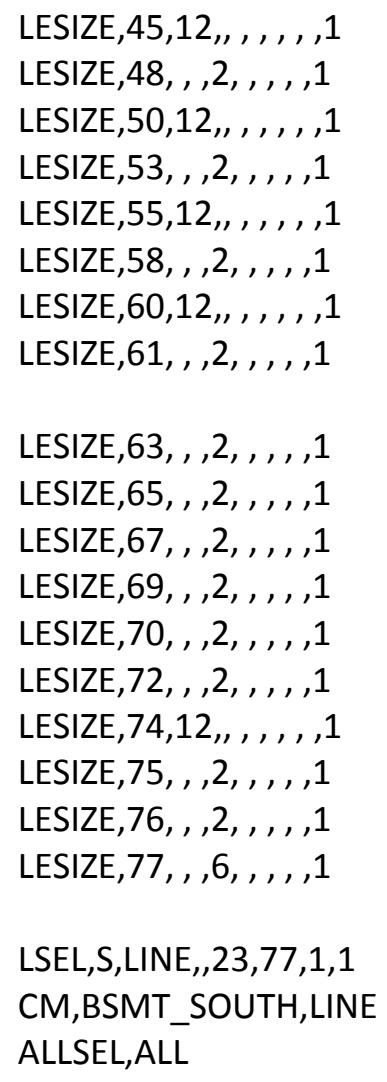

! ORIENTATION KP FOR LOADING STRIPS 


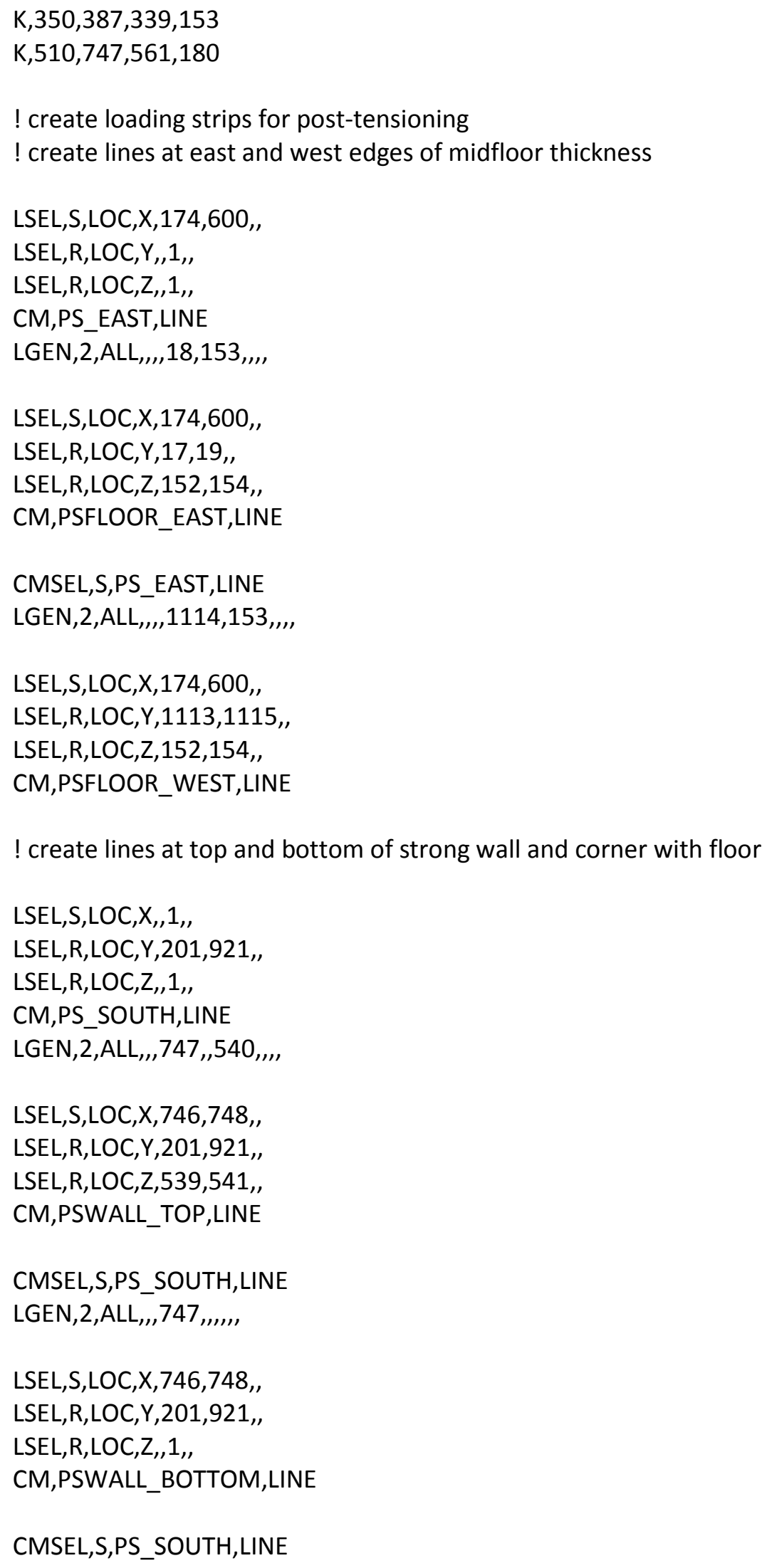




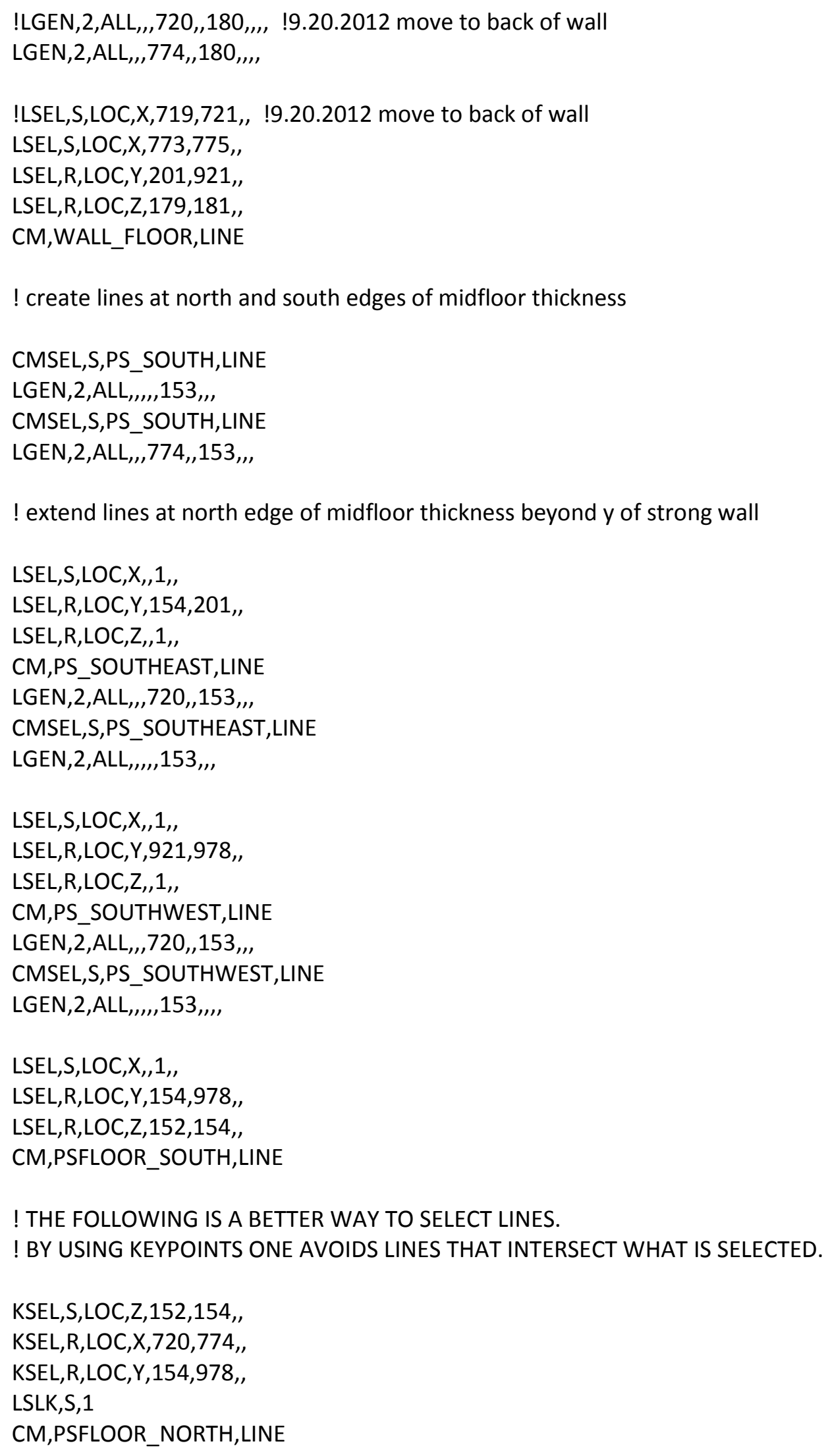

! THE FOLLOWING IS A BETTER WAY TO SELECT LINES.

! BY USING KEYPOINTS ONE AVOIDS LINES THAT INTERSECT WHAT IS SELECTED.

KSEL,S,LOC,Z,152,154,, KSEL,R,LOC,X,720,774,, KSEL,R,LOC,Y,154,978, LSLK,S, 1

CM,PSFLOOR_NORTH,LINE 
! construct basement bottom

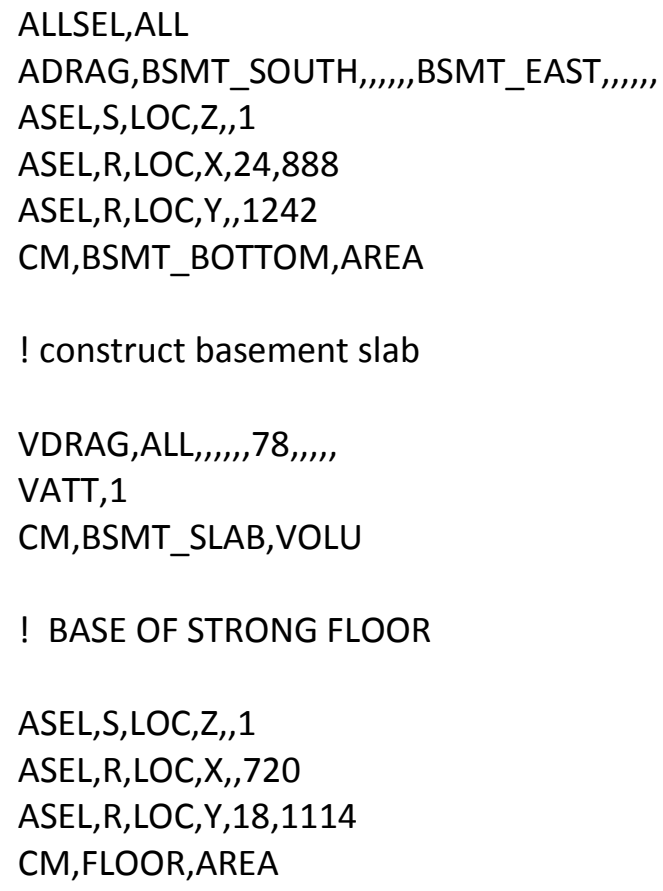


ASEL,S,LOC,Z,23,25

ASEL,R,LOC, X,56,174

ASEL,R,LOC, Y,618,634

CM,BASE_SOUTH6,AREA

ASEL,S,LOC,Z,23,25

ASEL,R,LOC, X,56,174

ASEL,R,LOC,Y,738,754

CM,BASE_SOUTH7,AREA

ASEL,S,LOC,Z,23,25

ASEL,R,LOC, X,56,174

ASEL,R,LOC, $Y, 858,874$

CM,BASE_SOUTH8,AREA

ASEL,S,LOC,Z,23,25

ASEL,R,LOC,X,56,174

ASEL,R,LOC,Y,978,994

CM,BASE_SOUTH9,AREA

CMGRP,BASE_SOUTH_WEST,BASE_SOUTH6,BASE_SOUTH7,BASE_SOUTH8,BASE_SOUTH9

! bases of 4 north-South walls, middle east part

ASEL,S,LOC,Z,23,25

ASEL,R,LOC,X,234,540

ASEL,R,LOC,Y,138,154

CM,BASE_MIDDLE2,AREA

ASEL,S,LOC,Z,23,25

ASEL,R,LOC,X,234,540

ASEL,R,LOC,Y,258,274

CM,BASE_MIDDLE3,AREA

ASEL,S,LOC,Z,23,25

ASEL,R,LOC,X,234,540

ASEL,R,LOC,Y,378,394

CM,BASE_MIDDLE4,AREA

ASEL,S,LOC,Z,23,25

ASEL,R,LOC,X,234,540

ASEL,R,LOC,Y,498,514

CM,BASE_MIDDLE5,AREA

CMGRP,BASE_MIDDLE_EAST,BASE_MIDDLE2,BASE_MIDDLE3,BASE_MIDDLE4,BASE_MIDDLE5

! bases of 5 north-South walls, middle west part 
ASEL,S,LOC,Z,23,25

ASEL, R,LOC, $X, 234,540$

ASEL,R,LOC,Y,618,634

CM,BASE_MIDDLE6,AREA

ASEL,S,LOC,Z,23,25

ASEL,R,LOC,X,234,540

ASEL,R,LOC,Y,738,754

CM,BASE_MIDDLE7,AREA

ASEL,S,LOC,Z,23,25

ASEL,R,LOC,X,234,540

ASEL, R,LOC,Y,858,874

CM,BASE_MIDDLE8,AREA

ASEL,S,LOC,Z,23,25

ASEL,R,LOC,X,234,540

ASEL,R,LOC,Y,978,994

CM,BASE_MIDDLE9,AREA

ASEL,S,LOC,Z,23,25

ASEL,R,LOC,X,174,408

ASEL,R,LOC,Y,1098,1114

CM,BASE_MIDDLE10,AREA

CMGRP,BASE_MIDDLE_WEST,BASE_MIDDLE6,BASE_MIDDLE7,BASE_MIDDLE8,BASE_MIDDLE9,BASE_MI DDLE10

! bases of 4 north-South walls, NORTH east part

ASEL,S,LOC,Z,23,25

ASEL,R,LOC,X,600,720

ASEL,R,LOC,Y,138,154

CM,BASE_NORTH2,AREA

ASEL,S,LOC,Z,23,25

ASEL,R,LOC,X,600,720

ASEL,R,LOC,Y,258,274

CM,BASE_NORTH3,AREA

ASEL,S,LOC,Z,23,25

ASEL,R,LOC,X,600,720

ASEL, R,LOC,Y,378,394

CM,BASE_NORTH4,AREA

ASEL,S,LOC,Z,23,25

ASEL, R,LOC,X,600,720

ASEL,R,LOC,Y,498,514 


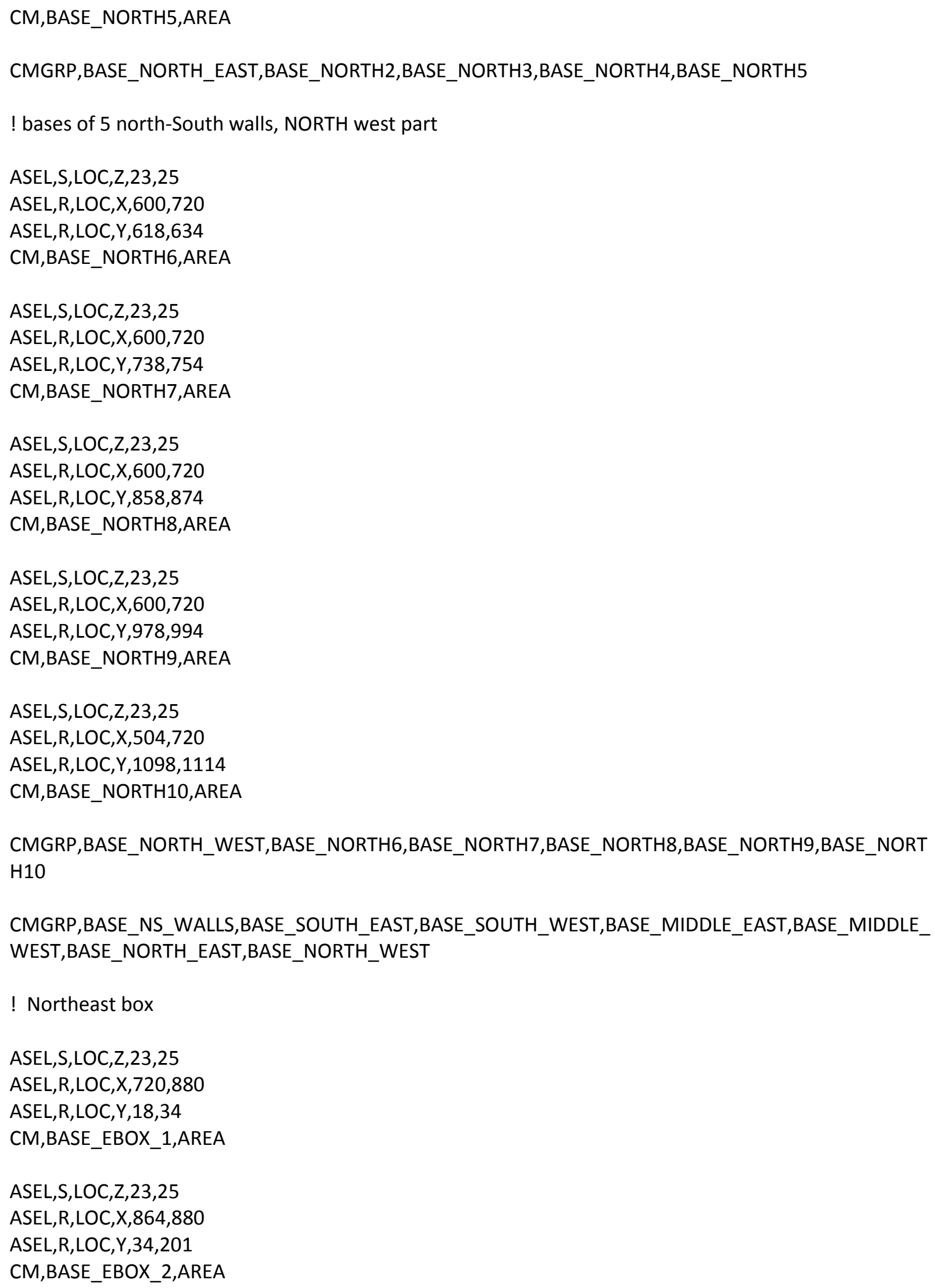


ASEL,S,LOC,Z,23,25

ASEL,R,LOC, $X, 774,880$

ASEL,R,LOC,Y,201,217

CM,BASE_EBOX_3,AREA

ASEL,S,LOC,Z,23,25

ASEL,R,LOC,X,720,774

ASEL,R,LOC,Y,18,201

CM,BASE_EBOX_4,AREA

ASEL,S,LOC,Z,23,25

ASEL,R,LOC, $X, 774,880$

ASEL,R,LOC,Y,18,217

CM,BASE_EBOX_5,AREA

! NorthWest box

ASEL,S,LOC,Z,23,25

ASEL,R,LOC, $X, 720,880$

ASEL,R,LOC,Y,1098,1114

CM,BASE_WBOX_1,AREA

ASEL,S,LOC,Z,23,25

ASEL,R,LOC,X,864,880

ASEL,R,LOC,Y,921,1098

CM,BASE_WBOX_2,AREA

ASEL,S,LOC,Z,23,25

ASEL,R,LOC,X,774,880

ASEL,R,LOC,Y,905,921

CM,BASE_WBOX_3,AREA

ASEL,S,LOC,Z,23,25

ASEL,R,LOC,X,720,774

ASEL,R,LOC,Y,921,1114

CM,BASE_WBOX_4,AREA

ASEL,S,LOC,Z,23,25

ASEL, R,LOC, $X, 774,880$

ASEL,R,LOC,Y,905,1114

CM,BASE_WBOX_5,AREA

CMGRP,BASE_BOX_WALLS,BASE_EBOX_1,BASE_EBOX_2,BASE_EBOX_3,BASE_WBOX_1,BASE_WBOX_2, BASE_WBOX_3

CMGRP,BASE_BOXES,BASE_EBOX_4,BASE_EBOX_5,BASE_WBOX_4,BASE_WBOX_5

! EAST WALL 
ASEL,S,LOC,Z,23,25

ASEL,R,LOC, X,56,720

ASEL,R,LOC,Y,18,34

CM,BASE_EASTWALL,AREA

! SOUTH WALL

ASEL,S,LOC,Z,23,25

ASEL, R,LOC, $X, 42,56$

ASEL,R,LOC,Y,18,1114

CM,BASE_SOUTHWALL,AREA

! STRONG WALL

ASEL,S,LOC,Z,23,25

ASEL,R,LOC,X,720,774

ASEL,R,LOC,Y,201,921

CM,BASE_STRONGWALL,AREA

! BUILD BOX TOPS

ALLSEL,ALL

CMSEL,S,BASE_BOXES,AREA

AGEN,2,ALL,,,,,150,,,

ASEL,S,LOC,Z,173,175,,1

VDRAG,ALL,,,,,,81,,,,,

! BUILD STRONG FLOOR

ALLSEL,ALL

CMSEL,S,FLOOR,AREA

AGEN,2,ALL,,,,,132,,,

ASEL,S,LOC,Z,131,133

VDRAG,ALL,,,,,,80,81,,,,

vSEL,S,LOC,Z,132,180,,1

VATT, 5

CM,STRONG_FLOOR,VOLU

! RAISE 26 WALLS

ALLSEL,ALL

CMSEL,S,BASE_NS_WALLS,AREA

VDRAG,ALL,,,,,,,79,,,,,

vSEL,S,LOC,Z,24,132,,1

!VSEL,U,VOLU,,STRONG_FLOOR,,, VATT, 3

CM,THIRTY_WALLS,VOLU 


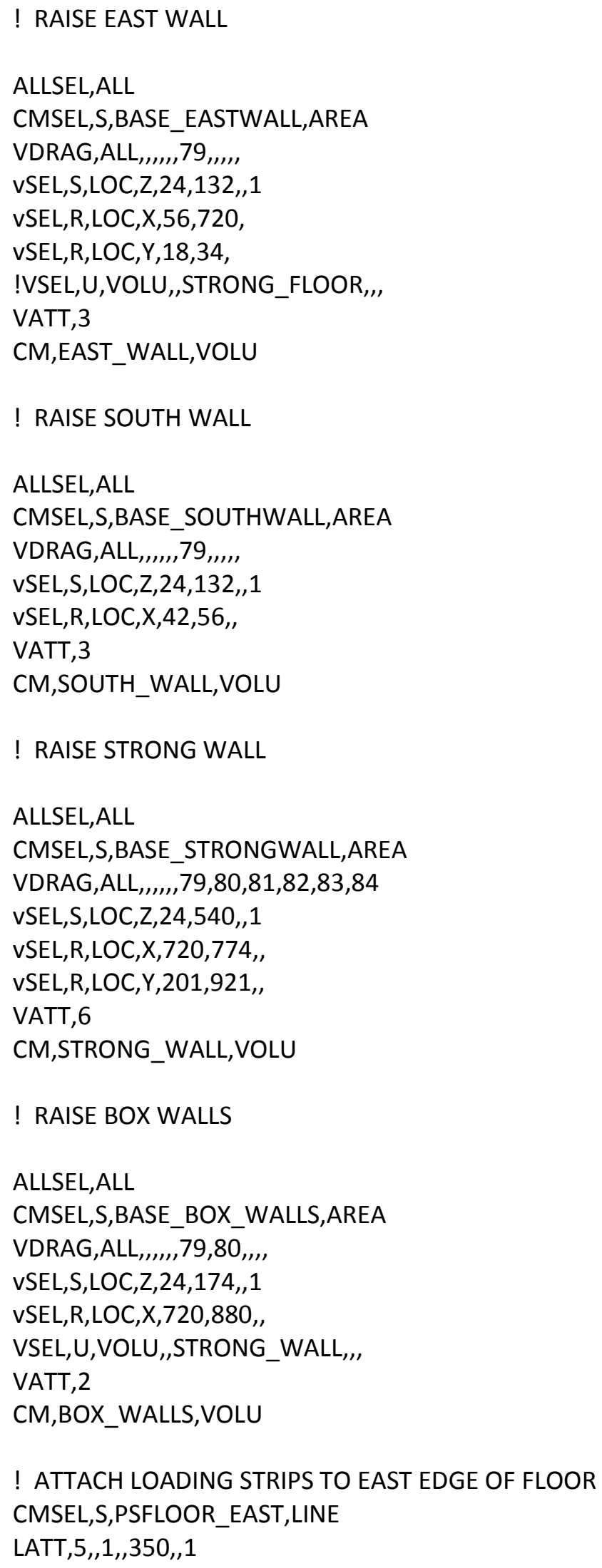


LMESH,ALL

! ATTACH TO WEST EDGE OF FLOOR

CMSEL,S,PSFLOOR_WEST,LINE

LATT,5,1,,350,,1

LMESH,ALL

! ATTACH TO TOP OF STRONG WALL

CMSEL,S,PSWALL_TOP,LINE

LATT, $6,1,, 510,1$

LMESH,ALL

! ATTACH TO BOTTOM OF STRONG WALL

CMSEL,S,PSWALL_BOTTOM,LINE

LATT,1,1,,510,,1

LMESH,ALL

! ATTACH TO SOUTH EDGE OF FLOOR

CMSEL,S,PSFLOOR_SOUTH,LINE

LATT $, 5,1,, 350,, 1$

LMESH,ALL

! ATTACH TO NORTH EDGE OF FLOOR VIA STRONG WALL

CMSEL,S,PSFLOOR_NORTH,LINE

LATT,5,1,,350,,1

LMESH,ALL

! ATTACH TO CORNER OF STRONG WALL AND FLOOR TO REACT SHEAR ON TOP OF FLOOR CMSEL,S,WALL_FLOOR,LINE

LATT $, 5,1,, 510,1$

LMESH,ALL

! mesh all volumes and merge all coincident items

ALLSEL,ALL

VMESH,ALL

NUMMRG,ALL,,,,

SAVE

/SOLU

ANTYPE,0 ! static analysis

! constrain bottom of slab

ASEL,S,LOC,Z,0,1

ASEL,R,LOC,X,24,888

$D A, A L L, U X, 0$

$D A, A L L, U Y, 0$

DA,ALL,UZ,0 
! release constraints at prestressing anchors

CMSEL,S,PSWALL_BOTTOM,LINE

DLDELE,ALL,ALL

! constrain axial rotation of loading beams as SOLID185 has no rotation DOF

$N=\operatorname{NODE}(174,18,153) \quad !$ PSFLOOR_EAST

$D, N, R O T X, \ldots, \ldots, \ldots,$,

$N=\operatorname{NODE}(174,1114,153) \quad !$ PSFLOOR_WEST

$D, N, R O T X, \ldots, \ldots, \ldots,$,

$\mathrm{N}=\operatorname{NODE}(747,921,540) \quad$ ! PSWALL_TOP

$\mathrm{D}, \mathrm{N}, \mathrm{ROTY}, \ldots, \ldots, \ldots,,$,

$\mathrm{N}=\operatorname{NODE}(747,921,0) \quad$ ! PSWALL_BOTTOM

$\mathrm{D}, \mathrm{N}, \mathrm{ROTY}, \ldots, \ldots, \ldots, \ldots$,

$N=\operatorname{NODE}(0,978,153) \quad !$ PSFLOOR_SOUTH

$\mathrm{D}, \mathrm{N}, \mathrm{ROTY}, \ldots, \ldots, \ldots, \ldots$,

$N=\operatorname{NODE}(720,978,153) \quad !$ PSFLOOR_NORTHWEST

$\mathrm{D}, \mathrm{N}, \mathrm{ROTY},,,,,,,,,$,

$N=\operatorname{NODE}(774,921,153) \quad !$ PSFLOOR_NORTHCENTRAL

$\mathrm{D}, \mathrm{N}, \mathrm{ROTY},,,,,,,,,$,

$N=\operatorname{NODE}(720,201,153) \quad$ ! PSFLOOR_NORTHEAST

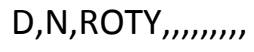

$N=\operatorname{NODE}(720,201,180) \quad$ ! WALL_FLOOR

$\mathrm{D}, \mathrm{N}, \mathrm{ROTY}, \ldots, \ldots, \ldots, \ldots$,

! LS1 = GRAVITY ONLY

ALLSEL,ALL

TIME,1

LSWRITE,1

! LS2 = APPLY UNBALANCED POST-TENSIONING LINE PRESSURE ON BEAMS

! GRAVITY UNTOUCHED AND REMAINS

CMSEL,S,PSWALL_TOP,LINE

ESLL,S

CM,TOP_BEAM,ELEM

SFBEAM,ALL,1,PRES,-19.65,-19.65, , , , ,

CMSEL,S,PSFLOOR_EAST,LINE

ESLL,S

CM,EAST_BEAM,ELEM

SFBEAM,ALL,1,PRES, $-4.08,-4.08$, , , , , , 


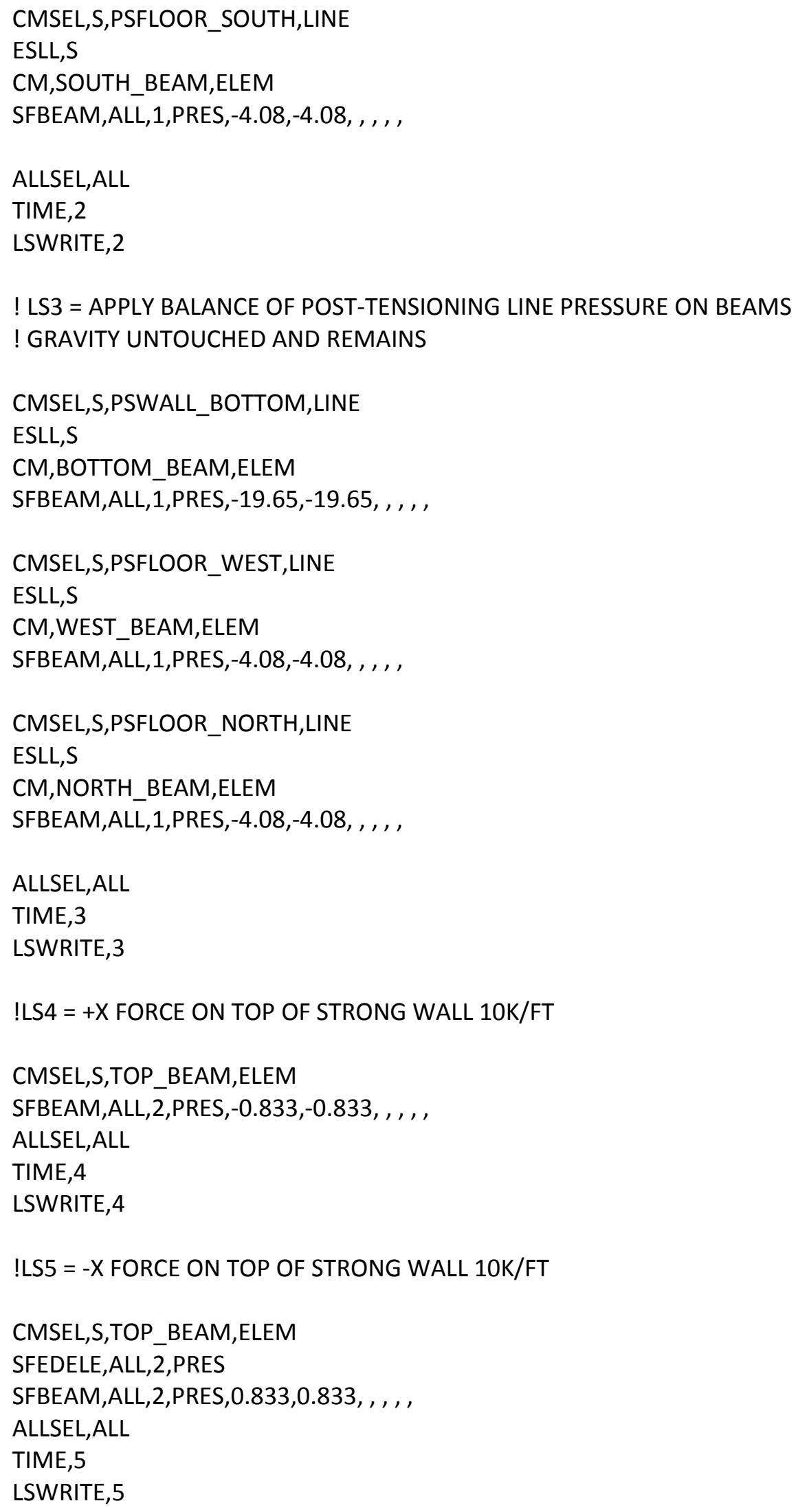




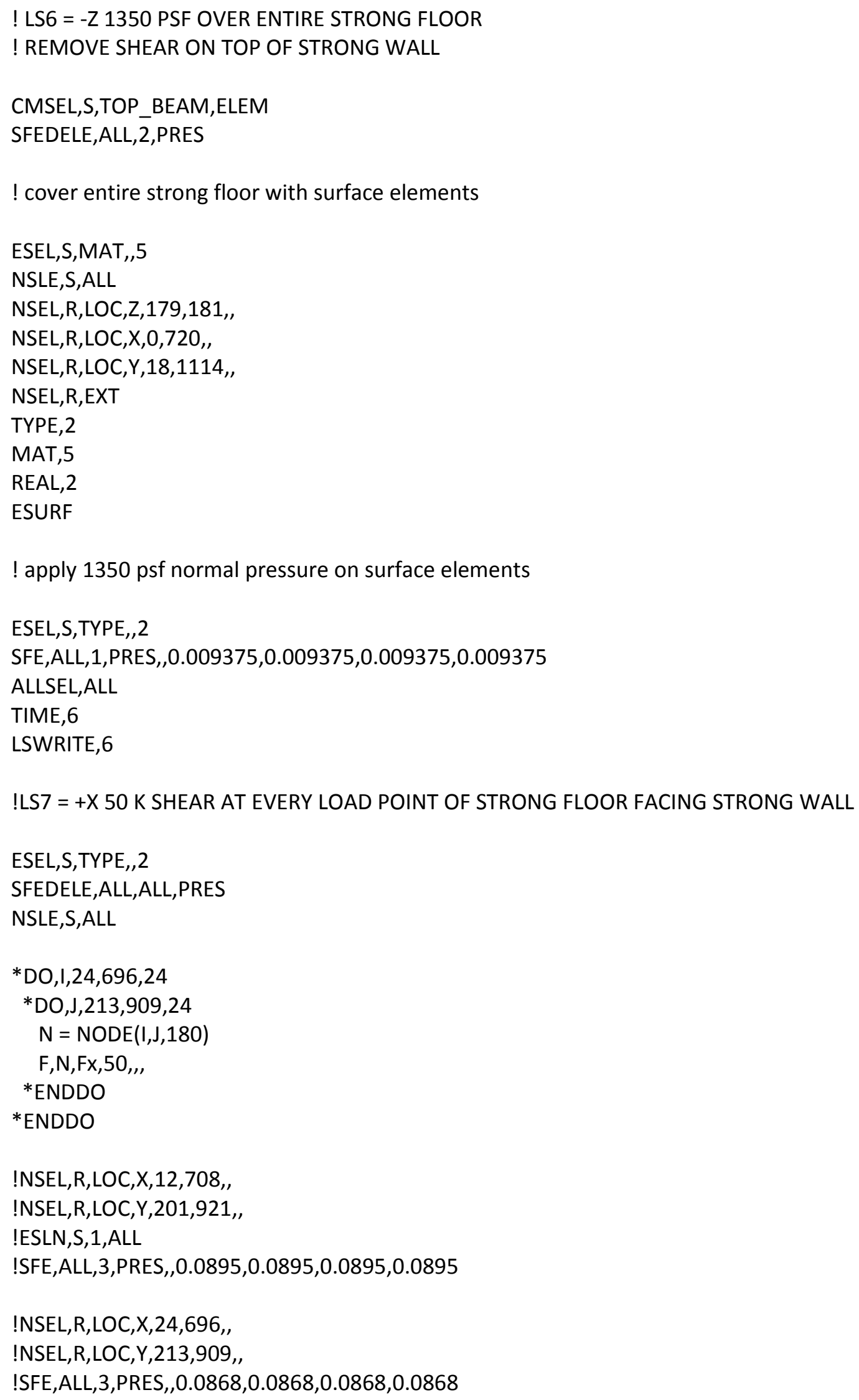




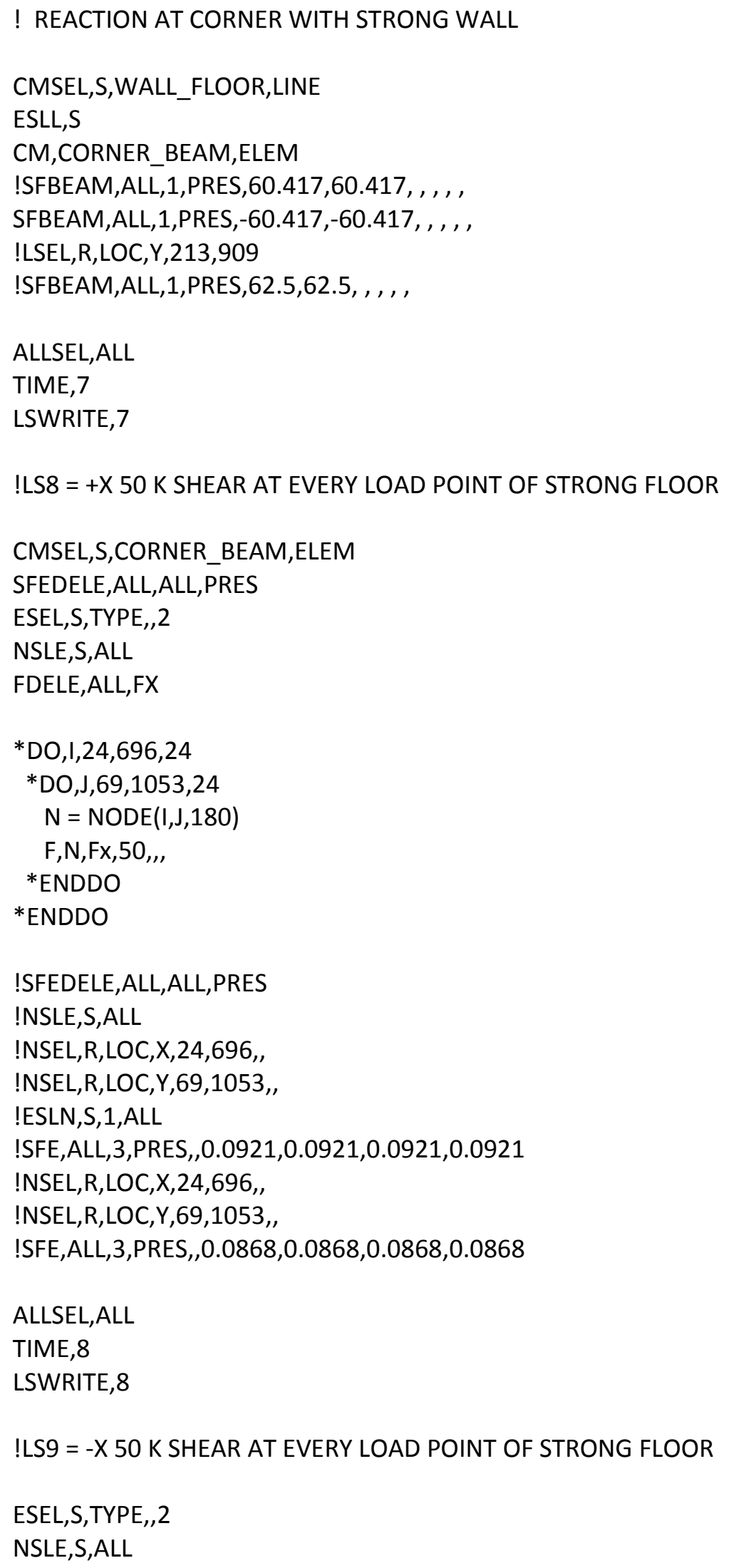




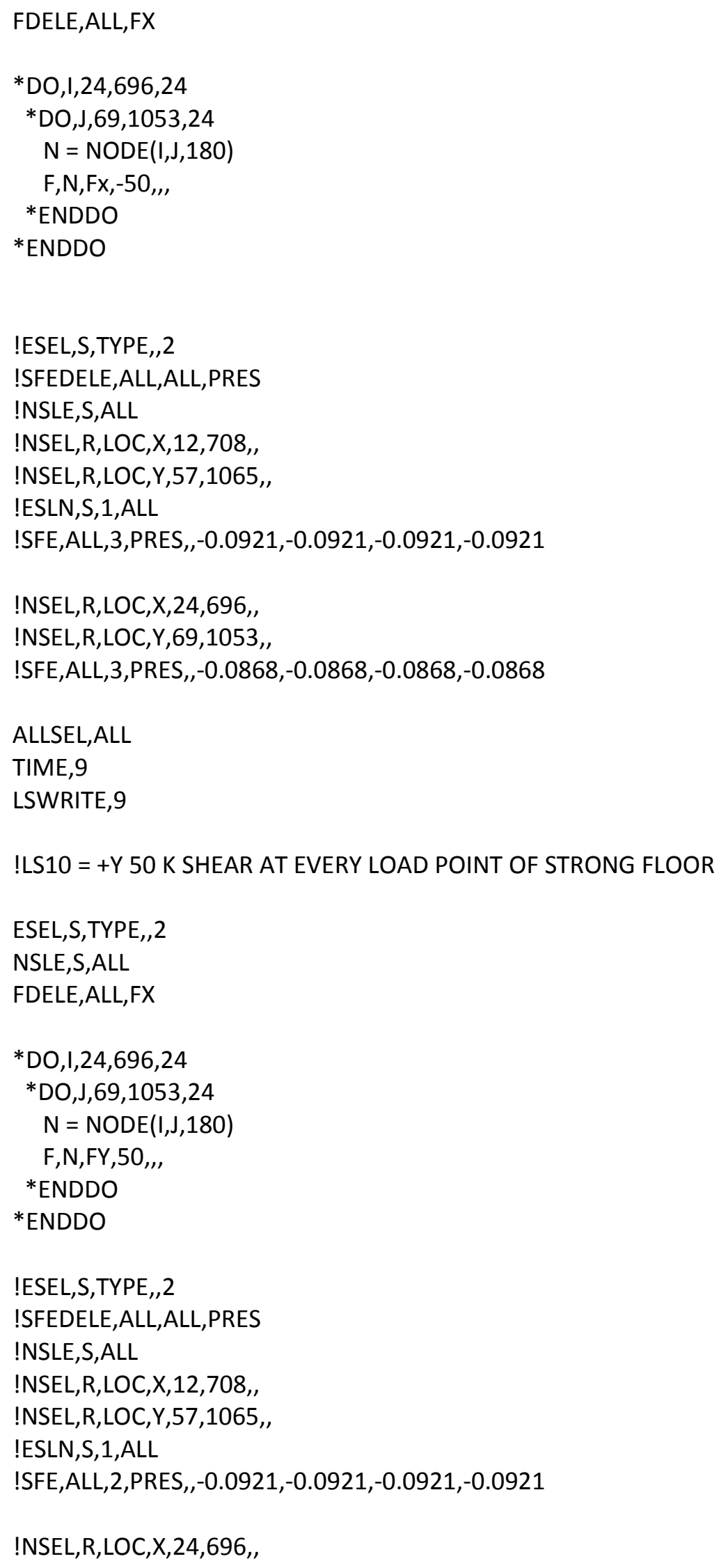




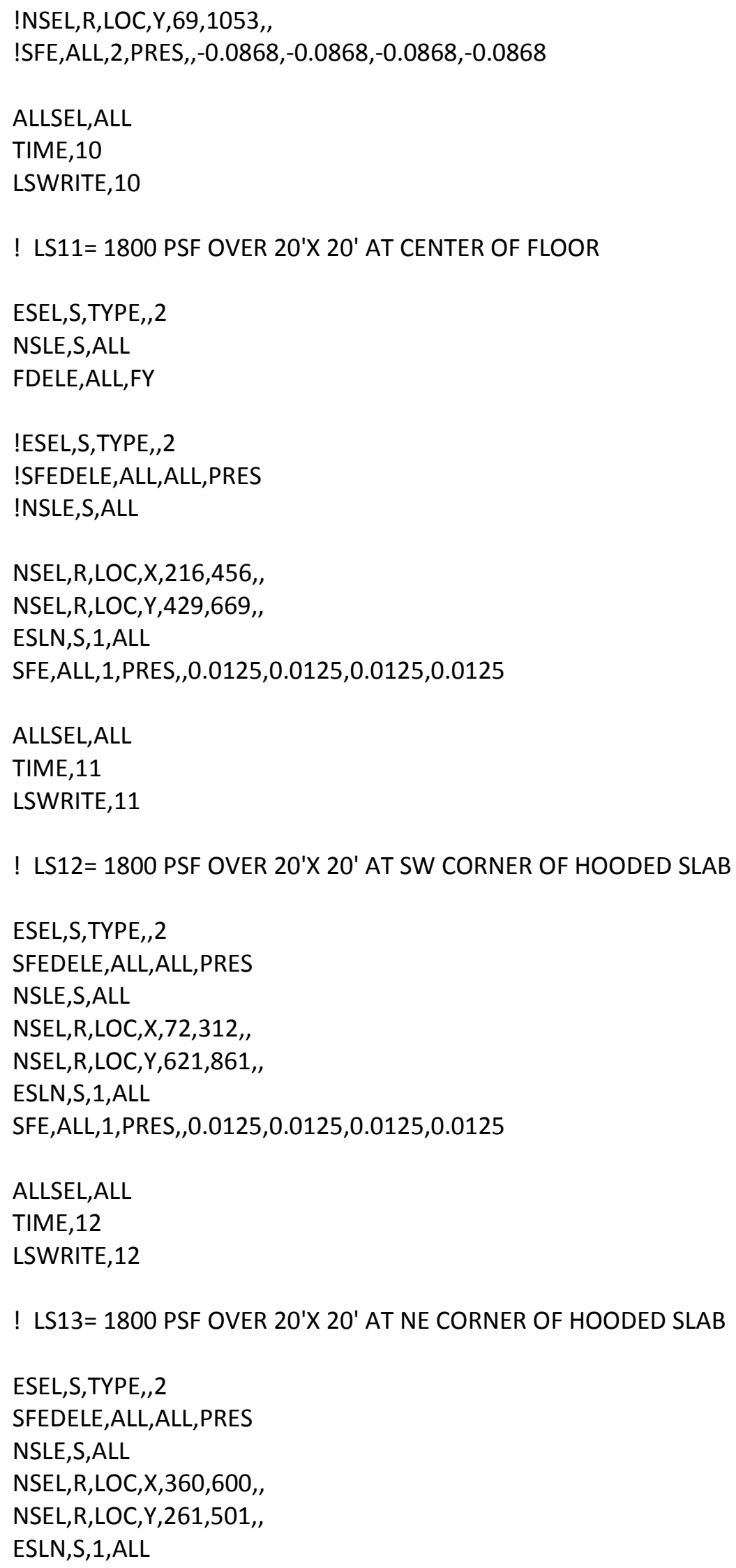




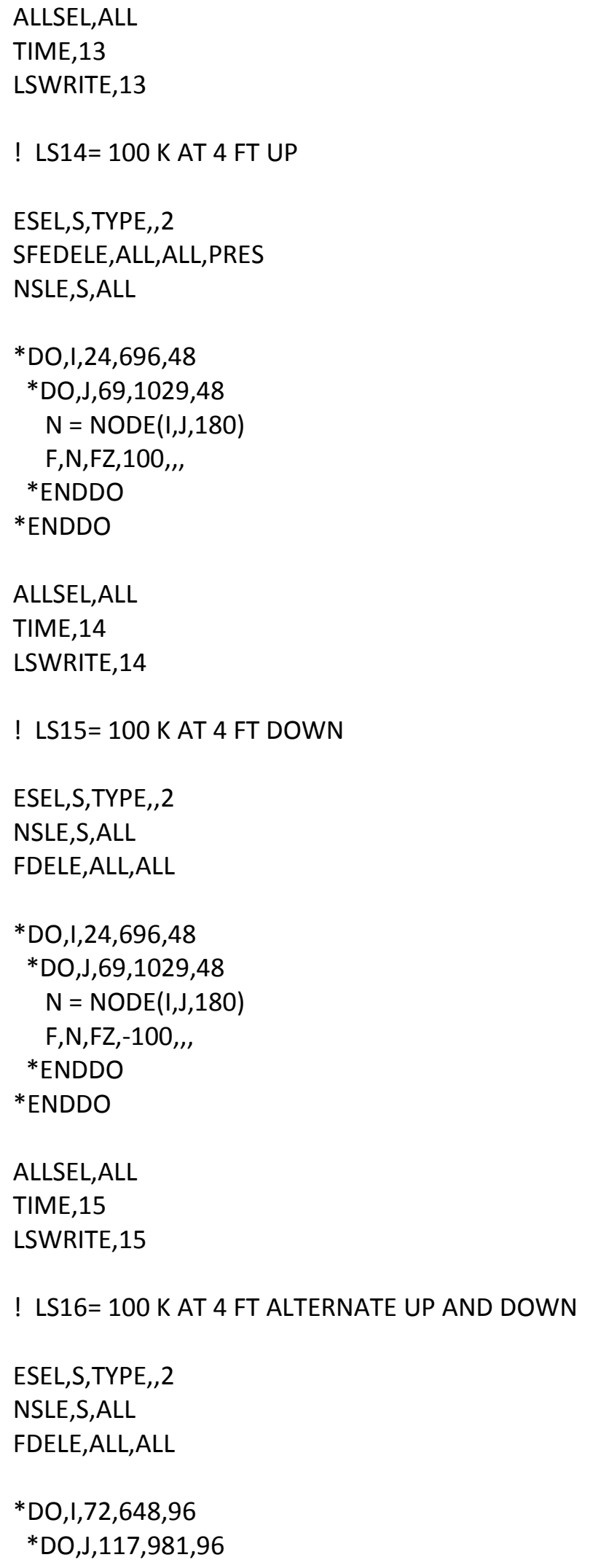




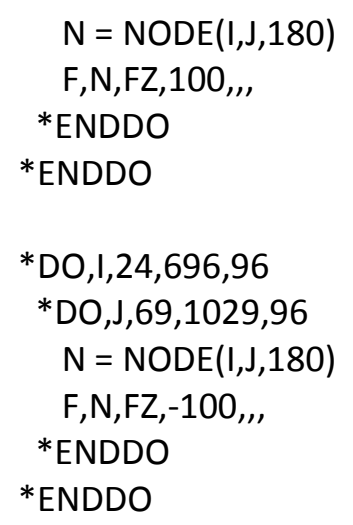

ALLSEL,ALL

TIME,17

LSWRITE,17 
! LS18 $=\mathrm{LS} 17+\mathrm{LS} 8=100 \mathrm{~K}$ up AT $6 \mathrm{FT}$ AND DOWN at $2 \mathrm{ft}$ centered $+\mathrm{X} 50 \mathrm{~K}$ SHEAR AT EVERY LOAD POINT

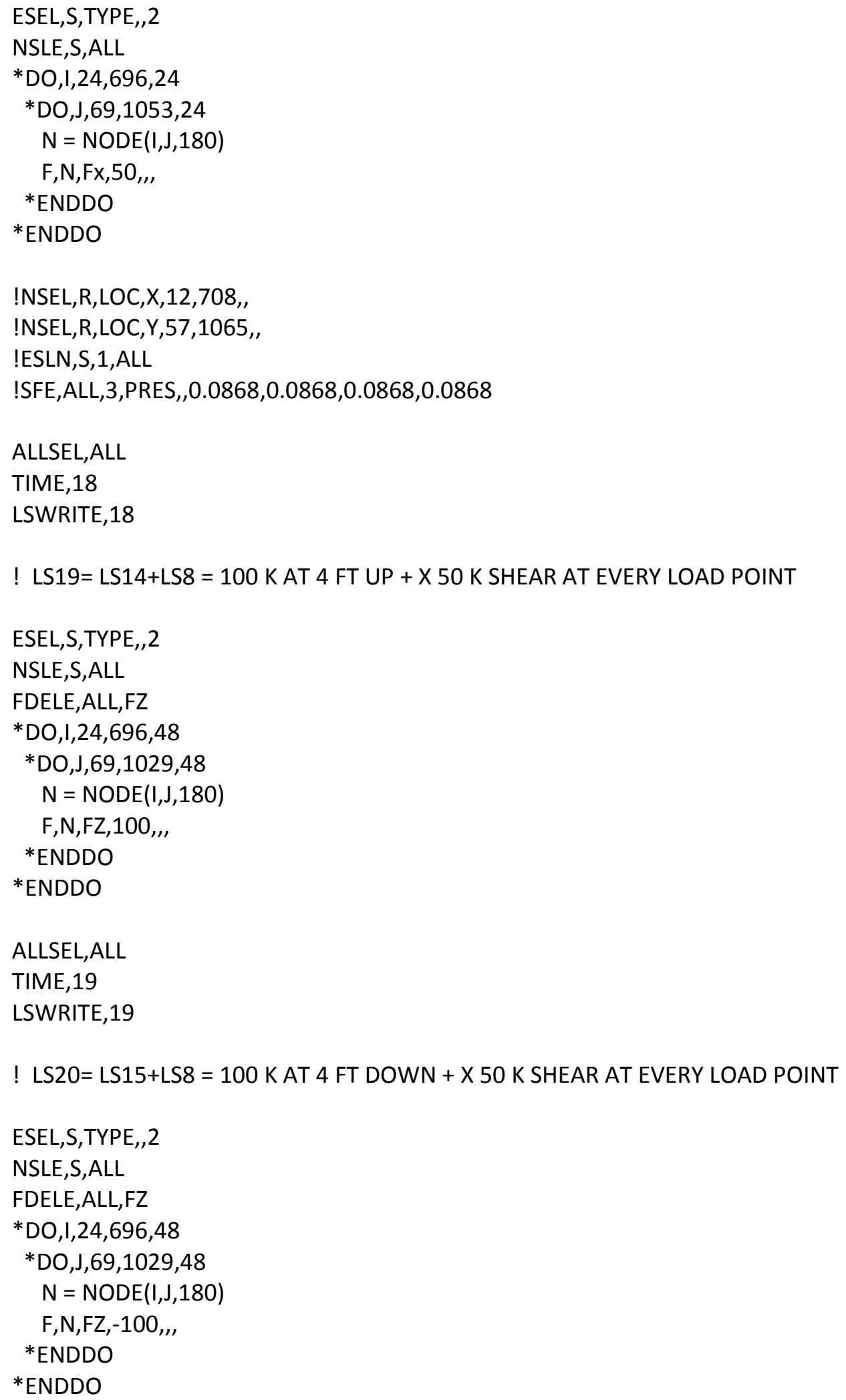




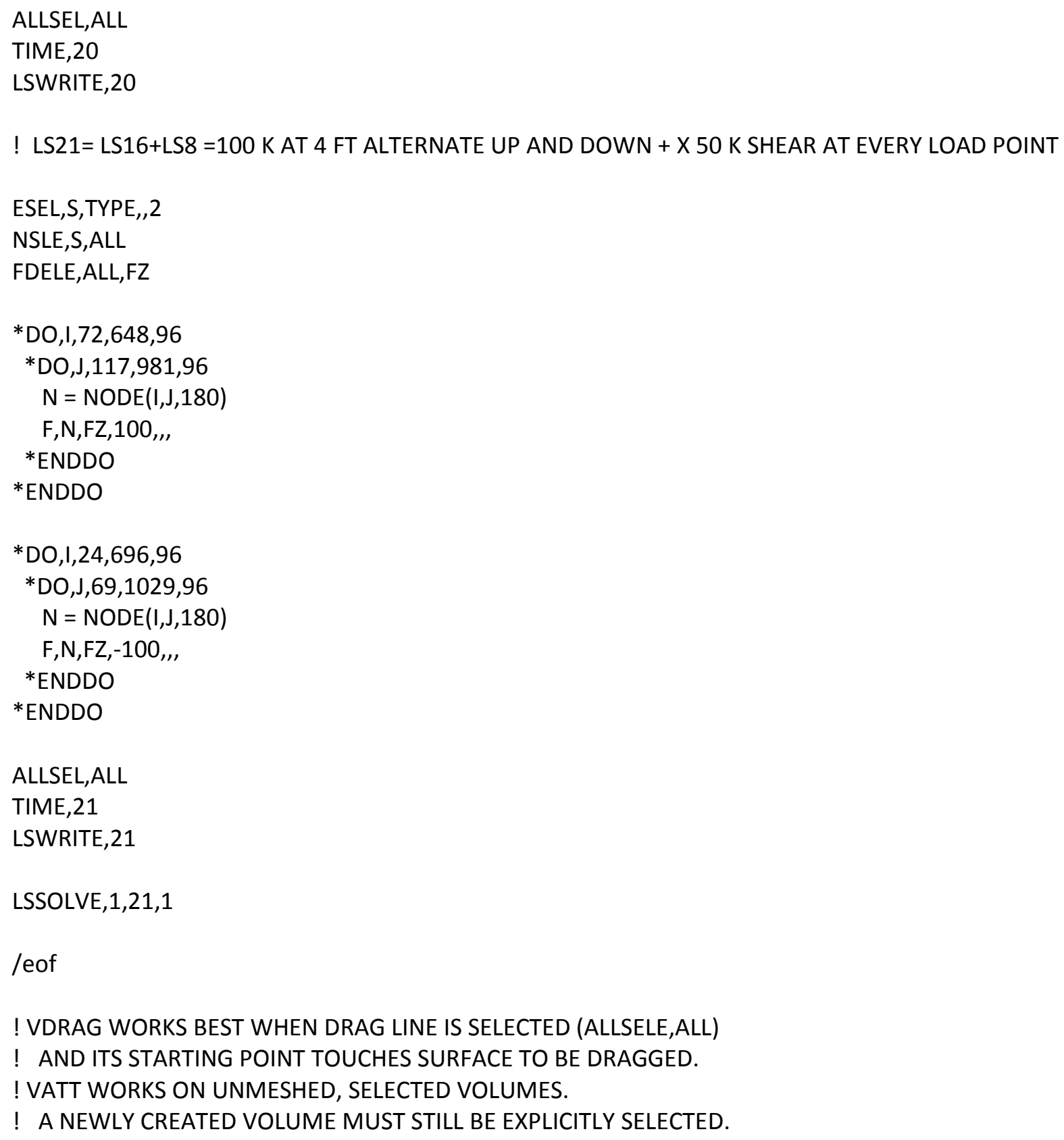




\section{Part 2 Modular Support Structure}

In the $2^{\text {nd }}$ part of this report, a FEM of the modular support structure is described component by component, then assembled and load tested in an example. The models enable a future user of the NFRL to add a potential test structure and perform thermal and structural simulations of the test. This report thus serves as a user's manual for the future NFRL experimenter. Each component is selfcontained, with its own coordinate and numbering system, and can be load tested on its own. In the assembly process, materials, element type, sections only need to be defined once. The numbering system for all ANSYS geometric entities (keypoints, lines, areas, volumes, elements and nodes) is designed so the components can be assembled with the concrete structure without interference. More details about the assembly process can be found in Chapter 13. Typical element size is 3 inches (76 mm), which is the distance between adjacent bolt holes.

Most structural components are defined in two versions: a structural version that uses ANSYS SHELL 181 (structural, 4 nodes) to model steel sections; and a thermal version that uses ANSYS SHELL 131 (thermal, 4 nodes) to model steel sections and a thermal blanket or wrap. Steel thermal properties are taken from Buchanan (2001), whereas the thermal blanket properties default to those of Blazeshield insulation material (NCSTAR 1) and a thickness of one inch $(25 \mathrm{~mm})$. The user can specify other properties.

Table 2.1 Steel properties

\begin{tabular}{|l|l|l|l|l|l|l|l|l|l|l|l|l|}
\hline$T^{\circ} \mathrm{C}$ & 25 & 50 & 100 & 150 & 200 & 300 & 400 & 500 & 600 & 700 & 800 & 1200 \\
\hline$E$ & 30000 & & 30000 & & 27000 & 24000 & 21000 & 18000 & 9300 & 3900 & 2700 & \\
\hline$K$ & 1.37 & & & & & & & & & & 0.693 & 0.693 \\
\hline$C$ & 199.5 & 208.5 & 221.2 & 231.5 & 240.3 & & 274.8 & 302.3 & 344.7 & & & \\
\hline$\rho$ & .2636 & & & & & & & & & & & \\
\hline$\varepsilon$ & 0.9 & & & & & & & & & & & \\
\hline
\end{tabular}

$T$ temperature ${ }^{\circ} \mathrm{C}$; $E$ Young's modulus ksi; $K$ conductivity $\mathrm{W} /(\mathrm{in} \cdot \mathrm{C})$; $C$ Heat capacity J/(lbm.C); $\rho$ mass density $\mathrm{lbm} / \mathrm{in}^{3} ; \varepsilon$ emissivity.

Table 2.2 Insulation properties

\begin{tabular}{|c|c|c|c|}
\hline$T^{\circ} \mathrm{C}$ & 25 & 377 & 677 \\
\hline$K$ & .0013 & .0025 & .0051 \\
\hline$C$ & 362.88 & & 907.2 \\
\hline$\rho$ & .00751 & & \\
\hline$\varepsilon$ & 0.9 & & \\
\hline$t$ & 1.0 & & \\
\hline
\end{tabular}

$t$ thickness inch

\section{References}

Buchanan, A.H. (2001) "Structural Design for Fire Safety," Wiley, New York, NY 10158

NCSTAR 1 (2005) "Final Report on the Collapse of the World Trade Center," National Construction Safety Team Act Report 1, National Institute of Standards and Technology, Gaithersburg, MD 20899 


\section{Chapter 2 Column-foot}

Table 2.3 Properties

\begin{tabular}{|l|l|}
\hline Column-foot & Starting numbers \\
\hline *Keypoints KP1 & 8000 \\
\hline *Lines LIN1 & 19000 \\
\hline *Areas ARE1 & 15000 \\
\hline *Volumes VOL1 & 5000 \\
\hline *Elements ELE1 & 67000 \\
\hline *Nodes NOD1 & 85000 \\
\hline $\begin{array}{l}\text { *Local coordinates } \\
\text { system 11 }\end{array}$ & $\begin{array}{l}\text { x parallel to flanges, point north; } \\
\text { y parallel to web, point west; } \\
\text { z points up; } \\
\text { origin : center of foot (Fig. 2.1) }\end{array}$ \\
\hline Element type & 5 Shell 181 \\
\hline Sections & $\begin{array}{l}\text { *Column HEIGHT } \\
2 \text { (2 inch foot) } \\
3 \text { (flange W12x106) } \\
4 \text { (web W12x106) }\end{array}$ \\
\hline Materials & $\begin{array}{l}10 \text { (W section) } \\
11 \text { (foot) }\end{array}$ \\
\hline *user input or default option.
\end{tabular}

LINES

LINE NUM

AUG $16 \quad 2012$

$17: 54: 27$

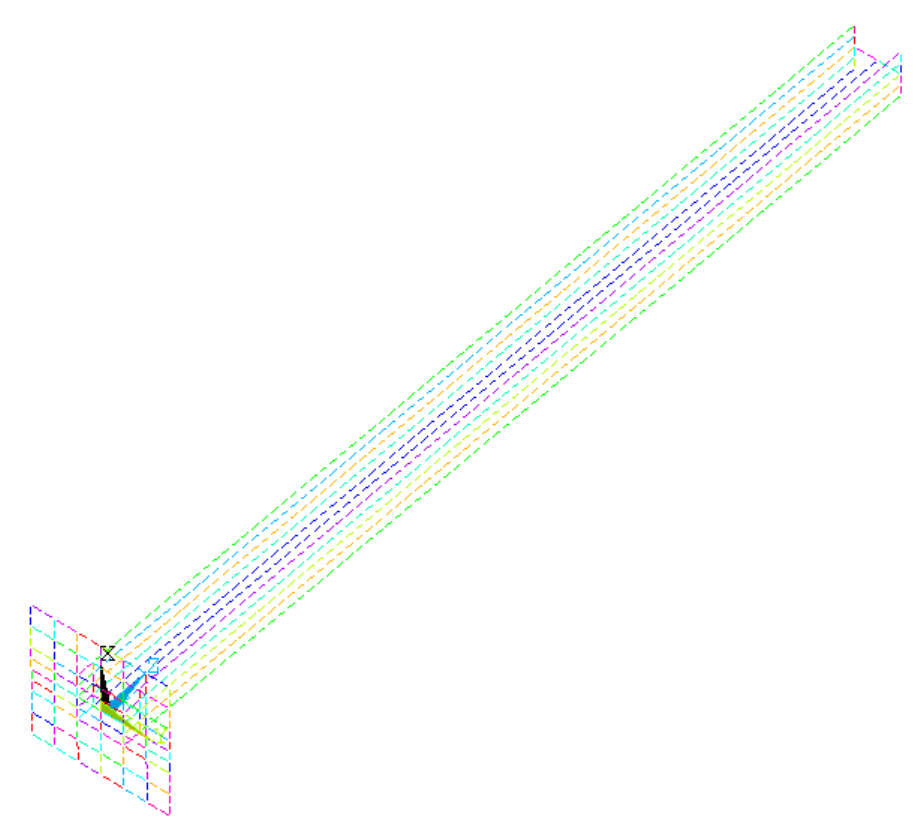

NERL BARE COLUMN AND FOOT

Fig.2.1 column-foot coordinate system 


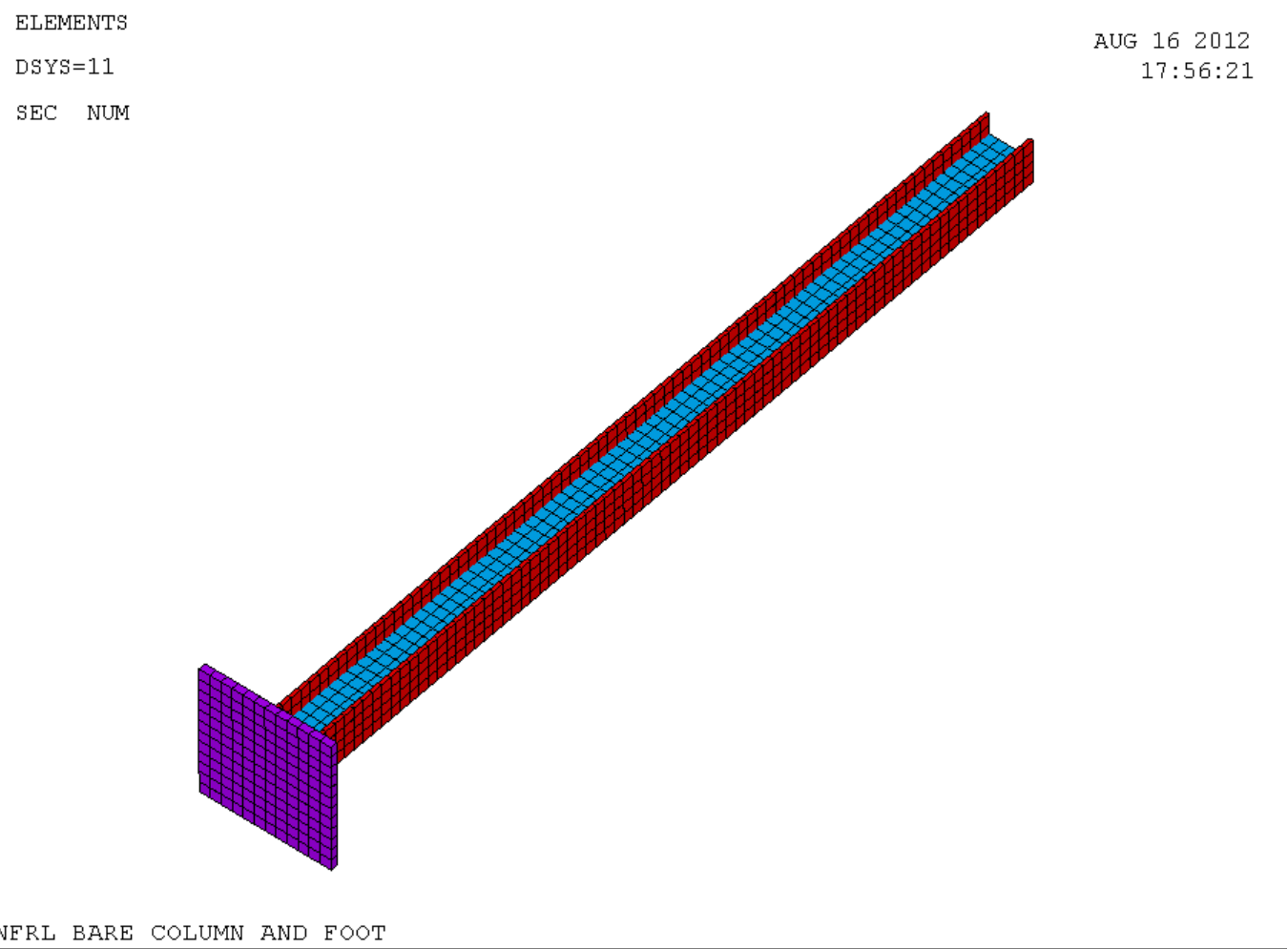

Fig. 2.2 Column-foot

\section{! Column-foot}

FINISH

/CLEAR

/CWD, 'C: \StrongFireLab'

/FILNAME,20120705D,0

/TITLE,NFRL BARE COLUMN AND FOOT

/REPLOT

KEYW,PR_SET,1

KEYW,PR_STRUC,1

KEYW,PR_THERM,0

KEYW,PR_FLUID,0

KEYW,PR_MULTI,0

/PREP7

! UNITS SI for thermal W, J. degree C

! UNITS CUSTOMARY for structural in, kipm, kipf, ksi

! \#\#\#\# material properties

! \# Material \# 10 - Steel for W sections, 50 KSI STEEL

! thermal properties may be unnecessary for structural analysis 


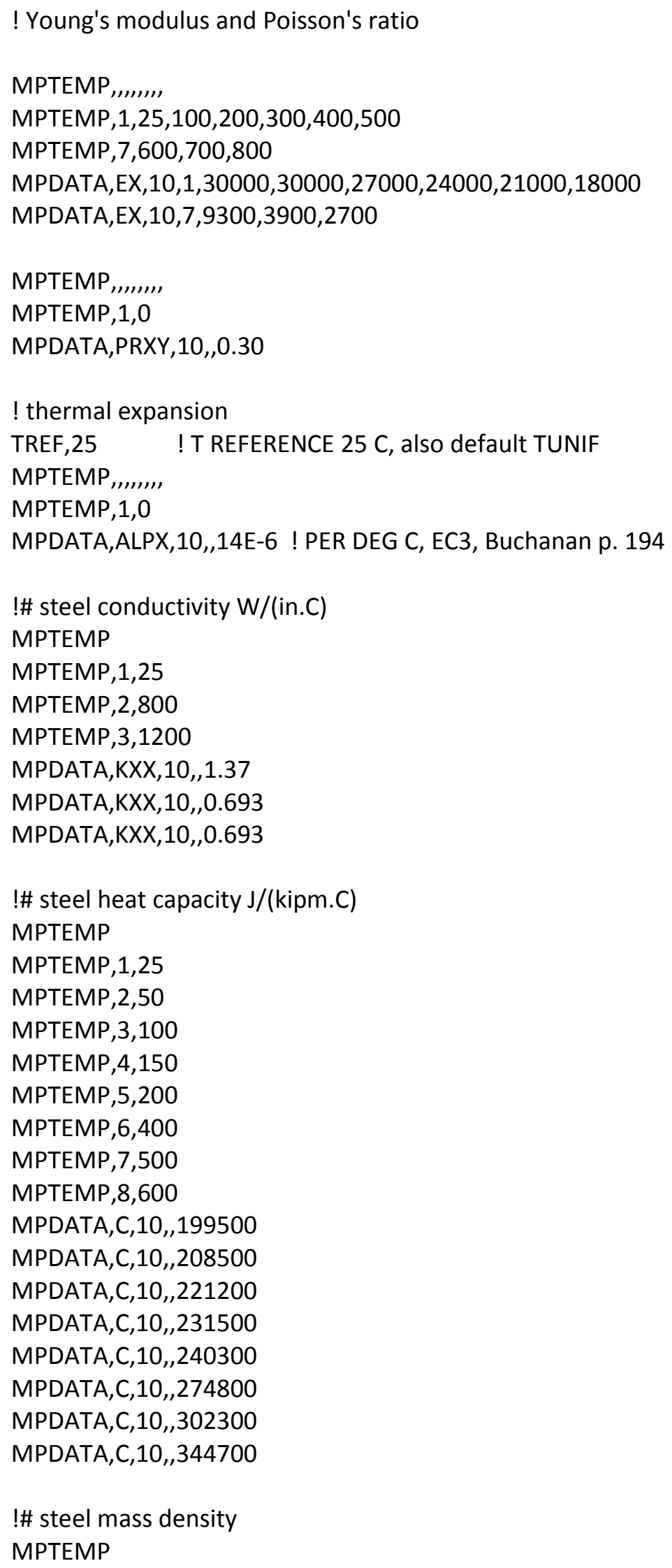




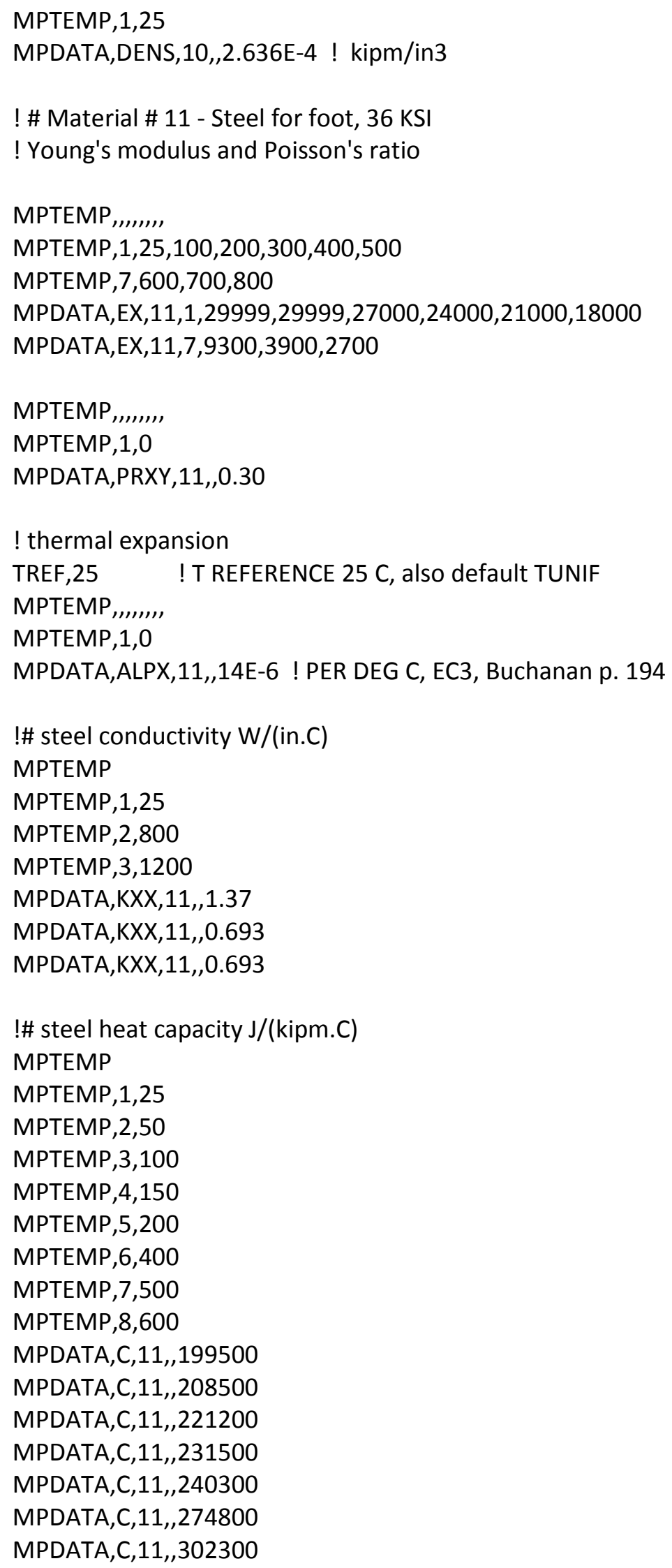


MPDATA,C,11,,344700

!\# steel mass density

MPTEMP

MPTEMP, 1,25

MPDATA,DENS,11,2.636E-4 ! kipm/in3

ET,5,SHELL181

KEYOPT,5,1,0 ! bending and membrane stiffness

KEYOPT,5,3,2 ! full integration with incompatible modes

KEYOPT, 5,8,0 ! store data at bottom of bottom layer and top of top layer KEYOPT,5,9,0 ! no user subroutine to define thickness

KEYOPT,5,10,0 ! no initial stress

sect,2, shell,,foot

secdata, $2,11,0,3$

secoffset,MID

seccontrol,0,0,0, 0, 1, 1, 1

sect,3,shell,,12106f

secdata, $0.99,10,0,3$

secoffset,MID

seccontrol,0,0,0, 0, 1, 1, 1

sect,4,shell,,12106W

secdata, $0.61,10,0,3$

secoffset,MID

seccontrol,0,0,0, 0, 1, 1, 1

! USER INPUT: STARTING NUMBERS, SUB 1============================

*SET,KP1,8000

*SET,LIN1,19000

*SET,ARE1,15000

*SET, VOL1,5000

*SET,ELE1,67000

*SET,NOD1,85000

! \# define model geometry, sheet $11 / 15$ lower left fig

! Local $X$ parallel to flanges, point North.

! Local Y parallel to web, point West.

! Local Z points up.

! user input: origin of local coordinates $=$ CENTER OF FOOT

LOCAL,11,0,100,100,180, , , ,1,1,

CSYS,11,

HEIGHT=240 ! COLUMN HEIGHT INCLUDES FOOTING, INCHES

$\mathrm{H}=\mathrm{HEIGHT}-1$

! END OF user input 


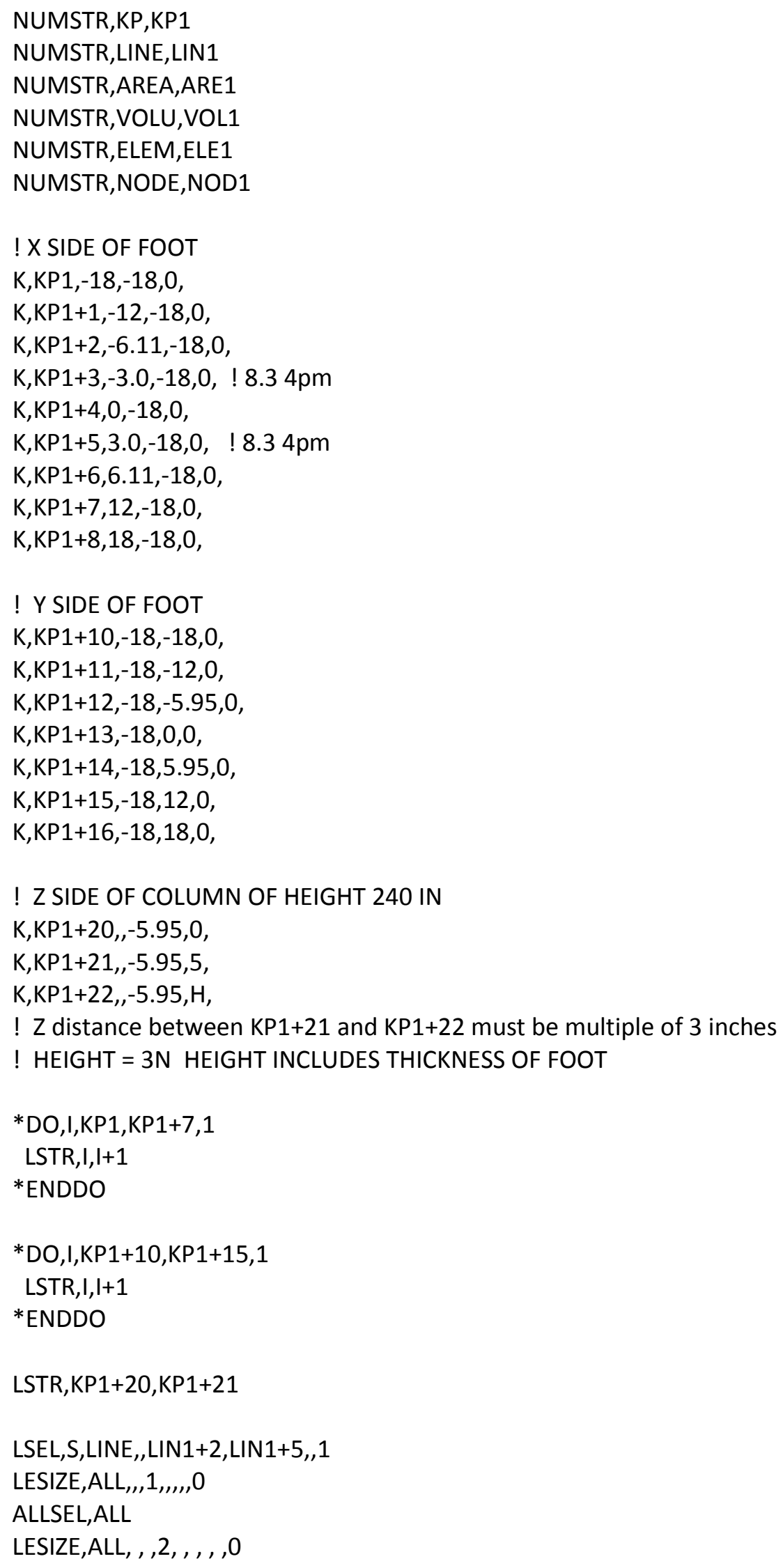


LSTR,KP1+21,KP1+22

!KSEL,S,KP , KP1+21,KP1+22, ,

!LSLK,S,1

!*get, aa,line,0,num,min

!LESIZE, aa,3, , , , , , , 1

LESIZE,LIN1+15,3, , , , , , , 1

! BASE OF FOOT

KSEL,S,LOC,X,-18.1,18.1,, KSEL,R,LOC,Y,-18.1,-17.9,, KSEL,R,LOC,Z,,0.1,,

LSLK,S,1

CM,FOOTX,LINE

KSEL,S,LOC,X,-18.1,-17.9,, KSEL,R,LOC,Y,-18.1,18.1,, KSEL,R,LOC,Z,0,0.1,,

LSLK,S,1

CM,FOOTY,LINE

! height of column

KSEL,S,LOC,X,-0.1,0.1,

KSEL,R,LOC,Y,-6.0,-5.90,,

KSEL,R,LOC,Z,,$H_{\text {, }}$

LSLK,S,1

CM,COLUMNZ,LINE

! foot area

ALLSEL,ALL

ADRAG,FOOTX,,,,,,FOOTY,,,,,,

! columnn flange 1

LSEL,S,LOC,X,-6.10,6.10,

LSEL,R,LOC,Y,-6.0,-5.9,,

LSEL,R,LOC,Z,,0.1,,

CM,COLFLAN1,LINE

! columnn flange 2

LSEL,S,LOC,X,-6.10,6.10,,

LSEL,R,LOC,Y,5.9,6.0,,

LSEL,R,LOC,Z,,0.1,,

CM,COLFLAN2,LINE

! columnn WEB

LSEL,S,LOC,X,-..5,.5, , LSEL,R,LOC,Y,-5.9,5.9,, 


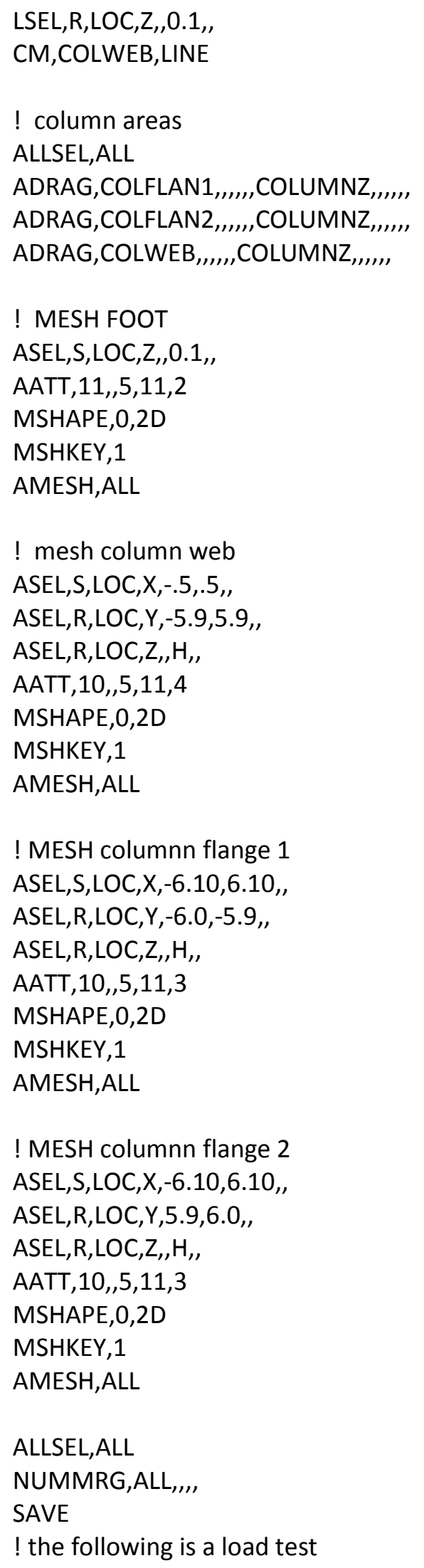


/SOLU

ANTYPE,0 ! static analysis

! constrain $\mathrm{Z}=0$ end

NSEL,S,LOC,Z,0,0.1,

$D, A L L, U X, \ldots, \ldots, \ldots, \ldots$,

$D, A L L, U Y, \ldots, \ldots, \ldots, \ldots$,

$\mathrm{D}, \mathrm{ALL}, \mathrm{UZ}, \ldots, \ldots, \ldots, \ldots$,

! LOAD UPPER END Z=HEIGHT

ALLSEL,ALL

$\mathrm{H} 1=\mathrm{H}-1$

$\mathrm{H} 2=\mathrm{H}+.1$

NSEL,S,LOC,Z,H1,H2, ,

$F, A L L, F Y, 1,$,

ALLSEL,ALL

OUTPR,STAT

OUTRES,STAT

SOLVE

/EOF 


\section{Chapter 3 Wrapped column-foot}

Table 3.1 Properties

\begin{tabular}{|c|c|}
\hline Wrapped column-foot & Starting numbers \\
\hline *Keypoints KP1 & 8000 \\
\hline *Lines LIN1 & 19000 \\
\hline${ }^{*}$ Areas ARE1 & 15000 \\
\hline *Volumes VOL1 & 5000 \\
\hline *Elements ELE1 & 67000 \\
\hline *Nodes NOD1 & 85000 \\
\hline $\begin{array}{l}\text { *Local coordinates } \\
\text { system } 11\end{array}$ & $\begin{array}{l}\text { x parallel to flanges, point north; } \\
\text { y parallel to web, point west; } \\
\text { z points up; } \\
\text { origin : center of foot (Fig. } 2.1 \text { ) }\end{array}$ \\
\hline Element type & 5 Shell 131 \\
\hline Sections & $\begin{array}{l}\text { *Column HEIGHT } \\
2 \text { ( } 2 \text { in foot) } \\
3 \text { (flange W12×106) } \\
4 \text { (web W12x106) } \\
\text { *5 (1 inch foot wrap) } \\
\text { *6 (1 inch flange wrap) } \\
* 7 \text { ( } 1 \text { inch side wrap) }\end{array}$ \\
\hline Materials & $\begin{array}{l}10 \text { (W section) } \\
11 \text { (foot) } \\
* 13 \text { (insulation) }\end{array}$ \\
\hline
\end{tabular}




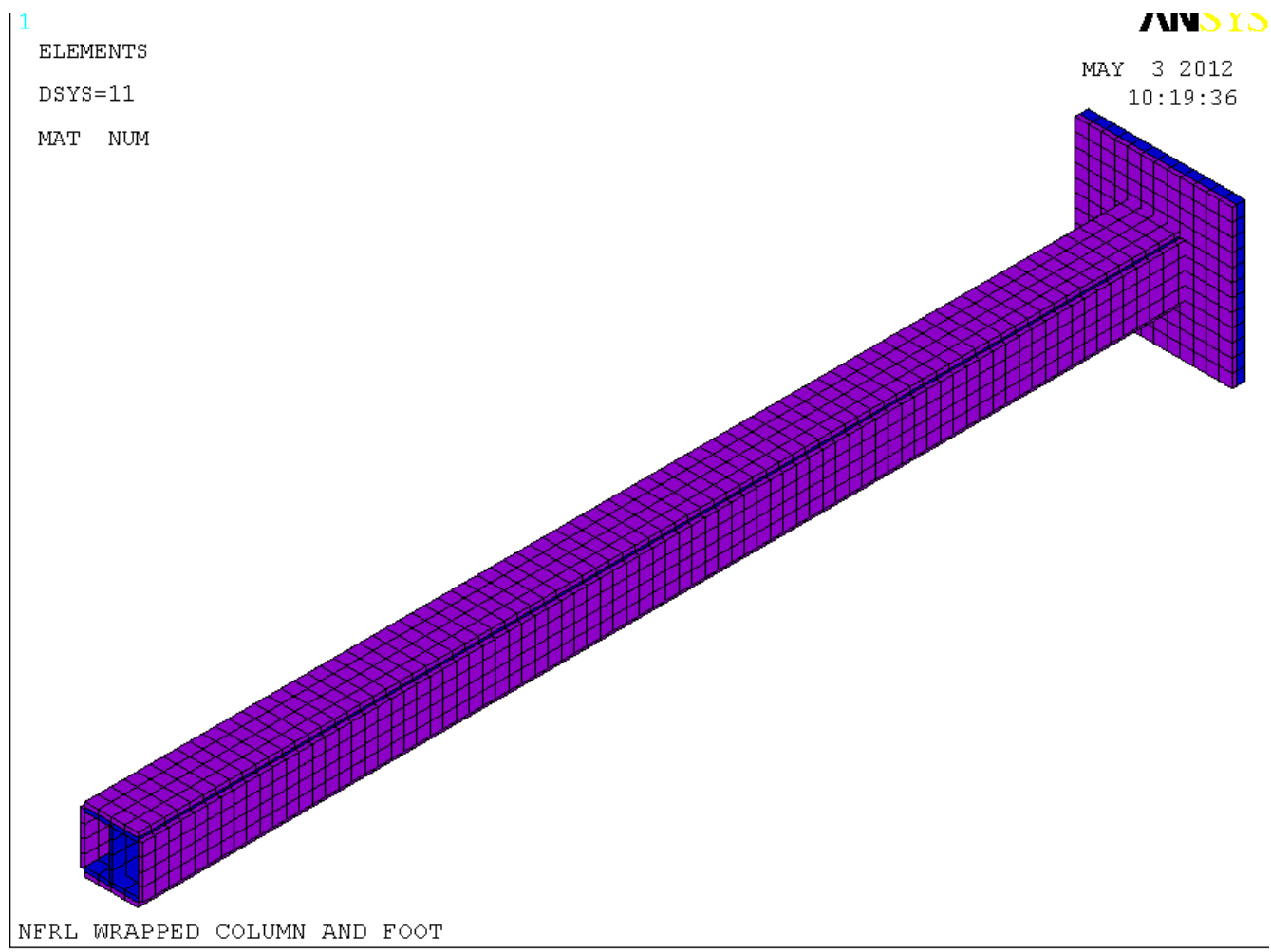

Fig. 3.1 Wrapped column-foot

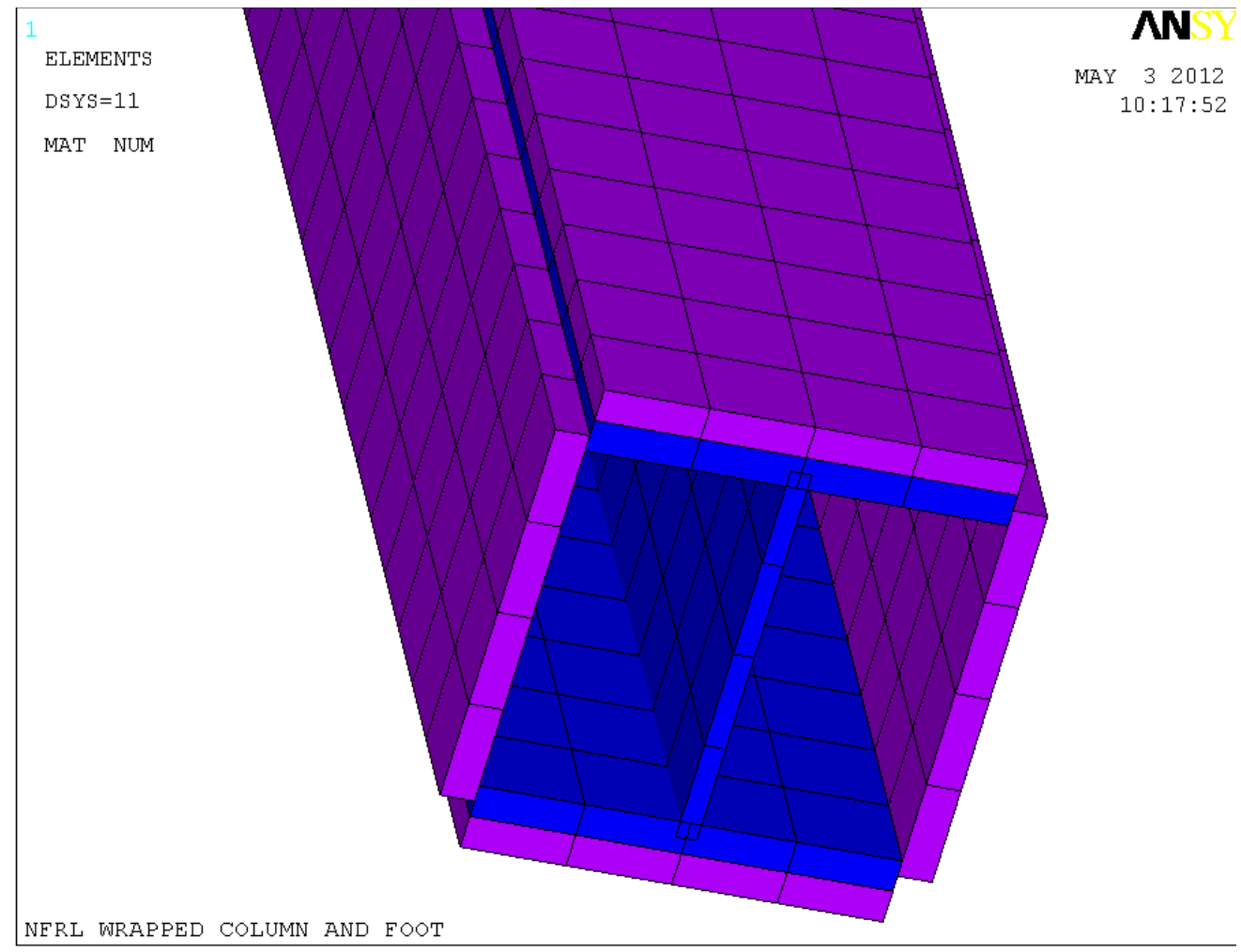

Fig. 3.2 Detail of wrapped column-foot 


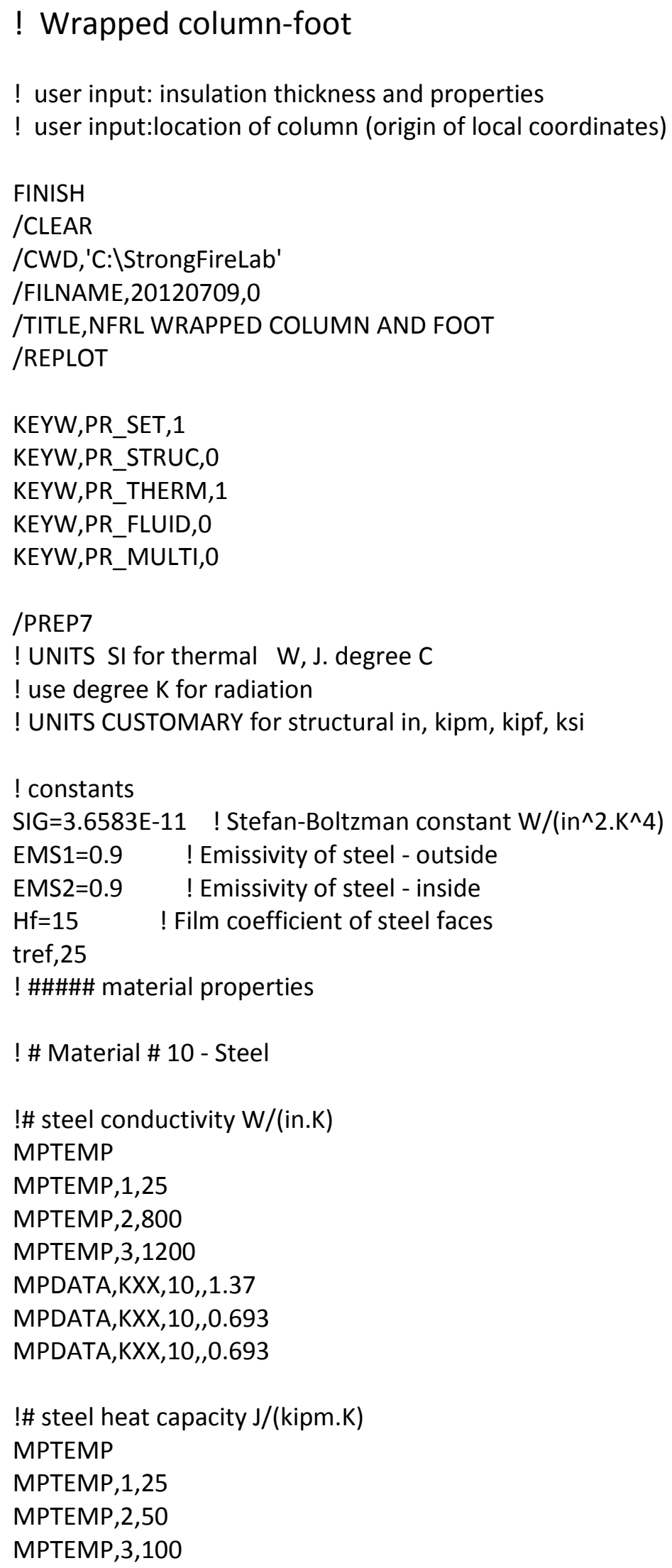




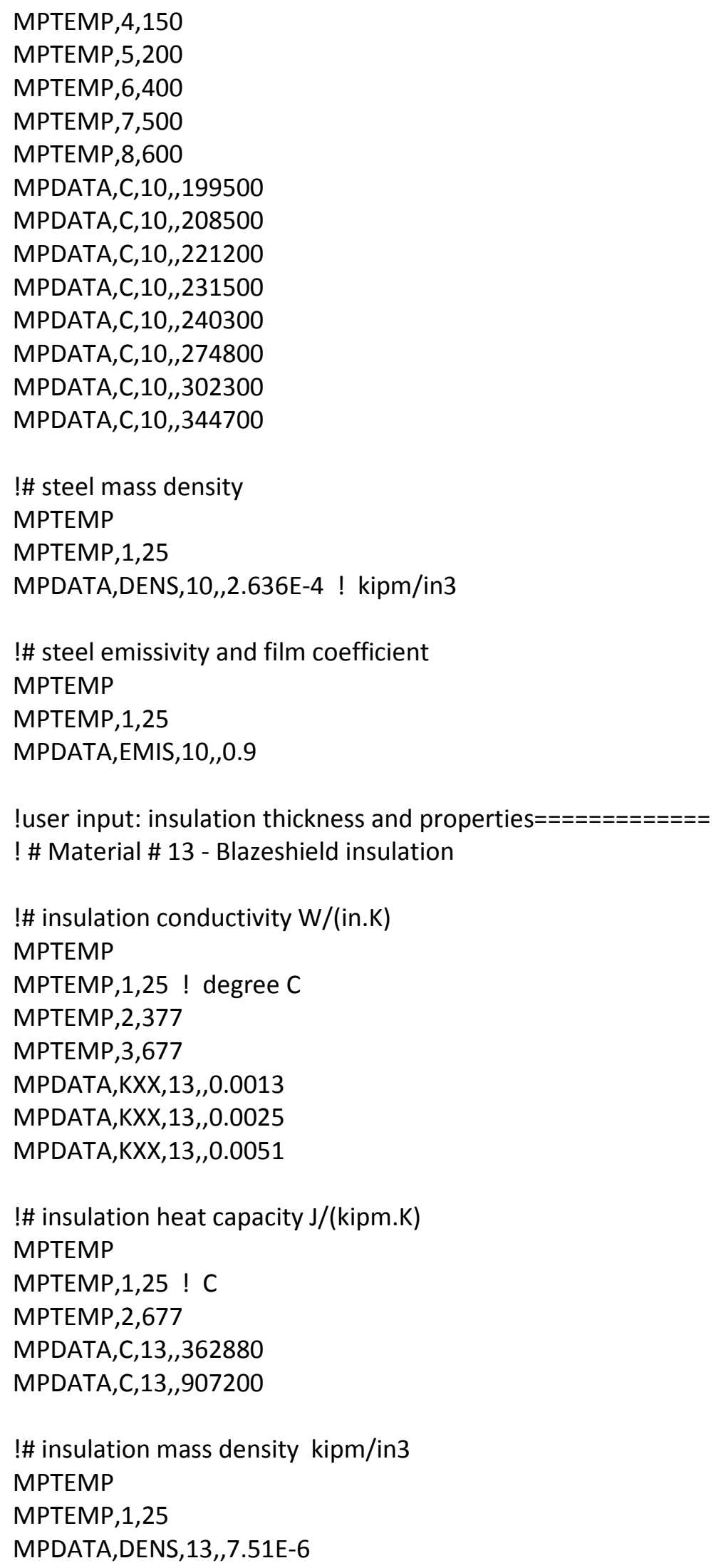


!\# insulation emissivity

MPTEMP

MPTEMP,1,25

MPDATA,EMIS,13,,0.9

! Insulation blanket thickness is $\mathrm{T}$

$\mathrm{T}=1$

! careful that same emissivity can be used for steel and insulation

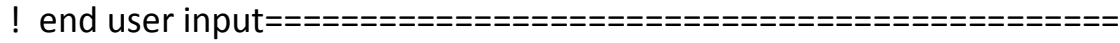

ET,5,SHELL131

KEYOPT,5,2,0 ! average film temperature

KEYOPT,5,3,0 ! quadratic temperature distribution through thickness

KEYOPT, $5,4,0$ ! use sect defined above

KEYOPT,5,6,0 ! no paint

!KEYOPT,5,8,2 use this option when insulation is present- data at top, bottom and mid of all layers !SHELL,MID use this option when insulation is present-results calculated at mid, not averaged from top and bottom

sect,2,shell,,foot ! bare steel

secdata, 2,10,0,3

secoffset,MID

seccontrol,0,0,0, 0, 1, 1, 1

sect,3,shell,,12106f ! bare steel

secdata, $0.99,10,0,3$

secoffset,MID

seccontrol,0,0,0, 0, 1, 1, 1

sect,4,shell,,12106W ! bare steel

secdata, $0.61,10,0,3$

secoffset,MID

seccontrol,0,0,0, 0, 1, 1, 1

sect,5, shell,,footw

secdata,2,10,0,3 ! steel layer 1 at bottom

secdata, $T, 13,0,3$

secoffset, user,-T/2 ! relative to midthickness of shell

seccontrol,0,0,0, 0, 1, 1, 1

sect,6,shell,,flangew

secdata,0.99,10,0,3 ! steel layer 1 at bottom

secdata, $\mathrm{T}, 13,0,3$

secoffset, user,-T/2 ! relative to midthickness of shell

seccontrol,0,0,0, 0, 1, 1, 1

sect,7,shell,,sidew ! blanket

secdata, T,13,0,3 
secoffset, bot

seccontrol,0,0,0, 0, 1, 1, 1

! USER INPUT: STARTING NUMBERS, SUB 1=============================

*SET,KP1,8000

*SET,LIN1,19000

*SET,ARE1,15000

*SET, VOL1,5000

*SET,ELE1,67000

*SET,NOD1,85000

! \# define model geometry, sheet 11/15 lower left fig

! Local $X$ parallel to flanges, point North.

! Local Y parallel to web, point West.

! Local Z points up.

! user input: origin of local coordinates $=$ CENTER OF FOOT

LOCAL,11,0,100,100,180, , , ,1,1,

CSYS,11,

HEIGHT=240 ! COLUMN HEIGHT INCLUDES FOOTING, INCHES

$\mathrm{H}=\mathrm{HEIGHT}-1$

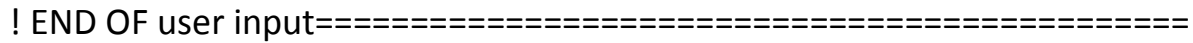

TREF, 25

NUMSTR, KP,KP1

NUMSTR,LINE,LIN1

NUMSTR,AREA,ARE1

NUMSTR,VOLU,VOL1

NUMSTR,ELEM,ELE1

NUMSTR,NODE,NOD1

! X SIDE OF FOOT

$\mathrm{K}, \mathrm{KP} 1,-18,-18,0$,

K,KP1+1,-12,-18,0,

$\mathrm{K}, \mathrm{KP} 1+2,-6.11,-18,0$,

$\mathrm{K}, \mathrm{KP} 1+3,-3.0,-18,0, \quad ! 8.34 \mathrm{pm}$

$\mathrm{K}, \mathrm{KP} 1+4,0,-18,0$,

$\mathrm{K}, \mathrm{KP} 1+5,3.0,-18,0, \quad ! 8.34 \mathrm{pm}$

$\mathrm{K}, \mathrm{KP} 1+6,6.11,-18,0$,

$\mathrm{K}, \mathrm{KP} 1+7,12,-18,0$,

$\mathrm{K}, \mathrm{KP} 1+8,18,-18,0$,

! Y SIDE OF FOOT

$\mathrm{K}, \mathrm{KP} 1+10,-18,-18,0$,

$\mathrm{K}, \mathrm{KP} 1+11,-18,-12,0$,

K,KP1+12,-18,-5.95,0,

$\mathrm{K}, \mathrm{KP} 1+13,-18,0,0$, 


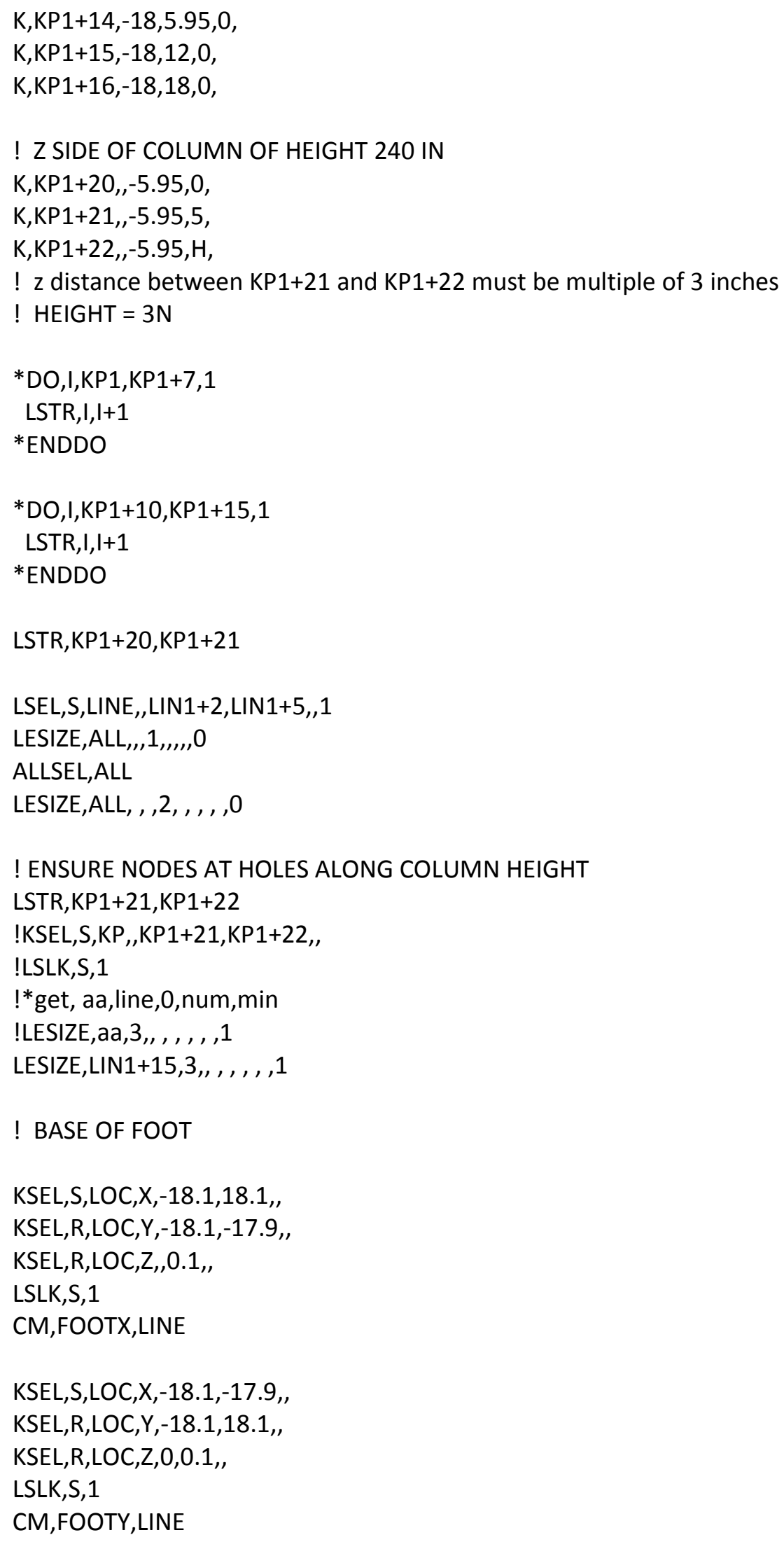




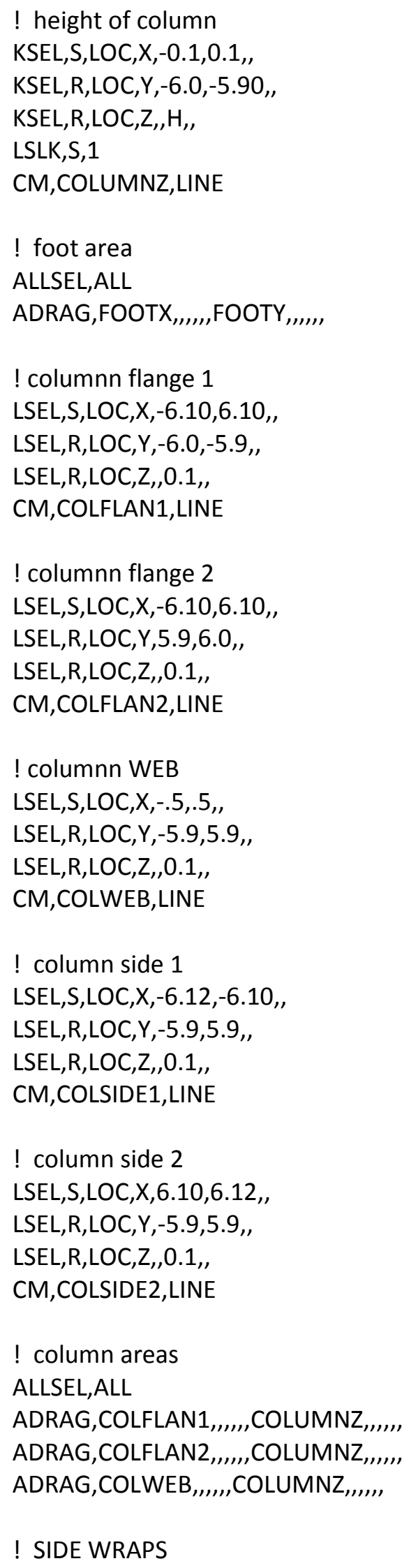




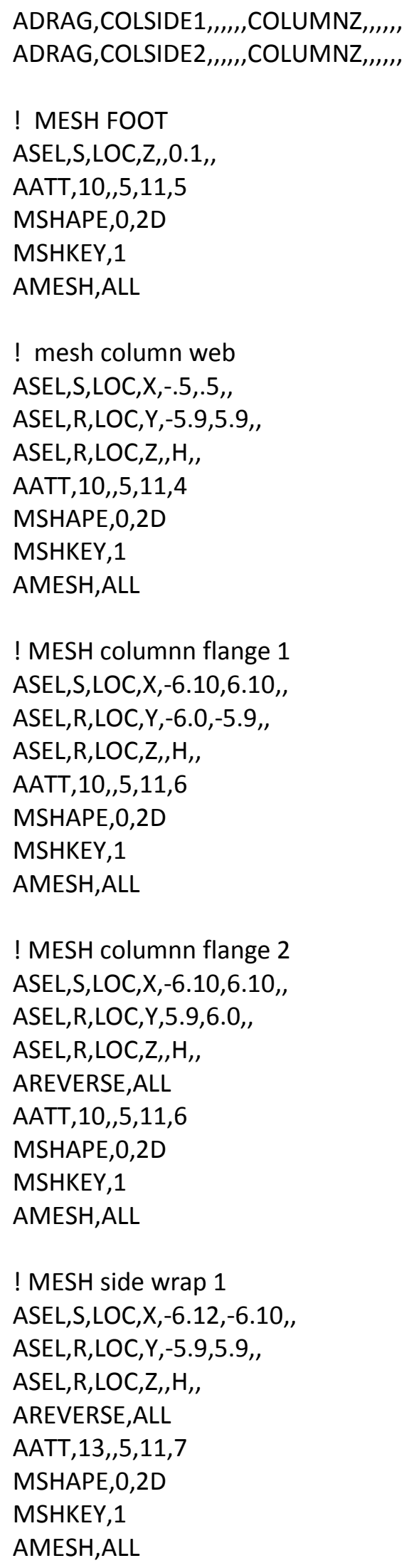




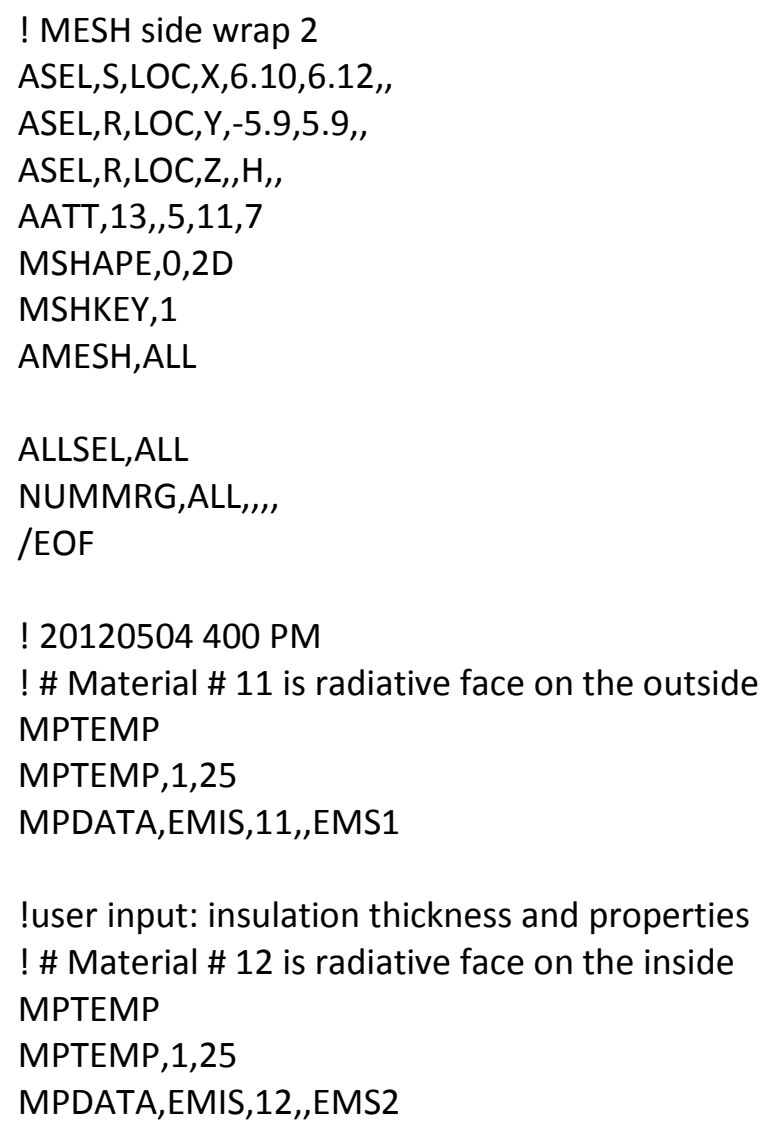




\section{Chapter 4 Wall-column}

Table 4.1 Properties

\begin{tabular}{|c|c|}
\hline Wall-column & Starting numbers \\
\hline *Keypoints KPO & 8100 \\
\hline *Lines LINO & 19200 \\
\hline *Areas ARE0 & 15100 \\
\hline *Volumes VOLO & 5000 \\
\hline *Elements ELEO & 69000 \\
\hline *Nodes NOD0 & 88000 \\
\hline $\begin{array}{l}\text { *Local coordinates } \\
\text { system } 12\end{array}$ & $\begin{array}{l}\text { x parallel to flanges, points North; } \\
\text { y parallel to web, points West; } \\
\text { z points up; } \\
\text { origin: center of I-section at base (Fig. 4.1). }\end{array}$ \\
\hline Element type & 5 Shell 181 \\
\hline Sections & 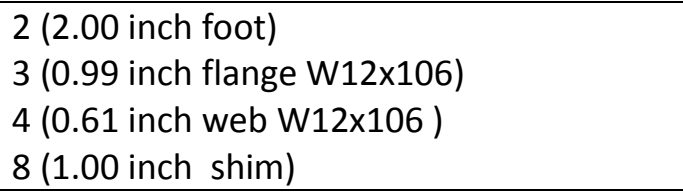 \\
\hline Materials & $\begin{array}{l}10,11 \text { (W sections) } \\
12(\text { shim) }\end{array}$ \\
\hline
\end{tabular}

\section{$\mathbf{N}$}

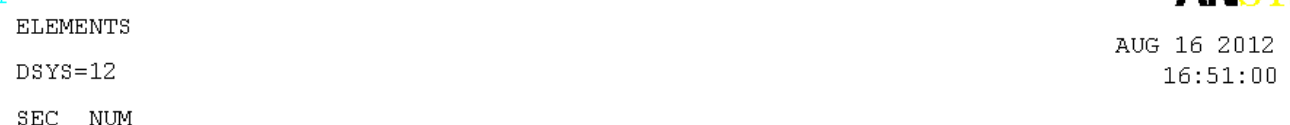

SEC NUM

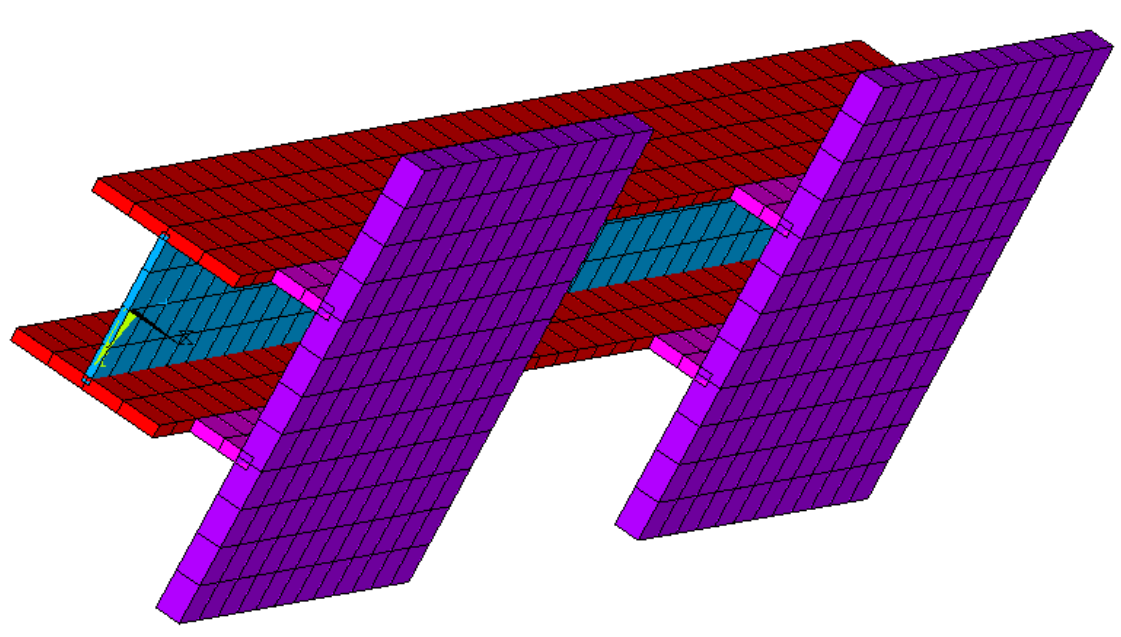

NFRL WALL COLUMN AND FOOT

Fig. 4.1 Wall-column 
! Wall-column LAST TESTED 7.20.2012

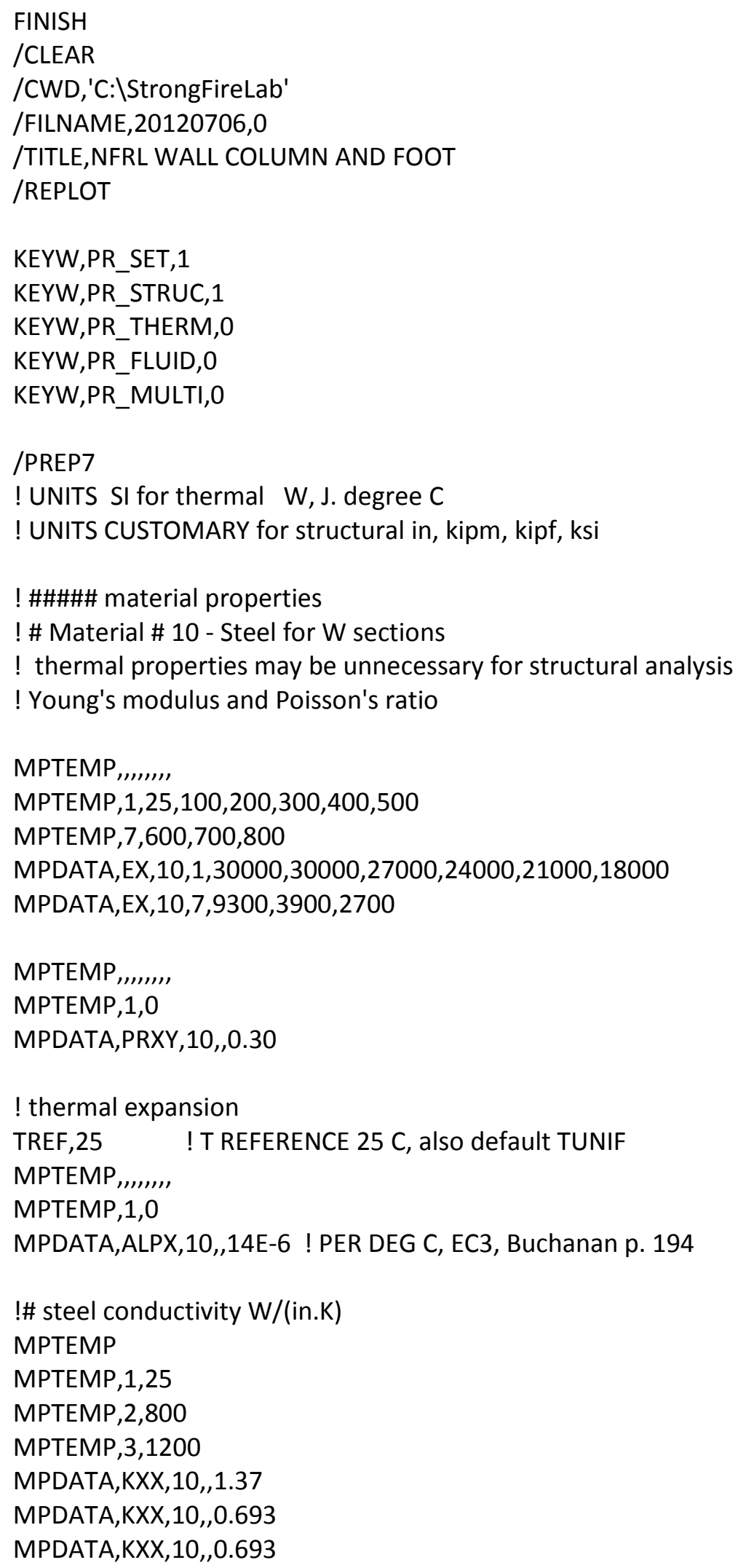




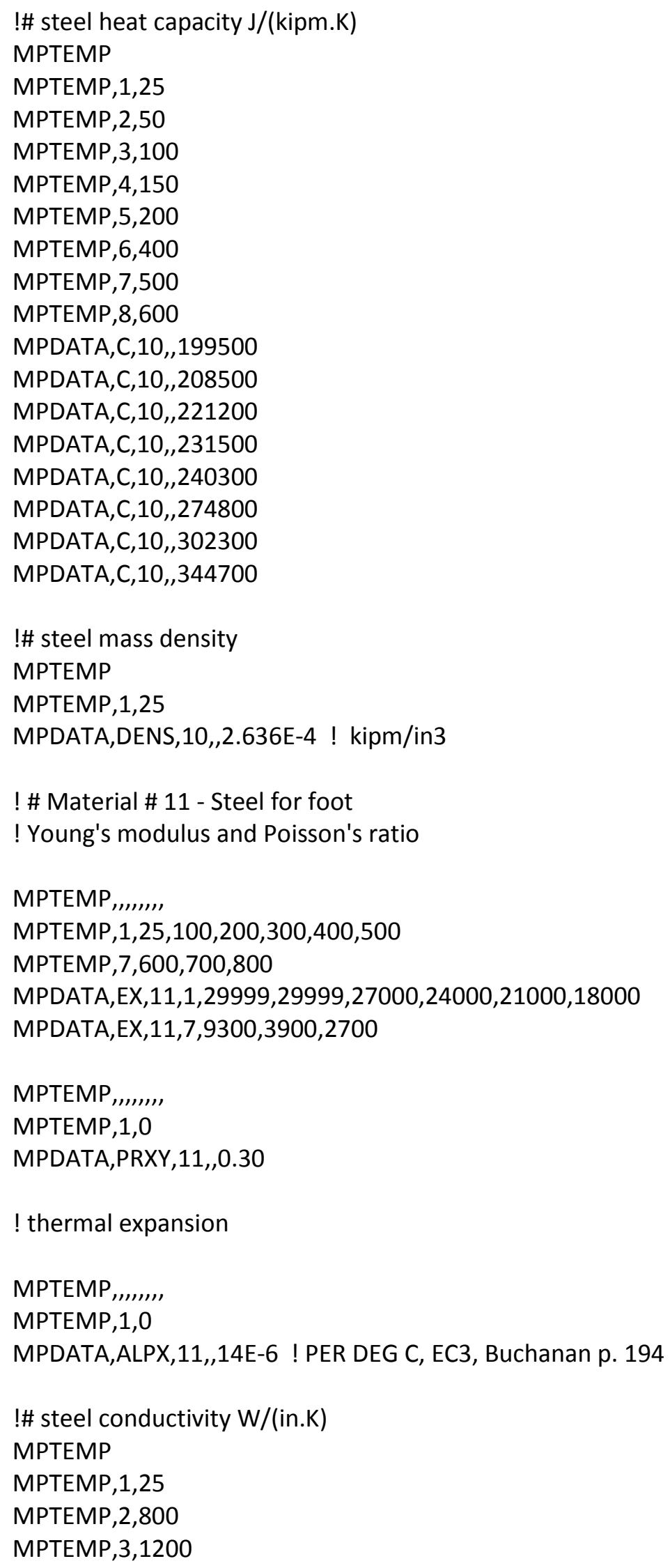




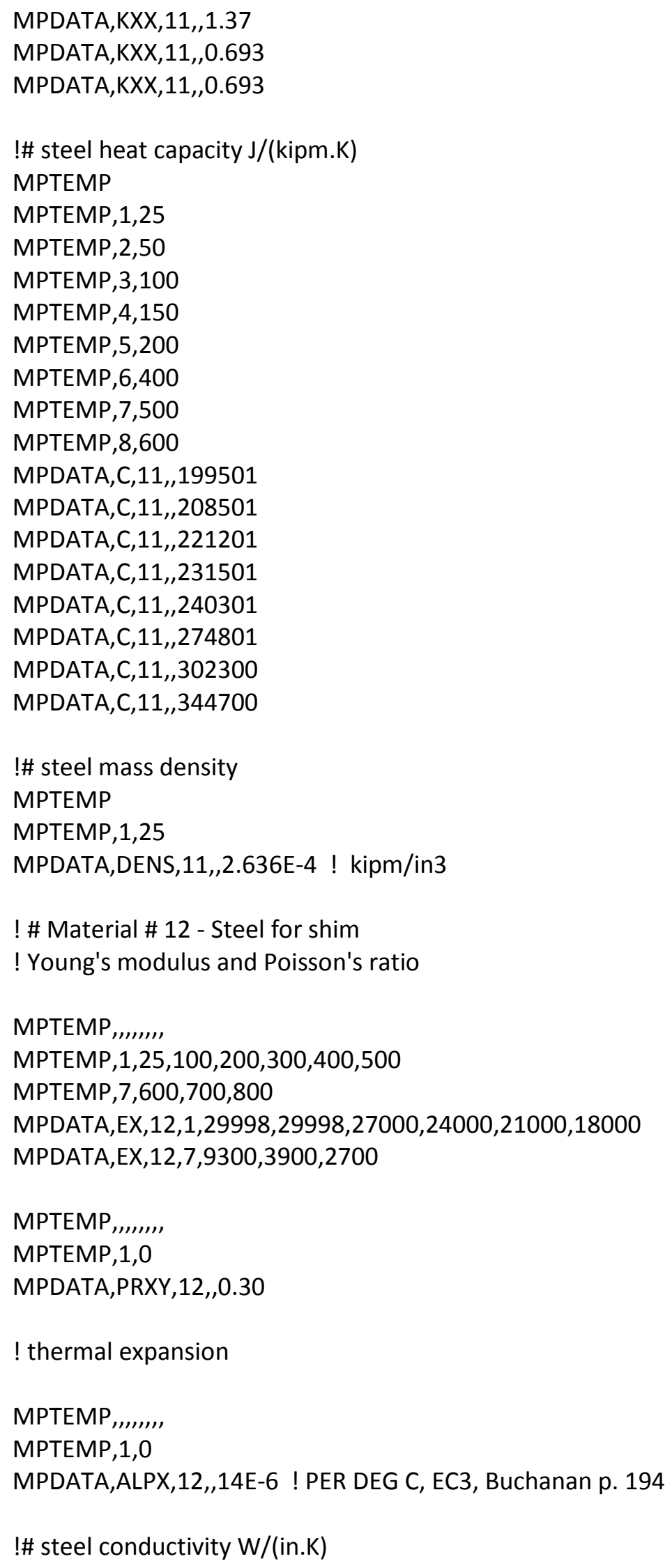




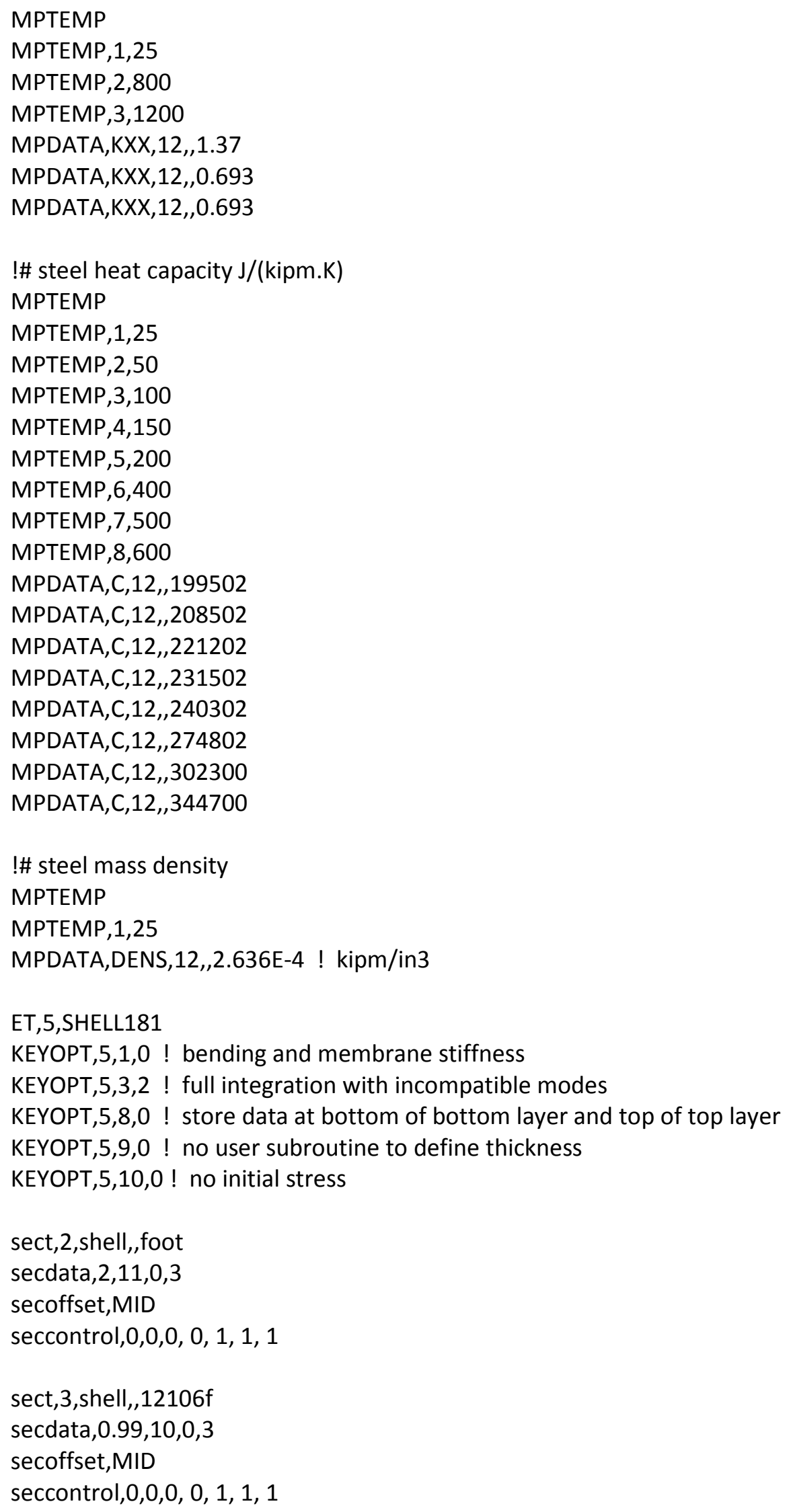




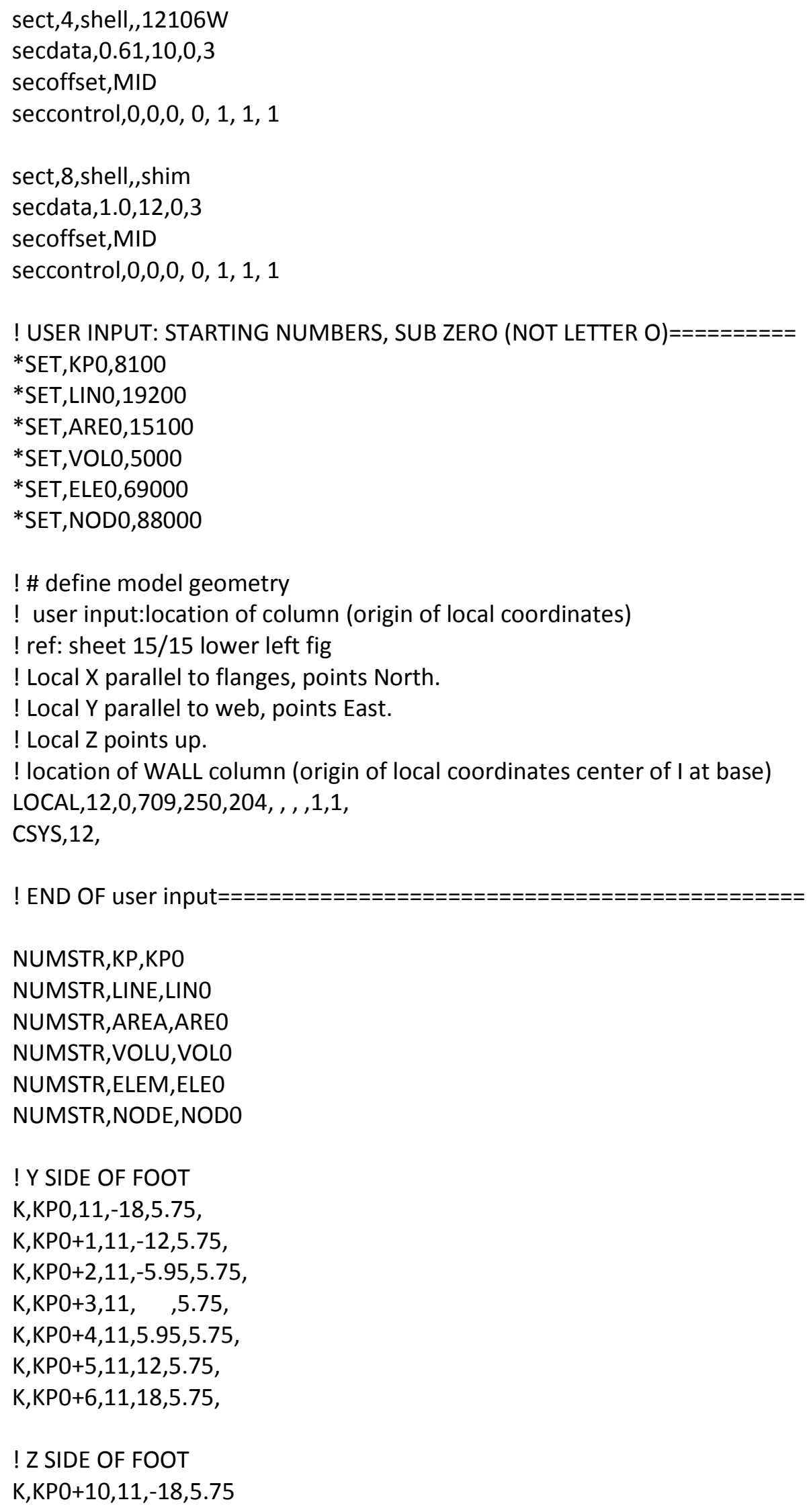


K,KP0+11,11,-18,11.75

$\mathrm{K}, \mathrm{KPO}+12,11,-18,35.75$

K,KPO+13,11,-18,41.75

*DO,I,KPO,KPO+5,1

LSTR,I,I+1

*ENDDO

*DO,I,KP0+10,KP0+12,1

LSTR,I,I+1

*ENDDO

KSEL,S,KP, ,KPO,KPO+13, , LSLK,S,1

LESIZE,ALL, , ,2, , , , , 1 $\mathrm{KSEL}, \mathrm{S}, \mathrm{KP}$, KPO+11,KPO+12,

LSLK,S,1

*get, aa,line,0,num, min

LESIZE, aa, 3, , , , , , , 1

KSEL,S,KP, KPO,KPO+6,

LSLK,S,1

CM,WALLFOOTY,LINE

KSEL,S,KP, ,KPO+10,KP0+13, LSLK,S,1

CM,WALLFOOTZ,LINE

! X SIDE OF SHIM

$\mathrm{K}, \mathrm{KPO}+20,6.11,5.95,5.75$

$\mathrm{K}, \mathrm{KPO}+21,11,5.95,5.75$

LSTR,KPO+20,KPO+21

$\mathrm{KSEL}, \mathrm{S}, \mathrm{KP}$, ,KPO+20,KPO+21,

LSLK,S,1

LESIZE,ALL, , ,2, , , , , 1

CM,SHIMX,LINE

! Z SIDE OF SHIM

$\mathrm{K}, \mathrm{KPO}+30,6.11,5.95,5.75$

$\mathrm{K}, \mathrm{KPO}+31,6.11,5.95,41.75$

LSTR,KPO+30,KPO+31

$\mathrm{KSEL}, \mathrm{S}, \mathrm{KP}$,,KPO+30,KPO+31,

LSLK,S,1

LESIZE,ALL, $3,$, , , , , , , 1

CM,SHIMZ,LINE

! base of columnn web

$\mathrm{K}, \mathrm{KPO}+40,-5.95$, 


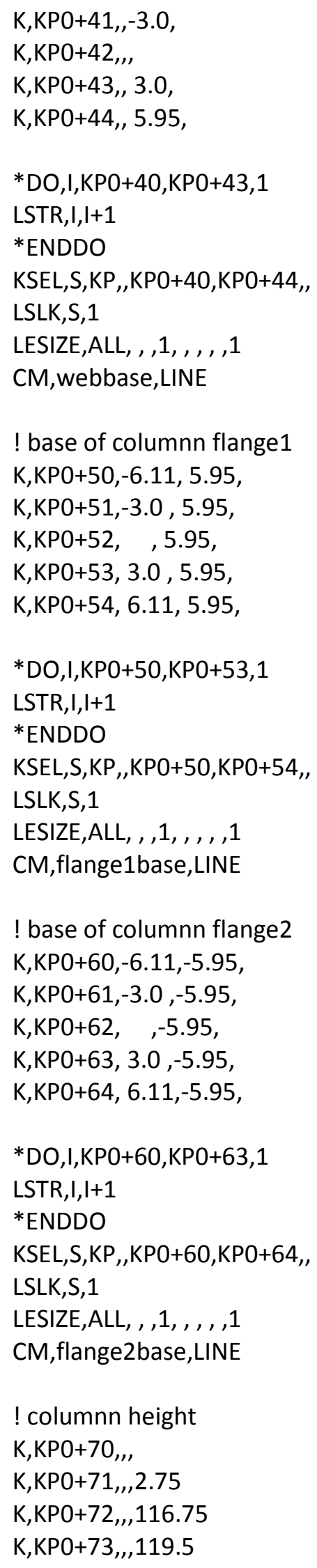




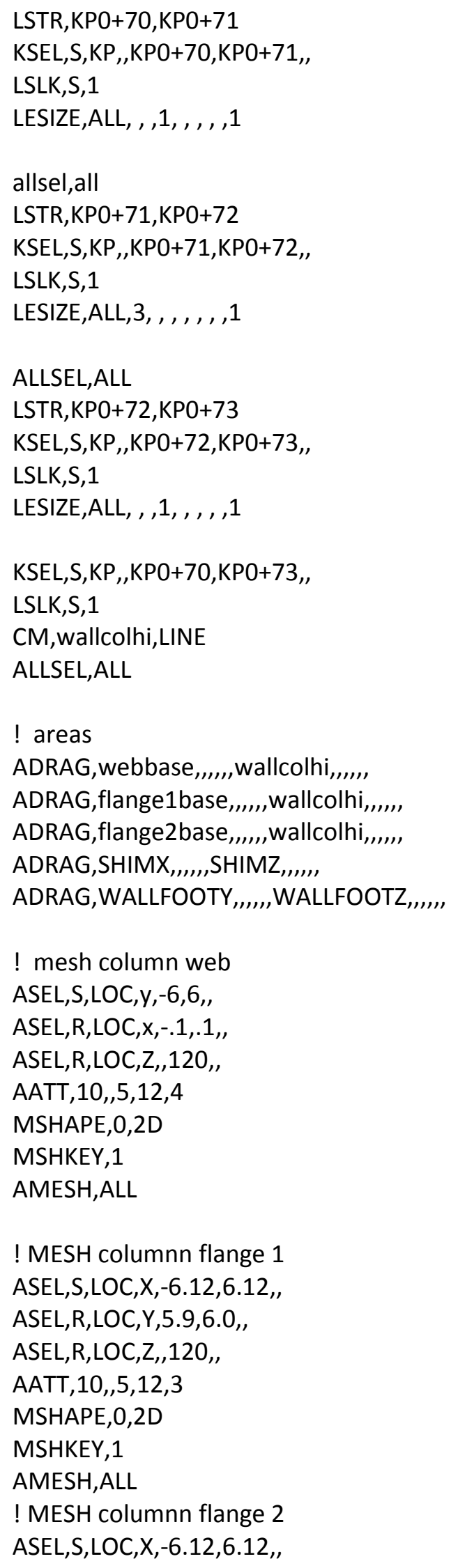




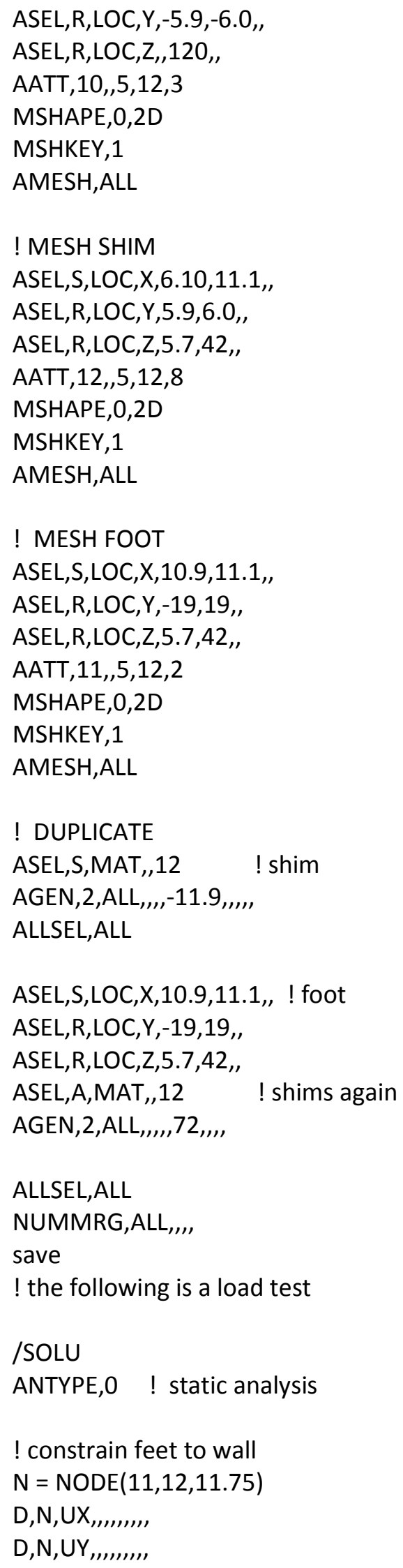




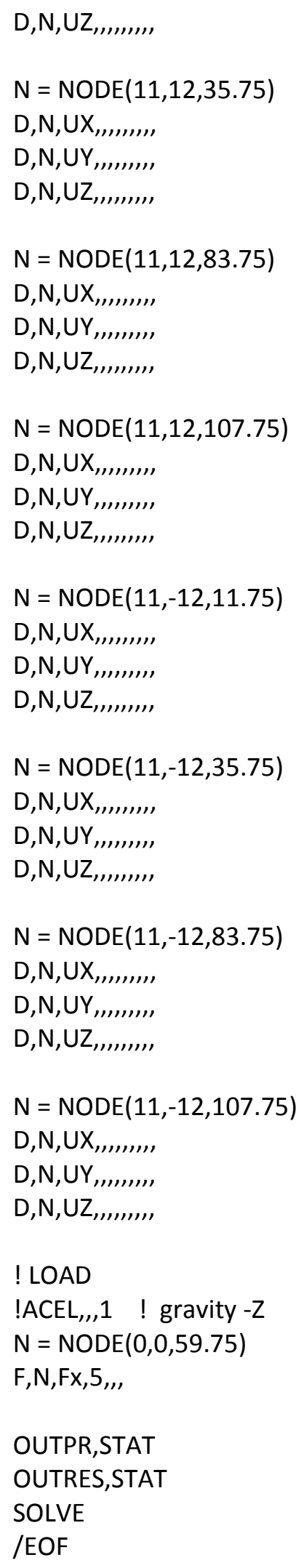




\section{Chapter 5 Wrapped wall-column}

Table 5.1 Properties

\begin{tabular}{|c|c|}
\hline Wrapped wall-column & Starting numbers \\
\hline *Keypoints KPO & 8100 \\
\hline *Lines LINO & 19200 \\
\hline${ }^{*}$ Areas AREO & 15100 \\
\hline *Volumes VOLO & 5000 \\
\hline *Elements ELE0 & 69000 \\
\hline *Nodes NOD0 & 88000 \\
\hline $\begin{array}{l}\text { *Local coordinates } \\
\text { system } 12\end{array}$ & $\begin{array}{l}\text { x parallel to flanges, points North; } \\
\text { y parallel to web, points West; } \\
\text { z points up; } \\
\text { origin: center of I-section at base (Fig. 4.1). }\end{array}$ \\
\hline Element type & 5 Shell 131 \\
\hline Sections & 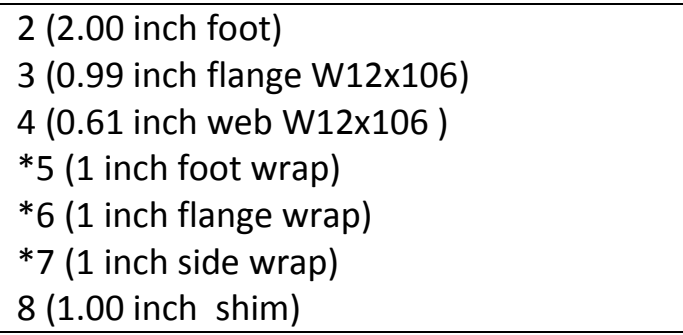 \\
\hline Materials & $\begin{array}{l}\text { 10, } 11 \text { (W sections) } \\
12 \text { (shim) } \\
* 13 \text { (insulation) }\end{array}$ \\
\hline
\end{tabular}




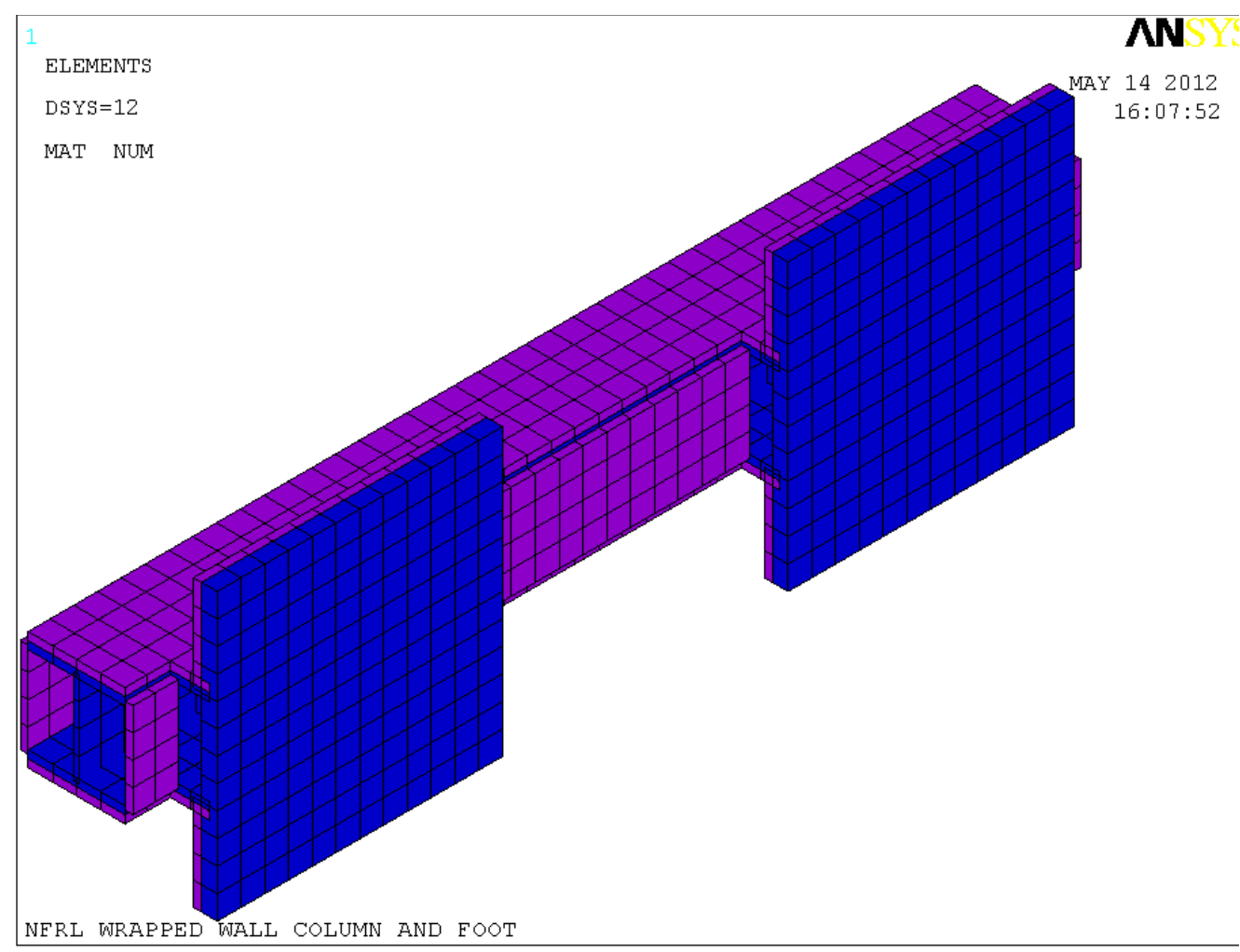

Fig. 5.1 Wrapped wall-column

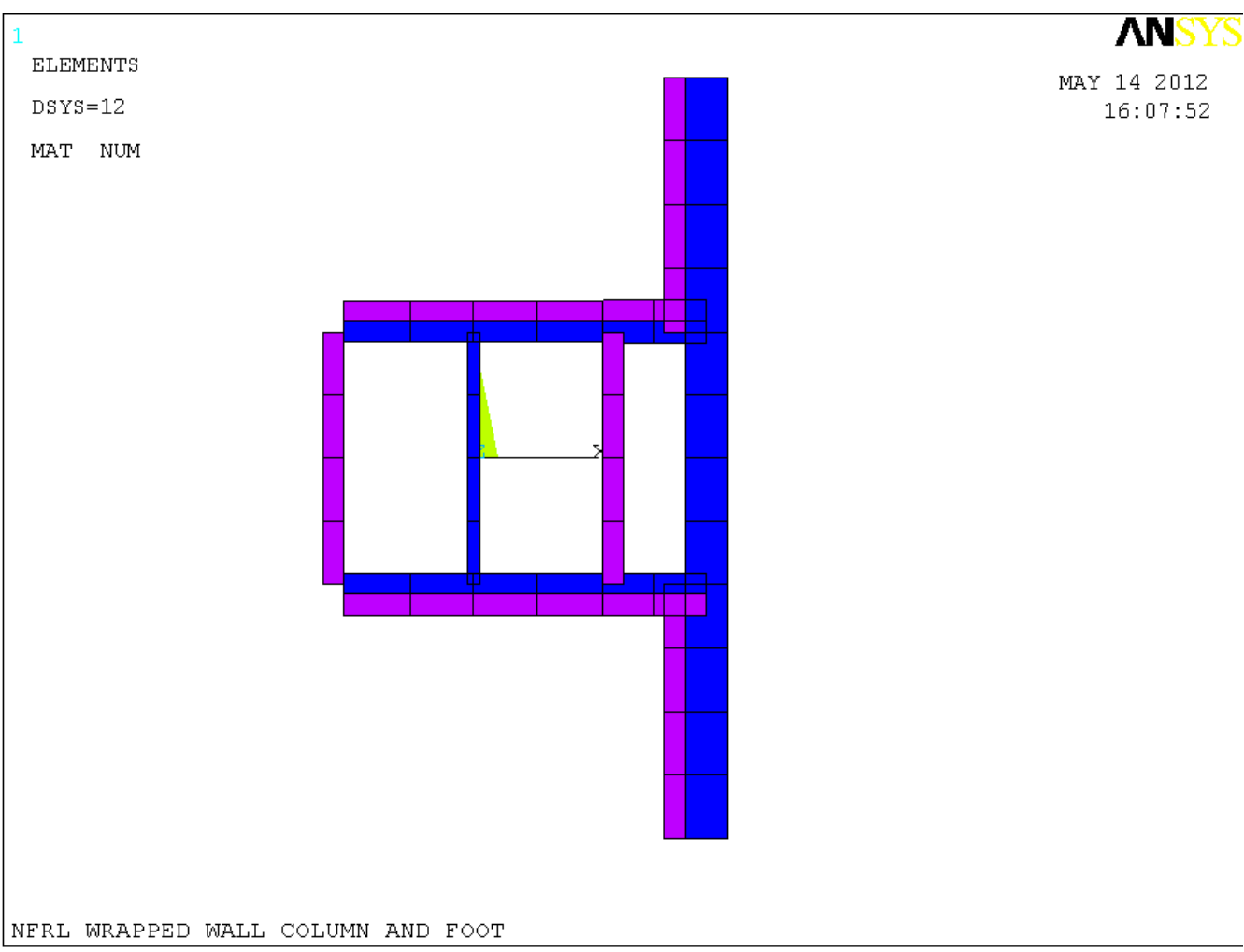

Fig. 5.2 Detail of wrapped wall-column 


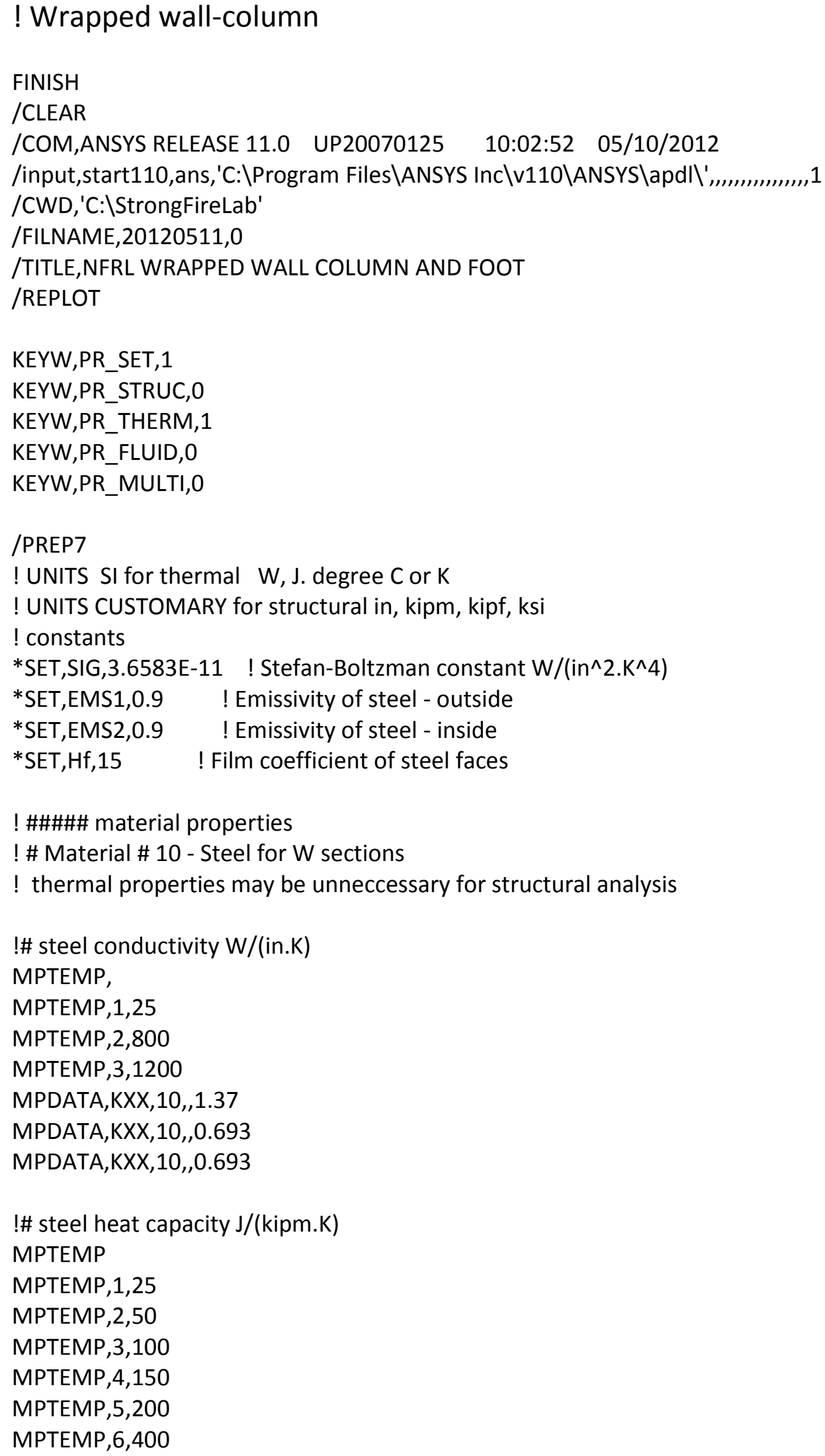




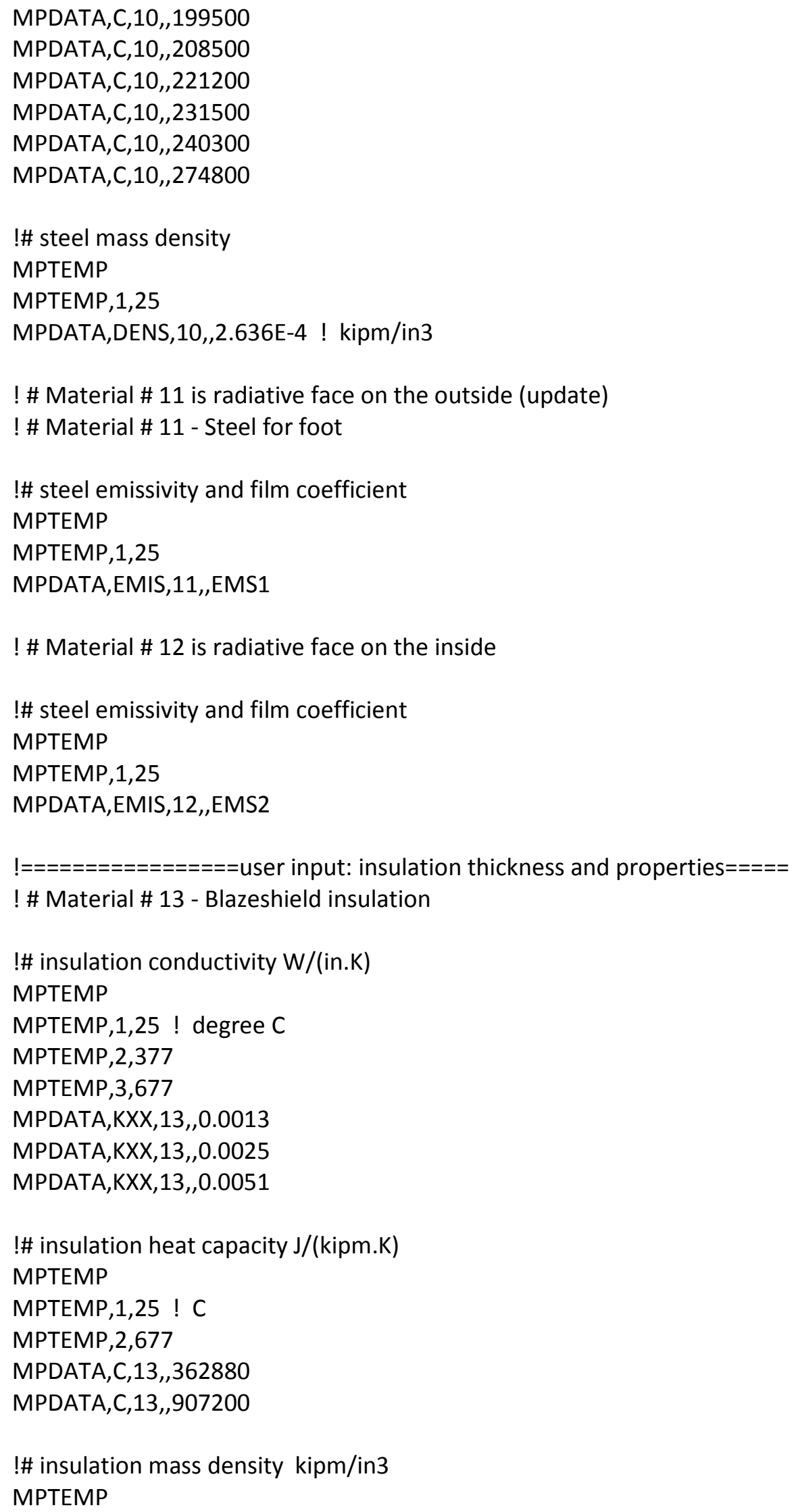




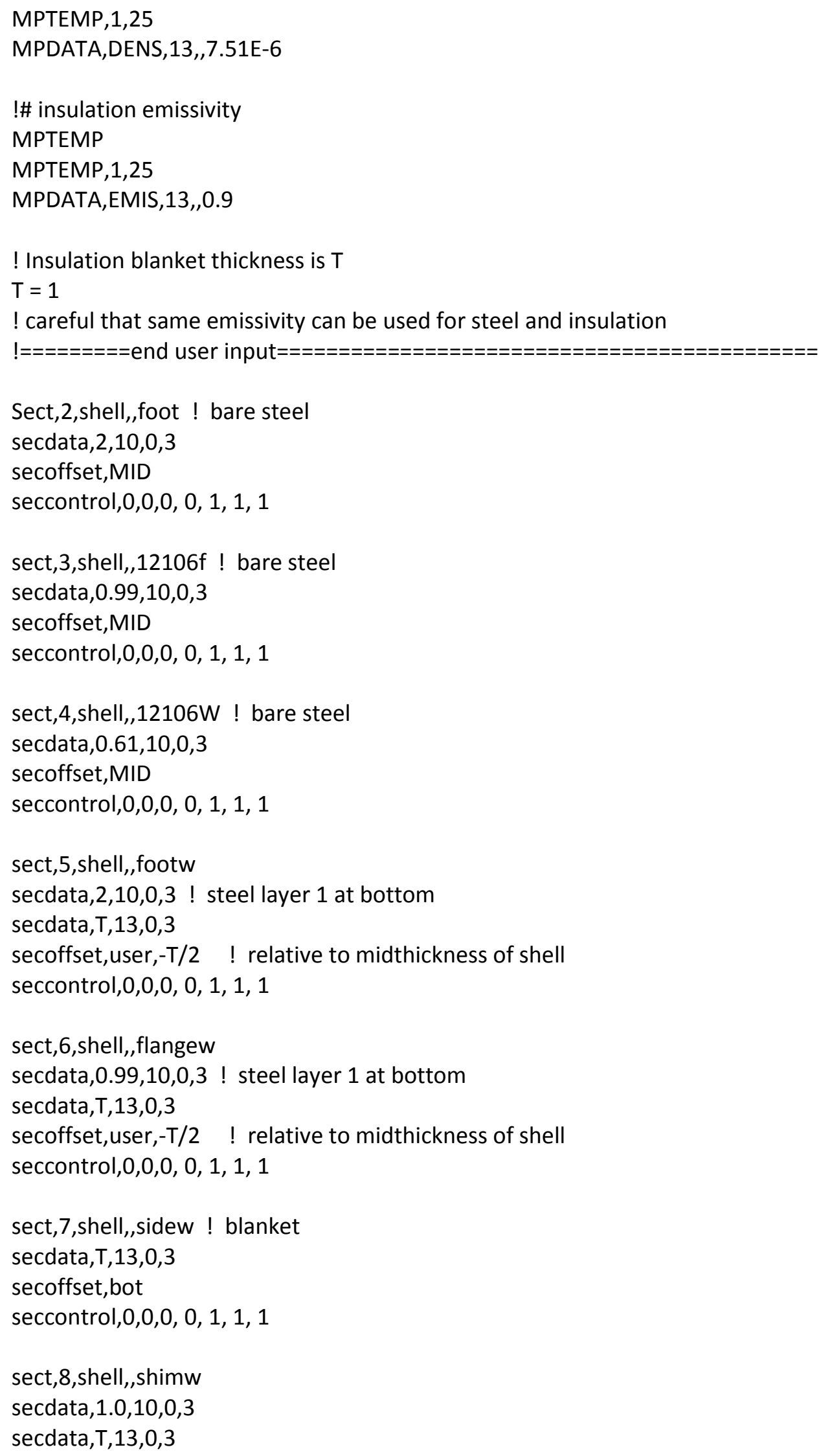


secoffset, user,-T/2 ! relative to midthickness of shell

seccontrol,0,0,0, 0, 1, 1, 1

ET,5,SHELL131

KEYOPT,5,2,0 ! average film temperature

KEYOPT,5,3,0 ! quadratic temperature distribution through thickness

KEYOPT,5,4,0 ! use sect defined above

KEYOPT,5,6,0 ! no paint

!KEYOPT,5,8,2 use this option when insulation is present- data at top, bottom and mid of all layers !SHELL,MID use this option when insulation is present-results calculated at mid, not averaged from top and bottom

! USER INPUT: STARTING NUMBERS, SUB ZERO (NOT LETTER O)==========

*SET,KPO,8100

*SET,LINO,19200

*SET,ARE0,15100

*SET, VOLO,5000

*SET,ELE0,69000

*SET,NOD0,88000

! \# define model geometry

! user input:location of column (origin of local coordinates)

! ref: sheet 15/15 lower left fig

! Local X parallel to flanges, points North.

! Local Y parallel to web, points West.

! Local Z points up.

! location of WALL column (origin of local coordinates center of I at base)

LOCAL,12,0,709,250,204, , , ,1,1,

CSYS,12,

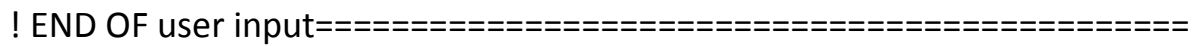

TREF,25

NUMSTR,KP,KPO

NUMSTR,LINE,LINO

NUMSTR,AREA,AREO

NUMSTR,VOLU,VOLO

NUMSTR,ELEM,ELEO

NUMSTR,NODE,NODO

! Y SIDE OF FOOT

$\mathrm{K}, \mathrm{KPO}, 11,-18,5.75$,

$\mathrm{K}, \mathrm{KPO}+1,11,-12,5.75$,

$\mathrm{K}, \mathrm{KPO}+2,11,-5.95,5.75$,

$\mathrm{K}, \mathrm{KPO}+3,11, \quad, 5.75$,

$\mathrm{K}, \mathrm{KPO}+4,11,5.95,5.75$,

$\mathrm{K}, \mathrm{KPO}+5,11,12,5.75$,

K,KP0+6,11,18,5.75, 


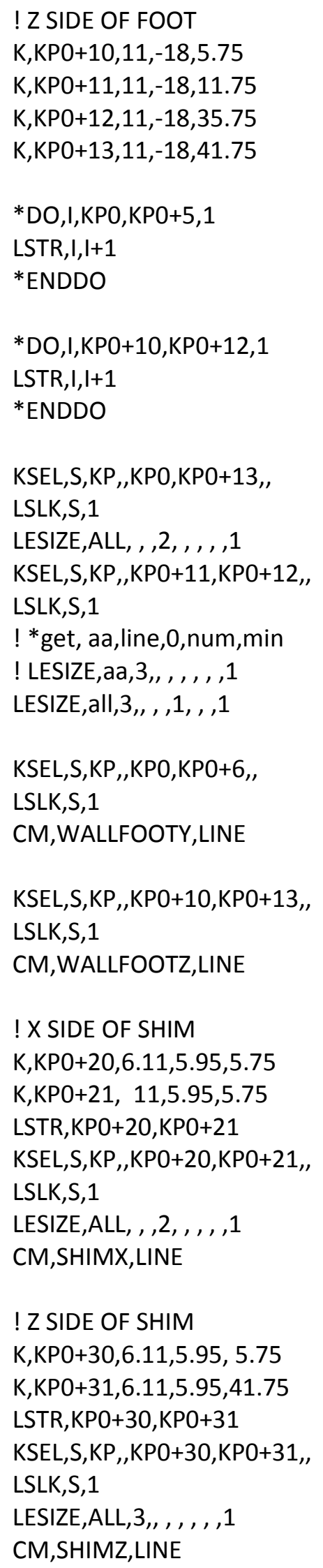




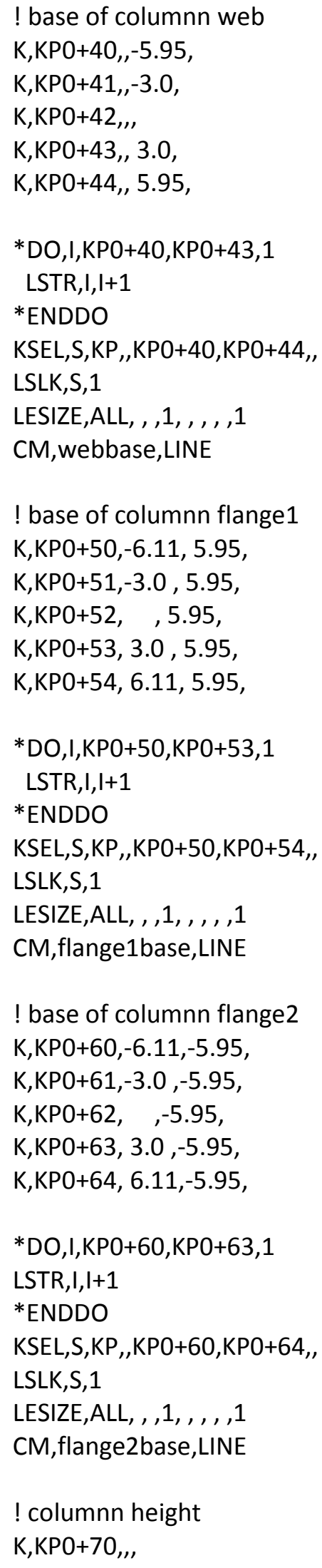




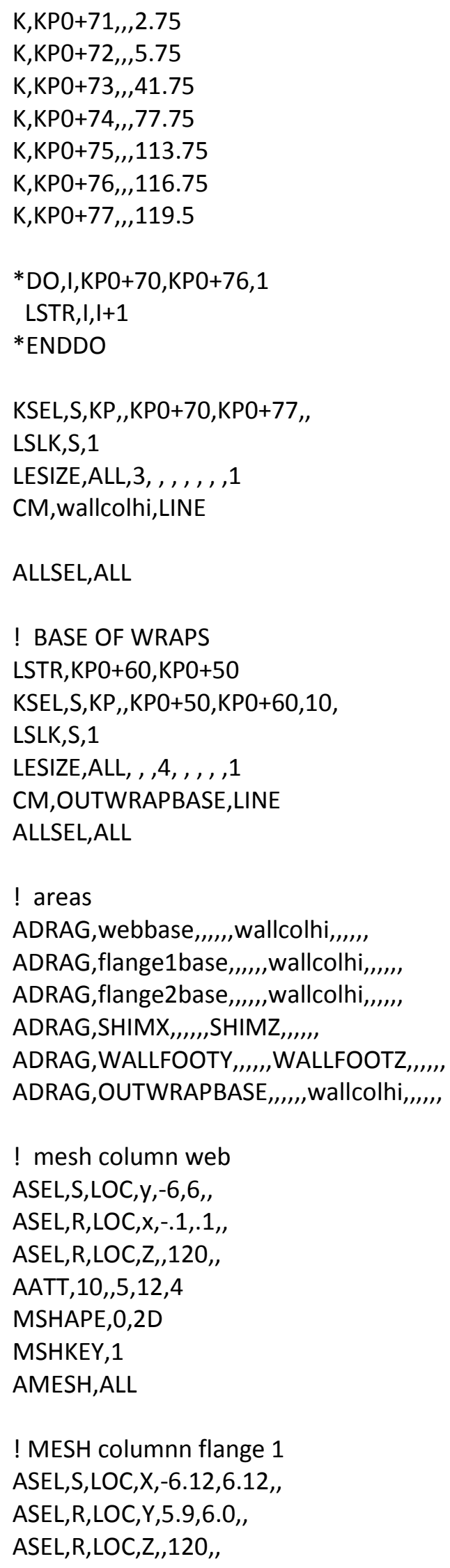


AREVERSE,ALL

AATT, 10, 5, 12,6

MSHAPE,0,2D

MSHKEY,1

AMESH,ALL

! MESH columnn flange 2

ASEL,S,LOC,X,-6.12,6.12,,

ASEL,R,LOC,Y,-5.9,-6.0,,

ASEL,R,LOC,Z,,120,,

AATT, 10,,5,12,6

MSHAPE,0,2D

MSHKEY,1

AMESH,ALL

! MESH SHIM1

ASEL,S,LOC,X,6.10,11.1,,

ASEL,R,LOC,Y,5.9,6.0,

ASEL,R,LOC,Z,5.7,42,,

AATT, 10, ,5,12,8

MSHAPE,0,2D

MSHKEY,1

AMESH,ALL

! MESH FOOT LEFT

ASEL,S,LOC,X,10.9,11.1,, ASEL,R,LOC,Y,-18.0,-5.9, ASEL,R,LOC,Z,5.7,42,, AREVERSE,ALL

AATT, 10,,5,12,5

MSHAPE,0,2D

MSHKEY,1

AMESH,ALL

! MESH FOOT RIGHT

ASEL,S,LOC,X,10.9,11.1,, ASEL,R,LOC,Y,5.9,18.0, ASEL,R,LOC,Z,5.7,42,, AREVERSE,ALL

AATT, 10,,5,12,5

MSHAPE,0,2D

MSHKEY,1

AMESH,ALL

! MESH FOOT MID

ASEL,S,LOC,X,10.9,11.1,, ASEL,R,LOC,Y,-6.0,6.0,, ASEL,R,LOC,Z,5.7,42,, 
AREVERSE,ALL

AATT,10,,5,12,2

MSHAPE,0,2D

MSHKEY,1

AMESH,ALL

! MESH ouside wrap

ASEL,S,LOC,X,-6.5,-6.0,, ASEL,R,LOC,Y,-6.0,6.0,, ASEL,R,LOC,Z,,120, AATT,13, 5, 12,7

MSHAPE,0,2D

MSHKEY,1

AMESH,ALL

! COPY SHIM

ASEL,S,LOC,X,6.10,11.1,, ASEL,R,LOC,Y,5.9,6.0,, ASEL,R,LOC,Z,5.7,42,, AGEN,2,ALL,,,,-11.9,,,,,

! REORIENT SHIM1 ASEL,S,LOC,X,6.10,11.1, ASEL,R,LOC,Y,5.9,6.0, ASEL,R,LOC,Z,5.7,42,, AREVERSE,ALL

! COPY FOOT AND SHIMS ASEL,S,LOC,X,6.10,11.1,, ASEL,R,LOC,Y,-18,18.0," ASEL,R,LOC,Z,5.7,42,, AGEN,2,ALL,,,,,,72,,,,

! COPY LOW WRAP ASEL,S,LOC,X,-6.5,-6.0,, ASEL,R,LOC,Y,-6.0,6.0,, ASEL,R,LOC,Z,,5.75,, AGEN,2,ALL,,,12.22,,,,,",

! COPY MID WRAP ASEL,S,LOC,X,-6.5,-6.0,, ASEL,R,LOC,Y,-6.0,6.0,, ASEL,R,LOC,Z,41,75,77.75,, AGEN,2,ALL,,,12.22,,,,,,

! COPY HIGH WRAP ASEL,S,LOC,X,-6.5,-6.0, ASEL,R,LOC,Y,-6.0,6.0,, 
ASEL,R,LOC,Z,113.75,119.5,,

AGEN,2,ALL,,,12.22,,,,,,

! REORIENT ouside wrap

ASEL,S,LOC,X,-6.5,-6.0,,

ASEL,R,LOC,Y,-6.0,6.0,,

ASEL,R,LOC,Z,,120,,

AREVERSE,ALL

ALLSEL,ALL

NUMMRG,ALL,,,,

/EOF

! 430pm MON 5/14 


\section{Chapter 6 Frame}

Table 6.1 Properties

\begin{tabular}{|l|l|}
\hline Frame & Starting numbers \\
\hline *Keypoints KP2 & 8400 \\
\hline *Lines LIN2 & 19600 \\
\hline *Areas ARE2 & 15300 \\
\hline *Volumes VOL2 & 5000 \\
\hline *Elements ELE2 & 70200 \\
\hline *Nodes NOD2 & 89500 \\
\hline $\begin{array}{l}\text { *Local coordinates } \\
\text { system 13 }\end{array}$ & $\begin{array}{l}\text { x along bottom of lowest horizontal member (web); } \\
\text { y perpendicular to web, right hand system; } \\
\text { z along left edge of left vertical member (flange); } \\
\text { origin: base of frame, at intersection of horizontal, } \\
\text { vertical and diagonal, lower left corner (Fig. 6.1). }\end{array}$ \\
\hline Element type & 5 Shell 181 \\
\hline Sections & $\begin{array}{l}3 \text { (0.99 inch flange W12x106) } \\
4 \text { (0.61 inch web W12x106) }\end{array}$ \\
\hline Materials & \begin{tabular}{l} 
* (W sections) \\
\hline
\end{tabular} \\
\hline
\end{tabular}

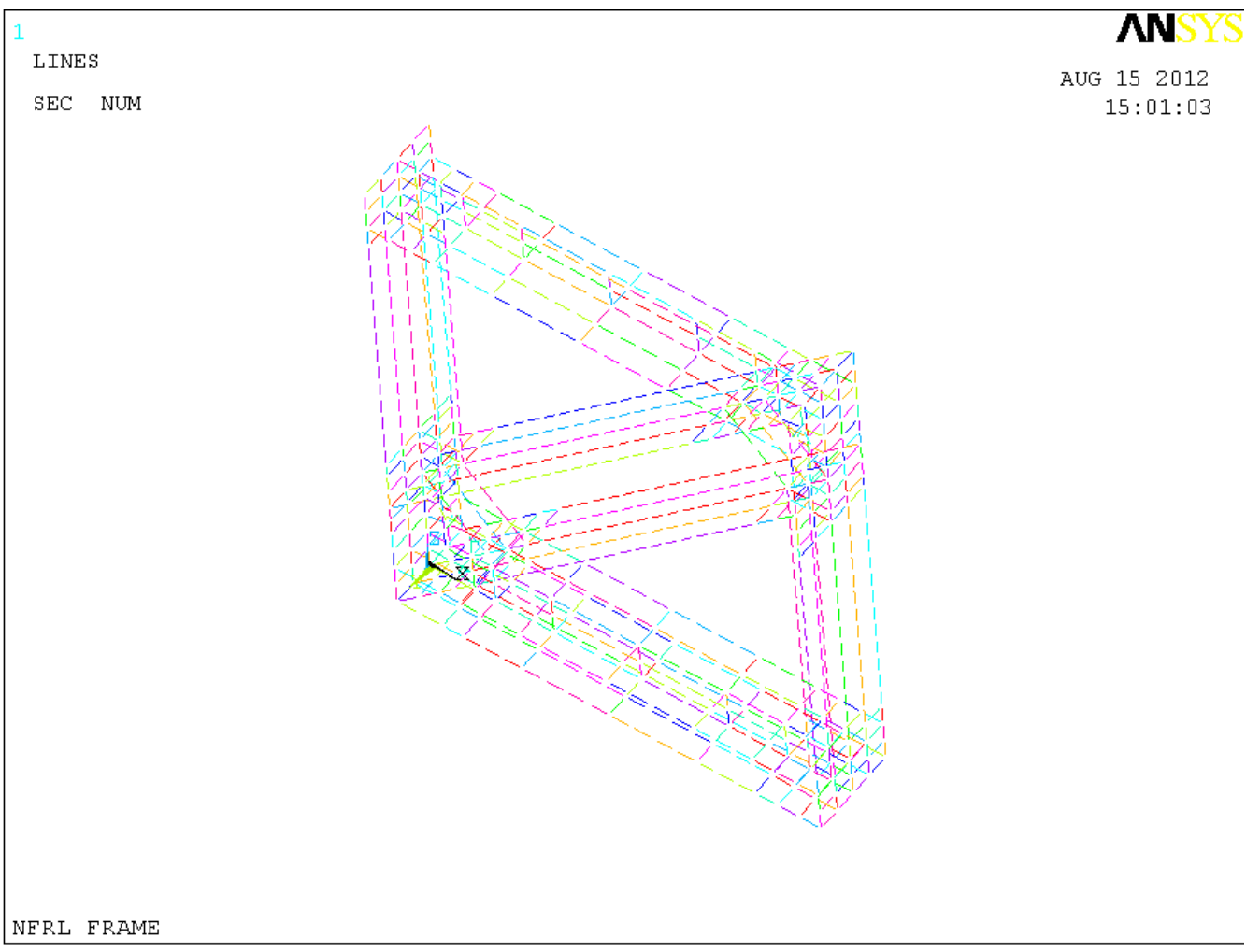

Fig. 6.1 Local coordinate system 13 of Frame 


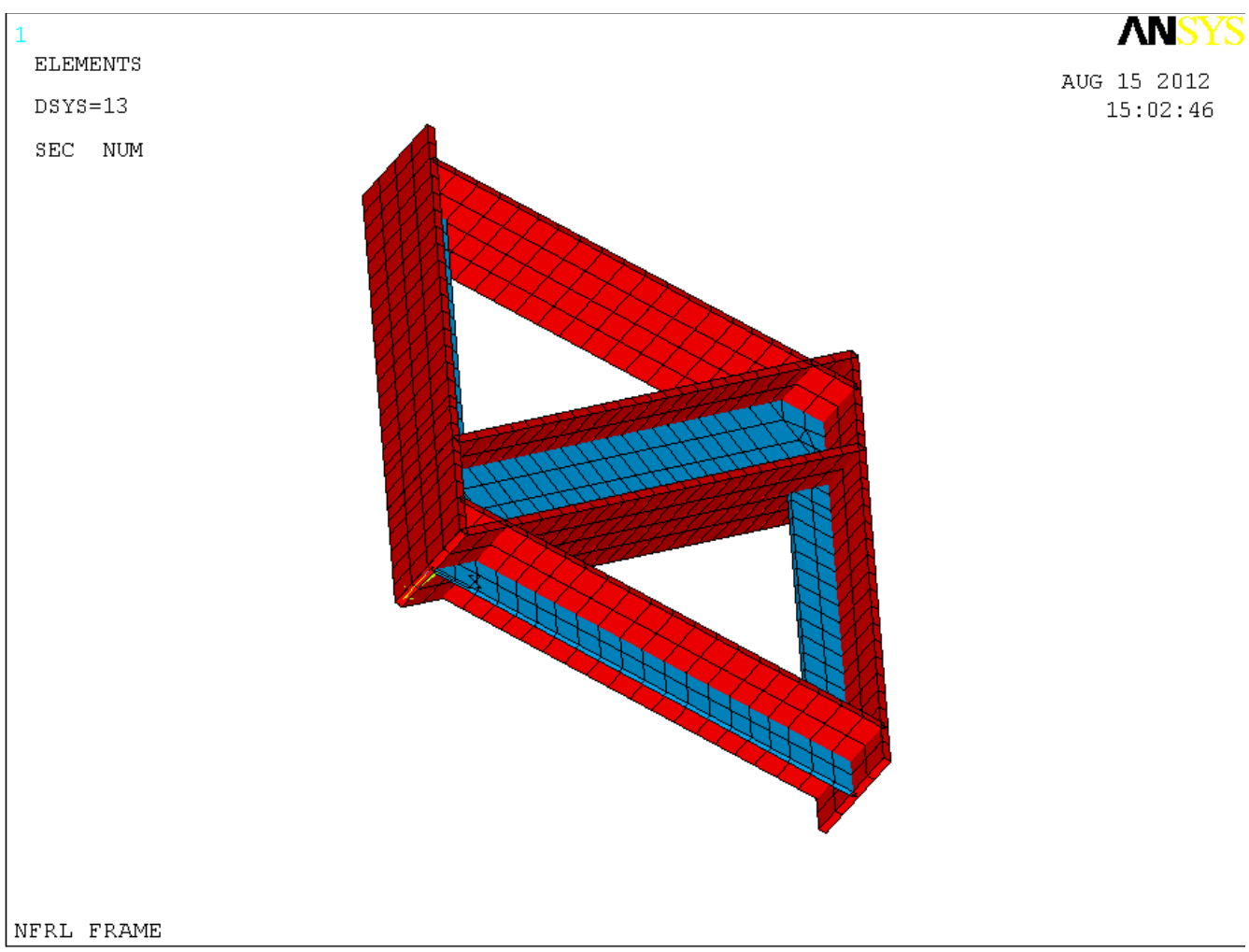

Fig. 6.2 3D view of Frame

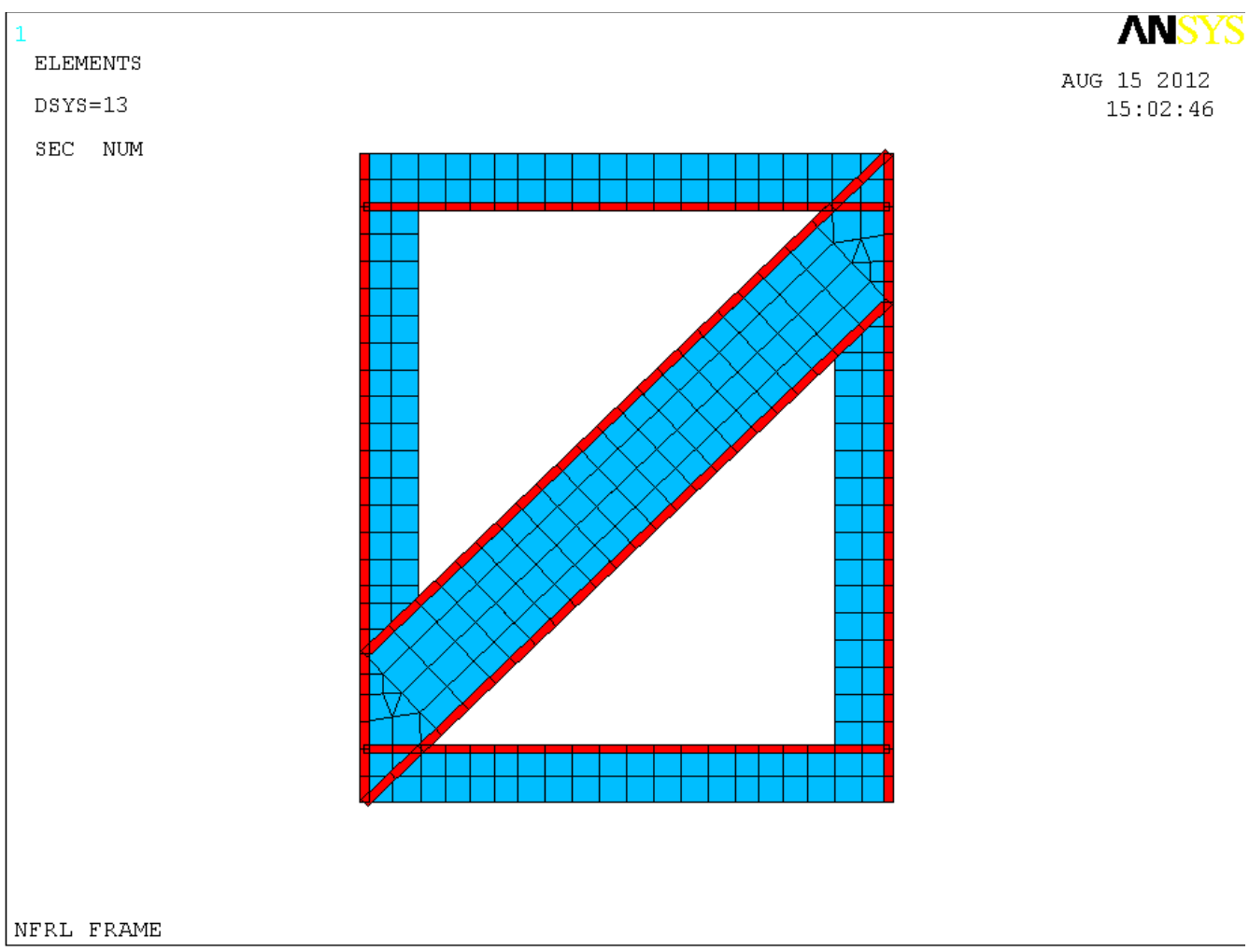

Fig. 6.3 Elevation view of Frame 
! Structural model - Frame LAST TESTED on Thu 7.20.20124 pm

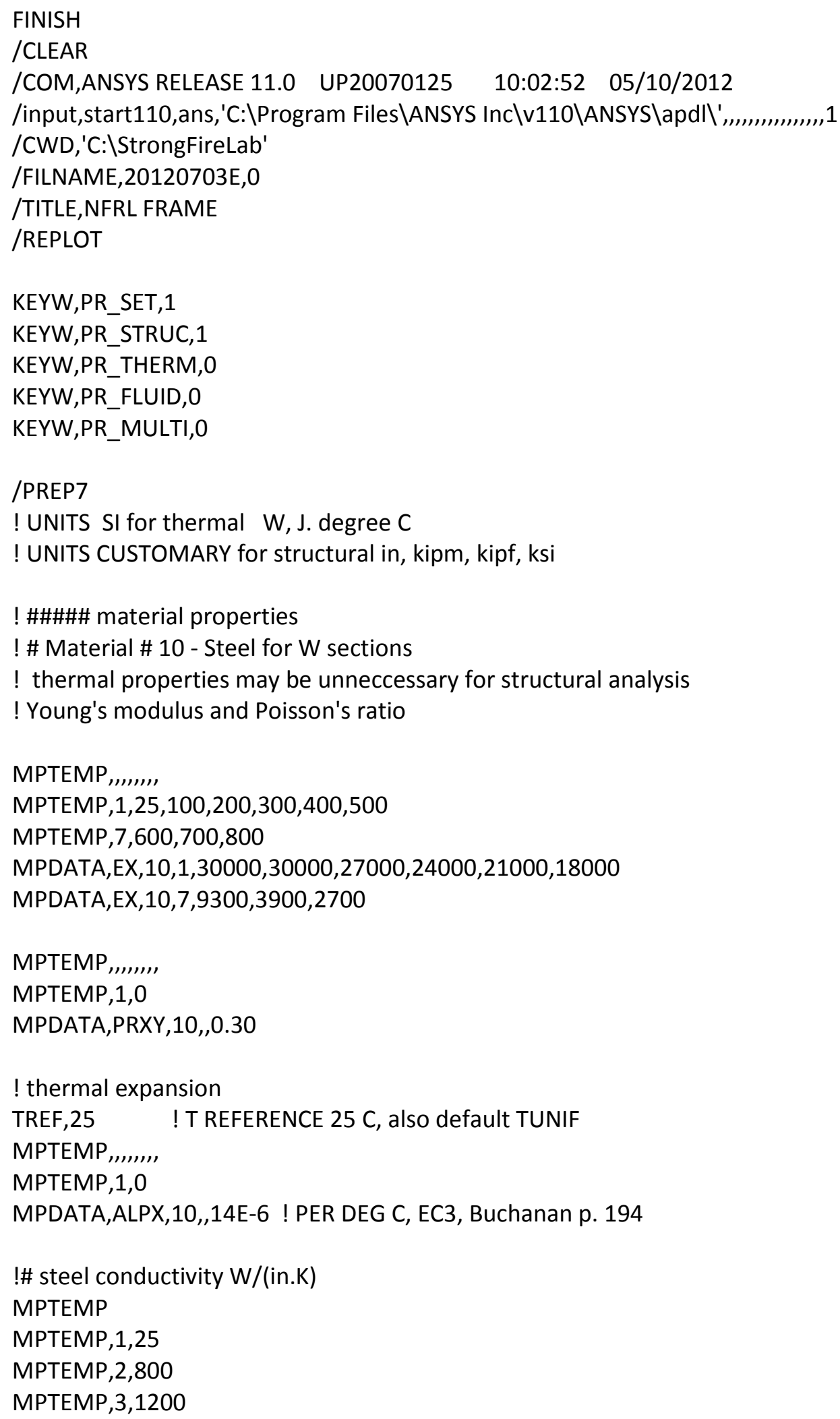




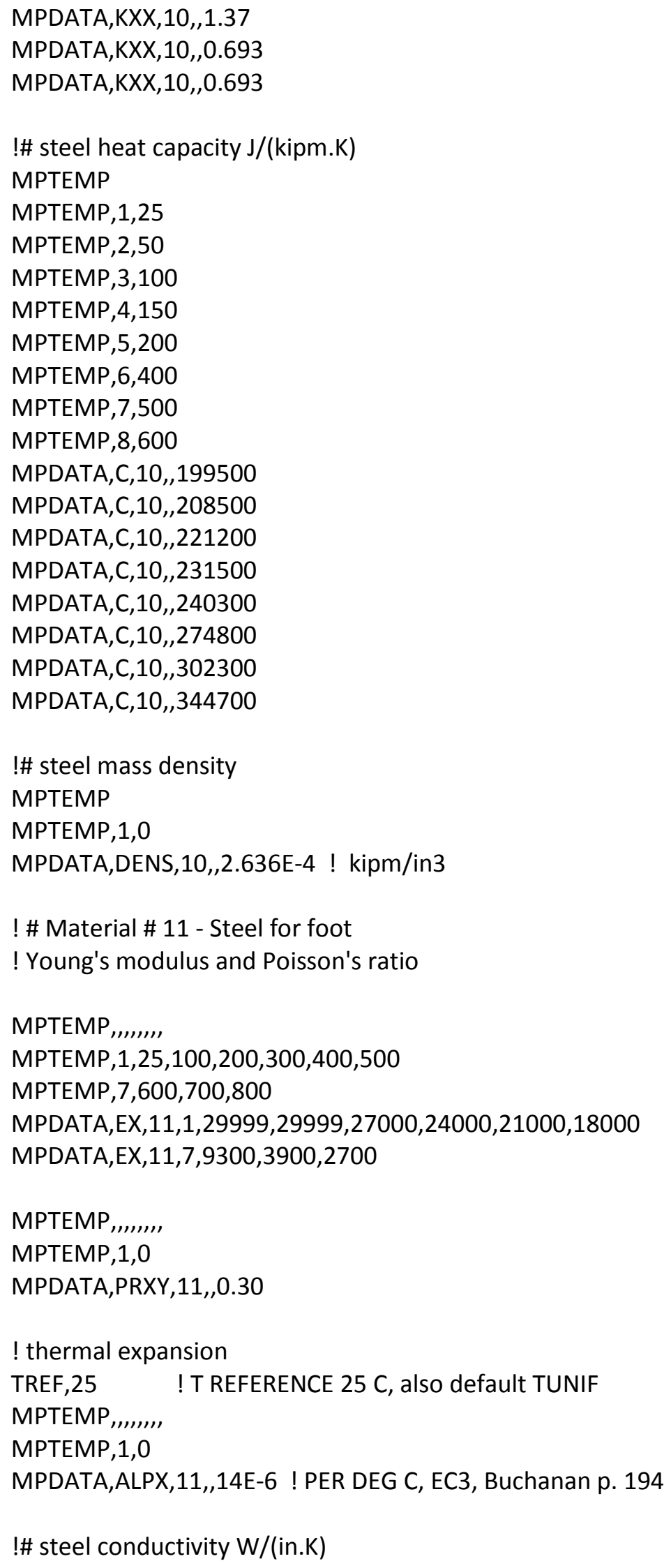




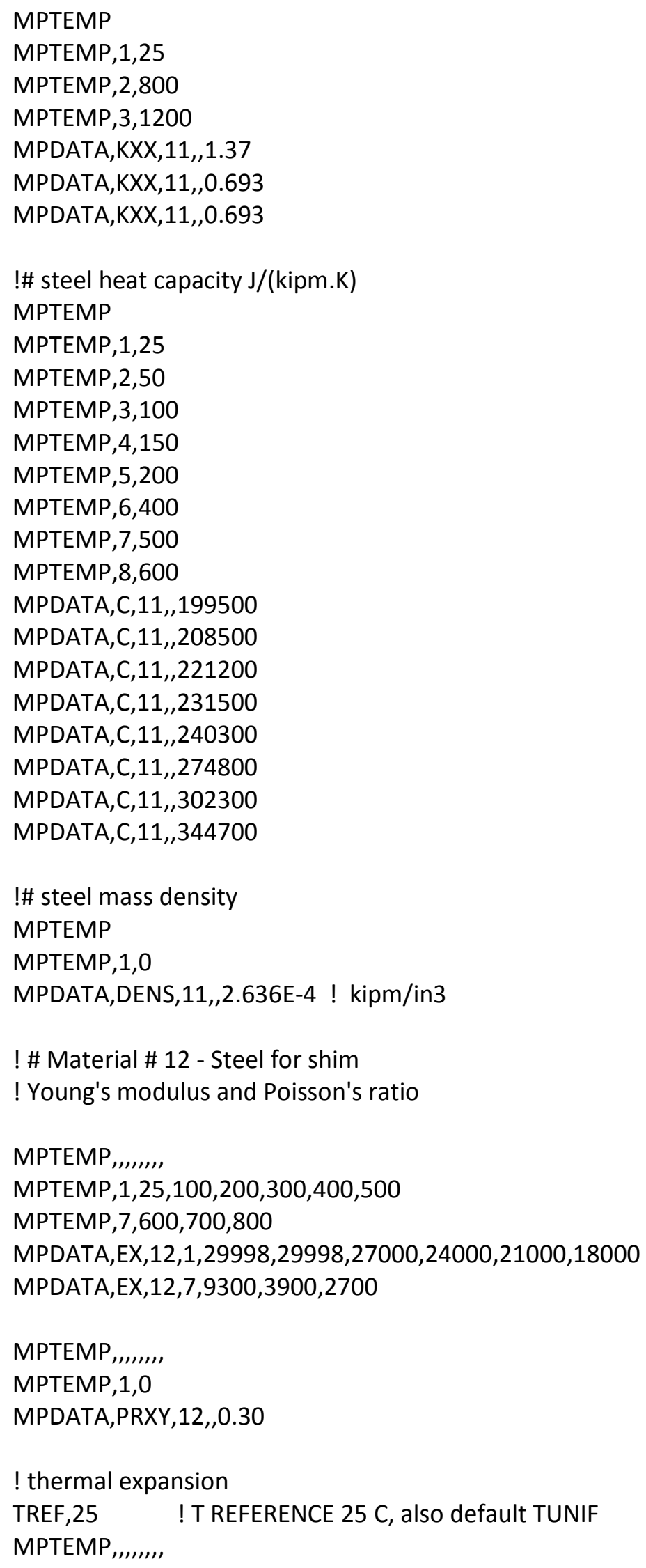




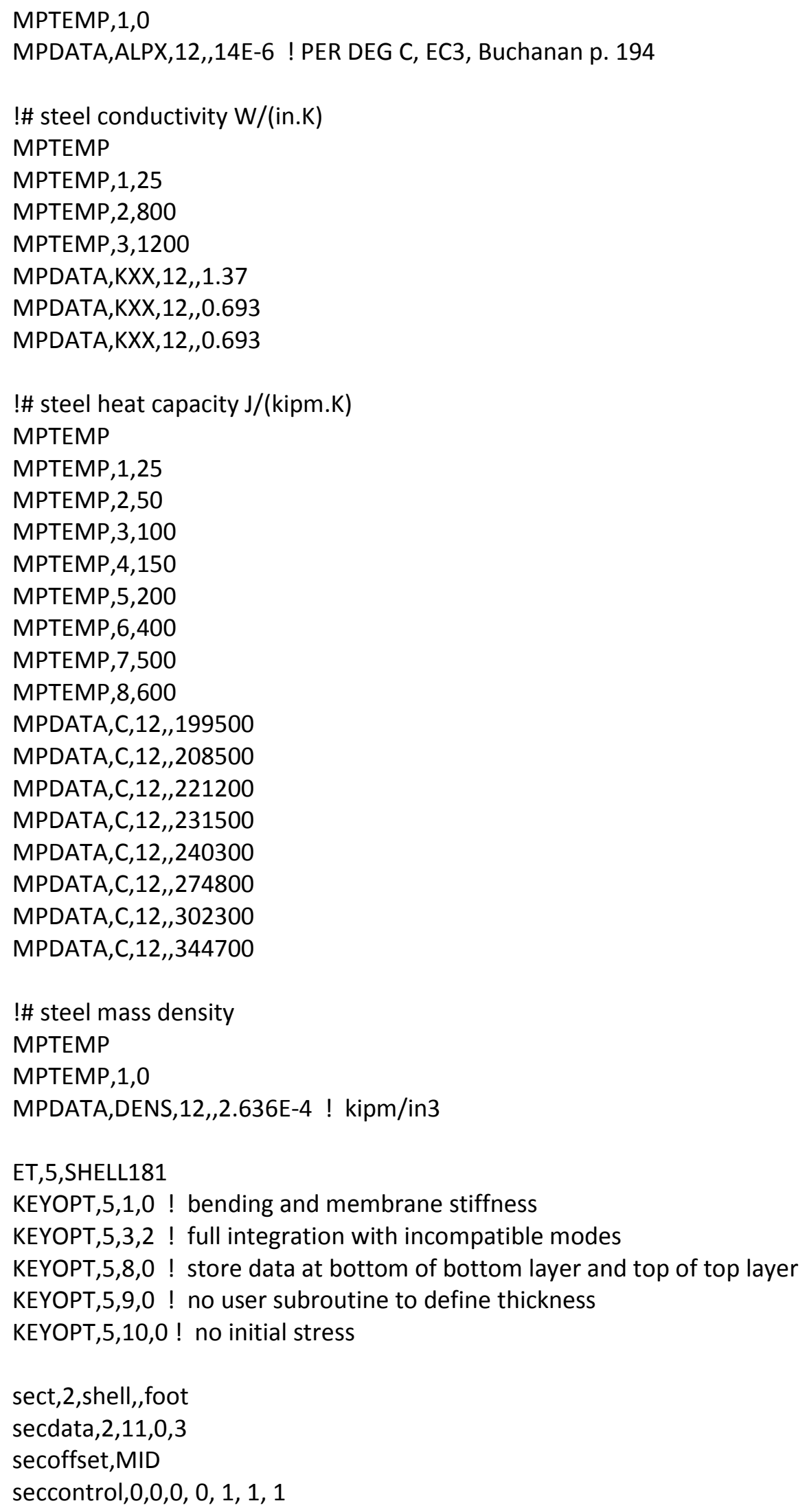


sect,3,shell,,12106f

secdata, $0.99,10,0,3$

secoffset,MID

seccontrol,0,0,0, 0, 1, 1, 1

sect, 4, shell,,12106W

secdata, $0.61,10,0,3$

secoffset,MID

seccontrol,0,0,0, 0, 1, 1, 1

sect, 8 , shell,,shim

secdata,1.0,12,0,3

secoffset, MID

seccontrol,0,0,0, 0, 1, 1, 1

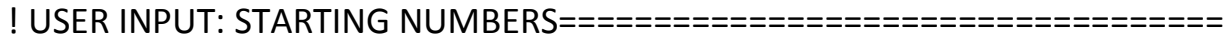

*SET,KP2,8400

*SET,LIN2,19600

*SET,ARE2,15300

*SET, VOL2,5000

*SET,ELE2,70200

*SET,NOD2,89500

! \# define model geometry

! user input:local coord system and location of frame

! ref: SHEET 1/15 LOWER LEFT FIG

! Local X ALONG EDGE OF LOWEST HORIZ MEMBER.

! Local Y perpendicular to web, right hand system.

! Local Z ALONG RIGHT EDGE OF RIGHT VERTICAL MEMBER.

! location of FRAME (origin of local coordinates: BASE OF FRAME,

! INTERSECTION OF HORIZ, VERT AND DIAG,LOWER RIGHT CORNER)

LOCAL,13,0,200,200,200, , , ,1,1

CSYS,13,

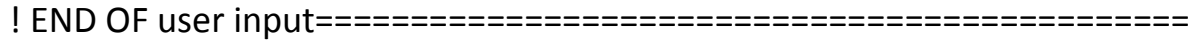

NUMSTR,KP,KP2

NUMSTR,LINE,LIN2

NUMSTR,AREA,ARE2

NUMSTR,VOLU,VOL2

NUMSTR,ELEM,ELE2

NUMSTR,NODE,NOD2

! $\mathrm{x}$ horiz edge of bottom web

$\mathrm{K}, \mathrm{KP} 2$, ,,

$\mathrm{K}, \mathrm{KP} 2+1,6.233$, , ! diag at 43.693 deg to horiz

$\mathrm{K}, \mathrm{KP} 2+2,9.06$, 


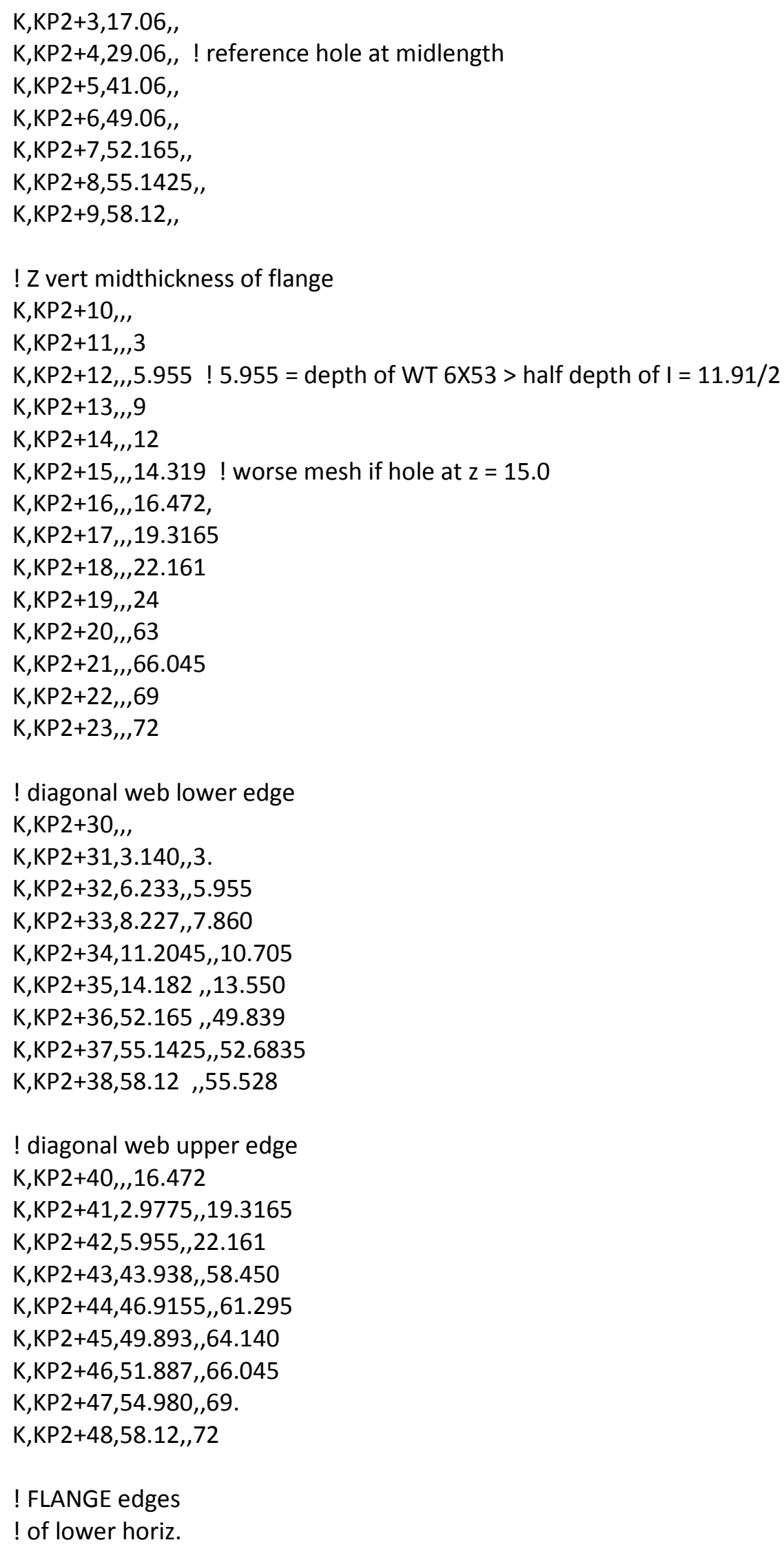




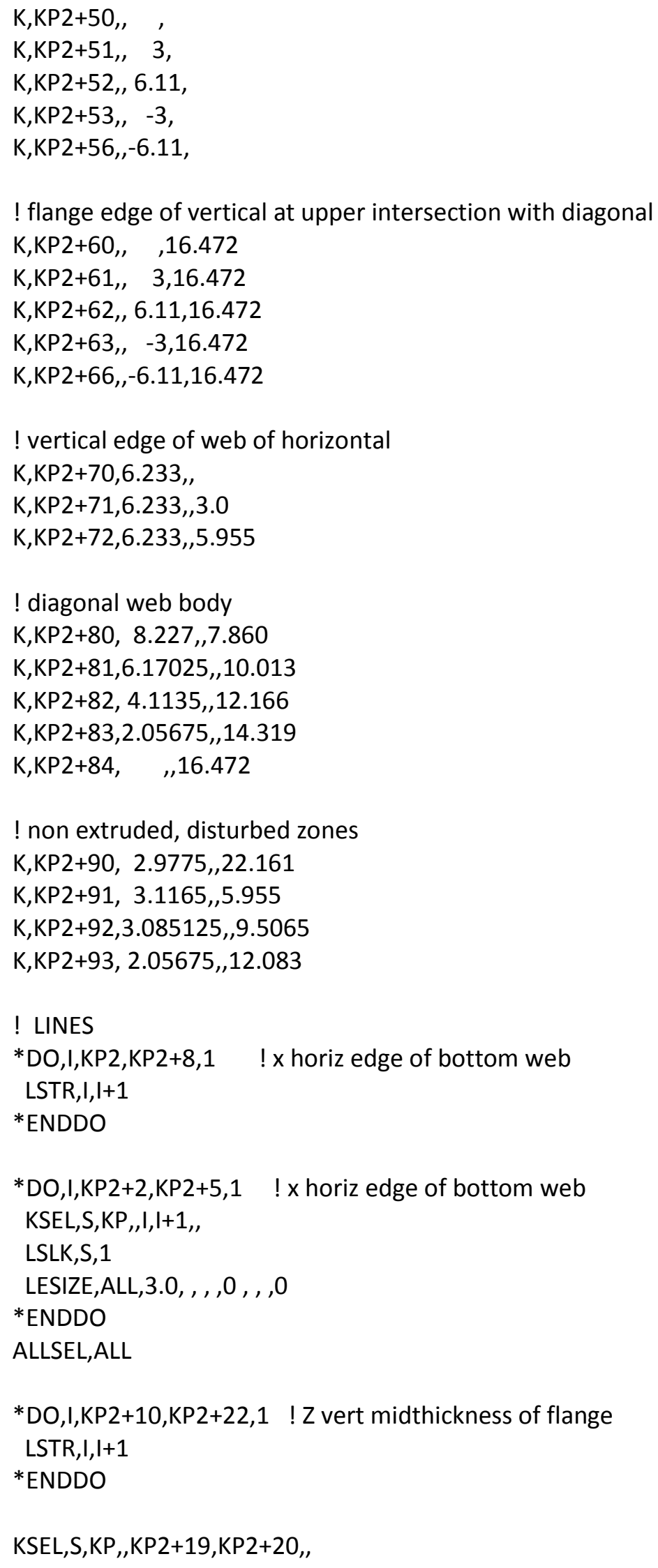




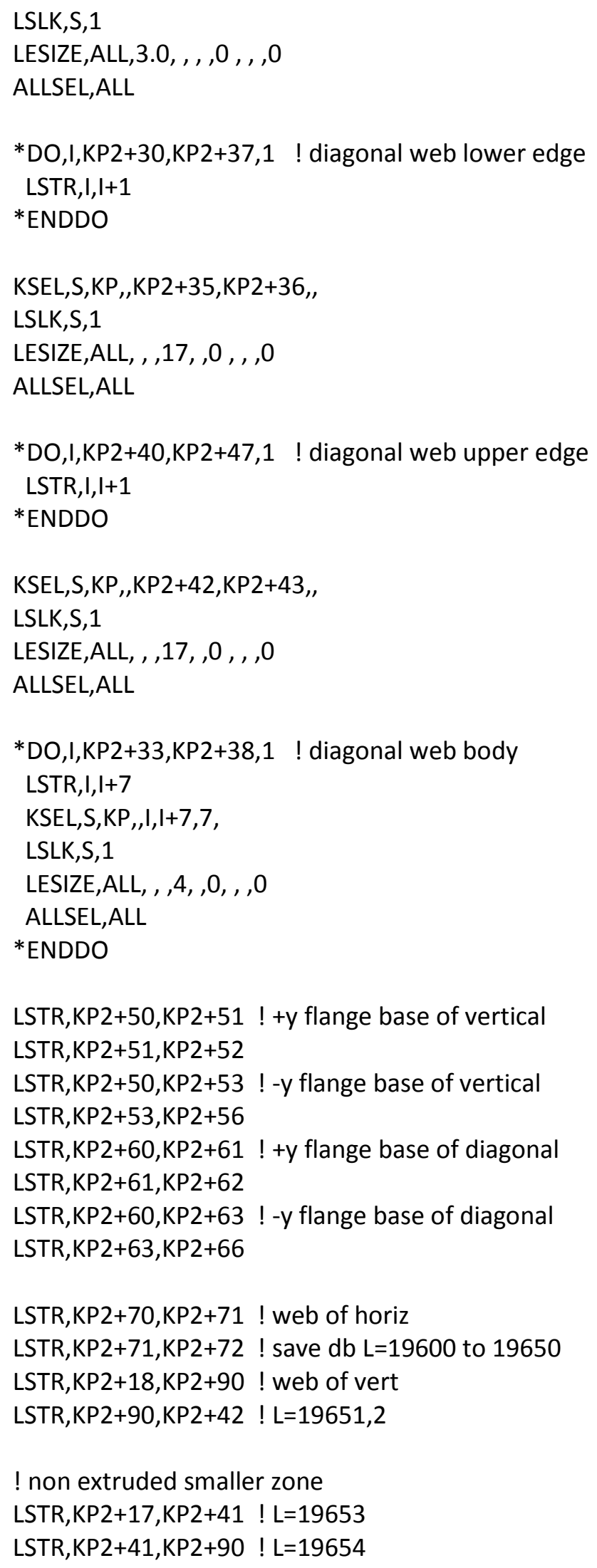




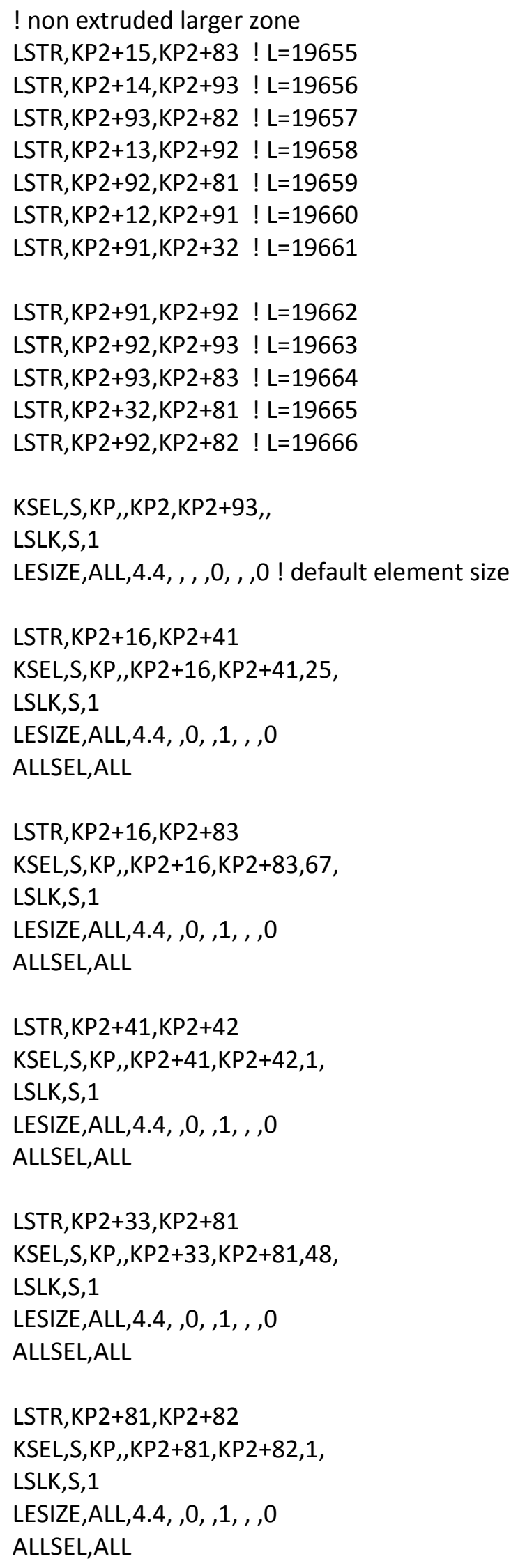




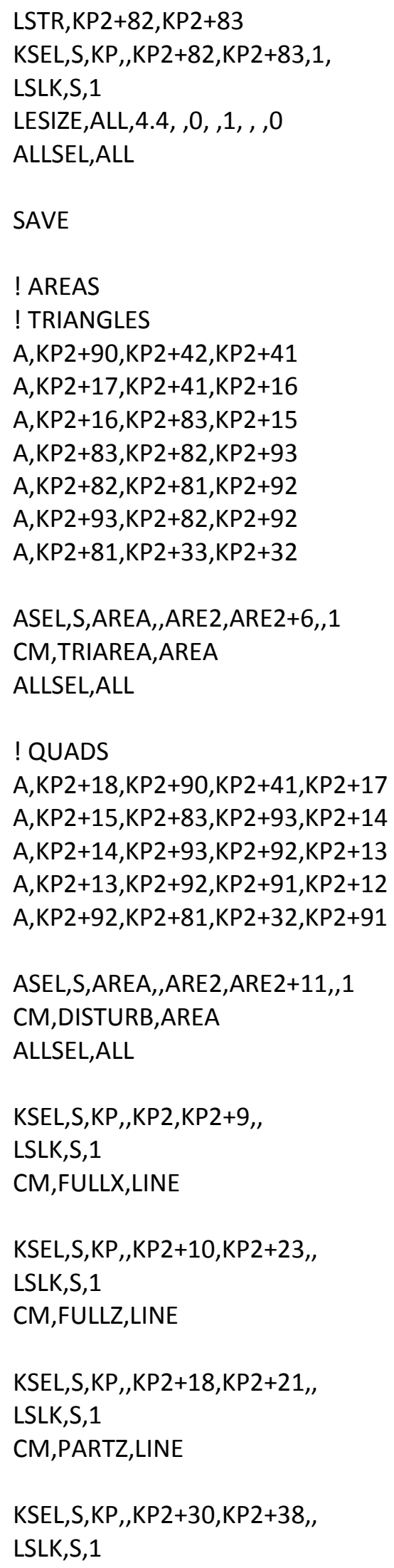


CM,lodiag,LINE

KSEL,S,KP, ,KP2+40,KP2+48,,

LSLK,S,1

CM,hidiag,LINE

KSEL,S,KP, ,KP2+50,KP2+52,1,

LSLK,S,1

CM,flangeplus, LINE

KSEL,S,KP ,KP2+50,KP2+56,3,

LSLK,S,1

CM,flangeminus, LINE

KSEL,S,KP, ,KP2+60,KP2+62,1,

LSLK,S,1

CM,flandiagplus, LINE

KSEL,S,KP, ,KP2+60,KP2+66,3,

LSLK,S,1

$\mathrm{CM}$,flandiagminus, LINE

KSEL,S,KP, ,KP2+70,KP2+72,1,

LSLK,S,1

CM, webhoriz,LINE

KSEL,S,KP ,KP2+18,KP2+42,24,

KSEL,A,KP ,KP2+90

LSLK,S,1

CM, webvert,LINE

ALLSEL,ALL

!!*GET,AMAX1,AREA,0,NUM,MAX

! diagonal web areas

$\mathrm{A}, \mathrm{KP} 2+33, \mathrm{KP} 2+34, \mathrm{KP} 2+41, \mathrm{KP} 2+40$

$* \mathrm{DO}, \mathrm{I}, \mathrm{KP} 2+34, \mathrm{KP} 2+37,1$

$A, I, I+1, I+8, I+7$

*ENDDO

ASEL,S,AREA,,ARE2,ARE2+16,,1

CM,DIAWEBTURB,AREA ! DIAGONAL WEB AND DISTURBED AREAS=D

ALLSEL,ALL

! areas

ADRAG, lodiag, ,,,,,, flangeplus,,,,,,,

ASEL,S,AREA,,ARE2,ARE2+32,,1 
CM,DLOPLUS,AREA ! DIAGONAL WEB,DISTURBED AND LOW PLUS DIAG FLANGE AREAS

ALLSEL,ALL

ADRAG,lodiag, ,,,,, flangeminus, ,,, ,

ASEL,S,AREA,,ARE2,ARE2+48,,1

CM,DLODIAG,AREA ! DIAGONAL WEB,DISTURBED AND LOW DIAG FLANGE AREAS

ALLSEL,ALL

ADRAG, hidiag, ,,,,, flandiagplus,,,,,,

ASEL,S,AREA,,ARE2,ARE2+64,,1

CM,DLOHIPLUS,AREA ! DIAGONAL WEB,DISTURBED,LOW DIAG FLANGE AND HIGH PLUS DIAG FLANGE

AREAS

ALLSEL,ALL

ADRAG, hidiag, ,,,,,, flandiagminus, ,,,,,

ASEL,S,AREA,,ARE2,ARE2+80,,1

CM,DLOHI,AREA ! DIAGONAL WEB,DISTURBED,LOW AND HIGH DIAG FLANGE AREAS

ALLSEL,ALL

ADRAG,FULLZ,,,,,,,flangeplus,,,,,,,

ADRAG,FULLZ,,,,,,,flangeminus,,,,,,

ADRAG, partz,,,,,,, webvert, $,, \ldots, \ldots$,

ADRAG, fullx,,,,,,,flangeplus,,,,,,,

ADRAG, full $x,,, \ldots,$, flangeminus, ,,,,,,

ADRAG, full $x,,, \ldots,$, WEBHORIZ,,,,,,,

ALLSEL,ALL

SAVE

! mesh webS

! MESH TRIANGLES

! much simpler to define elements by commands NKPT and E

CMSEL,S,TRIAREA,AREA

AATT, 10, 5, 13,4

MSHAPE,1,2D

MSHKEY,0

AMESH,ALL

! MESH QUADS

CMSEL,S,DISTURB,AREA

CMSEL,U,TRIAREA,AREA

AATT, 10,,5,13,4

MSHAPE, 0,2D

MSHKEY,1

AMESH,ALL

! MESH DIAG WEB

CMSEL,S,DIAWEBTURB,AREA 
CMSEL,U,DISTURB,AREA

AATT, 10,,5,13,4

MSHAPE,0,2D

MSHKEY,1

AMESH,ALL

! MESH DIAG FLANGES

CMSEL,S,DLOHI,AREA

CMSEL,U,DIAWEBTURB,AREA

AATT, 10,,5,13,3

MSHAPE,0,2D

MSHKEY,1

AMESH,ALL

! MESH VERTICAL flange

ASEL,S,LOC, $X, .1$,

ASEL,R,LOC,Y,-6.2,6.2,,

ASEL,R,LOC,Z,,72,,

AATT, 10, ,5,13,3

MSHAPE,0,2D

MSHKEY,2

AMESH,ALL

! mesh VERTICAL web

ASEL,S,LOC,y,-.1,.1,,

ASEL,R,LOC, $x$, ,6.0,,

ASEL,R,LOC,Z,22.0,72.0,,

AATT, 10,,5,13,4

MSHAPE,0,2D

MSHKEY,1

AMESH,ALL

! mesh horizontal web

ASEL,S,LOC,y,-.1,.1,,

ASEL,R,LOC, $x$, ,59.0,

ASEL,R,LOC,Z,0,6.0,,

AATT,10,,5,13,4

MSHAPE,0,2D

MSHKEY,1

AMESH,ALL

! INVERT BOTTOM T

ASEL,S,LOC,Z,,0.1,,

AGEN,2,ALL,,,,,5.955,,,

! MESH HORIZONTAL FLANGE

ASEL,S,LOC,Z,5.9,6.0,,

AATT, 10,,5,13,3 


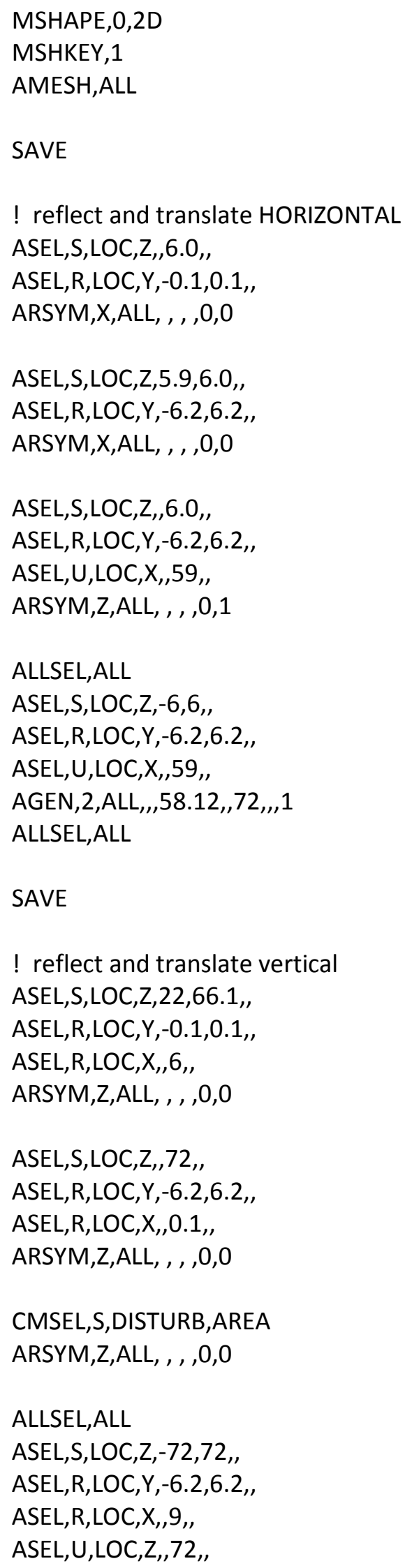




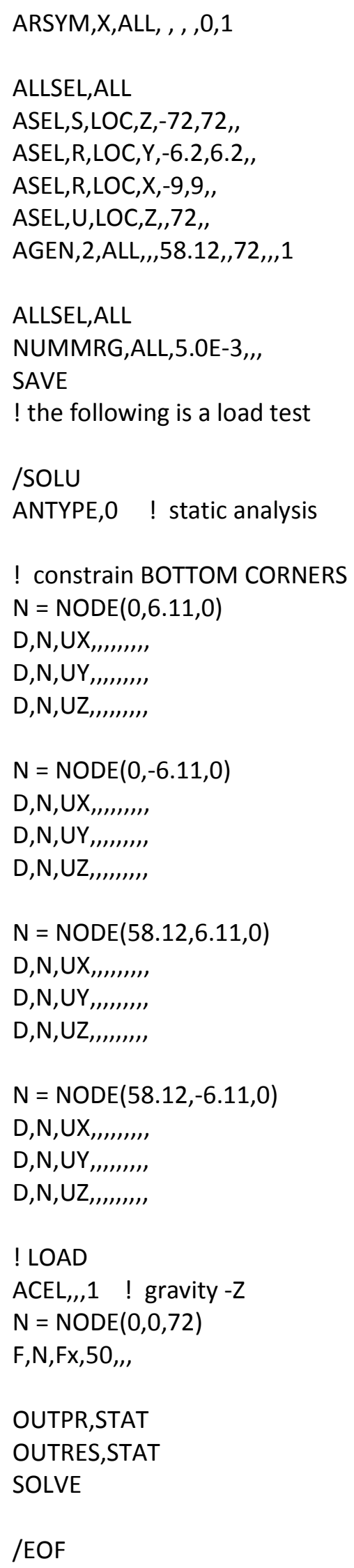




\section{Chapter 7 Wrapped frame}

Table 7.1 Properties

\begin{tabular}{|l|l|}
\hline Wrapped frame & Starting numbers \\
\hline *Keypoints KP2 & 8400 \\
\hline *Lines LIN2 & 19600 \\
\hline *Areas ARE2 & 15300 \\
\hline *Volumes VOL2 & 5000 \\
\hline *Elements ELE2 & 70200 \\
\hline *Nodes NOD2 & 89500 \\
\hline $\begin{array}{l}\text { *Local coordinates } \\
\text { system 13 }\end{array}$ & $\begin{array}{l}\text { x along bottom of lowest horizontal member (web); } \\
\text { y perpendicular to web, right hand system; } \\
\text { z along left edge of left vertical member (flange); } \\
\text { origin: base of frame, at intersection of horizontal, } \\
\text { vertical and diagonal, lower left corner (Fig. 6.1). }\end{array}$ \\
\hline Element type & $\begin{array}{l}5 \text { Shell 131 } \\
3 \text { (0.99 inch flange W12x106) } \\
4 \text { (0.61 inch web W12x106 ) } \\
* 6 \text { (1 inch flange wrap) } \\
* 7 \text { (1 inch side wrap) }\end{array}$ \\
\hline Sections & $\begin{array}{l}10 \text { (W sections) } \\
* 13 \text { (insulation) }\end{array}$ \\
\hline Materials & *user input or default option.
\end{tabular}

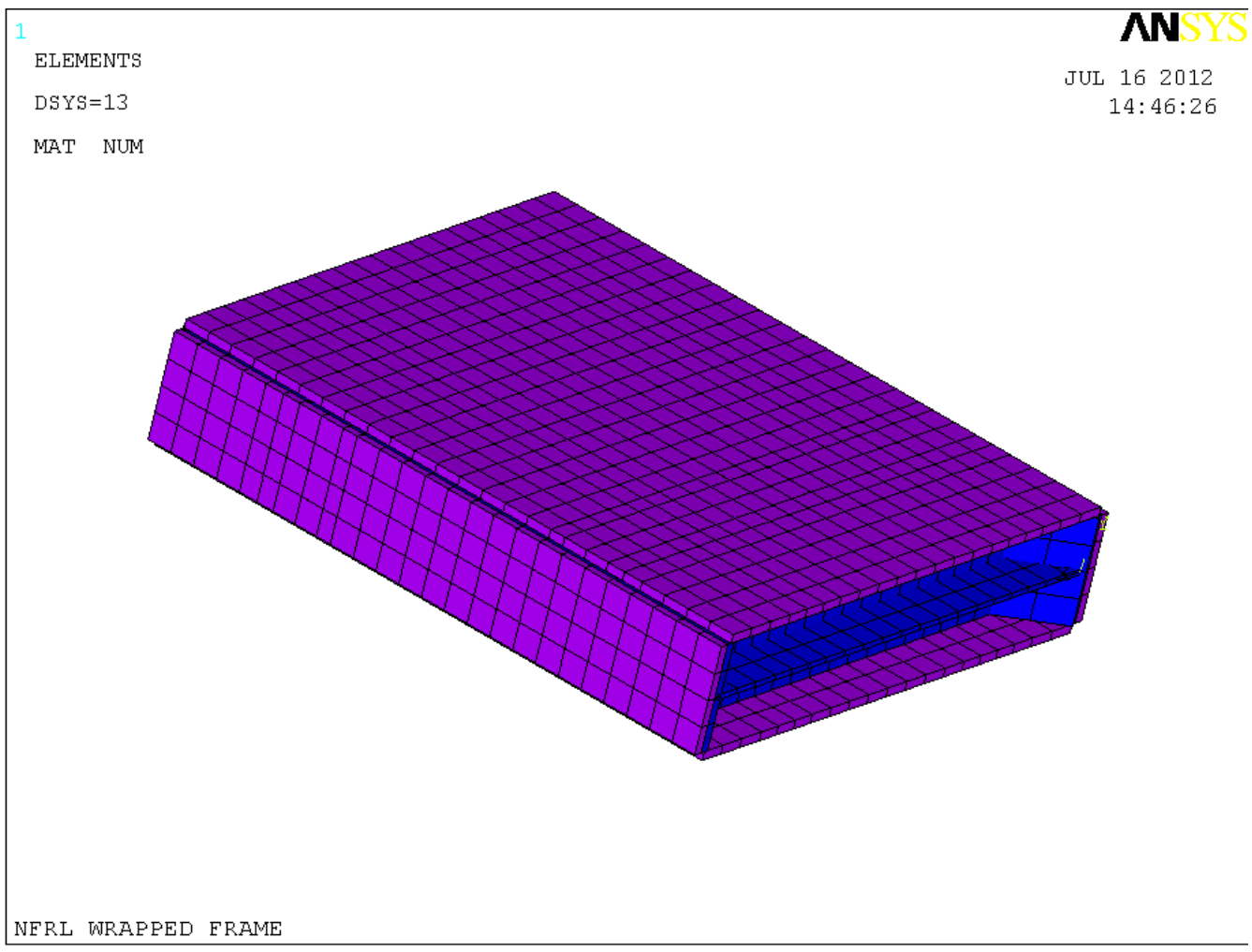

Fig. 7.1 Wrapped frame 


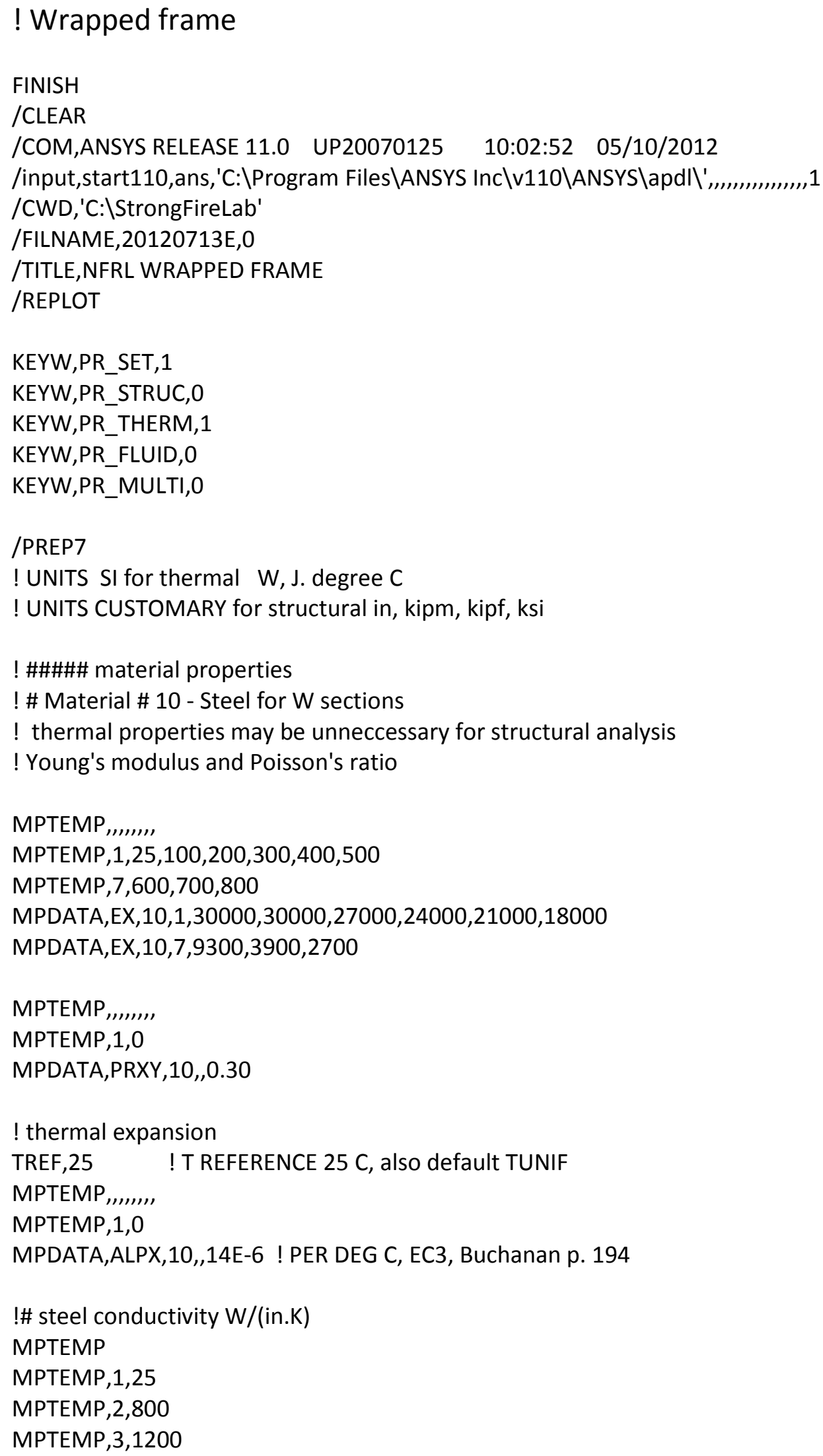




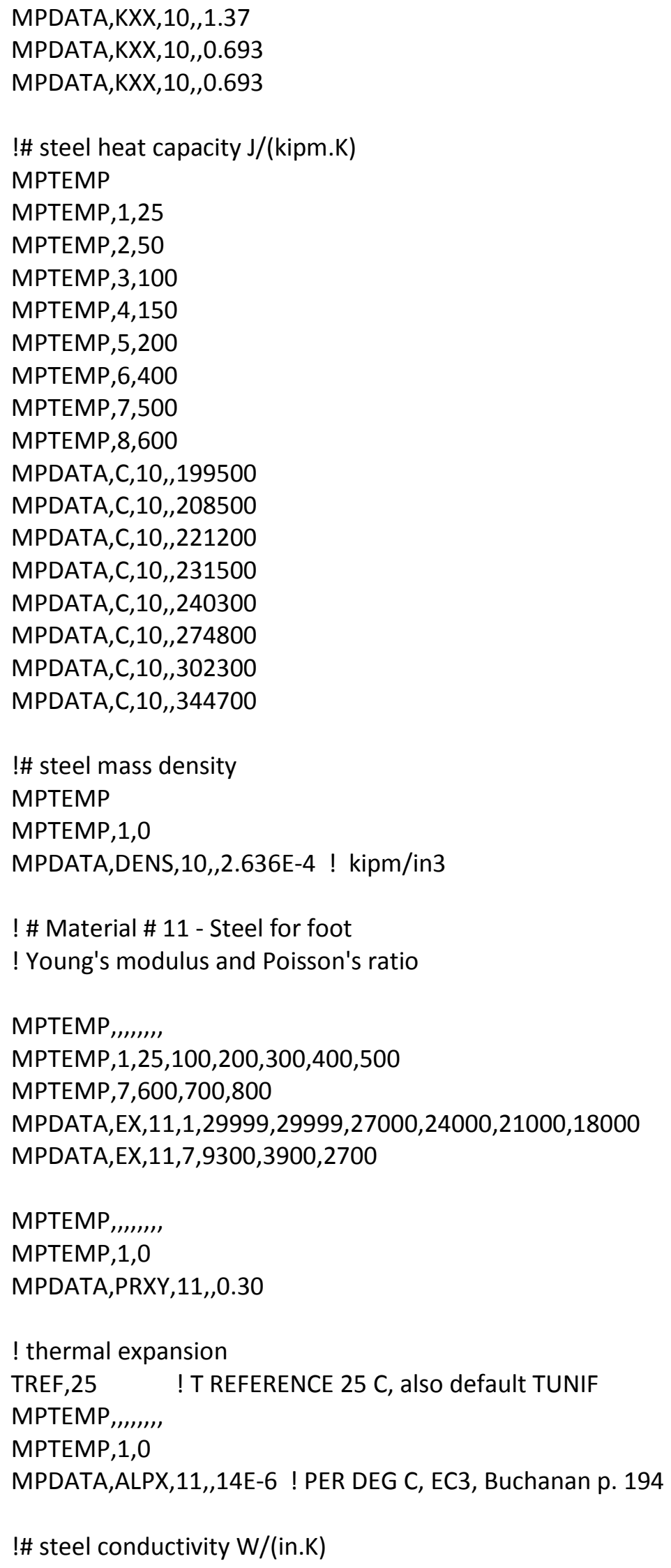




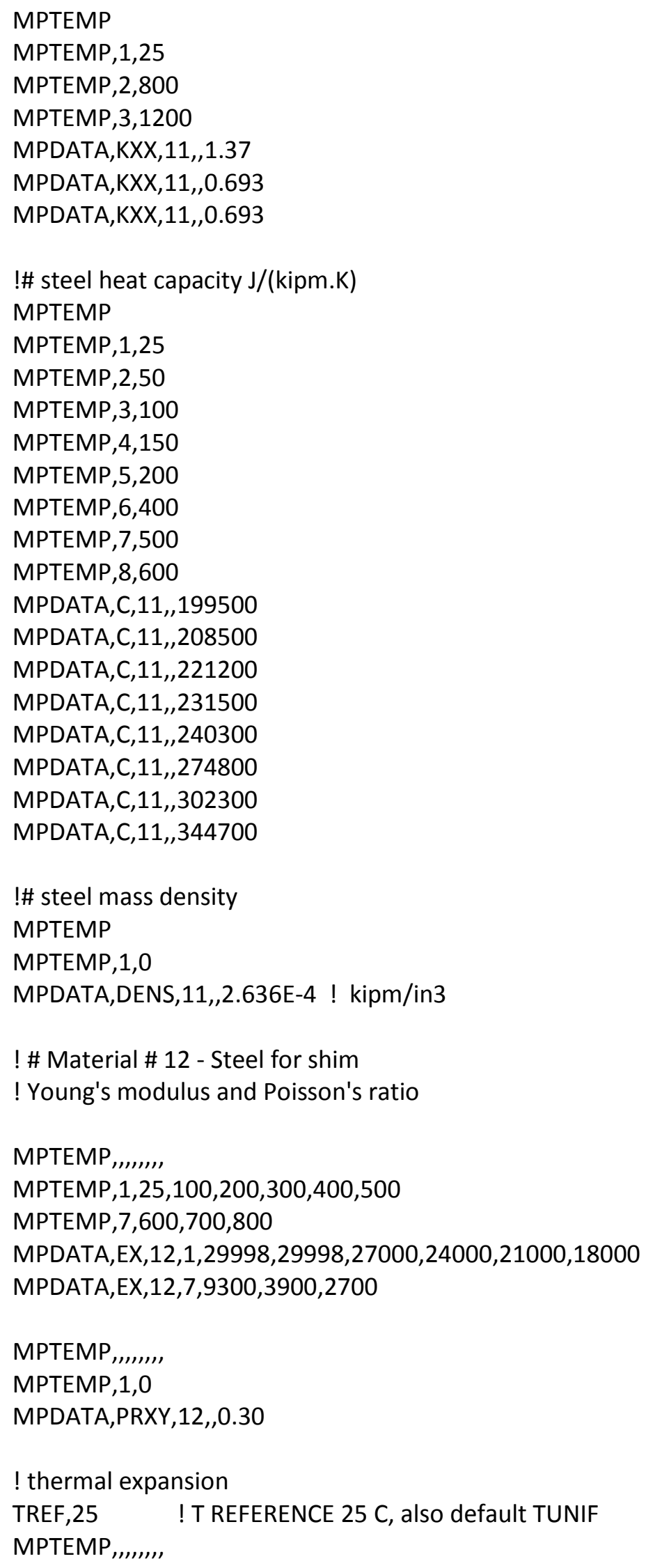


MPTEMP, 1,0

MPDATA,ALPX,12,,14E-6 ! PER DEG C, EC3, Buchanan p. 194

!\# steel conductivity W/(in.K)

MPTEMP

MPTEMP, 1,25

MPTEMP, 2,800

MPTEMP, 3,1200

MPDATA,KXX,12,,1.37

MPDATA,KXX,12,,0.693

MPDATA, KXX,12,,0.693

!\# steel heat capacity J/(kipm.K)

MPTEMP

MPTEMP, 1,25

MPTEMP, 2,50

MPTEMP, 3,100

MPTEMP,4,150

MPTEMP, 5,200

MPTEMP, 6,400

MPTEMP,7,500

MPTEMP,8,600

MPDATA,C,12,,199500

MPDATA,C,12,,208500

MPDATA,C,12,,221200

MPDATA,C,12,,231500

MPDATA,C,12,,240300

MPDATA,C,12,274800

MPDATA,C,12,,302300

MPDATA,C,12,,344700

!\# steel mass density

MPTEMP

MPTEMP, 1,0

MPDATA,DENS,12,,2.636E-4 ! kipm/in3

ET,5,SHELL131

KEYOPT,5,2,0 ! average film temperature

$\mathrm{KEYOPT}, 5,3,0$ ! quadratic temperature distribution through thickness

KEYOPT,5,4,0 ! use sect defined above

KEYOPT,5,6,0 ! no paint

!KEYOPT,5,8,2 use this option when insulation is present- data at top, bottom and mid of all layers !SHELL,MID use this option when insulation is present-results calculated at mid, not averaged from top and bottom

sect,2,shell, foot

secdata, $2,11,0,3$

secoffset,MID 


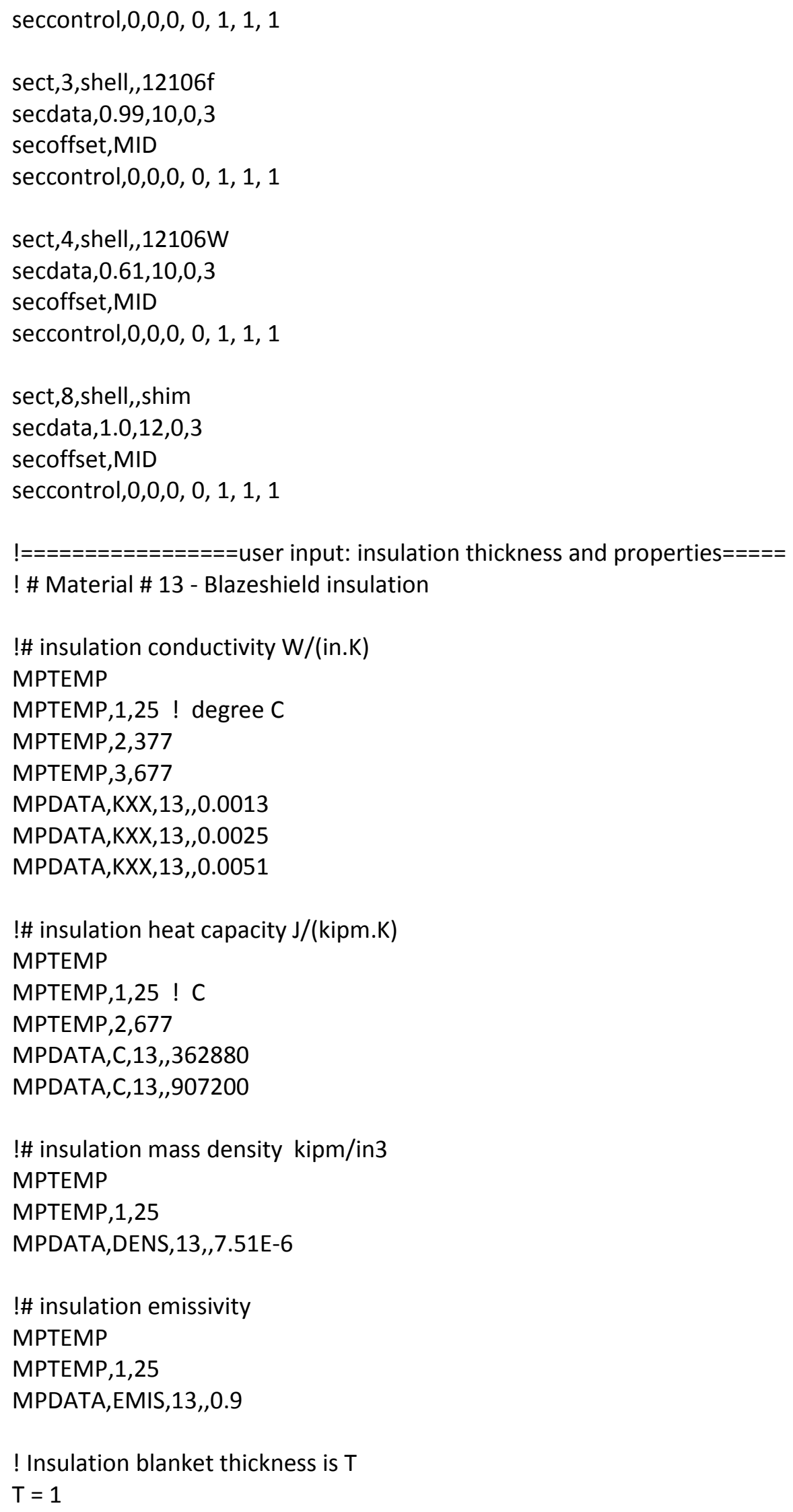


! careful that same emissivity can be used for steel and insulation

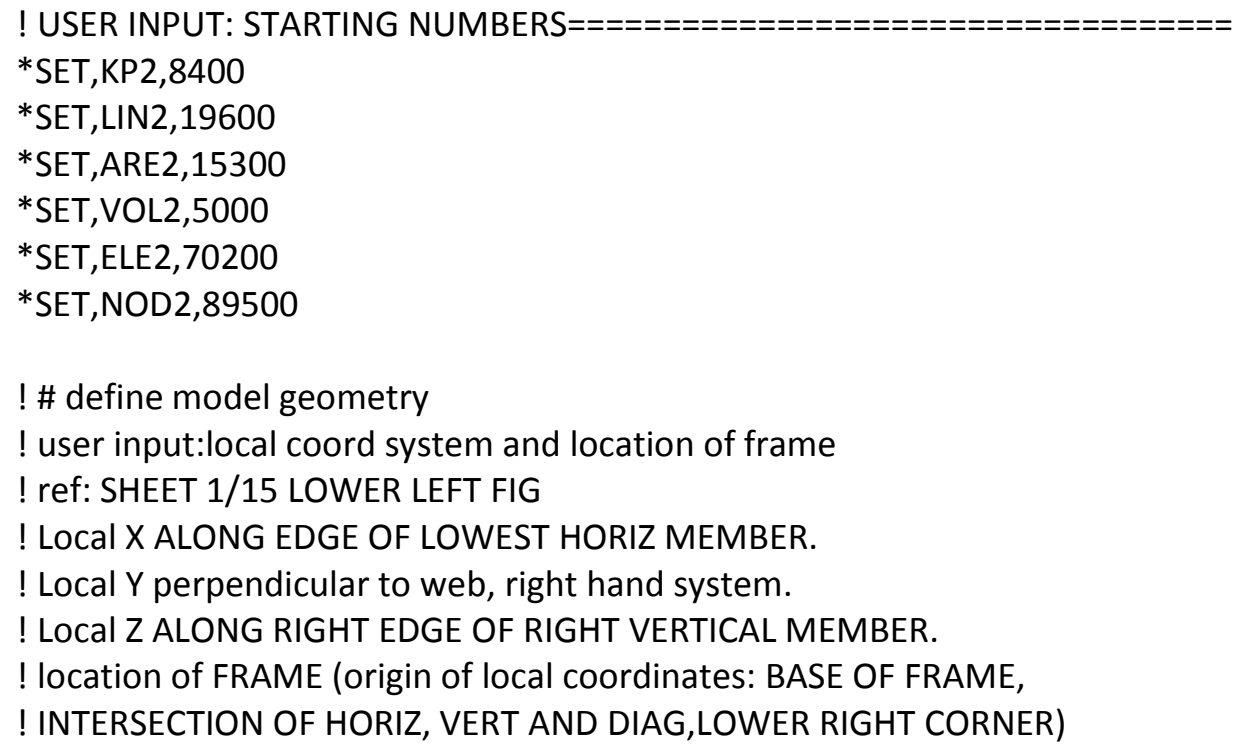




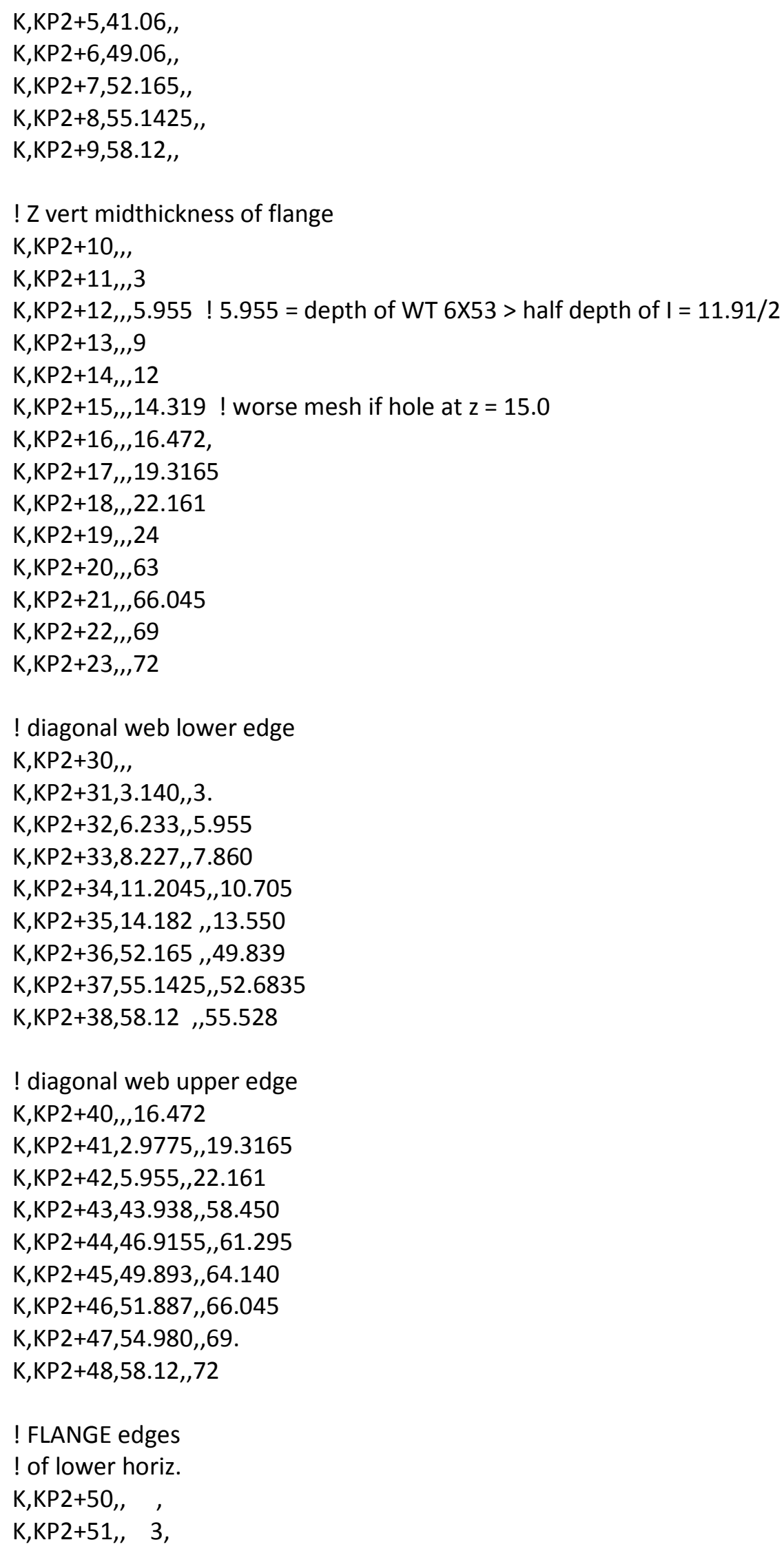




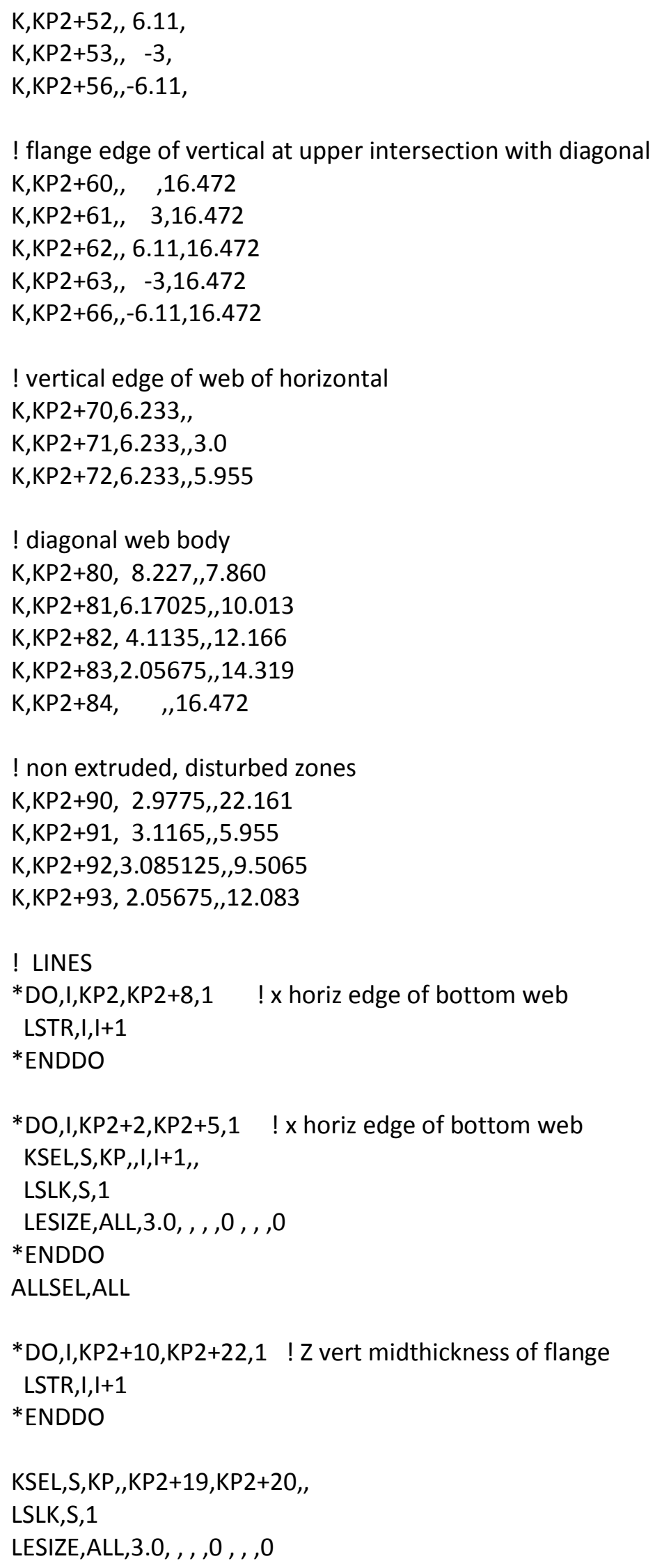


ALLSEL,ALL

*DO,I,KP2+30,KP2+37,1 ! diagonal web lower edge LSTR,I,I+1

*ENDDO

KSEL,S,KP, ,KP2+35,KP2+36, ,

LSLK,S,1

LESIZE,ALL, , , 17, , 0 , , ,0

ALLSEL,ALL

*DO,I,KP2+40,KP2+47,1 ! diagonal web upper edge LSTR, I,I+1

*ENDDO

KSEL,S,KP,,KP2+42,KP2+43,

LSLK,S,1

LESIZE,ALL, , ,17, ,0 , , ,0

ALLSEL,ALL

*DO,I,KP2+33,KP2+38,1 ! diagonal web body

LSTR,I,I+7

KSEL,S,KP, ,I,I+7,7,

LSLK,S,1

LESIZE,ALL, , , 4, , 0, , ,0

ALLSEL,ALL

*ENDDO

LSTR, KP2 +50,KP2+51 !+y flange base of vertical LSTR, KP2+51,KP2+52

LSTR, KP2 $+50, K P 2+53$ ! -y flange base of vertical

LSTR, KP2+53,KP2+56

LSTR, KP2 $+60, K P 2+61 !+y$ flange base of diagonal

LSTR, KP2+61,KP2+62

LSTR,KP2+60,KP2+63 ! -y flange base of diagonal

LSTR,KP2+63,KP2+66

LSTR, KP2 $+70, \mathrm{KP} 2+71$ ! web of horiz

LSTR,KP2+71,KP2+72 ! save db L=19600 to 19650

LSTR,KP2+18,KP2+90! web of vert

LSTR,KP2+90,KP2+42 ! L=19651,2

! non extruded smaller zone

LSTR,KP2+17,KP2+41 ! L=19653

LSTR,KP2+41,KP2+90 ! L=19654

! non extruded larger zone

LSTR,KP2+15,KP2+83 ! L=19655 


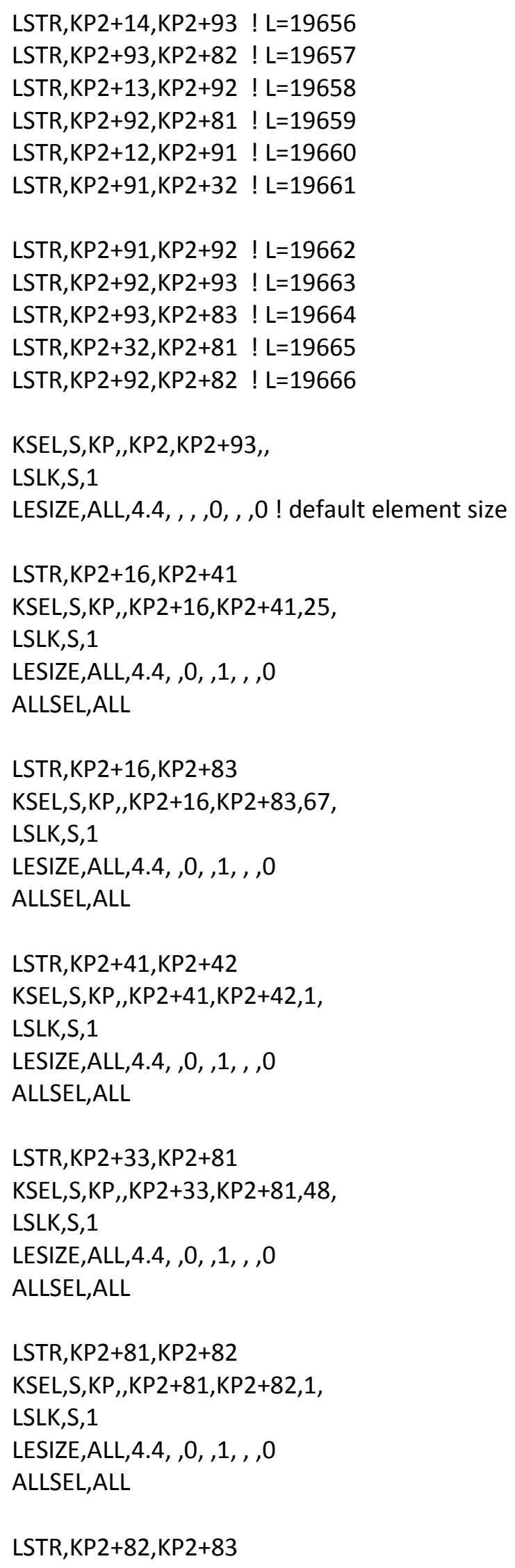




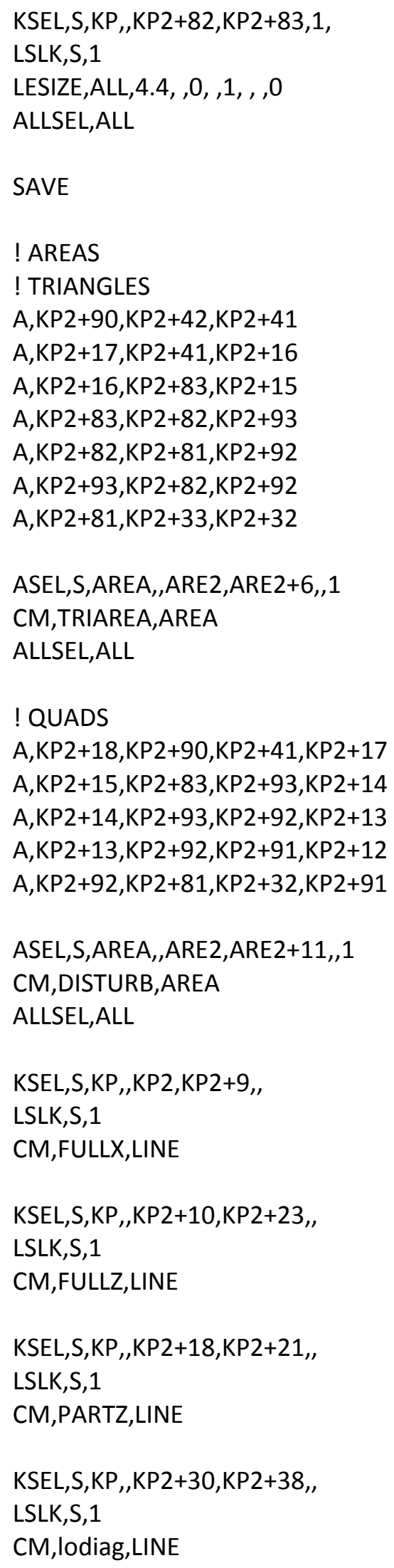


$\mathrm{KSEL}, \mathrm{S}, \mathrm{KP}$, ,KP2+40,KP2+48,

LSLK,S,1

CM,hidiag,LINE

KSEL,S,KP, ,KP2+50,KP2+52,1,

LSLK,S,1

CM,flangeplus,LINE

KSEL,S,KP, ,KP2+50,KP2+56,3,

LSLK,S,1

$\mathrm{CM}$, flangeminus, LINE

KSEL,S,KP, ,KP2+60,KP2+62,1, LSLK,S,1

$\mathrm{CM}$, flandiagplus, LINE

KSEL,S,KP, KP2+60,KP2+66,3,

LSLK,S,1

CM,flandiagminus, LINE

KSEL,S,KP, ,KP2+70,KP2+72,1,

LSLK,S,1

CM, webhoriz,LINE

KSEL,S,KP, KP2+18,KP2+42,24,

KSEL,A,KP, ,KP2+90

LSLK,S, 1

CM, webvert, LINE

ALLSEL,ALL

!!*GET,AMAX1,AREA,0,NUM,MAX

! diagonal web areas

$\mathrm{A}, \mathrm{KP} 2+33, \mathrm{KP} 2+34, \mathrm{KP} 2+41, \mathrm{KP} 2+40$

$* \mathrm{DO}, \mathrm{I}, \mathrm{KP} 2+34, \mathrm{KP} 2+37,1$

$A, I, I+1, I+8, I+7$

*ENDDO

ASEL,S,AREA,,ARE2,ARE2+16,,1

CM,DIAWEBTURB,AREA ! DIAGONAL WEB AND DISTURBED AREAS=D

ALLSEL,ALL

! areas

ADRAG, lodiag, ,,,,,, flangeplus,,,,,,

ASEL,S,AREA,,ARE2,ARE2+32,,1

CM,DLOPLUS,AREA ! DIAGONAL WEB,DISTURBED AND LOW PLUS DIAG FLANGE AREAS

ALLSEL,ALL 
ADRAG, lodiag, ,,,,,,flangeminus,,,,,,

ASEL,S,AREA,,ARE2,ARE2+48,,1

CM,DLODIAG,AREA ! DIAGONAL WEB,DISTURBED AND LOW DIAG FLANGE AREAS

ALLSEL,ALL

ADRAG, hidiag, ,,,,,,flandiagplus,,,,,,

ASEL,S,AREA,,ARE2,ARE2+64,,1

CM,DLOHIPLUS,AREA ! DIAGONAL WEB,DISTURBED,LOW DIAG FLANGE AND HIGH PLUS DIAG FLANGE

AREAS

ALLSEL,ALL

ADRAG, hidiag,,,,,,, flandiagminus,,,,,,

ASEL,S,AREA,,ARE2,ARE2+80,

CM,DLOHI,AREA ! DIAGONAL WEB,DISTURBED,LOW AND HIGH DIAG FLANGE AREAS

ALLSEL,ALL

ADRAG,FULLZ,,,,,,,flangeplus,,,,,,

ADRAG,FULLZ,,,,,,,flangeminus,,,,,,

ADRAG,partz,,,,,,, webvert,,,,,,

ADRAG,fullx,,,,,,,flangeplus,,,,,,,

ADRAG, fullx,,,,,, flangeminus,,,,,,

ADRAG, fullx,,,,,W,WEBHORIZ,,,,,,

ALLSEL,ALL

SAVE

! mesh webS

! MESH TRIANGLES

! much simpler to define elements by commands NKPT and E

CMSEL,S,TRIAREA,AREA

AATT, 10, 5, 13,4

MSHAPE,1,2D

MSHKEY,0

AMESH,ALL

! MESH QUADS

CMSEL,S,DISTURB,AREA

CMSEL,U,TRIAREA,AREA

AATT, 10, 5, 13,4

MSHAPE,0,2D

MSHKEY,1

AMESH,ALL

! MESH DIAG WEB

CMSEL,S,DIAWEBTURB,AREA

CMSEL,U,DISTURB,AREA

AATT, 10,,5,13,4

MSHAPE,0,2D 


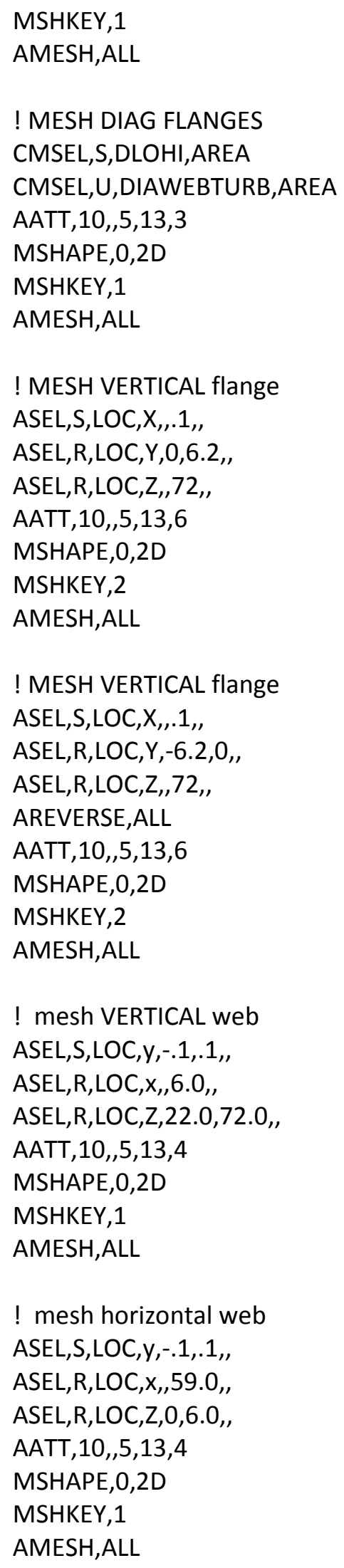




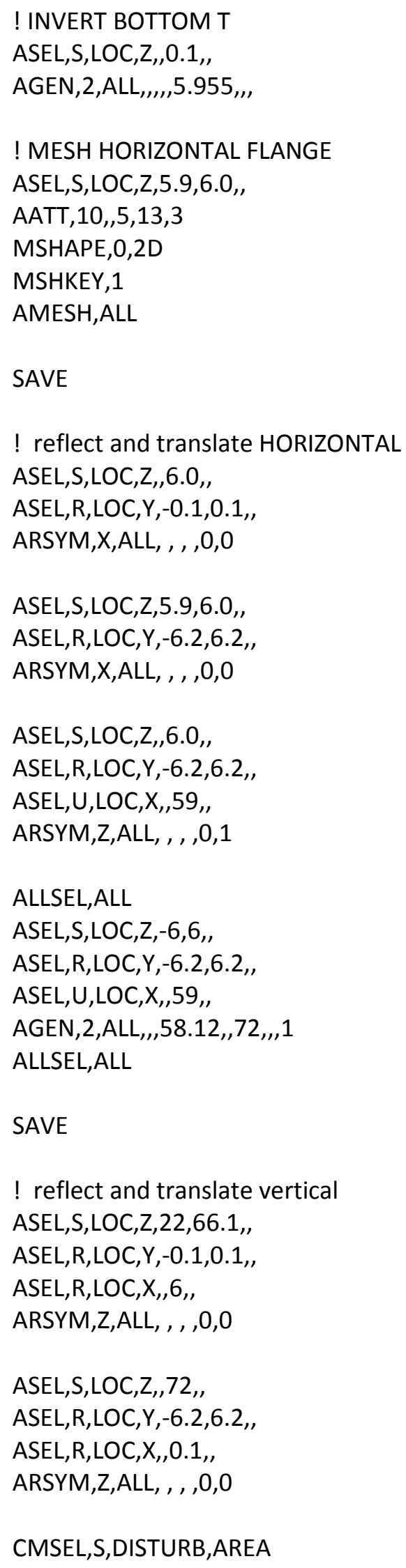




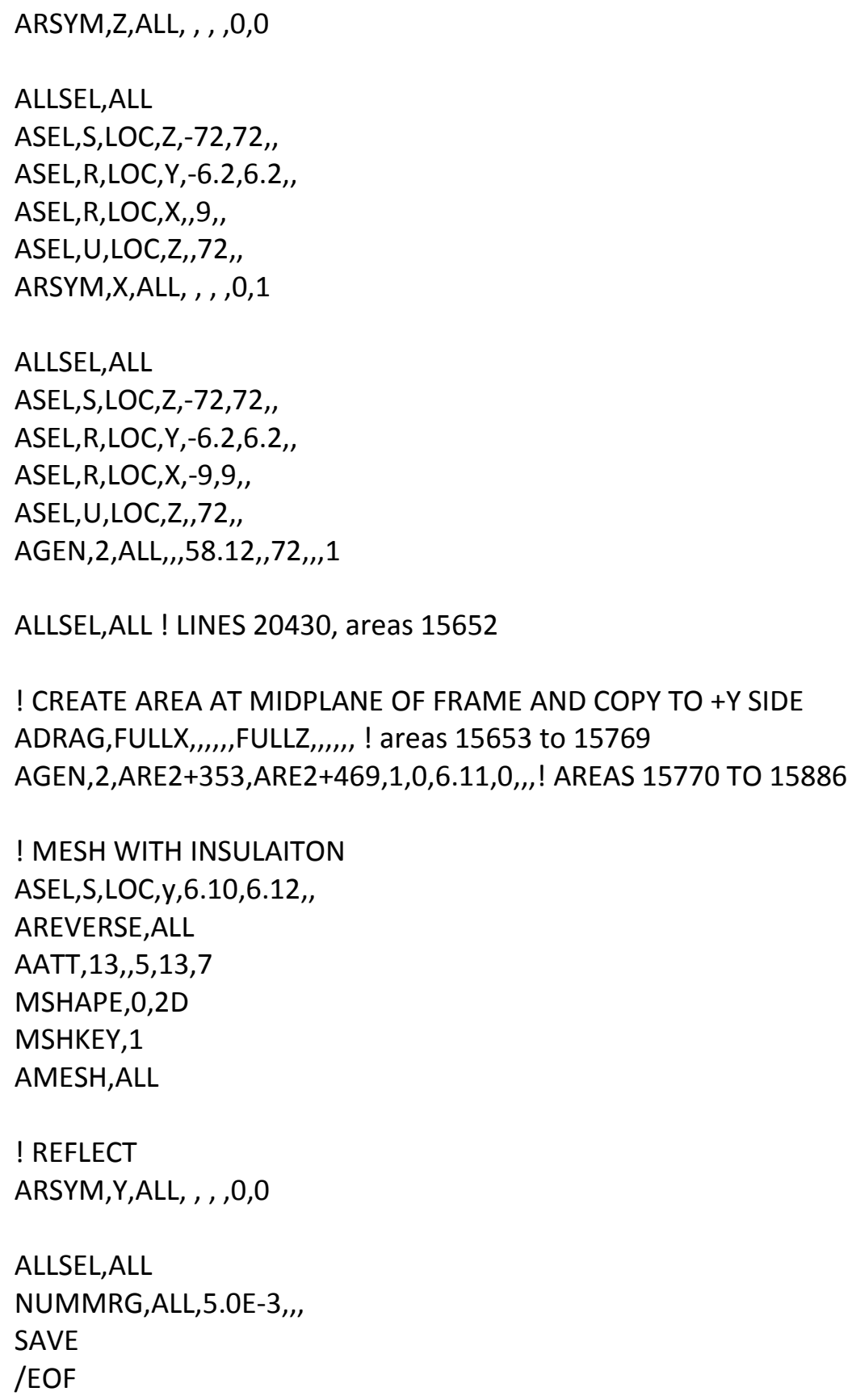




\section{Chapter 8 Web splice}

Table 8.1 Structural components

\begin{tabular}{|l|l|}
\hline Web splice & Starting numbers \\
\hline *Keypoints KP3 & 9350 \\
\hline *Lines LIN3 & 21300 \\
\hline *Areas ARE3 & 16100 \\
\hline${ }^{*}$ Volumes VOL3 & 5000 \\
\hline *Elements ELE3 & 72100 \\
\hline *Nodes NOD3 & 91700 \\
\hline $\begin{array}{l}\text { *Local coordinates } \\
\text { system 14 }\end{array}$ & $\begin{array}{l}\text { x along lower edge of splice; } \\
\text { y perpendicular to web, right hand system; } \\
\text { zalong left vertical edge of splice (Fig. 8.1); } \\
\text { connected by 3 holes to upper web of frame of } \\
\text { coordinates 13. }\end{array}$ \\
\hline Element type & 5 Shell 181 \\
\hline Sections & 9 (0.313 in) \\
\hline Materials & \begin{tabular}{l} 
* (W sections) \\
\hline
\end{tabular} \\
\hline
\end{tabular}
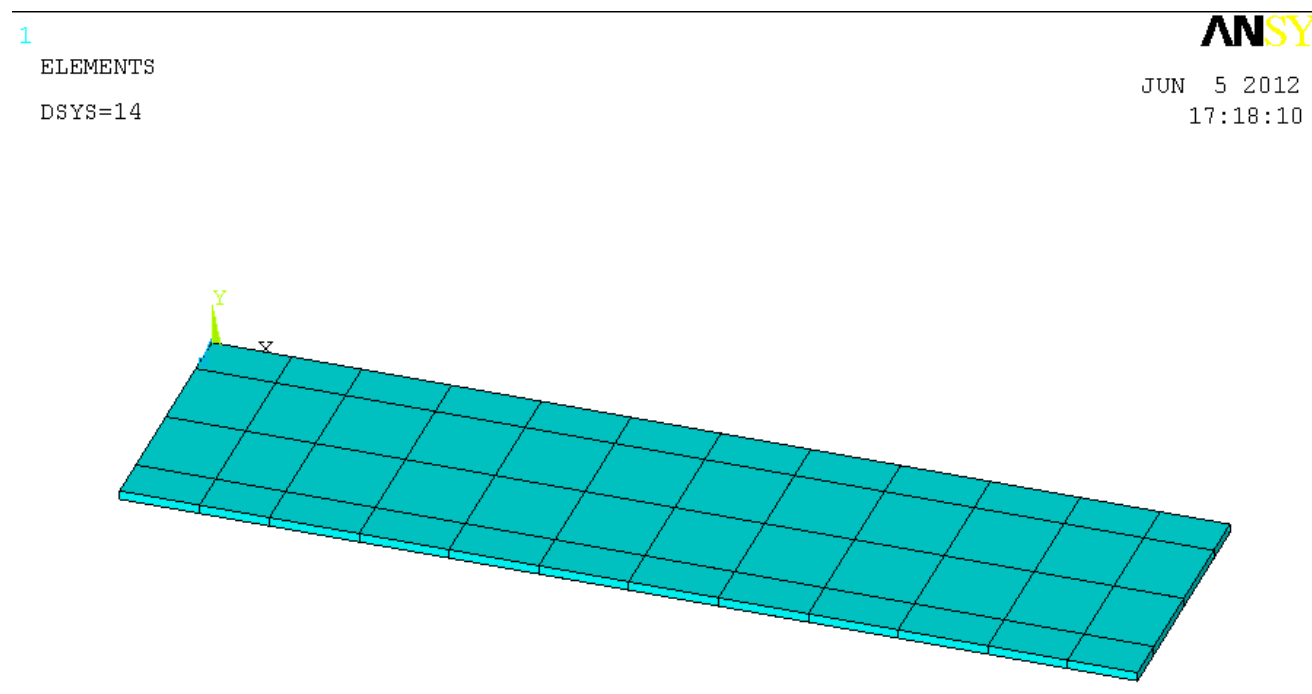

NERL WEB SPLICE

Fig. 8.1 Web splice 


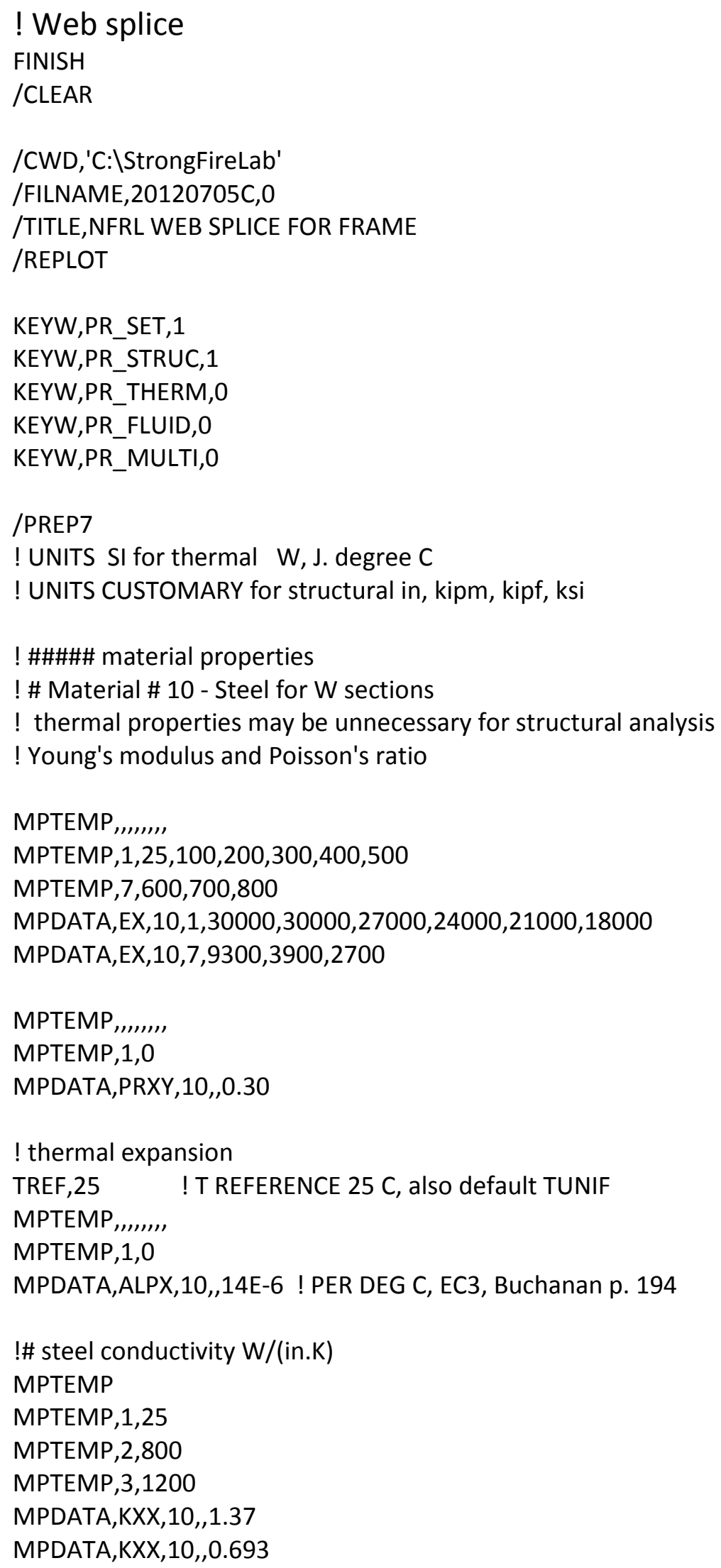




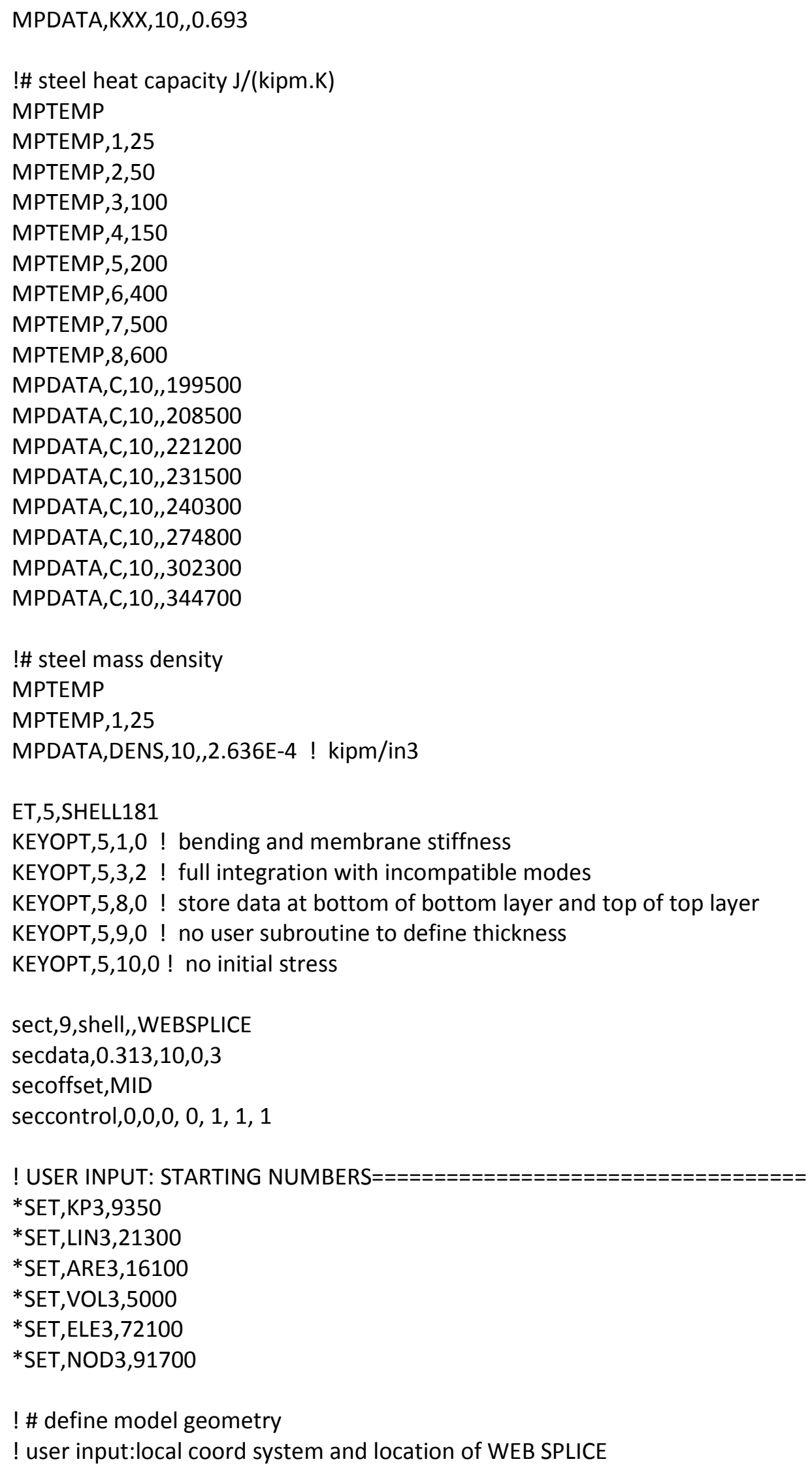


! ref: SHEET 10/15 LOWER LEFT FIG

! Local X ALONG LOWER EDGE OF SPLICE.

! Local Y perpendicular to web, right hand system.

! Local Z ALONG LEFT VERTICAL EDGE OF SPLICE.

! location of SPLICE (origin of local coordinates 14:

! CONNECTED BY 3 HOLES TO UPPER WEB OF FRAME OF COORD 13)

LOCAL,14,0,212.06,200,267.375, , , ,1,1

CSYS,14,

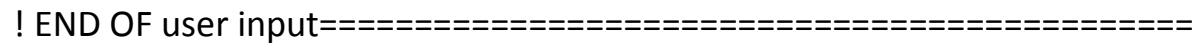

NUMSTR,KP,KP3

NUMSTR,LINE,LIN3

NUMSTR,AREA,ARE3

NUMSTR,VOLU,VOL3

NUMSTR,ELEM,ELE3

NUMSTR,NODE,NOD3

! $\mathrm{x}$ horiz edge of SPLICE bottom==================================

$\mathrm{K}, \mathrm{KP} 3,$,

$\mathrm{K}, \mathrm{KP} 3+1,2.6667$, ,

$\mathrm{K}, \mathrm{KP} 3+2,5.0$,

$\mathrm{K}, \mathrm{KP} 3+3,17.0$,

$\mathrm{K}, \mathrm{KP} 3+4,29.0$,

$\mathrm{K}, \mathrm{KP} 3+5,31.6667$,

$\mathrm{K}, \mathrm{KP} 3+6,34.0$,

! Z vert midthickness of flange

$\mathrm{K}, \mathrm{KP} 3+10,$,

$\mathrm{K}, \mathrm{KP} 3+11,,, 1.625$

$\mathrm{K}, \mathrm{KP} 3+12,,, 7.625$

$\mathrm{K}, \mathrm{KP} 3+13,,, 9.25$

! LINES

*DO,I,KP3,KP3+5,1 ! $x$ horiz bottom edge of SPLICE LSTR,I,I+1

*ENDDO

KSEL,S,KP,,KP3,KP3+6,,

LSLK,S,1

LESIZE,ALL,3.0, , , , 0 , , , 0

CM,SPLICEX,LINE

ALLSEL,ALL

*DO,I,KP3+10,KP3+12,1 ! Z vert LEFT EDGE OF SPLICE

LSTR,I,I+1 
*ENDDO

KSEL,S,KP, ,KP3+10,KP3+13,,

LSLK,S,1

LESIZE,ALL,3.0, , , , 0 , , , 0

CM,SPLICEZ,LINE

! area

ALLSEL,ALL

ADRAG,SPLICEX ,,,,,,SPLICEZ,,,,,,

asel,s, area,,are3,are3+17,,1

AATT,10,,5,14,9

MSHAPE,0,2D

MSHKEY,1

AMESH,ALL

ALLSEL,ALL

NUMMRG,ALL

SAVE

! the following is a load test

/SOLU

ANTYPE, 0 ! static analysis

! constrain $X$ ends

NSEL,S,LOC, X,0,0.1,

NSEL,A,LOC,X,33.9,34.1,

$D, A L L, U X, \ldots, \ldots, \ldots$,

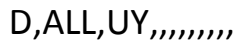

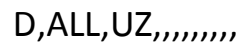

! LOAD MIDDLE $\mathrm{x}=17$

ALLSEL,ALL

NSEL,S,LOC,X,16.9,17.1,

$F, A L L, F Y,-1,,$,

ALLSEL,ALL

OUTPR,STAT

OUTRES,STAT

SOLVE

/EOF 


\section{Chapter 9 Girder}

Table 9.1 Properties

\begin{tabular}{|l|l|}
\hline Girder & Starting numbers \\
\hline *Keypoints KP4 & 9400 \\
\hline *Lines LIN4 & 21700 \\
\hline *Areas ARE4 & 16300 \\
\hline *Volumes VOL4 & 5000 \\
\hline *Elements ELE4 & 72400 \\
\hline *Nodes NOD4 & 92100 \\
\hline $\begin{array}{l}\text { *Local coordinates } \\
\text { system 15 }\end{array}$ & $\begin{array}{l}\text { x along midheight of web; } \\
\text { y perpendicular to web, right hand system; } \\
\text { z along left vertical edge of web; } \\
\text { origin: end of girder (Figs. 9.1 and 9.2) }\end{array}$ \\
\hline Element type & 5 Shell 181 \\
\hline Sections & $\begin{array}{l}\text { *Girder LENGTH } \\
10 \text { (0.55 inch web W 24x103) } \\
11 \text { (0.98 inch flange W 24x103) }\end{array}$ \\
\hline Materials & 10 (W sections) \\
\hline
\end{tabular}

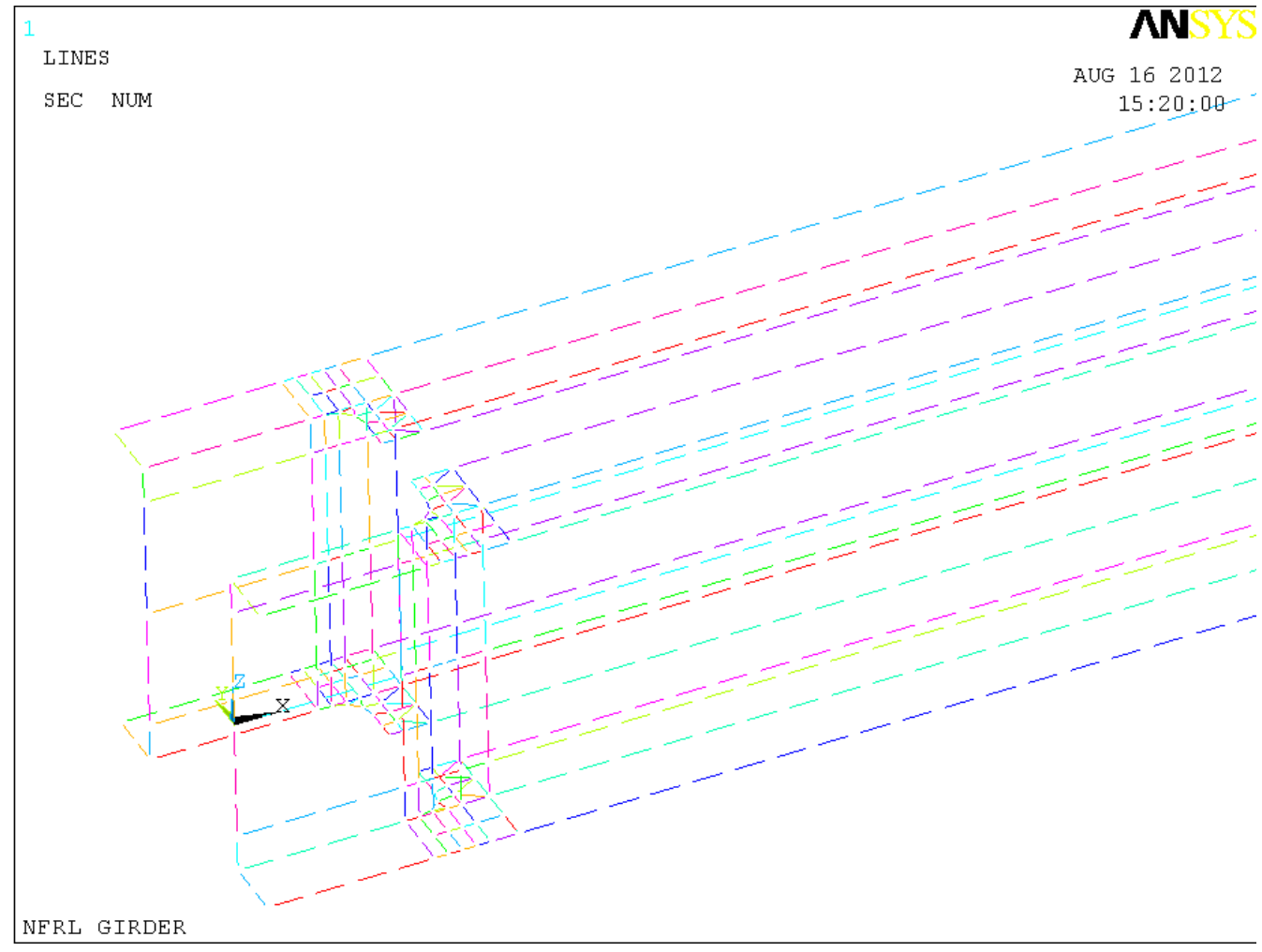

Fig. 9.1 Girder lines showing local coordinates 


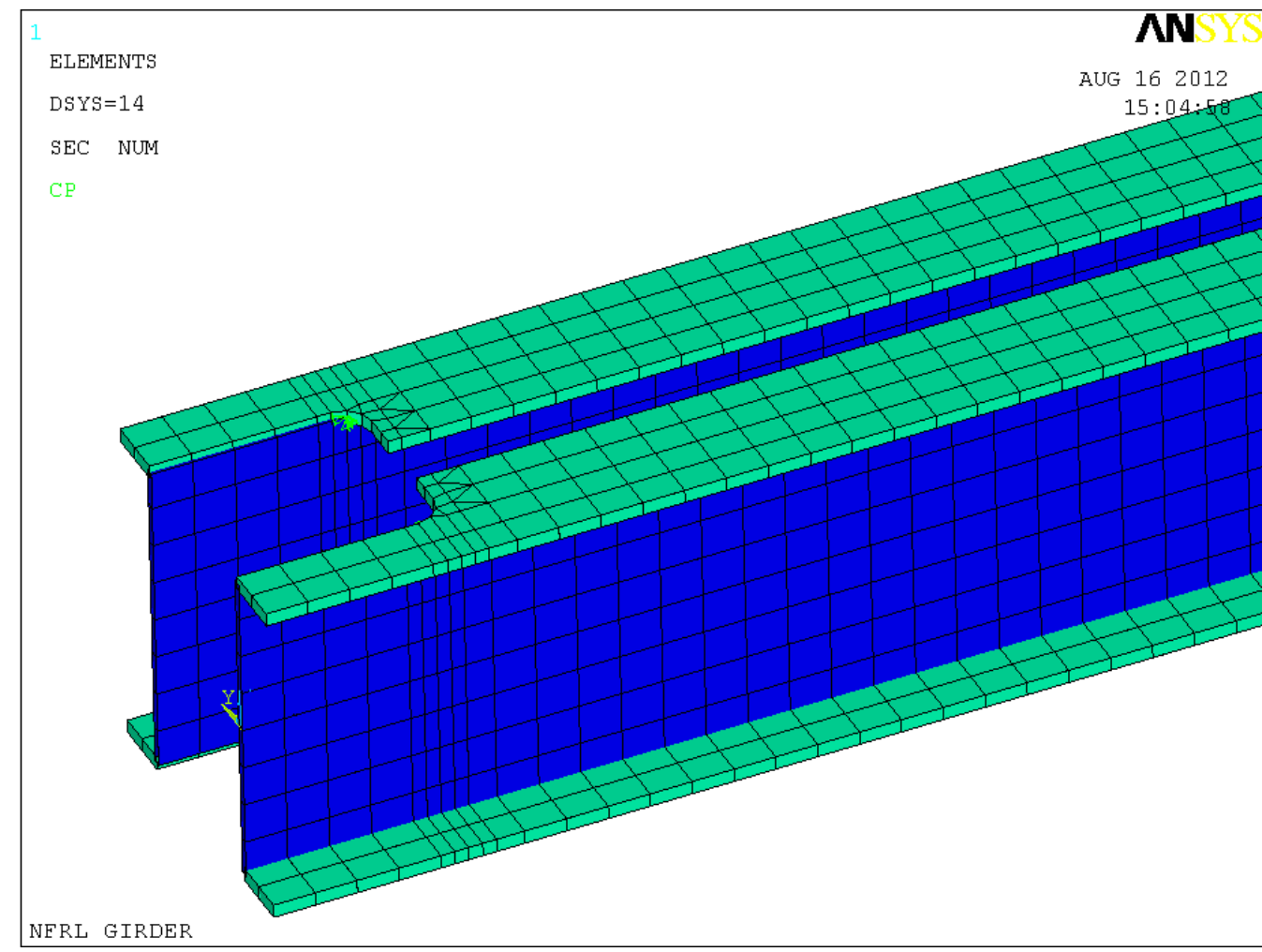

Fig. 9.2 Girder elements showing coordinate system

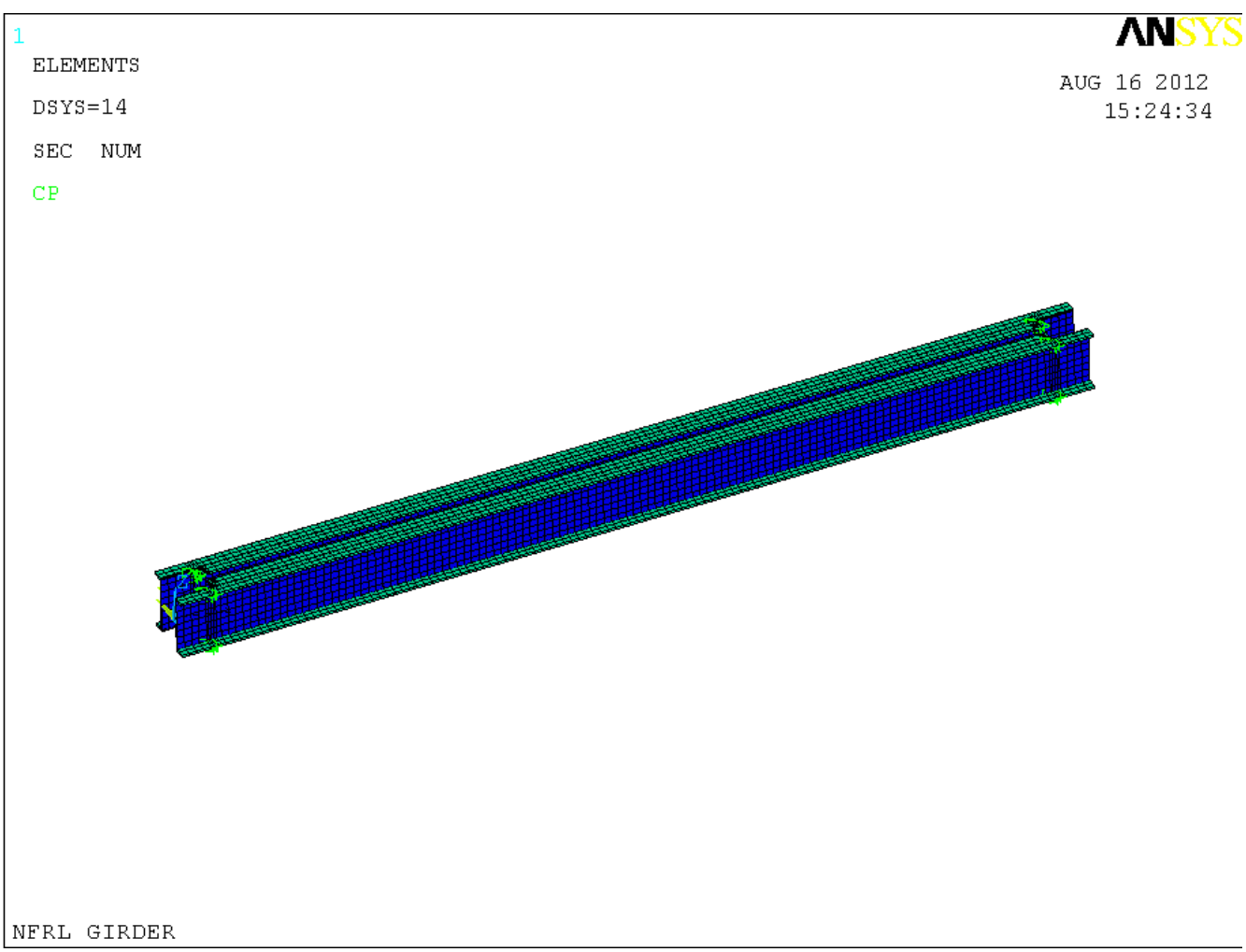

Fig. 9.3 Entire girder 


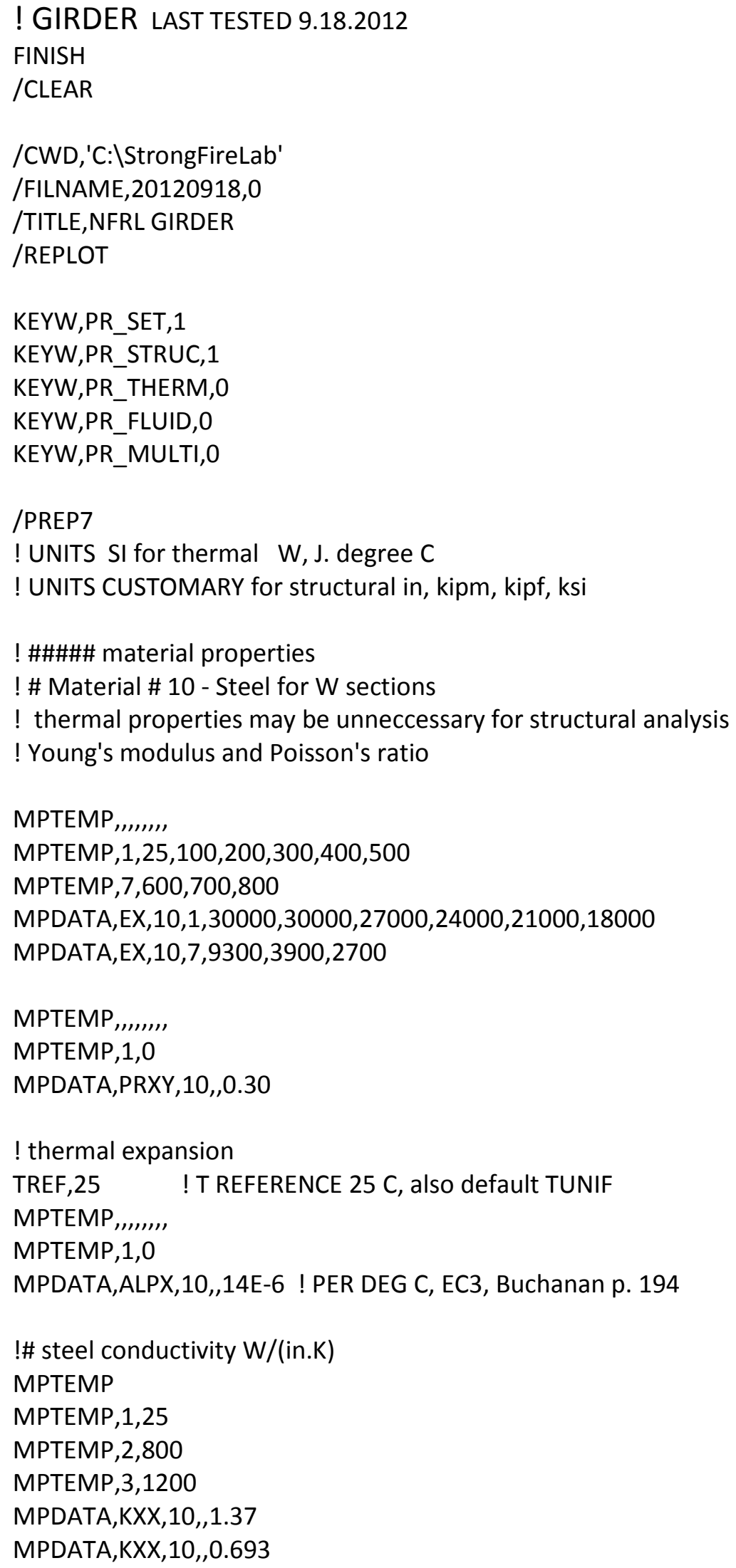


MPDATA,KXX,10,,0.693

!\# steel heat capacity J/(kipm.K)

MPTEMP

MPTEMP, 1,25

MPTEMP, 2,50

MPTEMP, 3,100

MPTEMP,4,150

MPTEMP,5,200

MPTEMP, 6,400

MPTEMP $, 7,500$

MPTEMP, 8,600

MPDATA,C,10,,199500

MPDATA,C,10,,208500

MPDATA,C,10,,221200

MPDATA,C,10,,231500

MPDATA,C,10,,240300

MPDATA,C,10,,274800

MPDATA,C,10,,302300

MPDATA,C,10,,344700

!\# steel mass density

MPTEMP

MPTEMP, 1,25

MPDATA,DENS,10,,2.636E-4 ! kipm/in3

ET,5,SHELL181

KEYOPT,5,1,0 ! bending and membrane stiffness

KEYOPT,5,3,2 ! full integration with incompatible modes

KEYOPT, 5,8,0 ! store data at bottom of bottom layer and top of top layer KEYOPT,5,9,0 ! no user subroutine to define thickness

KEYOPT,5,10,0 ! no initial stress

sect,10,shell,,girderweb24W103

secdata, $0.550,10,0,3$

secoffset,MID

seccontrol,0,0,0, 0, 1, 1, 1

sect,11,shell,,girderflange24W103

secdata, $0.980,10,0,3$

secoffset,MID

seccontrol,0,0,0, 0, 1, 1, 1

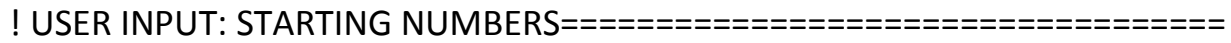

*SET,KP4,9400

*SET, LIN4, 21700

*SET,ARE4,16300

*SET, VOL4,5000 
*SET,ELE4,72400

*SET,NOD4,92100

! \# define model geometry

! user input:local coord system and location of girder

! ref: SHEET 12/15

! Local X ALONG midheight of web.

! Local Y perpendicular to web, right hand system.

! Local Z ALONG left vertical EDGE OF web.

LOCAL,15,0,10,100,200, , , ,1,1

CSYS,15,

LENGTH $=186.0$ !half - length of girder, must be $>126$ and multiple of 3 inches

! If length $>186$, user may have to increase element and node starting numbers

! of subsequent components.

!LENGTH $=162.0$

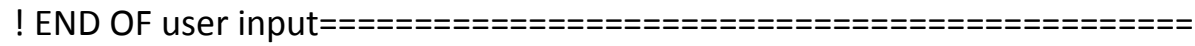

NUMSTR,KP,KP4

NUMSTR,LINE,LIN4

NUMSTR,AREA,ARE4

NUMSTR, VOLU,VOL4

NUMSTR,ELEM,ELE4

NUMSTR,NODE,NOD4

! $\mathrm{x}$ horiz midheight of web, halfspan

$\mathrm{K}, \mathrm{KP} 4,$,

$\mathrm{K}, \mathrm{KP} 4+1,12.0$,

$\mathrm{K}, \mathrm{KP} 4+2,13.0$,

$\mathrm{K}, \mathrm{KP} 4+3,14.0$,

$\mathrm{K}, \mathrm{KP} 4+4,16.0$,

$\mathrm{K}, \mathrm{KP} 4+5,18.0$,

K,KP4+6,LENGTH,, !***

! Z vert midthickness of web

$\mathrm{K}, \mathrm{KP} 4+10,$, ,

$\mathrm{K}, \mathrm{KP} 4+11,, 9$

!K,KP4+11,,,2.775

$\mathrm{K}, \mathrm{KP} 4+12,,, 11.775$

! y width of half flange

K,KP4+20,,,11.775

$\mathrm{K}, \mathrm{KP} 4+21,,-4.5,11.775$

! y width of full flange

K,KP4+30,18,4.5,11.775

K,KP4+31,18,-4.5,11.775 


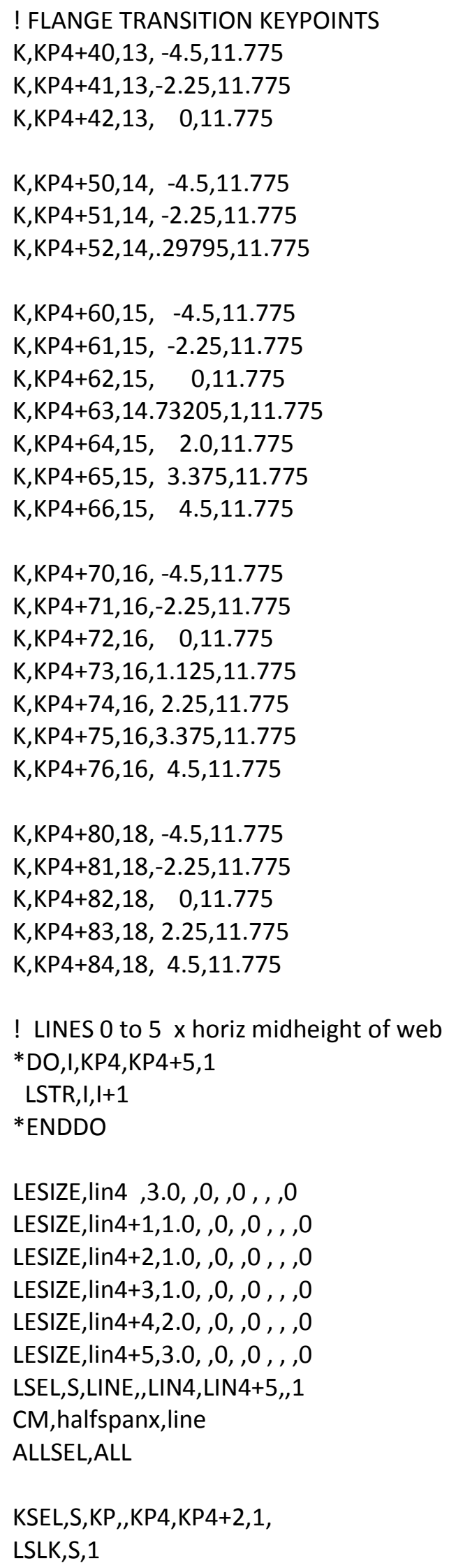


CM,cutoffx,line

allsel,all

KSEL,S,KP, ,KP4+5,KP4+6,1,

LSLK,S,1

$\mathrm{CM}$, fullwidthx, line

allsel,all

! LINES 6 to $7 \mathrm{z}$ vert midthickness of half web

*DO,I,KP4+10,KP4+11,1

LSTR,I,I+1

*ENDDO

LESIZE, lin4+6,3.0, ,0, , 0, , , 0

LESIZE,lin4+7, $0,1,1,0$, , , 0

!LESIZE,lin4+6, $0,1,, 0$, , , 0

!LESIZE, lin4+7,3.0, , $0,0,0,0$

KSEL,S,KP, ,KP4+10,KP4+12,1,

LSLK,S,1

CM, webdepthz,line

allsel,all

LSTR,KP4+20,KP4+21 ! L8 y width of half flange

LESIZE, lin4+8,0, ,2, , 0, , , 0

LSEL,S,LINE,,LIN4+8,,,1

$\mathrm{CM}$, halfwidthy, line

allsel,all

LSTR,KP4+30,KP4+31 ! L9 y width of full flange

LESIZE,lin4+9,0, ,4, , 0 , , , 0

allsel,all

LSEL,S,LINE,,LIN4+9,,,1

$\mathrm{CM}$, fullwidthy, line

ALLSEL,ALL

CMSEL,S, cutoffx, line

LGEN,2,ALL,,,,,11.775,,, ! L10 TO 11

LSEL,S,LINE,,LIN4+10,LIN4+11,1,1

CM,cutoff $\times 2$, line

allsel,all

CMSEL,S, fullwidthx,line

LGEN,2,ALL,,,,4.5,11.775,,,, ! L12 TO 13

LSEL,S,LINE,,LIN4+12,LIN4+13,,1

CM,fullwidthx2,line

allsel,all

LSEL,S,LINE,,LIN4,LIN4+12,1,1 


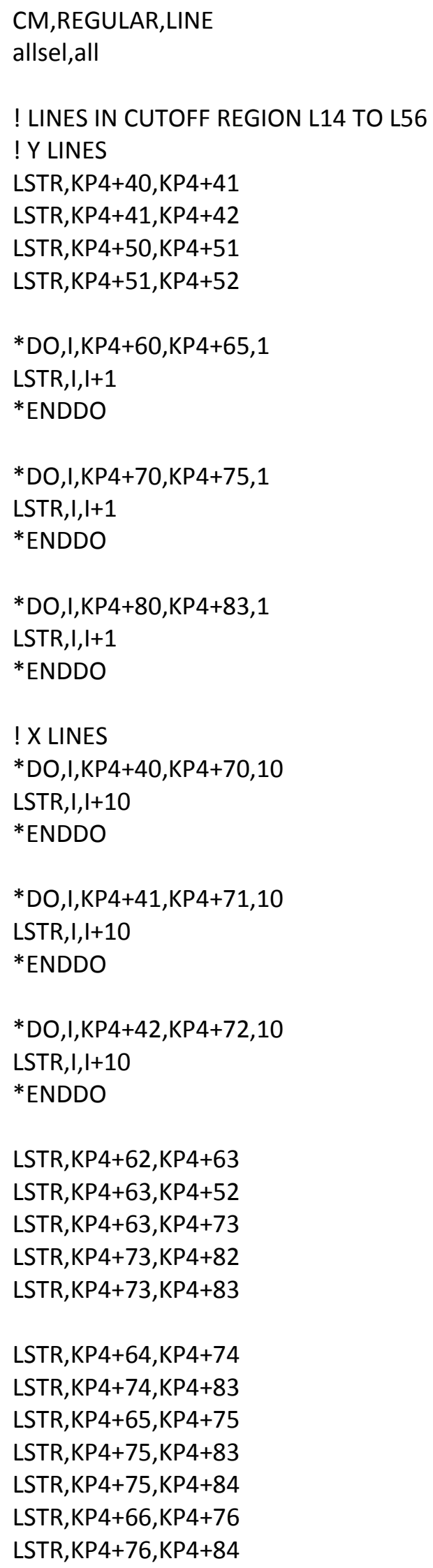


ALLSEL,ALL

LSEL,S,LINE,,LIN4,LIN4+55,,1

CM,ALLINES,LINES

CMSEL,S,ALLINES,LINE

CMSEL,U,REGULAR,LINE

LESIZE,ALL,0, , 1, , 0 , , ,0

CM,IRREGULAR,LINE

ALLSEL,ALL

! areas

*DO,I,KP4+40,KP4+70,10

$A, I, I+10, I+11, I+1$

*ENDDO

*DO,I,KP4+41,KP4+71,10

A, I,I+10,I+11,I+1

*ENDDO

$\mathrm{A}, \mathrm{KP} 4+52, \mathrm{KP} 4+62, \mathrm{KP} 4+63$

$\mathrm{A}, \mathrm{KP} 4+62, \mathrm{KP} 4+72, \mathrm{KP} 4+73, \mathrm{KP} 4+63$

$\mathrm{A}, \mathrm{KP} 4+72, \mathrm{KP} 4+82, \mathrm{KP} 4+73$

$\mathrm{A}, \mathrm{KP} 4+73, \mathrm{KP} 4+82, \mathrm{KP} 4+83$

$\mathrm{A}, \mathrm{KP} 4+63, \mathrm{KP} 4+73, \mathrm{KP} 4+74, \mathrm{KP} 4+64$

$\mathrm{A}, \mathrm{KP} 4+73, \mathrm{KP} 4+83, \mathrm{KP} 4+74$

$\mathrm{A}, \mathrm{KP} 4+64, \mathrm{KP} 4+74, \mathrm{KP} 4+75, \mathrm{KP} 4+65$

$\mathrm{A}, \mathrm{KP} 4+74, \mathrm{KP} 4+83, \mathrm{KP} 4+75$

$\mathrm{A}, \mathrm{KP} 4+75, \mathrm{KP} 4+83, \mathrm{KP} 4+84$

$\mathrm{A}, \mathrm{KP} 4+65, \mathrm{KP} 4+75, \mathrm{KP} 4+76, \mathrm{KP} 4+66$

$\mathrm{A}, \mathrm{KP} 4+75, \mathrm{KP} 4+84, \mathrm{KP} 4+76$

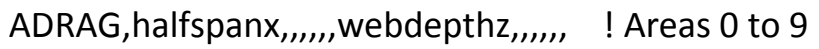

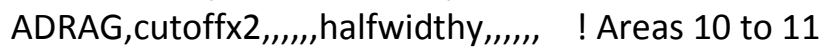

ADRAG, fullwidthx2,,,,,,, fullwidthy,,,,,, ! Areas 12 to 13

! MESH WEB

ASEL,S,LOC,Y,-0.1,0.1,,1

ASEL,R,LOC,Z,0,11.7,"

AATT, 10, 5, 15,10

MSHAPE,0,2D

MSHKEY,1

AMESH,ALL

ALLSEL,ALL

! MESH FLANGE

ASEL,S,LOC,Z,11.7,11.8,1 


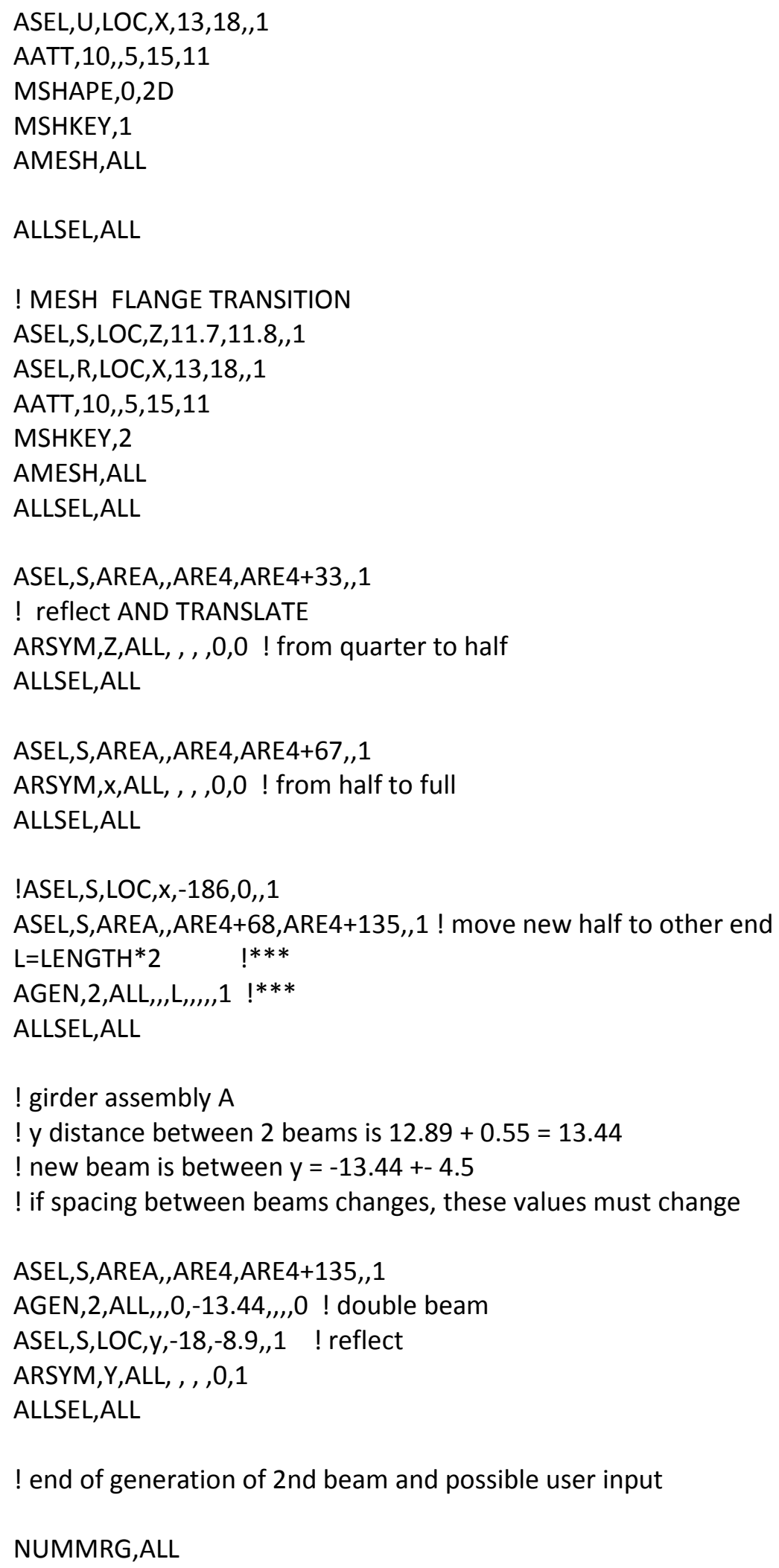




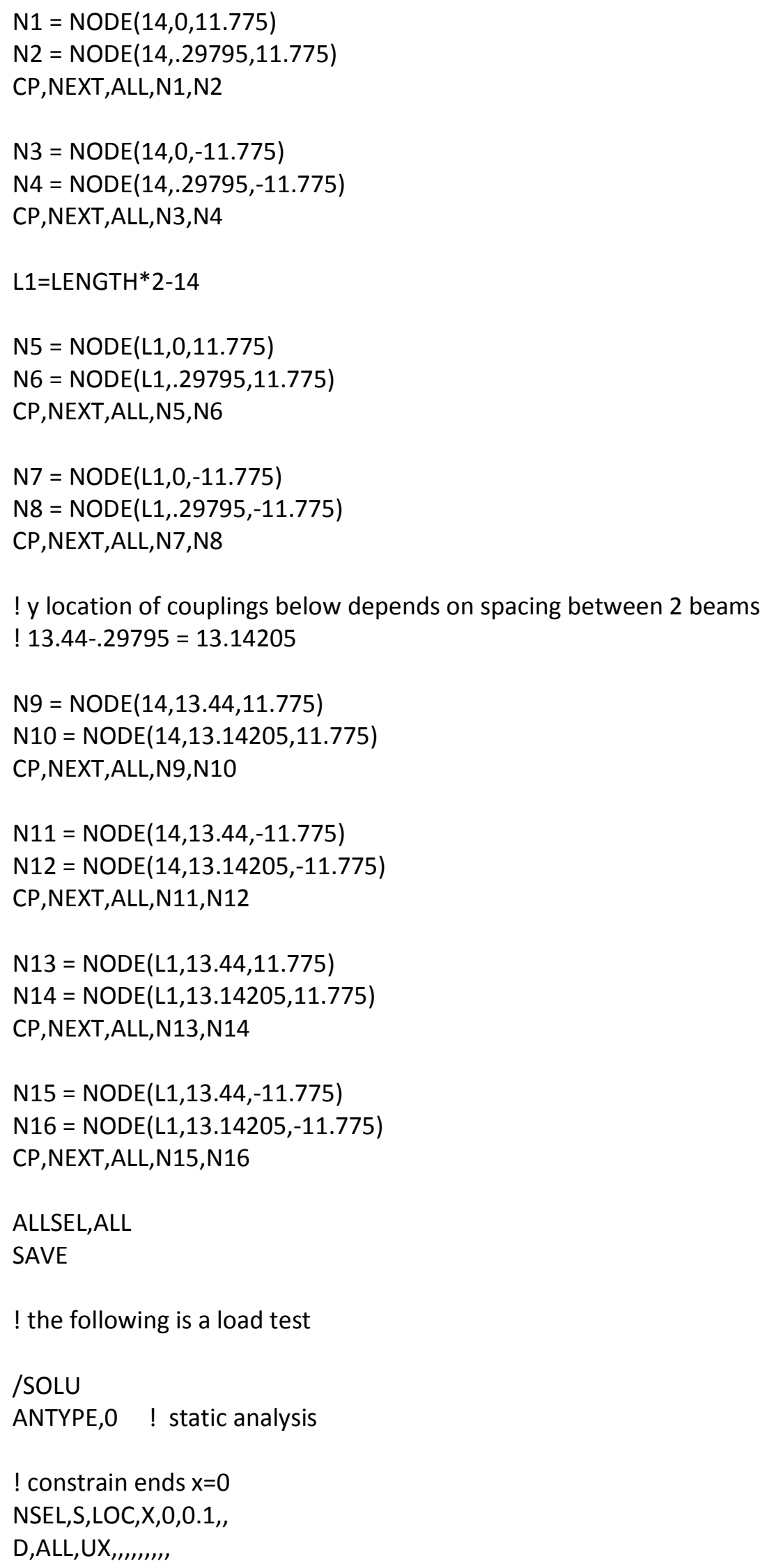




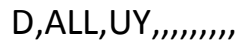

D,ALL,UZ,,,,,,,,,,

! LOAD ends $x=372$

ALLSEL,ALL

L3=LENGTH*2-0.1

L4=LENGTH*2+0.1

NSEL,S,LOC,X,L3,L4,

$F, A L L, F Z,-1$,,,

ALLSEL,ALL

OUTPR,STAT

OUTRES,STAT

SOLVE

/eof 


\section{Chapter 10 Wrapped girder}

\section{Table 10.1 Properties}

\begin{tabular}{|l|l|}
\hline Wrapped girder & Starting numbers \\
\hline *Keypoints KP4 & 9400 \\
\hline *Lines LIN4 & 21700 \\
\hline *Areas ARE4 & 16300 \\
\hline *Volumes VOL4 & 5000 \\
\hline *Elements ELE4 & 72400 \\
\hline *Nodes NOD4 & 92100 \\
\hline $\begin{array}{l}\text { *Local coordinates } \\
\text { system 15 }\end{array}$ & $\begin{array}{l}\text { x along midheight of web; } \\
\text { y perpendicular to web, right hand system; } \\
\text { z along left vertical edge of web; } \\
\text { origin: end of girder (Figs. 9.1 and 9.2) }\end{array}$ \\
\hline Element type & $\begin{array}{l}\text { * Shell 131 } \\
* 7 \text { (1 inch side wrap) } \\
10 \text { (0.55 inch web W 24x103) } \\
11 \text { (0.98 inch flange W 24x103) } \\
* 13 \text { (1 inch flange wrap) } \\
* 14 \text { (1 inch side wrap) }\end{array}$ \\
\hline $\begin{array}{l}10 \text { (W sections) } \\
* 13 \text { (insulation) }\end{array}$ \\
\hline Materials
\end{tabular}

*user input or default option.

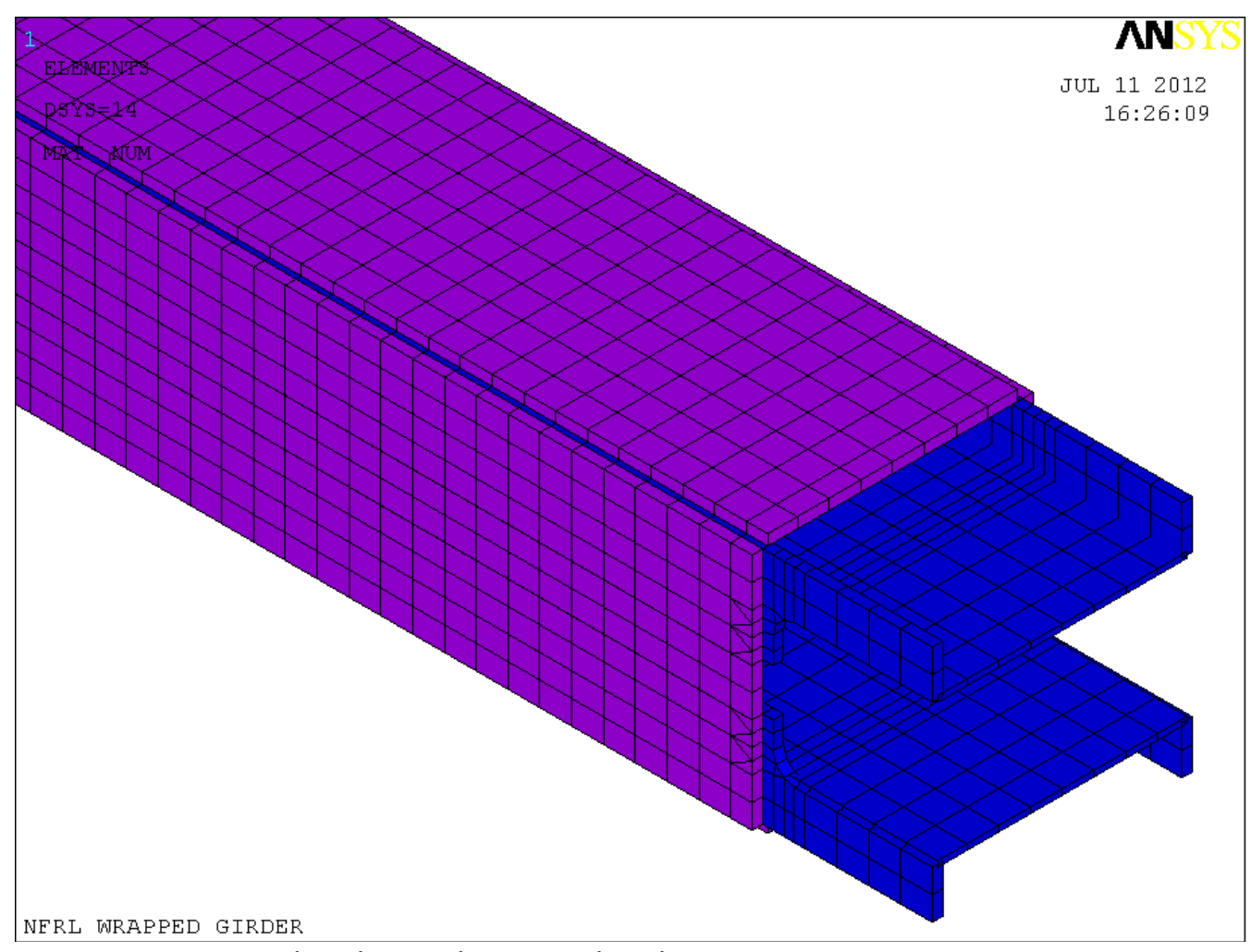

Fig. 10.1 Wrapped girder, with exposed end 
MAT NUM

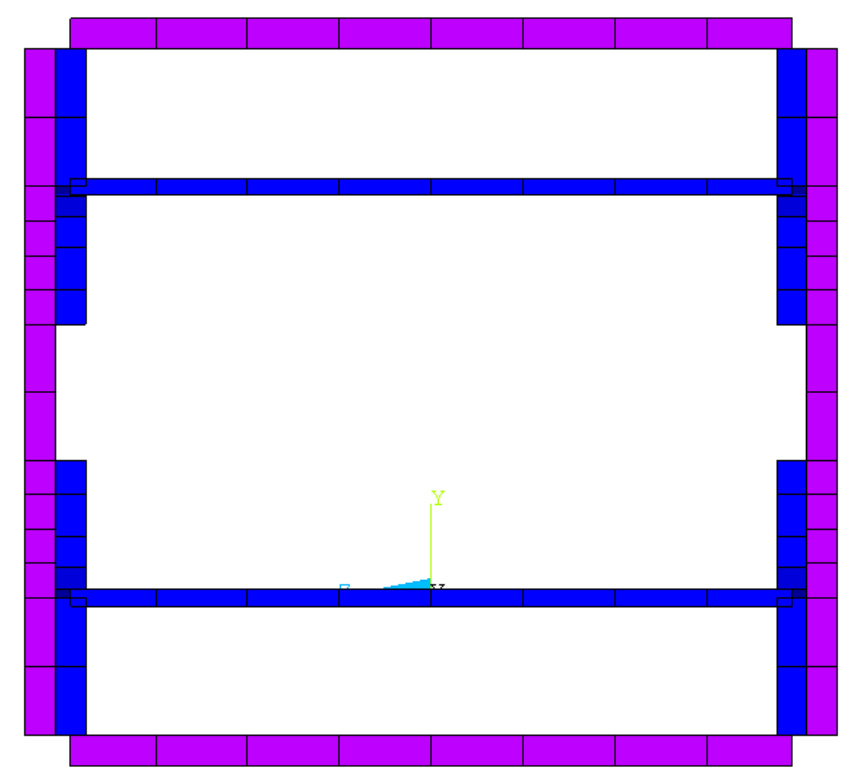

NFRL WRAPPED GIRDER

Fig. 10.2 Wrapped girder, end view

\section{! Wrapped girder}

! last tested 9.19.2012

FINISH

/CLEAR

/CWD, 'C:\StrongFireLab'

/FILNAME,20120919,0

/TITLE,NFRL WRAPPED GIRDER

/REPLOT

KEYW,PR_SET,1

KEYW,PR_STRUC,0

KEYW,PR_THERM,1

KEYW,PR_FLUID, 0

KEYW,PR_MULTI,0

\section{/PREP7}

! UNITS SI for thermal W, J. degree C

! UNITS CUSTOMARY for structural in, kipm, kipf, ksi

! \#\#\#\#\# material properties

! \# Material \# 10 - Steel for W sections

! thermal properties may be unneccessary for structural analysis 
! Young's modulus and Poisson's ratio

! constants

*SET,SIG,3.6583E-11 ! Stefan-Boltzman constant W/(in^2.K^4)

*SET,EMS1,0.9 ! Emissivity of steel - outside

*SET,EMS2,0.9 ! Emissivity of steel - inside

*SET,Hf,15 ! Film coefficient of steel faces

MPTEMP, ,,, , , , ,

MPTEMP,1,25,100,200,300,400,500

MPTEMP,7,600,700,800

MPDATA,EX,10,1,30000,30000,27000,24000,21000,18000

MPDATA,EX,10,7,9300,3900,2700

MPTEMP,,,,,,!,

MPTEMP,1,0

MPDATA,PRXY,10,,0.30

! thermal expansion

TREF,25 ! T REFERENCE $25 \mathrm{C}$, also default TUNIF

MPTEMP,,,, , , ,

MPTEMP, 1,0

MPDATA,ALPX,10,,14E-6 ! PER DEG C, EC3, Buchanan p. 194

!\# steel conductivity W/(in.K)

MPTEMP

MPTEMP,1,25

MPTEMP, 2,800

MPTEMP, 3,1200

MPDATA,KXX,10,1.37

MPDATA,KXX,10,,0.693

MPDATA,KXX,10,,0.693

!\# steel heat capacity J/(kipm.K)

MPTEMP

MPTEMP, 1,25

MPTEMP, 2,50

MPTEMP,3,100

MPTEMP,4,150

MPTEMP,5,200

MPTEMP, 6,400

MPTEMP,7,500

MPTEMP, 8,600

MPDATA,C,10,,199500

MPDATA,C,10,,208500

MPDATA,C,10,221200

MPDATA,C,10,,231500

MPDATA,C,10,,240300

MPDATA,C,10,,274800 
MPDATA, C,10,,302300

MPDATA,C,10,,344700

!\# steel mass density

MPTEMP

MPTEMP, 1,25

MPDATA,DENS,10,,2.636E-4 ! kipm/in3

\section{ET,5,SHELL131}

KEYOPT,5,2,0 ! average film temperature

KEYOPT,5,3,0 ! quadratic temperature distribution through thickness

KEYOPT, $5,4,0$ ! use sect defined above

KEYOPT, $5,6,0$ ! no paint

!KEYOPT,5,8,2 use this option when insulation is present- data at top, bottom and mid of all layers !SHELL,MID use this option when insulation is present-results calculated at mid, not averaged from top and bottom

sect,10,shell,,girderweb24W103

secdata, $0.550,10,0,3$

secoffset,MID

seccontrol,0,0,0, 0, 1, 1, 1

sect,11,shell,,girderflange24W103

secdata, $0.980,10,0,3$

secoffset,MID

seccontrol,0,0,0, 0, 1, 1, 1

!=================user input: insulation thickness and properties=====

! \# Material \# 13 - Blazeshield insulation

!\# insulation conductivity W/(in.K)

MPTEMP

MPTEMP,1,25 ! degree C

MPTEMP,2,377

MPTEMP, 3,677

MPDATA,KXX,13,,0.0013

MPDATA,KXX,13,0.0025

MPDATA,KXX,13,,0.0051

!\# insulation heat capacity J/(kipm.K)

MPTEMP

MPTEMP,1,25 ! C

MPTEMP $, 2,677$

MPDATA,C,13,,362880

MPDATA,C,13,,907200

!\# insulation mass density kipm/in3

MPTEMP 
MPTEMP,1,25

MPDATA,DENS,13,,7.51E-6

!\# insulation emissivity

MPTEMP

MPTEMP, 1,25

MPDATA,EMIS,13,,0.9

! Insulation blanket thickness is $\mathrm{T}$

$\mathrm{T}=1$

! careful that same emissivity can be used for steel and insulation

! USER INPUT: STARTING NUMBERS======================================

*SET,KP4,9400

*SET,LIN4,21700

*SET,ARE4,16300

*SET, VOL4,5000

*SET,ELE4,72400

*SET,NOD4,92100

! \# define model geometry

! user input:local coord system and location of girder

! ref: SHEET 12/15

! Local X ALONG midheight of web.

! Local Y perpendicular to web, right hand system.

! Local Z ALONG left vertical EDGE OF web.

LOCAL,15,0,10,100,200, , , ,1,1

CSYS, 15,

DSYS,15, ! display

LENGTH=186.0 ! half length of girder, must be $>126$ and multiple of 3 inches

! If length $>186$, user may have to increase element and node starting numbers ! of subsequent components.

!LENGTH $=162.0$

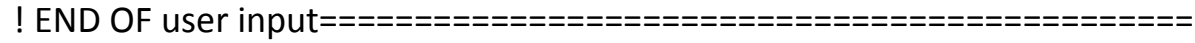

sect,13, shell,,flangew

secdata,0.98,10,0,3 ! steel layer 1 at bottom, NEEDS UPDATING

secdata, $\mathrm{T}, 13,0,3$

secoffset, user,-T/2 ! relative to midthickness of shell

seccontrol,0,0,0, 0, 1, 1, 1

sect,7,shell,,sidew ! blanket

secdata, $\mathrm{T}, 13,0,3$

secoffset, bot

seccontrol,0,0,0, 0, 1, 1, 1

sect,14,shell,,sidew ! blanket 


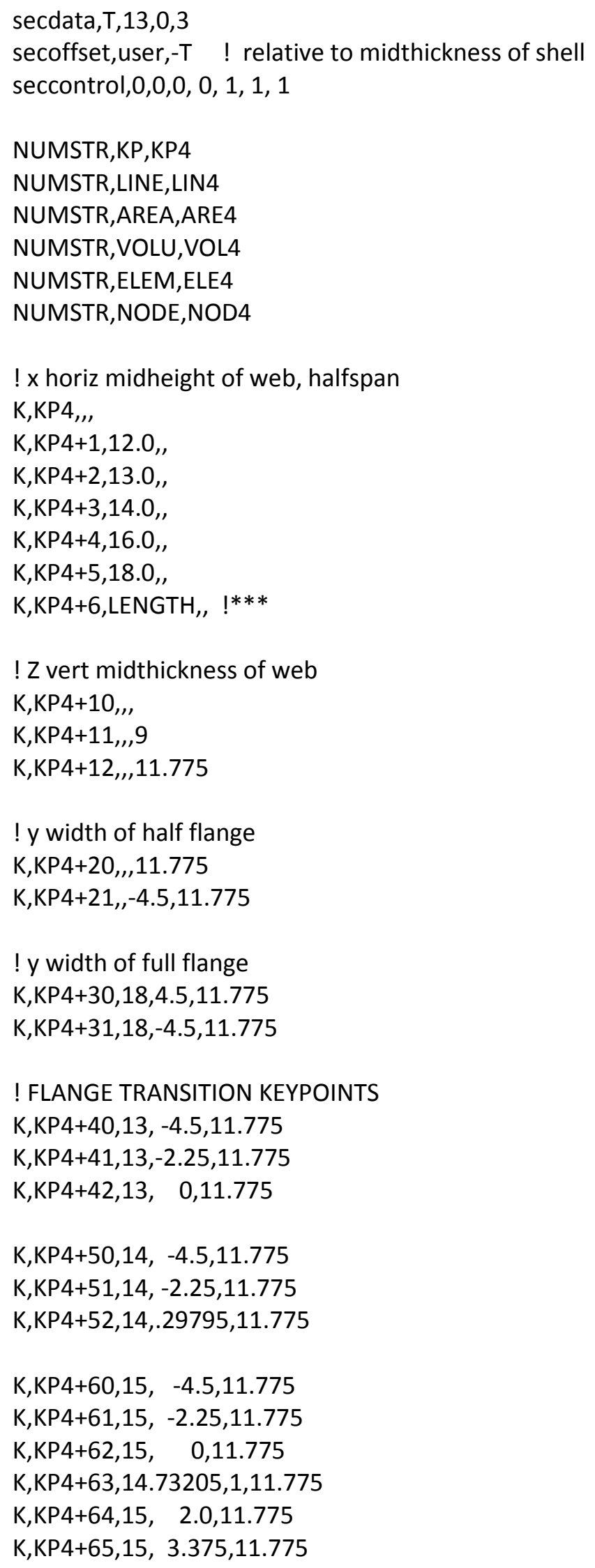




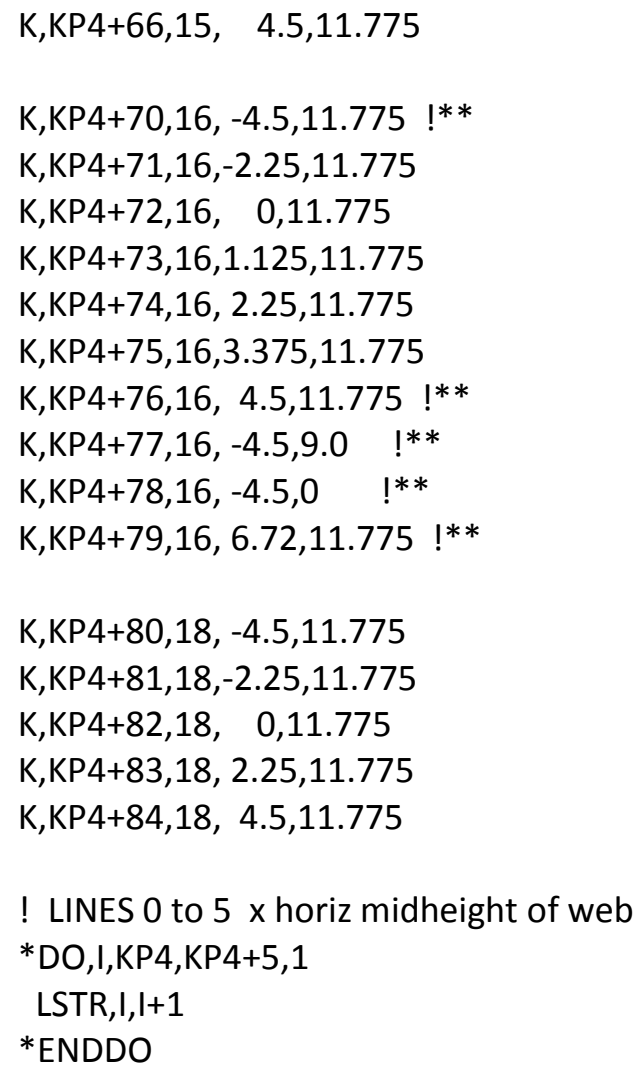


LESIZE,lin4+6,3.0, , $0,0,0,0$

LESIZE, lin4+7, $0,, 1,0,0,0$

KSEL,S,KP, ,KP4+10,KP4+12,1,

LSLK,S,1

CM, webdepthz,line

allsel,all

LSTR,KP4 $+20, \mathrm{KP} 4+21$ ! L8 y width of half flange

LESIZE, lin4+8,0, ,2, , 0 , , , 0

LSEL,S,LINE,,LIN4+8,,,1

$\mathrm{CM}$, halfwidthy, line

allsel,all

LSTR,KP4+30,KP4+31 ! L9 y width of full flange

LESIZE, lin4+9,0, , 4,0 , , , 0

allsel,all

LSEL,S,LINE,,LIN4+9,,,1

$\mathrm{CM}$, fullwidthy, line

ALLSEL,ALL

CMSEL,S, cutoffx, line

LGEN,2,ALL,,,,,11.775,,,, ! L10 TO 11

LSEL,S,LINE,,LIN4+10,LIN4+11,1,1

$\mathrm{CM}$,cutoff 2 , line

allsel,all

CMSEL,S, fullwidthx, line

LGEN,2,ALL,,,, 4.5,11.775,,,, ! L12 TO 13

LSEL,S,LINE,,LIN4+12,LIN4+13,,1

CM,fullwidthx2,line

allsel,all

LSEL,S,LINE,,LIN4,LIN4+12,1,1

CM,REGULAR,LINE

allsel,all

! LINES IN CUTOFF REGION L14 TO L56

! Y LINES

LSTR,KP4+40,KP4+41

LSTR,KP4+41,KP4+42

LSTR,KP4+50,KP4+51

LSTR,KP4+51,KP4+52

*DO,I,KP4+60,KP4+65,1

LSTR,I,I+1

*ENDDO 
*DO,I,KP4+70,KP4+75,1

LSTR,I,I+1

*ENDDO

*DO,I,KP4+80,KP4+83,1

LSTR,I,I+1

*ENDDO

! X LINES

*DO,I,KP4+40,KP4+70,10

LSTR, I,I+10

*ENDDO

*DO,I,KP4+41,KP4+71,10

LSTR, I,I+10

*ENDDO

*DO,I,KP4+42,KP4+72,10

LSTR, I,I+10

*ENDDO

LSTR, KP4+62,KP4+63

LSTR,KP4+63,KP4+52

LSTR, KP4+63, KP4+73

LSTR, KP4+73,KP4+82

LSTR,KP4+73,KP4+83

LSTR,KP4+64,KP4+74

LSTR,KP4+74,KP4+83

LSTR, KP4+65, KP4+75

LSTR,KP4+75,KP4+83

LSTR,KP4+75, KP4+84

LSTR,KP4+66,KP4+76

LSTR, KP4+76,KP4+84

ALLSEL,ALL

LSEL,S,LINE,,LIN4,LIN4+55,,1

CM,ALLINES,LINES

CMSEL,S,ALLINES,LINE

CMSEL,U,REGULAR,LINE

LESIZE,ALL, 0,1, , 0 , , , 0

CM,IRREGULAR,LINE

allsel,all ! L21055

! generate new lines for insulation

LGEN,2,LIN4+4,LIN4+5,1,0,6.72,11.775,,,! L21056,7 insulation flange edge

LGEN,2,LIN4+4,LIN4+5,1,0,-4.5,11.775,,,! L21058,9 flange edge 
LSTR,KP4+70,KP4+77 ! L21060 side cover LSTR,KP4+77,KP4+78 ! L21061 side cover LSTR,KP4+76,KP4+79! L21062 flange cover

LESIZE, lin4+60,0, , 1, , 0, , , 0

LESIZE, lin4+61,3.0, , $0,0,0,0$

LESIZE, lin4+62,0, , 1, , 0 , , , 0

ALLSEL,ALL

! areas

*DO,I,KP4+40,KP4+70,10

$A, I, I+10, I+11, I+1$ ! regular part of transition zone

*ENDDO ! A16000-3

*DO,I,KP4+41,KP4+71,10

$A, I, I+10, I+11, I+1$ ! next part of flange in $+y$ direction

*ENDDO ! A16004-7

$\mathrm{A}, \mathrm{KP} 4+52, \mathrm{KP} 4+62, \mathrm{KP} 4+63$

$\mathrm{A}, \mathrm{KP} 4+62, \mathrm{KP} 4+72, \mathrm{KP} 4+73, \mathrm{KP} 4+63$

$\mathrm{A}, \mathrm{KP} 4+72, \mathrm{KP} 4+82, \mathrm{KP} 4+73$

$\mathrm{A}, \mathrm{KP} 4+73, \mathrm{KP} 4+82, \mathrm{KP} 4+83$

$\mathrm{A}, \mathrm{KP} 4+63, \mathrm{KP} 4+73, \mathrm{KP} 4+74, \mathrm{KP} 4+64$

$\mathrm{A}, \mathrm{KP} 4+73, \mathrm{KP} 4+83, \mathrm{KP} 4+74$

$\mathrm{A}, \mathrm{KP} 4+64, \mathrm{KP} 4+74, \mathrm{KP} 4+75, \mathrm{KP} 4+65$

$\mathrm{A}, \mathrm{KP} 4+74, \mathrm{KP} 4+83, \mathrm{KP} 4+75$

$\mathrm{A}, \mathrm{KP} 4+75, \mathrm{KP} 4+83, \mathrm{KP} 4+84$

$\mathrm{A}, \mathrm{KP} 4+65, \mathrm{KP} 4+75, \mathrm{KP} 4+76, \mathrm{KP} 4+66$

$\mathrm{A}, \mathrm{KP} 4+75, \mathrm{KP} 4+84, \mathrm{KP} 4+76$ ! rest of flange transition A16008-18

ADRAG, halfspanx,,,,,,,webdepthz,,,,!, ! ! Web areas A16019-30

ADRAG,cutoffx2,,,,,,, halfwidthy,,,,,, ! flange areas end-transition A16031-2

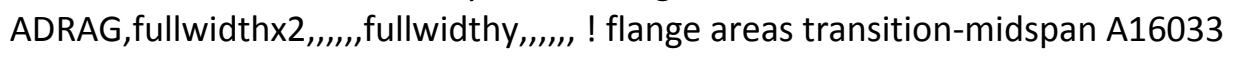

ADRAG,lin4+62,,,,,,lin4+58,lin4+59,,,,, ! ! flange cover extension A16034-5

ADRAG, lin4+60, lin4+61,,,,,lin4+56,lin4+57,,,,,! ! flange cover extension A16036-9

! MESH WEB, BARE STEEL

ASEL,S,LOC,Y,-0.1,0.1,1

ASEL,R,LOC,Z,0,11.7,"

AATT $, 10,5,15,10$

MSHAPE,0,2D

MSHKEY,1

AMESH,ALL

ALLSEL,ALL

! MESH FLANGE END, BARE STEEL

ASEL,S,LOC,Z,11.7,11.8,,1

ASEL,R,LOC,X,0,13,,1 
!ASEL,U,LOC,X,13,18,,1

AATT,10,5, 15,11

MSHAPE,0,2D

MSHKEY,1

AMESH,ALL

ALLSEL,ALL

! MESH FLANGE TRANSITION, BARE STEEL

ASEL,S,LOC,Z,11.7,11.8,,1

ASEL, R,LOC,X,13,16, ,1

AATT, $10,5,15,11$

MSHKEY,2

AMESH,ALL

ALLSEL,ALL

! MESH FLANGE TRANSITION, INSULATED STEEL

ASEL,S,LOC,Z,11.7,11.8,,1

ASEL,R,LOC,X,16,18,,1

ASEL,R,LOC,Y,-4.5,4.5,,1

AATT, 10, 5, 15,13

MSHKEY,2

AMESH,ALL

ALLSEL,ALL

! MESH FLANGE MIDDLE, INSULATED STEEL

ASEL,S,LOC,Z,11.7,11.8,,1

ASEL,R,LOC,X,18,LENGTH,,1 !**

ASEL,R,LOC,Y,-4.5,4.5,,1

AREVERSE,ALL

AATT, 10, 5, 15,13

MSHAPE,0,2D

MSHKEY,1

AMESH,ALL

ALLSEL,ALL

! MESH FLANGE INSULATION BETWEEN BEAMS

ASEL,S,LOC,Z,11.7,11.8,,1

ASEL,R,LOC,X,16,LENGTH,,1 !**

ASEL,R,LOC,Y,4.5,6.72,,1

AREVERSE,ALL

AATT,13, 5, 15,14

MSHAPE,0,2D

MSHKEY,1

AMESH,ALL

ALLSEL,ALL

! MESH SIDE INSULATION PARALLEL TO WEB

ASEL,S,LOC,Z,0,11.8,,1 


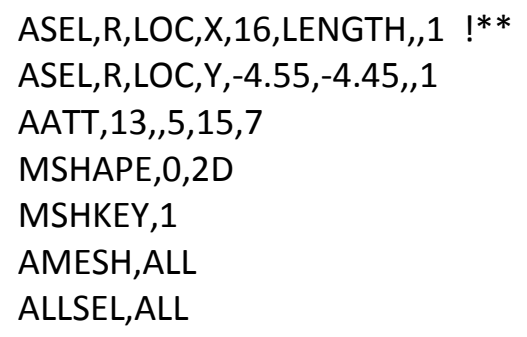




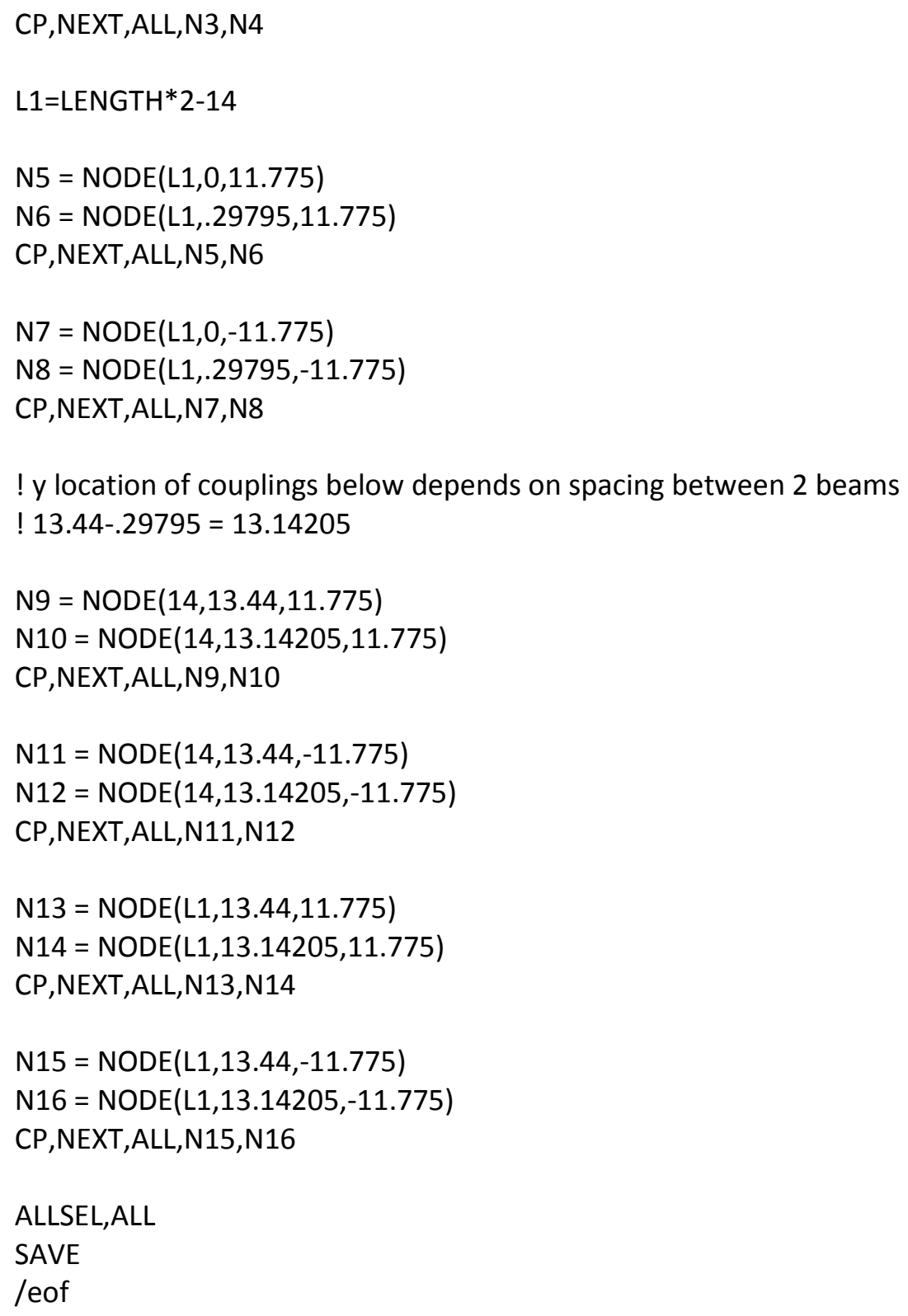




\section{Chapter 11 Girder Angle}

\section{Table 11.1 Properties}

\begin{tabular}{|l|l|}
\hline Girder angle & Starting numbers \\
\hline *Keypoints KP5 & 10000 \\
\hline *Lines LIN5 & 22700 \\
\hline *Areas ARE5 & 16700 \\
\hline *Volumes VOL5 & 5000 \\
\hline *Elements ELE5 & 79000 \\
\hline *Nodes NOD5 & 101100 \\
\hline $\begin{array}{l}\text { *Local coordinates } \\
\text { system 16 }\end{array}$ & $\begin{array}{l}\text { x along leg with holes near middle; } \\
\text { y along leg with holes closer to edge; } \\
\text { z along length of angle; } \\
\text { origin at corner (Fig. 11.1). }\end{array}$ \\
\hline Element type & 5 Shell 181 \\
\hline Sections & 12 (0.75 in) \\
\hline Materials & 10 \\
\hline
\end{tabular}

LINES

TYPE NUM
NN

AUG 202012 $14: 20: 19$

NERL GIRDER ANGLE B

Fig. 11.1 Girder angle coordinates 


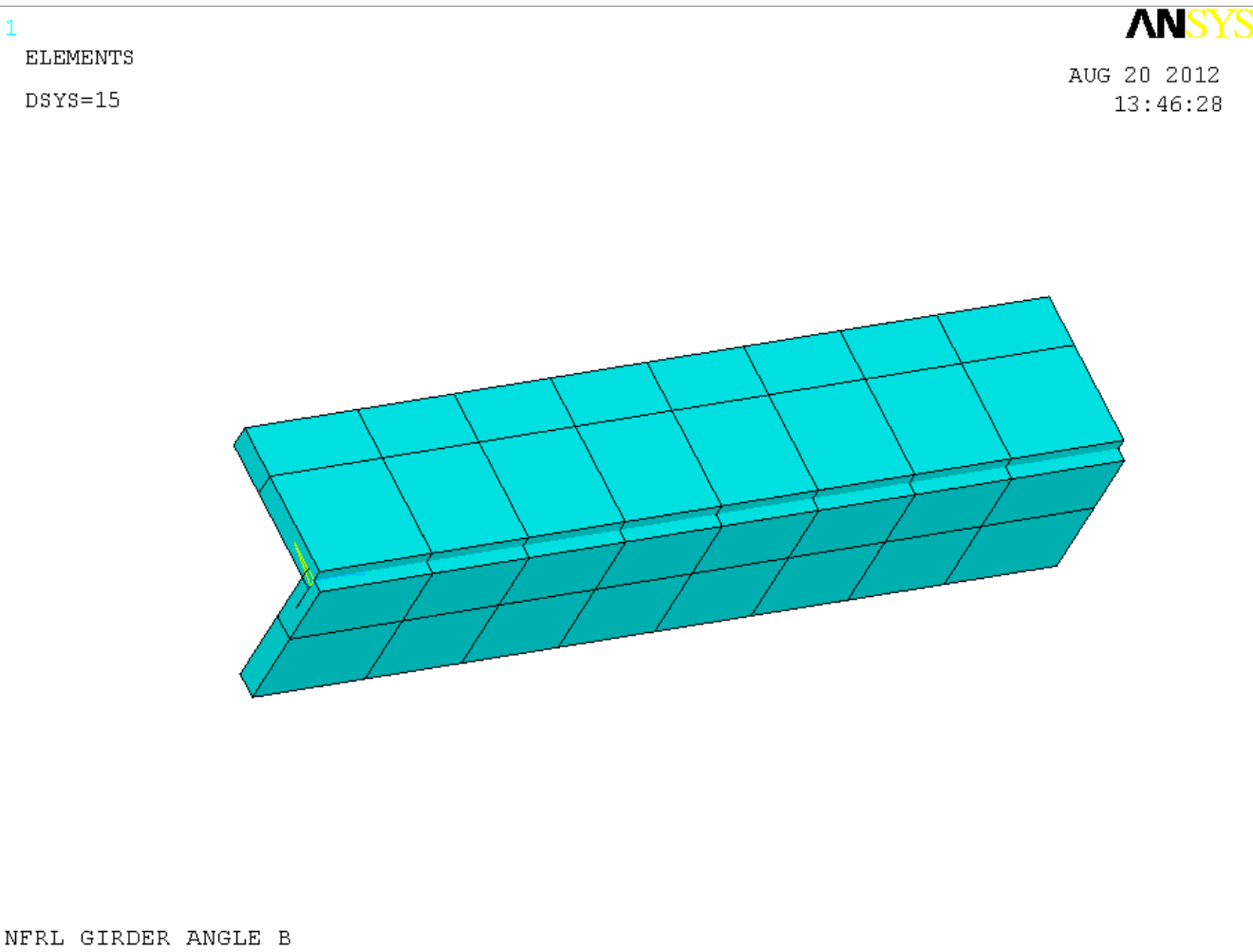

Fig. 11.2 Girder angle

\section{! Girder angle}

\section{FINISH}

/CLEAR

/CWD, 'C: \StrongFireLab'

/FILNAME,20120705,0

/TITLE,NFRL GIRDER ANGLE B

/REPLOT

\section{KEYW,PR_SET,1}

KEYW,PR_STRUC,1

KEYW,PR_THERM,0

KEYW,PR_FLUID, 0

KEYW,PR_MULTI,0

/PREP7

! UNITS SI for thermal W, J. degree C

! UNITS CUSTOMARY for structural in, kipm, kipf, ksi

! \#\#\#\# material properties

! \# Material \# 10 - Steel for W sections

! thermal properties may be unneccessary for structural analysis 


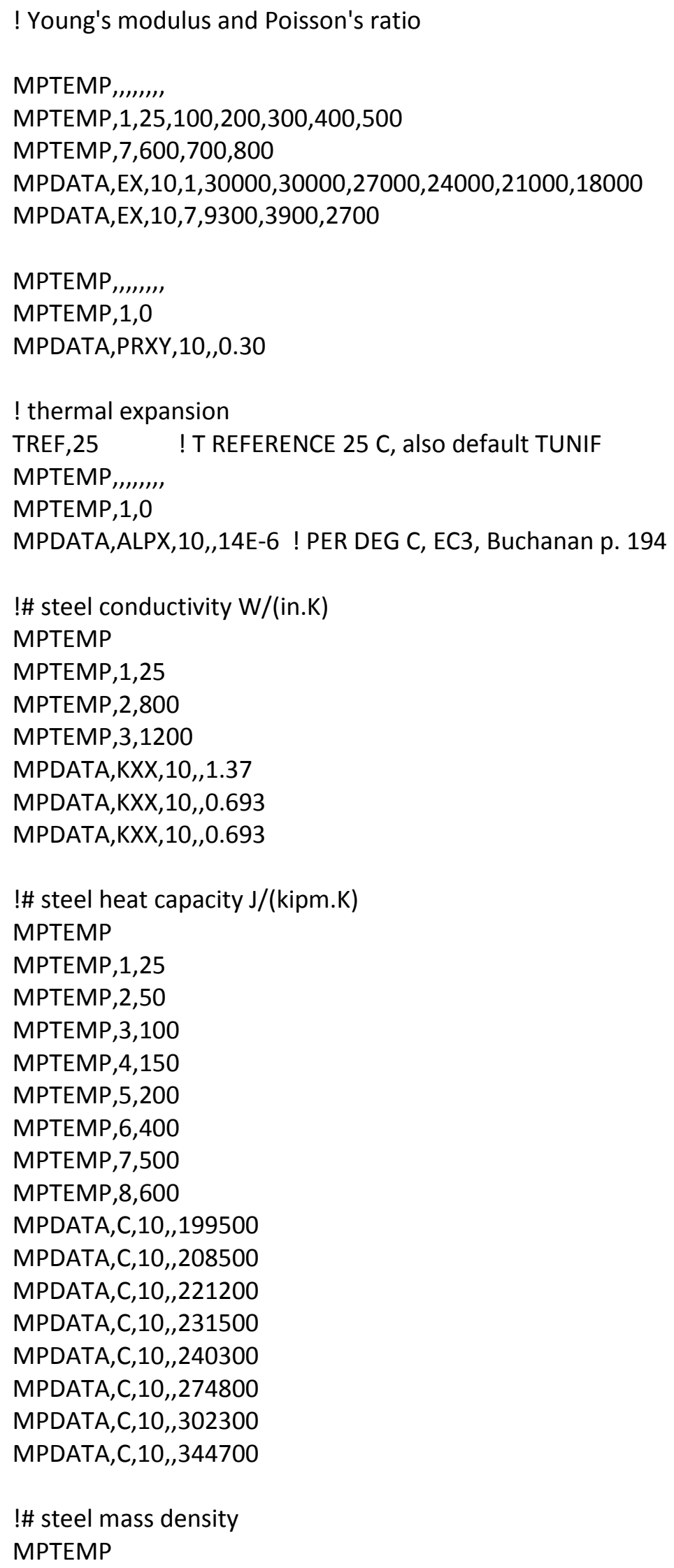


MPTEMP, 1,0

MPDATA,DENS,10,,2.636E-4 ! kipm/in3

ET,5,SHELL181

KEYOPT,5,1,0 ! bending and membrane stiffness

KEYOPT,5,3,2 ! full integration with incompatible modes

KEYOPT, $5,8,0$ ! store data at bottom of bottom layer and top of top layer

KEYOPT,5,9,0 ! no user subroutine to define thickness

KEYOPT,5,10,0 ! no initial stress

sect, 12 , shell,,girderangle $\mathrm{L} 5 \times 5 \times 0.75$

secdata, $0.75,10,0,3$

secoffset,MID

seccontrol,0,0,0, 0, 1, 1, 1

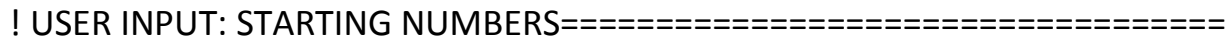

*SET,KP5,10000

*SET,LIN5,22700

*SET,ARE5, 16700

*SET, VOL5,5000

*SET,ELE5,79000

*SET,NOD5,101100

! \# define model geometry

! user input:local coord system and location of girder

! ref: SHEET 13/15

! Local Y ALONG leg with holes closer to edge.

! Local $X$ along leg with holes near middle.

! Local Z ALONG length of angle.

! origin at corner.

LOCAL,16,0,20,120,180, , , ,1,1

CSYS,16,

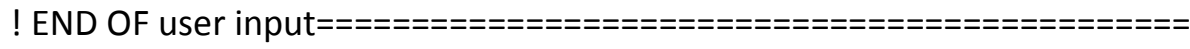

NUMSTR,KP,KP5

NUMSTR,LINE,LIN5

NUMSTR,AREA,ARE5

NUMSTR, VOLU,VOL5

NUMSTR,ELEM,ELE5

NUMSTR,NODE,NOD5

$\mathrm{K}, \mathrm{KP5}$,,,

$\mathrm{K}, \mathrm{KP} 5+1,2.08$,

$\mathrm{K}, \mathrm{KP} 5+2,4.625$,

$\mathrm{K}, \mathrm{KP} 5+3,3.07$,

$\mathrm{K}, \mathrm{KP} 5+4,4.625$, 


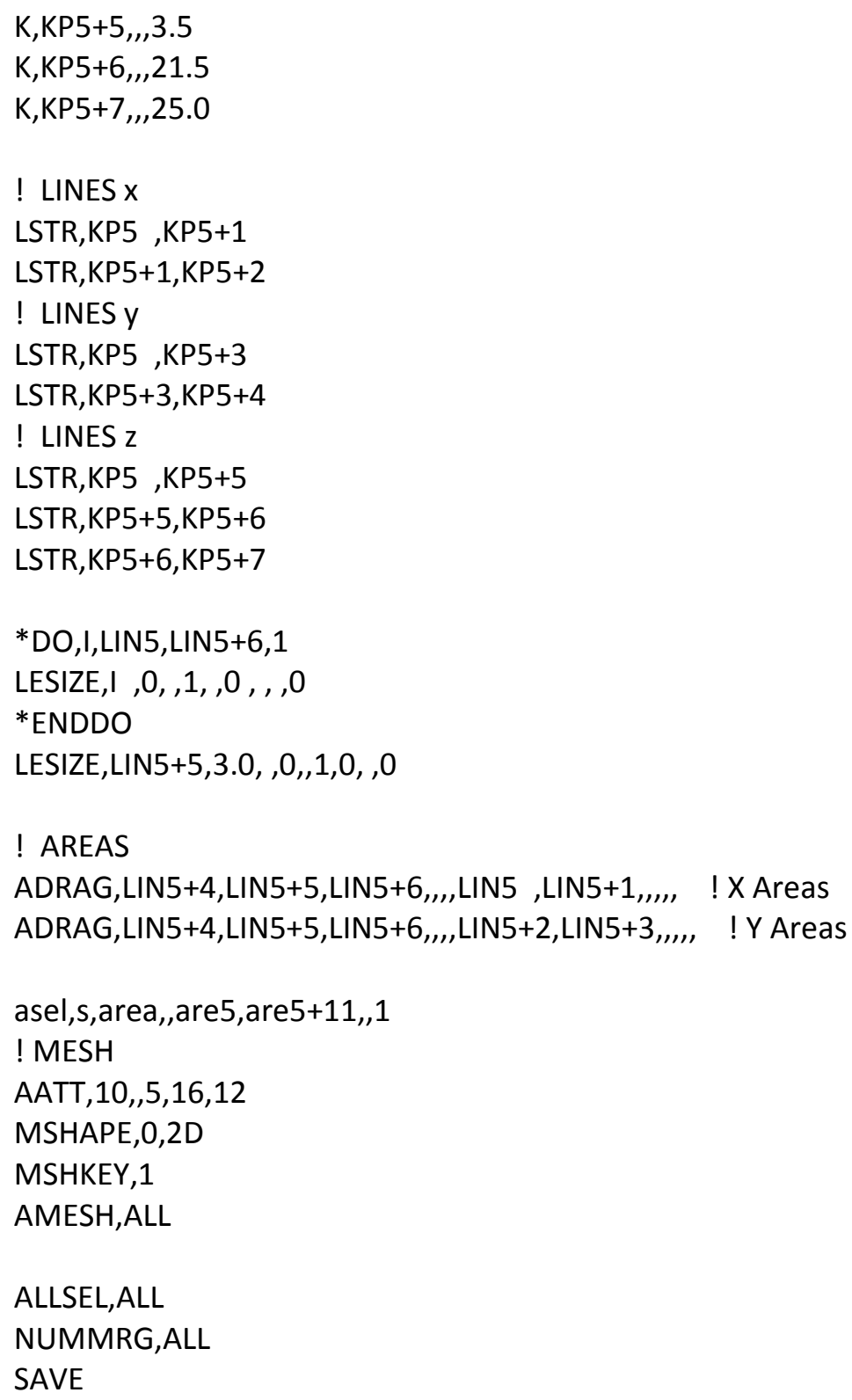




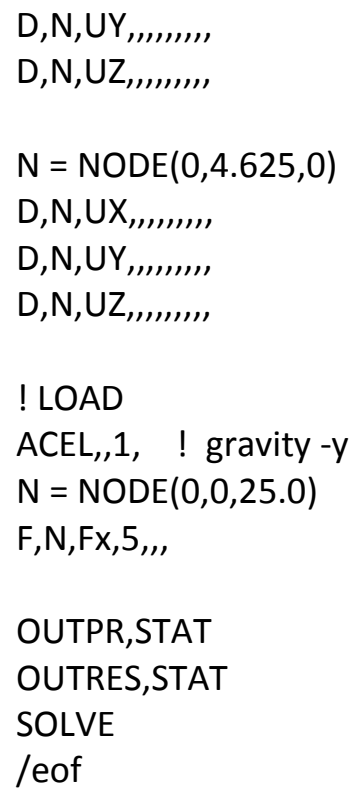




\section{Chapter 12 Girder spacer}

Table 12.1 Properties

\begin{tabular}{|l|l|}
\hline Girder spacer & Starting numbers \\
\hline *Keypoints KP6 & 10100 \\
\hline *Lines LIN6 & 22800 \\
\hline${ }^{*}$ Areas ARE6 & 16800 \\
\hline *Volumes VOL6 & 5000 \\
\hline *Elements ELE6 & 79100 \\
\hline *Nodes NOD6 & 101200 \\
\hline $\begin{array}{l}\text { *Local coordinates } \\
\text { system 17 }\end{array}$ & $\begin{array}{l}\text { x along flange; } \\
\text { y along web; } \\
\text { z along length of spacer; } \\
\text { origin: midthickness of junction web-flange (Fig. 12.1). }\end{array}$ \\
\hline Element type & $\begin{array}{l}5 \text { Shell 181 } \\
6 \text { (Beam 188) }\end{array}$ \\
\hline Sections & $\begin{array}{l}3 \text { (0.99 inch flange W12x106) } \\
15 \text { (tube 80) }\end{array}$ \\
\hline Materials & 10 (W sections) \\
\hline
\end{tabular}




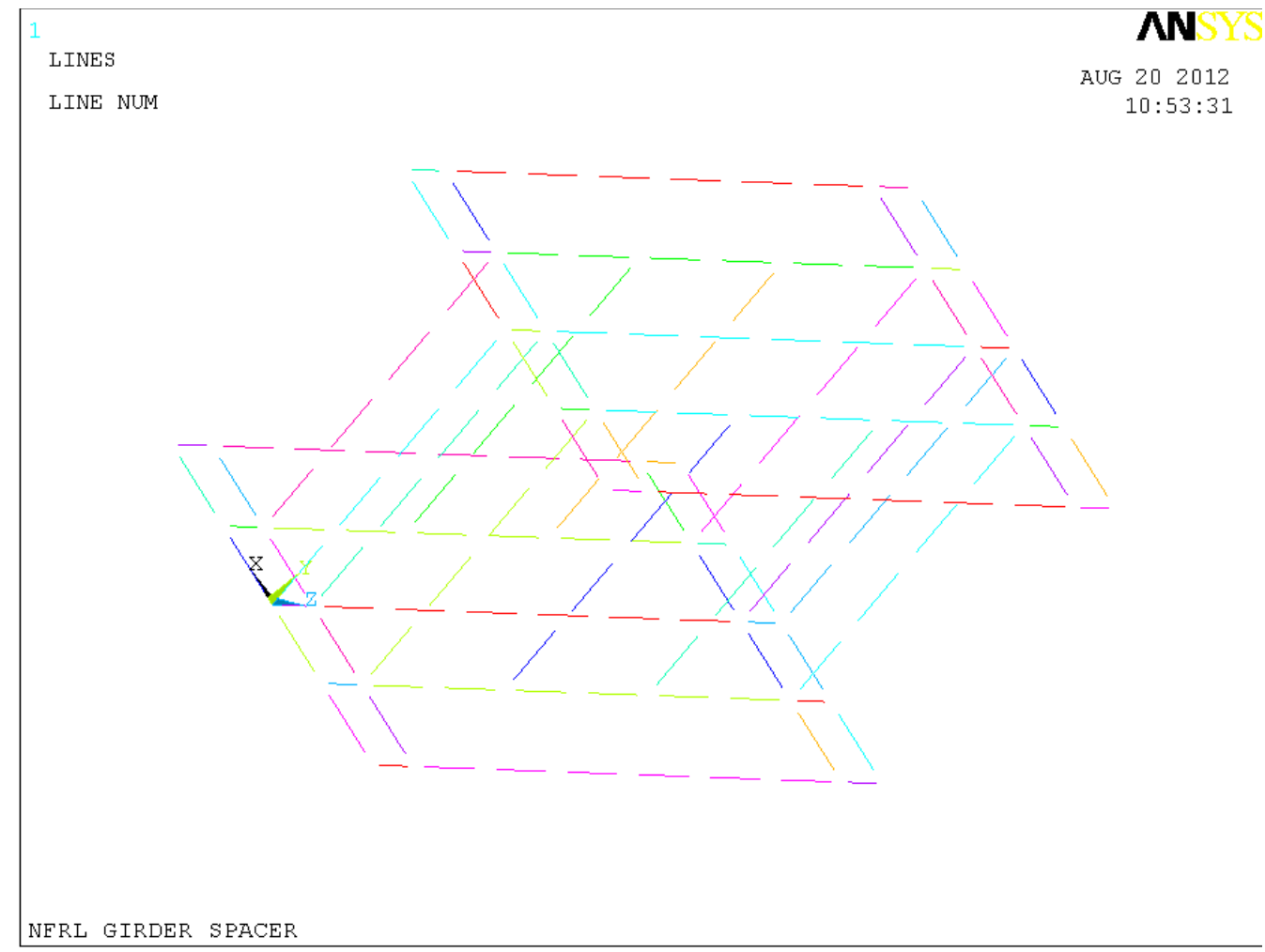

Fig. 12.1 Girder spacer coordinate system

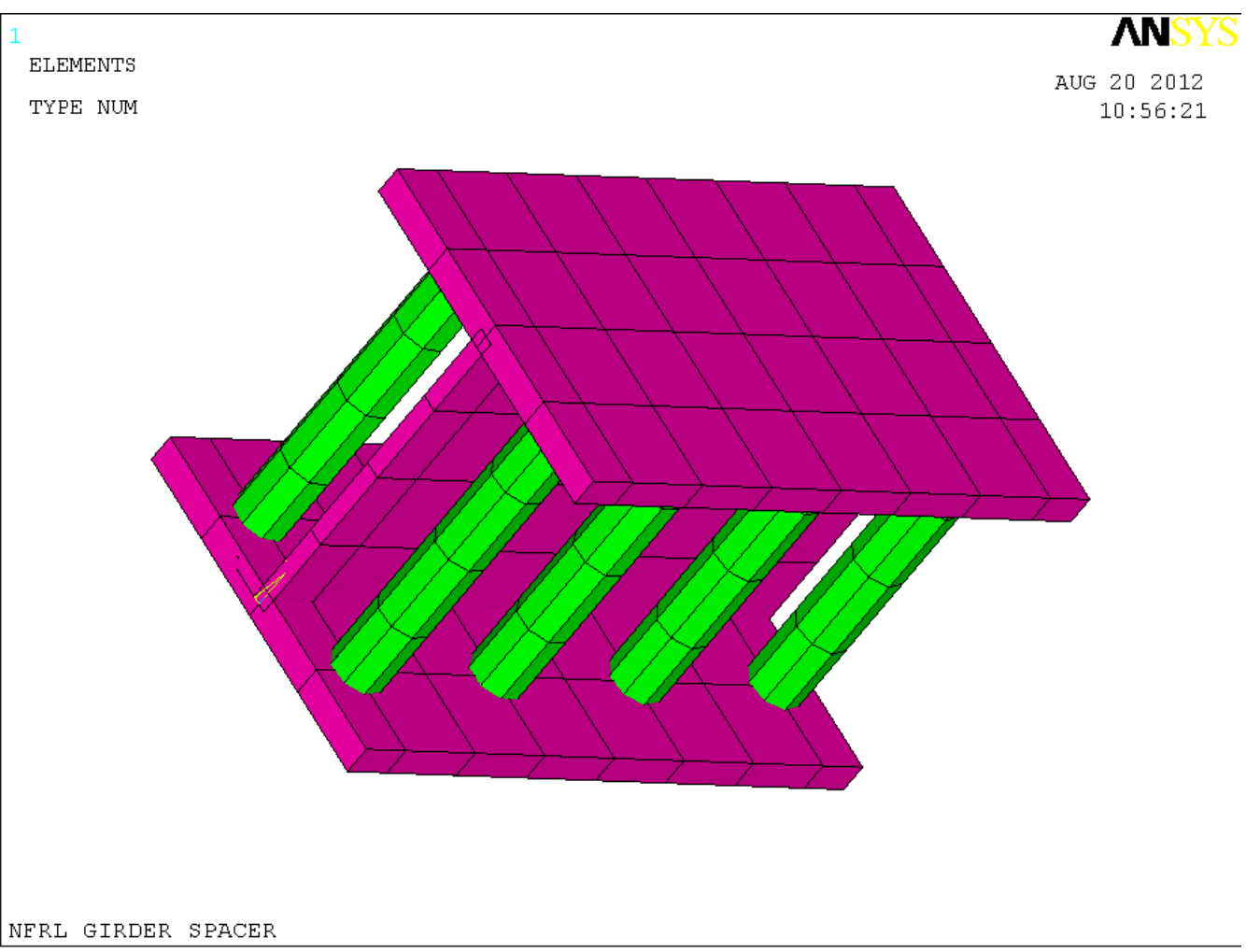

Fig. 12.2 Girder spacer 


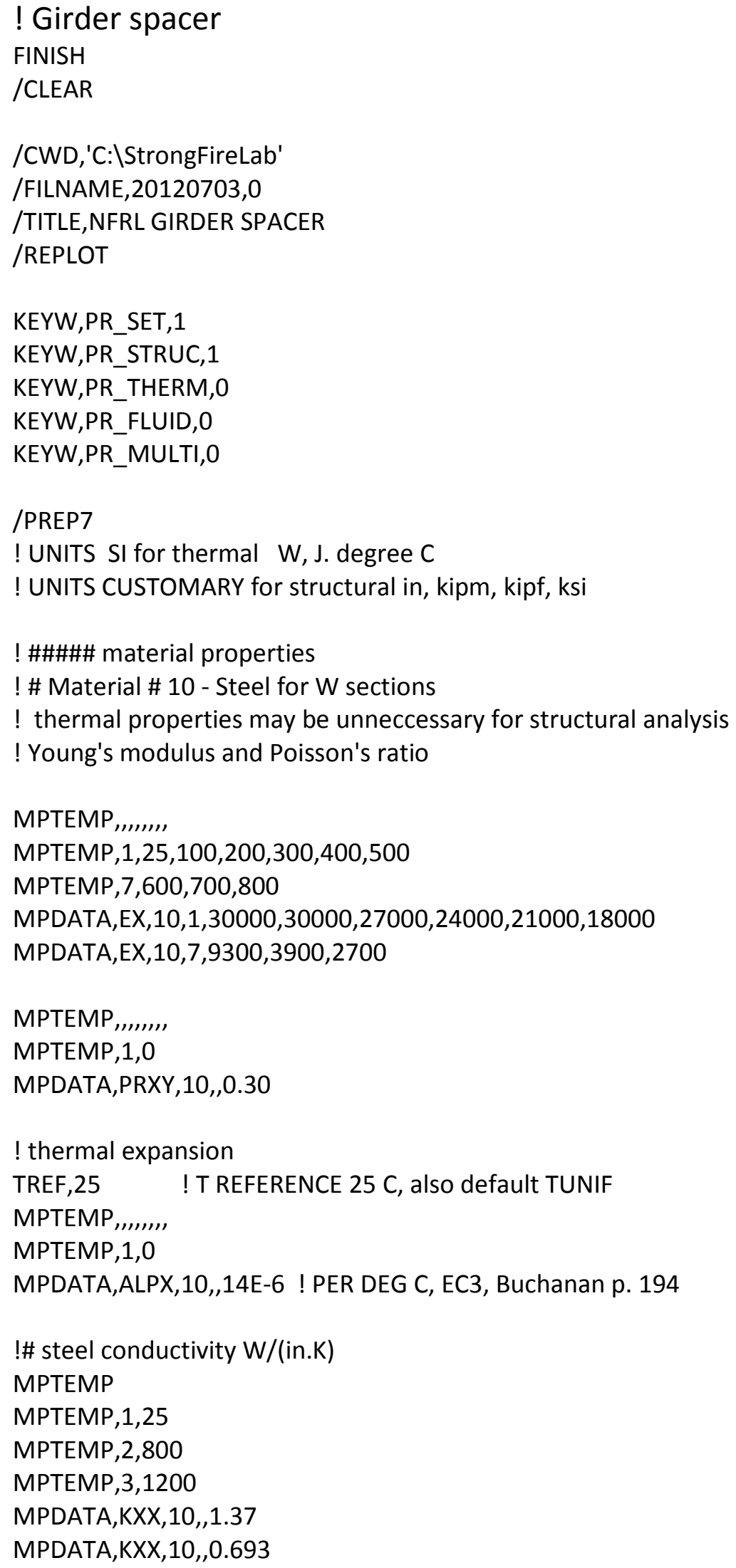


MPDATA,KXX,10,,0.693

!\# steel heat capacity J/(kipm.K)

MPTEMP

MPTEMP, 1,25

MPTEMP, 2,50

MPTEMP, 3,100

MPTEMP,4,150

MPTEMP,5,200

MPTEMP, 6,400

MPTEMP $, 7,500$

MPTEMP, 8,600

MPDATA,C,10,,199500

MPDATA,C,10,,208500

MPDATA,C,10,,221200

MPDATA,C,10,,231500

MPDATA,C,10,,240300

MPDATA,C,10,,274800

MPDATA,C,10,,302300

MPDATA,C,10,,344700

!\# steel mass density

MPTEMP

MPTEMP, 1,0

MPDATA,DENS,10,,2.636E-4！ kipm/in3

ET,5,SHELL181

KEYOPT,5,1,0 ! bending and membrane stiffness

KEYOPT,5,3,2 ! full integration with incompatible modes

KEYOPT, $5,8,0$ ! store data at bottom of bottom layer and top of top layer

!KEYOPT, 5,8,2 use this option when insulation is present- data at top, bottom and mid of all layers

!SHELL,MID use this option when insulation is present-results calculated at mid, not averaged from top and bottom

KEYOPT,5,9,0 ! no user subroutine to define thickness

KEYOPT,5,10,0 ! no initial stress

sect,3,shell,,12106f

secdata, $0.99,10,0,3$

secoffset,MID

seccontrol,0,0,0, 0, 1, 1, 1

sect,4,shell,,12106W

secdata, $0.61,10,0,3$

secoffset,MID

seccontrol,0,0,0, 0, 1, 1, 1

ET,6,BEAM188

SECTYPE, 15, BEAM, CTUBE, SCH80, 0 


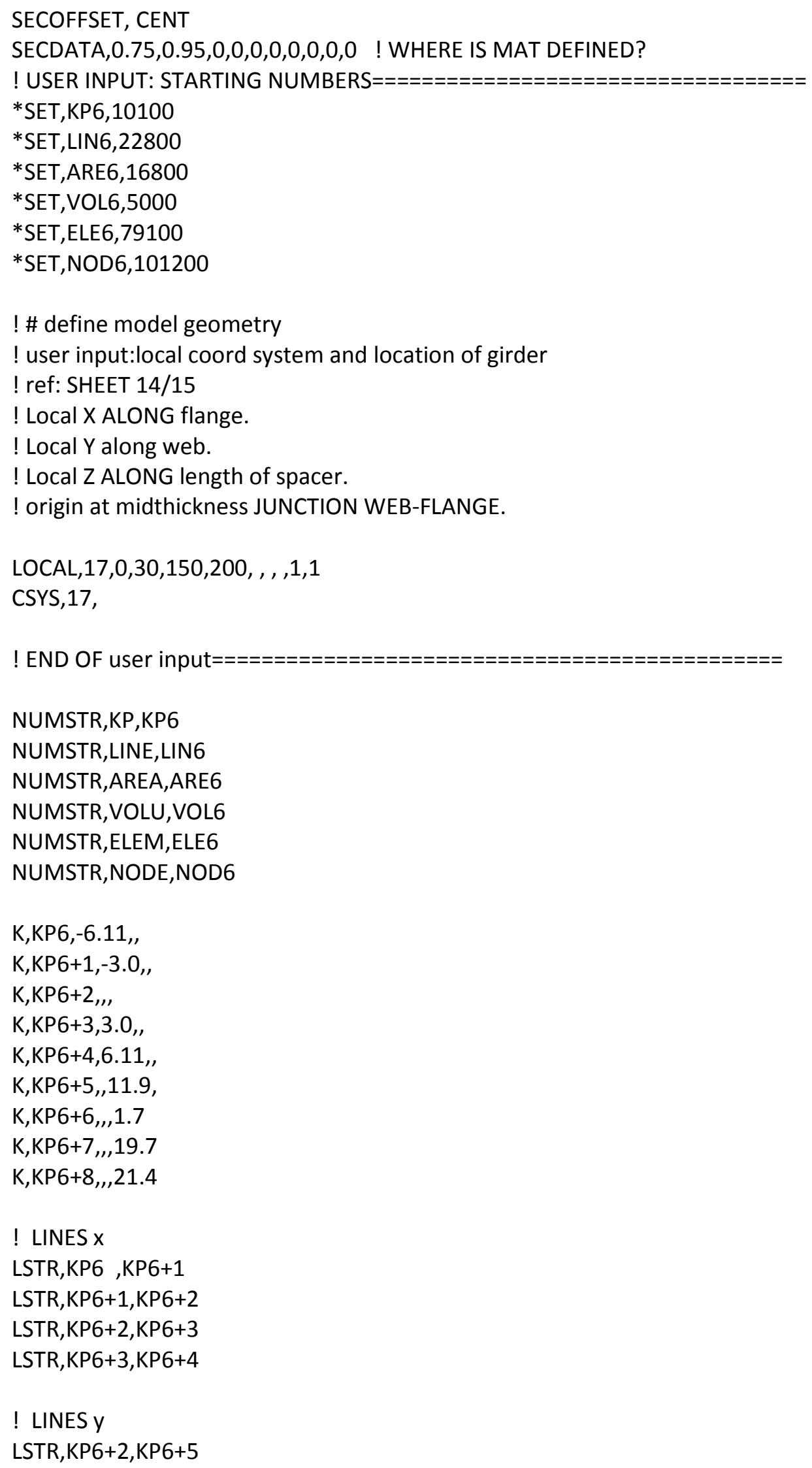




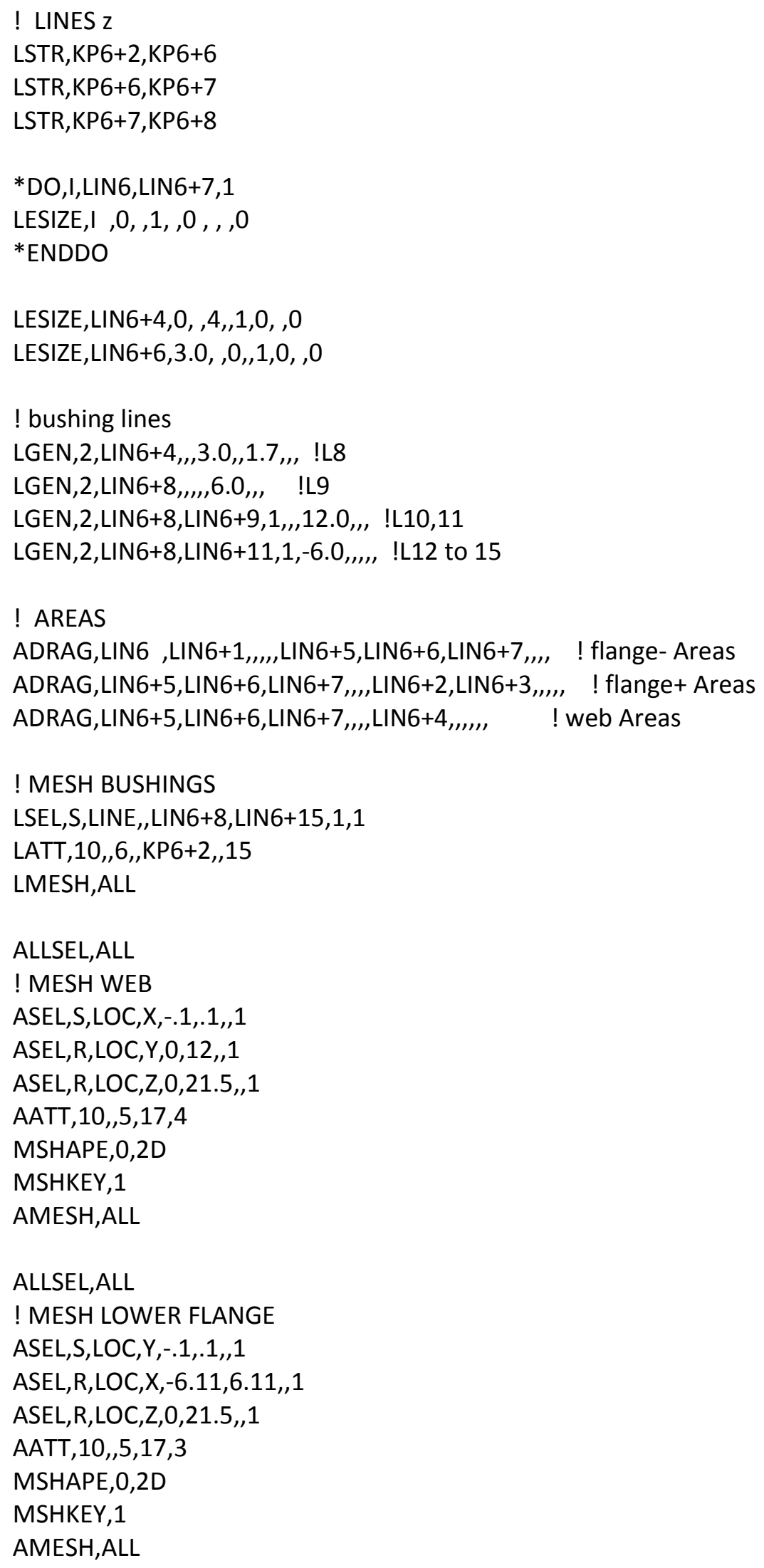




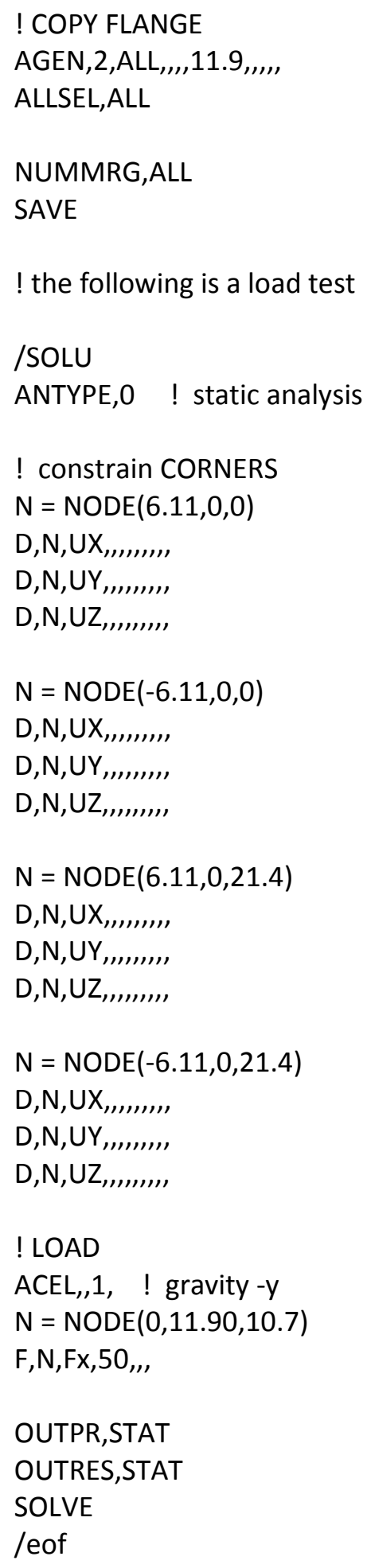




\section{Chapter 13 Assembling the Support Structure}

To assemble various structural components, the following procedure is recommended:

1. Deploy: Bring onto the assembly site (global coordinate system) various structural components in order of increasing keypoints (see Table 13.1). Just as in a real construction site, components should be placed some distance apart to ensure there is no interference during construction of the individual sets (the ANSYS command used to select various keypoints, lines or areas uses a volume of space that is bigger than the actual objects). It is recommended to place the girder in its final location from the start, as its formulation contains constraints that are cumbersome to move. See Fig. 13.1.

2. Move: The user can translate and rotate the various structural components into their final locations (Fig. 13.2) by using the AGEN and ATRAN commands respectively. As the ATRAN command operates from the current coordinate system to a destination coordinate system specified in the command, it is a good idea to explicitly define the current coordinate system again before issuing the ATRAN command if there is any doubt. This is particularly important as the LOCAL command not only defines the local coordinate system in terms of the global one, but also takes the user to the newly defined local system. The girder spacer can be duplicated by creating new elements in a new location, but the original cannot be moved (translation or rotation is performed in one step, but the spacer consists of area and line elements that would be movable in two steps, except they are connected). The original girder spacer used in constructing the assembly is selected out before starting the solution.

3. Number: Before creating new structural components by duplicating, the user should specify starting numbers (NUMSTR) for keypoints, lines, areas, volumes, elements and nodes at higher values than the maximum currently used. If this is not done, ANSYS starts numbering the newly created entities at the lowest available numbers, and thus fills in the gaps between the previous numbers. This can cause a numbering interference problem if, in step 1, the user did not bring in all the components needed. When the missing component is brought in later, it may find its numbers already occupied. The user should issue NUMSTR after bringing in additional components.

4. Copy: Duplication is performed by AGEN for area elements and LGEN for line elements (Figs. 13.3 and 13.4).

5. Fit: Correct placement of all components should be verified to ensure mesh lines and nodes, i.e., bolts and bolt holes match (Figs. 13.5 - 13.12).

6. Constrain: As shell elements are defined at their midplanes, perfectly matched adjacent steel plates are separated by half of their combined thicknesses. The nodes thus cannot be merged and must be constrained individually. This is the virtual equivalent of tightening bolts, and is performed in three steps by constraining the three degrees of translation for each pair of nodes.

7. Restrain: The assembly is bolted to the strong floor and strong wall at the appropriate nodes. The support conditions do not account for the prestress in the anchor rods, nor the involvement of the rest of a foot (contact with the floor) or a pad (contact with the wall). The resultant reaction at a foot or a pad is correct, but the compressive forces are concentrated at a couple of 
bolt locations rather than distributed over, say, half of a foot or a pad. As compression is not a concern, this approximation only needs to be refined if the tension at any anchor exceeds the prestress and partial liftoff occurs. A detailed local model can be performed then of the foot or pad and portions of the concrete floor or wall, using properly prestressed anchors and contact elements.

8. Solve: Select out the construction girder spacer, load and run the model. The APDL (ANSYS Programming Design Language) commands are included at the end of this chapter for the example illustrated.

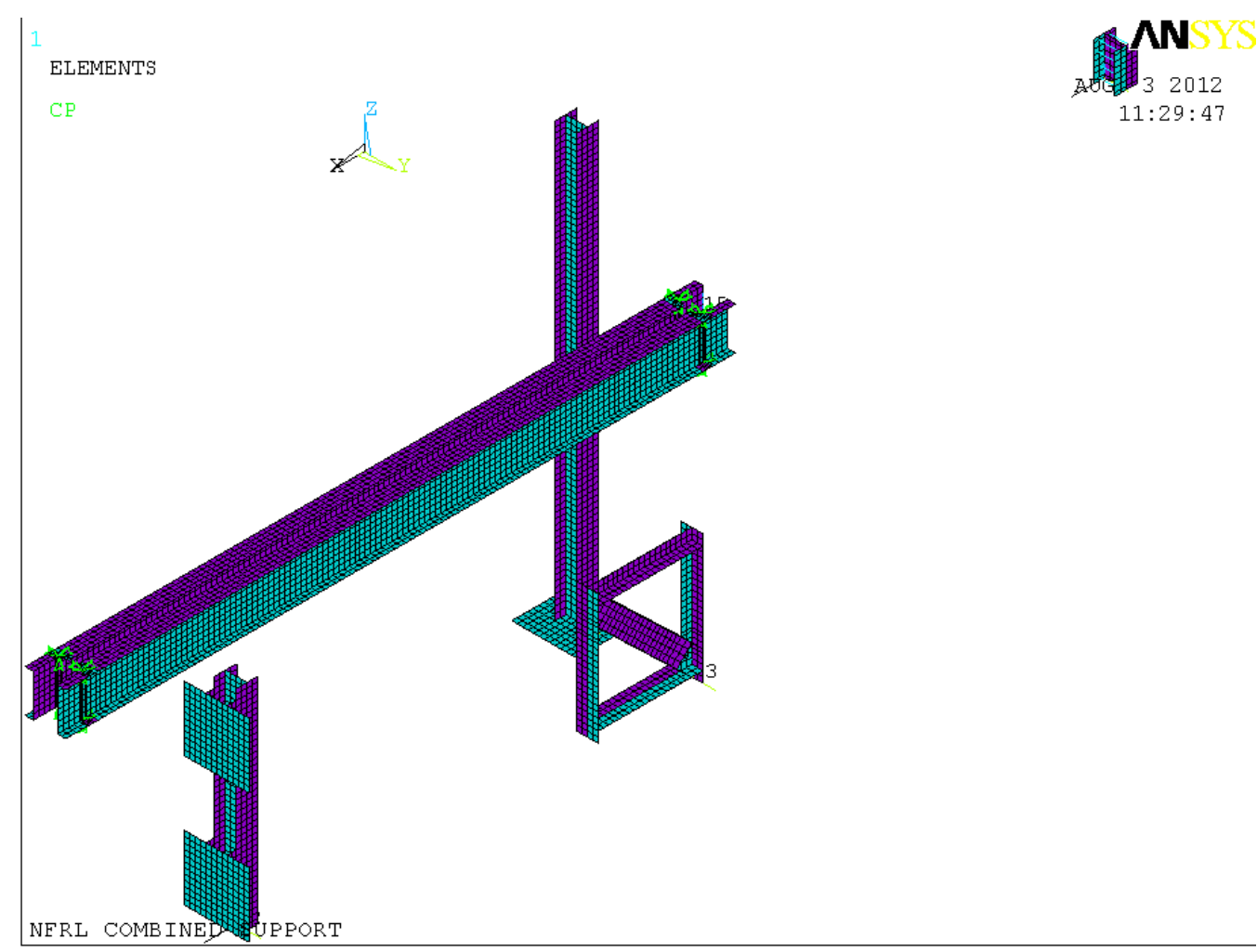

Fig. 13.1 Step 1: Deploy structural components onto construction site 
Table 13.1 Structural components

\begin{tabular}{|c|c|c|c|c|c|c|c|}
\hline $\begin{array}{l}\text { Starting } \\
\text { numbers }\end{array}$ & $\begin{array}{l}\text { Column-foot } \\
\text { (index 1) }\end{array}$ & Wall-column (0) & Frame (2) & $\begin{array}{l}\text { Web splice } \\
\text { (3) }\end{array}$ & Girder (4) & $\begin{array}{l}\text { B2 Girder } \\
\text { assembly (5) }\end{array}$ & $\begin{array}{l}\text { Girder spacer } \\
(6)\end{array}$ \\
\hline Keypoints & 8000 & 8100 & 8400 & 9350 & 9400 & 10000 & 10100 \\
\hline Lines & 19000 & 19200 & 19600 & 21300 & 21700 & 22700 & 22800 \\
\hline Areas & 15000 & 15100 & 15300 & 16100 & 16300 & 16700 & 16800 \\
\hline Volumes & 5000 & 5000 & 5000 & 5000 & 5000 & 5000 & 5000 \\
\hline Elements & 67000 & 69000 & 70200 & 72100 & 72400 & 79000 & 79100 \\
\hline Nodes & 85000 & 88000 & 89500 & 91700 & 92100 & 101100 & 101200 \\
\hline $\begin{array}{l}\text { Local } \\
\text { coordinates } \\
\text { system }\end{array}$ & 11 & 12 & 13 & 14 & 15 & 16 & 17 \\
\hline Element type & $\begin{array}{l}5 \\
\text { Shell } 181 \\
\end{array}$ & 5 & 5 & 5 & 5 & 5 & $\begin{array}{l}5 \\
6 \text { (Beam 188) }\end{array}$ \\
\hline Sections & 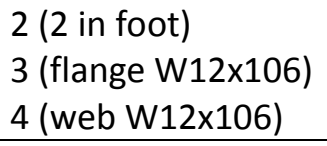 & $\begin{array}{l}2,3,4 \\
8 \text { (shim) }\end{array}$ & 3,4 & 9 (0.313 in) & $\begin{array}{l}10 \text { (web W 24×103) } \\
11 \text { (flange W 24×103) }\end{array}$ & 12 (0.75 in) & $\begin{array}{l}3 \\
15 \text { (tube } 80 \text { ) }\end{array}$ \\
\hline Materials & $\begin{array}{l}10 \text { (W section) } \\
11 \text { (foot) }\end{array}$ & $\begin{array}{l}\text { 10, 11, } \\
12 \text { (shim) }\end{array}$ & 10 & 10 & 10 & & 10 \\
\hline
\end{tabular}


ELEMENTS

$\mathrm{CE}$

\section{$\mathbf{N}$}

AUG 32012

$11: 47: 30$

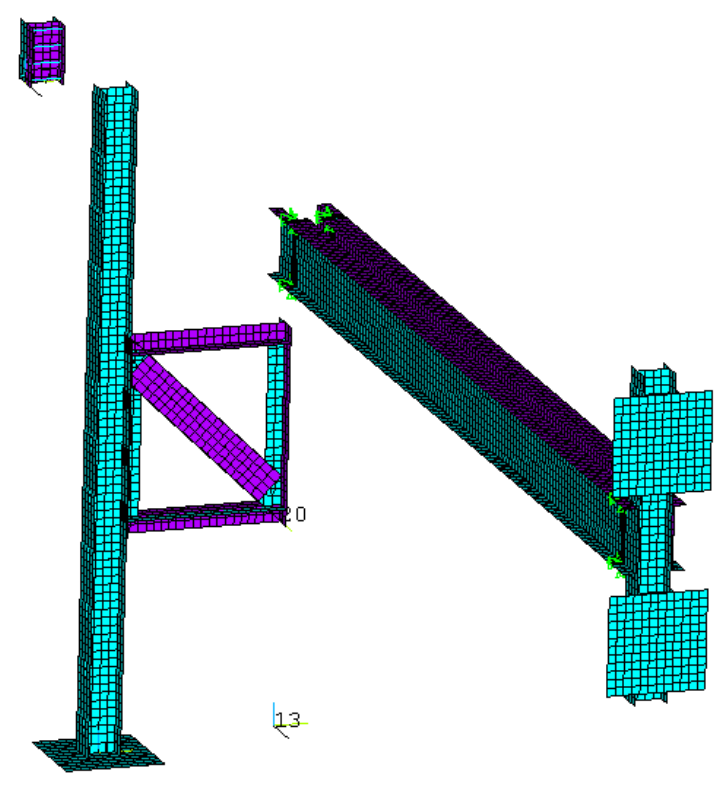

NERL COMBINED SUPPORT

Fig. 13.2 Step 2: Move components into place

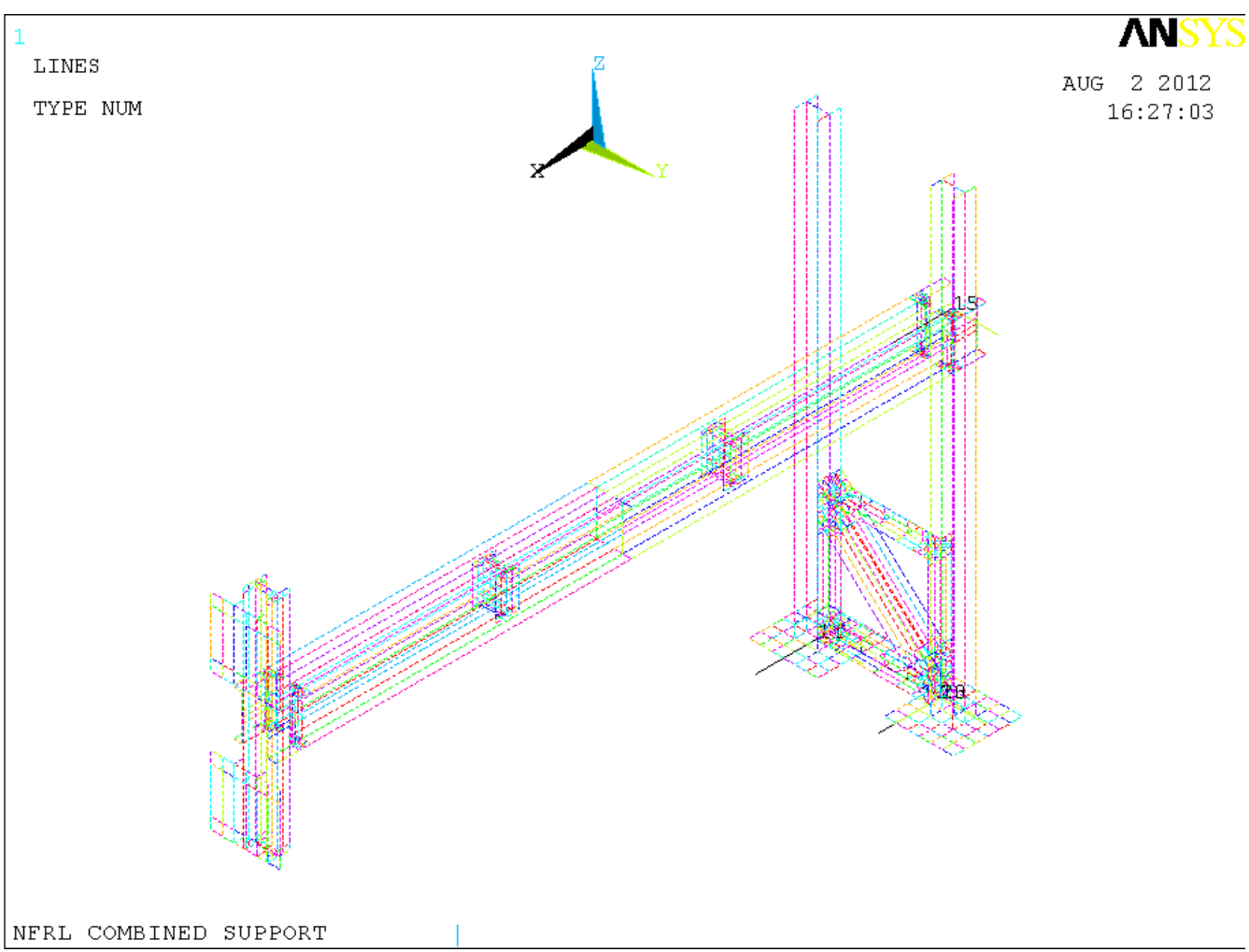


Fig. 13.3 Step 4: Copy components into place

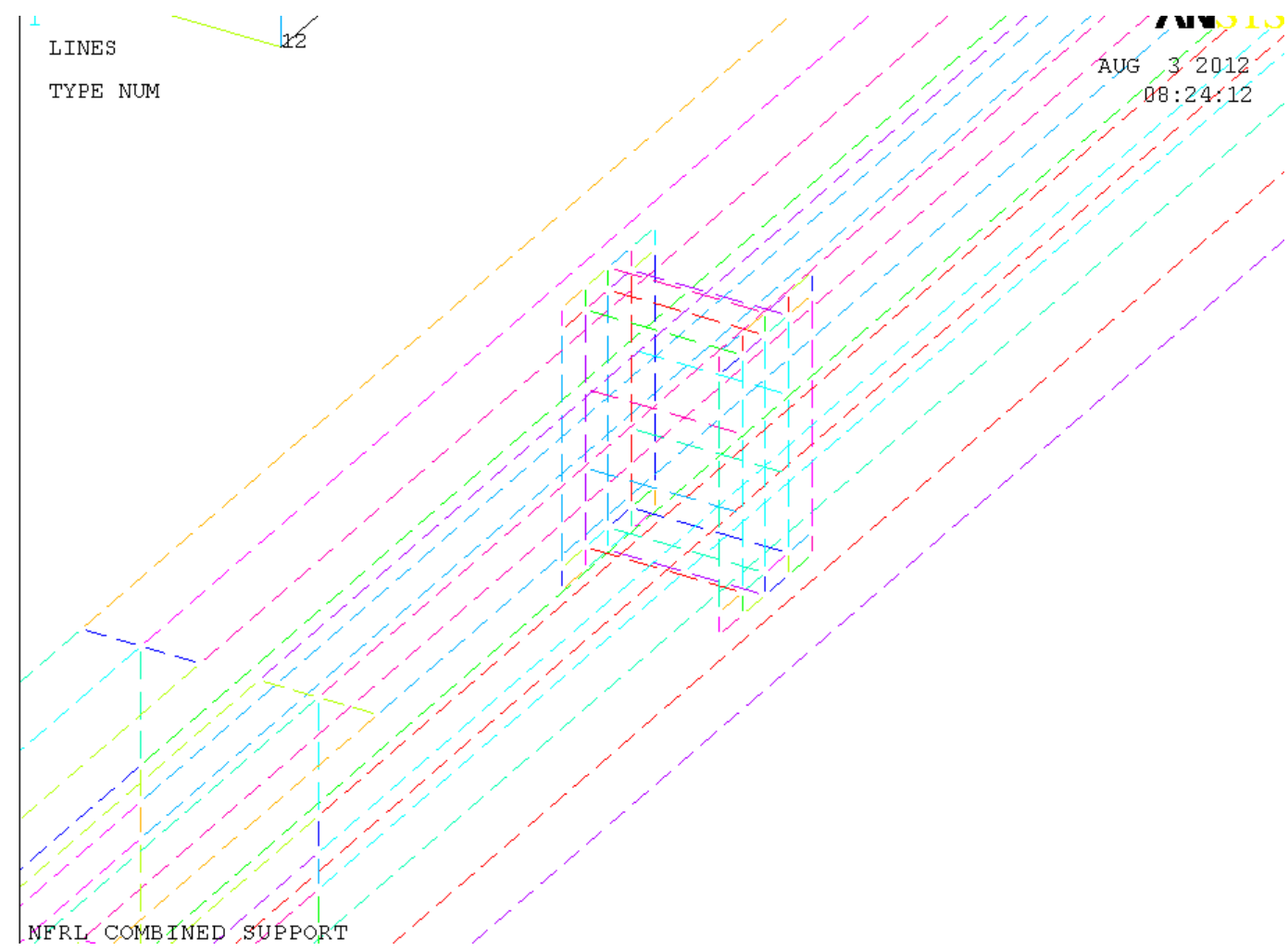

Fig. 13.4 Step 4 Copy: Detail of girder spacer in place

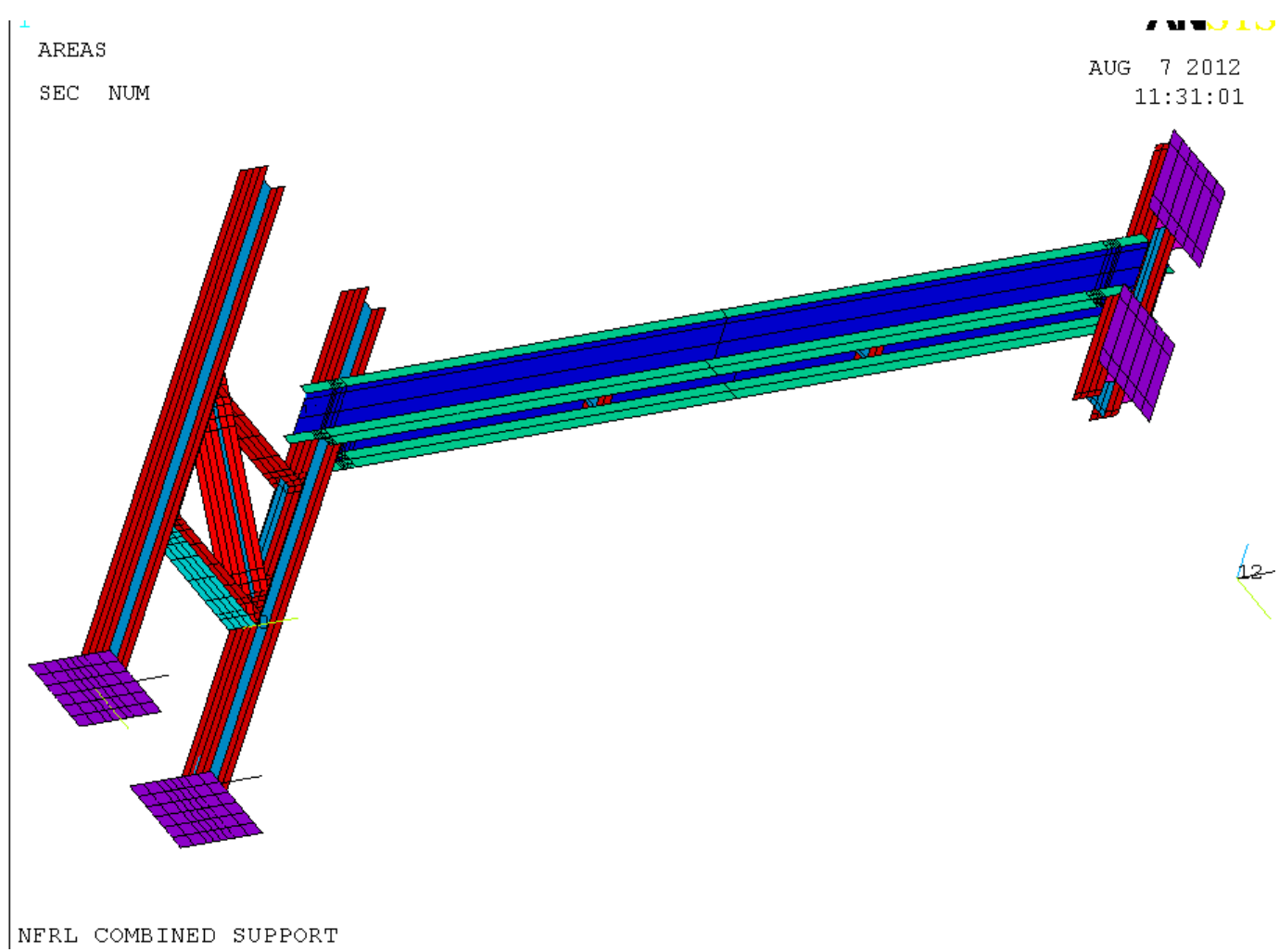


Fig. 13.5 Step 5: Fit components together

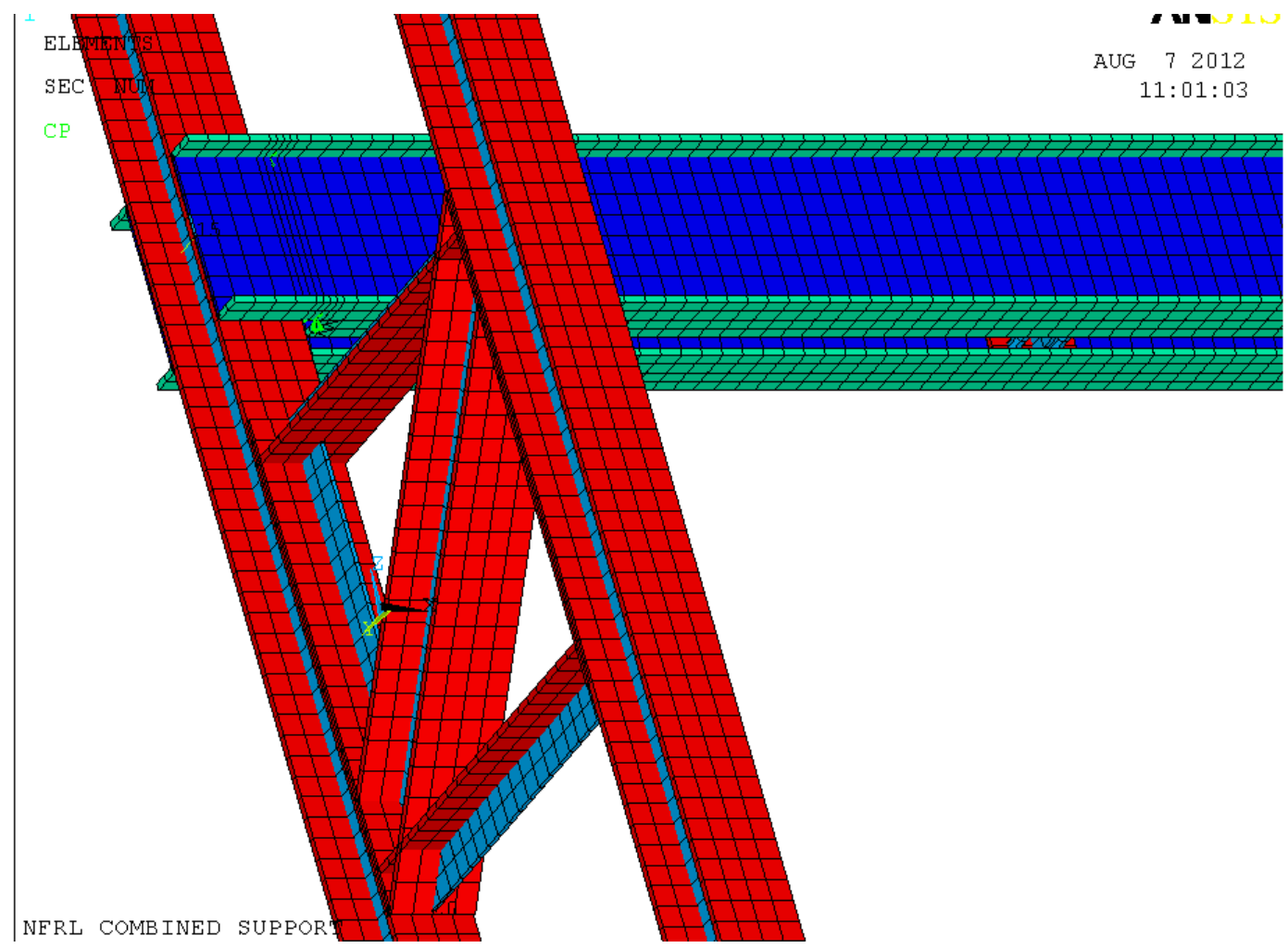

Fig. 13.6 Step 5 Fit: 3D detail of foot-columns, frame and girder

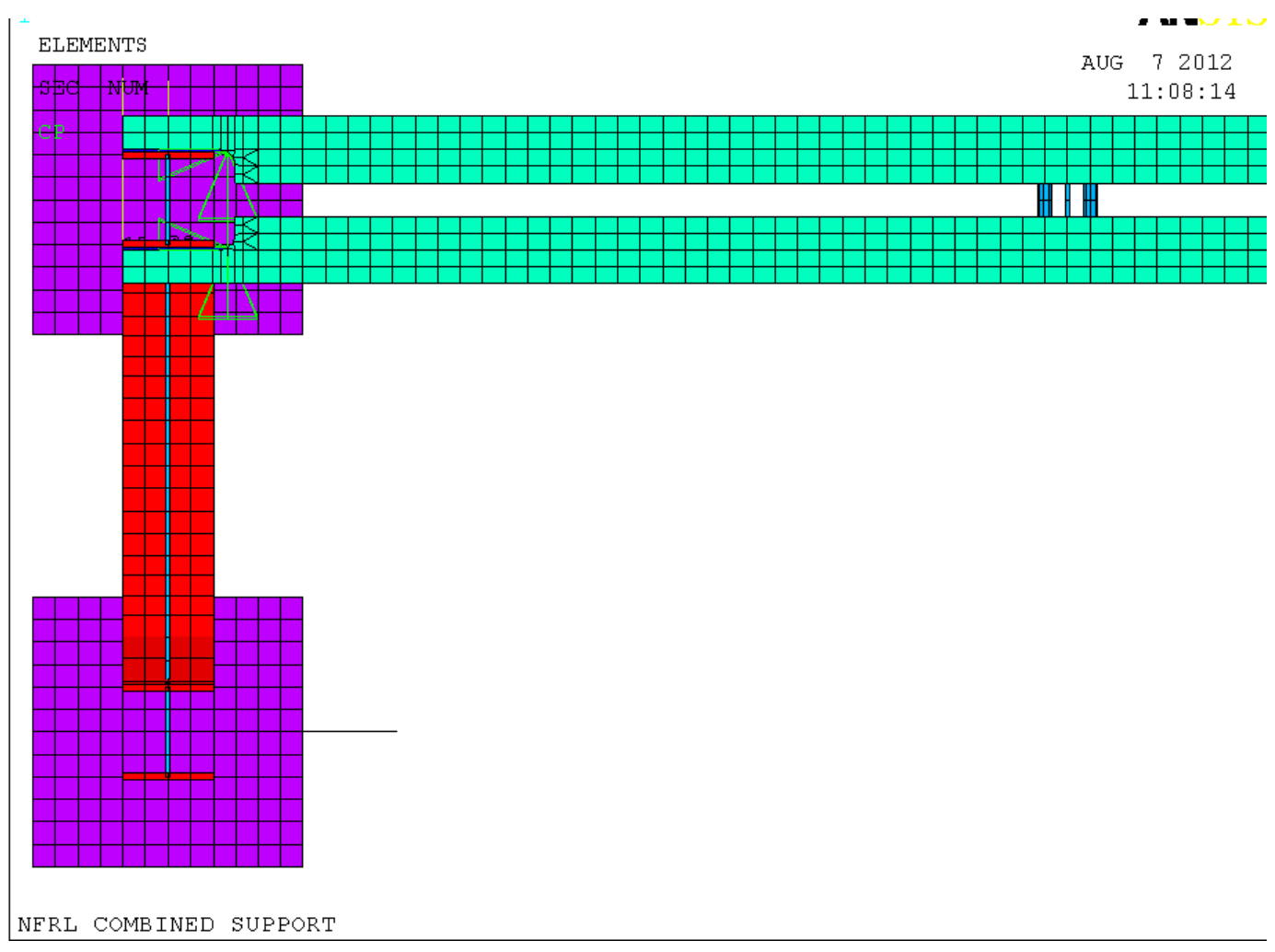


Fig. 13.7 Step 5 Fit: Top view of detail of foot-columns, frame, girder and spacer

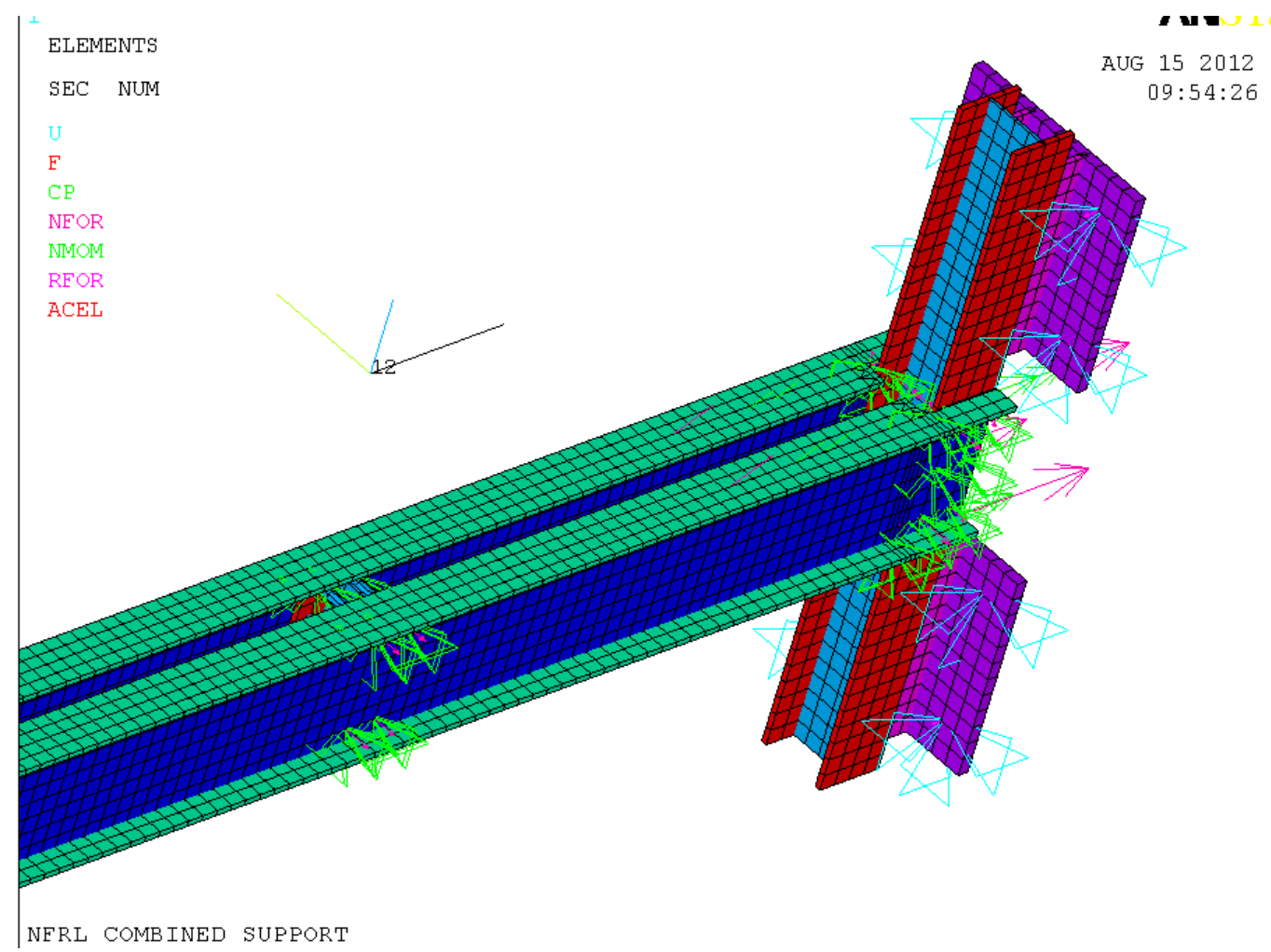

Fig. 13.8 Step 5 Fit: 3D detail of wall-column and girder

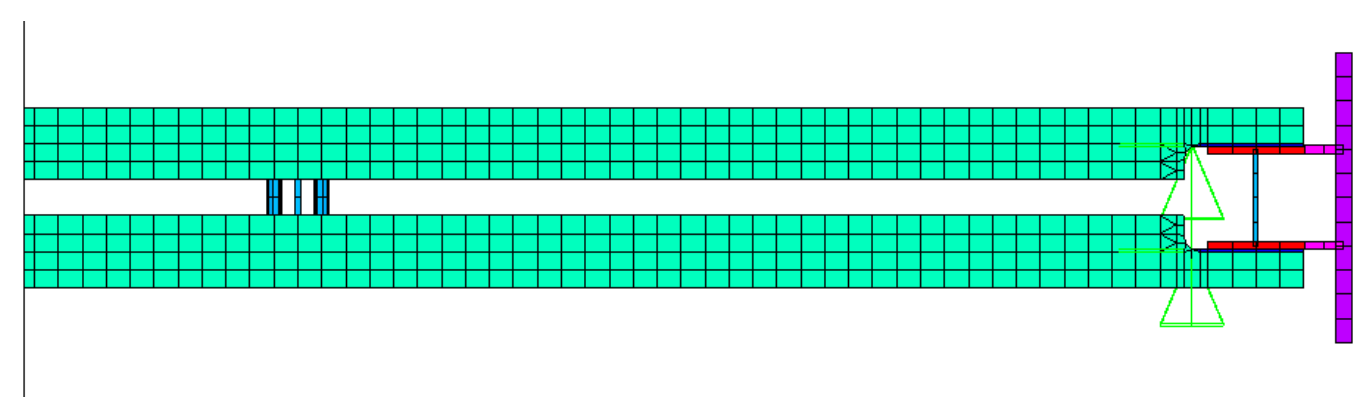

Fig. 13.9 Step 5 Fit: Top view of detail of wall-column, girder and spacer 


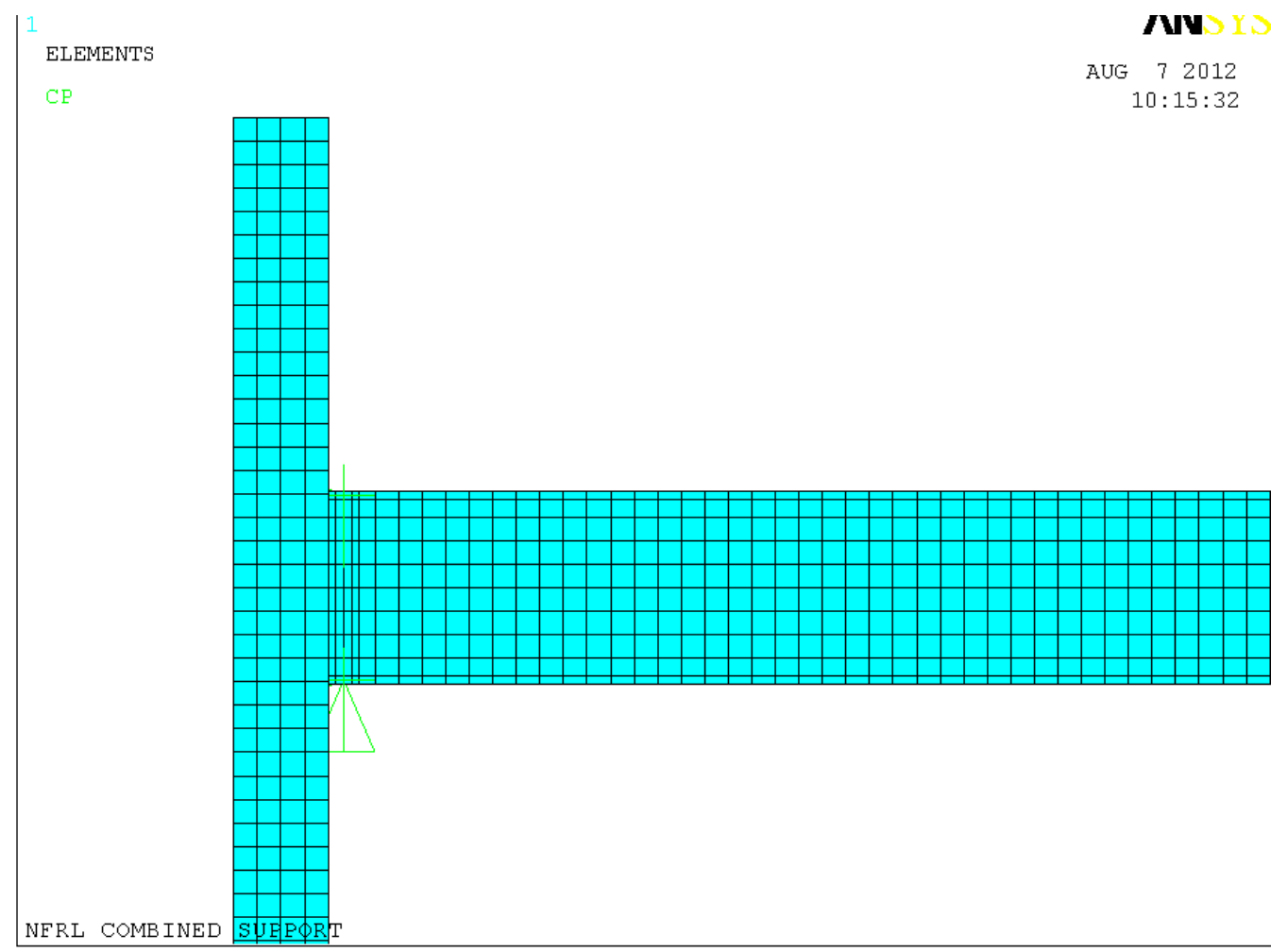

Fig. 13.10 Step 5 Fit: elevation view of girder and foot-column

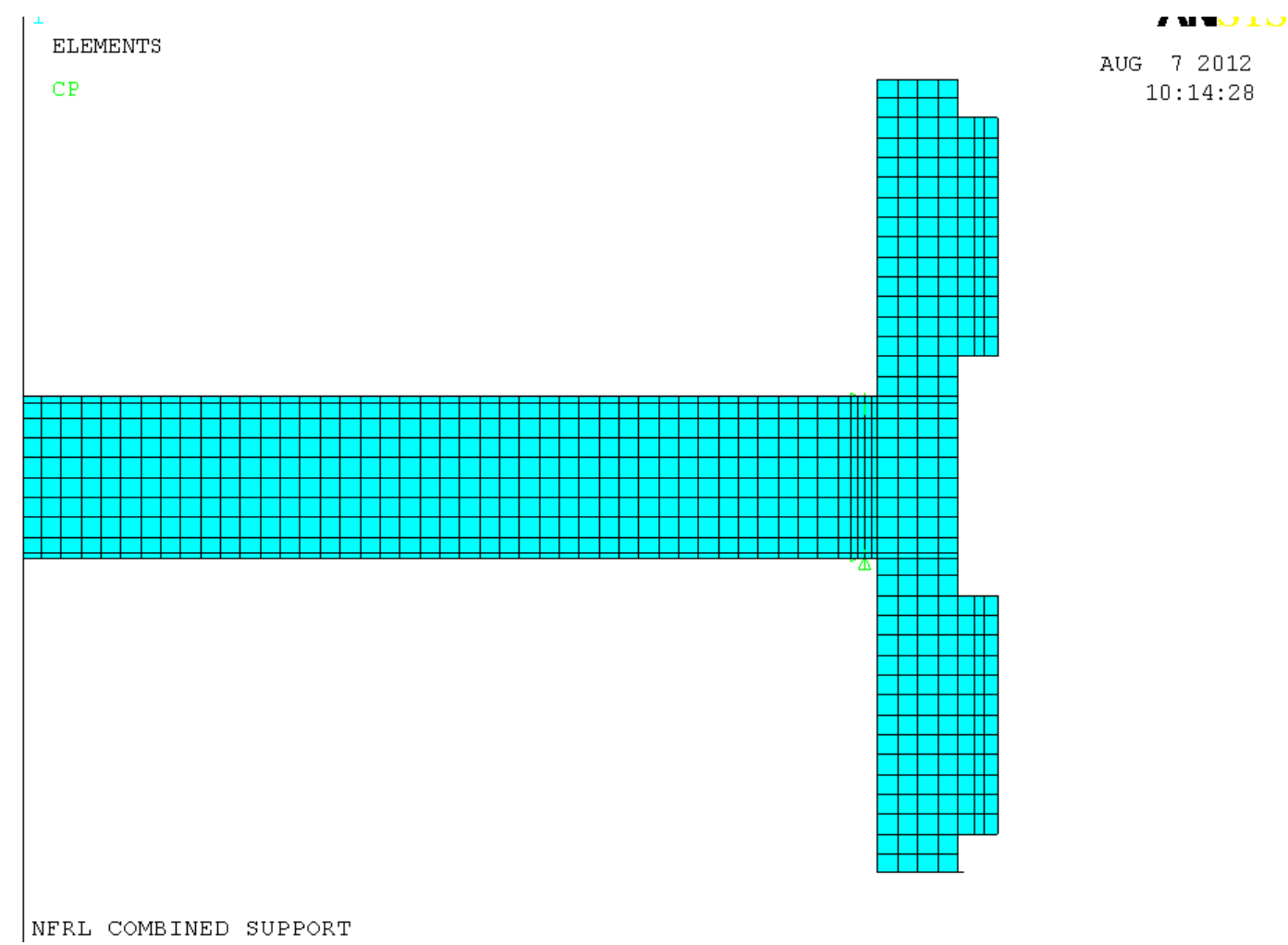

Fig. 13.11 Step5 Fit: elevation view of girder and wall-column 


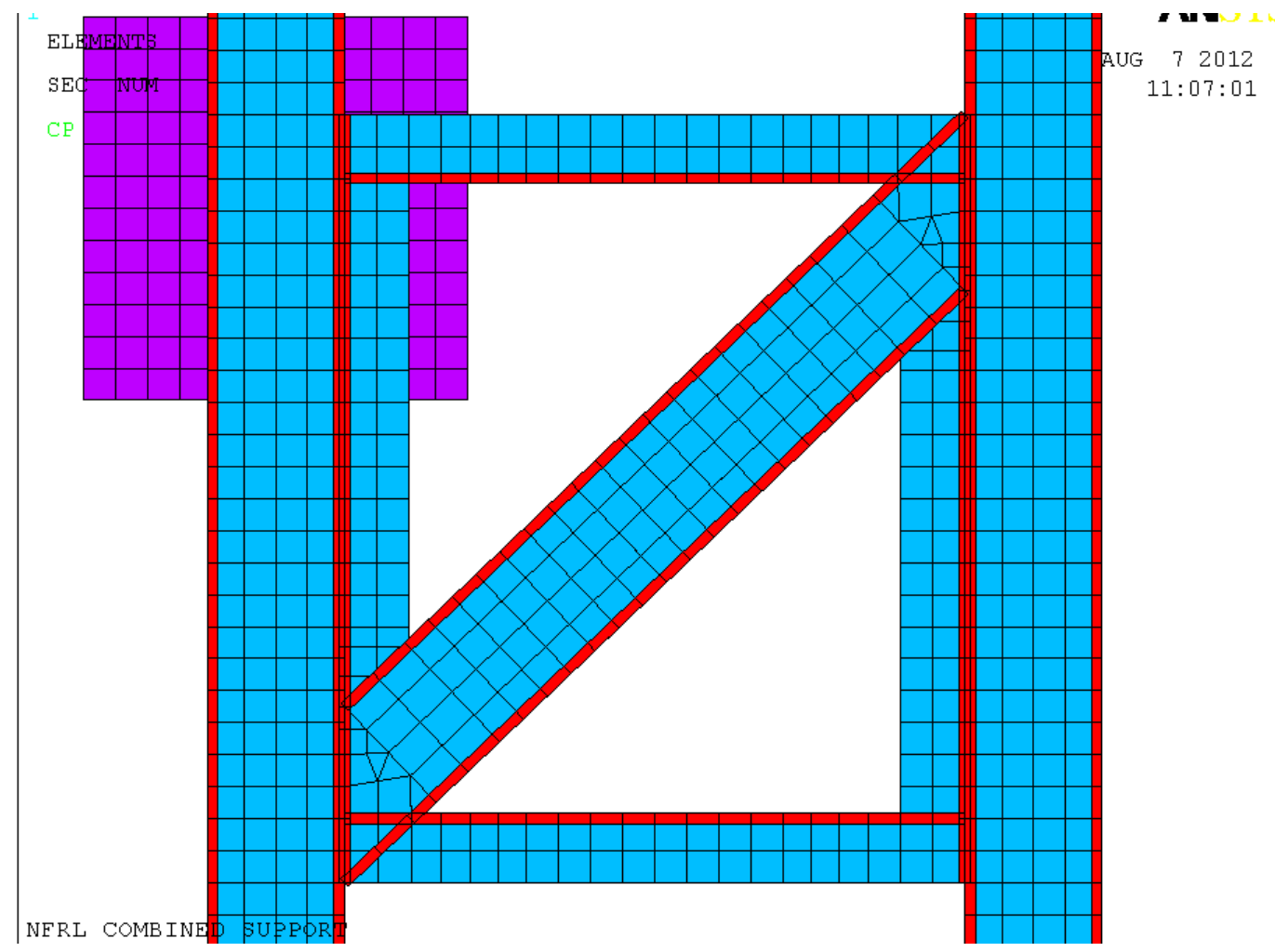

Fig. 13.12 Step 5 Fit: Detail of frame and foot-columns 


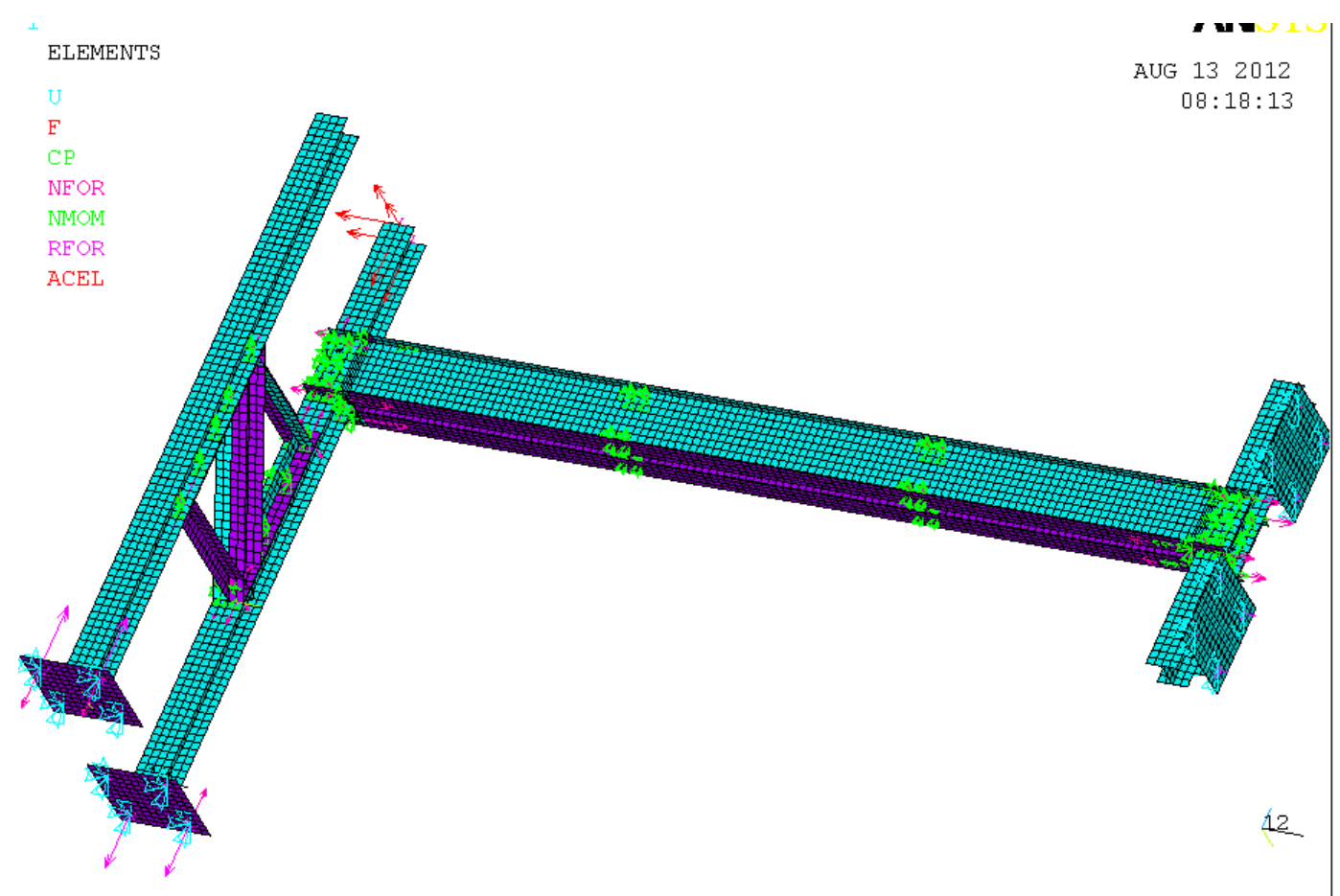

NERL COMBINED SUPPORT

Fig. 13.13 Steps 6 Constrain, 7 Restrain, 8 Load: Bolt components together, to wall and floor, and load

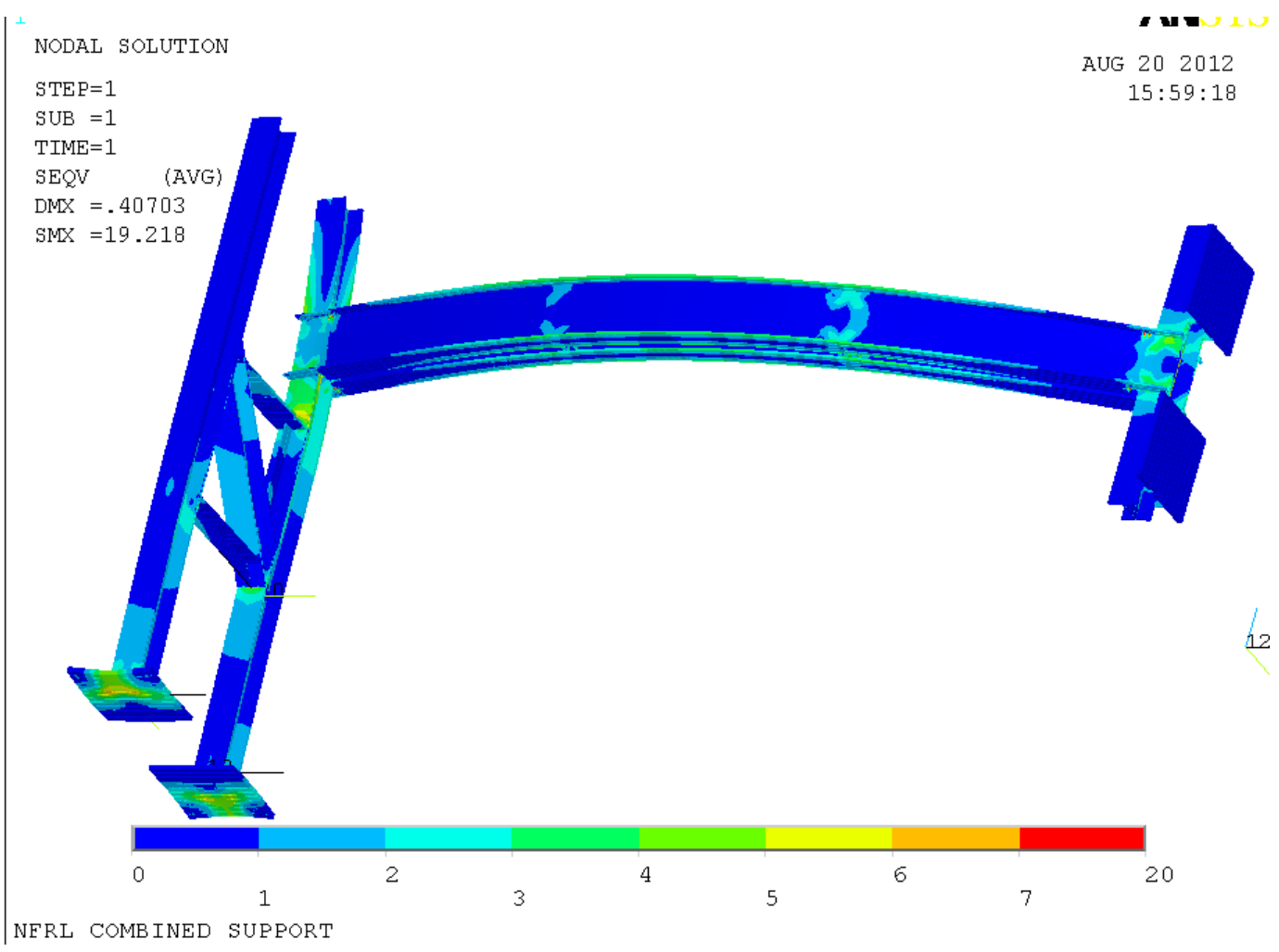

Fig. 13.14 Deformed shape and Von Mises stress (1 ksi=6.89 MPa) 


\section{! Combined structural support}

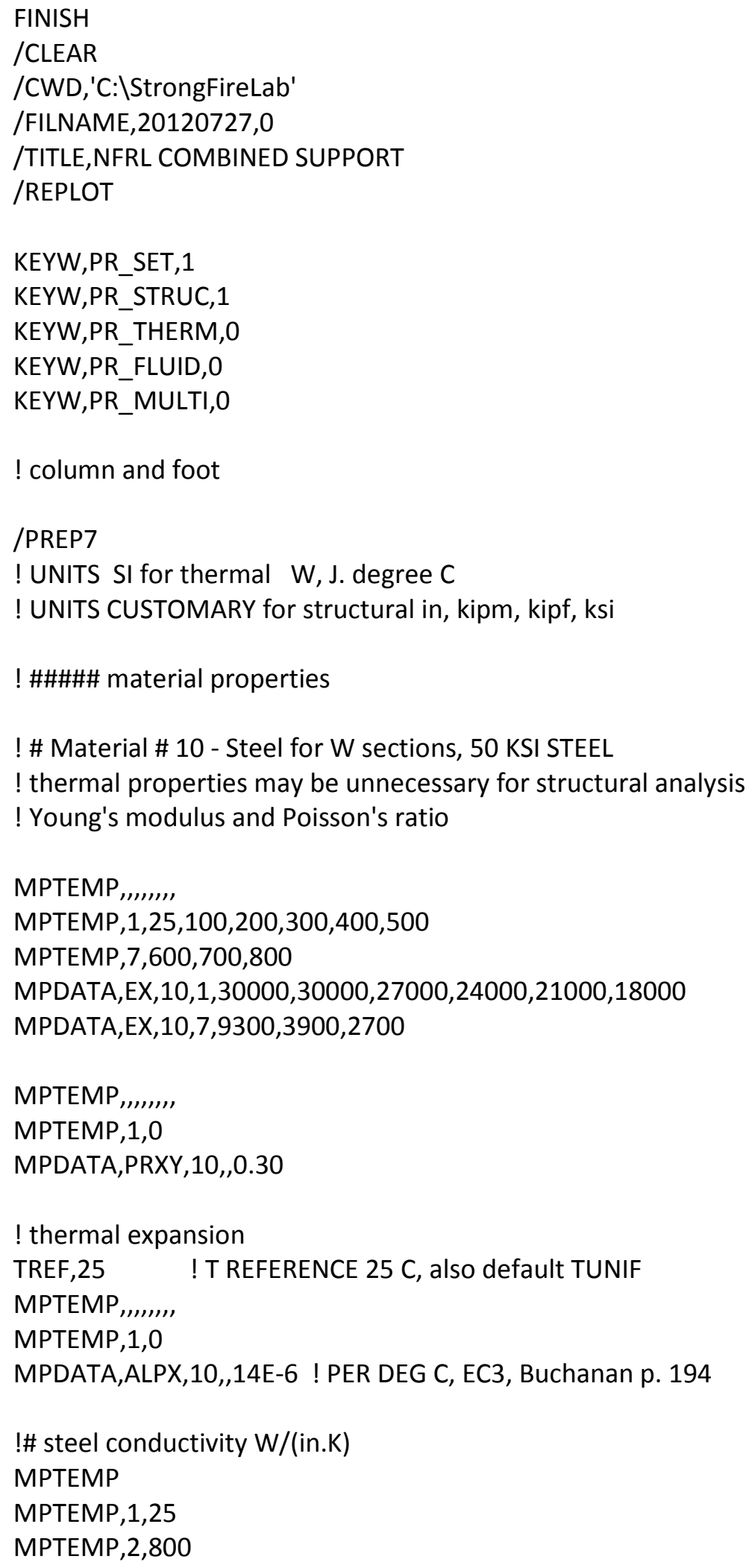




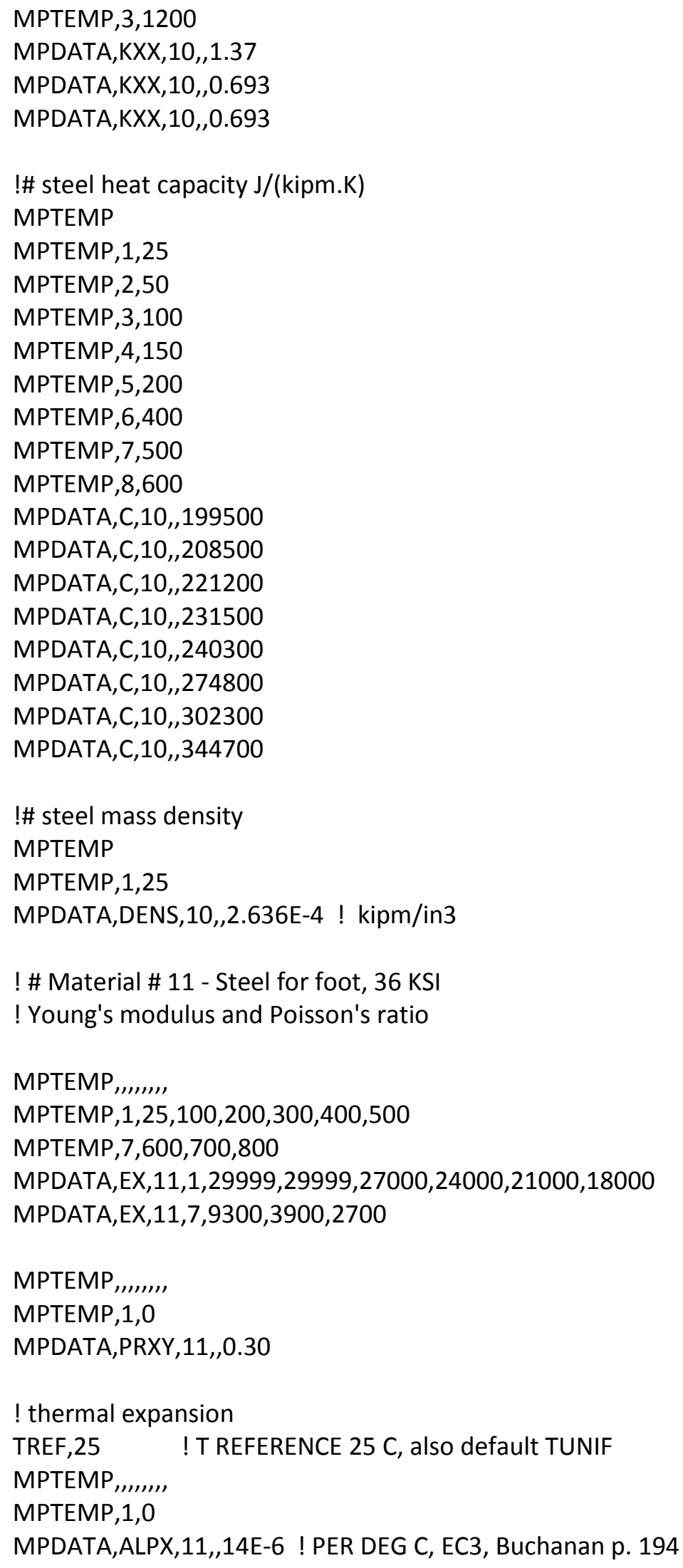




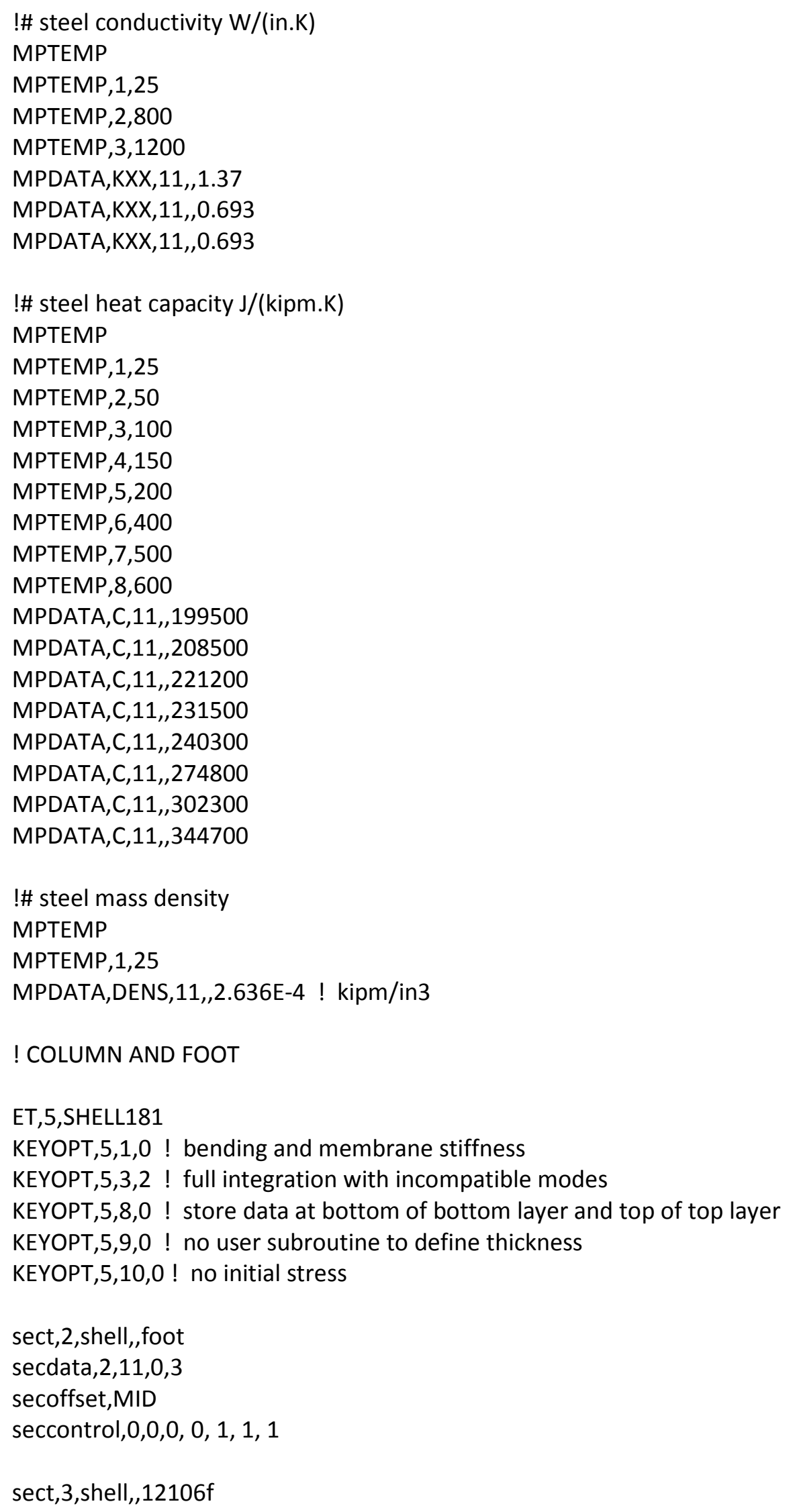


secdata, $0.99,10,0,3$

secoffset,MID

seccontrol,0,0,0, 0, 1, 1, 1

sect,4,shell,,12106W

secdata, $0.61,10,0,3$

secoffset,MID

seccontrol,0,0,0, 0, 1, 1, 1

! USER INPUT: STARTING NUMBERS, SUB 1============================

*SET, KP1,8000

*SET,LIN1,19000

*SET,ARE1,15000

*SET, VOL1,5000

*SET,ELE1,67000

*SET,NOD1,85000

! \# define model geometry, sheet $11 / 15$ lower left fig

! Local X parallel to flanges, point North.

! Local Y parallel to web, point West.

! Local Z points up.

! user input: origin of local coordinates $=$ CENTER OF FOOT

LOCAL,11,0,348,465.98,182, , , ,1,1,

CSYS,11,

HEIGHT=240 ! COLUMN HEIGHT INCLUDES FOOTING, INCHES

$\mathrm{H}=\mathrm{HEIGHT}-1$

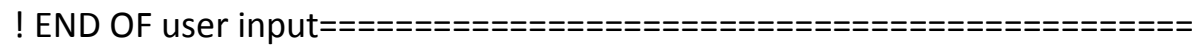

NUMSTR,KP,KP1

NUMSTR,LINE,LIN1

NUMSTR,AREA,ARE1

NUMSTR, VOLU,VOL1

NUMSTR,ELEM,ELE1

NUMSTR,NODE,NOD1

! X SIDE OF FOOT

$\mathrm{K}, \mathrm{KP} 1,-18,-18,0$,

K,KP1+1,-12,-18,0,

$\mathrm{K}, \mathrm{KP} 1+2,-6.11,-18,0$,

$\mathrm{K}, \mathrm{KP} 1+3,-3.0,-18,0, \quad ! 8.34 \mathrm{pm}$

$\mathrm{K}, \mathrm{KP} 1+4,0,-18,0$,

$\mathrm{K}, \mathrm{KP} 1+5,3.0,-18,0, \quad ! 8.34 \mathrm{pm}$

$\mathrm{K}, \mathrm{KP} 1+6,6.11,-18,0$,

$\mathrm{K}, \mathrm{KP} 1+7,12,-18,0$, 


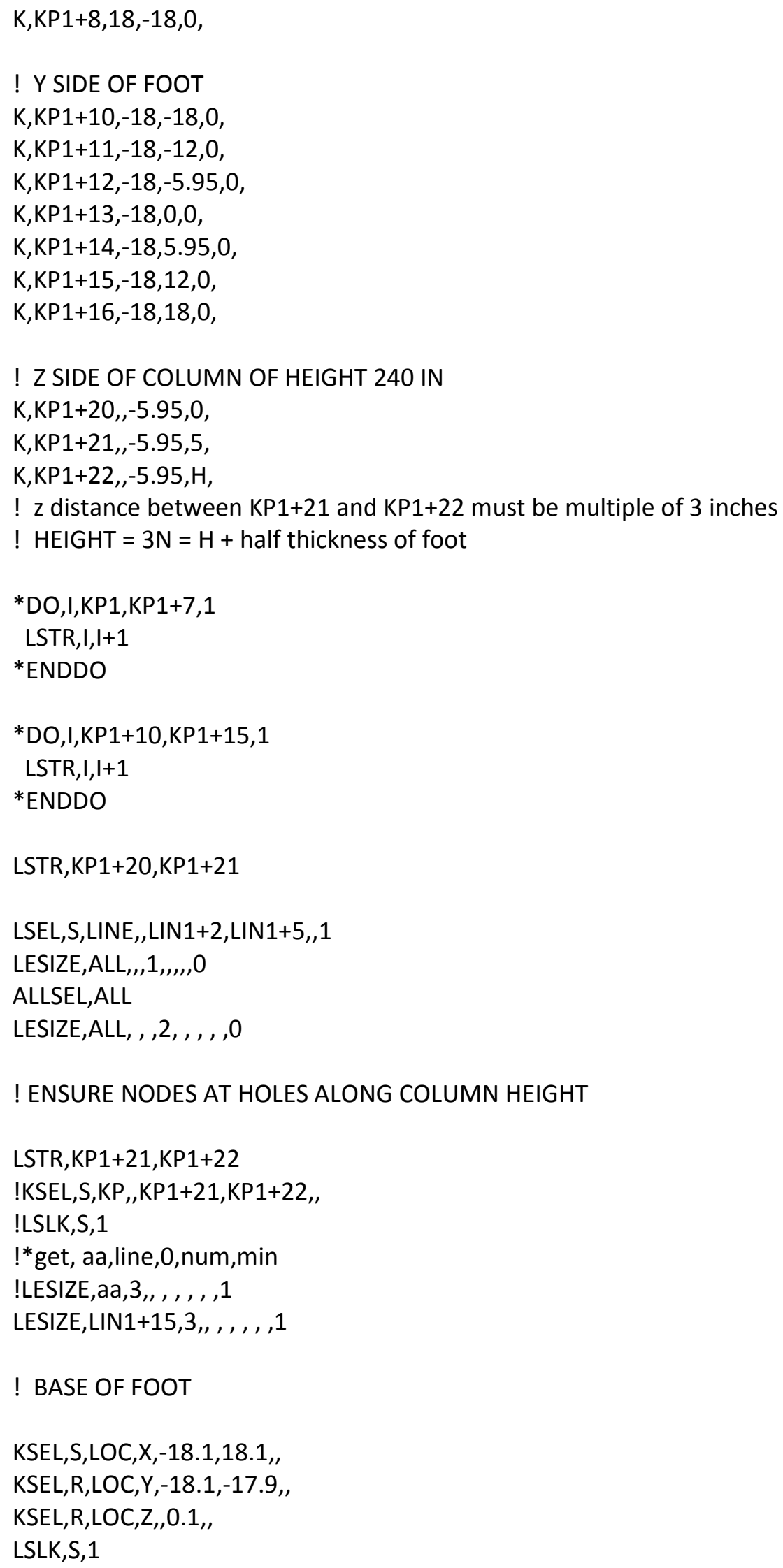




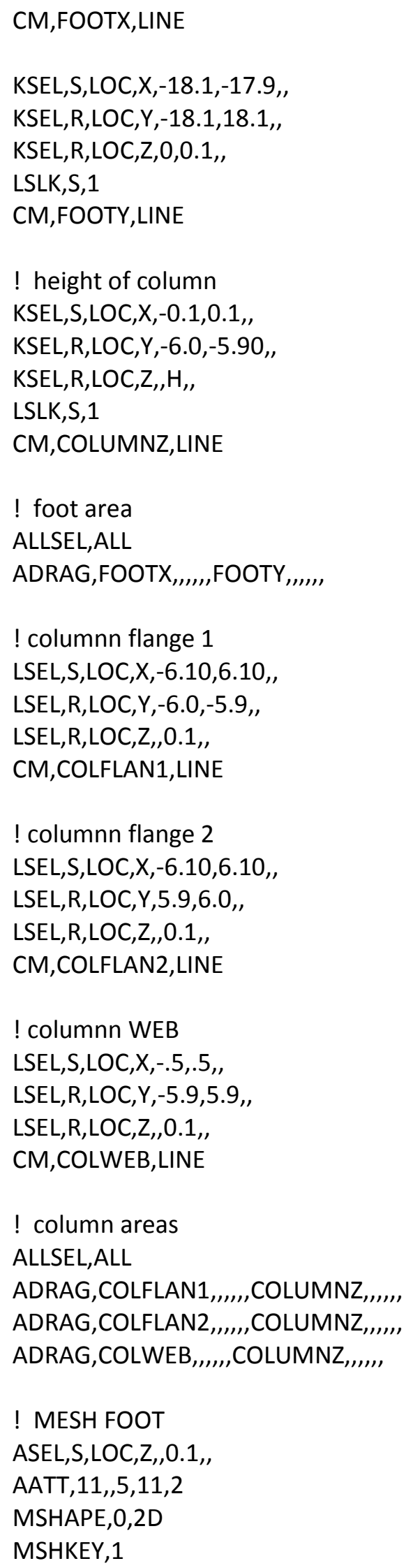




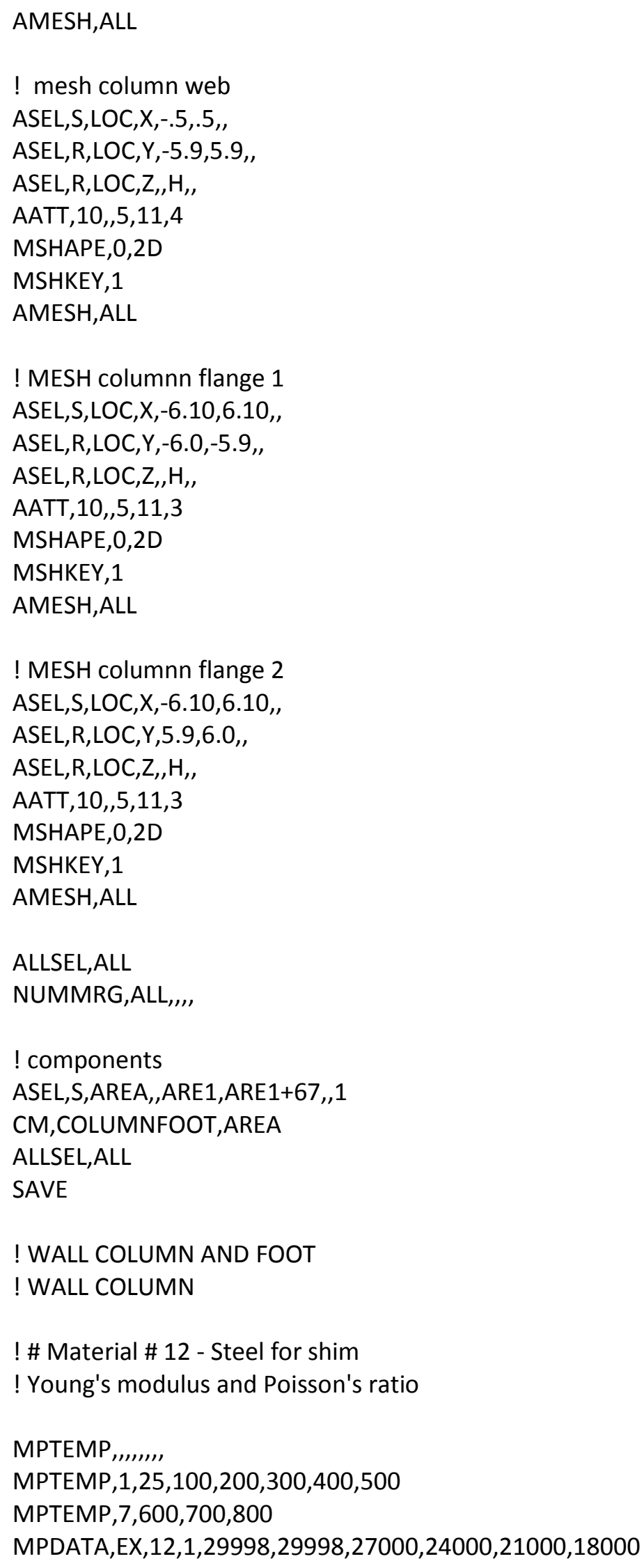




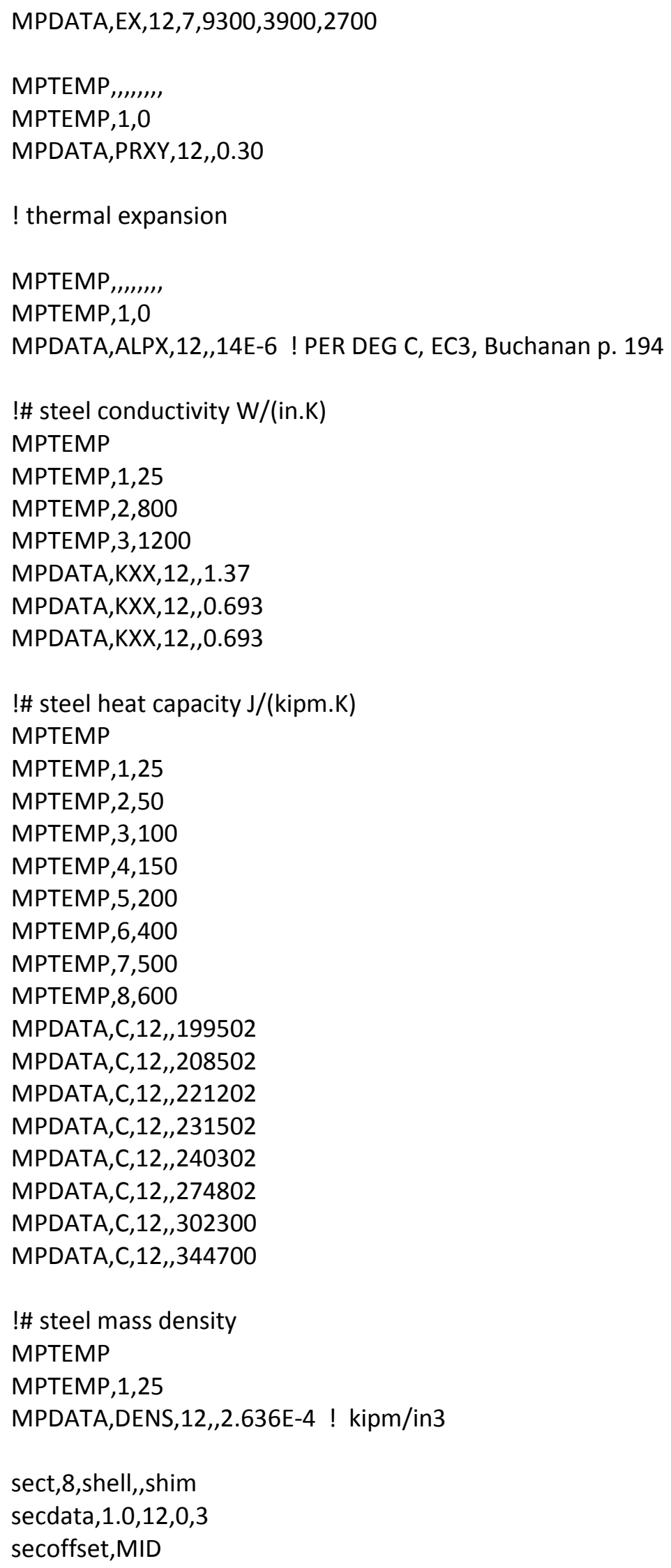


seccontrol,0,0,0, 0, 1, 1, 1

! USER INPUT: STARTING NUMBERS, SUB ZERO (NOT LETTER O)==========

*SET, KPO, 8100

*SET,LINO,19200

*SET,ARE0,15100

*SET, VOLO,5000

*SET,ELE0,69000

*SET,NOD0,88000

! \# define model geometry

! user input:location of column (origin of local coordinates)

! ref: sheet 15/15 lower left fig

! Local X parallel to flanges, points North.

! Local Y parallel to web, points West.

! Local Z points up.

! location of WALL column (origin of local coordinates center of I at base)

LOCAL,12,0,708,637,301.48, , , ,1,1,

! move back by $d y=-100$

CSYS,12,

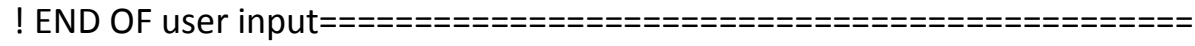

NUMSTR,KP,KPO

NUMSTR,LINE,LINO

NUMSTR,AREA,AREO

NUMSTR,VOLU,VOLO

NUMSTR,ELEM,ELEO

NUMSTR,NODE,NODO

! Y SIDE OF FOOT

$\mathrm{K}, \mathrm{KPO}, 11,-18,5.75$,

$\mathrm{K}, \mathrm{KPO}+1,11,-12,5.75$,

$\mathrm{K}, \mathrm{KPO}+2,11,-5.95,5.75$,

$\mathrm{K}, \mathrm{KPO}+3,11, \quad, 5.75$,

$\mathrm{K}, \mathrm{KPO}+4,11,5.95,5.75$,

$\mathrm{K}, \mathrm{KPO}+5,11,12,5.75$,

K,KPO+6,11,18,5.75,

! Z SIDE OF FOOT

K,KPO+10,11,-18,5.75

K,KPO+11,11,-18,11.75

K,KPO+12,11,-18,35.75

K,KPO+13,11,-18,41.75

*DO,I,KPO,KPO+5, 1

LSTR,I,I+1 
*ENDDO

*DO,I,KP0+10,KP0+12,1

LSTR,I,I+1

*ENDDO

KSEL,S,KP, ,KPO,KPO+13, ,

LSLK,S,1

LESIZE,ALL, , ,2, , , , , 1

KSEL,S,KP ,KPO+11,KP0+12,

LSLK,S,1

*get, aa,line,0,num, min

LESIZE, aa, 3 , , , , , , ,1

KSEL,S,KP, ,KPO,KPO+6, ,

LSLK,S,1

CM,WALLFOOTY,LINE

KSEL,S,KP ,KPO+10,KPO+13, ,

LSLK,S, 1

CM,WALLFOOTZ,LINE

! X SIDE OF SHIM

$\mathrm{K}, \mathrm{KPO}+20,6.11,5.95,5.75$

$\mathrm{K}, \mathrm{KPO}+21,11,5.95,5.75$

LSTR, KPO+20,KPO+21

KSEL,S,KP,,KPO+20,KPO+21,

LSLK,S,1

LESIZE,ALL, , ,2, , , , 1

CM,SHIMX,LINE

! Z SIDE OF SHIM

$\mathrm{K}, \mathrm{KPO}+30,6.11,5.95,5.75$

$\mathrm{K}, \mathrm{KPO}+31,6.11,5.95,41.75$

LSTR, KPO+30,KP0+31

$\mathrm{KSEL}, \mathrm{S}, \mathrm{KP}$, ,KP0+30,KP0+31,

LSLK,S,1

LESIZE,ALL, 3, , , , , , , , 1

CM,SHIMZ,LINE

! base of columnn web

$\mathrm{K}, \mathrm{KPO}+40,-5.95$,

$\mathrm{K}, \mathrm{KPO}+41,-3.0$,

$\mathrm{K}, \mathrm{KPO}+42,$,

$\mathrm{K}, \mathrm{KPO}+43,3.0$,

$\mathrm{K}, \mathrm{KPO}+44$, 5.95,

*DO,I,KPO+40,KPO+43,1 
LSTR,I,I+1

*ENDDO

$\mathrm{KSEL}, \mathrm{S}, \mathrm{KP}$, ,KPO+40,KPO+44,

LSLK,S,1

LESIZE,ALL, , ,1, , , , , 1

CM, webbase, LINE

! base of columnn flange1

$\mathrm{K}, \mathrm{KPO}+50,-6.11,5.95$,

$\mathrm{K}, \mathrm{KPO}+51,-3.0,5.95$,

$\mathrm{K}, \mathrm{KPO}+52, \quad, 5.95$,

$\mathrm{K}, \mathrm{KPO}+53,3.0,5.95$,

$\mathrm{K}, \mathrm{KPO}+54,6.11,5.95$,

*DO,I,KP0+50,KP0+53,1

LSTR,I,I+1

*ENDDO

KSEL,S,KP, ,KPO+50,KPO+54,,

LSLK,S,1

LESIZE,ALL, , ,1 , , , , ,1

$\mathrm{CM}$, flange1 base, LINE

! base of columnn flange2

K,KPO+60,-6.11,-5.95,

$\mathrm{K}, \mathrm{KPO}+61,-3.0,-5.95$,

$\mathrm{K}, \mathrm{KPO}+62, \quad,-5.95$,

K,KPO+63, $3.0,-5.95$,

$\mathrm{K}, \mathrm{KPO}+64,6.11,-5.95$,

*DO,I,KPO+60,KPO+63,1

LSTR,I,I+1

*ENDDO

KSEL,S,KP, ,KPO+60,KPO+64,, LSLK,S,1

LESIZE,ALL, , , 1, , , , , 1

$\mathrm{CM}$, flange2 base, LINE

! columnn height

$\mathrm{K}, \mathrm{KPO}+70,$,

$\mathrm{K}, \mathrm{KPO}+71,,, 2.75$

$\mathrm{K}, \mathrm{KPO}+72,,, 116.75$

$\mathrm{K}, \mathrm{KPO}+73,,, 119.5$

LSTR, KPO+70,KPO+71

$\mathrm{KSEL}, \mathrm{S}, \mathrm{KP}$, ,KPO+70,KPO+71,

LSLK,S, 1

LESIZE,ALL, , , 1, , , , , 1 


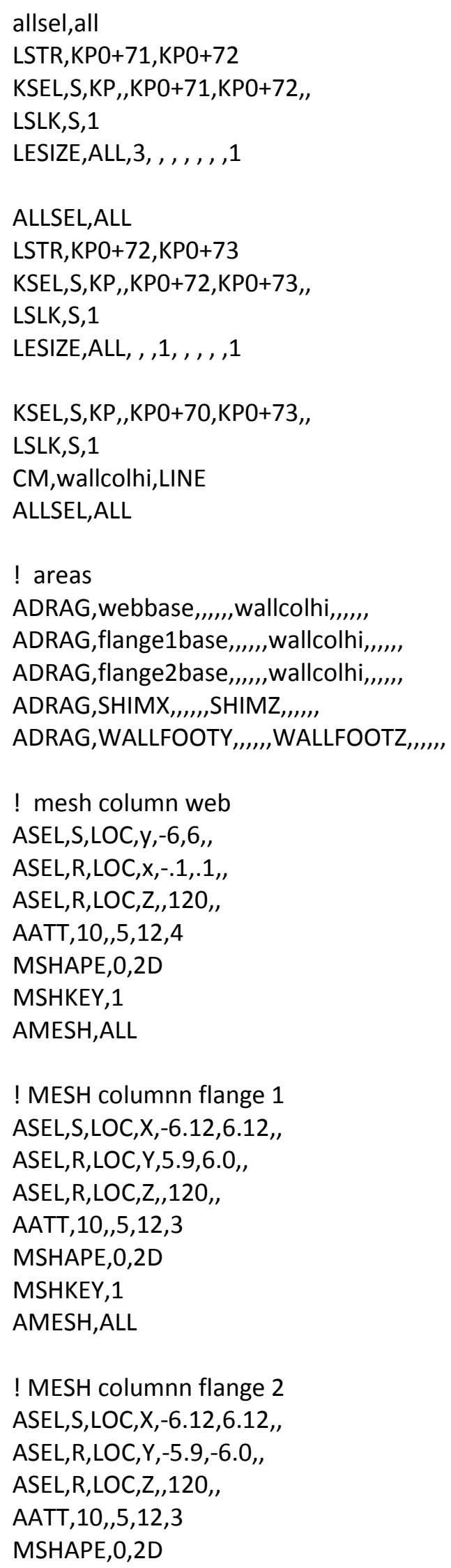




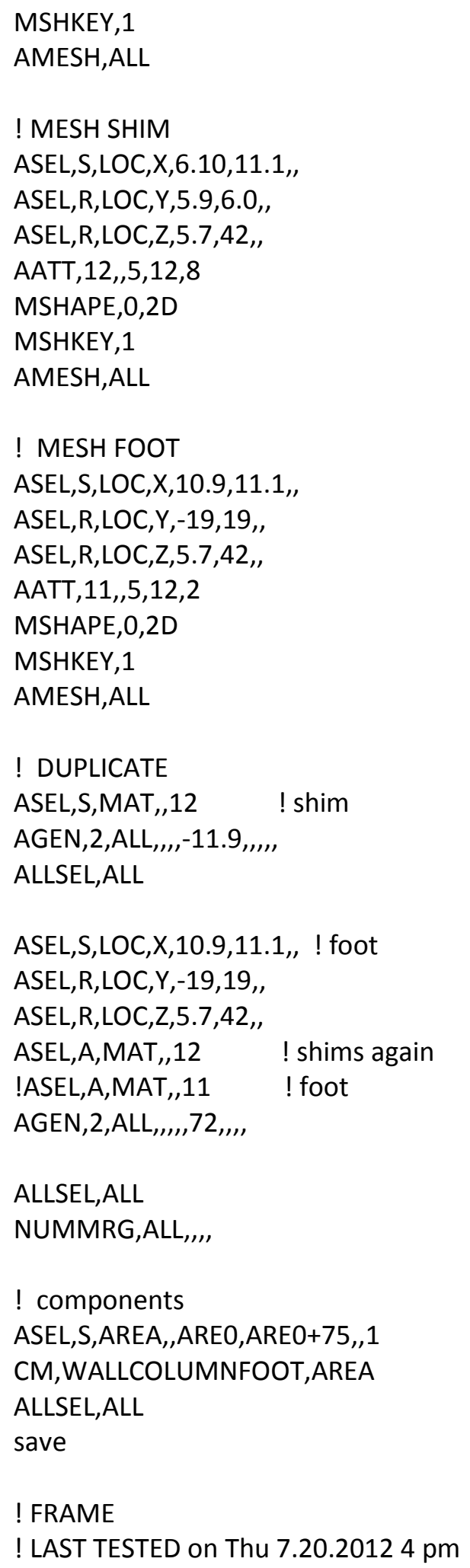




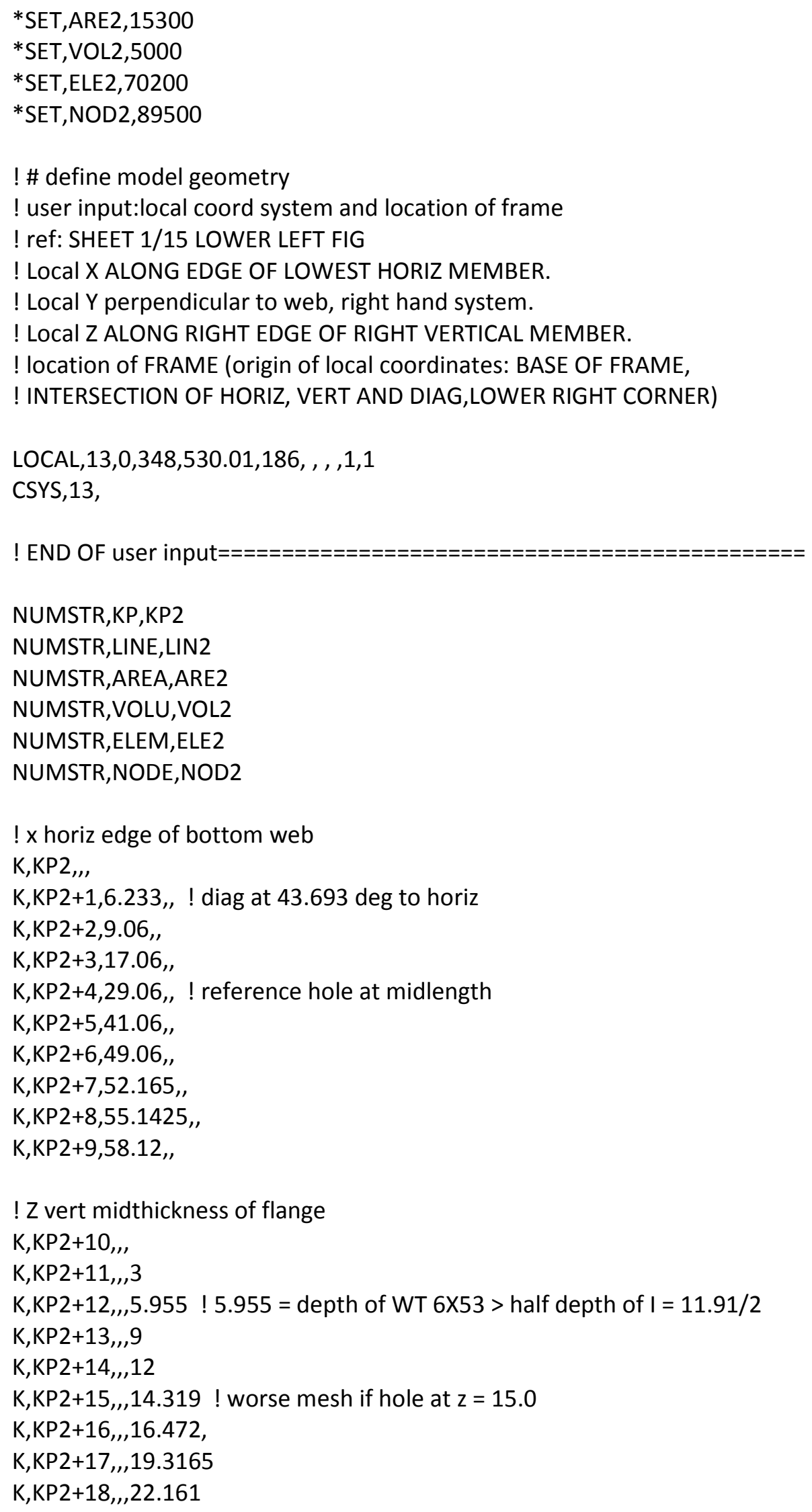




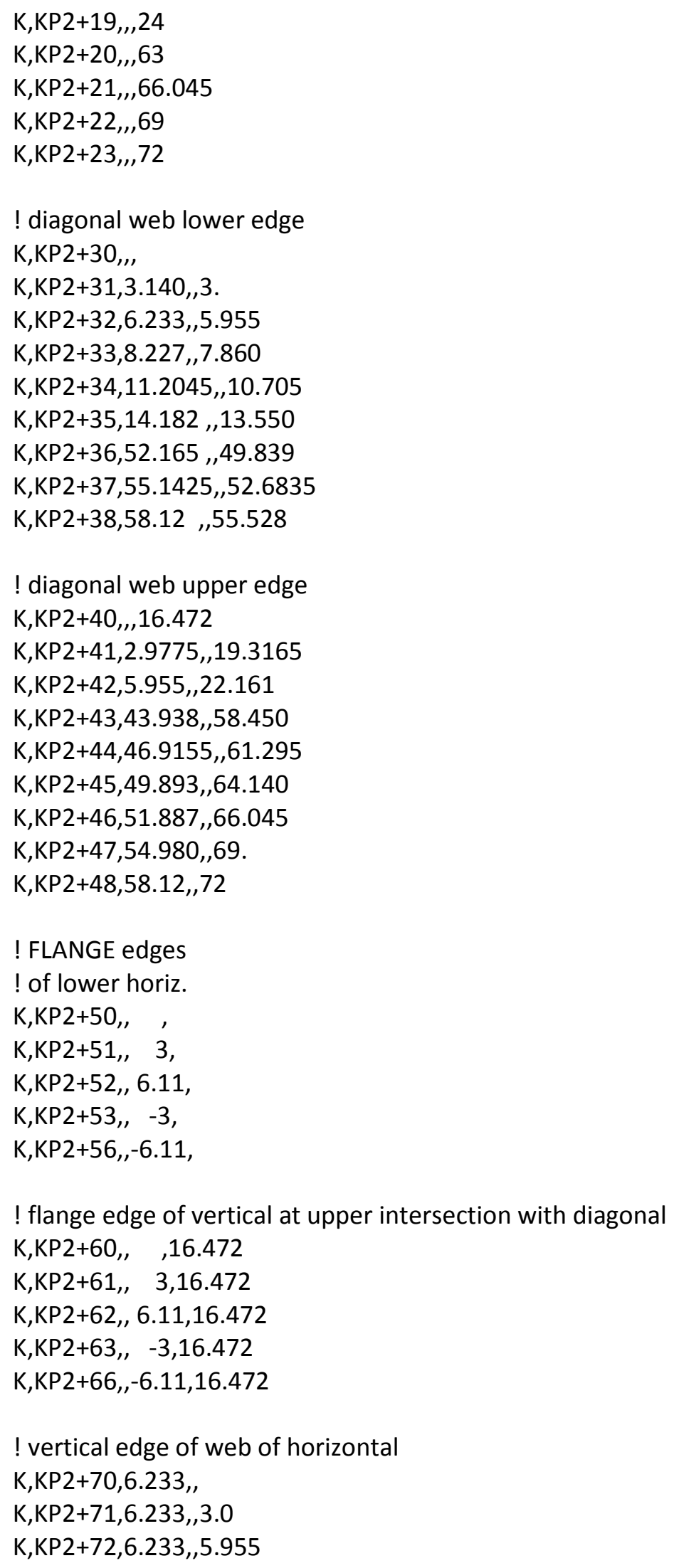


! diagonal web body

$\mathrm{K}, \mathrm{KP} 2+80,8.227,7.860$

$\mathrm{K}, \mathrm{KP} 2+81,6.17025,, 10.013$

$\mathrm{K}, \mathrm{KP} 2+82,4.1135,12.166$

$\mathrm{K}, \mathrm{KP} 2+83,2.05675,, 14.319$

$\mathrm{K}, \mathrm{KP} 2+84, \quad, 16.472$

! non extruded, disturbed zones

$\mathrm{K}, \mathrm{KP} 2+90,2.9775,, 22.161$

$\mathrm{K}, \mathrm{KP} 2+91,3.1165,, 5.955$

$K, K P 2+92,3.085125,, 9.5065$

$\mathrm{K}, \mathrm{KP} 2+93,2.05675,, 12.083$

! LINES

*DO,I,KP2,KP2+8,1 ! $x$ horiz edge of bottom web LSTR,I,I+1

*ENDDO

*DO,I,KP2+2,KP2+5,1 ! $x$ horiz edge of bottom web KSEL,S,KP, I, I+1,

LSLK,S,1

LESIZE,ALL,3.0, , , , 0 , , , 0

*ENDDO

ALLSEL,ALL

*DO,I,KP2+10,KP2+22,1 ! Z vert midthickness of flange LSTR,I,I+1

*ENDDO

KSEL,S,KP, ,KP2+19,KP2+20,

LSLK,S,1

LESIZE,ALL,3.0, , , , 0 , , , 0

ALLSEL,ALL

*DO,I,KP2+30,KP2+37,1 ! diagonal web lower edge LSTR,I,I+1

*ENDDO

$\mathrm{KSEL}, \mathrm{S}, \mathrm{KP}$, ,KP2+35,KP2+36,

LSLK,S,1

LESIZE,ALL, , ,17, ,0 , , ,0

ALLSEL,ALL

*DO,I,KP2+40,KP2+47,1 ! diagonal web upper edge LSTR, I,I+1

*ENDDO

KSEL,S,KP „,KP2+42,KP2+43, 


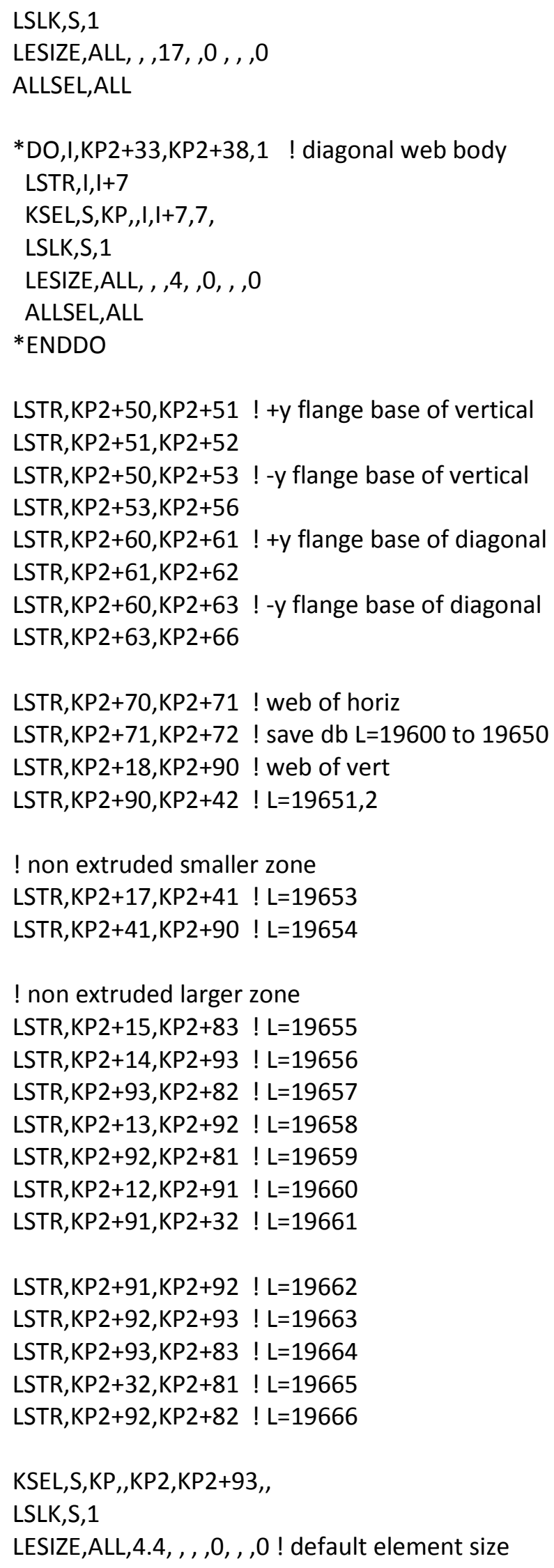




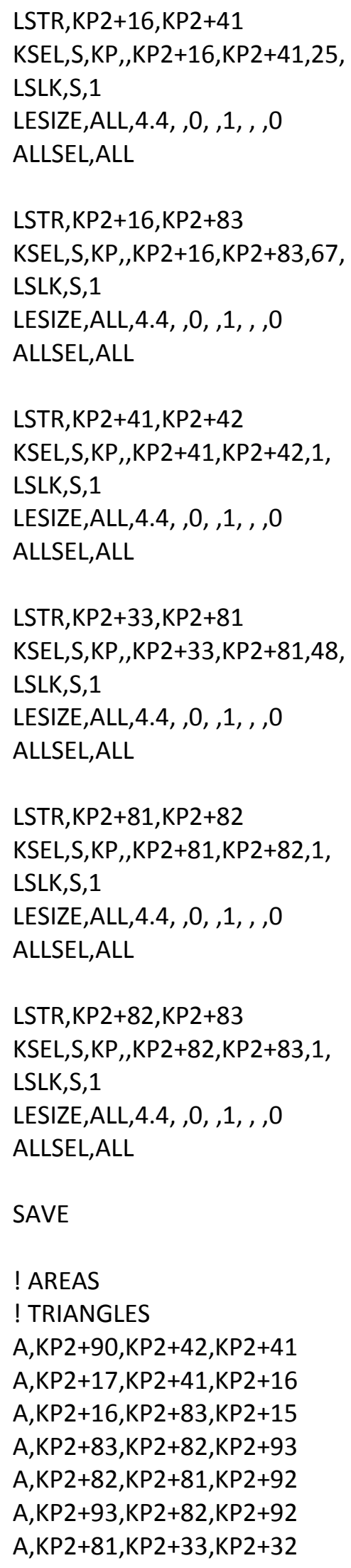


ASEL,S,AREA,,ARE2,ARE2+6,,1

CM,TRIAREA,AREA

ALLSEL,ALL

! QUADS

$\mathrm{A}, \mathrm{KP} 2+18, \mathrm{KP} 2+90, \mathrm{KP} 2+41, \mathrm{KP} 2+17$

$\mathrm{A}, \mathrm{KP} 2+15, \mathrm{KP} 2+83, \mathrm{KP} 2+93, \mathrm{KP} 2+14$

$\mathrm{A}, \mathrm{KP} 2+14, \mathrm{KP} 2+93, \mathrm{KP} 2+92, \mathrm{KP} 2+13$

$\mathrm{A}, \mathrm{KP} 2+13, \mathrm{KP} 2+92, \mathrm{KP} 2+91, \mathrm{KP} 2+12$

$\mathrm{A}, \mathrm{KP} 2+92, \mathrm{KP} 2+81, \mathrm{KP} 2+32, \mathrm{KP} 2+91$

ASEL,S,AREA,,ARE2,ARE2+11,,1

CM,DISTURB,AREA

ALLSEL,ALL

KSEL,S,KP, ,KP2,KP2+9,

LSLK,S,1

CM,FULLX,LINE

$\mathrm{KSEL}, \mathrm{S}, \mathrm{KP}$, ,KP2+10,KP2+23,

LSLK,S,1

CM,FULLZ,LINE

KSEL,S,KP „KP2+18,KP2+21,

LSLK,S, 1

CM,PARTZ,LINE

KSEL,S,KP, ,KP2+30,KP2+38,

LSLK,S,1

CM,lodiag,LINE

KSEL,S,KP ,KP2+40,KP2+48,

LSLK,S,1

CM,hidiag,LINE

KSEL,S,KP, ,KP2+50,KP2+52,1,

LSLK,S,1

CM,flangeplus, LINE

KSEL,S,KP, ,KP2+50,KP2+56,3,

LSLK,S,1

$\mathrm{CM}$,flangeminus, LINE

KSEL,S,KP, ,KP2+60,KP2+62,1,

LSLK,S,1

CM,flandiagplus, LINE 
$\mathrm{KSEL}, \mathrm{S}, \mathrm{KP}$, ,KP2+60,KP2+66,3,

LSLK,S,1

$\mathrm{CM}$,flandiagminus, LINE

$\mathrm{KSEL}, \mathrm{S}, \mathrm{KP}$, ,KP2+70,KP2+72,1,

LSLK,S,1

CM, webhoriz,LINE

$\mathrm{KSEL}, \mathrm{S}, \mathrm{KP}$, ,KP2+18,KP2+42,24,

KSEL,A,KP, KP2+90

LSLK,S,1

CM, webvert, LINE

ALLSEL,ALL

!!*GET,AMAX1,AREA,0,NUM,MAX

! diagonal web areas

$\mathrm{A}, \mathrm{KP} 2+33, \mathrm{KP} 2+34, \mathrm{KP} 2+41, \mathrm{KP} 2+40$

$* \mathrm{DO}, \mathrm{I}, \mathrm{KP} 2+34, \mathrm{KP} 2+37,1$

$A, I, I+1, I+8, I+7$

*ENDDO

ASEL,S,AREA,,ARE2,ARE2+16,,1

CM,DIAWEBTURB,AREA ! DIAGONAL WEB AND DISTURBED AREAS=D

ALLSEL,ALL

! areas

ADRAG,lodiag,,,,,,, flangeplus,,,,,

ASEL,S,AREA,,ARE2,ARE2+32,,1

CM,DLOPLUS,AREA ! DIAGONAL WEB,DISTURBED AND LOW PLUS DIAG FLANGE AREAS

ALLSEL,ALL

ADRAG, lodiag, ,,,,,, flangeminus,,,,,,

ASEL,S,AREA,,ARE2,ARE2+48,,1

CM,DLODIAG,AREA ! DIAGONAL WEB,DISTURBED AND LOW DIAG FLANGE AREAS

ALLSEL,ALL

ADRAG, hidiag, ,,,,, flandiagplus, ,,, ,

ASEL,S,AREA,,ARE2,ARE2+64,,1

CM,DLOHIPLUS,AREA ! DIAGONAL WEB,DISTURBED,LOW DIAG FLANGE AND HIGH PLUS DIAG FLANGE AREAS

ALLSEL,ALL

ADRAG, hidiag,,,,,,, flandiagminus,,,,,!,

ASEL,S,AREA,,ARE2,ARE2+80,,1

CM,DLOHI,AREA ! DIAGONAL WEB,DISTURBED,LOW AND HIGH DIAG FLANGE AREAS

ALLSEL,ALL 


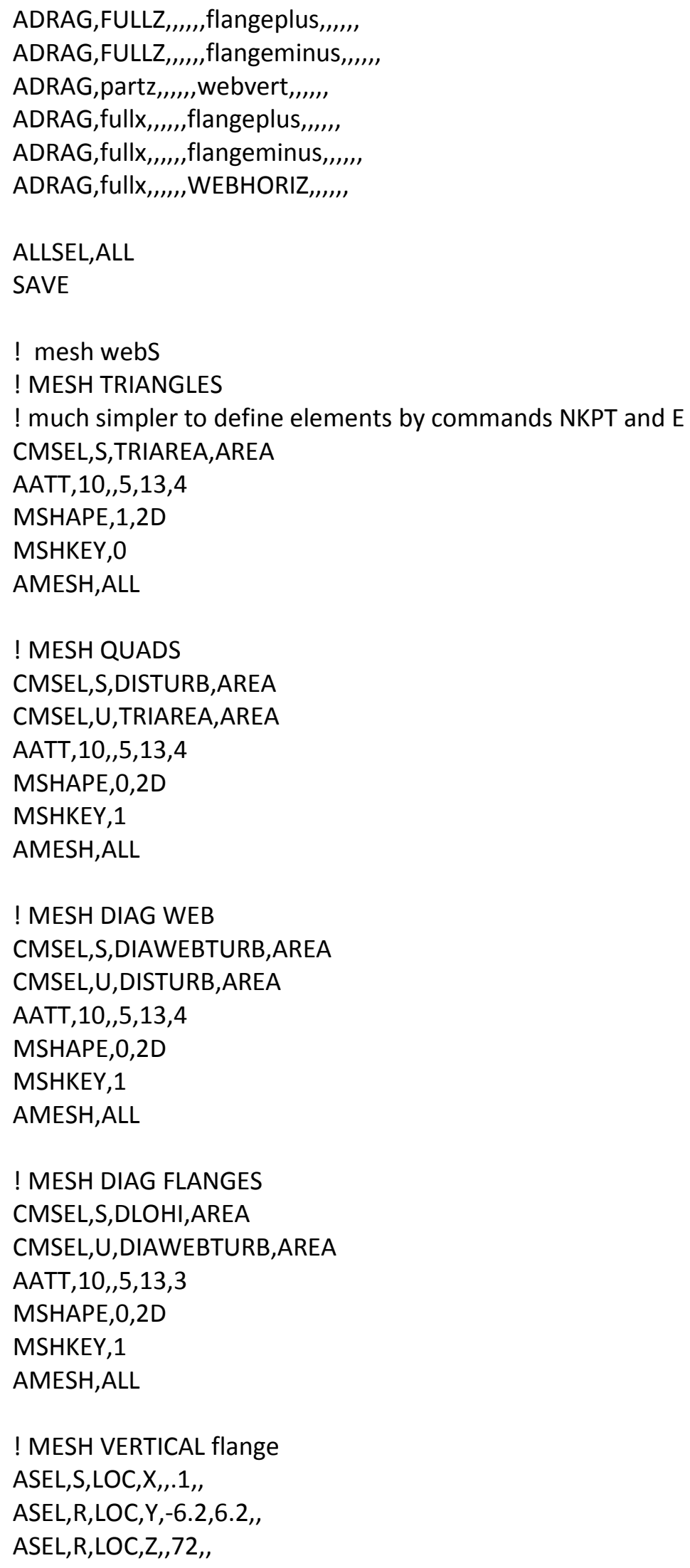




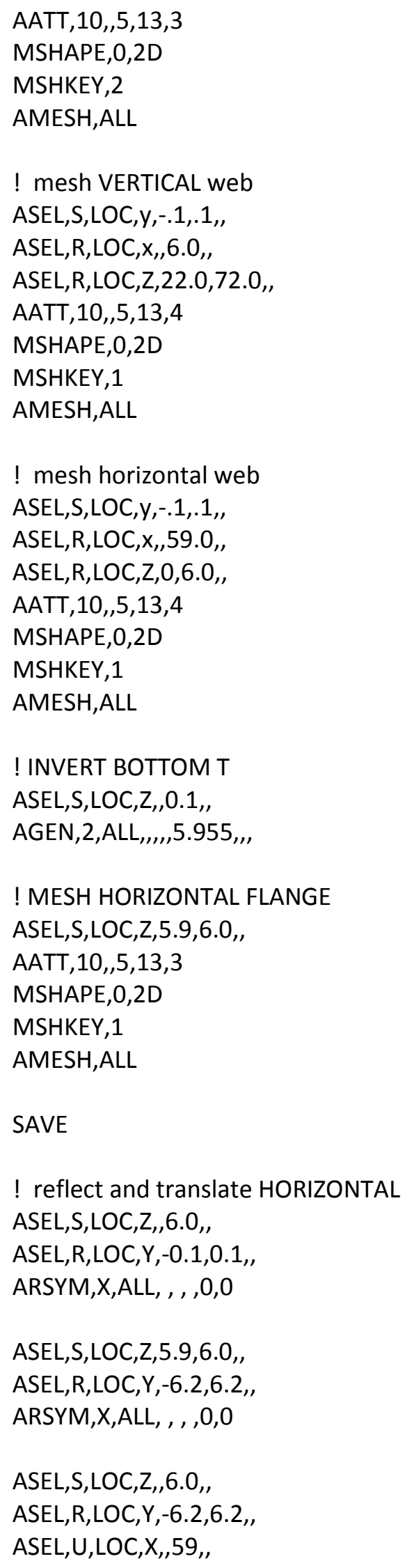




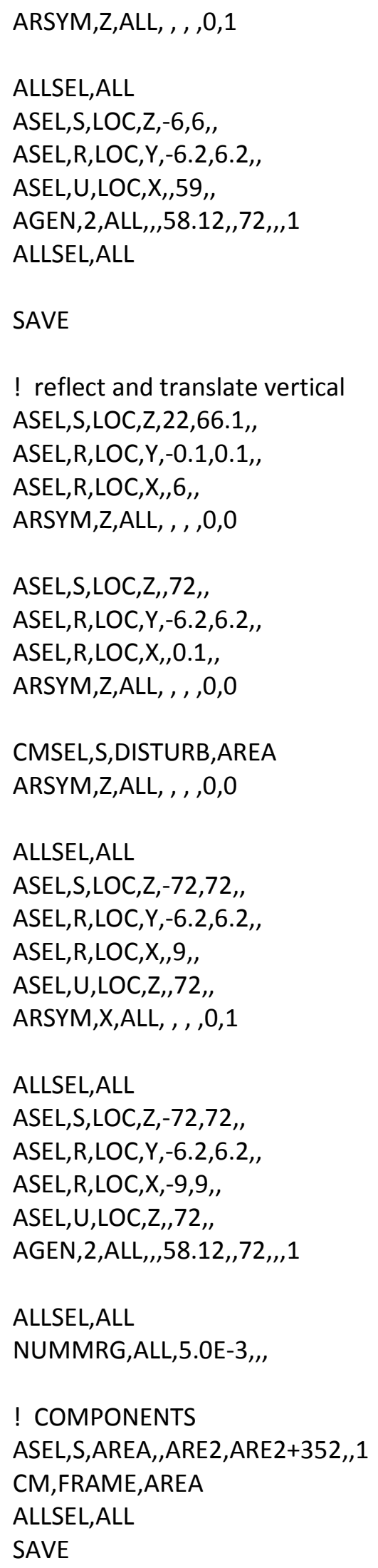


! girder LAST TESTED 9.19.2012

sect,10,shell,,girderweb24W103

secdata, $0.550,10,0,3$

secoffset,MID

seccontrol,0,0,0, 0, 1, 1, 1

sect,11,shell,,girderflange24W103

secdata, $0.980,10,0,3$

secoffset,MID

seccontrol,0,0,0, 0, 1, 1, 1

! USER INPUT: STARTING NUMBERS======================================

*SET,KP4,9400

*SET,LIN4,21700

*SET,ARE4,16300

*SET, VOL4,5000

*SET,ELE4,72400

*SET,NOD4,92100

! \# define model geometry

! user input:local coord system and location of girder

! ref: SHEET 12/15

! Local X ALONG midheight of web.

! Local Y perpendicular to web, right hand system.

! Local Z ALONG left vertical EDGE OF web.

LOCAL,15,0,342,530.28,361, , , ,1,1

CSYS, 15,

LENGTH=186.0 ! half length of girder, must be $>126$ and multiple of 3 inches.

! If length $>186$, user may have to increase element and node starting numbers ! of subsequent components.

!LENGTH $=162.0$

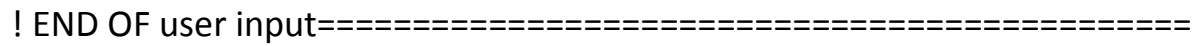

NUMSTR,KP,KP4

NUMSTR,LINE,LIN4

NUMSTR,AREA,ARE4

NUMSTR,VOLU,VOL4

NUMSTR,ELEM,ELE4

NUMSTR,NODE,NOD4

! x horiz midheight of web, halfspan

$\mathrm{K}, \mathrm{KP} 4,$,

K,KP4+1,12.0,

$\mathrm{K}, \mathrm{KP} 4+2,13.0$,

$\mathrm{K}, \mathrm{KP} 4+3,14.0$,

K,KP4+4,16.0, 


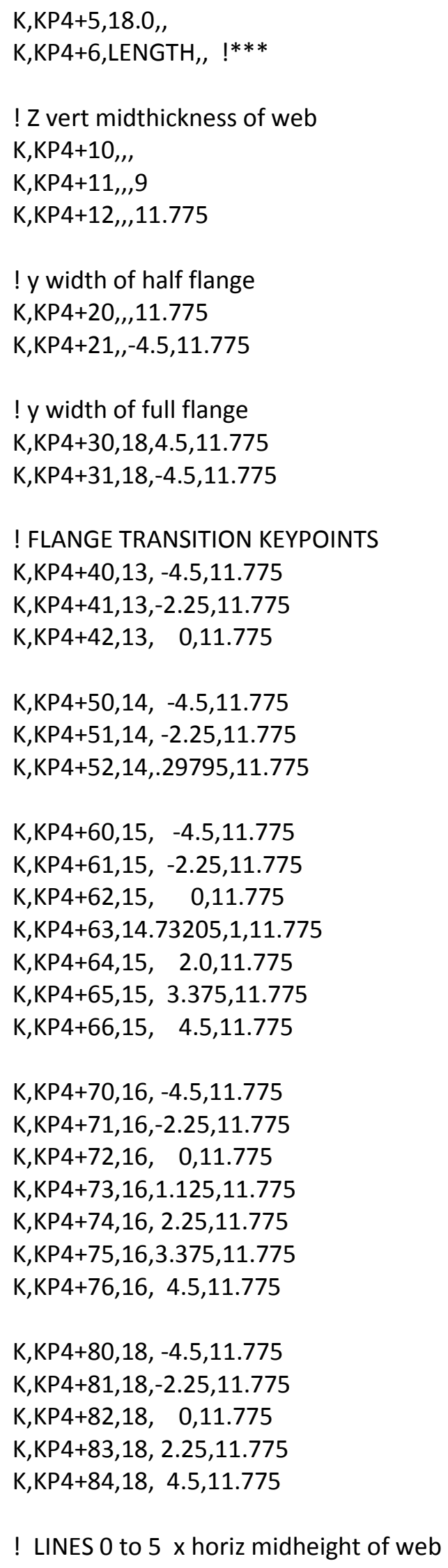


*DO,I,KP4,KP4+5, 1

LSTR,I,I+1

*ENDDO

LESIZE,lin4 ,3.0, , $0,, 0,0$

LESIZE,lin4+1,1.0, , $0,0,0$

LESIZE,lin4+2,1.0, , $0,0,0$

LESIZE,lin4+3,1.0, ,0, ,0 , , ,0

LESIZE, lin4+4,2.0, ,0, ,0 , , ,0

LESIZE, lin4+5,3.0, , 0, , 0 , , ,0

LSEL,S,LINE,,LIN4,LIN4+5,,1

$\mathrm{CM}$, halfspanx, line

ALLSEL,ALL

$\mathrm{KSEL}, \mathrm{S}, \mathrm{KP}$, ,KP4,KP4+2,1,

LSLK,S,1

CM,cutoffx,line

allsel,all

KSEL,S,KP, ,KP4+5,KP4+6,1,

LSLK,S,1

CM,fullwidthx,line

allsel,all

! LINES 6 to $7 \mathrm{z}$ vert midthickness of half web

*DO,I,KP4+10,KP4+11,1

LSTR,I,I+1

*ENDDO

LESIZE,lin4+6,3.0, , 0, , 0, , , 0

LESIZE, lin4+7, $0,1,1,0$, , , 0

KSEL,S,KP, ,KP4+10,KP4+12,1,

LSLK,S,1

CM, webdepthz,line

allsel,all

LSTR,KP4+20,KP4+21 ! L8 y width of half flange

LESIZE, lin4+8,0, , 2, , 0, , , 0

LSEL,S,LINE,,LIN4+8,,,1

$\mathrm{CM}$, halfwidthy, line

allsel,all

LSTR,KP4+30,KP4+31 ! L9 y width of full flange LESIZE, lin4+9,0, , 4, , 0 , , ,0

allsel,all

LSEL,S,LINE,,LIN4+9,,,1

$\mathrm{CM}$, fullwidthy, line 


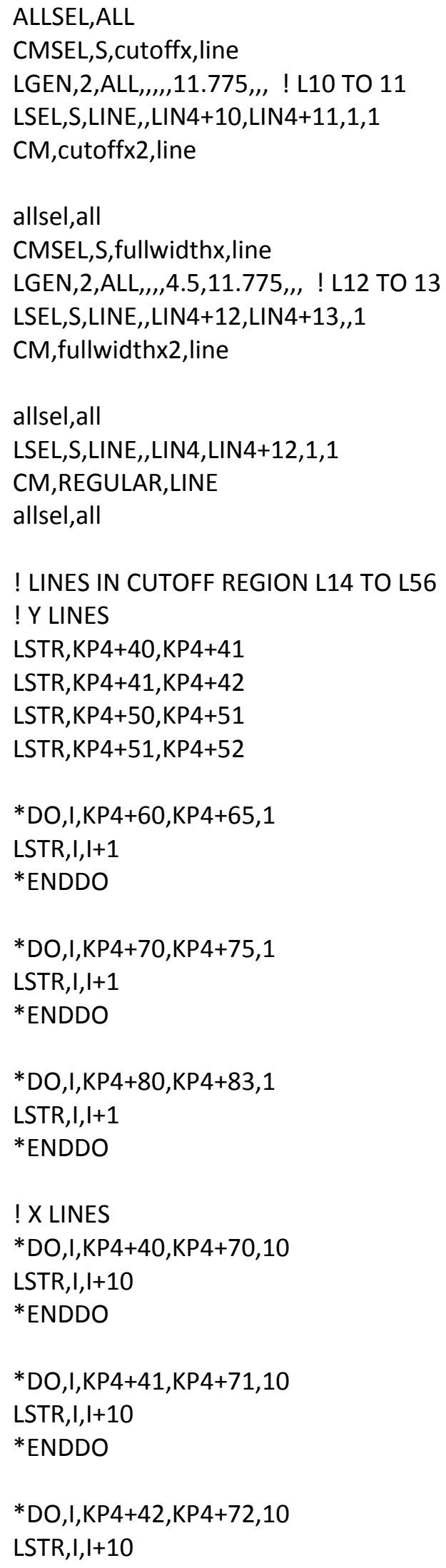


*ENDDO

LSTR,KP4+62,KP4+63

LSTR,KP4+63,KP4+52

LSTR,KP4+63,KP4+73

LSTR,KP4+73,KP4+82

LSTR,KP4+73,KP4+83

LSTR,KP4+64,KP4+74

LSTR, KP4+74,KP4+83

LSTR,KP4+65, KP4+75

LSTR,KP4+75,KP4+83

LSTR,KP4+75,KP4+84

LSTR,KP4+66,KP4+76

LSTR,KP4+76,KP4+84

ALLSEL,ALL

LSEL,S,LINE,,LIN4,LIN4+55,,1

CM,ALLINES, LINES

CMSEL,S,ALLINES,LINE

CMSEL,U,REGULAR,LINE

LESIZE,ALL,0, , 1, , 0 , , , 0

CM,IRREGULAR,LINE

ALLSEL,ALL

! areas

*DO,I,KP4+40,KP4+70,10

A, I,I+10,I+11,I+1

*ENDDO

*DO,I,KP4+41,KP4+71,10

A,I,I+10,I+11,I+1

*ENDDO

$\mathrm{A}, \mathrm{KP} 4+52, \mathrm{KP} 4+62, \mathrm{KP} 4+63$

$\mathrm{A}, \mathrm{KP} 4+62, \mathrm{KP} 4+72, \mathrm{KP} 4+73, \mathrm{KP} 4+63$

$\mathrm{A}, \mathrm{KP} 4+72, \mathrm{KP} 4+82, \mathrm{KP} 4+73$

$\mathrm{A}, \mathrm{KP} 4+73, \mathrm{KP} 4+82, \mathrm{KP} 4+83$

$\mathrm{A}, \mathrm{KP} 4+63, \mathrm{KP} 4+73, \mathrm{KP} 4+74, \mathrm{KP} 4+64$

$\mathrm{A}, \mathrm{KP} 4+73, \mathrm{KP} 4+83, \mathrm{KP} 4+74$

A,KP4+64,KP4+74,KP4+75,KP4+65

$\mathrm{A}, \mathrm{KP} 4+74, \mathrm{KP} 4+83, \mathrm{KP} 4+75$

$\mathrm{A}, \mathrm{KP} 4+75, \mathrm{KP} 4+83, \mathrm{KP} 4+84$

$\mathrm{A}, \mathrm{KP} 4+65, \mathrm{KP} 4+75, \mathrm{KP} 4+76, \mathrm{KP} 4+66$

$\mathrm{A}, \mathrm{KP} 4+75, \mathrm{KP} 4+84, \mathrm{KP} 4+76$

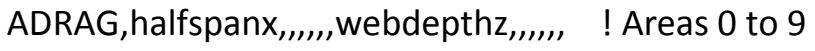


ADRAG,cutoff $x 2,, \ldots,$, , halfwidthy, $, \ldots, \ldots$, ! Areas 10 to 11

ADRAG, fullwidth $x 2,, \ldots,,$, fullwidthy,,,,,, ! Areas 12 to 13

! MESH WEB

ASEL,S,LOC,Y,-0.1,0.1,1

ASEL,R,LOC,Z,0,11.7,"

AATT,10, 5, 15,10

MSHAPE,0,2D

MSHKEY,1

AMESH,ALL

ALLSEL,ALL

! MESH FLANGE

ASEL,S,LOC,Z,11.7,11.8,,1

ASEL,U,LOC,X,13,18,,1

AATT, 10, 5, 15,11

MSHAPE,0,2D

MSHKEY,1

AMESH,ALL

ALLSEL,ALL

! MESH FLANGE TRANSITION

ASEL,S,LOC,Z,11.7,11.8,,1

ASEL,R,LOC,X,13,18,,1

AATT, 10, 5, 15,11

MSHKEY,2

AMESH,ALL

ALLSEL,ALL

ASEL,S,AREA,,ARE4,ARE4+33,,1

! reflect AND TRANSLATE

ARSYM,Z,ALL, , , ,0,0 ! from quarter to half

ALLSEL,ALL

ASEL,S,AREA,,ARE4,ARE4+67,,1

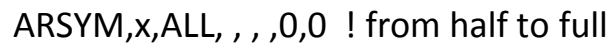

ALLSEL,ALL

!ASEL,S,LOC, $x,-186,0,1$

ASEL,S,AREA,,ARE4+68,ARE4+135,,1 ! move new half to other end $\mathrm{L}=\mathrm{LENGTH}{ }^{*} 2$ !***

AGEN,2,ALL,,,L,,,,,,1 ! !***

ALLSEL,ALL

! girder assembly A

! y distance between 2 beams is $12.89+0.55=13.44$ 
! new beam is between $y=-13.44+-4.5$

! if spacing between beams changes, these values must change

ASEL,S,AREA,,ARE4,ARE4+135,,1

AGEN,2,ALL,,,0,-13.44,,,,0 ! double beam

ASEL,S,LOC,y,-18,-8.9,,1 ! reflect

ARSYM,Y,ALL, , , , 0,1

ALLSEL,ALL

! end of generation of 2 nd beam and possible user input

NUMMRG,ALL

! CONSTRAIN TOP OF WEB WITH BEGINNING OF FULL FLANGE.

! moving or duplicating girder by AGEN may be difficult because of constraints.

! best to move or duplicate girder without constraints, then reapply constraints at new location.

$\mathrm{N} 1=\operatorname{NODE}(14,0,11.775)$

$\mathrm{N} 2=\operatorname{NODE}(14, .29795,11.775)$

CP,NEXT,ALL,N1,N2

$\mathrm{N} 3=\operatorname{NODE}(14,0,-11.775)$

N4 $=\operatorname{NODE}(14, .29795,-11.775)$

CP,NEXT,ALL,N3,N4

L1=LENGTH*2-14

N5 $=\operatorname{NODE}(L 1,0,11.775)$

N6 $=\operatorname{NODE}(\operatorname{L1}, .29795,11.775)$

CP,NEXT,ALL,N5,N6

N7 $=\operatorname{NODE}(\operatorname{L1}, 0,-11.775)$

$\mathrm{N} 8=\operatorname{NODE}(\mathrm{L} 1, .29795,-11.775)$

CP,NEXT,ALL,N7,N8

! y location of couplings below depends on spacing between 2 beams

! 13.44-.29795 = 13.14205

$\mathrm{N} 9=\operatorname{NODE}(14,13.44,11.775)$

$\mathrm{N} 10=\operatorname{NODE}(14,13.14205,11.775)$

CP,NEXT,ALL,N9,N10

$\mathrm{N} 11=\operatorname{NODE}(14,13.44,-11.775)$

$\mathrm{N} 12=\operatorname{NODE}(14,13.14205,-11.775)$

CP,NEXT,ALL,N11,N12

$\mathrm{N} 13=\operatorname{NODE}(\mathrm{L} 1,13.44,11.775)$

N14 $=$ NODE $(L 1,13.14205,11.775)$ 
CP,NEXT,ALL,N13,N14

$\mathrm{N} 15=\operatorname{NODE}(\mathrm{L} 1,13.44,-11.775)$

$\mathrm{N} 16=\mathrm{NODE}(\mathrm{L} 1,13.14205,-11.775)$

CP,NEXT,ALL,N15,N16 ! set 48 last

ALLSEL,ALL

ASEL,S,AREA,,ARE4,ARE4+271,,1

CM,GIRDER,AREA

ALLSEL,ALL

SAVE

! girder spacer

ET,6,BEAM188

SECTYPE, 15, BEAM, CTUBE, SCH80, 0

SECOFFSET, CENT

SECDATA, $0.75,0.95,0,0,0,0,0,0,0,0$ ! WHERE IS MAT DEFINED?

! USER INPUT: STARTING NUMBERS====================================="

*SET,KP6,10100

*SET,LIN6,22800

*SET,ARE6,16800

*SET, VOL6,5000

*SET,ELE6,79100

*SET,NOD6,101200

! \# define model geometry

! user input:local coord system and location of girder

! ref: SHEET 14/15

! Local X ALONG flange.

! Local Y along web.

! Local Z ALONG length of spacer.

! origin at midthickness JUNCTION WEB-FLANGE.

LOCAL,17,0,108,519.05,350.3, , , ,1,1

CSYS,17,

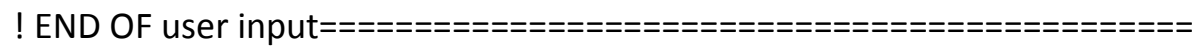

NUMSTR,KP,KP6

NUMSTR,LINE,LIN6

NUMSTR,AREA,ARE6

NUMSTR, VOLU,VOL6

NUMSTR,ELEM,ELE6

NUMSTR,NODE,NOD6 


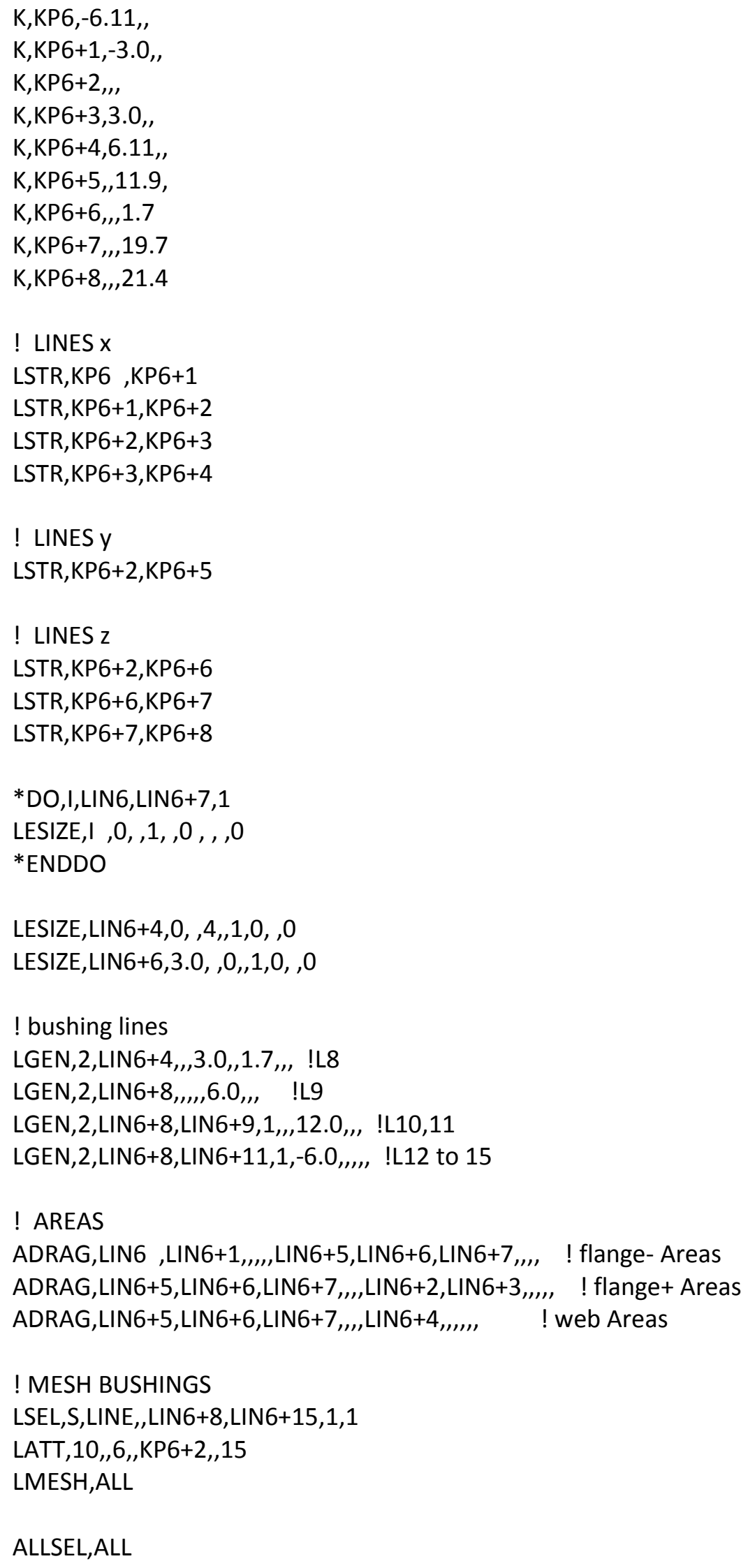




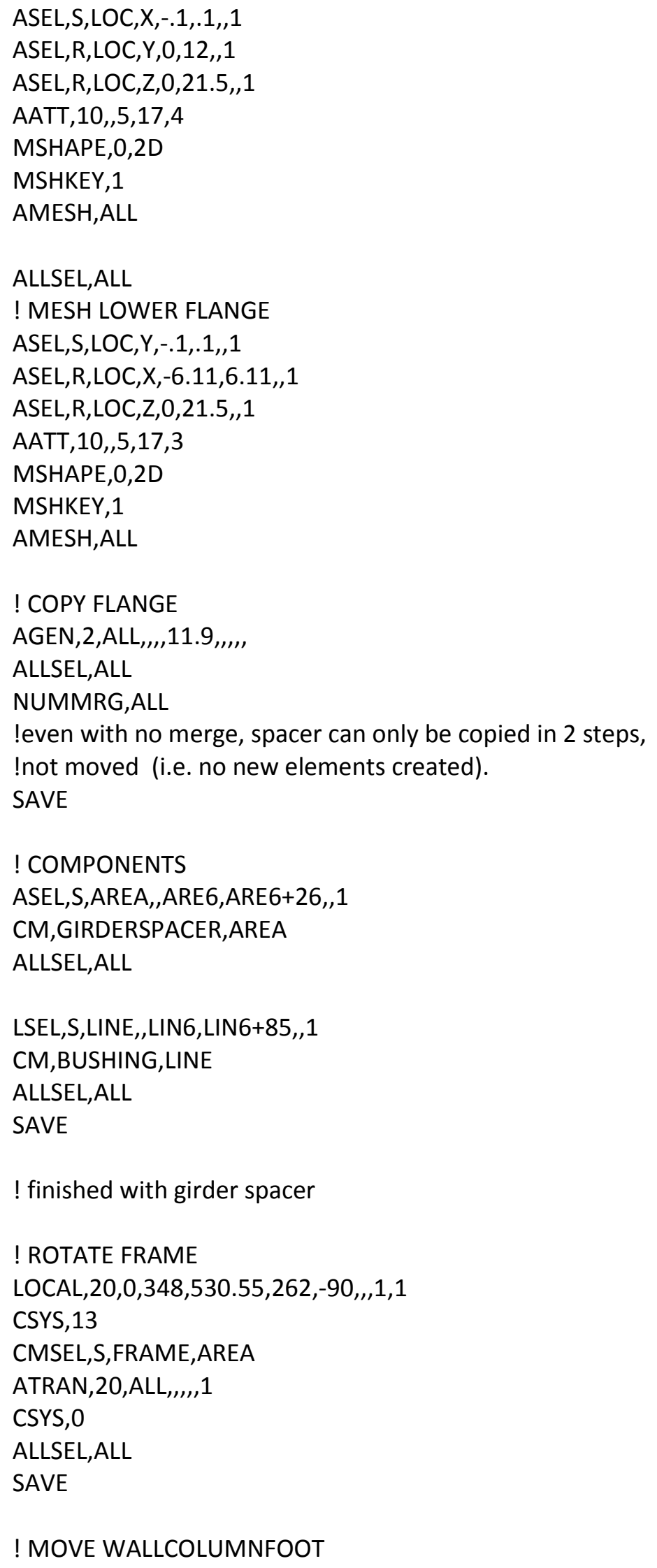


CMSEL,S,WALLCOLUMNFOOT,AREA

AGEN,2,ALL,,,0,-100.0,0,,,1

ALLSEL,ALL

SAVE

! COPY COLUMNFOOT

*SET, KP7, 10200

*SET,LIN7,22900

*SET,ARE7,16900

*SET, VOL7,5000

*SET,ELE7,79300

*SET,NOD7,101500

NUMSTR,KP,KP7

NUMSTR,LINE,LIN7

NUMSTR,AREA,ARE7

NUMSTR, VOLU,VOL7

NUMSTR,ELEM,ELE7

NUMSTR,NODE,NOD7

CSYS, 0

CMSEL,S,COLUMNFOOT,AREA

AGEN,2,ALL,,,0,71.02,0,,,

ALLSEL,ALL

! components

ASEL,S,AREA,,ARE7,ARE7+67,,1

CM,COLUMNFOOT2,AREA

ALLSEL,ALL

SAVE

! COPY SPACER 1

*SET,KP8, 10300

*SET,LIN8,23100

*SET,ARE8, 17000

*SET, VOL8,5000

*SET,ELE8,80450

*SET,NOD8,102750

NUMSTR,KP,KP8

NUMSTR,LINE,LIN8

NUMSTR,AREA,ARE8

NUMSTR, VOLU,VOL8

NUMSTR,ELEM,ELE8

NUMSTR,NODE,NOD8

CMSEL,S,BUSHING,LINE 
LGEN,2,ALL,,,360,12,0,,,0

ALLSEL,ALL

CMSEL,S,GIRDERSPACER,AREA

AGEN,2,ALL,,,360,12,0,,,0

ALLSEL,ALL

NUMMRG,ALL

! COMPONENTS

LSEL,S,LINE,,LIN8,LIN8+85,,1

CM,BUSHING1,LINE

ALLSEL,ALL

ASEL,S,AREA,,ARE8,ARE8+26,,1 CM,GIRDERSPACER1,AREA

ALLSEL,ALL

SAVE

! copy SPACER 2

*SET,KP9,10400

*SET,LIN9,23250

*SET,ARE9,17050

*SET, VOL9,5000

*SET,ELE9,81000

*SET,NOD9,103000

NUMSTR,KP,KP9

NUMSTR,LINE,LIN9

NUMSTR,AREA,ARE9

NUMSTR,VOLU,VOL9

NUMSTR,ELEM,ELE9

NUMSTR,NODE,NOD9

CMSEL,S,BUSHING,LINE

LGEN,2,ALL,,,480,12,0,,,0

ALLSEL,ALL

CMSEL,S,GIRDERSPACER,AREA

AGEN,2,ALL,,,480,12,0,,,0

ALLSEL,ALL

NUMMRG,ALL

! COMPONENTS

LSEL,S,LINE,,LIN9,LIN9+85,,1

CM,BUSHING2,LINE

ALLSEL,ALL

ASEL,S,AREA,,ARE9,ARE9+26,,1 


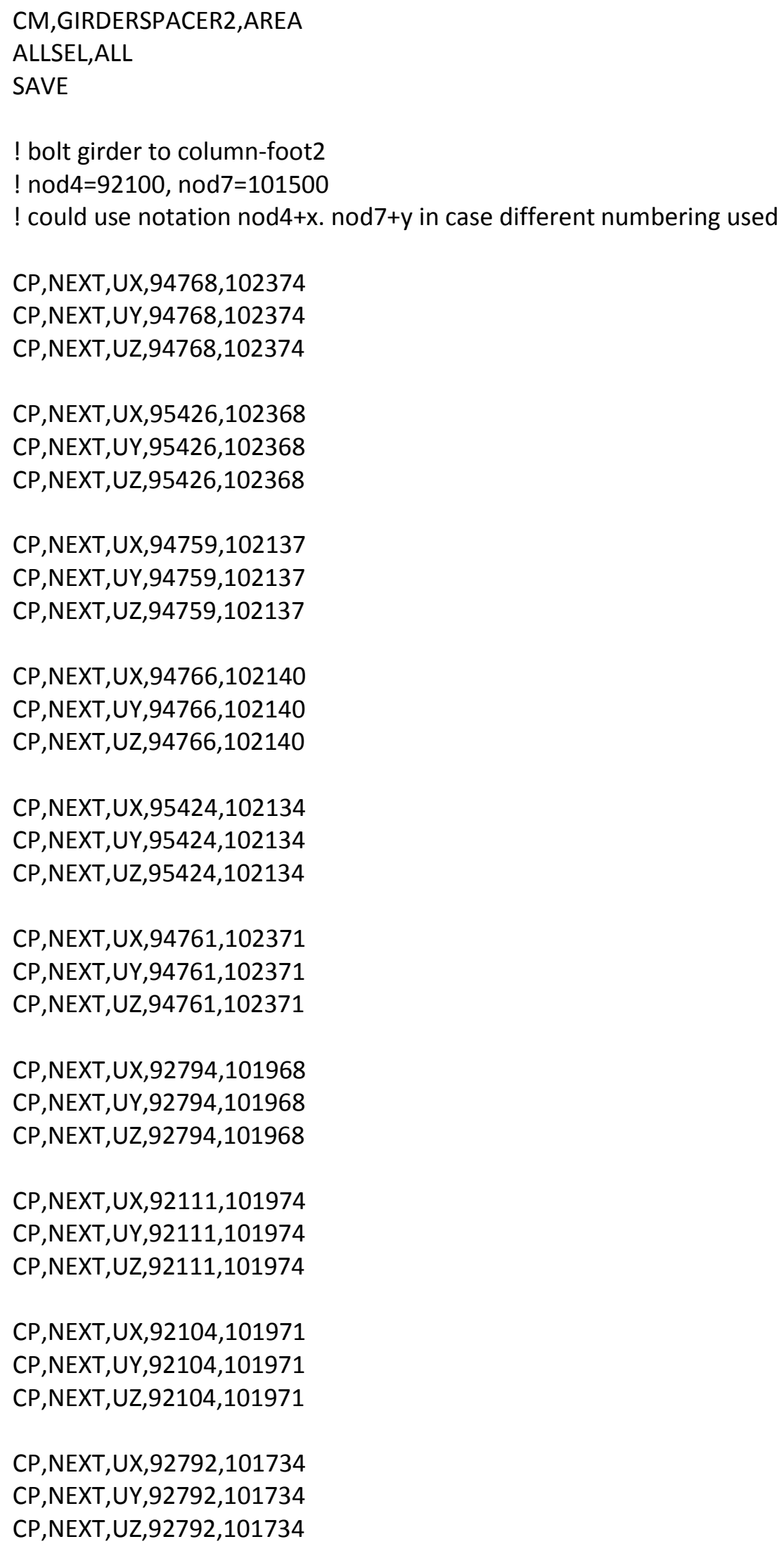




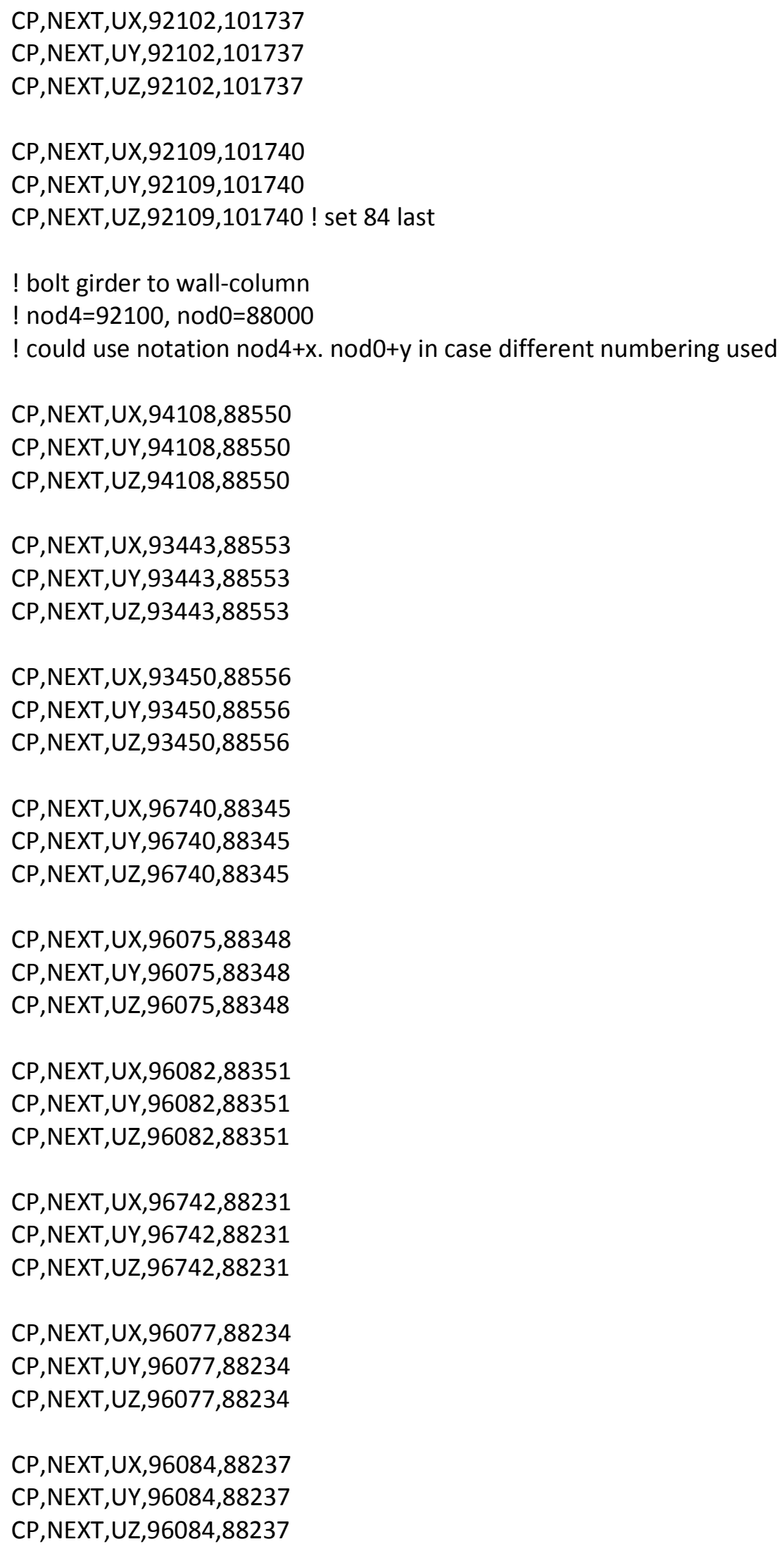




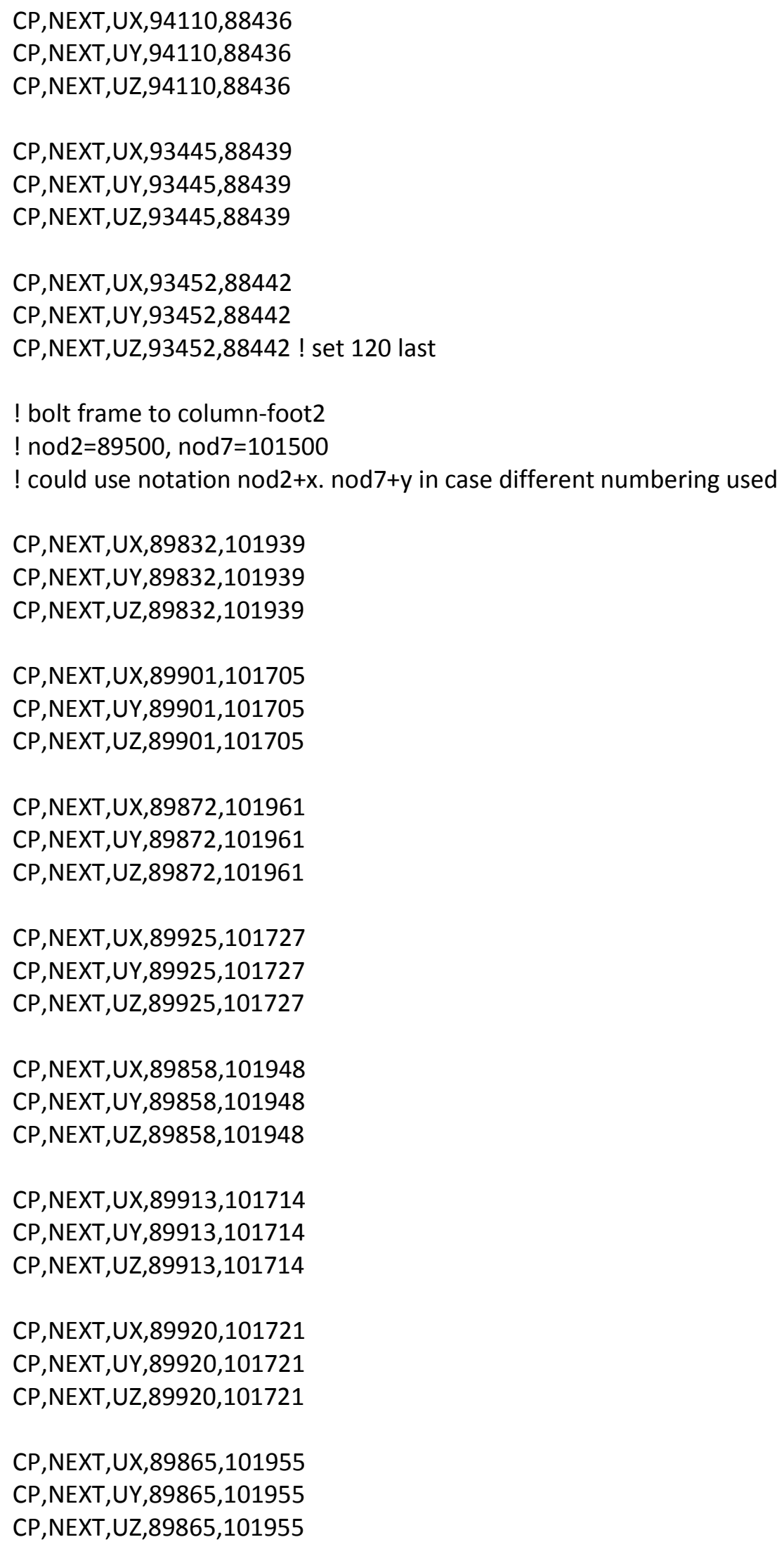




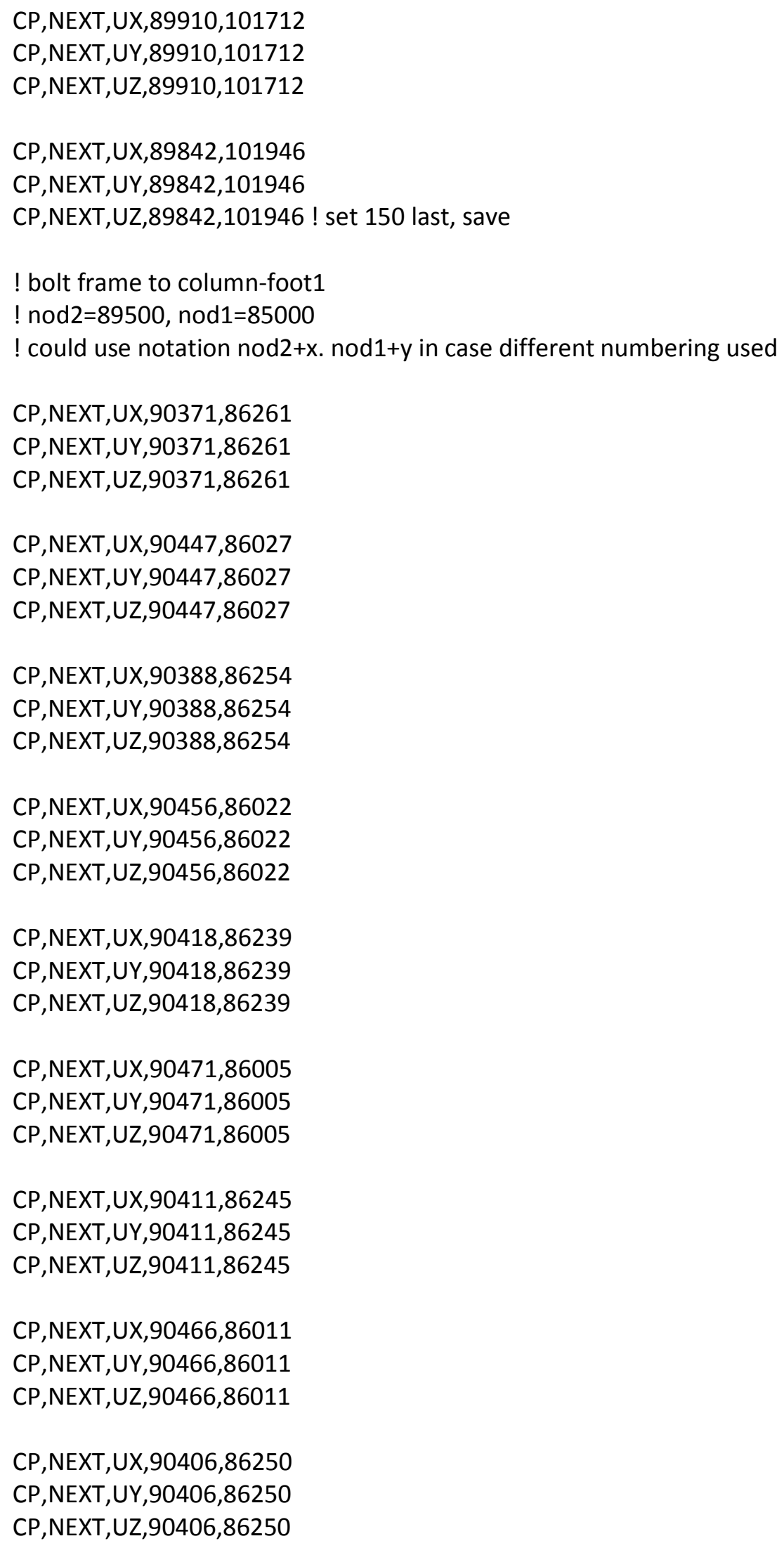




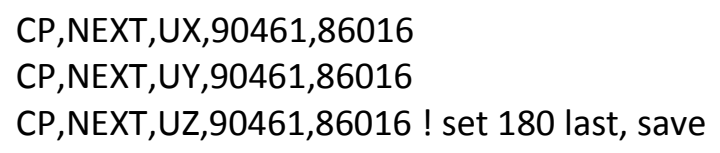


CP,NEXT,UX,93574,103027

CP,NEXT,UY,93574,103027

CP,NEXT,UZ,93574,103027

CP,NEXT,UX,96864,103001

CP,NEXT,UY,96864,103001

CP,NEXT,UZ,96864,103001

CP,NEXT,UX,94232,103000

CP,NEXT,UY, 94232,103000

CP,NEXT,UZ,94232,103000

CP,NEXT,UX,96208,103064

CP,NEXT,UY, 96208,103064

CP,NEXT,UZ,96208,103064

CP,NEXT,UX,96866,103037

CP,NEXT,UY, 96866,103037

CP,NEXT,UZ,96866,103037

CP,NEXT,UX,93576,103063

CP,NEXT,UY,93576,103063

CP,NEXT,UZ,93576,103063

CP,NEXT,UX,94234,103036

CP,NEXT,UY,94234,103036

CP,NEXT,UZ,94234,103036 ! set 228 last, save

! BOLT foot 2 to strong floor

! Nod7=101500 could be used in notation

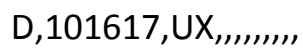

$\mathrm{D}, 101617, \mathrm{UY},, \ldots, \ldots, \ldots$,

D,101617,UZ,,,,,,,,,

D,101635,UX,,,,,,,,!,

D, 101635,UY,,,,, , , ,

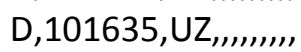

D,101529,UX,,,,,,,,",

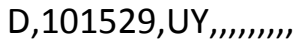

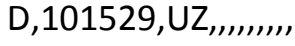

D,101503,UX,,,, , , , , ,

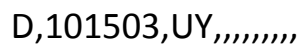

D,101503,UZ,,,,,,,,!,

! BOLT foot 1 to strong floor 
! Nod1 $1=85000$ could be used in notation

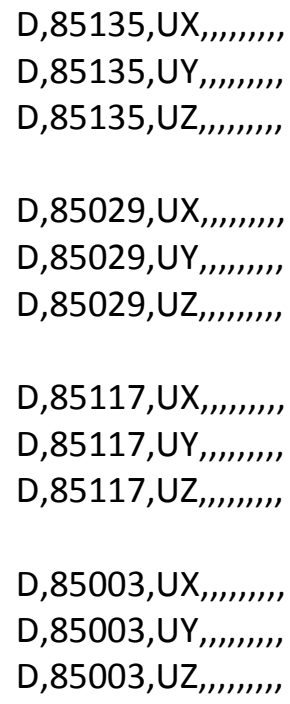

! bolt pad 1 to strong wall

! Nod $0=88000$ could be used in notation

$\mathrm{D}, 88683, \mathrm{UX}, \ldots, \ldots, \ldots, \ldots$

$\mathrm{D}, 88683, \mathrm{UY},,,,,,,,$,

$\mathrm{D}, 88683, \mathrm{UZ}, \ldots, \ldots, \ldots, \ldots$,

$D, 88657, U X, \ldots, \ldots, \ldots, \ldots$

$\mathrm{D}, 88657, \mathrm{UY}, \ldots, \ldots, \ldots, \ldots$

$\mathrm{D}, 88657, \mathrm{UZ}, \ldots, \ldots, \ldots, \ldots$

$\mathrm{D}, 88693, \mathrm{UX}, \ldots, \ldots, \ldots, \ldots$,

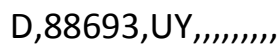

$\mathrm{D}, 88693, \mathrm{UZ}, \ldots, \ldots, \ldots, \ldots$

$\mathrm{D}, 88765, \mathrm{UX},, \ldots, \ldots, \ldots$,

$\mathrm{D}, 88765, \mathrm{UY},, \ldots, \ldots, \ldots$,

$\mathrm{D}, 88765, \mathrm{UZ}, \ldots, \ldots, \ldots, \ldots$

! bolt pad 2 to strong wall

! Nod $0=88000$ could be used in notation

$\mathrm{D}, 88930, \mathrm{UX}, \ldots, \ldots, \ldots, \ldots$

$\mathrm{D}, 88930, \mathrm{UY},,, \ldots, \ldots,,$,

$\mathrm{D}, 88930, \mathrm{UZ},,,, \ldots, \ldots,$,

$\mathrm{D}, 89012, \mathrm{UX},, \ldots, \ldots,,,$,

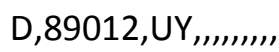

$\mathrm{D}, 89012, \mathrm{UZ}, \ldots, \ldots, \ldots, \ldots$, 


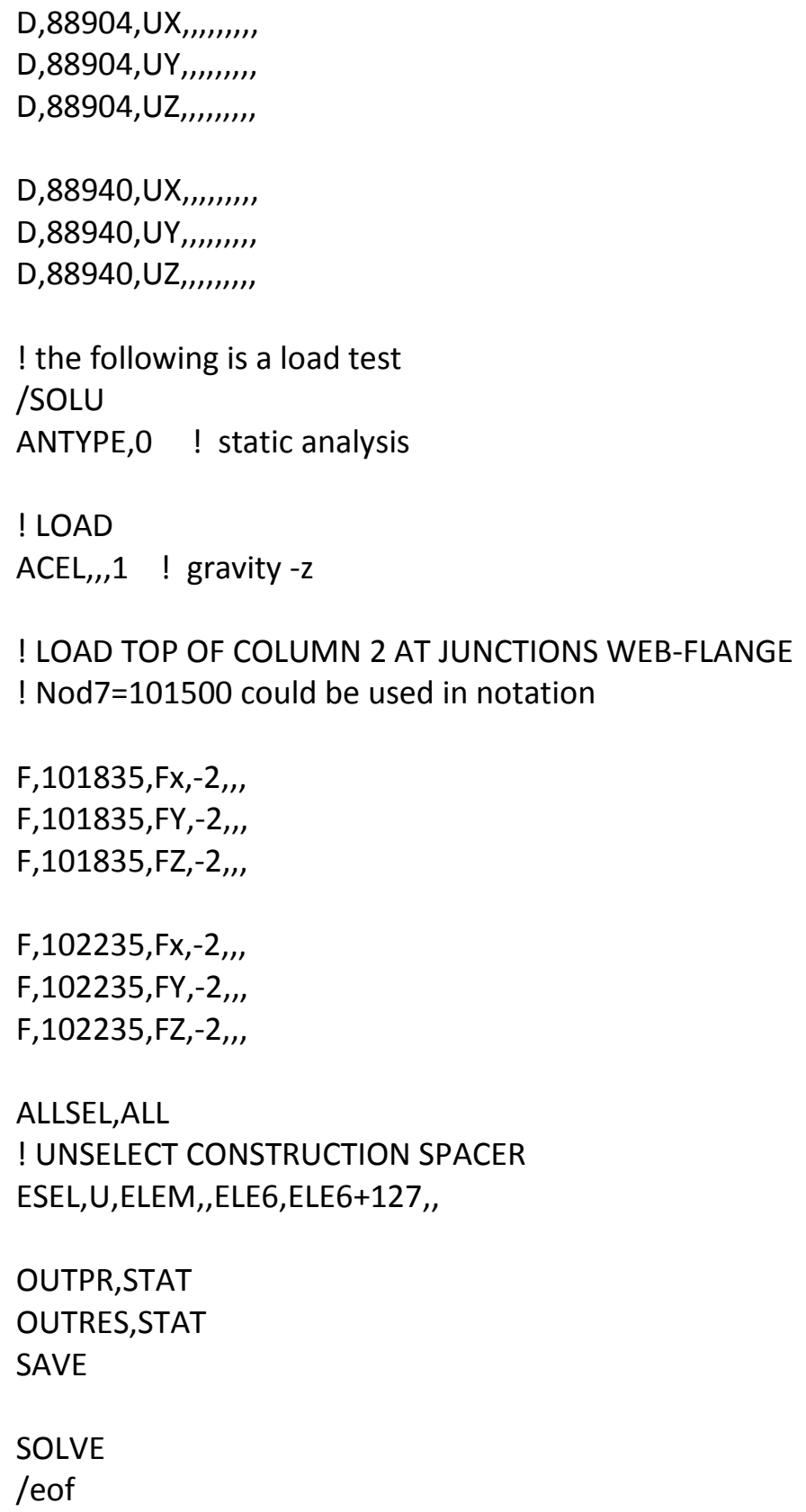




\section{Chapter 14 Assembling the Support and the Concrete Structures}

To assemble the modular support structure (and potential future experimental structures) inside the concrete structure with proper connections and restraints, the same procedure is used as in Chapter 13.

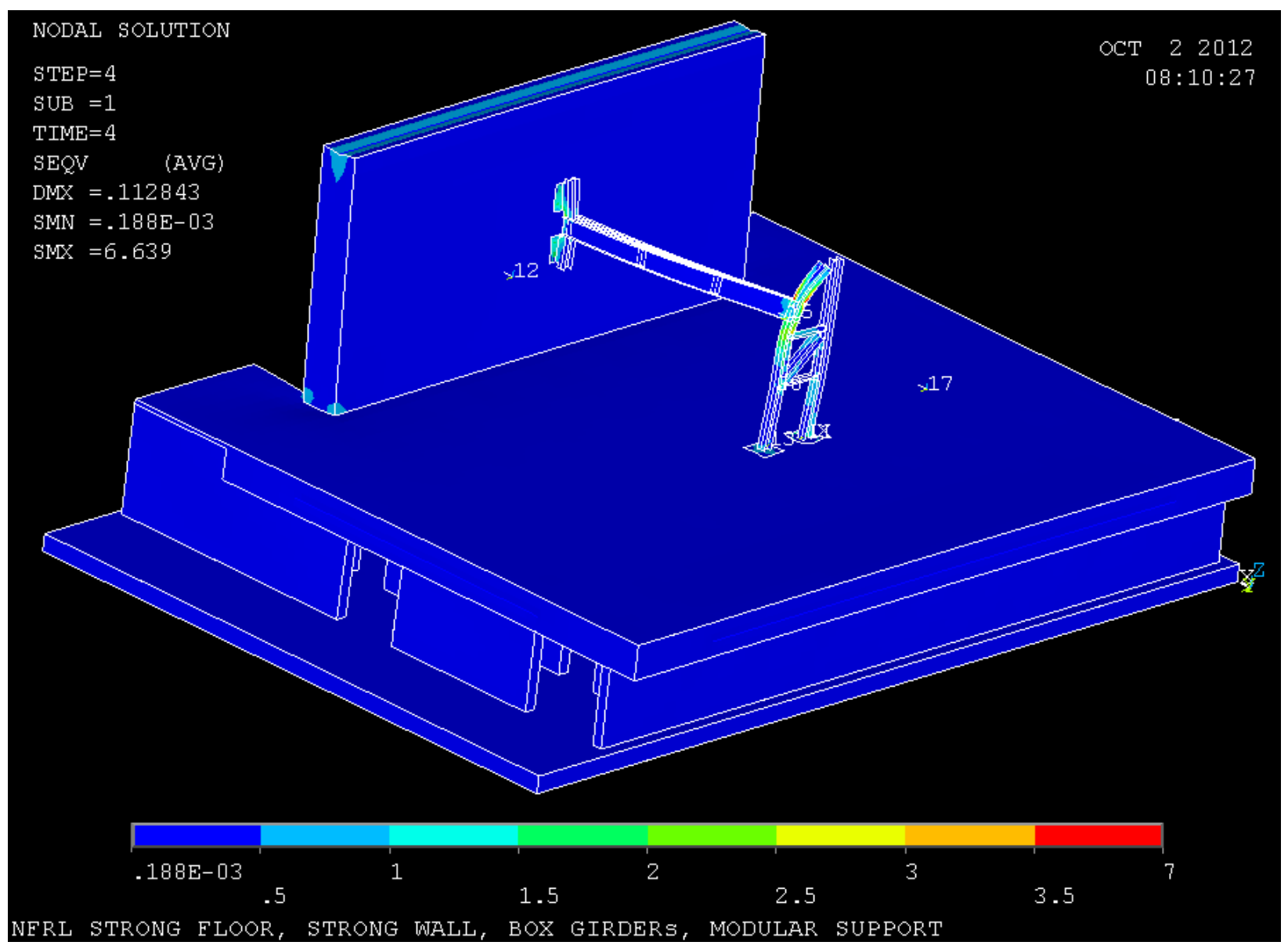

Fig. 14.1 Deformations and von Mises stress. Reaction is at base of slab. 


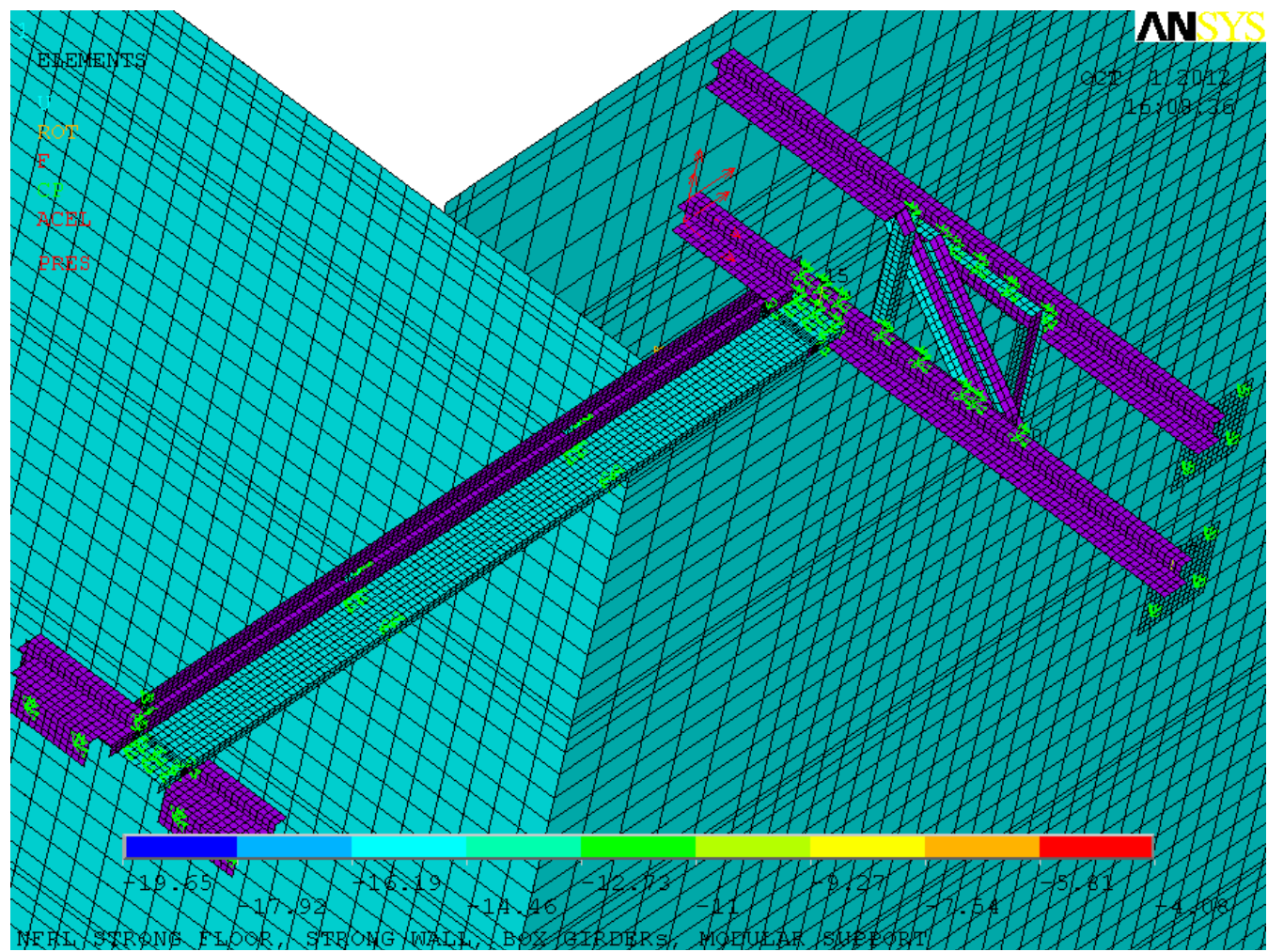

Fig. 14.2 Bolting the support structure together, to the strong floor and strong wall

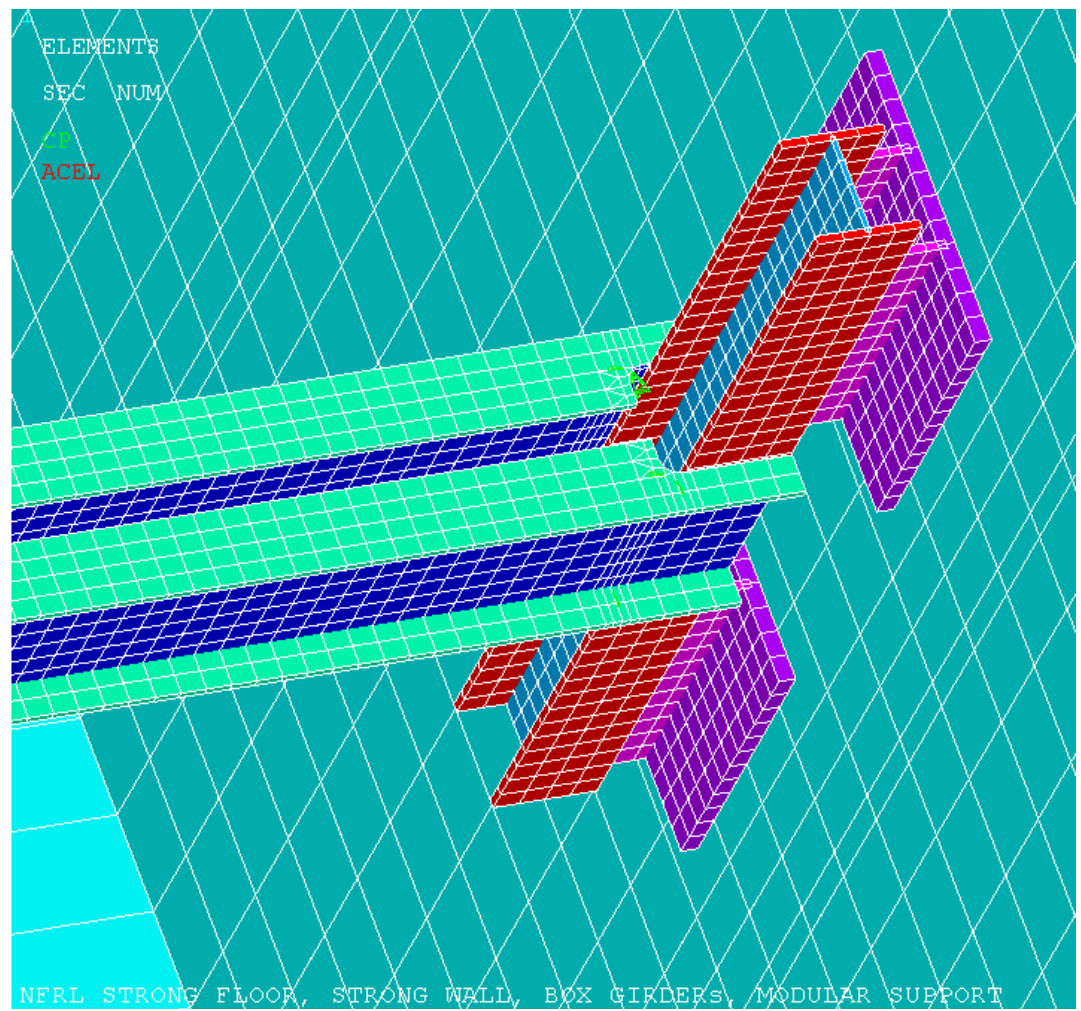

Fig. 14.3 Girder and wall-column at strong wall 


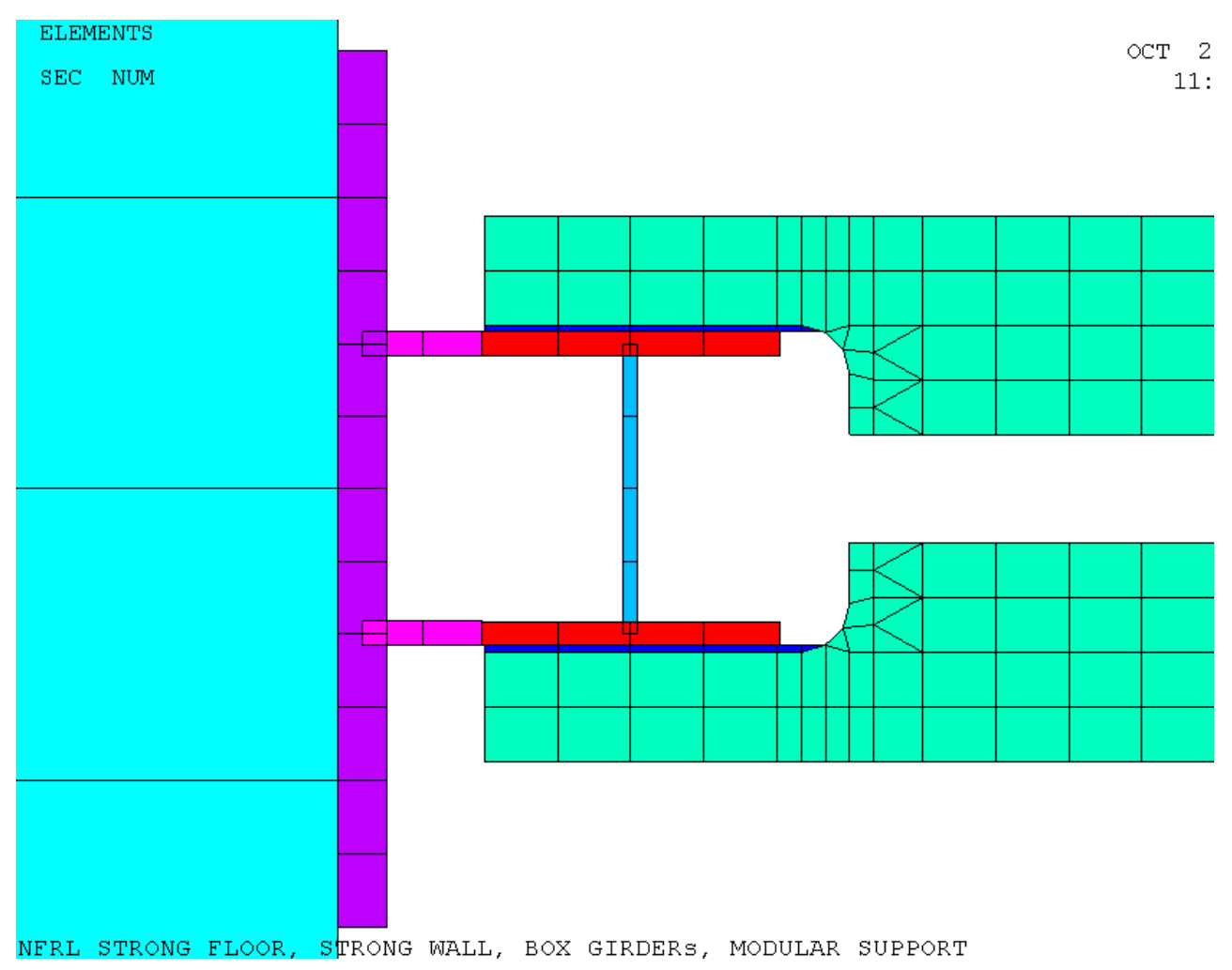

Fig. 14.4 Fitting wall-column to strong wall

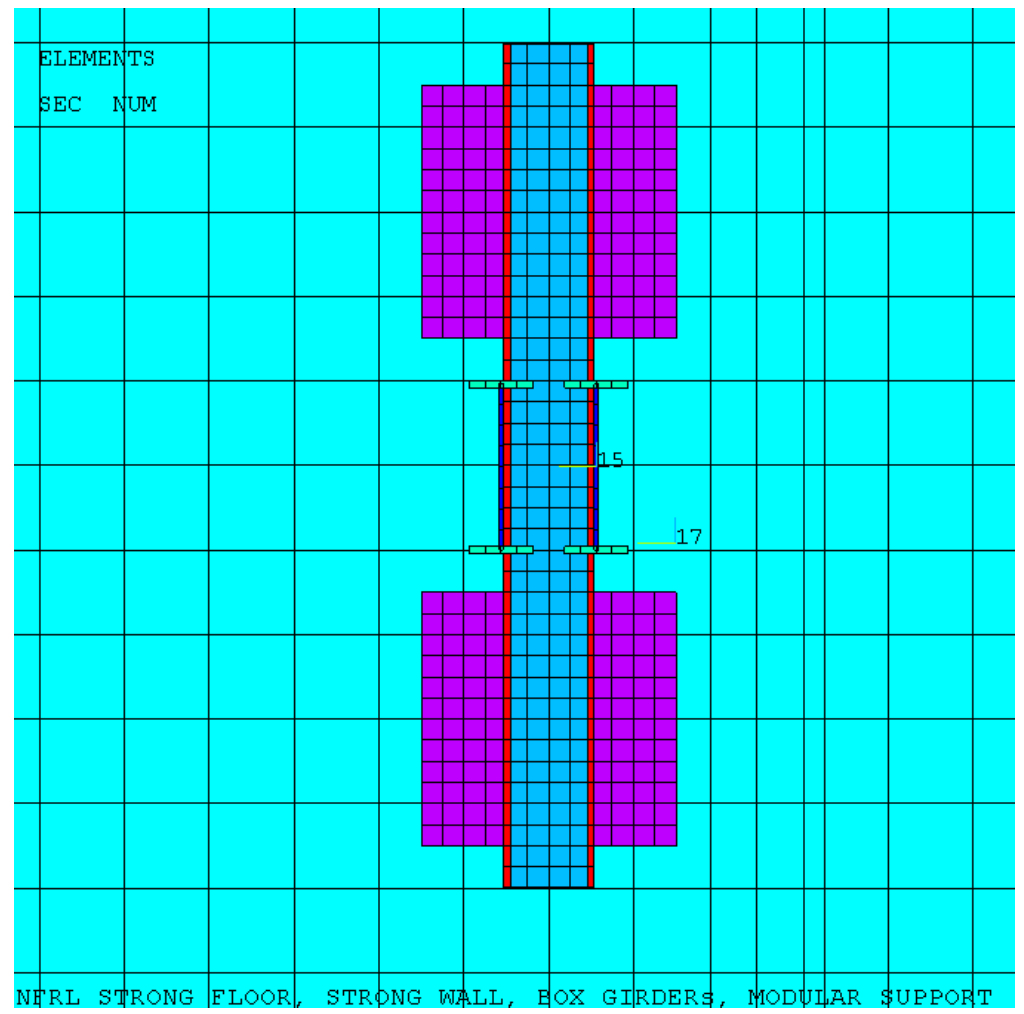

Fig. 14.5 Bolting wall-column to strong wall 


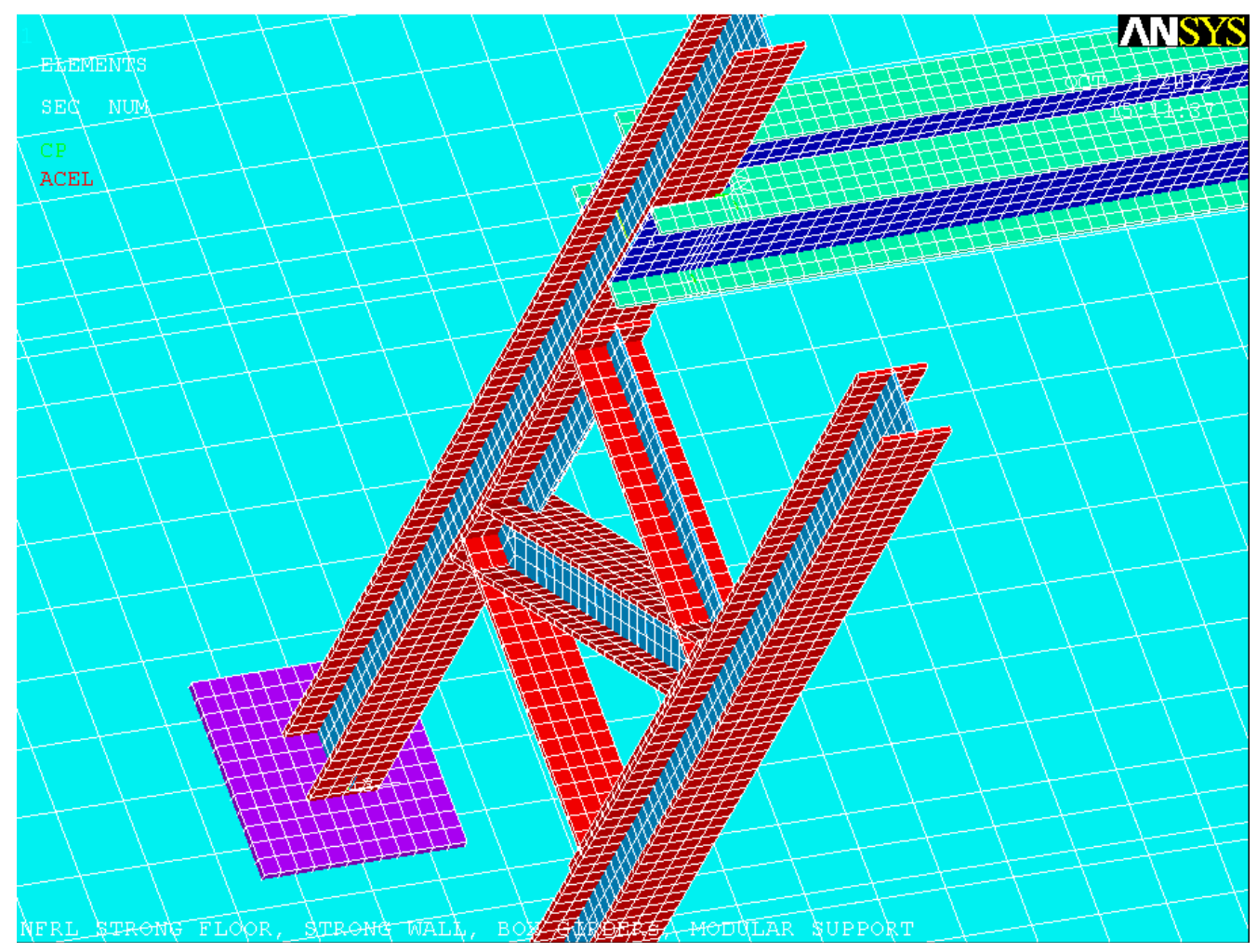

Fig. 14.6 Girder, column-foot and frame

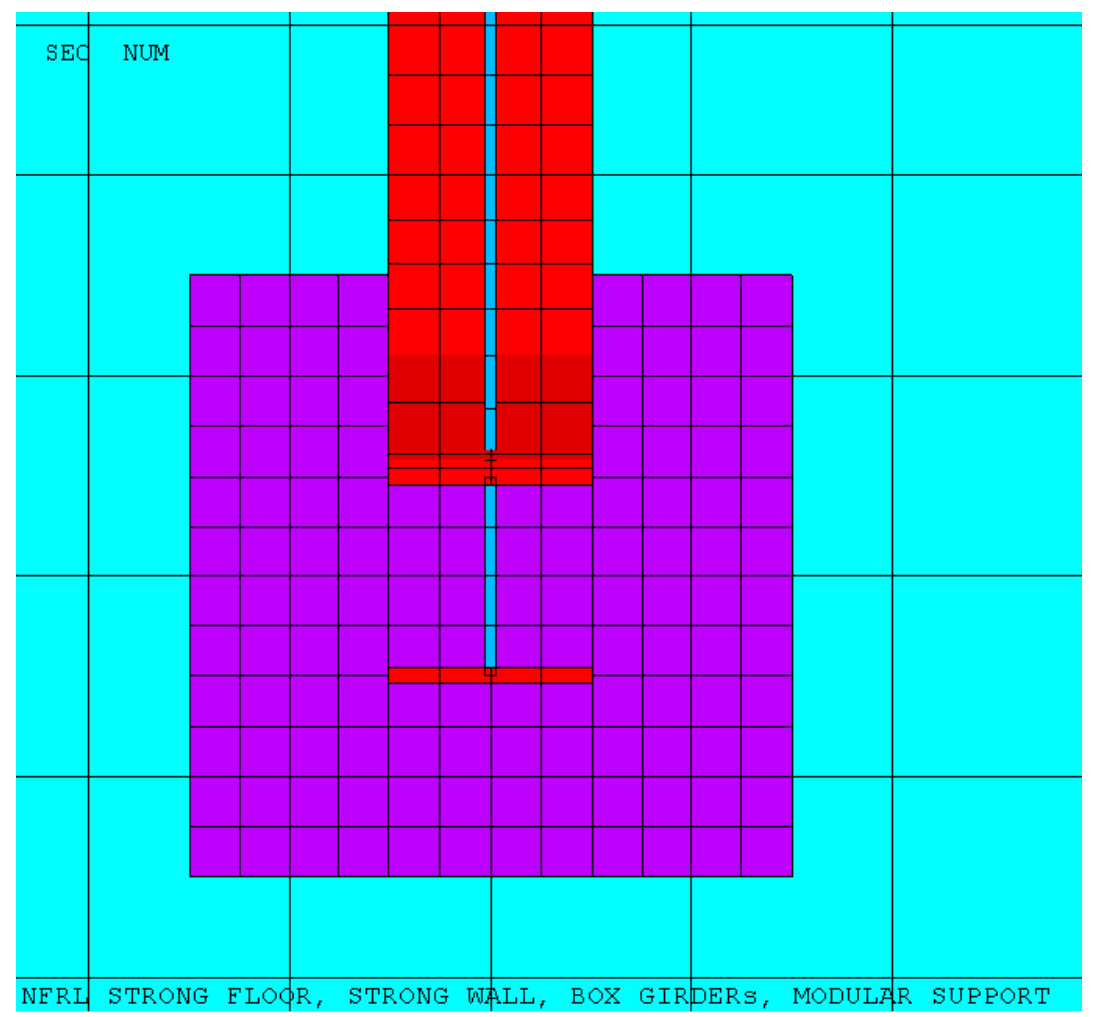

Fig. 14.7 Fitting foot 1 onto strong floor 


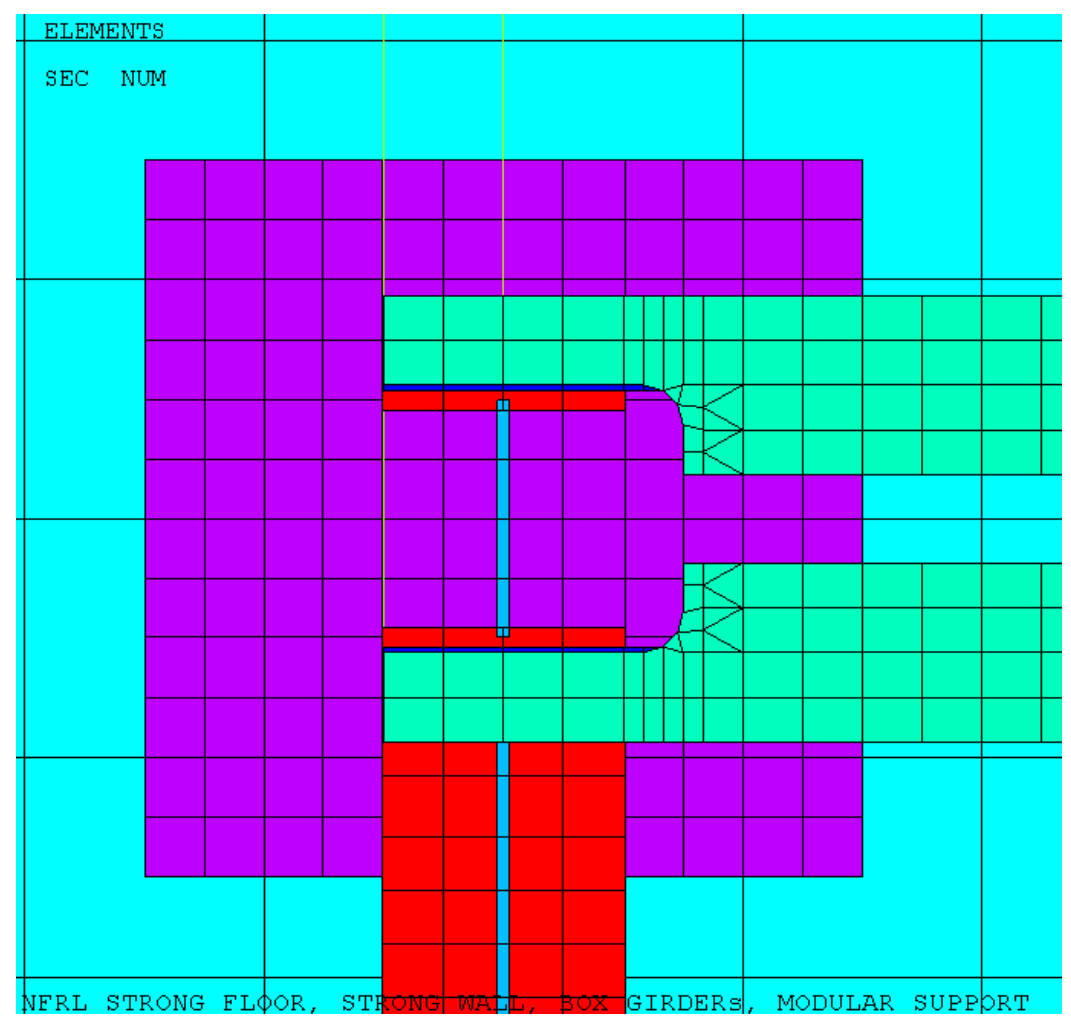

Fig. 14.8 Fitting foot 2 onto strong floor

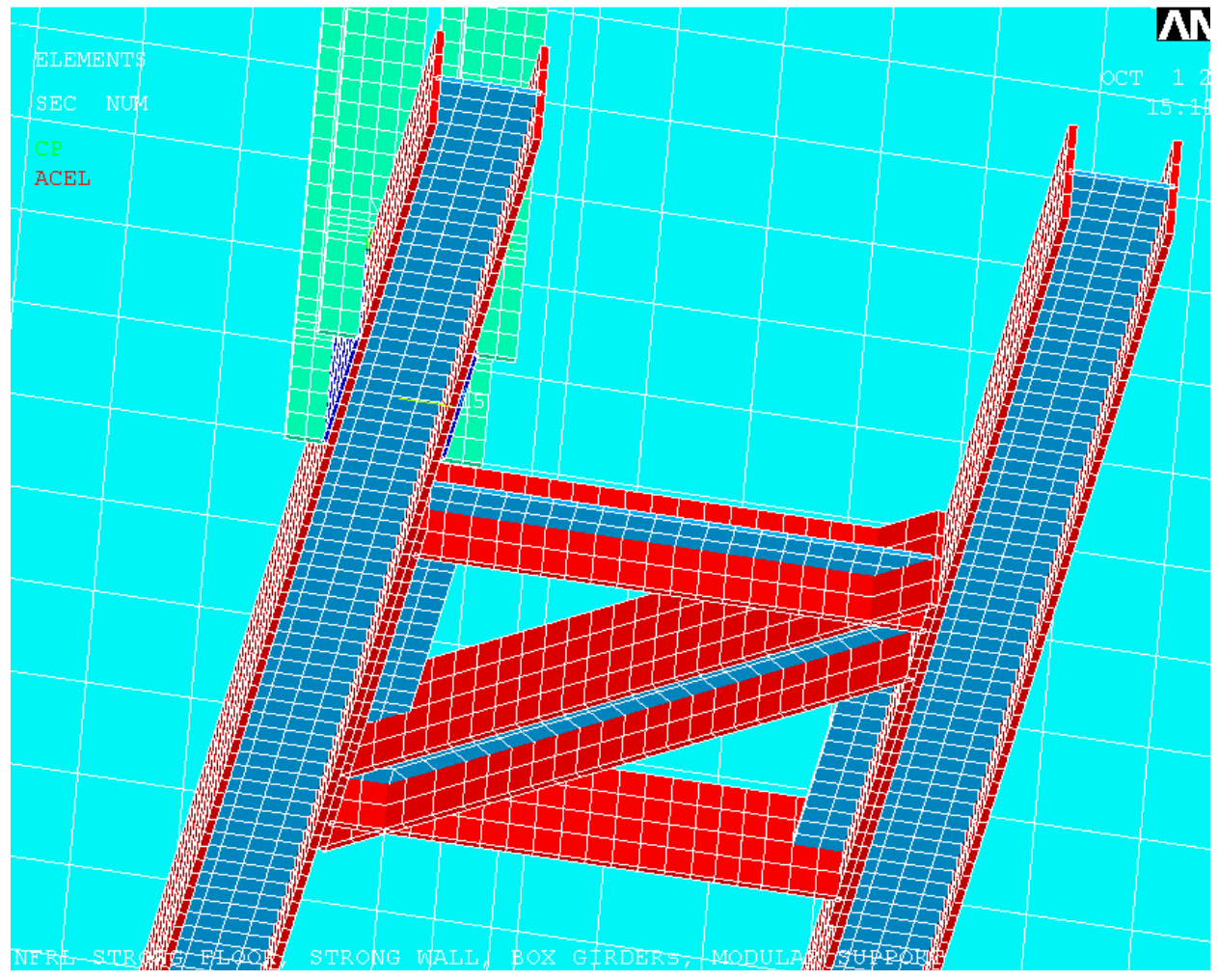

Fig. 14.9 Columns and frame 


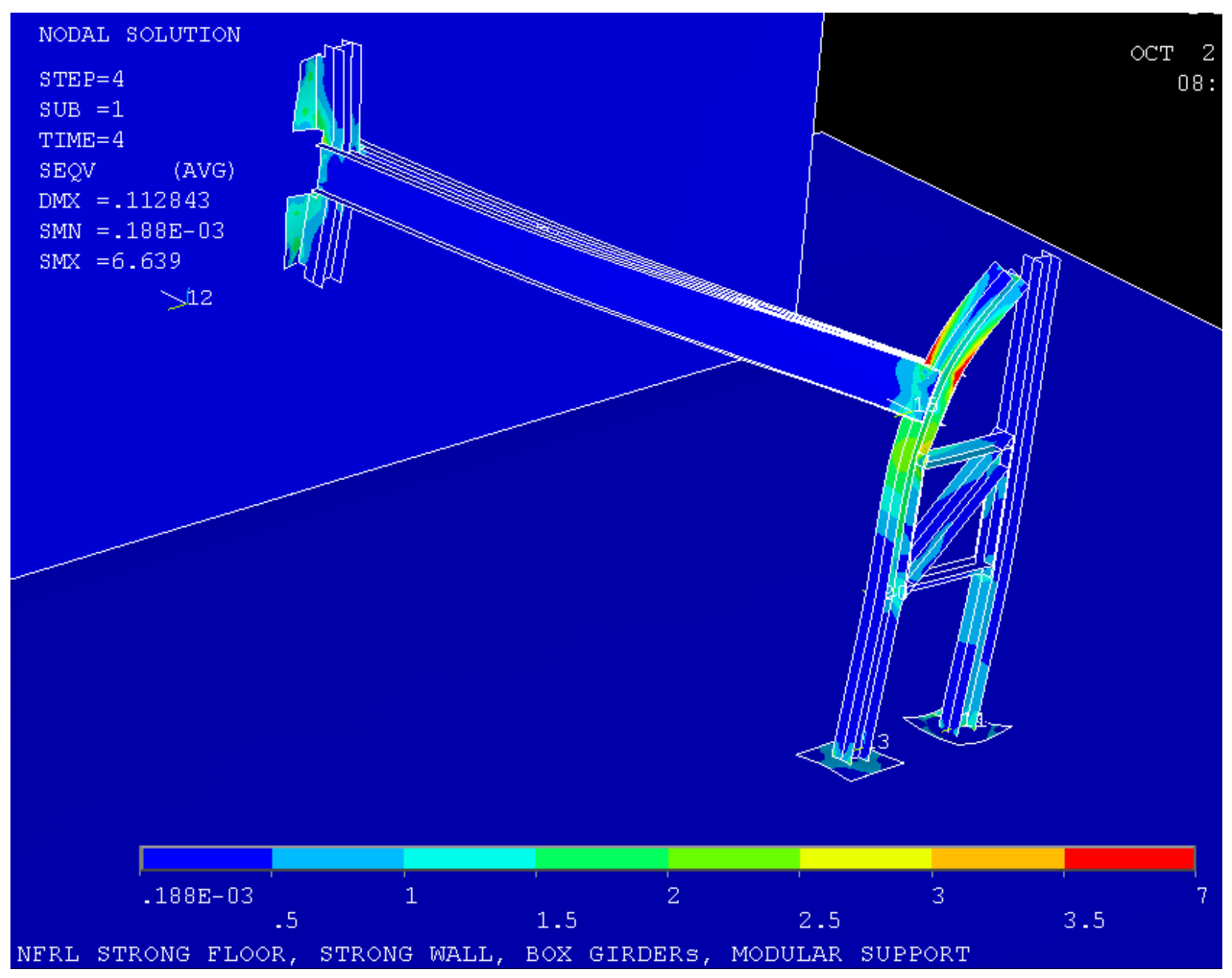

Fig. 14.10 Deformations and von Mises stress, detail. 
! begin slab at $x=0$. East wall closed. west wall special. West box correctly placed.

! all floor loading points at KP. prestressing KP both ways.

! input to run 20120319 3pm success, equilibrium satisfied.

! 20120418 constrained axial rotation of loading beams as SOLID 185 nodes have no rotation DOF.

! A good alternative to using BEAM188 as loading beam is to use SURF156,

! which has an orientation node in addition to 2 end nodes and possibly a middle node.

FINISH

/CLEAR

/CWD, 'C: \StrongFireLab'

/FILNAME,20121001,0

/TITLE,NFRL STRONG FLOOR, STRONG WALL, BOX GIRDERS, MODULAR SUPPORT

/REPLOT

KEYW,PR_SET,1

KEYW,PR_STRUC,1

KEYW,PR_THERM,0

KEYW,PR_FLUID,0

KEYW,PR_MULTI,0

/PREP7

ET,1,BEAM188

SECTYPE, 1, BEAM, RECT, , 0

SECOFFSET, CENT

SECDATA, 1, 0.5, 0, $0,0,0,0,0,0,0$

ET,2,SURF154

KEYOPT, 2,2,0

KEYOPT, $2,4,1$

KEYOPT, 2,6,0

KEYOPT, 2,11,0

KEYOPT, 2,12,0

$\mathrm{R}, 2, \ldots, \ldots$, , ,

RMORE,.01,.01,.01,.01,

\section{ET,3,SOLID185}

ACEL,,1 ! gravity

! $\mathrm{x}$ is $\mathrm{S}$ to $\mathrm{N}, \mathrm{y}$ is $\mathrm{E}$ to $\mathrm{W}, \mathrm{z}$ is down to up

! units, kips, inch, degree $C$

! Mat 1 is for slab on grade, $f^{\prime} c=3500$ psi

TREF,25 ! T REFERENCE 25 C, also default TUNIF 9/25/2012

MPTEMP, ,,, , , , ,

MPTEMP,1,0 


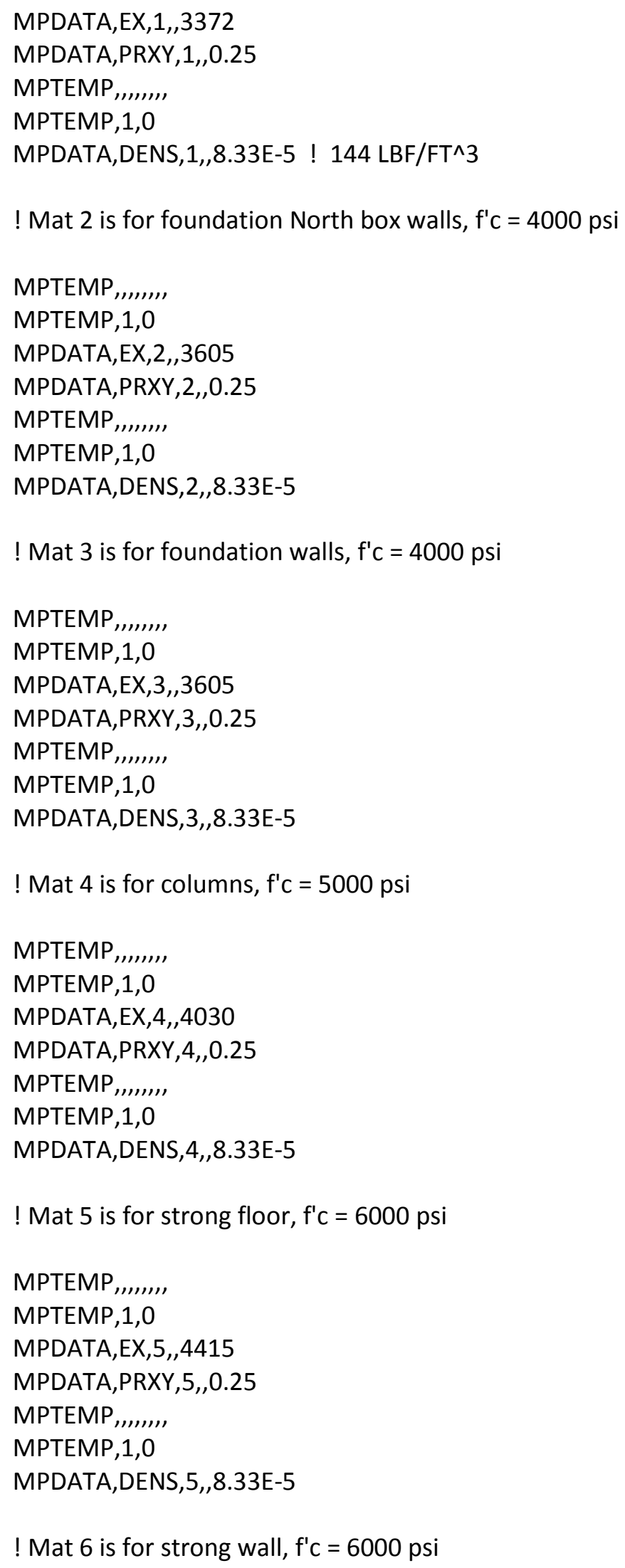

! Mat 6 is for strong wall, $\mathrm{f}^{\prime} \mathrm{c}=6000 \mathrm{psi}$ 


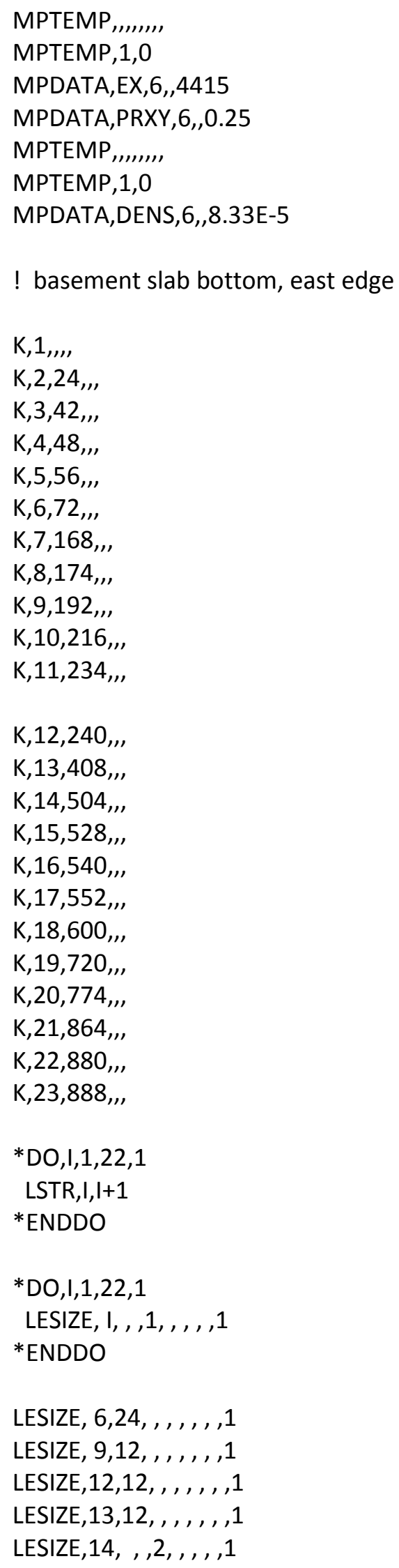




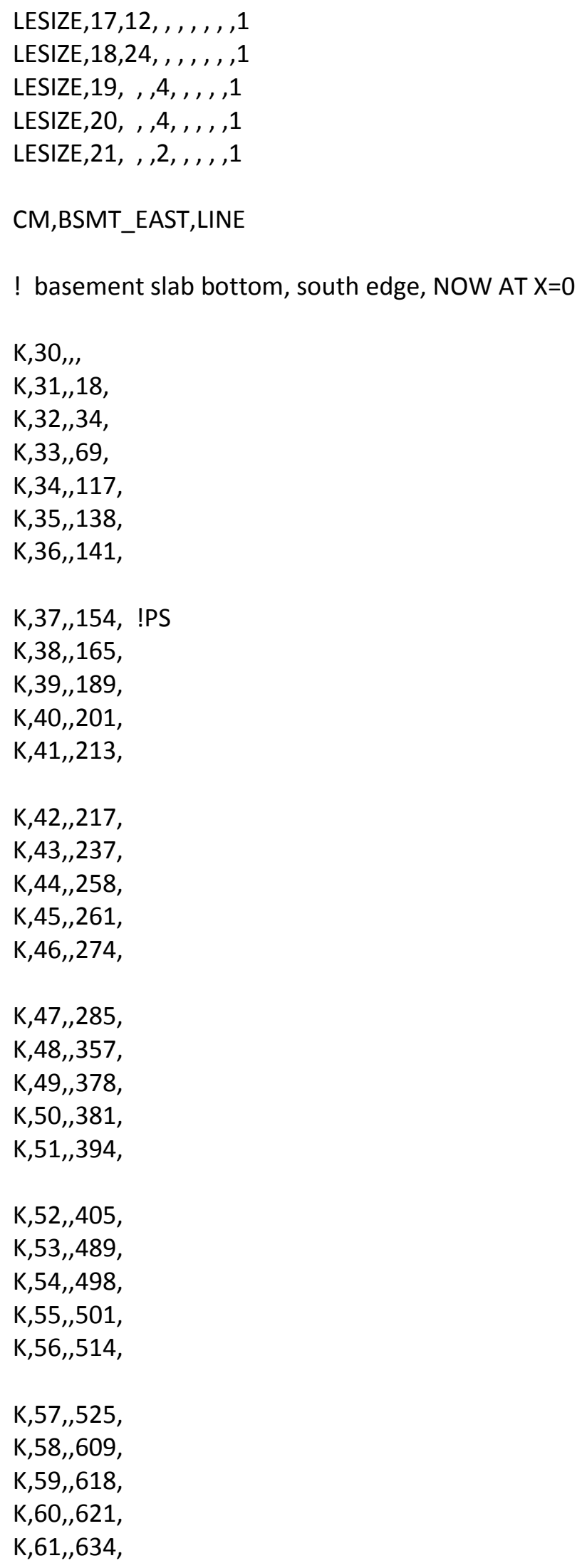


$\mathrm{K}, 62,645$,

$\mathrm{K}, 63,, 729$,

$\mathrm{K}, 64,, 738$,

$\mathrm{K}, 65,, 741$,

$\mathrm{K}, 66,754$,

$\mathrm{K}, 67,, 765$,

$\mathrm{K}, 68,, 837$,

$\mathrm{K}, 69,, 858$,

$\mathrm{K}, 70,861$,

$\mathrm{K}, 71,, 874$,

$\mathrm{K}, 72,885$,

$\mathrm{K}, 73,, 905$,

$\mathrm{K}, 74,, 909$,

$\mathrm{K}, 75,921$,

$\mathrm{K}, 76,, 933$,

$\mathrm{K}, 77,, 957$,

$\mathrm{K}, 78,, 978$, !PS

$\mathrm{K}, 79,, 981$,

$\mathrm{K}, 80,, 994$,

$\mathrm{K}, 81,, 1005$,

$\mathrm{K}, 82,, 1053$,

$\mathrm{K}, 83,, 1098$,

$\mathrm{K}, 84,1114$,

$\mathrm{K}, 85,, 1242$,

*DO,I,30,84,1

LSTR,I,I+1

*ENDDO

*DO,I,23,77,1

LESIZE, I, , ,1, , , , ,1

*ENDDO

LESIZE, 24, , , 2, , , , , 1

LESIZE, 25, , , 2, , , , , 1

LESIZE,26,24, , , , , , ,

LESIZE, 29, , , 2, , , , , 1

LESIZE, 31,12, , , , , , , 1

LESIZE, 33, , , 2, , , , ,

LESIZE,35, , , 2, , , , , 1

LESIZE,36, , , 2, , , , , 1

LESIZE, 38, , , 2, , , , , 1

LESIZE,40,12,, , , , , , 1

LESIZE,41, , , 2, , , , , 1 


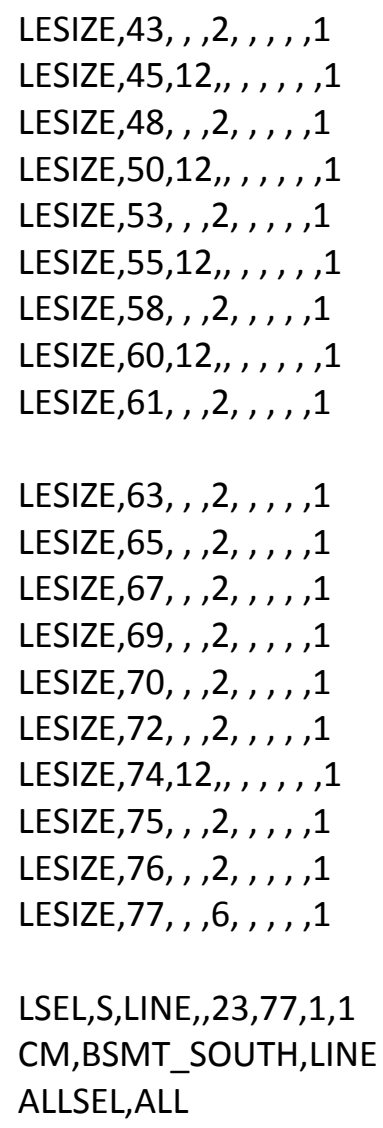




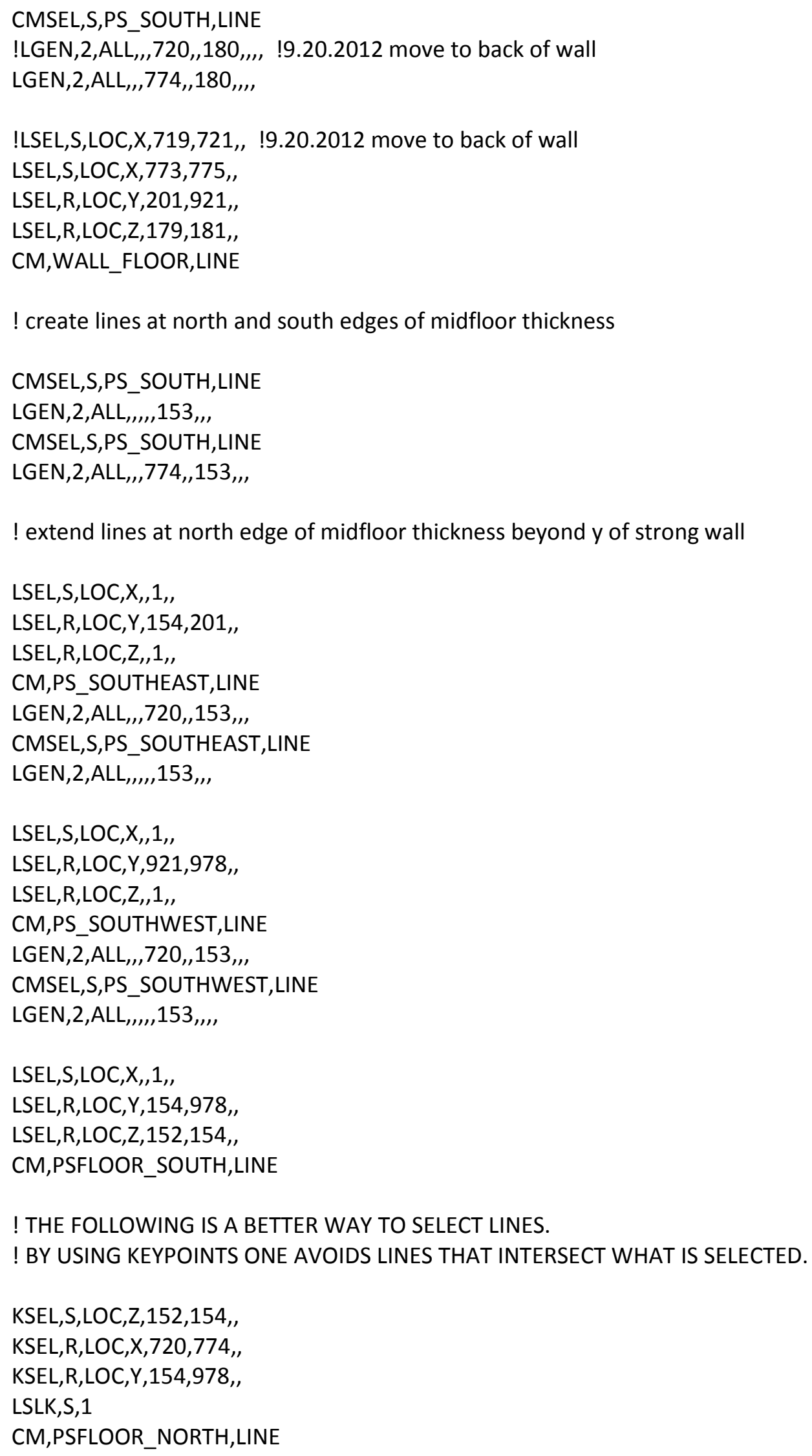

! THE FOLLOWING IS A BETTER WAY TO SELECT LINES. ! BY USING KEYPOINTS ONE AVOIDS LINES THAT INTERSECT WHAT IS SELECTED.

KSEL,S,LOC,Z,152,154,, KSEL,R,LOC,X,720,774, KSEL,R,LOC,Y,154,978,, LSLK,S,1 CM,PSFLOOR_NORTH,LINE 
! construct basement bottom

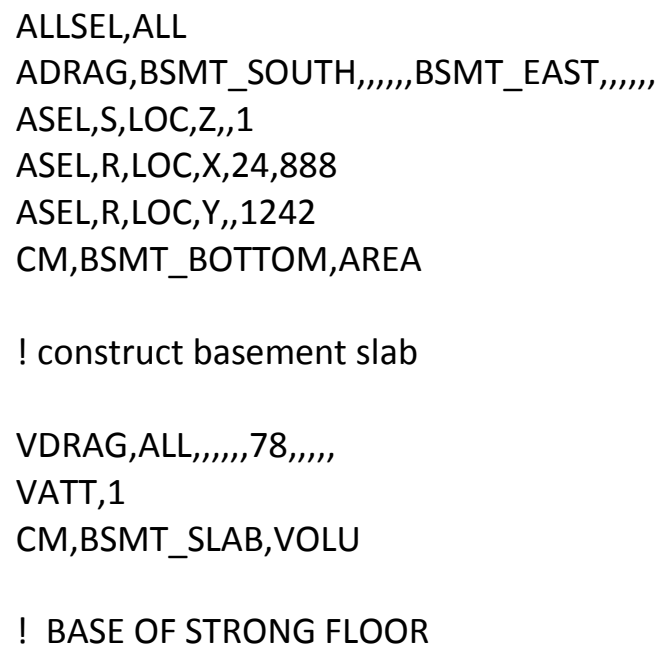


ASEL,S,LOC,Z,23,25

ASEL,R,LOC,X,56,174

ASEL,R,LOC,Y,618,634

CM,BASE_SOUTH6,AREA

ASEL,S,LOC,Z,23,25

ASEL,R,LOC,X,56,174

ASEL,R,LOC,Y,738,754

CM,BASE_SOUTH7,AREA

ASEL,S,LOC,Z,23,25

ASEL,R,LOC,X,56,174

ASEL,R,LOC,Y,858,874

CM,BASE_SOUTH8,AREA

ASEL,S,LOC,Z,23,25

ASEL,R,LOC,X,56,174

ASEL,R,LOC,Y,978,994

CM,BASE_SOUTH9,AREA

CMGRP,BASE_SOUTH_WEST,BASE_SOUTH6,BASE_SOUTH7,BASE_SOUTH8,BASE_SOUTH9

! bases of 4 north-South walls, middle east part

ASEL,S,LOC,Z,23,25

ASEL, R,LOC,X,234,540

ASEL,R,LOC,Y,138,154

CM,BASE_MIDDLE2,AREA

ASEL,S,LOC,Z,23,25

ASEL,R,LOC,X,234,540

ASEL,R,LOC,Y,258,274

CM,BASE_MIDDLE3,AREA

ASEL,S,LOC,Z,23,25

ASEL,R,LOC,X,234,540

ASEL,R,LOC,Y,378,394

CM,BASE_MIDDLE4,AREA

ASEL,S,LOC,Z,23,25

ASEL,R,LOC,X,234,540

ASEL,R,LOC,Y,498,514

CM,BASE_MIDDLE5,AREA

CMGRP,BASE_MIDDLE_EAST,BASE_MIDDLE2,BASE_MIDDLE3,BASE_MIDDLE4,BASE_MIDDLE5

! bases of 5 north-South walls, middle west part 
ASEL,S,LOC,Z,23,25

ASEL,R,LOC,X,234,540

ASEL,R,LOC,Y,618,634

CM,BASE_MIDDLE6,AREA

ASEL,S,LOC,Z,23,25

ASEL,R,LOC, $X, 234,540$

ASEL,R,LOC,Y,738,754

CM,BASE_MIDDLE7,AREA

ASEL,S,LOC,Z,23,25

ASEL,R,LOC, $X, 234,540$

ASEL,R,LOC,Y,858,874

CM,BASE_MIDDLE8,AREA

ASEL,S,LOC,Z,23,25

ASEL,R,LOC,X,234,540

ASEL,R,LOC,Y,978,994

CM,BASE_MIDDLE9,AREA

ASEL,S,LOC,Z,23,25

ASEL, R,LOC,X,174,408

ASEL,R,LOC,Y,1098,1114

CM,BASE_MIDDLE10,AREA

CMGRP,BASE_MIDDLE_WEST,BASE_MIDDLE6,BASE_MIDDLE7,BASE_MIDDLE8,BASE_MIDDLE9,BASE_MI DDLE10

! bases of 4 north-South walls, NORTH east part

ASEL,S,LOC,Z,23,25

ASEL,R,LOC,X,600,720

ASEL,R,LOC,Y,138,154

CM,BASE_NORTH2,AREA

ASEL,S,LOC,Z,23,25

ASEL,R,LOC,X,600,720

ASEL,R,LOC,Y,258,274

CM,BASE_NORTH3,AREA

ASEL,S,LOC,Z,23,25

ASEL,R,LOC, $X, 600,720$

ASEL,R,LOC,Y,378,394

CM,BASE_NORTH4,AREA

ASEL,S,LOC,Z,23,25

ASEL,R,LOC,X,600,720 
ASEL,R,LOC,Y,498,514

CM,BASE_NORTH5,AREA

CMGRP,BASE_NORTH_EAST,BASE_NORTH2,BASE_NORTH3,BASE_NORTH4,BASE_NORTH5

! bases of 5 north-South walls, NORTH west part

ASEL,S,LOC,Z,23,25

ASEL,R,LOC,X,600,720

ASEL,R,LOC,Y,618,634

CM,BASE_NORTH6,AREA

ASEL,S,LOC,Z,23,25

ASEL,R,LOC,X,600,720

ASEL,R,LOC,Y,738,754

CM,BASE_NORTH7,AREA

ASEL,S,LOC,Z,23,25

ASEL,R,LOC,X,600,720

ASEL,R,LOC,Y,858,874

CM,BASE_NORTH8,AREA

ASEL,S,LOC,Z,23,25

ASEL,R,LOC,X,600,720

ASEL,R,LOC,Y,978,994

CM,BASE_NORTH9,AREA

ASEL,S,LOC,Z,23,25

ASEL,R,LOC, $X, 504,720$

ASEL,R,LOC,Y,1098,1114

CM,BASE_NORTH10,AREA

CMGRP,BASE_NORTH_WEST,BASE_NORTH6,BASE_NORTH7,BASE_NORTH8,BASE_NORTH9,BASE_NORT $\mathrm{H} 10$

CMGRP,BASE_NS_WALLS,BASE_SOUTH_EAST,BASE_SOUTH_WEST,BASE_MIDDLE_EAST,BASE_MIDDLE_ WEST,BASE_NORTH_EAST,BASE_NORTH_WEST

! Northeast box

ASEL,S,LOC,Z,23,25

ASEL,R,LOC,X,720,880

ASEL,R,LOC,Y,18,34

CM,BASE_EBOX_1,AREA

ASEL,S,LOC,Z,23,25

ASEL,R,LOC,X,864,880

ASEL,R,LOC,Y,34,201 
CM,BASE_EBOX_2,AREA

ASEL,S,LOC,Z,23,25

ASEL,R,LOC,X,774,880

ASEL,R,LOC,Y,201,217

CM,BASE_EBOX_3,AREA

ASEL,S,LOC,Z,23,25

ASEL,R,LOC,X,720,774

ASEL,R,LOC,Y,18,201

CM,BASE_EBOX_4,AREA

ASEL,S,LOC,Z,23,25

ASEL,R,LOC,X,774,880

ASEL,R,LOC,Y,18,217

CM,BASE_EBOX_5,AREA

! NorthWest box

ASEL,S,LOC,Z,23,25

ASEL,R,LOC, $X, 720,880$

ASEL,R,LOC,Y,1098,1114

CM,BASE_WBOX_1,AREA

ASEL,S,LOC,Z,23,25

ASEL,R,LOC,X,864,880

ASEL,R,LOC,Y,921,1098

CM,BASE_WBOX_2,AREA

ASEL,S,LOC,Z,23,25

ASEL,R,LOC,X,774,880

ASEL,R,LOC,Y,905,921

CM,BASE_WBOX_3,AREA

ASEL,S,LOC,Z,23,25

ASEL,R,LOC,X,720,774

ASEL,R,LOC,Y,921,1114

CM,BASE_WBOX_4,AREA

ASEL,S,LOC,Z,23,25

ASEL,R,LOC,X,774,880

ASEL, R,LOC,Y,905,1114

CM,BASE_WBOX_5,AREA

CMGRP,BASE_BOX_WALLS,BASE_EBOX_1,BASE_EBOX_2,BASE_EBOX_3,BASE_WBOX_1,BASE_WBOX_2, BASE_WBOX_3

CMGRP,BASE_BOXES,BASE_EBOX_4,BASE_EBOX_5,BASE_WBOX_4,BASE_WBOX_5 


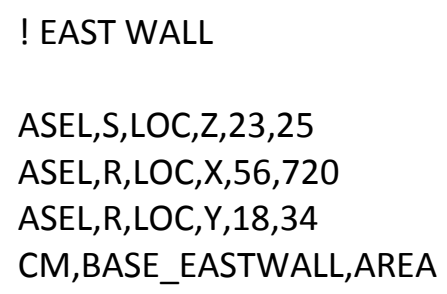




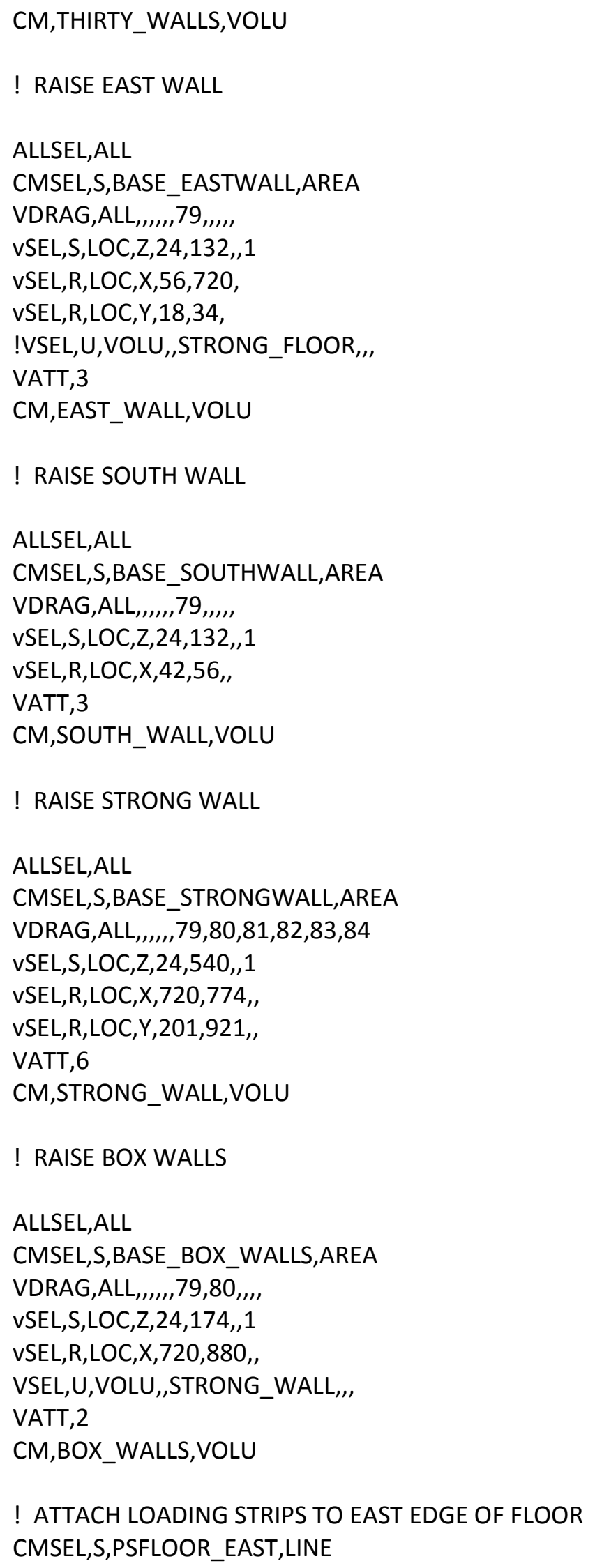




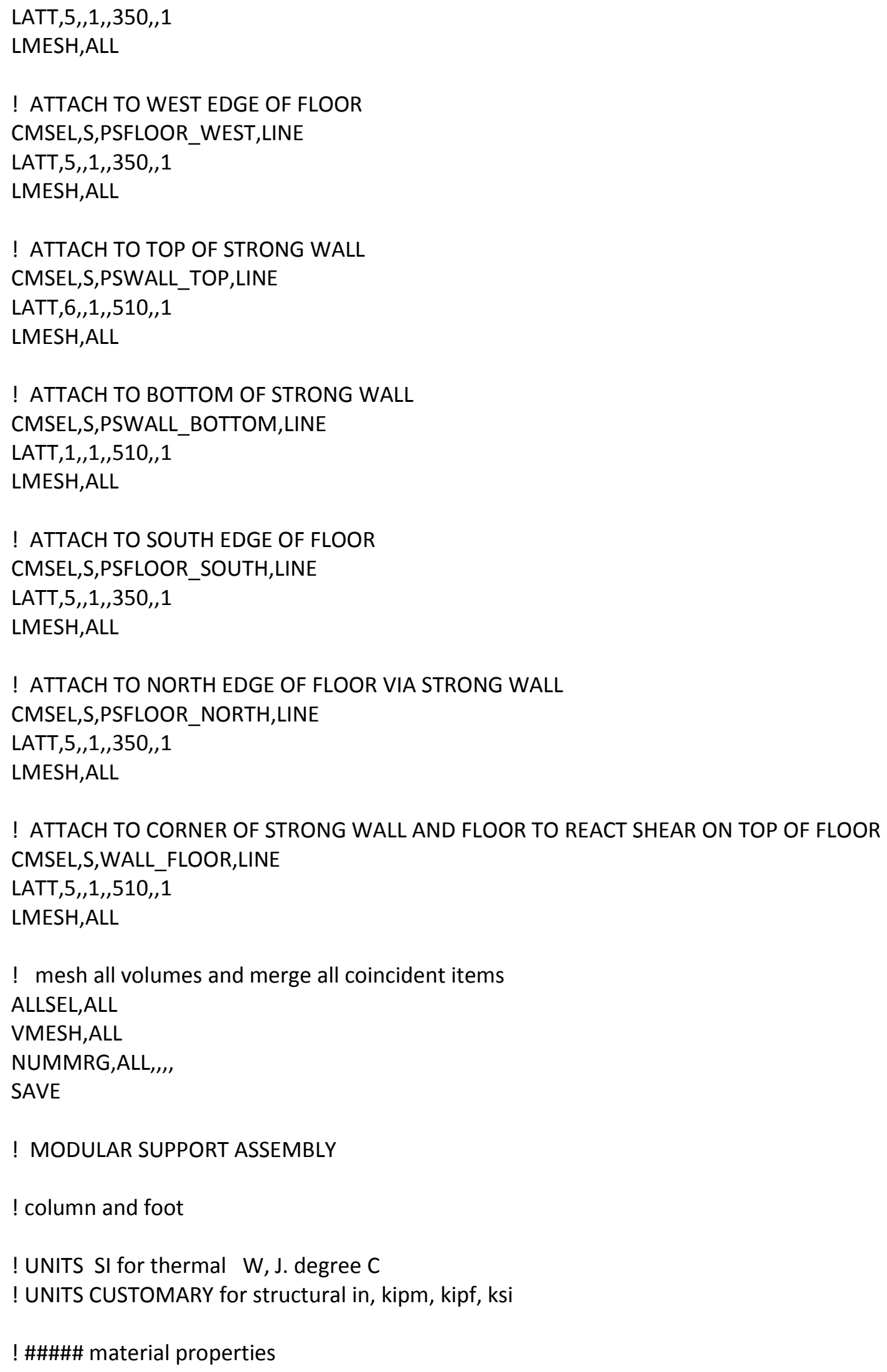


! \# Material \# 10 - Steel for W sections, 50 KSI STEEL

! thermal properties may be unnecessary for structural analysis

! Young's modulus and Poisson's ratio

MPTEMP,,,n,, ,

MPTEMP,1,25,100,200,300,400,500

MPTEMP, $7,600,700,800$

MPDATA,EX,10,1,30000,30000,27000,24000,21000,18000

MPDATA,EX,10,7,9300,3900,2700

MPTEMP, , , , , , ,

MPTEMP, 1,0

MPDATA,PRXY,10,,0.30

! thermal expansion

TREF,25 ! T REFERENCE $25 \mathrm{C}$, also default TUNIF

MPTEMP,,,,,,, ,

MPTEMP, 1,0

MPDATA,ALPX,10,,14E-6 ! PER DEG C, EC3, Buchanan p. 194

!\# steel conductivity W/(in.K)

MPTEMP

MPTEMP,1,25

MPTEMP, 2,800

MPTEMP, 3,1200

MPDATA,KXX,10,,1.37

MPDATA,KXX,10,,0.693

MPDATA,KXX,10,,0.693

!\# steel heat capacity J/(kipm.K)

MPTEMP

MPTEMP, 1,25

MPTEMP, 2,50

MPTEMP,3,100

MPTEMP, 4,150

MPTEMP,5,200

MPTEMP 6,400

MPTEMP,7,500

MPTEMP, 8,600

MPDATA,C,10,,199500

MPDATA,C,10,,208500

MPDATA,C,10,,221200

MPDATA,C,10,,231500

MPDATA,C,10,,240300

MPDATA,C,10,274800

MPDATA,C,10,,302300

MPDATA,C,10,,344700 


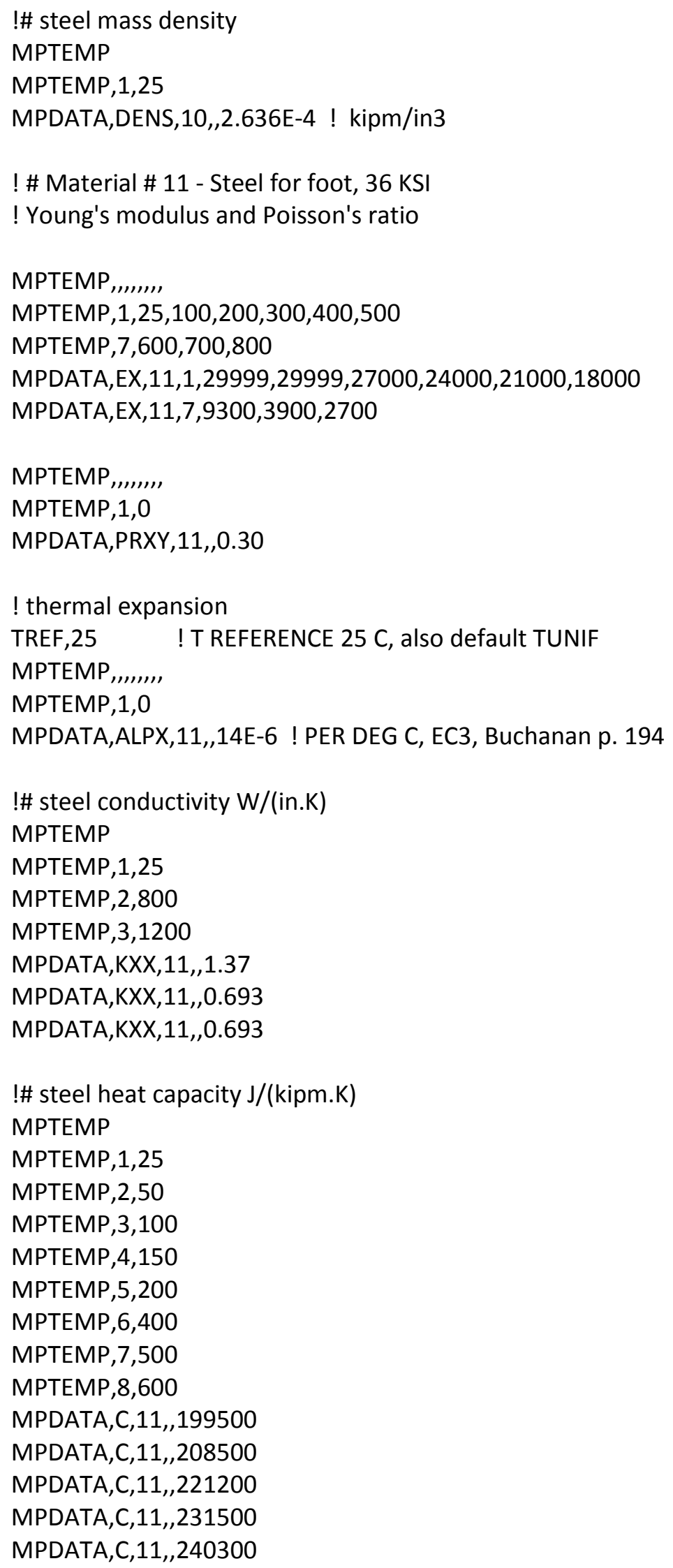


MPDATA,C,11,,274800

MPDATA,C,11,,302300

MPDATA,C,11,,344700

!\# steel mass density

MPTEMP

MPTEMP,1,25

MPDATA,DENS,11,2.636E-4！ kipm/in3

\section{! COLUMN AND FOOT}

\section{ET,5,SHELL181}

KEYOPT,5,1,0 ! bending and membrane stiffness

KEYOPT,5,3,2 ! full integration with incompatible modes

KEYOPT,5,8,0 ! store data at bottom of bottom layer and top of top layer KEYOPT,5,9,0 ! no user subroutine to define thickness

KEYOPT,5,10,0 ! no initial stress

sect,2,shell,,foot

secdata, $2,11,0,3$

secoffset,MID

seccontrol,0,0,0, 0, 1, 1, 1

sect,3,shell,,12106f

secdata,0.99,10,0,3

secoffset,MID

seccontrol,0,0,0, 0, 1, 1, 1

sect,4,shell,,12106W

secdata,0.61,10,0,3

secoffset,MID

seccontrol,0,0,0, 0, 1, 1, 1

! USER INPUT: STARTING NUMBERS, SUB 1============================

*SET,KP1,8000

*SET,LIN1,19000

*SET,ARE1,15000

*SET, VOL1,5000

*SET,ELE1,67000

*SET,NOD1,85000

! \# define model geometry, sheet $11 / 15$ lower left fig

! Local $X$ parallel to flanges, point North.

! Local Y parallel to web, point West.

! Local Z points up.

! user input: origin of local coordinates $=$ CENTER OF FOOT 


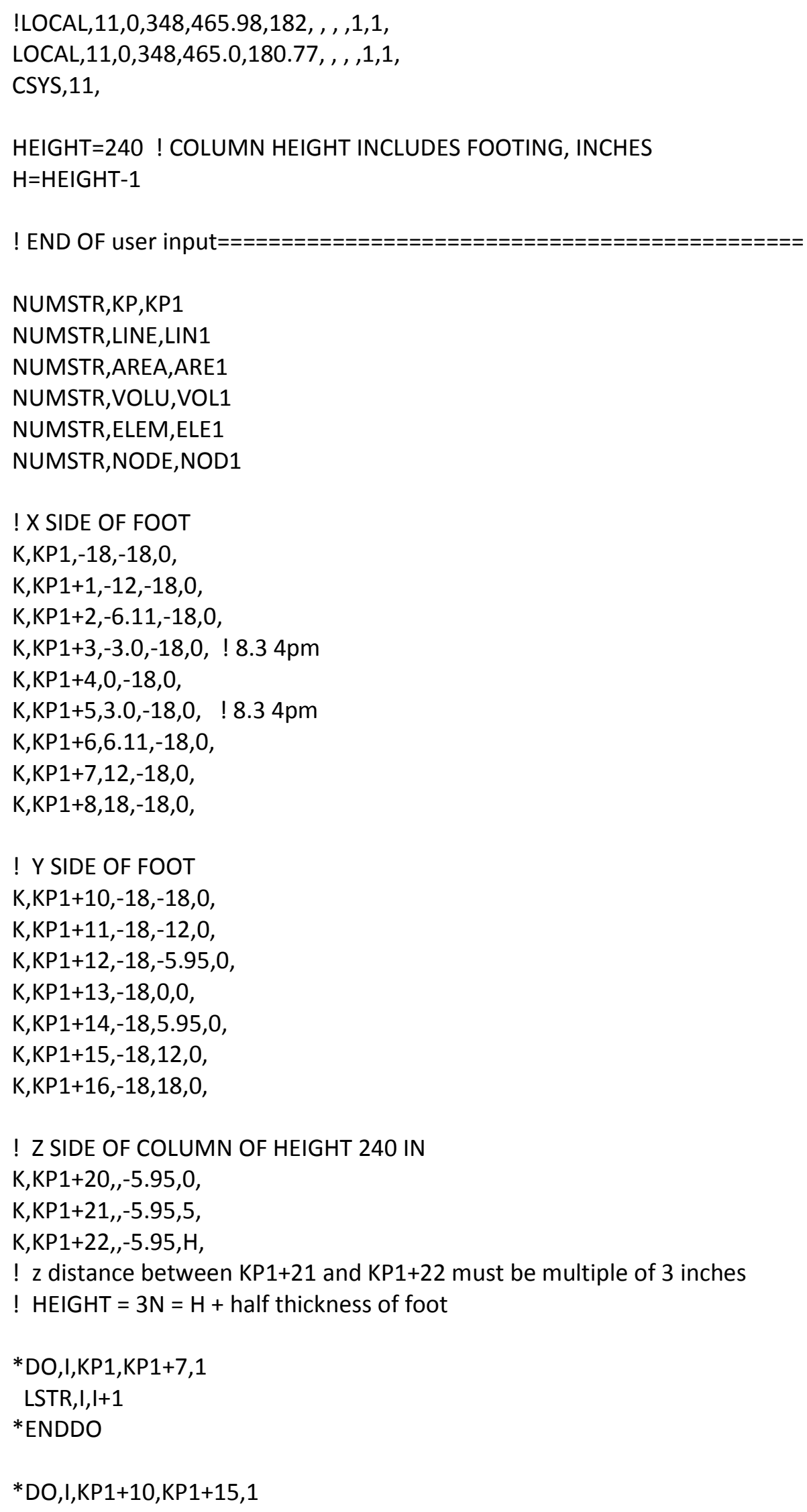




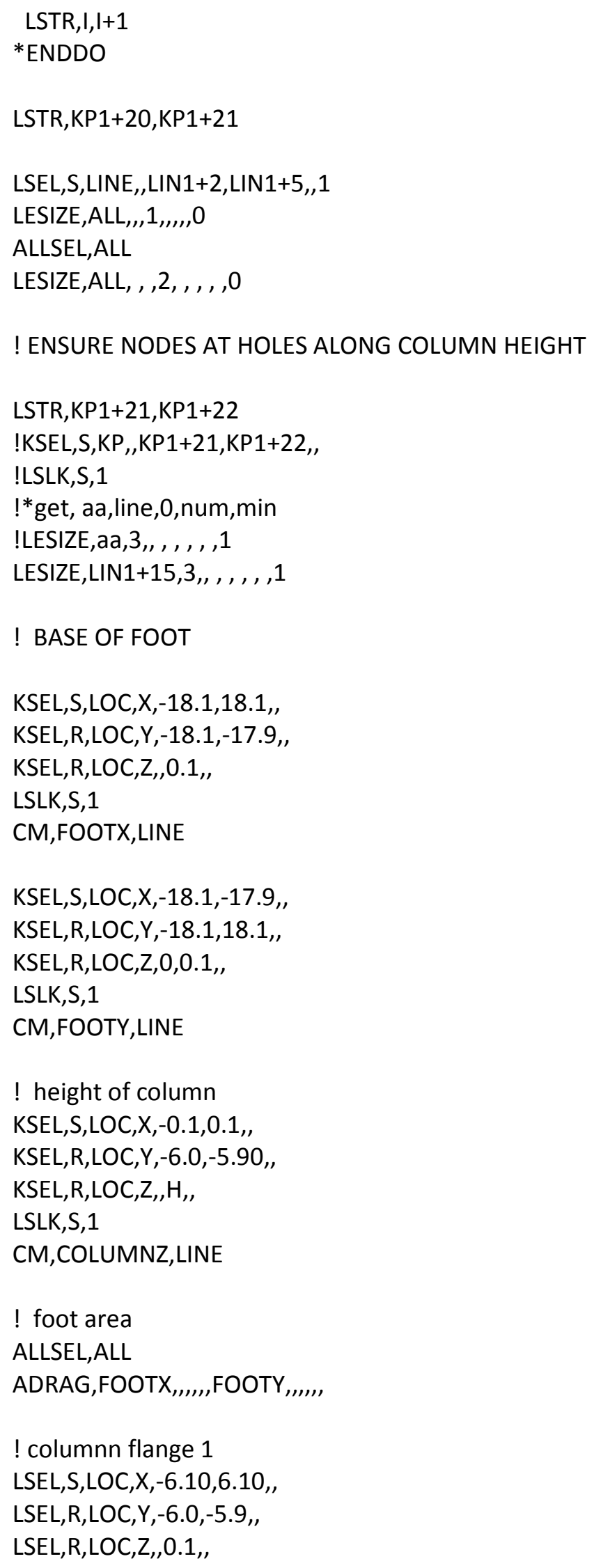




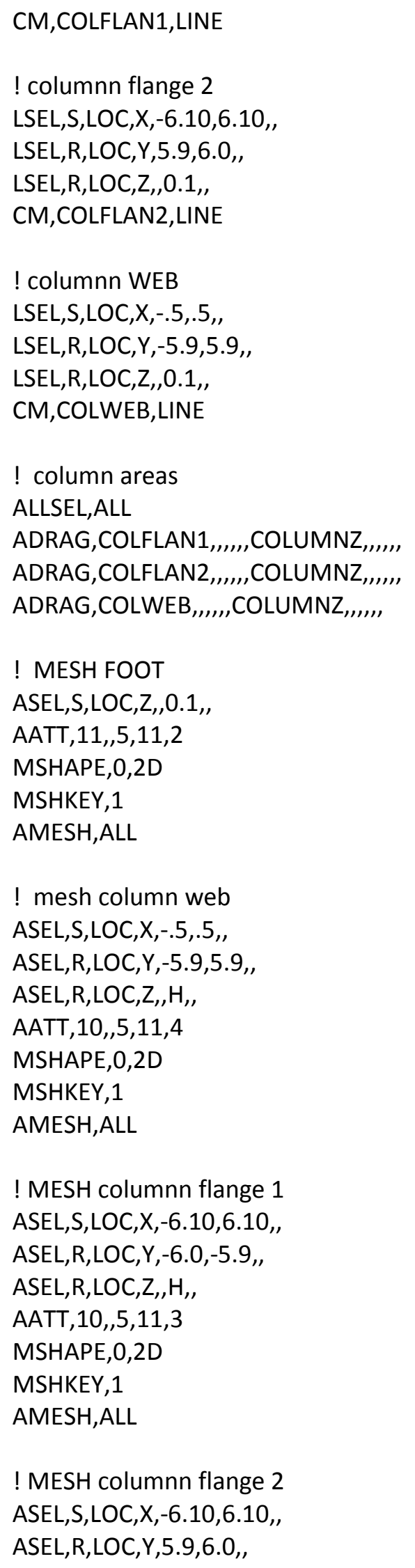




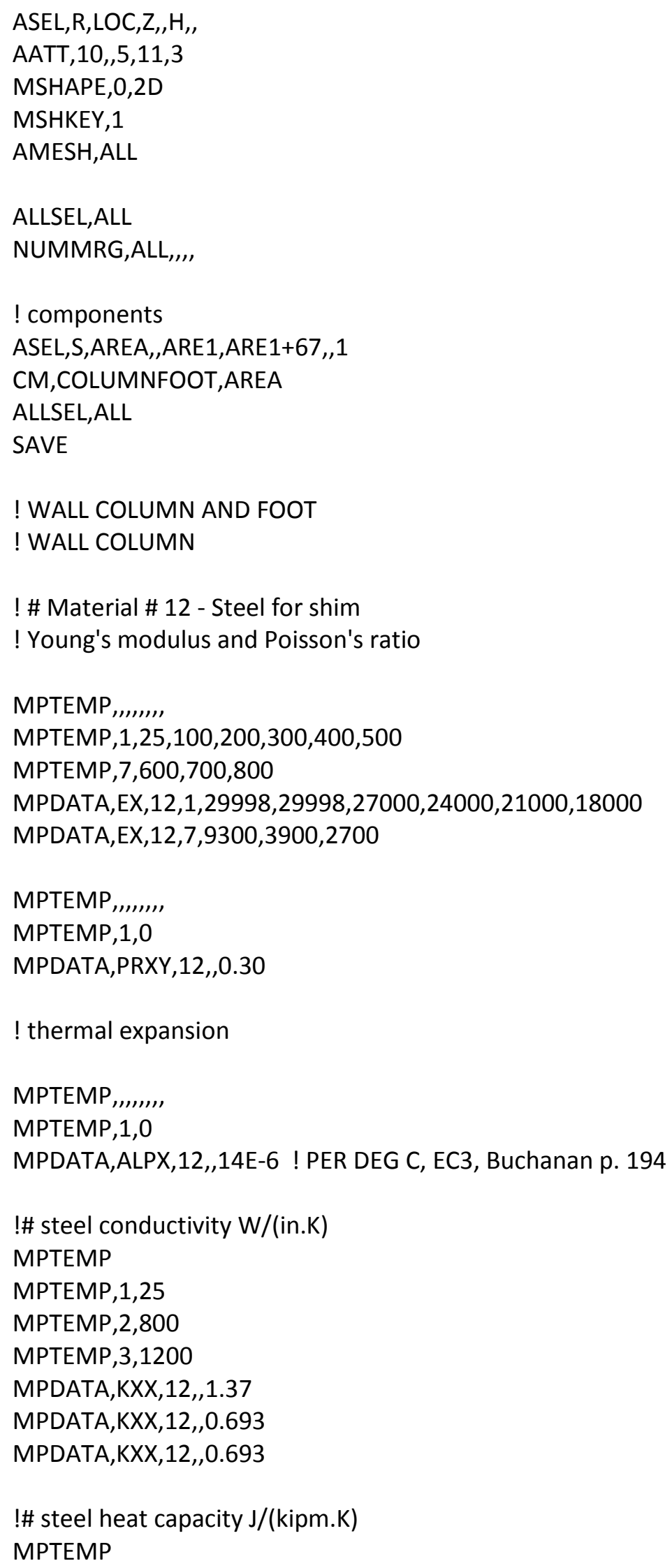




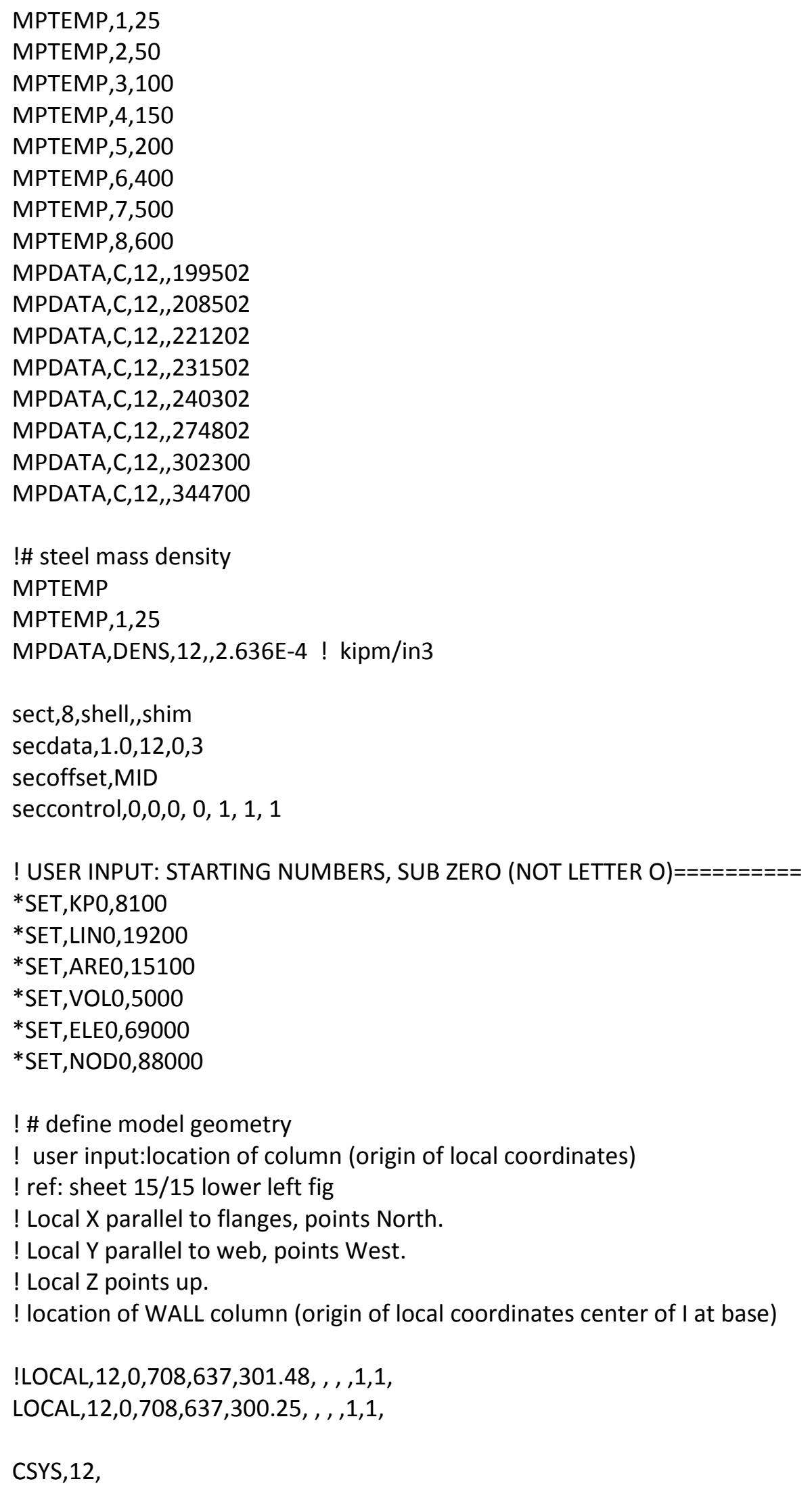




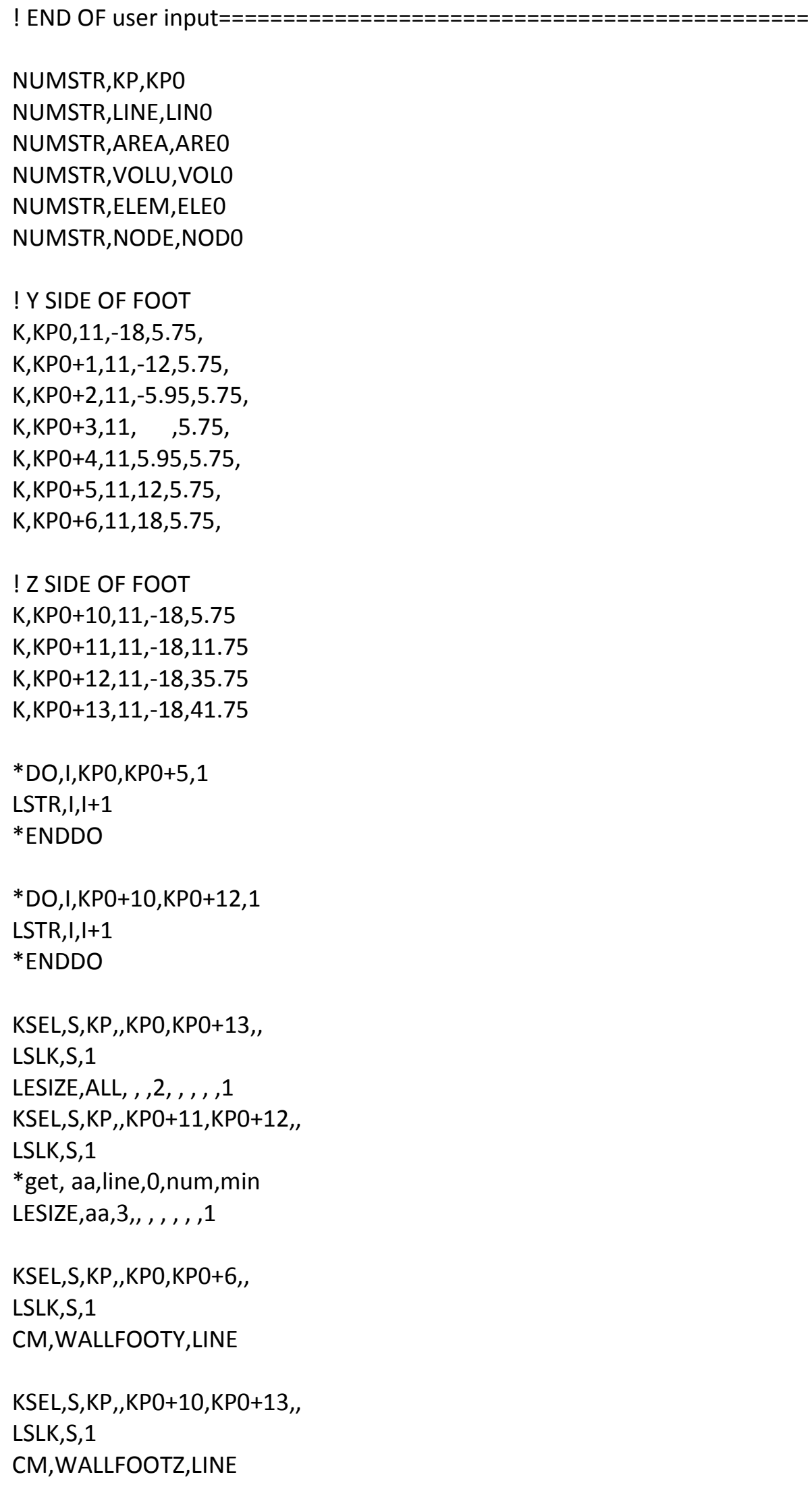




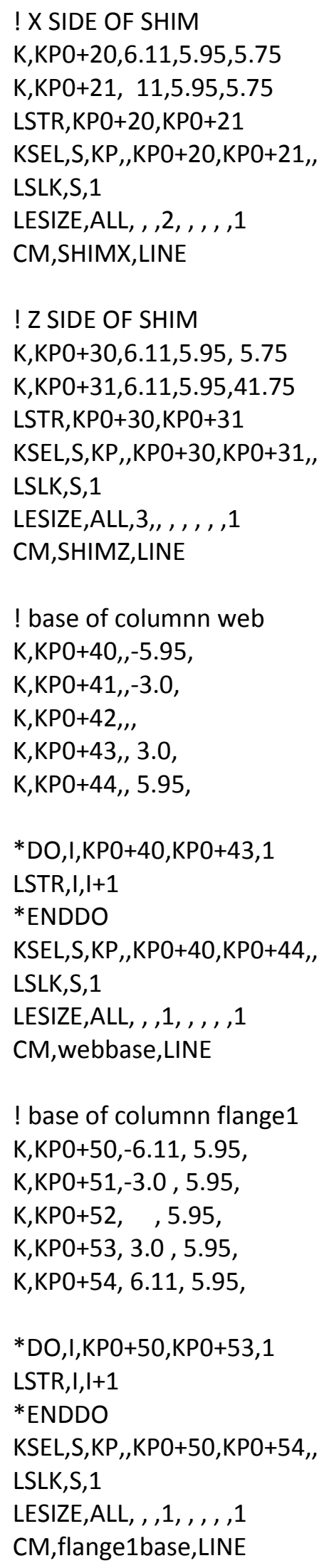




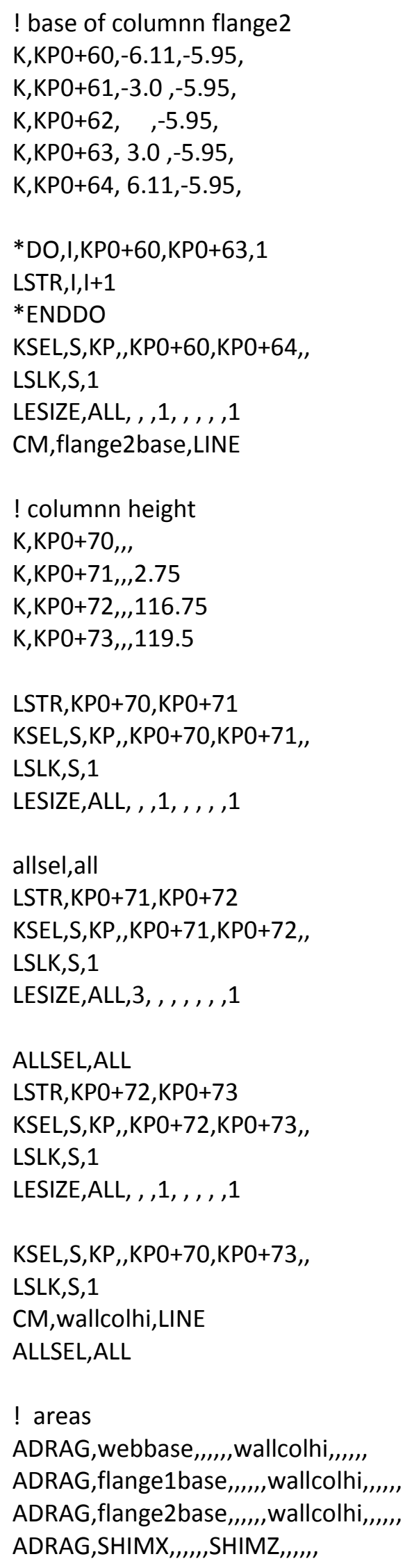




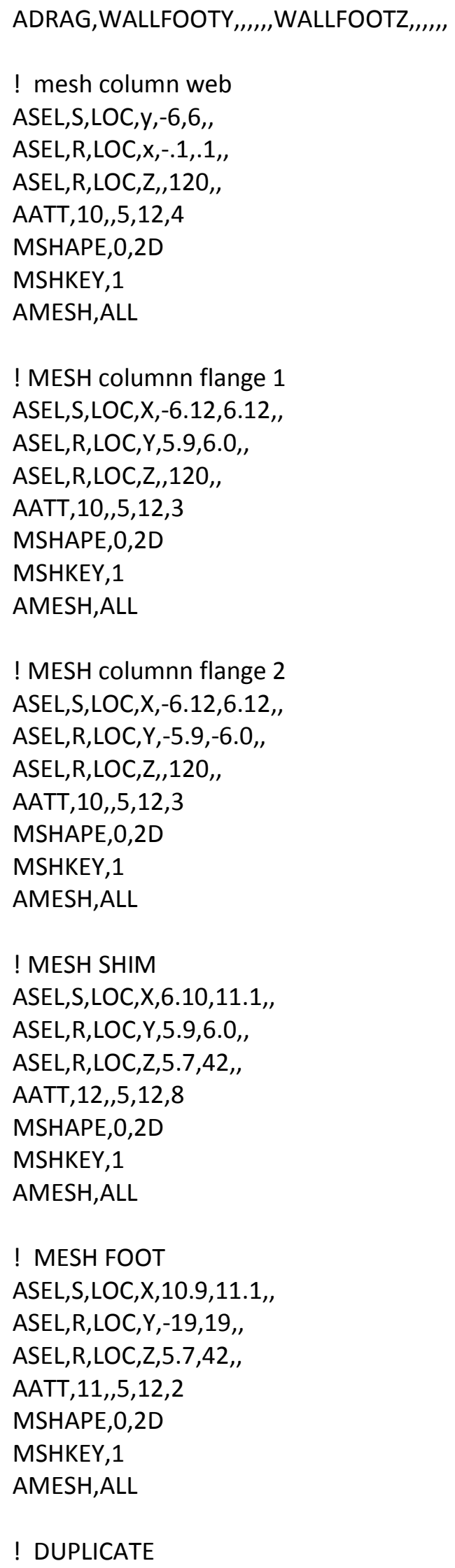




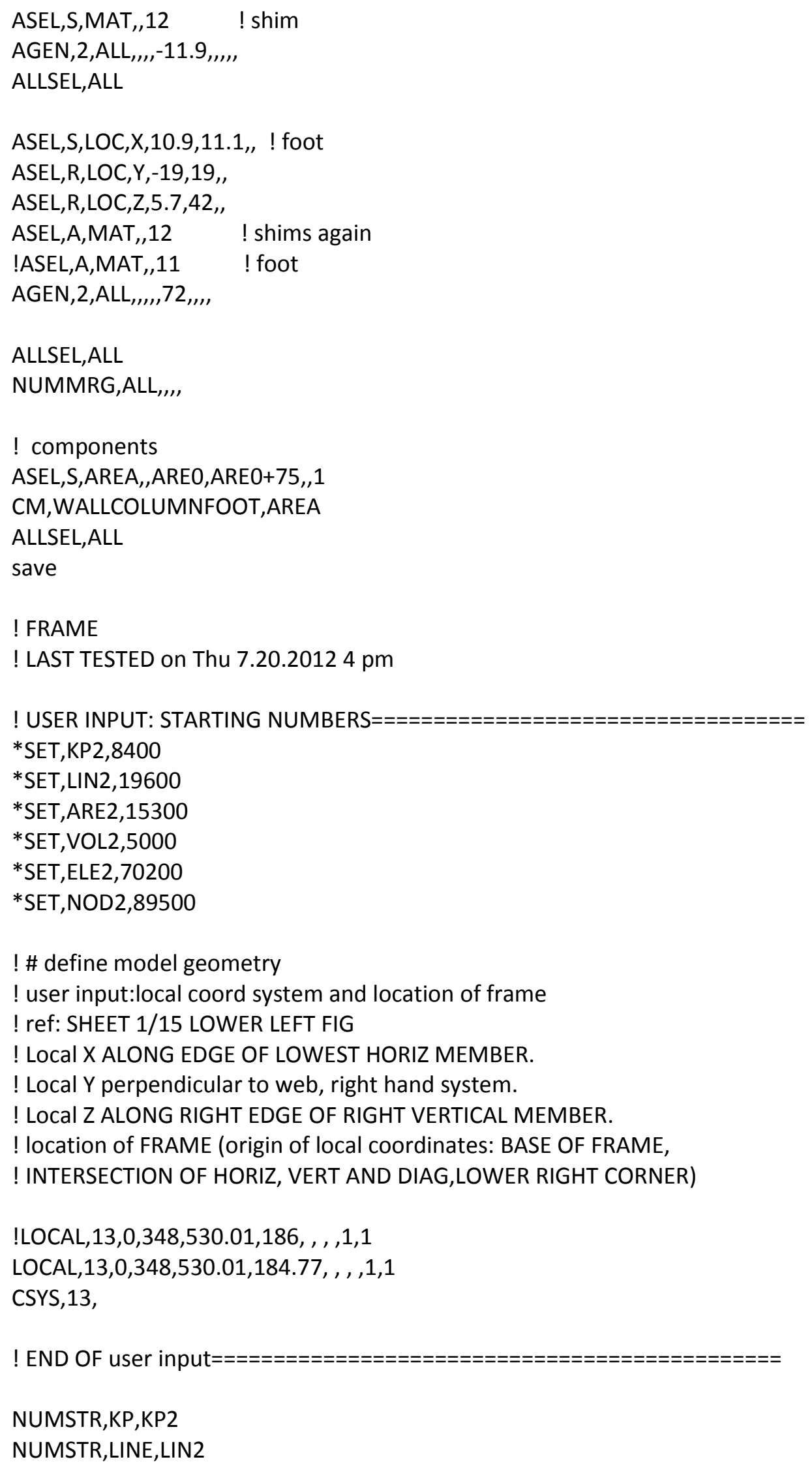




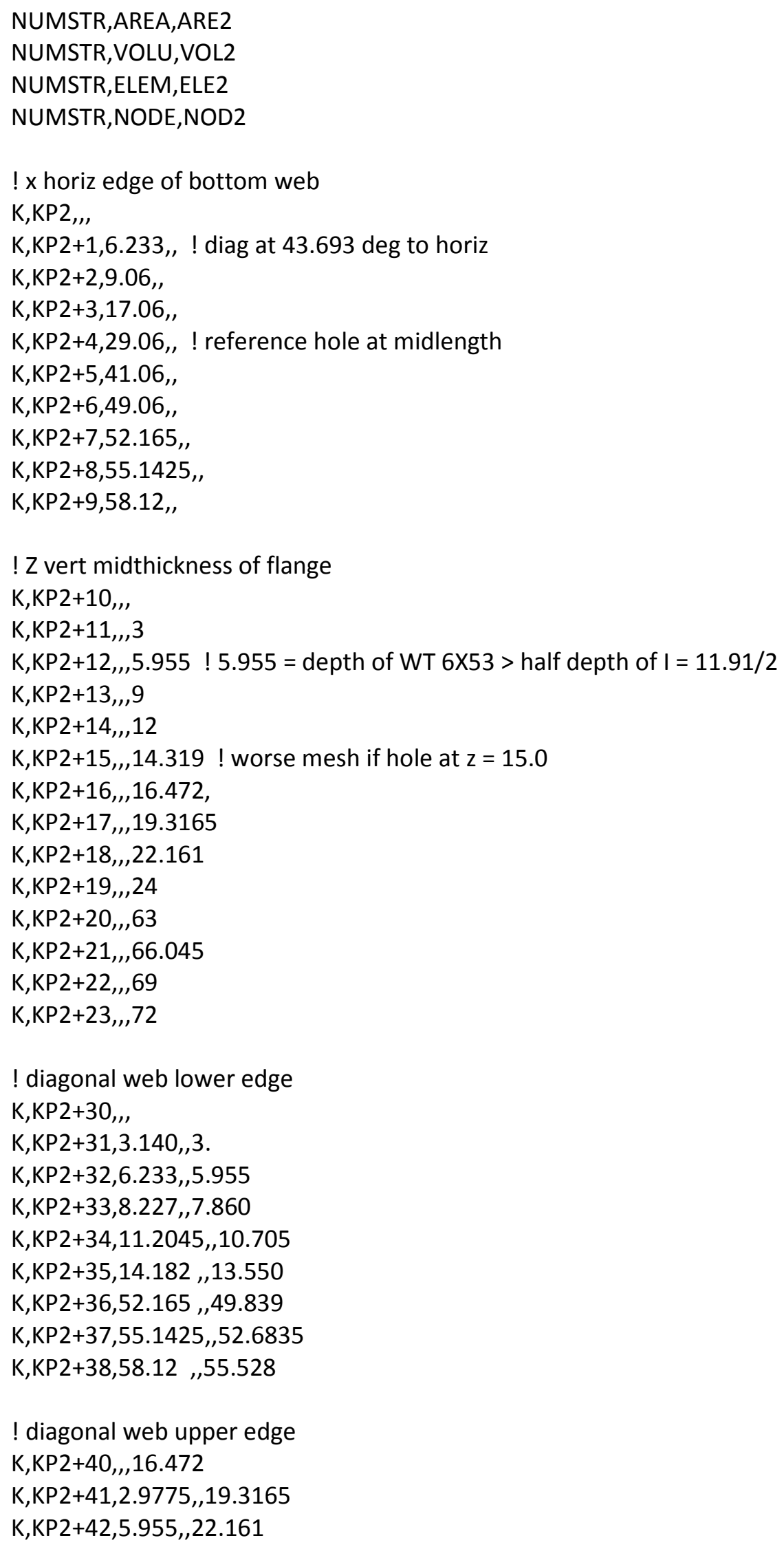




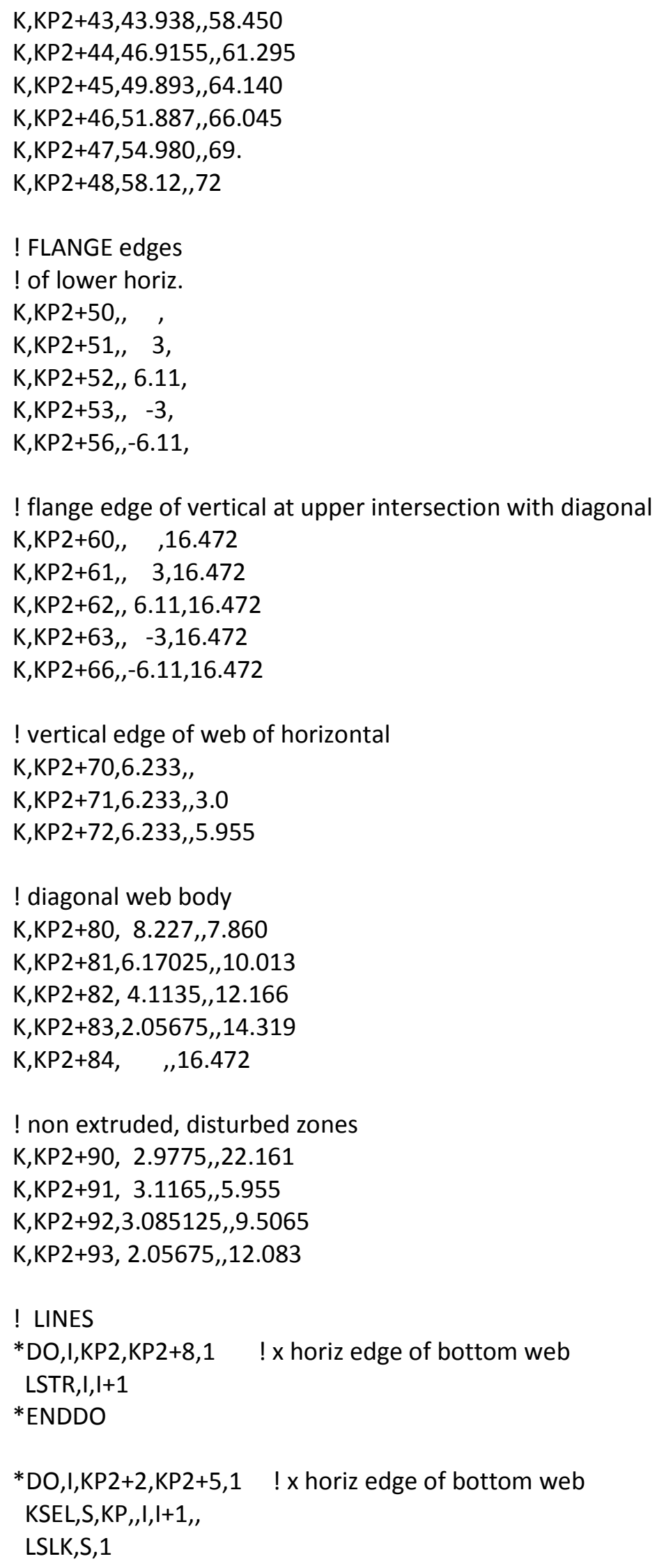


LESIZE,ALL,3.0, , , , 0 , , , 0

*ENDDO

ALLSEL,ALL

*DO,I,KP2+10,KP2+22,1 ! Z vert midthickness of flange

LSTR,I,I+1

*ENDDO

KSEL,S,KP, ,KP2+19,KP2+20,

LSLK,S,1

LESIZE,ALL,3.0, , , , 0 , , , 0

ALLSEL,ALL

*DO,I,KP2+30,KP2+37,1 ! diagonal web lower edge

LSTR,I,I+1

*ENDDO

KSEL,S,KP, ,KP2+35,KP2+36,

LSLK,S,1

LESIZE,ALL, , ,17, ,0 , , ,0

ALLSEL,ALL

*DO,I,KP2+40,KP2+47,1 ! diagonal web upper edge LSTR,I,I+1

*ENDDO

$\mathrm{KSEL}, \mathrm{S}, \mathrm{KP}$, ,KP2+42,KP2+43,

LSLK,S,1

LESIZE,ALL, , ,17, ,0 , , ,0

ALLSEL,ALL

*DO,I,KP2+33,KP2+38,1 ! diagonal web body

LSTR,I,I+7

$\mathrm{KSEL}, \mathrm{S}, \mathrm{KP}, \mathrm{I}, \mathrm{I}+7,7$,

LSLK,S,1

LESIZE,ALL, , ,4, ,0, , ,0

ALLSEL,ALL

*ENDDO

LSTR,KP2+50,KP2+51 !+y flange base of vertical LSTR, KP2+51,KP2+52

LSTR,KP2+50,KP2+53 ! -y flange base of vertical

LSTR, KP2+53,KP2+56

LSTR, KP2+60,KP2+61 !+y flange base of diagonal

LSTR, KP2+61, KP2+62

LSTR, KP2 $+60, K P 2+63$ ! -y flange base of diagonal

LSTR,KP2+63,KP2+66 
LSTR,KP2 +70,KP2+71 ! web of horiz

LSTR,KP2+71,KP2+72 ! save db L=19600 to 19650

LSTR,KP2+18,KP2+90! web of vert

LSTR,KP2+90,KP2+42 ! L=19651,2

! non extruded smaller zone

LSTR,KP2+17,KP2+41 ! L=19653

LSTR,KP2+41,KP2+90 ! L=19654

! non extruded larger zone

LSTR,KP2+15,KP2+83 ! L=19655

LSTR,KP2+14,KP2+93 ! L=19656

LSTR,KP2+93,KP2+82 ! L=19657

LSTR,KP2+13,KP2+92 ! L=19658

LSTR,KP2+92,KP2+81 ! L=19659

LSTR,KP2+12,KP2+91 ! L=19660

LSTR,KP2+91,KP2+32 ! L=19661

LSTR,KP2+91,KP2+92 ! L=19662

LSTR,KP2+92,KP2+93 ! L=19663

LSTR,KP2+93,KP2+83 ! L=19664

LSTR,KP2+32,KP2+81 ! L=19665

LSTR,KP2+92,KP2+82 ! L=19666

KSEL,S,KP, ,KP2,KP2+93, ,

LSLK,S, 1

LESIZE,ALL,4.4, , , ,0 , , ,0 ! default element size

LSTR, KP2+16,KP2+41

$\mathrm{KSEL}, \mathrm{S}, \mathrm{KP}$, ,KP2+16,KP2+41,25,

LSLK,S,1

LESIZE,ALL,4.4, ,0, ,1, , ,0

ALLSEL,ALL

LSTR, KP2+16,KP2+83

$\mathrm{KSEL}, \mathrm{S}, \mathrm{KP}$, ,KP2+16,KP2+83,67,

LSLK,S,1

LESIZE,ALL,4.4, ,0, ,1, , ,0

ALLSEL,ALL

LSTR,KP2+41,KP2+42

$\mathrm{KSEL}, \mathrm{S}, \mathrm{KP}$, ,KP2+41,KP2+42,1,

LSLK,S,1

LESIZE,ALL,4.4, , 0, ,1, , ,0

ALLSEL,ALL

LSTR,KP2+33,KP2+81

$\mathrm{KSEL}, \mathrm{S}, \mathrm{KP}$, ,KP2+33,KP2+81,48, 


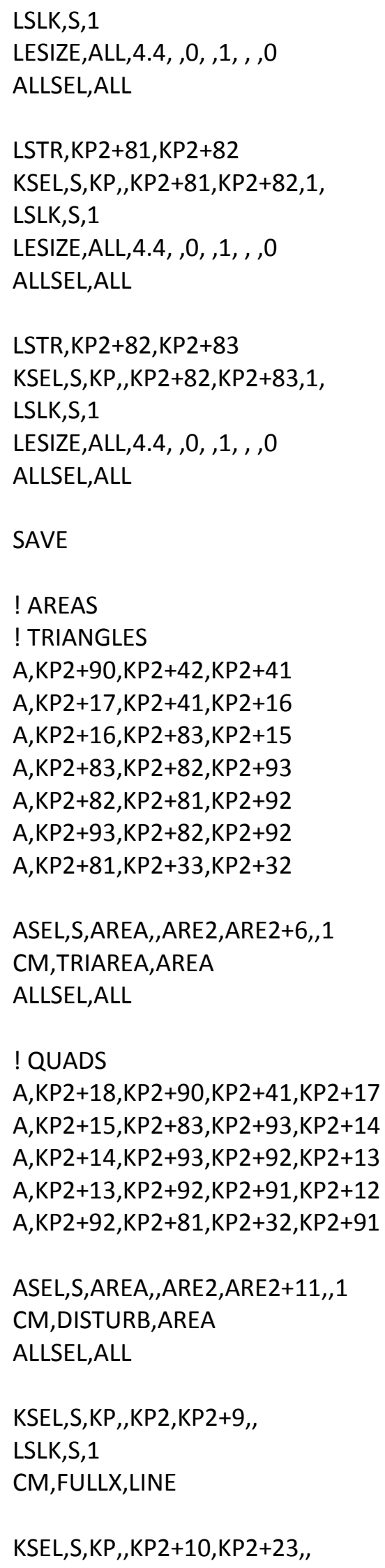




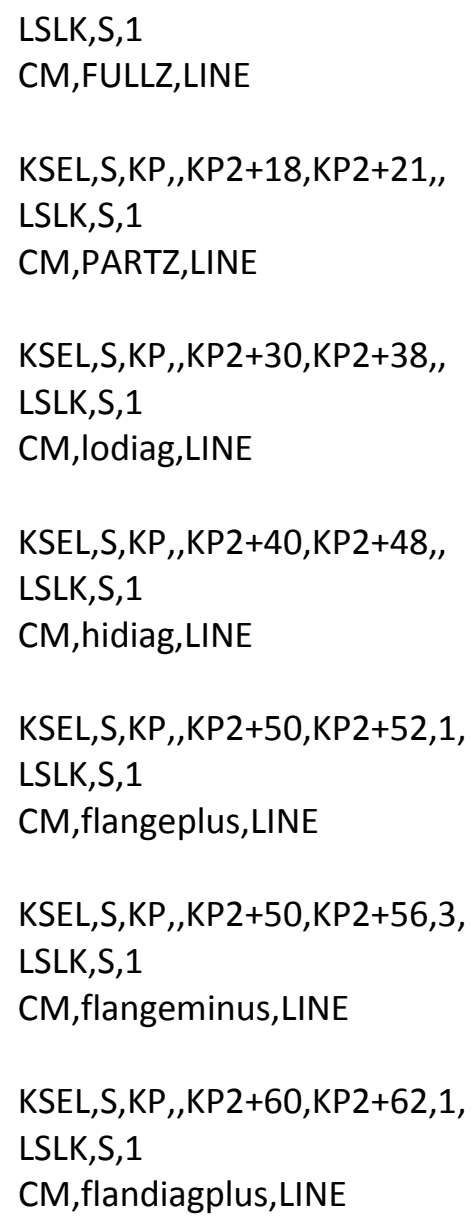


*ENDDO

ASEL,S,AREA,,ARE2,ARE2+16,,1

CM,DIAWEBTURB,AREA ! DIAGONAL WEB AND DISTURBED AREAS=D

ALLSEL,ALL

! areas

ADRAG,lodiag,,,,,,, flangeplus,,,,,,

ASEL,S,AREA,,ARE2,ARE2+32,,1

CM,DLOPLUS,AREA ! DIAGONAL WEB,DISTURBED AND LOW PLUS DIAG FLANGE AREAS

ALLSEL,ALL

ADRAG, lodiag, ,,,,, flangeminus,,,,,

ASEL,S,AREA,,ARE2,ARE2+48,,1

CM,DLODIAG,AREA ! DIAGONAL WEB,DISTURBED AND LOW DIAG FLANGE AREAS

ALLSEL,ALL

ADRAG, hidiag, ,,,,,, flandiagplus,,,,,,

ASEL,S,AREA,,ARE2,ARE2+64,,1

CM,DLOHIPLUS,AREA ! DIAGONAL WEB,DISTURBED,LOW DIAG FLANGE AND HIGH PLUS DIAG FLANGE AREAS

ALLSEL,ALL

ADRAG, hidiag, ,,,,,, flandiagminus,,,,,,

ASEL,S,AREA,,ARE2,ARE2+80,,1

CM,DLOHI,AREA ! DIAGONAL WEB,DISTURBED,LOW AND HIGH DIAG FLANGE AREAS

ALLSEL,ALL

ADRAG,FULLZ,,,,,,,flangeplus,,,,,,

ADRAG,FULLZ,,,,,, flangeminus,,,,,,

ADRAG,partz,,,,,,, webvert,,,,,,

ADRAG, full $x,,,,,$, , flangeplus,,,,,,

ADRAG, fullx,,,,,, flangeminus,,,,,,

ADRAG,fullx,,,,,,WEBHORIZ,,,,,,

ALLSEL,ALL

SAVE

! mesh webS

! MESH TRIANGLES

! much simpler to define elements by commands NKPT and $\mathrm{E}$

CMSEL,S,TRIAREA,AREA

AATT, 10, 5, 13,4

MSHAPE,1,2D

MSHKEY,0

AMESH,ALL

! MESH QUADS 


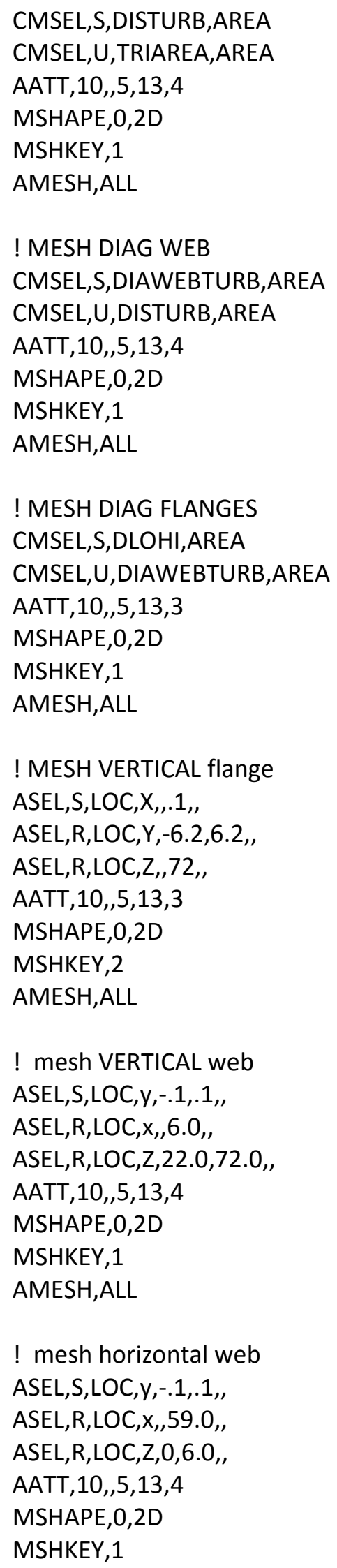




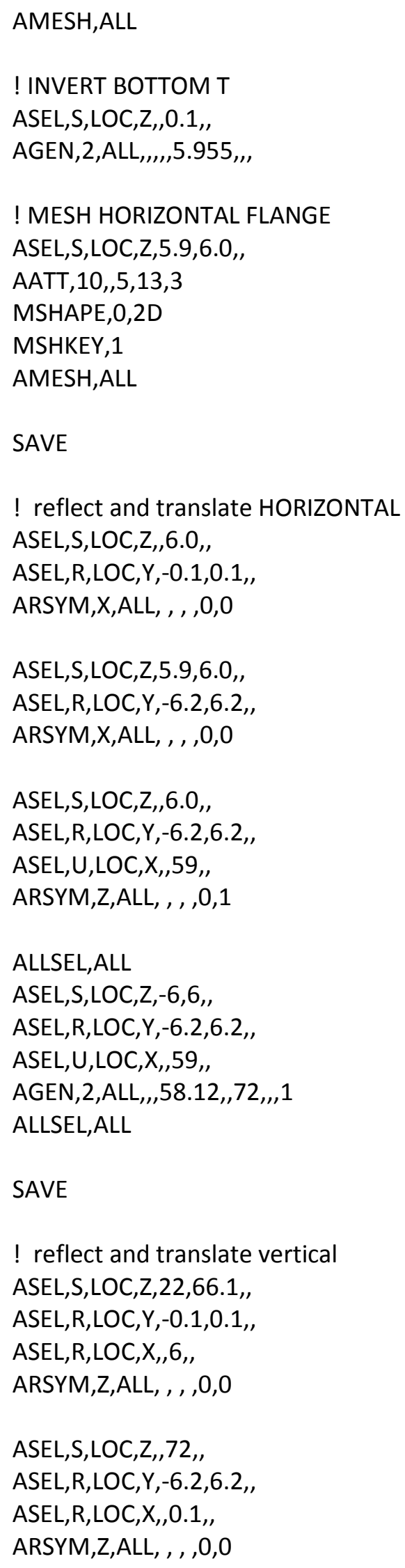




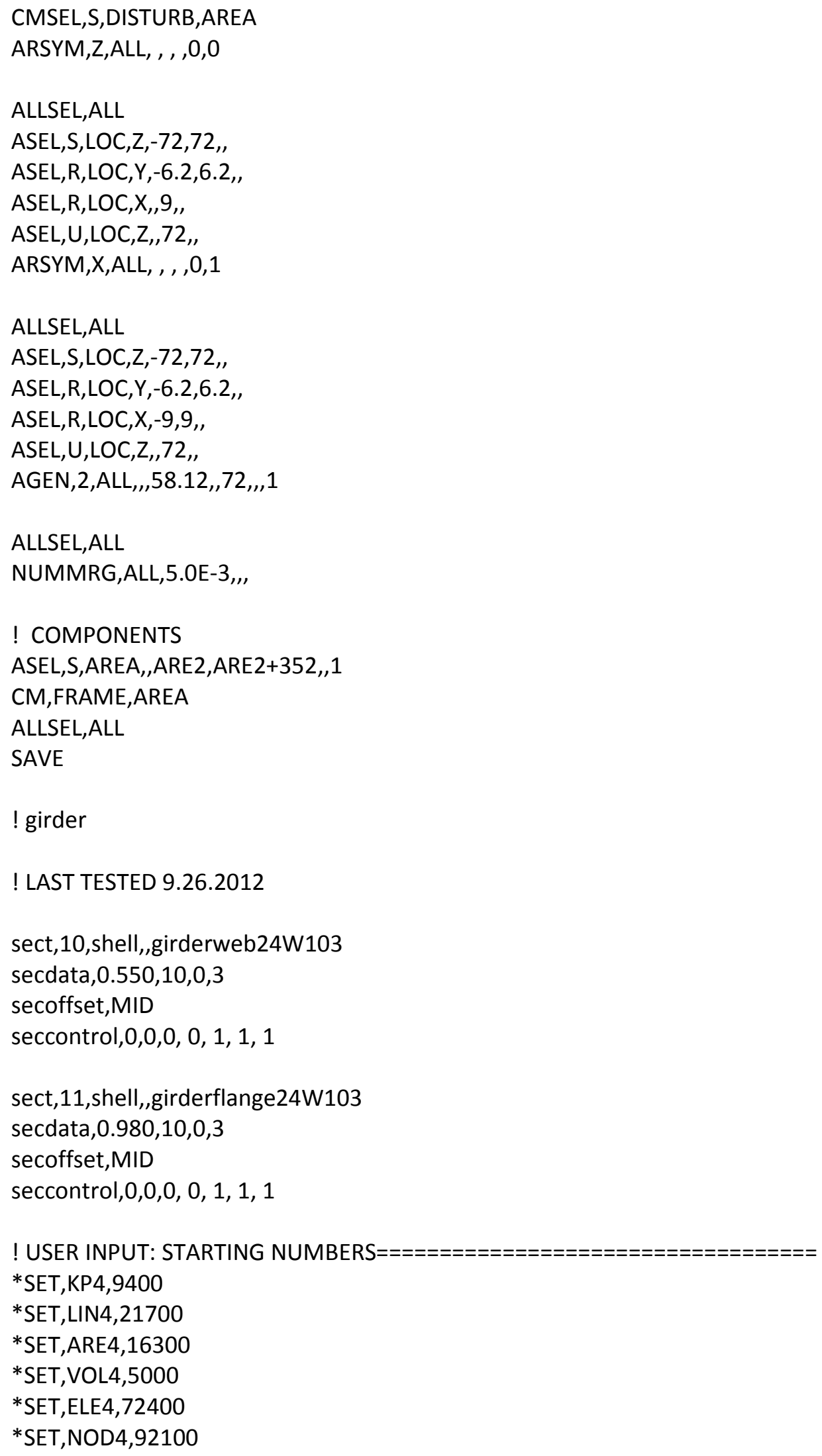


! \# define model geometry

! user input:local coord system and location of girder

! ref: SHEET 12/15

! Local X ALONG midheight of web.

! Local Y perpendicular to web, right hand system.

! Local Z ALONG left vertical EDGE OF web.

!LOCAL,15,0,342,530.28,361, , , ,1,1

LOCAL,15,0,342,530.28,359.77, , , , 1,1

CSYS,15,

LENGTH $=186.0$ !half length of girder, must be $>126$ and multiple of 3 inches.

!If length $>186$, user may have to increase element and node starting numbers of subsequent

components.

!LENGTH $=162.0$

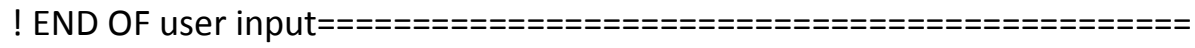

NUMSTR,KP,KP4

NUMSTR,LINE,LIN4

NUMSTR,AREA,ARE4

NUMSTR,VOLU,VOL4

NUMSTR,ELEM,ELE4

NUMSTR,NODE,NOD4

! $\mathrm{x}$ horiz midheight of web, halfspan

$\mathrm{K}, \mathrm{KP} 4,,$,

$\mathrm{K}, \mathrm{KP} 4+1,12.0$,

$\mathrm{K}, \mathrm{KP} 4+2,13.0$,

$\mathrm{K}, \mathrm{KP} 4+3,14.0$,

$\mathrm{K}, \mathrm{KP} 4+4,16.0$,

$\mathrm{K}, \mathrm{KP} 4+5,18.0$,

$\mathrm{K}, \mathrm{KP} 4+6, \mathrm{LENGTH}$, !***

! Z vert midthickness of web

$\mathrm{K}, \mathrm{KP} 4+10,,$,

$\mathrm{K}, \mathrm{KP} 4+11,,, 9$

$\mathrm{K}, \mathrm{KP} 4+12,,, 11.775$

! y width of half flange

K,KP4+20,,,11.775

$\mathrm{K}, \mathrm{KP} 4+21,,-4.5,11.775$

! y width of full flange

$\mathrm{K}, \mathrm{KP} 4+30,18,4.5,11.775$

$\mathrm{K}, \mathrm{KP} 4+31,18,-4.5,11.775$

! FLANGE TRANSITION KEYPOINTS

K,KP4+40,13, -4.5,11.775 


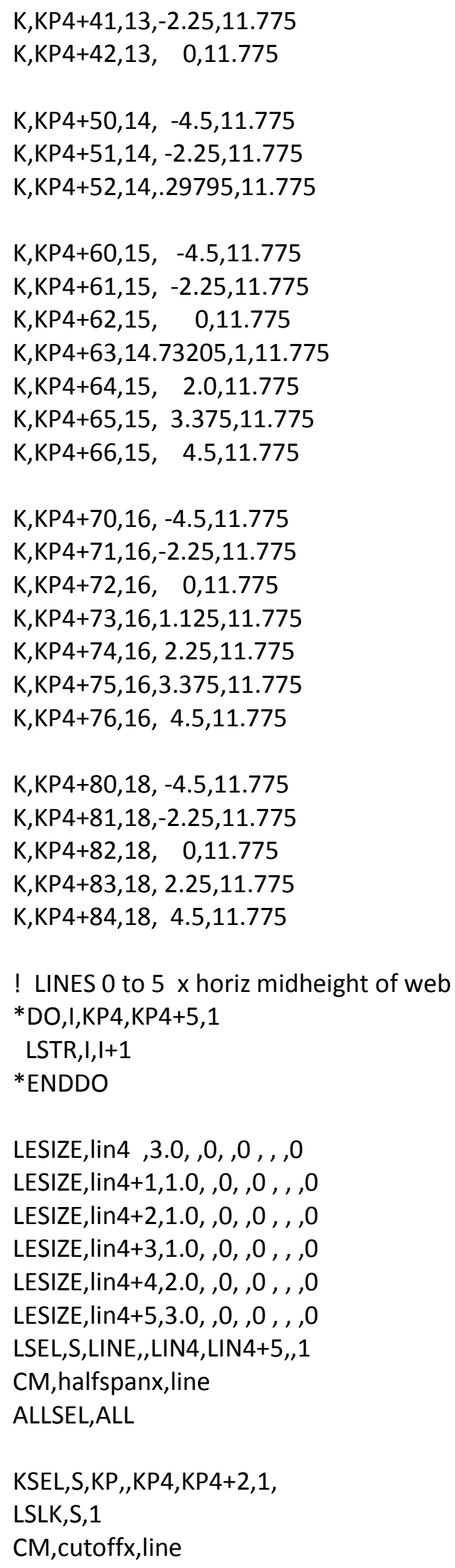


allsel,all

KSEL,S,KP, KP4+5,KP4+6,1,

LSLK,S,1

$\mathrm{CM}$,fullwidthx,line

allsel,all

! LINES 6 to $7 \mathrm{z}$ vert midthickness of half web

*DO,I,KP4+10,KP4+11,1

LSTR,I,I+1

*ENDDO

LESIZE, lin4+6,3.0, ,0, ,0 , , ,0

LESIZE,lin4+7, 0, ,1, ,0 , , ,0

KSEL,S,KP, KP4+10,KP4+12,1,

LSLK,S,1

CM, webdepthz,line

allsel,all

LSTR,KP4+20,KP4+21 ! L8 y width of half flange

LESIZE, lin4+8,0, ,2, , 0 , , , 0

LSEL,S,LINE,,LIN4+8,,,1

$\mathrm{CM}$, halfwidthy, line

allsel,all

LSTR,KP4+30,KP4+31 ! L9 y width of full flange

LESIZE, lin4+9,0, ,4, ,0 , , ,0

allsel,all

LSEL,S,LINE,,LIN4+9,,,1

$\mathrm{CM}$,fullwidthy, line

ALLSEL,ALL

CMSEL,S, cutoffx, line

LGEN,2,ALL,,,,,11.775,,, ! L10 TO 11

LSEL,S,LINE,,LIN4+10,LIN4+11,1,1

CM,cutoff $x 2$, line

allsel,all

CMSEL,S, fullwidthx,line

LGEN,2,ALL,,,,,4.5,11.775,,,, ! L12 TO 13

LSEL,S,LINE,,LIN4+12,LIN4+13,,1

$\mathrm{CM}$,fullwidthx2,line

allsel,all

LSEL,S,LINE,,LIN4,LIN4+12,1,1

CM,REGULAR,LINE

allsel,all 
! LINES IN CUTOFF REGION L14 TO L56

! Y LINES

LSTR,KP4+40,KP4+41

LSTR, KP4+41,KP4+42

LSTR,KP4+50,KP4+51

LSTR,KP4+51,KP4+52

*DO,I,KP4+60,KP4+65,1

LSTR,I,I+1

*ENDDO

*DO,I,KP4+70,KP4+75,1

LSTR,I,I+1

*ENDDO

*DO,I,KP4+80,KP4+83,1

LSTR,I,I+1

*ENDDO

! X LINES

*DO,I,KP4+40,KP4+70,10

LSTR, I,I+10

*ENDDO

*DO,I,KP4+41,KP4+71,10

LSTR, I,I+10

*ENDDO

*DO,I,KP4+42,KP4+72,10

LSTR, I,I+10

*ENDDO

LSTR,KP4+62,KP4+63

LSTR,KP4+63,KP4+52

LSTR,KP4+63,KP4+73

LSTR,KP4+73,KP4+82

LSTR,KP4+73,KP4+83

LSTR,KP4+64,KP4+74

LSTR,KP4+74,KP4+83

LSTR,KP4+65, KP4+75

LSTR,KP4+75,KP4+83

LSTR,KP4+75,KP4+84

LSTR,KP4+66,KP4+76

LSTR,KP4+76,KP4+84

ALLSEL,ALL 
LSEL,S,LINE,,LIN4,LIN4+55,,1

CM,ALLINES,LINES

CMSEL,S,ALLINES,LINE

CMSEL,U,REGULAR,LINE

LESIZE,ALL, $0,1,, 0$, , , 0

CM,IRREGULAR,LINE

ALLSEL,ALL

! areas

*DO,I,KP4+40,KP4+70,10

$A, I, I+10, I+11, I+1$

*ENDDO

*DO,I,KP4+41,KP4+71,10

$A, I, I+10, I+11, I+1$

*ENDDO

$\mathrm{A}, \mathrm{KP} 4+52, \mathrm{KP} 4+62, \mathrm{KP} 4+63$

$\mathrm{A}, \mathrm{KP} 4+62, \mathrm{KP} 4+72, \mathrm{KP} 4+73, \mathrm{KP} 4+63$

$\mathrm{A}, \mathrm{KP} 4+72, \mathrm{KP} 4+82, \mathrm{KP} 4+73$

A, KP4+73,KP4+82,KP4+83

$\mathrm{A}, \mathrm{KP} 4+63, \mathrm{KP} 4+73, \mathrm{KP} 4+74, \mathrm{KP} 4+64$

$\mathrm{A}, \mathrm{KP} 4+73, \mathrm{KP} 4+83, \mathrm{KP} 4+74$

$\mathrm{A}, \mathrm{KP} 4+64, \mathrm{KP} 4+74, \mathrm{KP} 4+75, \mathrm{KP} 4+65$

$\mathrm{A}, \mathrm{KP} 4+74, \mathrm{KP} 4+83, \mathrm{KP} 4+75$

$\mathrm{A}, \mathrm{KP} 4+75, \mathrm{KP} 4+83, \mathrm{KP} 4+84$

$\mathrm{A}, \mathrm{KP} 4+65, \mathrm{KP} 4+75, \mathrm{KP} 4+76, \mathrm{KP} 4+66$

A, KP4+75, KP4+84, KP4+76

ADRAG, halfspanx $,,, \ldots,$, , webdepthz,,,,,, ! ! Areas 0 to 9

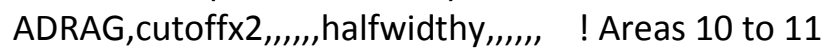

ADRAG,fullwidthx2,,,,,, fullwidthy,,,,,, ! Areas 12 to 13

! MESH WEB

ASEL,S,LOC,Y,-0.1,0.1,,1

ASEL,R,LOC,Z,0,11.7,,

AATT,10, 5, 15,10

MSHAPE,0,2D

MSHKEY,1

AMESH,ALL

ALLSEL,ALL

! MESH FLANGE

ASEL,S,LOC,Z,11.7,11.8,,1

ASEL,U,LOC,X,13,18,1

AATT, 10, 5, 15,11

MSHAPE,0,2D 


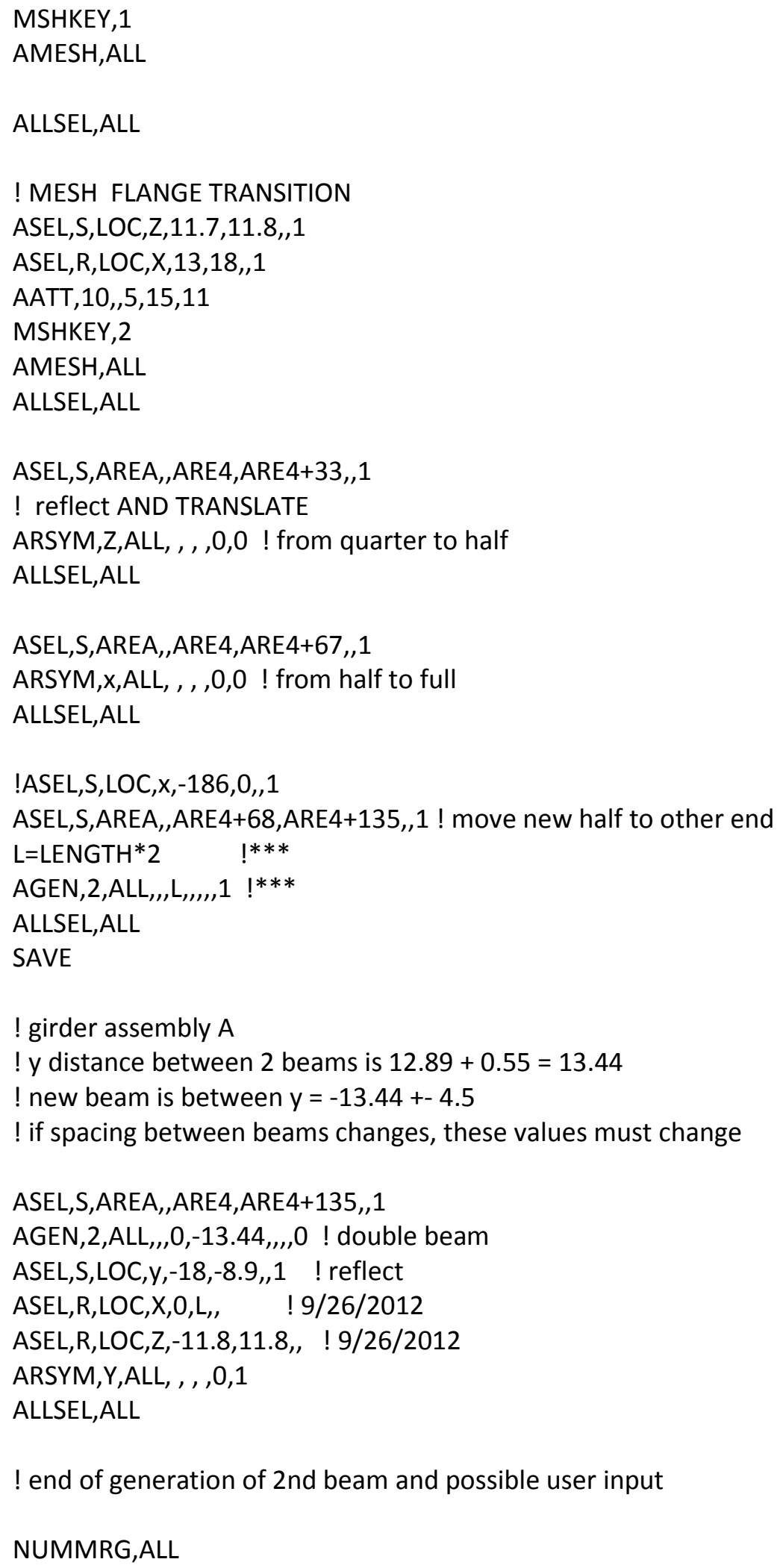


! best to move or duplicate girder without constraints, then reapply constraints at new location.

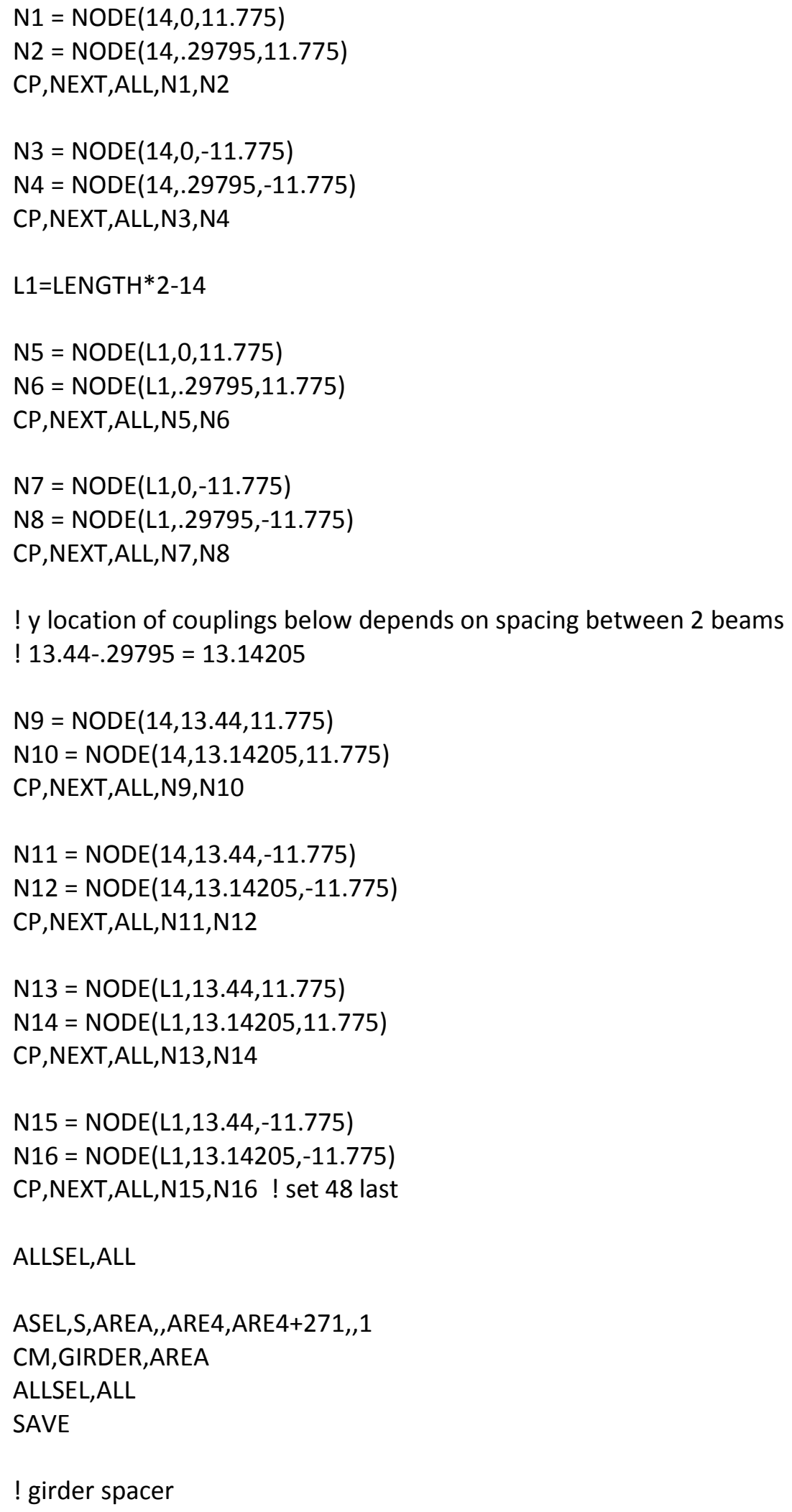


ET,6,BEAM188

SECTYPE, 15, BEAM, CTUBE, SCH80, 0

SECOFFSET, CENT

SECDATA $0.75,0.95,0,0,0,0,0,0,0,0$ ! WHERE IS MAT DEFINED?

! USER INPUT: STARTING NUMBERS=======================================

*SET,KP6,10100

*SET,LIN6,22800

*SET,ARE6,16800

*SET,VOL6,5000

*SET,ELE6,79100

*SET,NOD6,101200

! \# define model geometry

! user input:local coord system and location of girder

! ref: SHEET 14/15

! Local X ALONG flange.

! Local Y along web.

! Local Z ALONG length of spacer.

! origin at midthickness JUNCTION WEB-FLANGE.

!LOCAL,17,0,108,519.05,350.3, , , , 1,1

LOCAL,17,0,108,519.05,349.07, , , , 1,1

CSYS, 17,

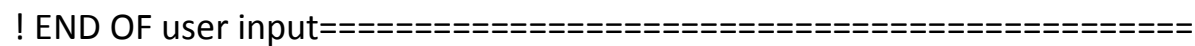

NUMSTR,KP,KP6

NUMSTR,LINE,LIN6

NUMSTR,AREA,ARE6

NUMSTR,VOLU,VOL6

NUMSTR,ELEM,ELE6

NUMSTR,NODE,NOD6

$\mathrm{K}, \mathrm{KP} 6,-6.11$,

K,KP6+1,-3.0,,

$\mathrm{K}, \mathrm{KP} 6+2,,$,

$\mathrm{K}, \mathrm{KP} 6+3,3.0$,

$\mathrm{K}, \mathrm{KP} 6+4,6.11$,

$\mathrm{K}, \mathrm{KP} 6+5,11.9$,

$\mathrm{K}, \mathrm{KP} 6+6,,, 1.7$

$\mathrm{K}, \mathrm{KP} 6+7,,, 19.7$

$\mathrm{K}, \mathrm{KP} 6+8,,, 21.4$

! LINES $\mathrm{X}$

LSTR,KP6 ,KP6+1

LSTR, KP6+1,KP6+2

LSTR,KP6+2,KP6+3 


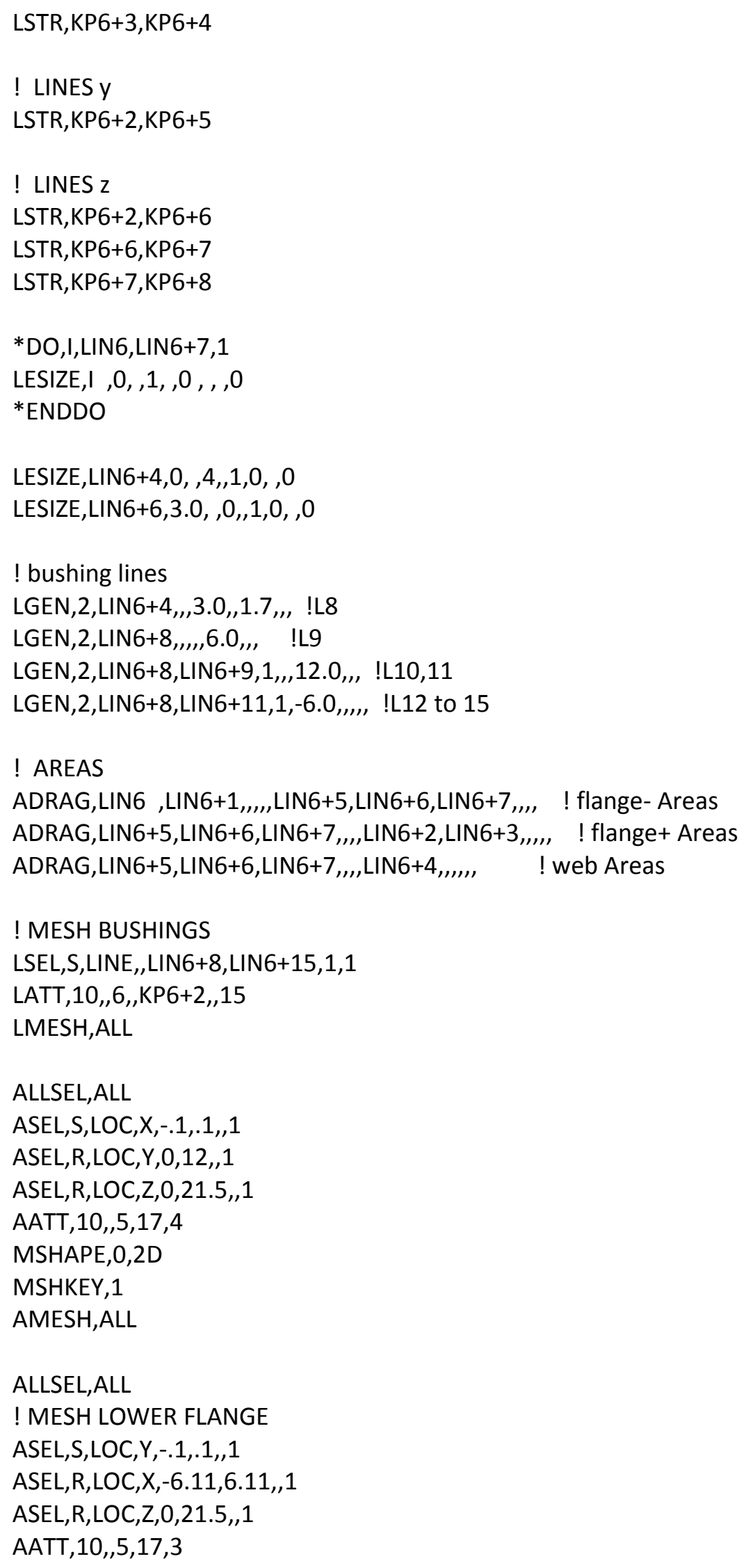




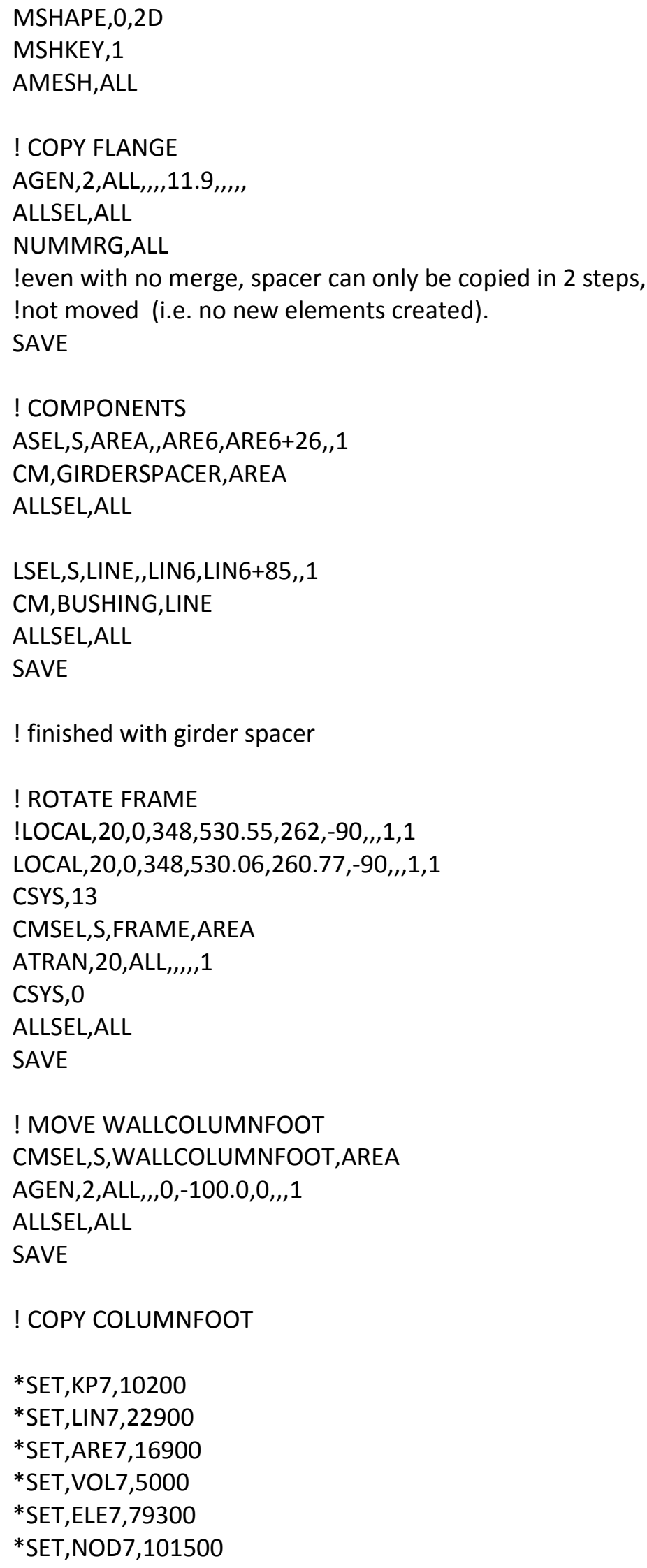




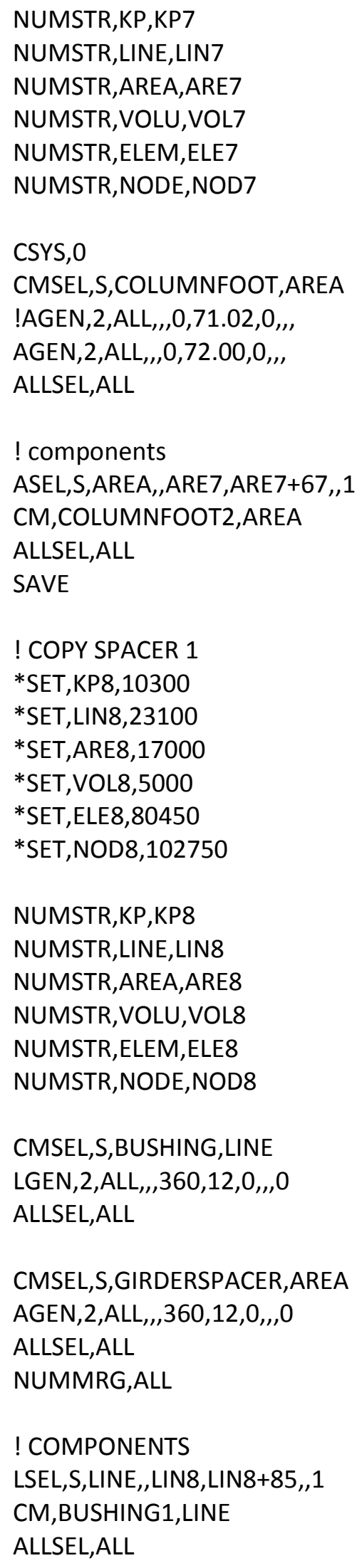




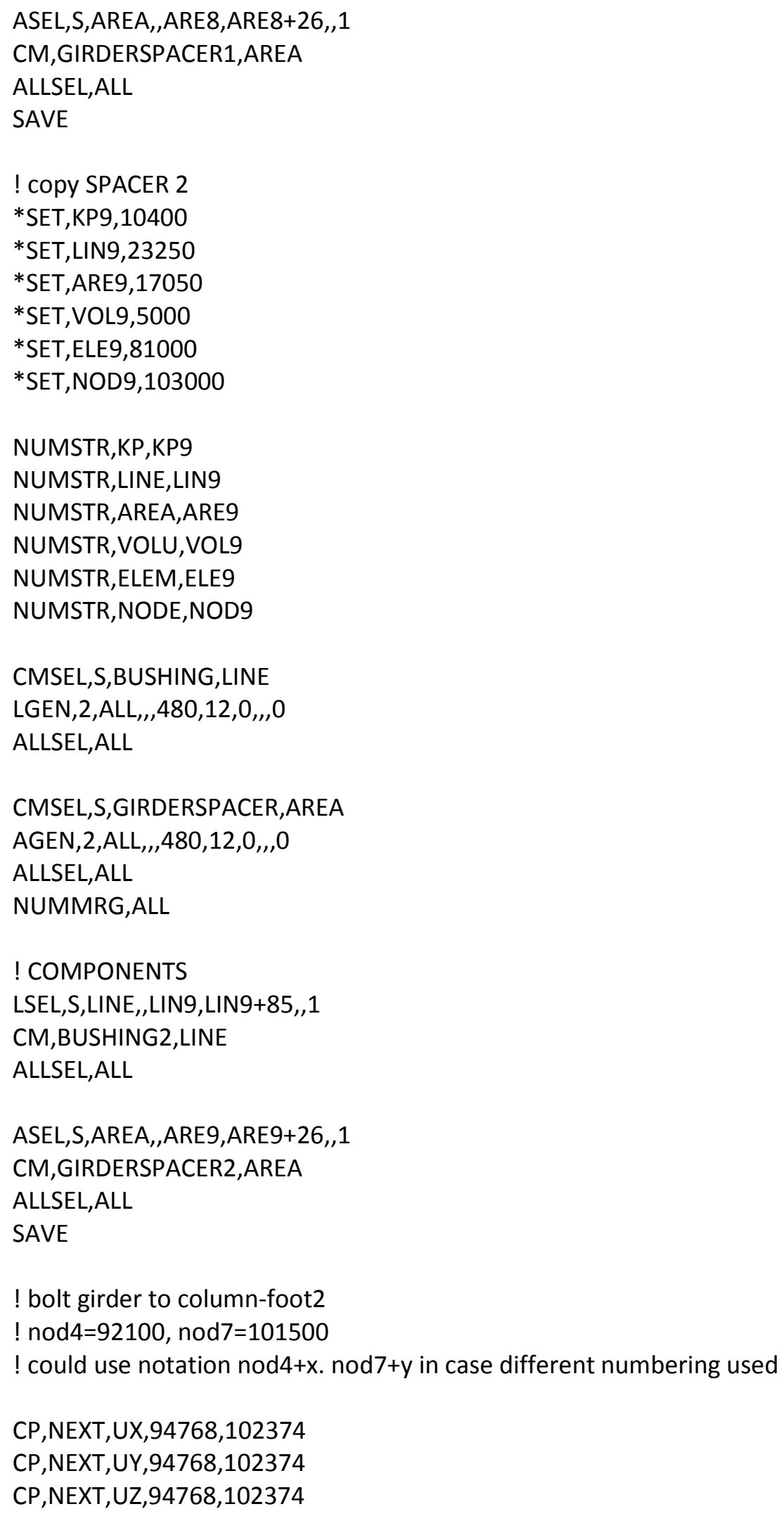


CP,NEXT,UX,95426,102368

CP,NEXT,UY,95426,102368

CP,NEXT,UZ,95426,102368

CP,NEXT,UX,94759,102137

CP,NEXT,UY,94759,102137

CP,NEXT,UZ,94759,102137

CP,NEXT,UX,94766,102140

CP,NEXT,UY, 94766,102140

CP,NEXT,UZ,94766,102140

CP,NEXT,UX,95424,102134

CP,NEXT,UY,95424,102134

CP,NEXT,UZ,95424,102134

CP,NEXT,UX,94761,102371

CP,NEXT,UY, 94761,102371

CP,NEXT,UZ,94761,102371

CP,NEXT,UX, 92794,101968

CP,NEXT,UY,92794,101968

CP,NEXT,UZ,92794,101968

CP,NEXT,UX,92111,101974

CP,NEXT,UY,92111,101974

CP,NEXT,UZ,92111,101974

CP,NEXT,UX,92104,101971

CP,NEXT,UY,92104,101971

CP,NEXT,UZ,92104,101971

CP,NEXT,UX,92792,101734

CP,NEXT,UY,92792,101734

CP,NEXT,UZ,92792,101734

CP,NEXT,UX,92102,101737

CP,NEXT,UY,92102,101737

CP,NEXT,UZ,92102,101737

CP,NEXT,UX,92109,101740

CP,NEXT,UY,92109,101740

CP,NEXT,UZ,92109,101740 ! set 84 last

! bolt girder to wall-column

$! \operatorname{nod} 4=92100, \operatorname{nod} 0=88000$

! could use notation nod $4+x$. nod $0+y$ in case different numbering used 
CP,NEXT,UX,94108,88550

CP,NEXT,UY, 94108,88550

CP,NEXT,UZ,94108,88550

CP,NEXT,UX, 93443,88553

CP,NEXT,UY, 93443,88553

CP,NEXT,UZ,93443,88553

CP,NEXT,UX,93450,88556

CP,NEXT,UY,93450,88556

CP,NEXT,UZ,93450,88556

CP,NEXT,UX,96740,88345

CP,NEXT,UY, 96740,88345

CP,NEXT,UZ,96740,88345

CP,NEXT,UX, 96075,88348

CP,NEXT,UY, 96075,88348

CP,NEXT,UZ, 96075,88348

CP,NEXT,UX,96082,88351

CP,NEXT,UY,96082,88351

CP,NEXT,UZ,96082,88351

CP,NEXT,UX,96742,88231

CP,NEXT,UY,96742,88231

CP,NEXT,UZ,96742,88231

CP,NEXT,UX,96077,88234

CP,NEXT,UY, 96077,88234

CP,NEXT,UZ,96077,88234

CP,NEXT,UX,96084,88237

CP,NEXT,UY, 96084,88237

CP,NEXT,UZ,96084,88237

CP,NEXT,UX, 94110,88436

CP,NEXT,UY,94110,88436

CP,NEXT,UZ,94110,88436

CP,NEXT, UX,93445,88439

CP,NEXT,UY, 93445,88439

CP,NEXT,UZ,93445,88439

CP,NEXT,UX, 93452,88442

CP,NEXT,UY, 93452,88442

CP,NEXT,UZ,93452,88442 ! set 120 last 


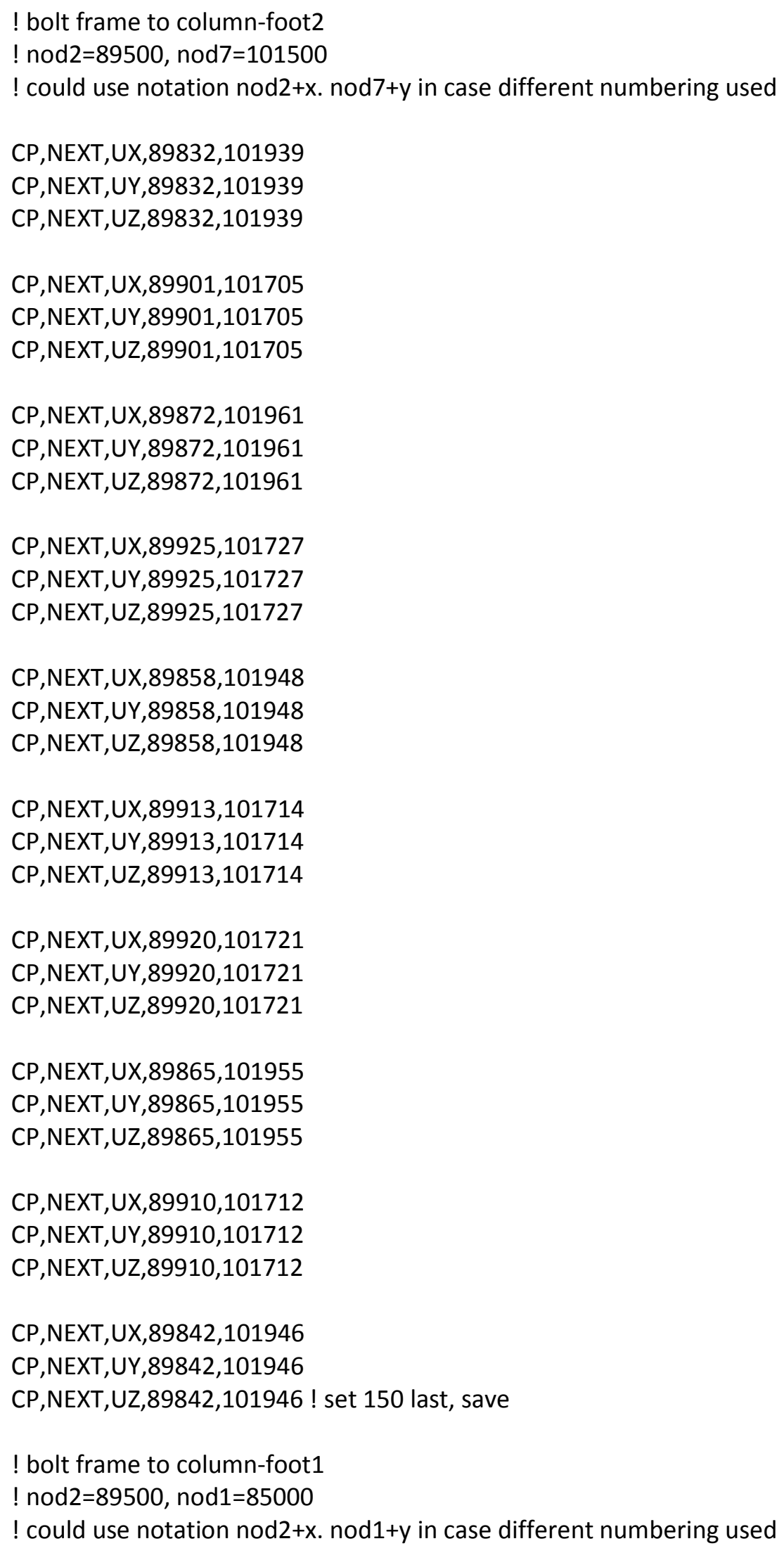


CP,NEXT,UX,90371,86261

CP,NEXT,UY,90371,86261

CP,NEXT,UZ,90371,86261

CP,NEXT,UX,90447,86027

CP,NEXT,UY, 90447,86027

CP,NEXT,UZ,90447,86027

CP,NEXT,UX,90388,86254

CP,NEXT,UY,90388,86254

CP,NEXT,UZ,90388,86254

CP,NEXT,UX, 90456,86022

CP,NEXT,UY, 90456,86022

CP,NEXT,UZ,90456,86022

CP,NEXT,UX,90418,86239

CP,NEXT,UY, 90418,86239

CP,NEXT,UZ,90418,86239

CP,NEXT,UX,90471,86005

CP,NEXT,UY,90471,86005

CP,NEXT,UZ,90471,86005

CP,NEXT,UX,90411,86245

CP,NEXT,UY, 90411,86245

CP,NEXT,UZ,90411,86245

CP,NEXT,UX,90466,86011

CP,NEXT,UY,90466,86011

CP,NEXT,UZ,90466,86011

CP,NEXT,UX,90406,86250

CP,NEXT,UY, 90406,86250

CP,NEXT,UZ,90406,86250

CP,NEXT,UX,90461,86016

CP,NEXT,UY,90461,86016

CP,NEXT,UZ,90461,86016 ! set 180 last, save

! connect girder to spacer 1

CP,NEXT,UX,92235,102777

CP,NEXT,UY, 92235,102777

CP,NEXT,UZ,92235,102777

CP,NEXT,UX, 92918,102750

CP,NEXT,UY,92918,102750 
CP,NEXT,UZ,92918,102750

CP,NEXT,UX,95550,102751

CP,NEXT,UY,95550,102751

CP,NEXT,UZ,95550,102751

CP,NEXT,UX,94892,102778

CP,NEXT,UY,94892,102778

CP,NEXT,UZ,94892,102778

CP,NEXT,UX,94890,102814

CP,NEXT,UY,94890,102814

CP,NEXT,UZ,94890,102814

CP,NEXT,UX,95548,102787

CP,NEXT,UY,95548,102787

CP,NEXT,UZ,95548,102787

CP,NEXT,UX,92916,102786

CP,NEXT,UY,92916,102786

CP,NEXT,UZ,92916,102786

CP,NEXT,UX, 92233,102813

CP,NEXT,UY, 92233,102813

CP,NEXT,UZ,92233,102813 ! set 204 last, save

! connect girder to spacer 2

CP,NEXT,UX,96206,103028

CP,NEXT,UY,96206,103028

CP,NEXT,UZ,96206,103028

CP,NEXT,UX,93574,103027

CP,NEXT,UY,93574,103027

CP,NEXT,UZ,93574,103027

CP,NEXT,UX,96864,103001

CP,NEXT,UY,96864,103001

CP,NEXT,UZ,96864,103001

CP,NEXT,UX,94232,103000

CP,NEXT,UY,94232,103000

CP,NEXT,UZ,94232,103000

CP,NEXT,UX,96208,103064

CP,NEXT,UY,96208,103064

CP,NEXT,UZ,96208,103064 
CP,NEXT,UX,96866,103037

CP,NEXT,UY,96866,103037

CP,NEXT,UZ,96866,103037

CP,NEXT,UX,93576,103063

CP,NEXT,UY, 93576,103063

CP,NEXT,UZ,93576,103063

CP,NEXT,UX,94234,103036

CP,NEXT,UY,94234,103036

CP,NEXT,UZ,94234,103036 ! set 228 last, save

save

!ET,7,MPC184

! BOLT foot 2 (at end of girder) to strong floor ! southwest

CP,NEXT,UX,101617,45796

CP,NEXT,UY,101617,45796

CP,NEXT,UZ,101617,45796

! northwest

CP,NEXT,UX,101635,45798

CP,NEXT,UY, 101635,45798

CP,NEXT,UZ,101635,45798

! southeast

CP,NEXT,UX,101503,45750

CP,NEXT,UY,101503,45750

CP,NEXT,UZ,101503,45750

! Northeast

CP,NEXT,UX,101529,45752

CP,NEXT,UY,101529,45752

CP,NEXT,UZ,101529,45752

! BOLT foot 1 to strong floor

! southwest

CP,NEXT,UX,85117,45680

CP,NEXT,UY, 85117,45680

CP,NEXT,UZ,85117, 45680

! northwest

CP,NEXT,UX,85135,45682

CP,NEXT,UY, 85135,45682

CP,NEXT,UZ,85135, 45682 
! southeast

CP,NEXT,UX,85003,45654

CP,NEXT,UY,85003,45654

CP,NEXT,UZ,85003,45654

! Northeast

CP,NEXT,UX,85029,45656

CP,NEXT,UY,85029,45656

CP,NEXT,UZ,85029,45656

! bolt (UPPER)pad 2 to strong wall

! downwest

CP,NEXT,UX,88930,76450

CP,NEXT,UY,88930,76450

CP,NEXT,UZ,88930,76450

! upwest

CP,NEXT,UX,89012,76452

CP,NEXT,UY,89012,76452

CP,NEXT,UZ,89012,76452

! downeast

CP,NEXT,UX,88904,76320

CP,NEXT,UY,88904,76320

CP,NEXT,UZ,88904,76320

! upeast

CP,NEXT,UX,88940,76322

CP,NEXT,UY,88940,76322

CP,NEXT,UZ,88940,76322

! bolt (LOWER)pad 1 to strong wall

! downwest

CP,NEXT,UX,88683,76444

CP,NEXT,UY,88683,76444

CP,NEXT,UZ,88683,76444

! upwest

CP,NEXT,UX,88765,76446

CP,NEXT,UY,88765,76446

CP,NEXT,UZ,88765,76446

! downeast

CP,NEXT,UX,88657,76314

CP,NEXT,UY,88657,76314

CP,NEXT,UZ,88657,76314

! upeast 
CP,NEXT,UX,88693,76316

CP,NEXT,UY,88693,76316

CP,NEXT,UZ,88693,76316

save

/SOLU

ANTYPE,0 ! static analysis

! constrain bottom of slab

ASEL,S,LOC,Z,0,1

ASEL, R, LOC, $X, 24,888$

$D A, A L L, U X, 0$

DA,ALL,UY,O

$\mathrm{DA}, \mathrm{ALL}, \mathrm{UZ}, 0$

! release constraints at prestressing anchors

CMSEL,S,PSWALL_BOTTOM,LINE

DLDELE,ALL,ALL

! constrain axial rotation of loading beams as SOLID185 has no rotation DOF

$N=\operatorname{NODE}(174,18,153) \quad !$ PSFLOOR_EAST

$D, N, R O T X, \ldots, \ldots, \ldots$,

$N=\operatorname{NODE}(174,1114,153) !$ PSFLOOR_WEST

$D, N, R O T X,,,,,,,,$,

$N=\operatorname{NODE}(747,921,540) \quad !$ PSWALL_TOP

$\mathrm{D}, \mathrm{N}, \mathrm{ROTY}, \ldots, \ldots, \ldots,$,

$\mathrm{N}=\operatorname{NODE}(747,921,0) \quad$ ! PSWALL_BOTTOM

D,N,ROTY,,,,,',,',

$N=\operatorname{NODE}(0,978,153) \quad !$ PSFLOOR_SOUTH

$\mathrm{D}, \mathrm{N}, \mathrm{ROTY},,,,,, \ldots,$,

$N=\operatorname{NODE}(720,978,153) \quad !$ PSFLOOR_NORTHWEST

D,N,ROTY,,,,,,,,, ,

$N=\operatorname{NODE}(774,921,153) \quad$ ! PSFLOOR_NORTHCENTRAL

$\mathrm{D}, \mathrm{N}, \mathrm{ROTY},,,,,,,,,$,

$N=\operatorname{NODE}(720,201,153) \quad !$ PSFLOOR_NORTHEAST

$\mathrm{D}, \mathrm{N}, \mathrm{ROTY},,,,, \ldots,,$,

$N=\operatorname{NODE}(720,201,180) \quad !$ WALL_FLOOR

$\mathrm{D}, \mathrm{N}, \mathrm{ROTY},,,,, \ldots,,$,

! LS1 = GRAVITY ONLY

ALLSEL,ALL 
TIME,1

LSWRITE,1

! LS2 = APPLY UNBALANCED POST-TENSIONING LINE PRESSURE ON BEAMS ! GRAVITY UNTOUCHED AND REMAINS

CMSEL,S,PSWALL_TOP,LINE

ESLL,S

CM,TOP_BEAM,ELEM

SFBEAM,ALL,1,PRES,-19.65,-19.65, , , , ,

CMSEL,S,PSFLOOR_EAST,LINE

ESLL,S

CM,EAST_BEAM,ELEM

SFBEAM,ALL,1,PRES, $-4.08,-4.08$, , , , ,

CMSEL,S,PSFLOOR_SOUTH,LINE

ESLL,S

CM,SOUTH_BEAM,ELEM

SFBEAM,ALL,1,PRES,-4.08,-4.08, , , , ,

ALLSEL,ALL

TIME,2

LSWRITE,2

! LS3 = APPLY BALANCE OF POST-TENSIONING LINE PRESSURE ON BEAMS

! GRAVITY UNTOUCHED AND REMAINS

CMSEL,S,PSWALL_BOTTOM,LINE

ESLL,S

CM,BOTTOM_BEAM,ELEM

SFBEAM,ALL,1,PRES,-19.65,-19.65, , , , ,

CMSEL,S,PSFLOOR_WEST,LINE

ESLL,S

CM,WEST_BEAM,ELEM

SFBEAM,ALL,1,PRES, $-4.08,-4.08$, , , , ,

CMSEL,S,PSFLOOR_NORTH,LINE

ESLL,S

CM,NORTH_BEAM,ELEM

SFBEAM,ALL,1,PRES, $-4.08,-4.08$, , , , ,

ALLSEL,ALL

TIME,3

LSWRITE,3

! LOAD support 
!ACEL,,,1 ! gravity $-\mathrm{z}$

! LOAD TOP OF COLUMN 2 AT JUNCTIONS WEB-FLANGE

! Nod7=101500 could be used in notation

$\mathrm{F}, 101835, \mathrm{Fx},-2,$,

$\mathrm{F}, 101835, \mathrm{FY},-2,,$,

$\mathrm{F}, 101835, \mathrm{FZ},-2,,$,

$\mathrm{F}, 102235, \mathrm{Fx},-2,,$,

$\mathrm{F}, 102235, \mathrm{FY},-2$, ,,

$F, 102235, F Z,-2$,,,

ALLSEL,ALL

TIME, 4

LSWRITE,4

! UNSELECT CONSTRUCTION SPACER

ESEL,U,ELEM,,ELE6,ELE6+127,,

OUTPR,STAT

OUTRES,STAT

SAVE

LSSOLVE,1,4,1

/eof 


\section{Appendix}

\section{Modular Support Drawings}



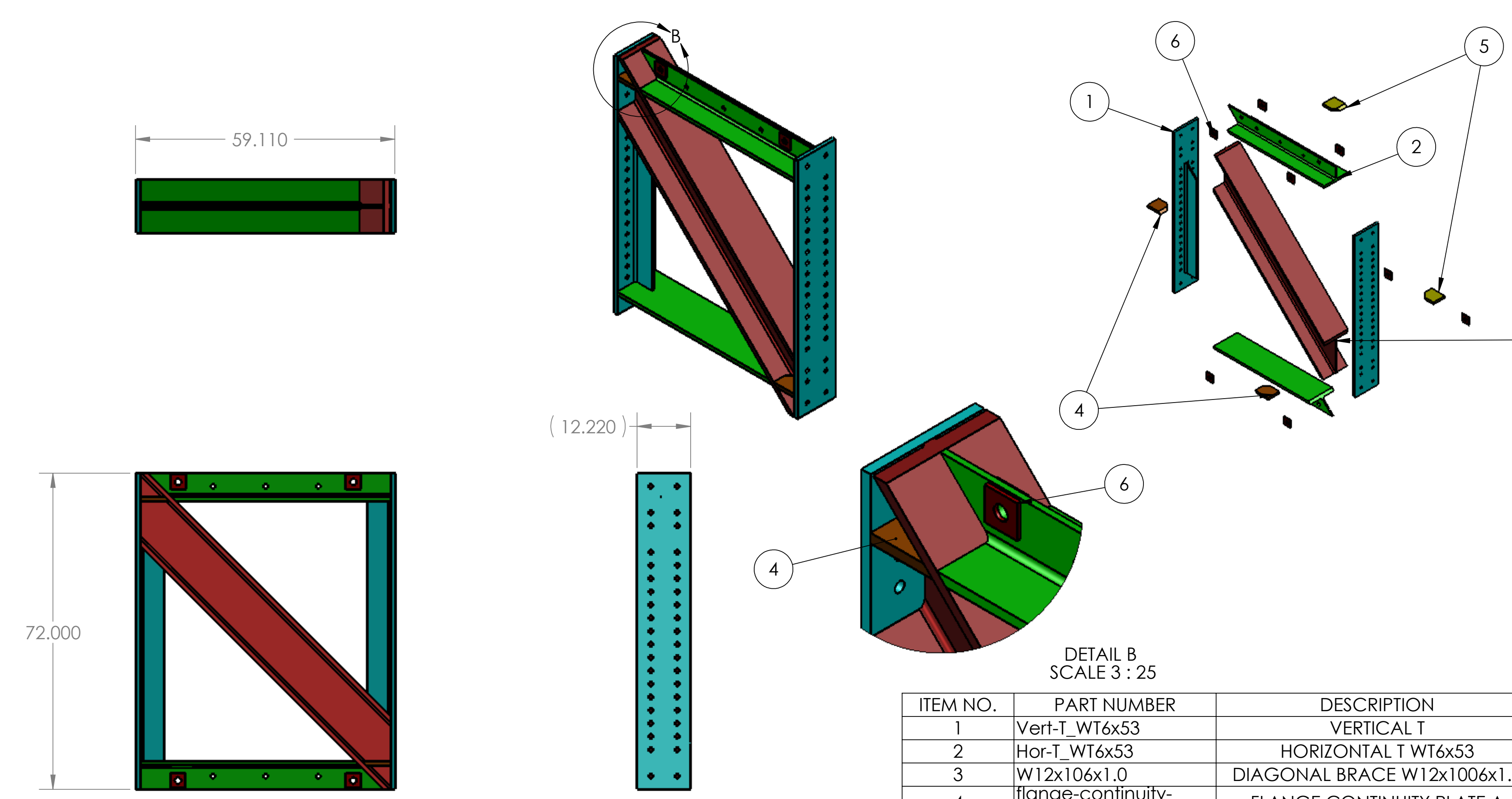

3

NOTES:

1) ALL PART DIMENSIONS DO NOT CONTAIN NECESSARY CLEARANCES FOR FABRICATION. -1
FABRICATION. TOLERANCES FOR PART, WELDMENT AND MODULAR REACTION FRAME SYSTEM ASSEMBLY. 2) APPROXIMATE WEIGHT OF ASSEMBLY IS 1,756 LBS.
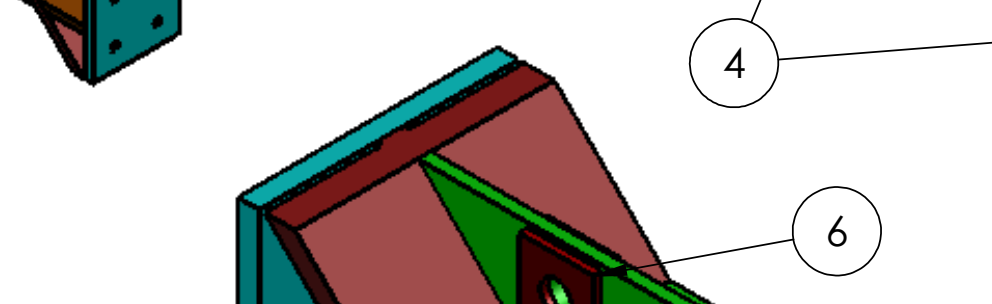

(6)

DETALL B
SCALE $3: 25$

PART NUMBE

\begin{tabular}{|c|c|c|c|}
\hline ITEM NO. & PART NUMBER & DESCRIPTION & QTY. \\
\hline 1 & Vert-T_WT6x53 & VERTICAL T & 2 \\
\hline 2 & Hor-T_WT6x53 & HORIZONTAL T WT6X53 & 2 \\
\hline 3 & W12x106x1.0 & DIAGONAL BRACE W12×1006×1.0 & 1 \\
\hline 4 & $\begin{array}{l}\text { flange-continuity- } \\
\text { plate_A }\end{array}$ & FLANGE CONTINUITY PLATE A & 2 \\
\hline 5 & $\begin{array}{l}\text { flange-eontinuity- } \\
\text { plate_B }\end{array}$ & FLANGE CONTINUITY PLATE B & 2 \\
\hline 6 & $\begin{array}{l}\text { Washer-plate_MRF- } \\
\text { brace-module }\end{array}$ & WASHER PLATE MRF BRACE MODULE & 8 \\
\hline
\end{tabular}
brace-module

DIMENSIONS ARE IN INCHES

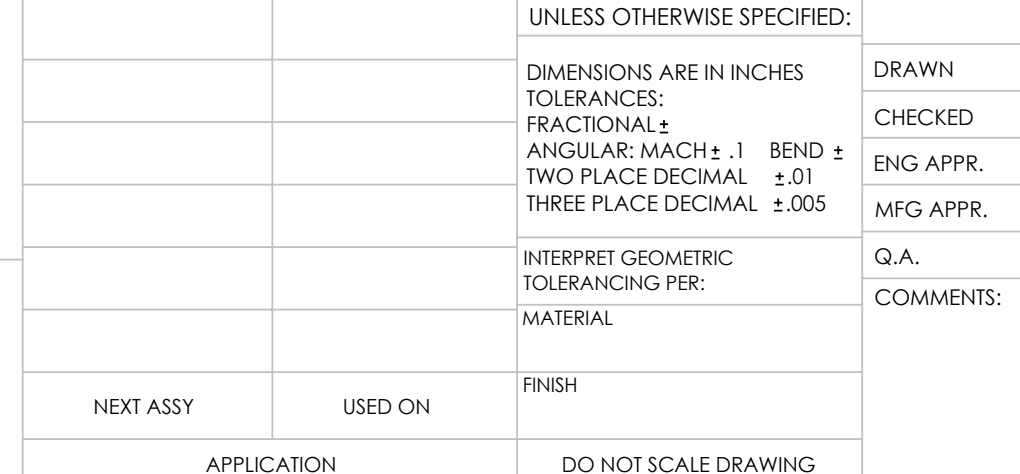

DRAWN $\begin{array}{llll}\text { ANGULAR: MACH.1 } & \text { BEND } & \text { ENG APPR. } \\ \text { WO PLACEDECIMAL } & \pm .01 & \text { EN } & \end{array}$ 005 MFG APPR. INTERRET GEOMERRC
TOLERANCING PER: Q.A. NAME DATE DO NOT SCALE DRAWING
TITLE:

\section{MRF BRACE MODULE}

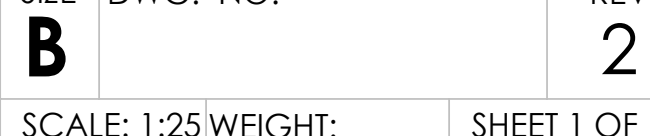



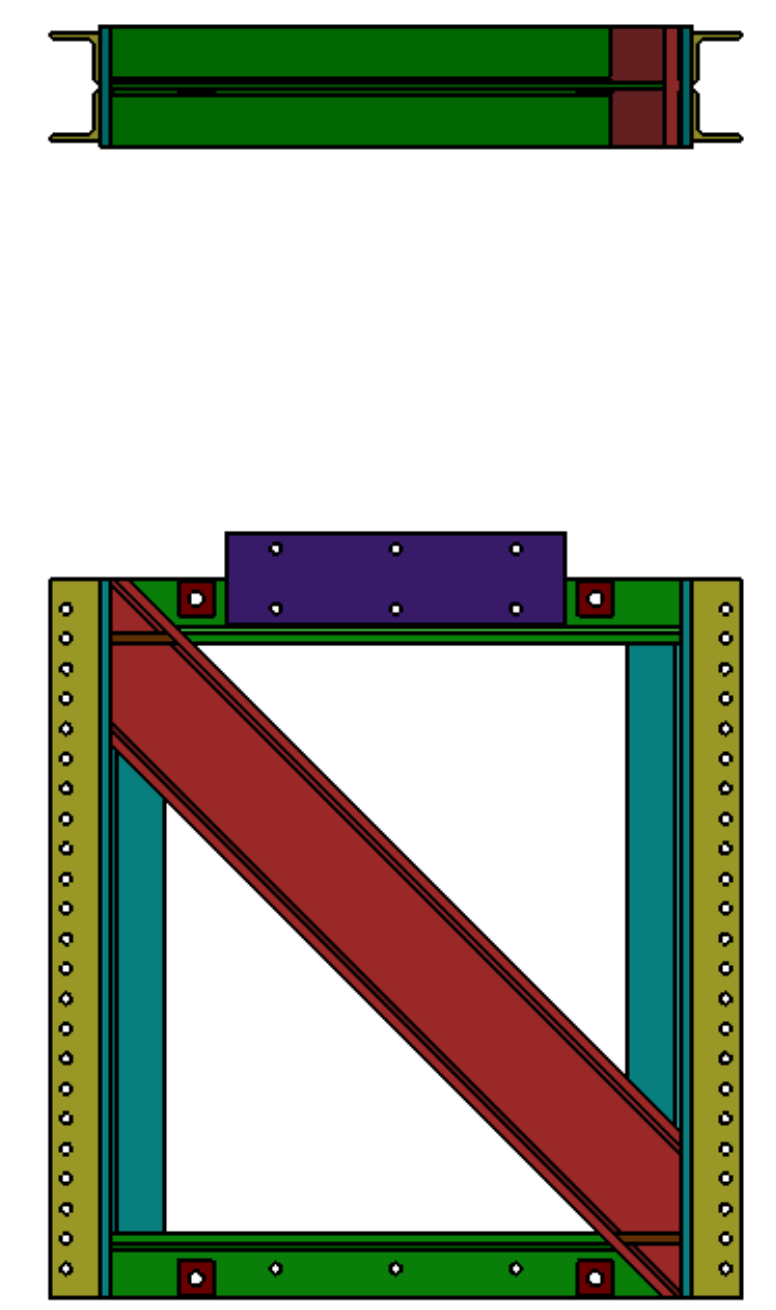

NOTES:

1) "BRACE MODULE ANGLE A ADAPTER" (7) ENABLES BRACE MODULE TO FASTEN TO COLUMN BETWEEN FLANGES. QUANTITY: 4X PER MODULE

2) “WEB SPLICE PLATE” (8) ENABLES BRACE MODULE TO FASTEN TO

ANOTHER VERTICALLY STACKED BRACE MODULE. QUANTITY $2 X$ PER MODULE

3) APPROXIMATE WEIGHT OF ASSEMBLY IS 2,346 LBS.
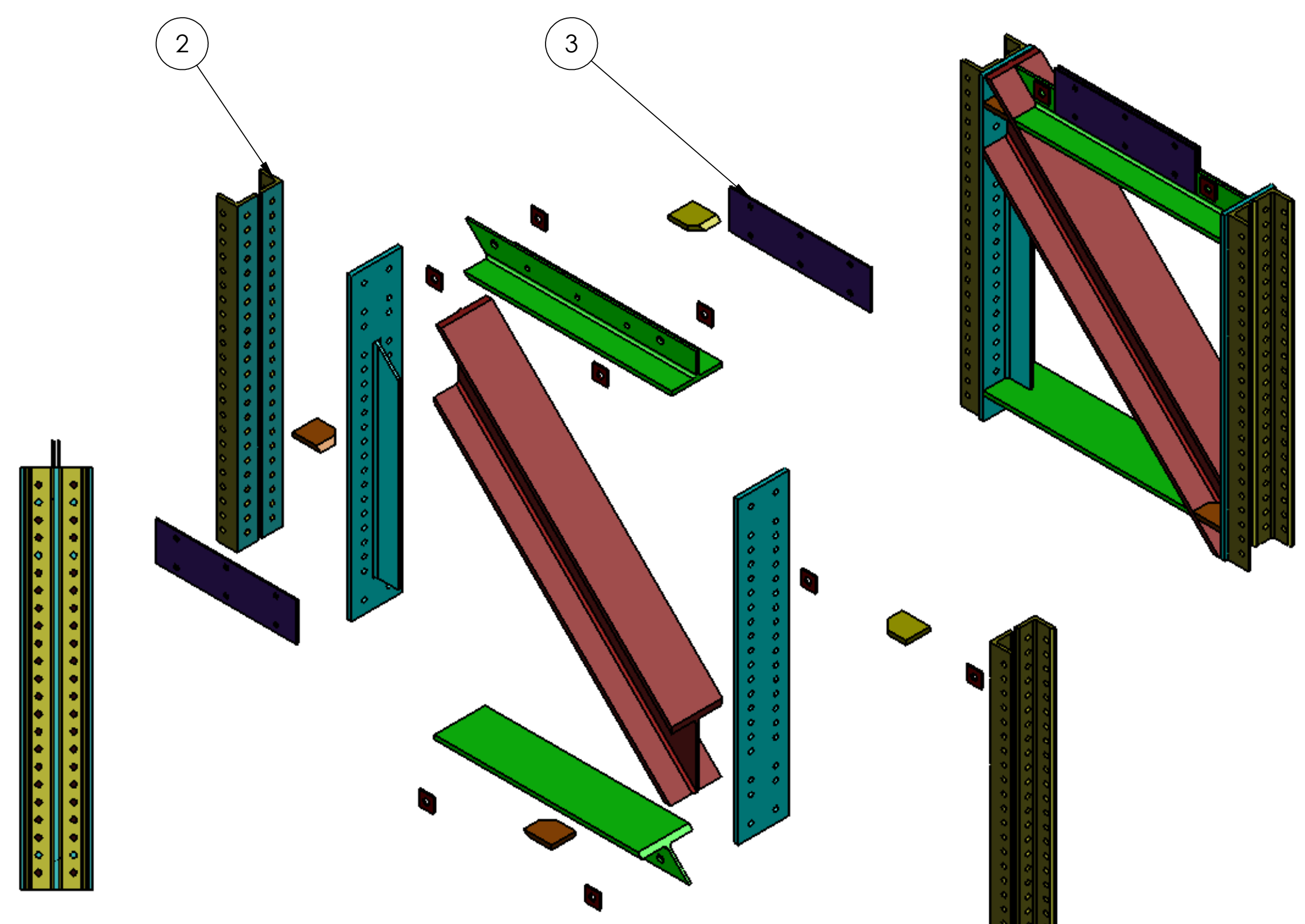

$\mathrm{D}$

C

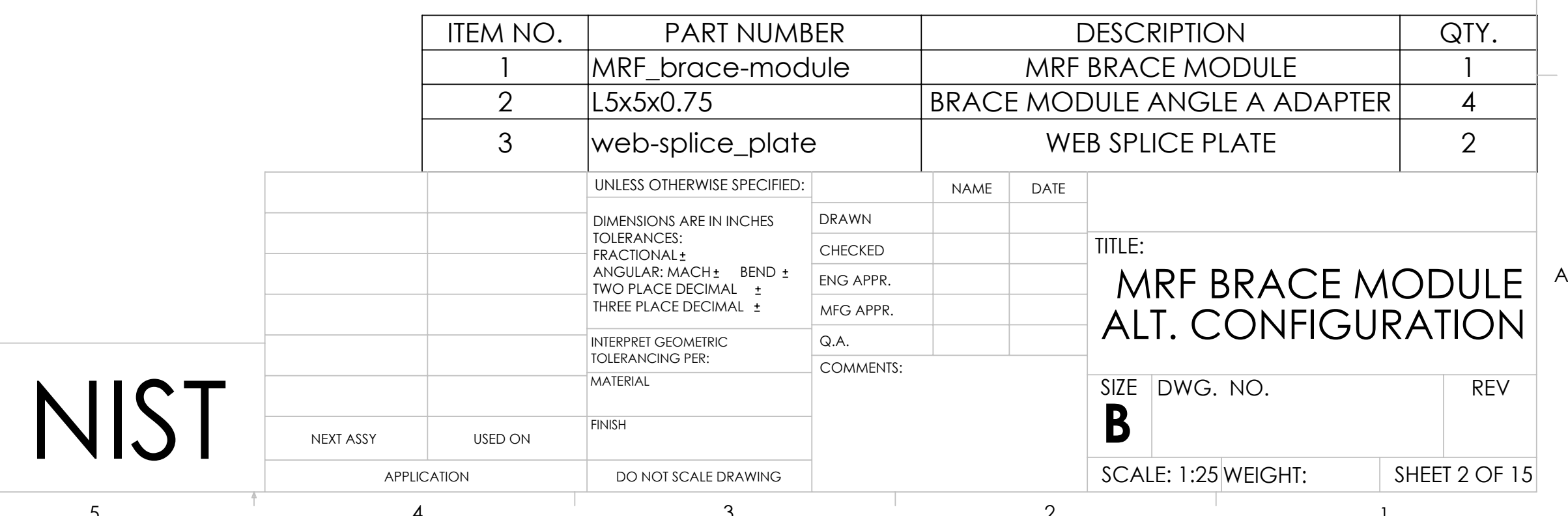



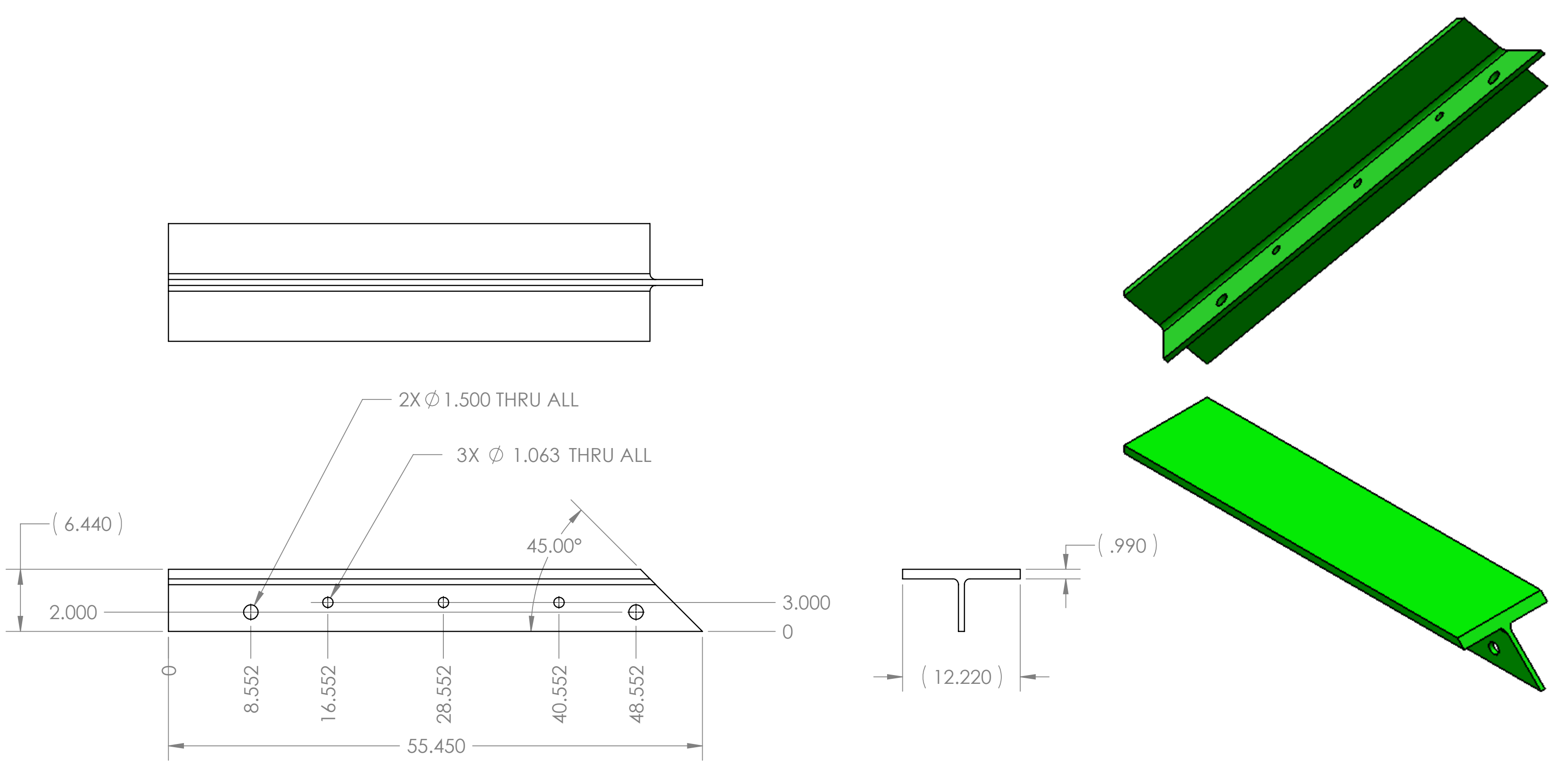

NOTES:

1) ALL PART DIMENSIONS DO NOT CONTAIN NECESSARY CLEARANCES FOR FABRICATION. VENDER IS RESPONSIBLE FOR DETERMINING ALL APPROPRIATE CLEARANCES AND
TOLERANCES FOR PART, WELDMENT AND MODULAR REACTION FRAME SYSTEM ASSEMBLY 2) APPROXIMATE WEIGHT OF PART IS 221 LBS.

3) MATERIAL: WT6 653

4) QUANTITY: SEE SHEET 1

NIST

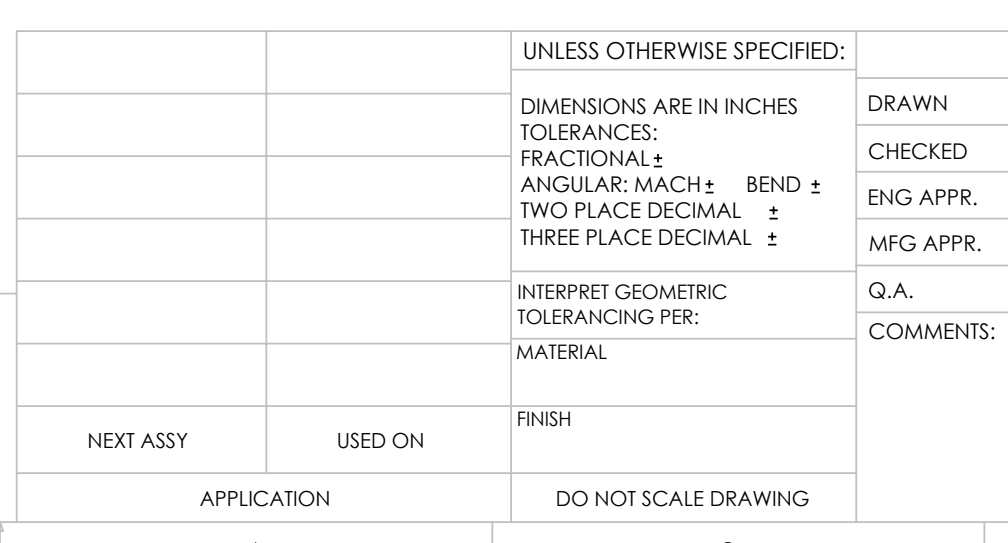

3

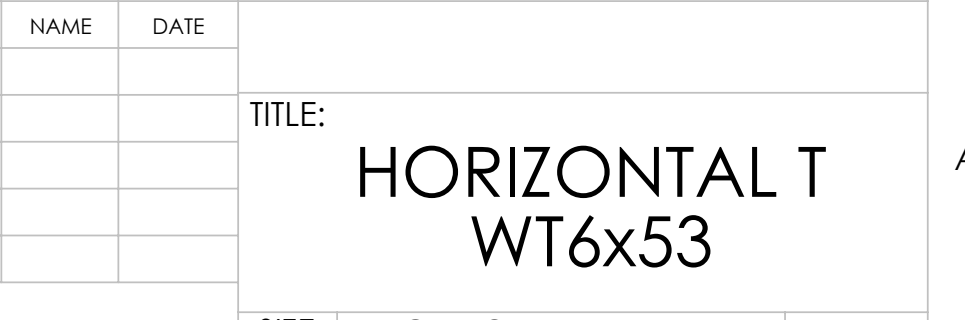

B 

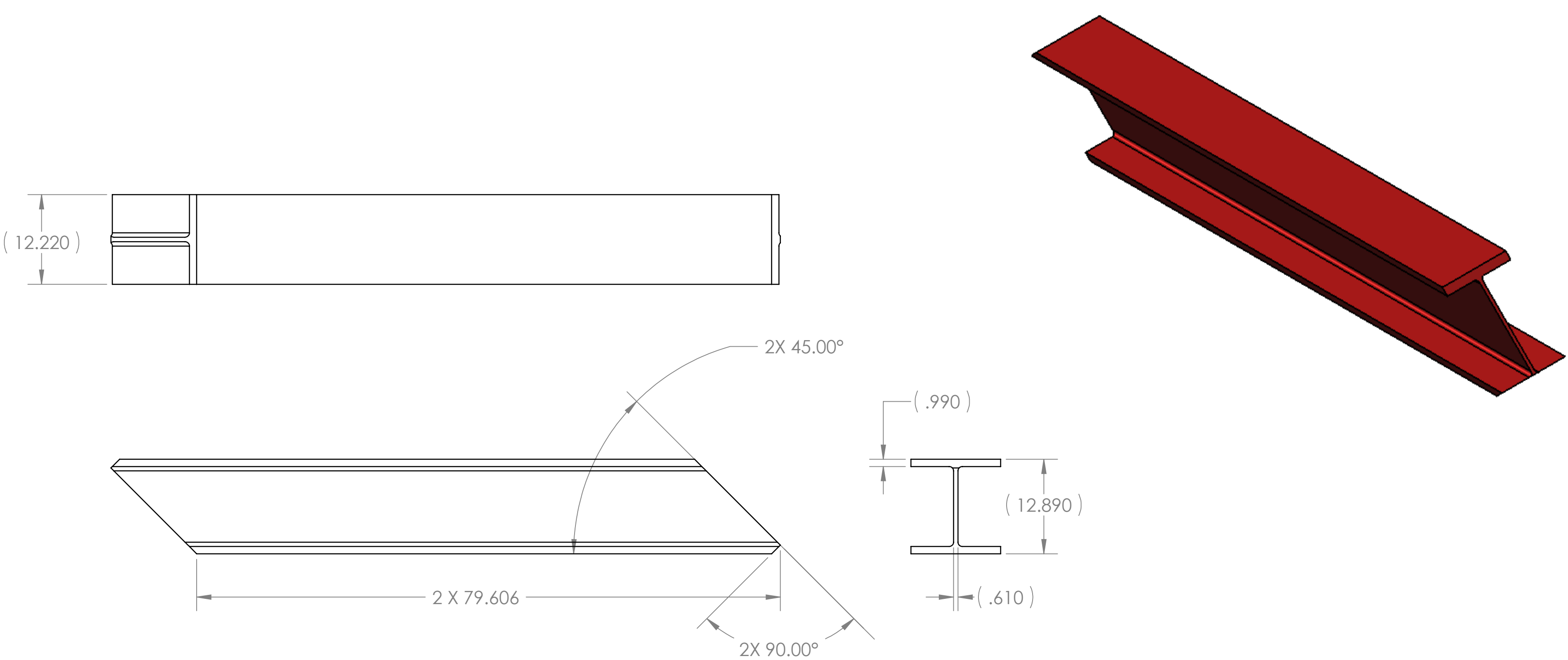

\section{NOTES:}

1) ALL PART DIMENSIONS DO NOT CONTAIN NECESSARY CLEARANCES FOR FABRICATION. VENDER IS RESPONSIBLE FOR DETERMINING ALL APPROPRIATE CLEARANCES AND 作

2) APPROXIMATE WEIGHT OF PART IS 704 LBS.

3) MATERIAL: W12×106

4) QUANTITY: SEE SHEET 1

\begin{tabular}{|c|c|c|}
\hline & & UNLESS OTHERWISE SPECIFEL \\
\hline & & 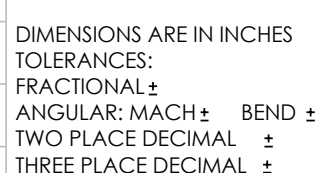 \\
\hline & & $\begin{array}{l}\text { INTERPRE GEOMERTRC } \\
\text { TOLERANGIG PRR: } \\
\text { TMERRAL }\end{array}$ \\
\hline NEXT ASSY & USED ON & FNSH \\
\hline \multicolumn{2}{|c|}{ APPLICATION } & DO NOT SCALE DRAWING \\
\hline
\end{tabular}

\begin{tabular}{|l|l|}
\hline DRAWN \\
\hline CHECKED \\
\hline ENG APPR. \\
\hline MFG APPR. \\
\hline Q.A. \\
\hline COMMENTS: \\
\hline
\end{tabular}

NAME DATE

TITLE:

DIAGONAL BRACE W12X106

SIZE

SCALE: 1:15 WEIGHT: SHEET 5 OF 15 

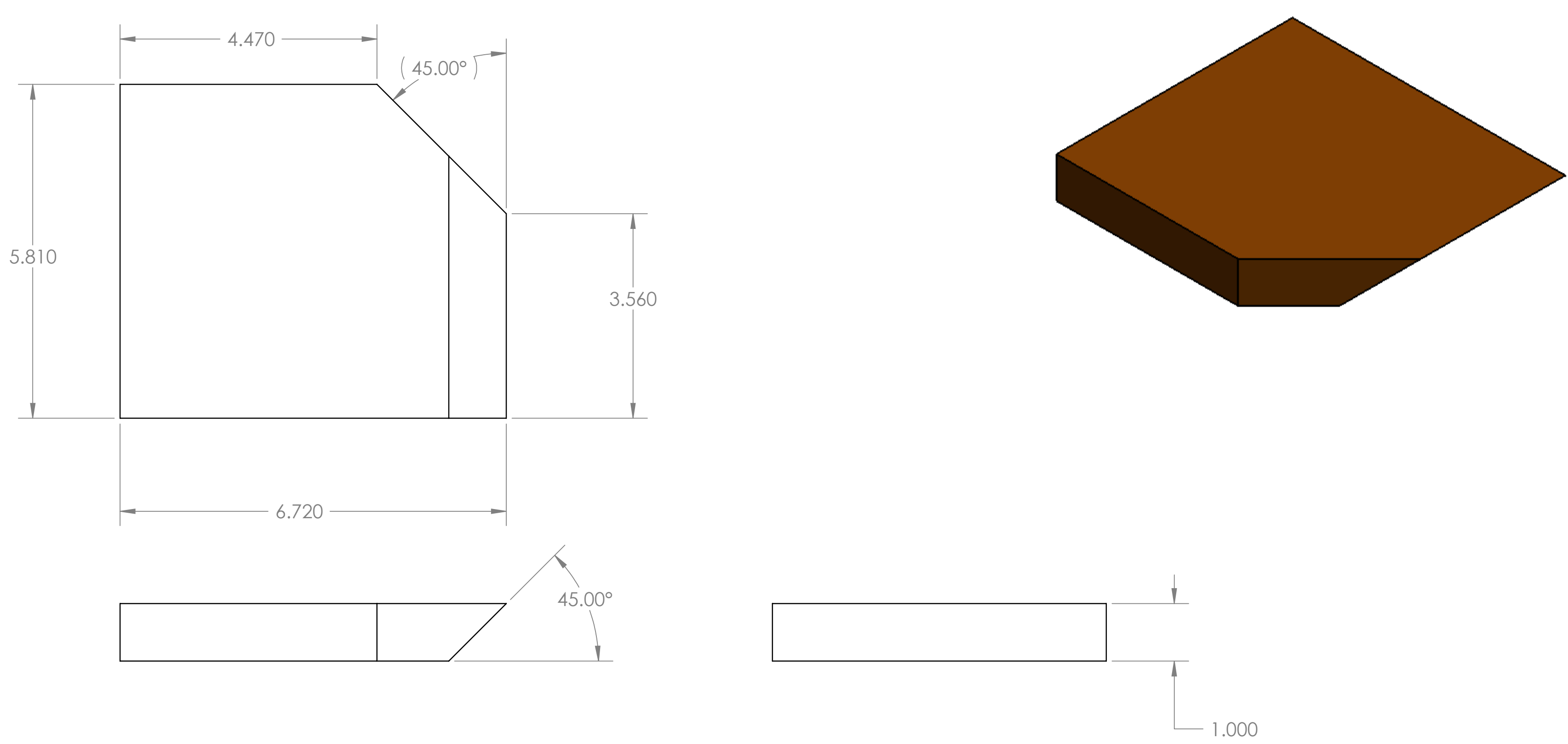

NOTES:

1) ALL PART DIMENSIONS DO NOT CONTAIN NECESSARY CLEARANCES FOR FABRICATION. VENDER IS RESPONSIBLE FOR DETERMINING ALL APPROPRIATE CLEARANCES AND

TOLERANCES FOR PART, WELDMENT AND MODULAR REACTION FRAME SYSTEM ASSEMBLY.
3) MATERIAL: A36 STEEL OR EQUIVALENT

4) QUANTITY: SEE SHEET 1
NIST

\begin{tabular}{|c|c|c|c|}
\hline & & UNLESS OTHERWIIE SPECIFIED: & \multirow{3}{*}{$\begin{array}{l}\text { DRAWN } \\
\text { CHECKED }\end{array}$} \\
\hline & & \multirow{4}{*}{ 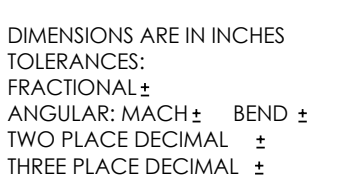 } & \\
\hline & & & \\
\hline & & & ENG APPR. \\
\hline & & & MFG APPR. \\
\hline & & $\begin{array}{l}\text { INTERRRET GEOMETRLC } \\
\text { TOLRANAING PER: } \\
\text { MATERAA }\end{array}$ & \multirow[t]{4}{*}{$\begin{array}{l}\text { Q.A. } \\
\text { COMMENTS: }\end{array}$} \\
\hline & & & \\
\hline NEXT ASSY & USED ON & FNISH & \\
\hline \multicolumn{2}{|c|}{ APPLICATION } & DO NOT SCALE DRAWING & \\
\hline
\end{tabular}

3

\begin{tabular}{|c|c|c|}
\hline NAME & DATE & \multirow{4}{*}{$\begin{array}{l}\text { TITLE: } \\
\text { FLANGE CONTINUITY } \\
\text { PLATE A }\end{array}$} \\
\hline & & \\
\hline & & \\
\hline & & \\
\hline
\end{tabular}

B

SCALE: 1:2 WEIGHT: SHEET 6 OF 15 

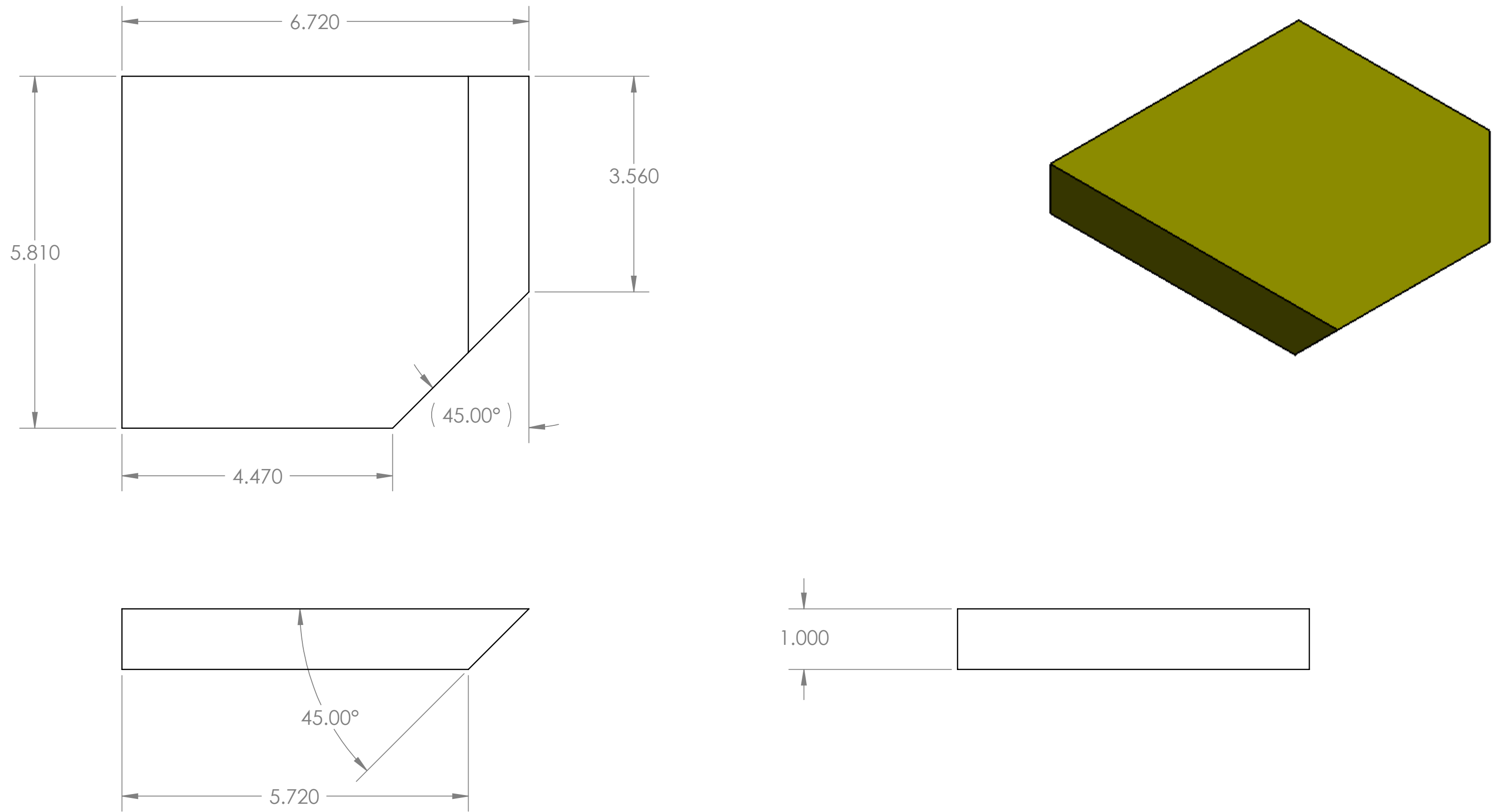

NOTES:

1) ALL PART DIMENSIONS DO NOT CONTAIN NECESSARY CLEARANCES FOR FABRICATION. VENDER IS RESPONSIBLE FOR DETERMINING ALL APPROPRIATE CLEARANCES AND
TOLERANCES FOR PART, WELDMENT AND MODULAR REACTION FRAME SYSTEM ASSEMBLY,

2) APPROXIMATE WEIGHT OF PART IS 10 LBS.

3) MATERIAL: A36 STEEL OR EQUIVALENT

4) QUANTITY: SEE SHEET 1

NIST

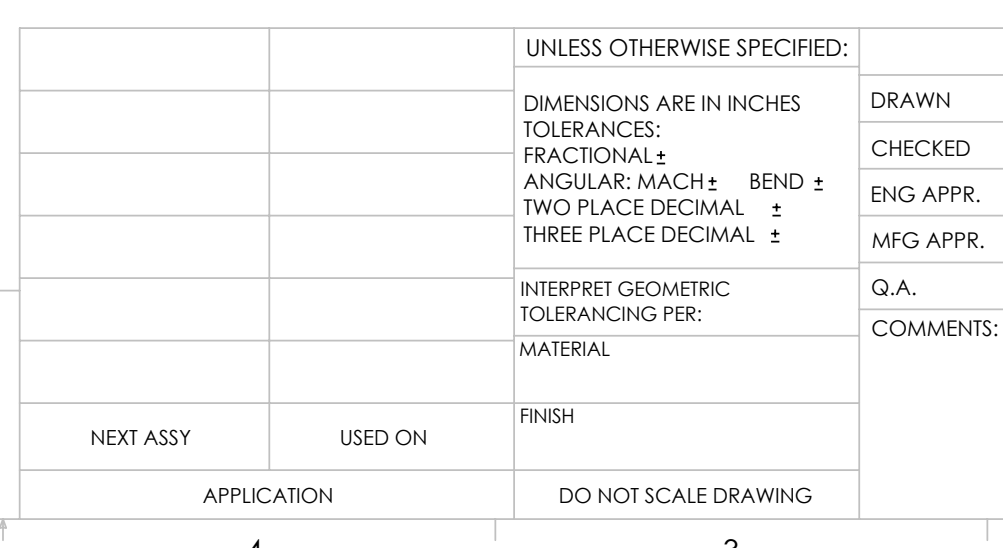

3

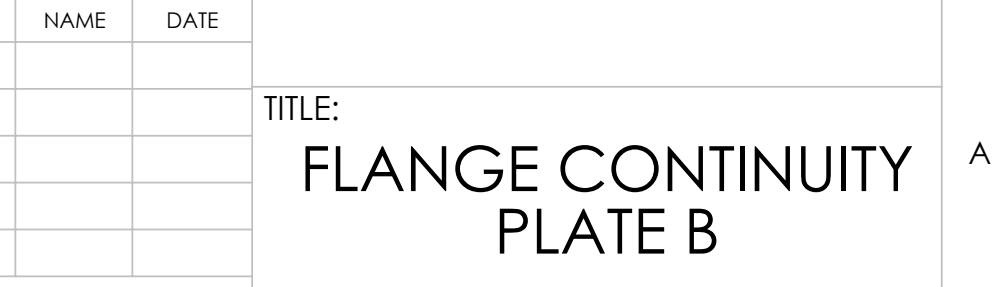

PLATE B

B

SCALE: 1:2 WEIGHT: SHEET 7 OF 15 
$\varnothing 1.500$ THRU ALL
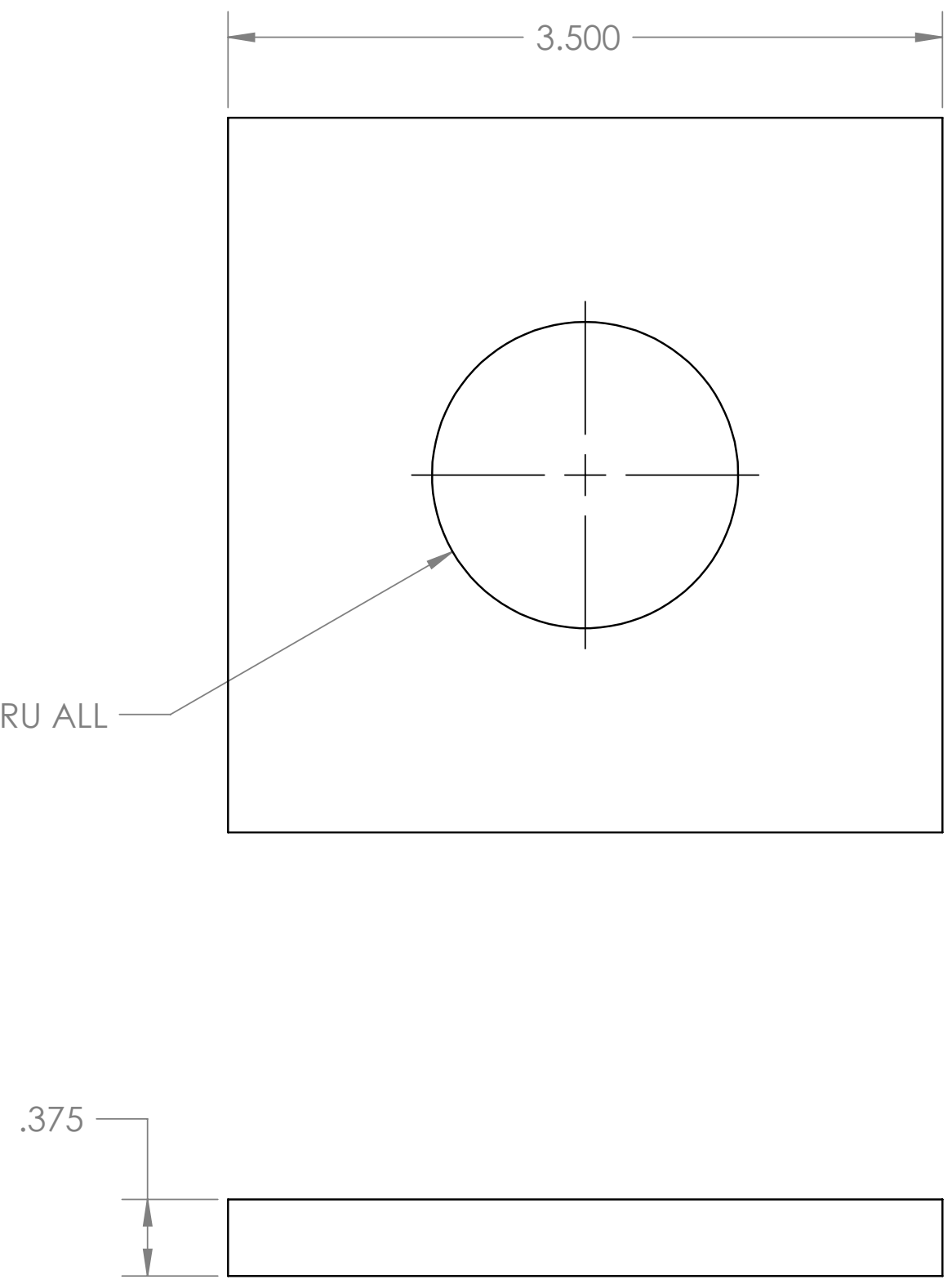
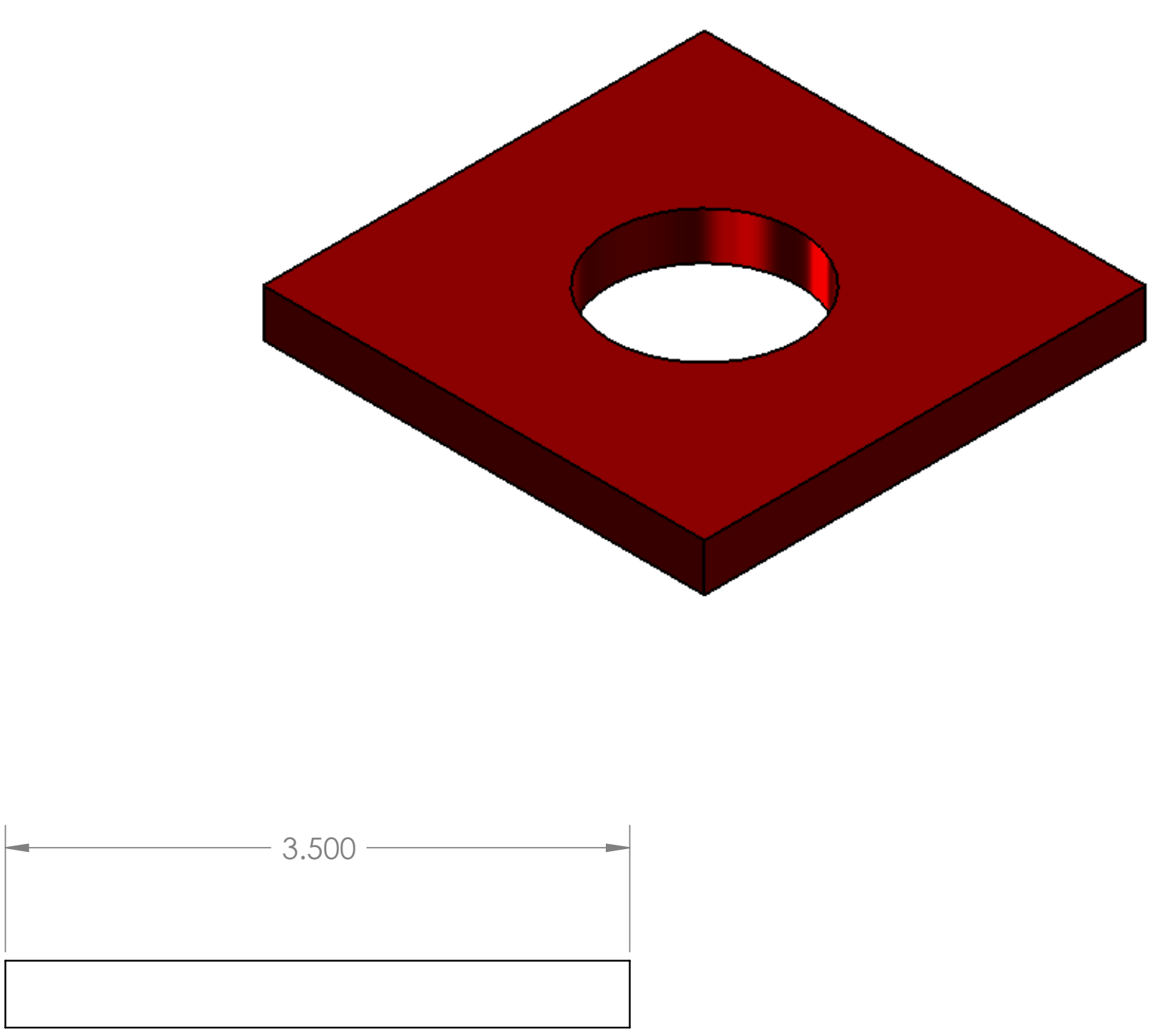

NOTES:

1) ALL PART DIMENSIONS DO NOT CONTAIN NECESSARY CLEARANCES FOR FABRICATION.

VENDER IS RESPONSIBLE FOR DETERMINING ALL APPROPRIATE CLEARANCES AND
TOLERANCES FOR PART, WELDMENT AND MODULAR REACTION FRAME SYSTEM ASSEMBLY

3) MATERIAL: A36 STEEL OR EQUIVALENT

4) QUANTITY: SEE SHEET 1

NIST

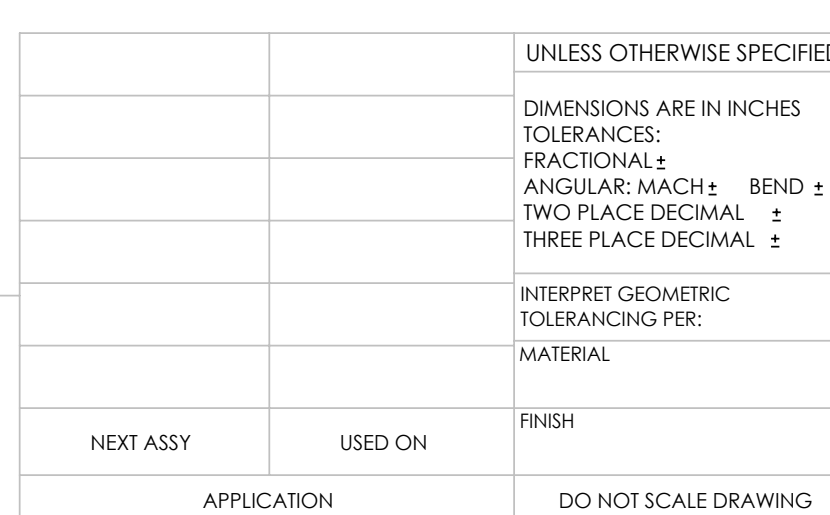

4

\begin{tabular}{|l|}
\hline DRAWN \\
\hline CHECKED \\
\hline ENG APPR. \\
\hline MFG APPR. \\
\hline Q.A. \\
\hline COMMENTS: \\
\hline
\end{tabular}

NAME DAtE

TITLE:

WASHER PLATE MRF BRACE MODULE

\section{B}




\section{$3.445 \longrightarrow+$}
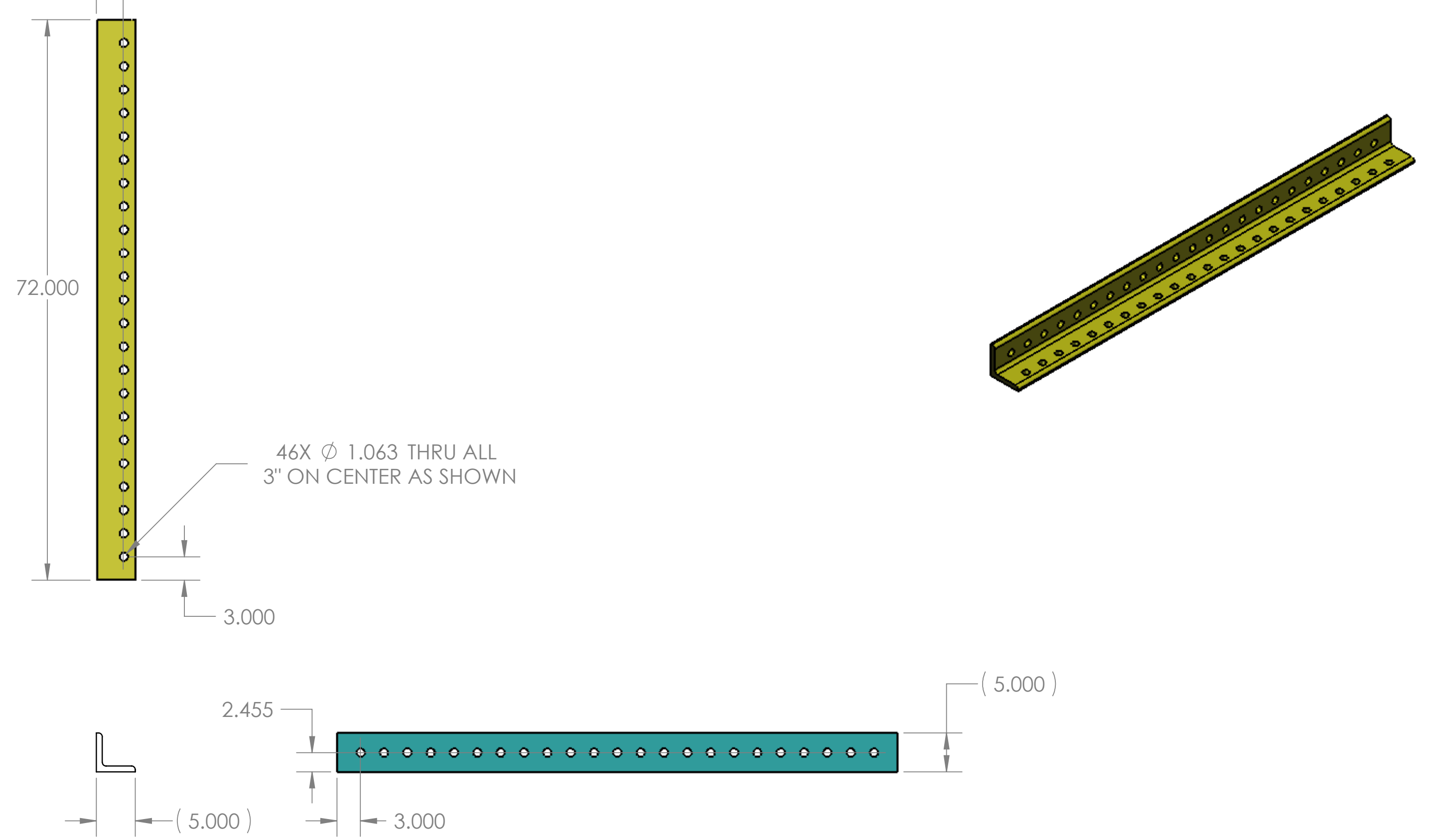

NOTES:

1) ALL PART DIMENSIONS DO NOT CONTAIN NECESSARY CLEARANCES FOR FABRICATION. VENDER IS RESPONSIBLE FOR DETERMINING ALL APPROPRIATE CLEARANCES AND
TOLERANCES FOR PART, WELDMENT AND MODULAR REACTION FRAME SYSTEM ASSEMBLY.

2) APPROXIMATE WEIGHT OF PART IS 132 LBS.

3) MATERIAL: L5 $\times 5 \times 0.75$ A36 STEEL OR EQUIVALENT

4) QUANTITY: SEE SHEET 1

5) SEE SHEET 2 FOR PROPER ASSEMBLY ORIENTATION VIA COLOR CODE

NIST

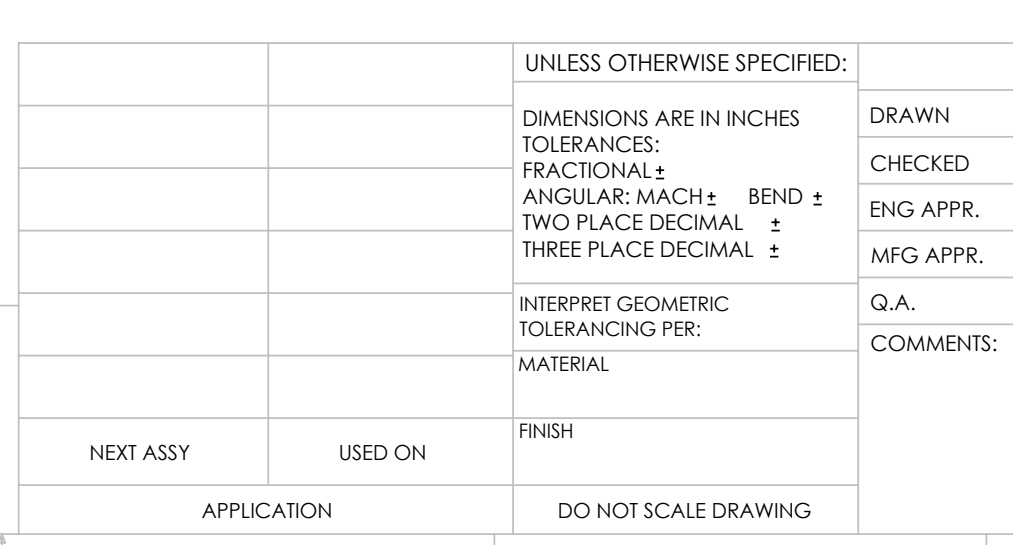

3

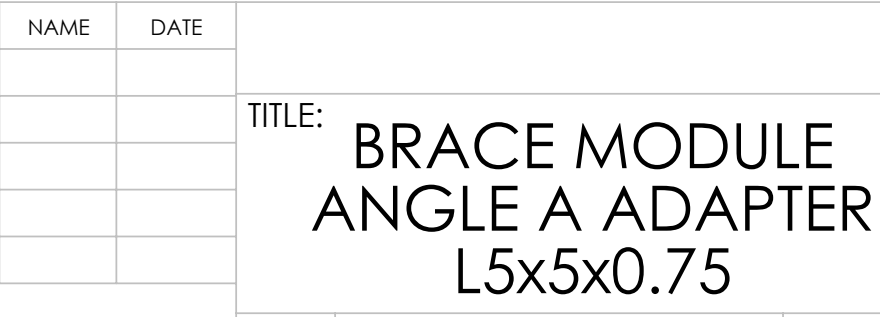
NAME DATE

TITLE: BRACE MODULE ANGLE A ADAPTER L5 $\times 5 \times 0.75$

B 


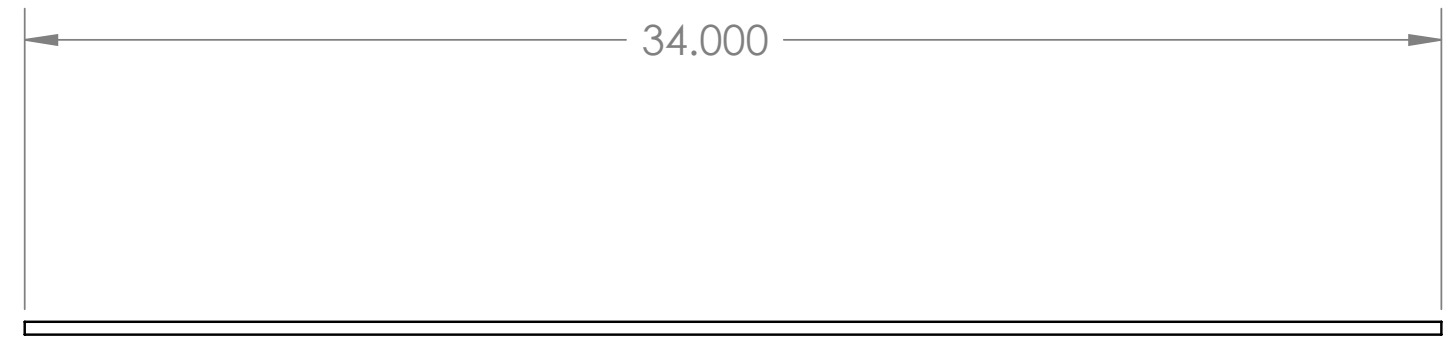

6X $\varnothing 1.063$ THRU ALL
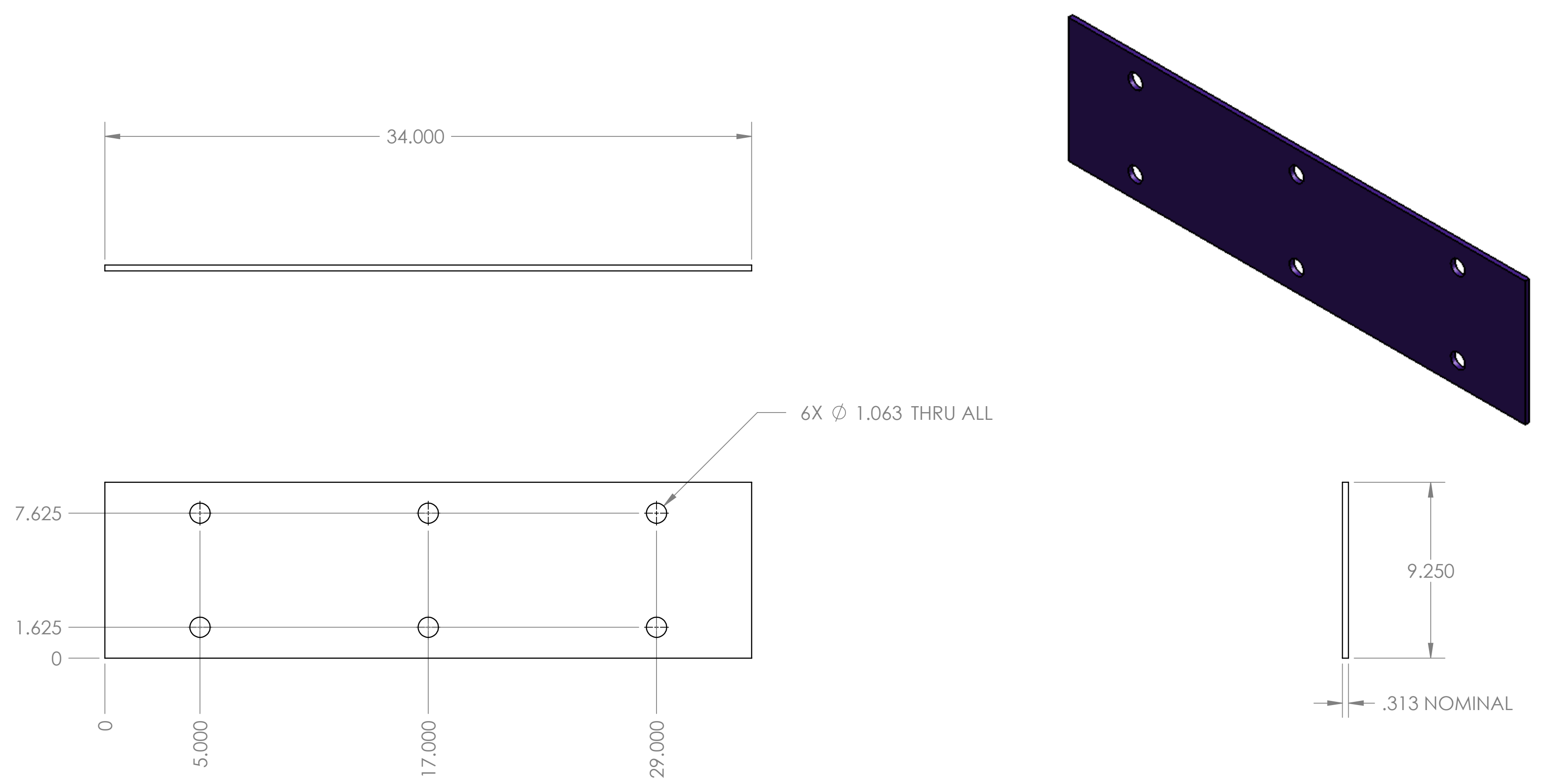

NOTES:

1) ALL PART DIMENSIONS DO NOT CONTAIN NECESSARY CLEARANCES FOR FABRICATION. VENDER IS RESPONSIBLE FOR DETERMINING ALL APPROPRIATE CLEARANCES AND
TOLERANCES FOR PART, WELDMENT AND MODULAR REACTION FRAME SYSTEM ASSEMBLY. 2) APPROXIMATE WEIGHT OF PART IS 27 LBS.

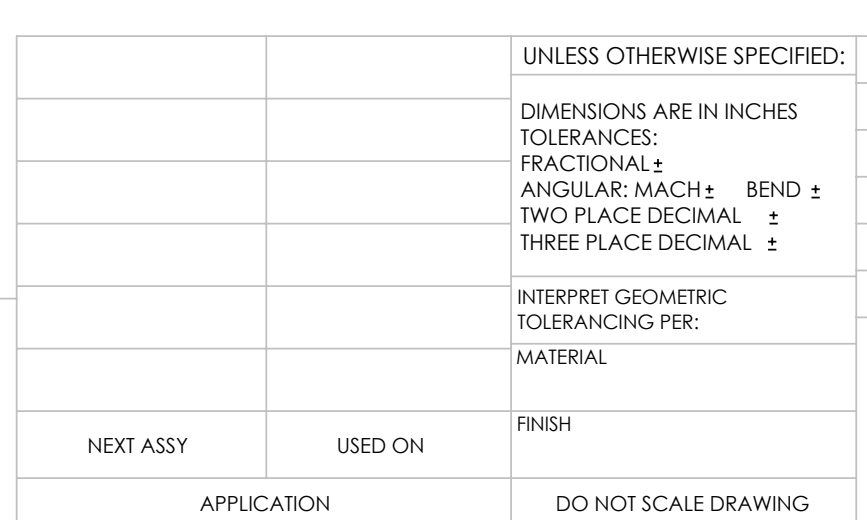

\begin{tabular}{|l|}
\hline DRAWN \\
\hline CHECKED \\
\hline ENG APPR. \\
\hline MFG APPR. \\
\hline Q.A. \\
\hline COMMENTS: \\
\hline
\end{tabular}

\begin{tabular}{|c|c|c|c|}
\hline NAME & DATE & & \\
\hline & & \multirow{2}{*}{\multicolumn{2}{|c|}{$\begin{array}{l}\text { TITLE: } \\
\text { WEB SPLCE PLATE }\end{array}$}} \\
\hline & & & \\
\hline & & $\begin{array}{l}\text { SIZE DWG. NO. } \\
\mathbf{B}\end{array}$ & REV \\
\hline & & SCALE: 1:6 WEIGHT: & SHEET 10 OF 15 \\
\hline
\end{tabular}




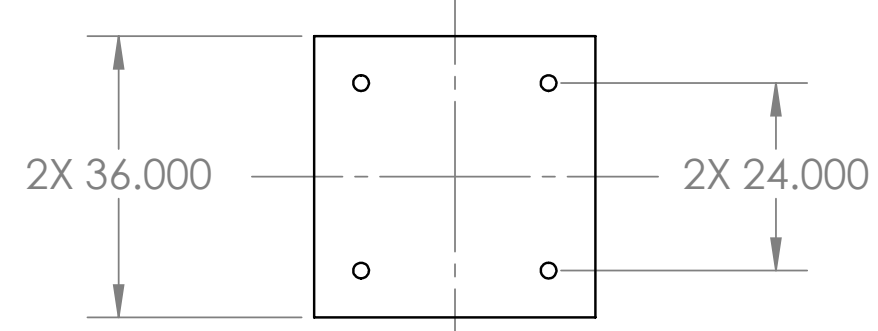
1

- ON CENTER AS SHOWN REPEAT AS REQUIRED
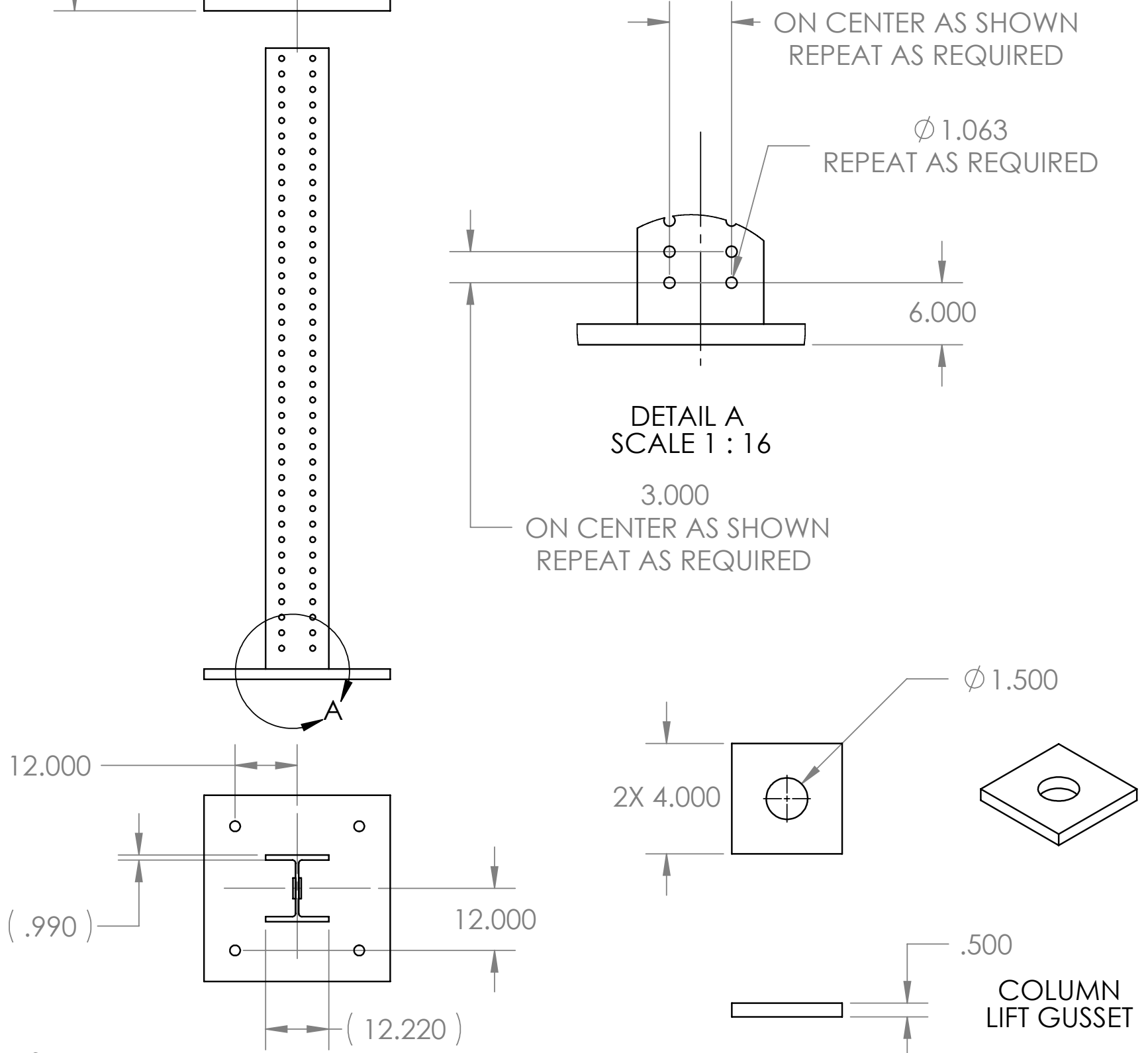

NOTES:

1) ALL PART DIMENSIONS DO NOT CONTAIN NECESSARY CLEARANCES FOR FABRICATION. VENDER IS RESPONSIBLE FOR DETERMIINING ALL APPROPRIATE CLEARANCES AND

TOLERANCES FOR PART, WELDMENT AND MODULAR REACTION FRAME SYSTEM ASSEMBLY.

A 2) COLUMN HEIGHT MAY VARY

3) GIRDER BEAM MATERIAL: W12×106

4) COLUMN FOOT MATERIAL: A36 STEEL OR EQUIVALENT

5) COLUMN GUSSET MATERIAL: A36 STEEL OR EQUIVALENT

3) APPROXIMATE WEIGHT OF 10' COLUMN ASSEMBLY IS 1,753 LBS

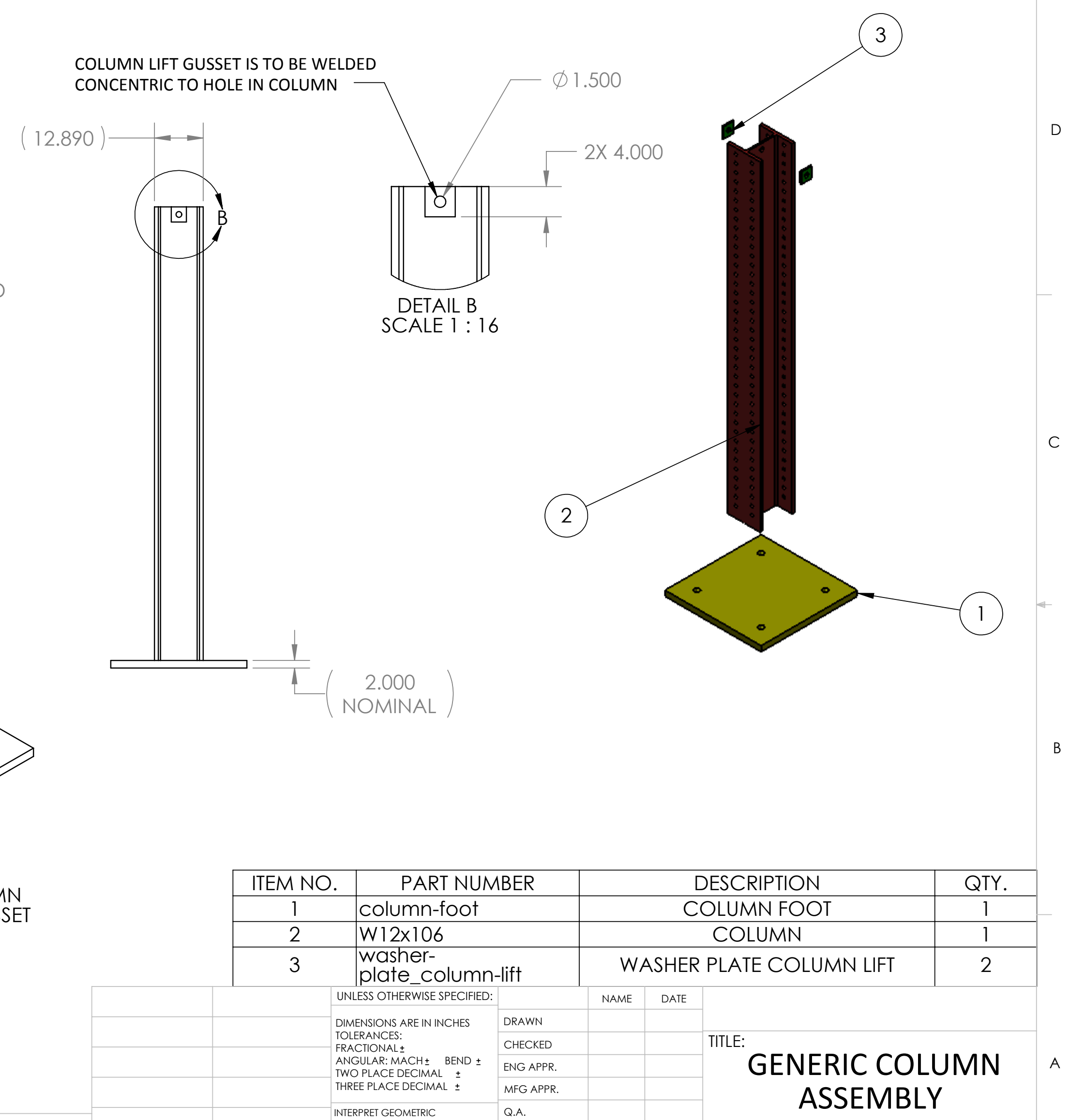

NIST

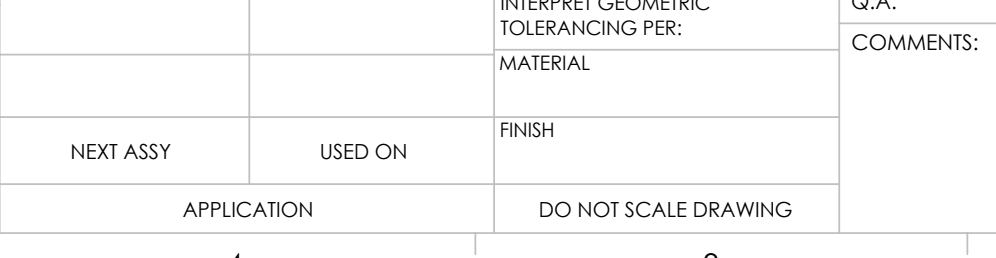

4
B

SCALE: 1:32 WEIGHT: $\quad$ SHEET 11 OF 15 

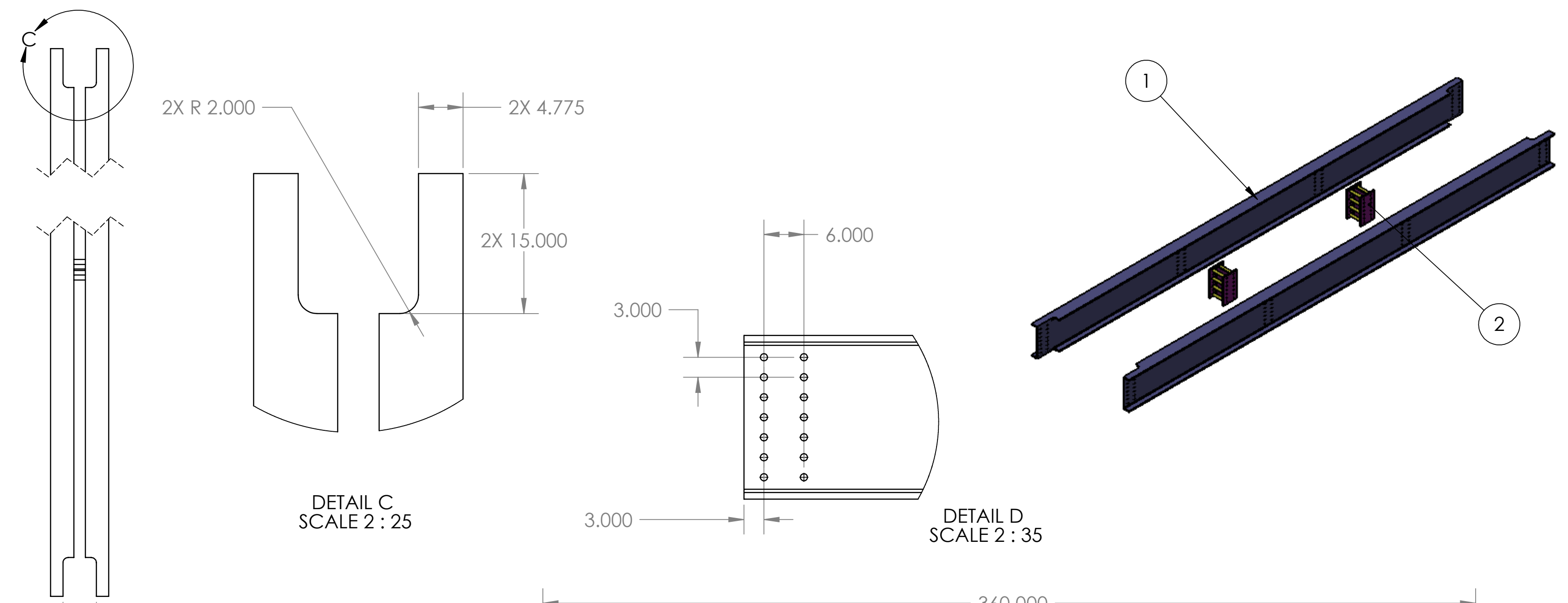

$$
\begin{aligned}
& \text { DETAIL C } \\
& \text { SCALE } 2: 25
\end{aligned}
$$
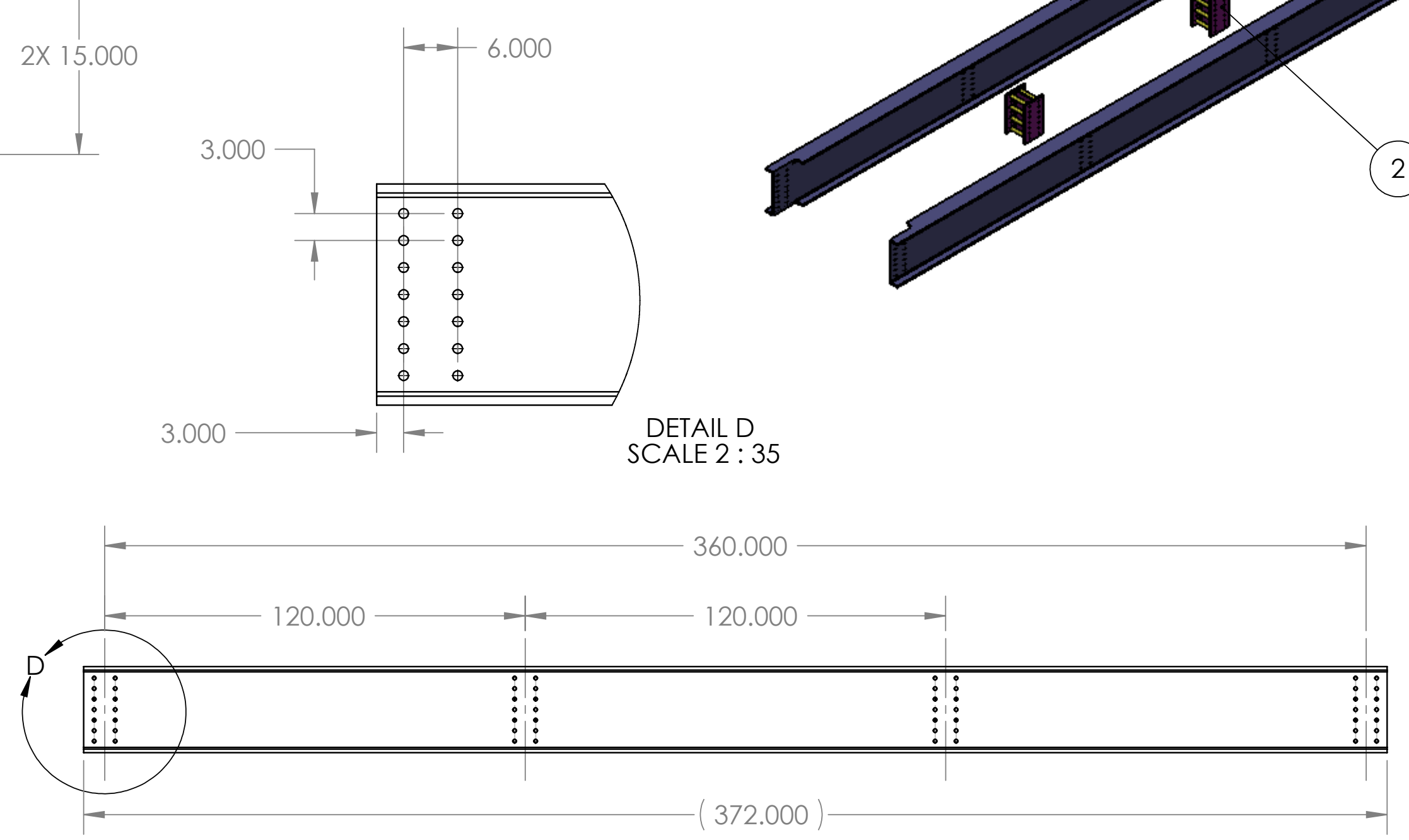

NOTES:

1) ALL PART DIMENSIONS DO NOT CONTAIN NECESSARY CLEARANCES FOR FABRICATION. VENDER IS RESPONSIBLE FOR DETERMIIING ALL APPROPRIATE CLEARANCES AND 2) GIRDER LENGTH MAY ONLY VARY IN 24" INCREMENTS A HOLE PATTERNS TO ACCOMMODATE GIRDER SPACER SHALL BE SPACED A MAXIMUM
OF 120" APART AS SHOWN

4) NO GIRDER SPACER NECESSARY FOR GIRDER SPAN OF 10

5) APPROXIMANT WEIGHT OF GIRDER A ASSEMBLY AS SHOWN (30') IS 6,657 LBS., FOR 20' 4,392 LBS., FOR 10' 1,783 LBS.

NIST

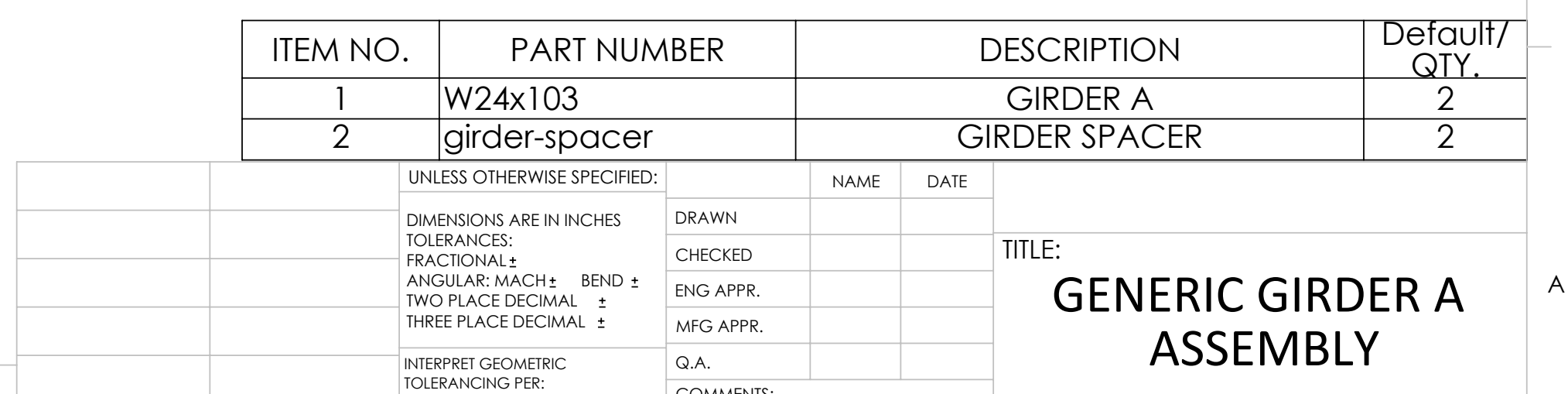

B 

SCALE $1: 15$
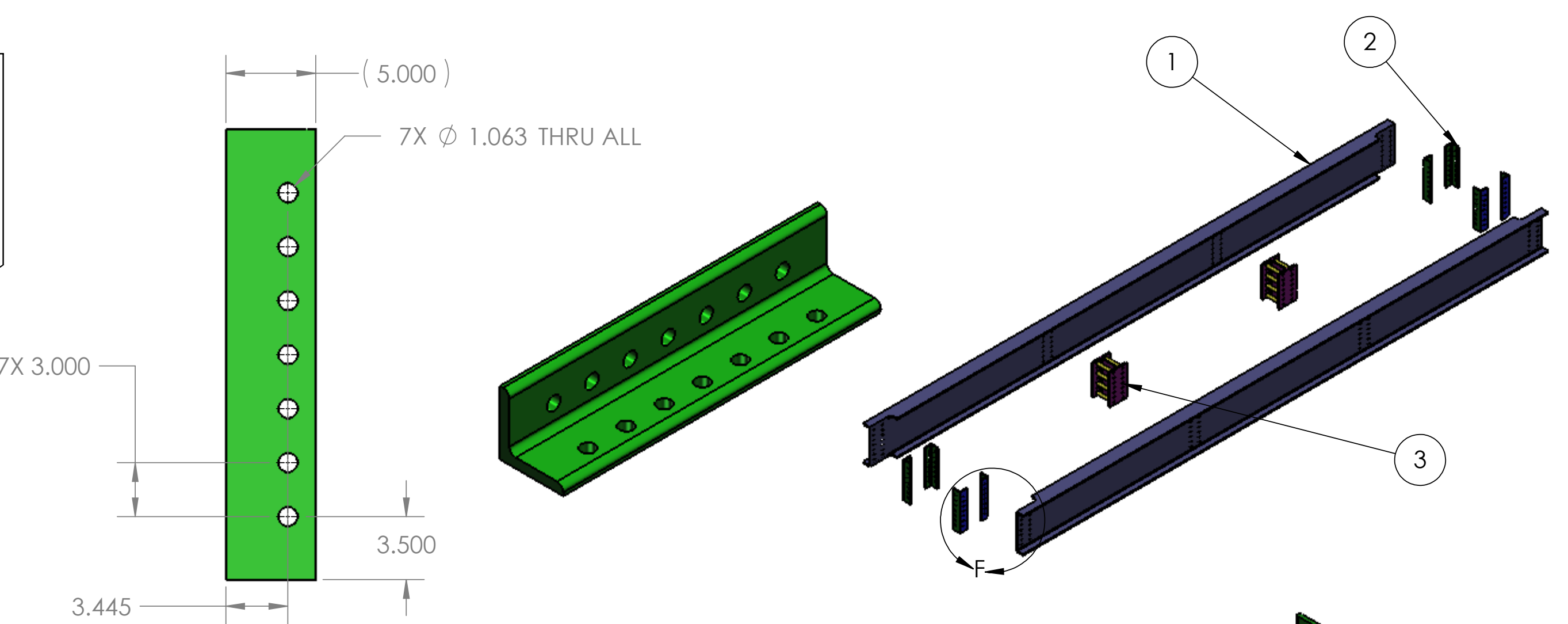

$$
3.445
$$
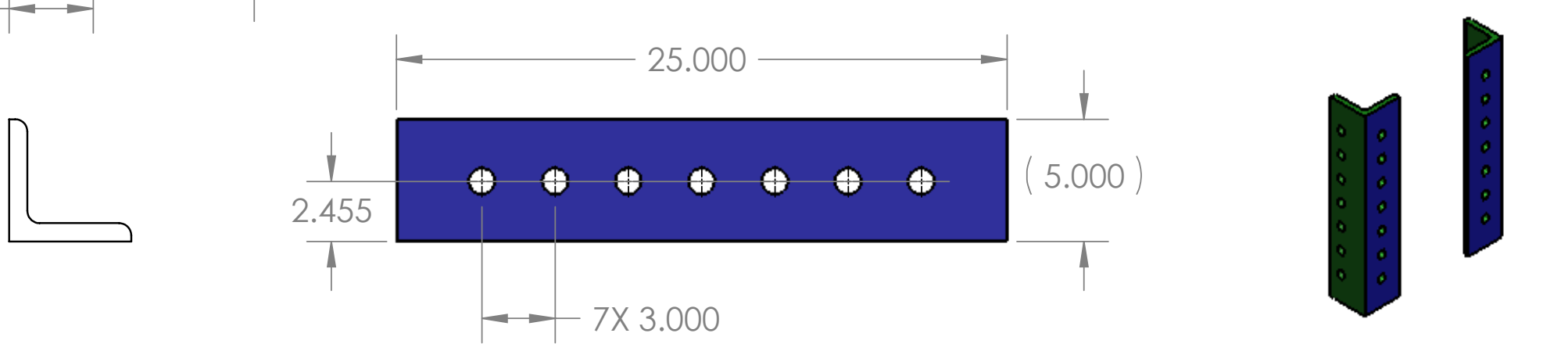

DETAIL F SCALE $1: 20$
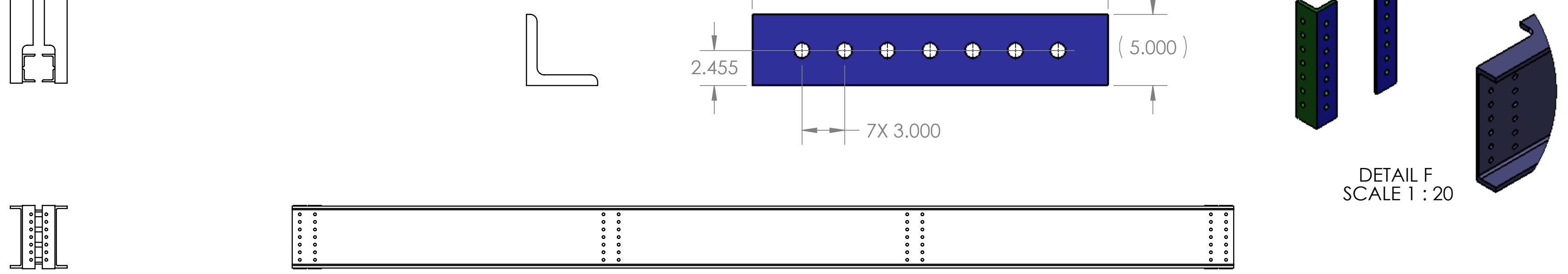

NOTES:

1) ALL PART DIMENSIONS DO NOT CONTAIN NECESSARY CLEARANCES FOR FABRICATION. VENDER IS RESPONSIBLE FOR DETERMINING ALL APPROPRIATE CLEARANCES AND

TOLERANCES FOR PART, WELDMENT AND MODULAR REACTION FRAME SYSTEM ASSEMBLY.

\section{2) GIRDER LENGTH MAY ONLY VARY IN 24" INCREMENTS} 3) HOLE PATTERNS TO ACCOMMODATE GIRDER SPACER SHALL BE SPACED A MAXIMUM

A 4) NO GIRDER SPACER NECESSARY FOR GIRDER SPAN OF 10'

5) GIRDER ANGLE B ADAPTER MATERIAL: L5 $\times 5 \times 0.75$

6) APPROXIMANT WEIGHT OF GIRDER B ADAPTER: 46 LBS.

5) APPROXIMANT WEIGHT OF GIRDER B ASSEMBLY AS SHOWN (30') IS 7,025 LBS., FOR 20' 4,760 LBS., FOR 10' 1,783 LBS.

NIST

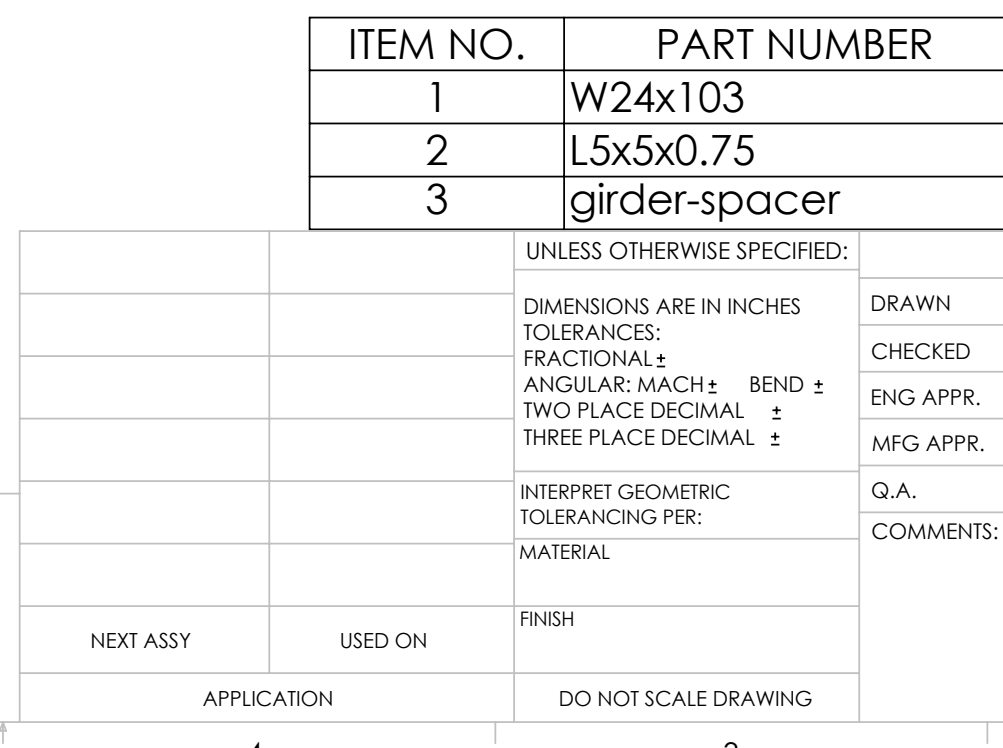

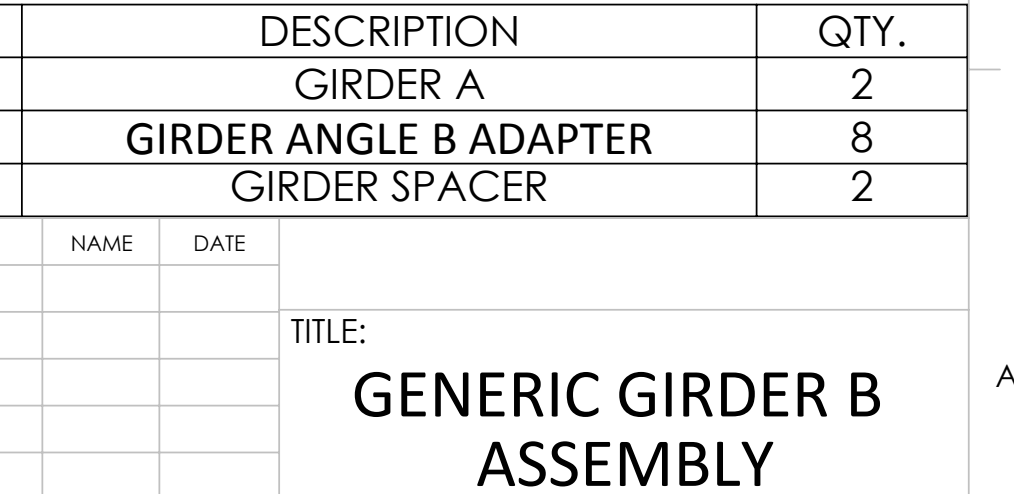

ASSEMBLY

B

SCALE: 1:45 WEIGHT: $\quad$ SHEET 13 OF 15 


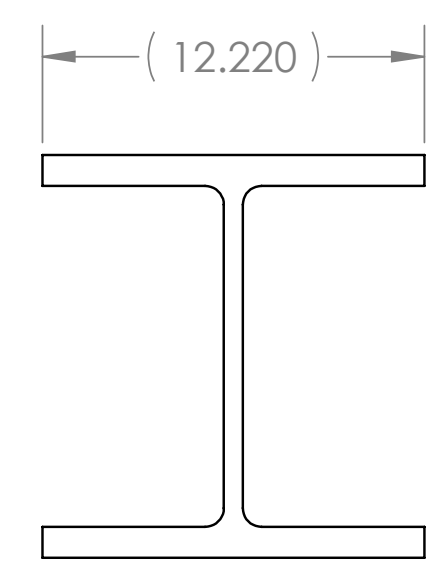

$14 X \varnothing 1.063$ THRU ALL
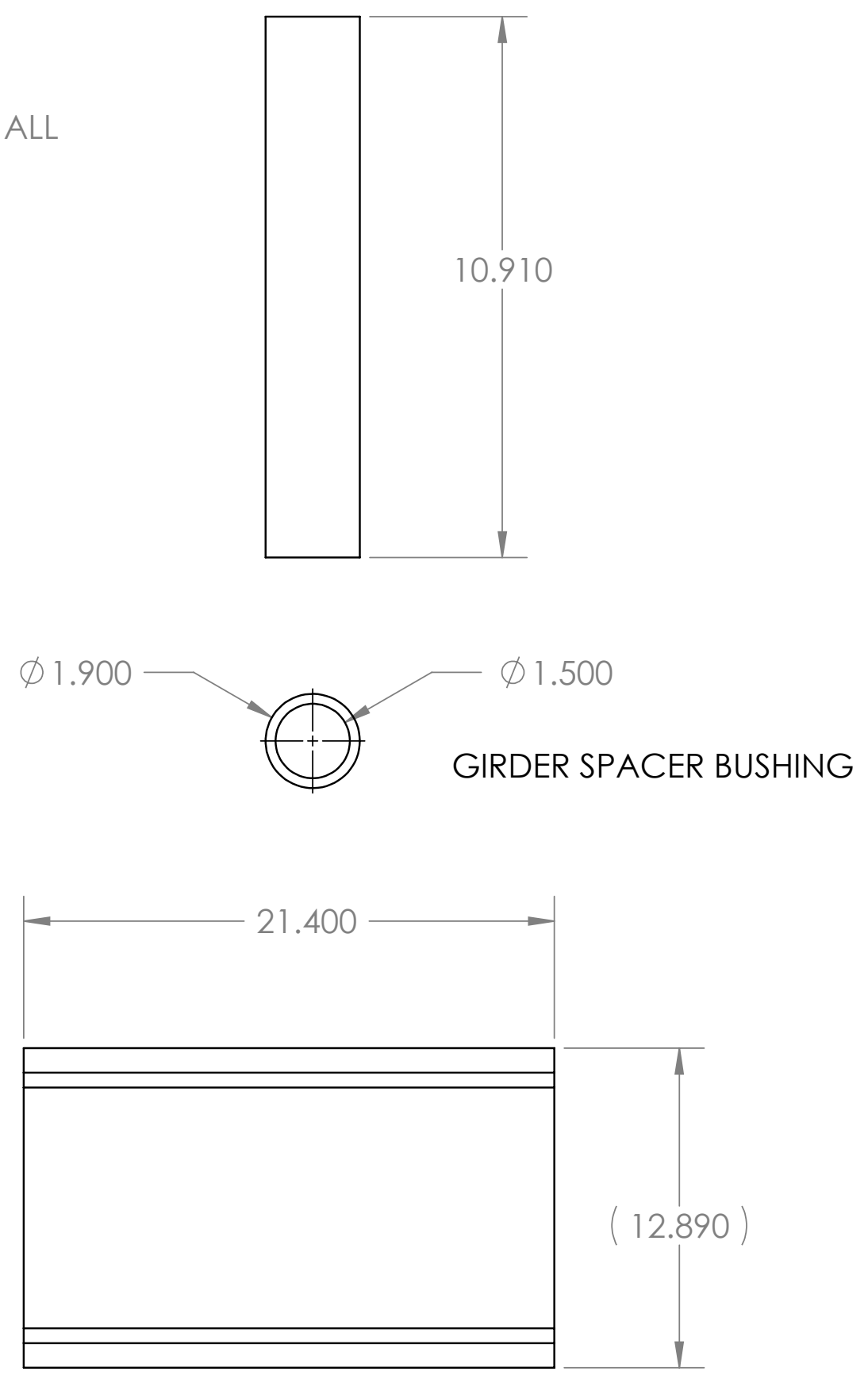

(2)

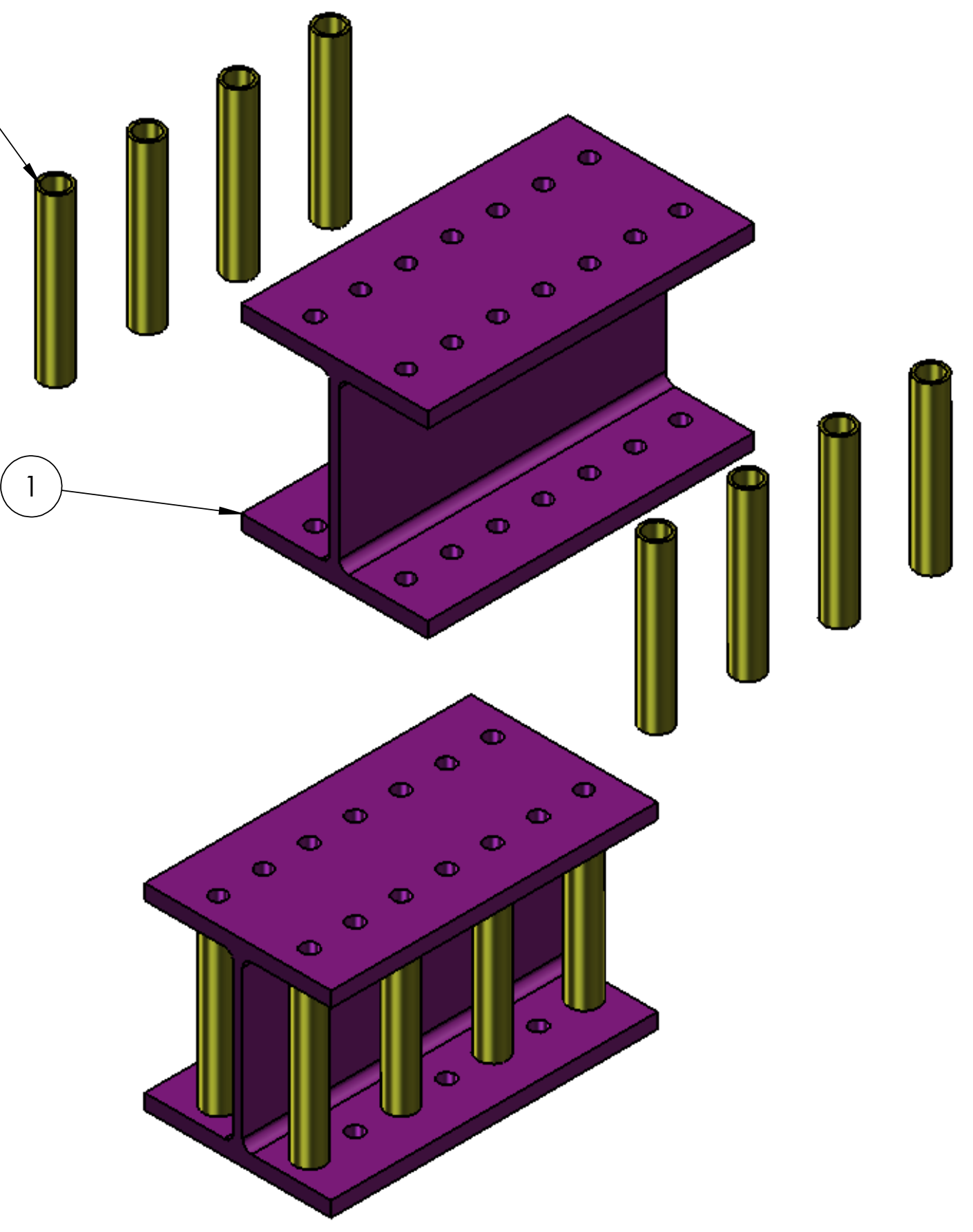

\begin{tabular}{|c|l|c|c|}
\hline ITEM NO. & \multicolumn{1}{|c|}{ PART NUMBER } & DESCRIPTION & QTY. \\
\hline 1 & W12x106 & GIRDER SPACER & 1 \\
\hline 2 & $\begin{array}{l}\text { girder_Spacer- } \\
\text { bushing_SCH-80 }\end{array}$ & GIRDER SPACER BUSHING & 8 \\
\hline
\end{tabular}

1) ALL PART DIMENSIONS DO NOT CONTAIN NECESSARY CLEARANCES FOR FABRICATION. VENDER IS RESPONSIBLE FOR DETERMINING ALL APPROPRIATE CLEARANCES AND TOLERANCES FOR PART, WELDMENT AND MODULAR REACTION FRAME SYSTEM ASSEMBLY.

2) GIRDER SPACER MATERIAL: W12×106

3) GIRDER SPACER BUSHING MATERIAL: $1.5 \times 1.9 \mathrm{SCH} 80$ PIPE 4) APPROXIMATE WEIGHT: 210 LBS
NIST

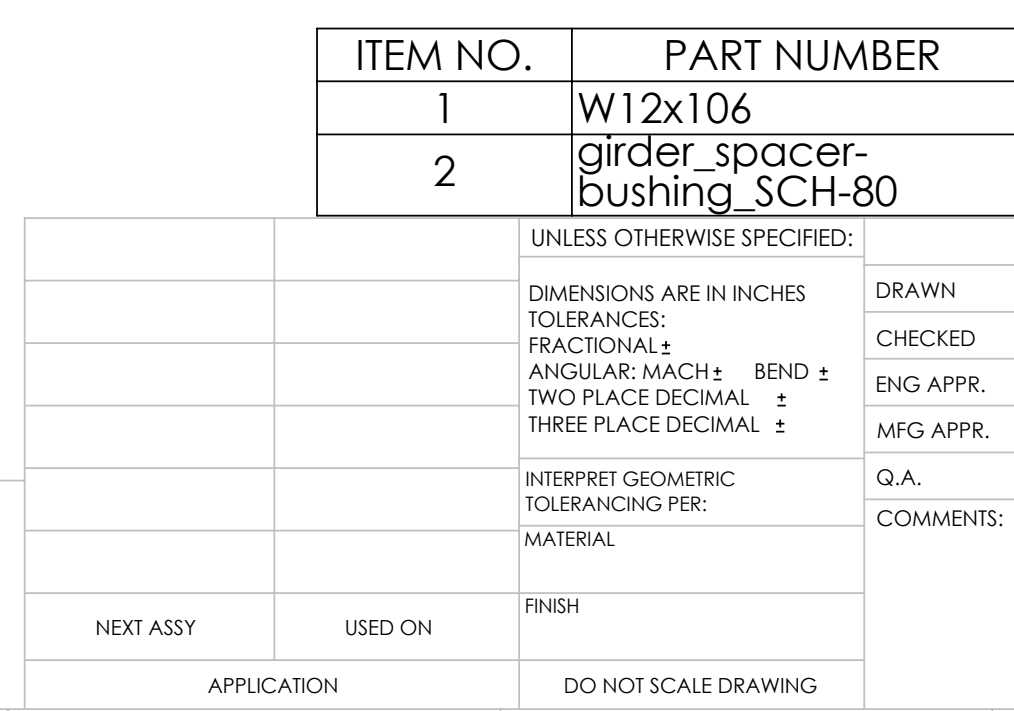

4

\section{GIRDER SPACER}

B

SCALE: 1:8 WEIGHT: SHEET 14 OF 15 

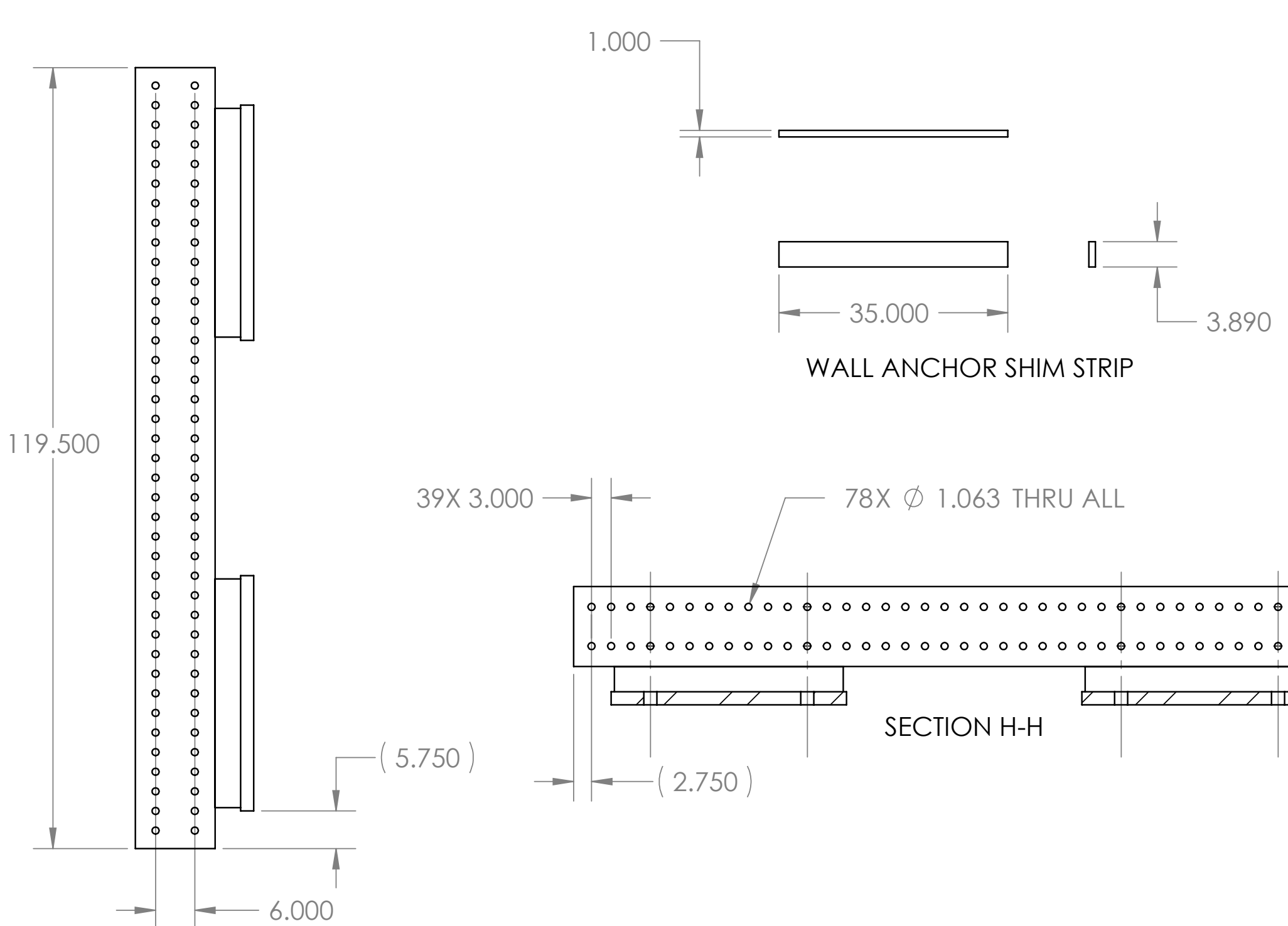

WALL ANCHOR SHIM STRIP
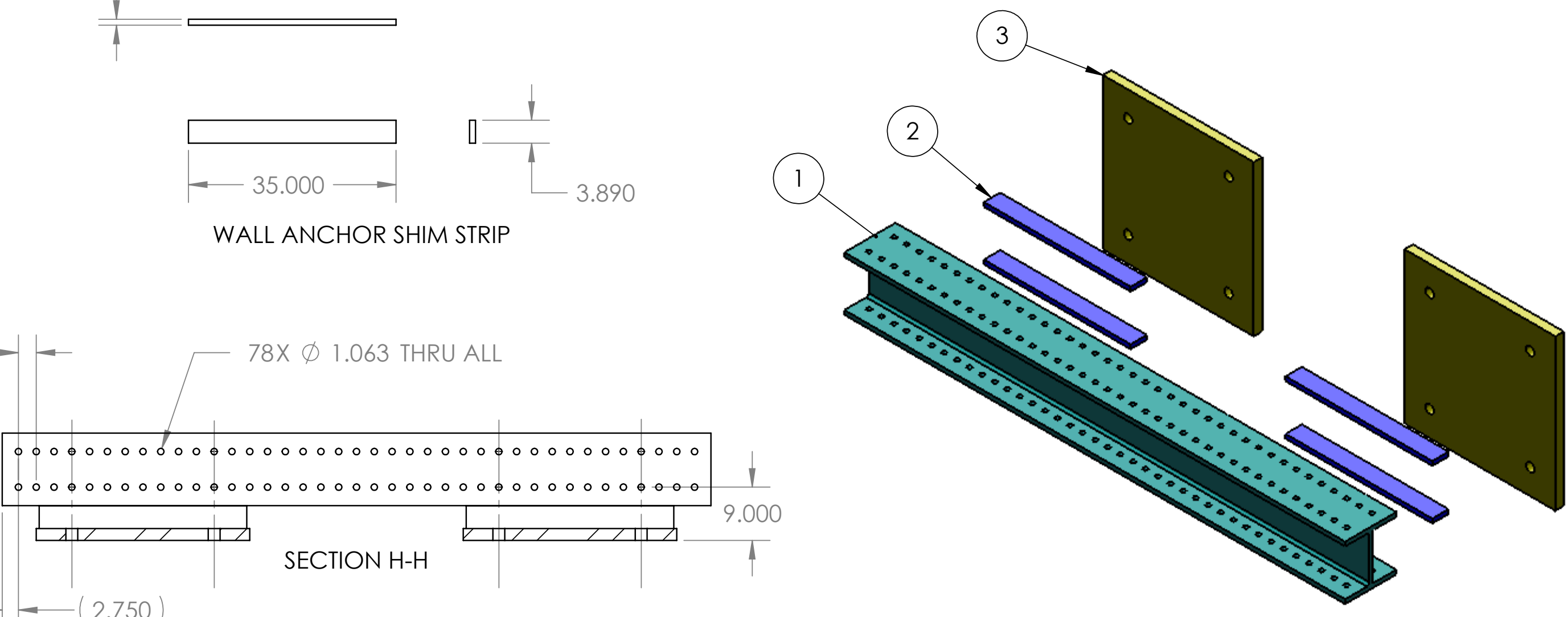

$=-6.000$

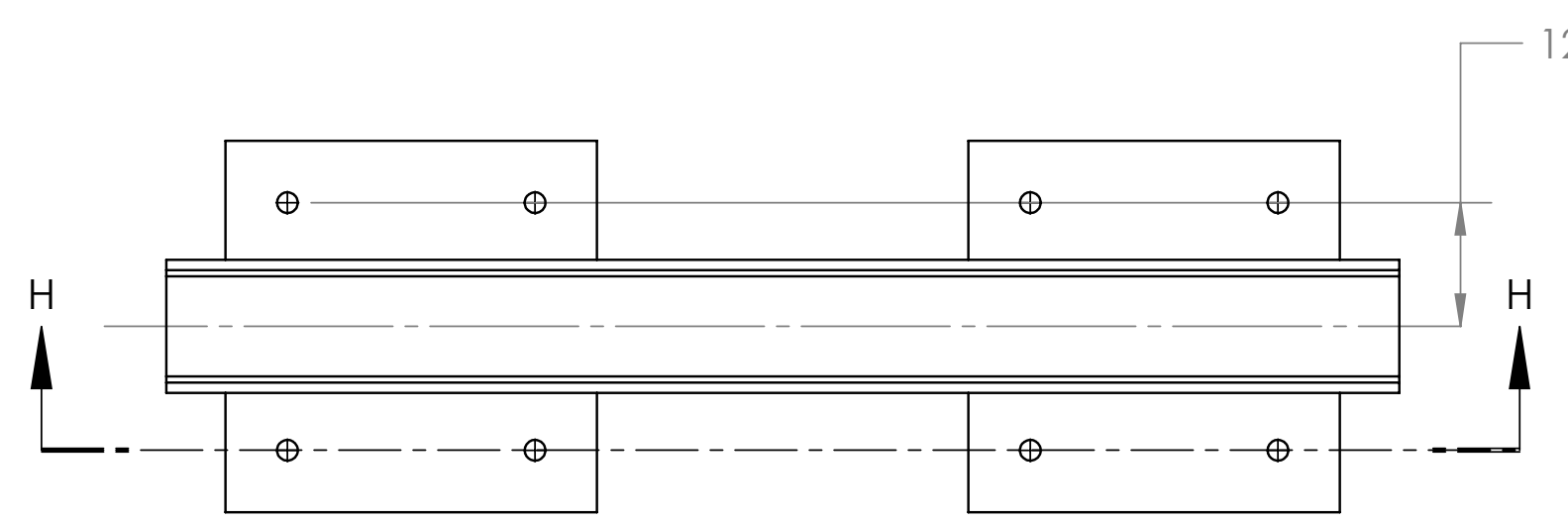

NOTES:

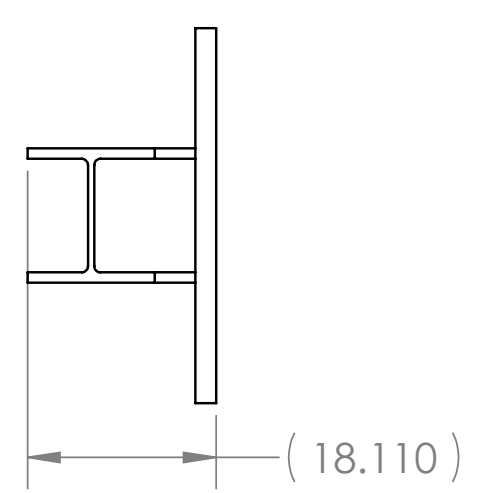

1) ALL PART DIMENSIONS DO NOT CONTAIN NECESSARY CLEARANCES FOR FABRICATION. VENDER IS RESPONSIBLE FOR DETERMINING ALL APPROPRIATE CLEARANCES AND
TOLERANCES FOR PART, WELDMENT AND MODULAR REACTION FRAME SYSTEM ASSEMBLY. 2) WALL COLUMN MATERIAL: W12×106

3) WALL ANCHOR SHIM STRIP MATERIAL: A36 OR EQIVELENT 4) APPROXIMATE WEIGHT: 2,634 LBS
NIST

\begin{tabular}{|c|l|c|c|}
\hline ITEM NO. & \multicolumn{1}{|c|}{ PART NUMBER } & DESCRIPTION & QTY. \\
\hline 1 & W12x106 & WALL COLUMN & 1 \\
\hline 2 & wall-anchor_shim-strip & WALL ANCHOR SHIM STRIP & 4 \\
\hline 3 & column-foot & COLUMN FOOT & 2 \\
\hline
\end{tabular}
UNLESS OTHERWISE SPECIFIED:

DIMENSIONS ARE IN INCHES DIMENSIONS
TOLERANCES
RRATIONAL:
R ANGULAR: MACH: BEND
TWO PACEDECIMAL
TWR A HREE PLACE DECIMAL

DRAWN CHECKED COMMENTS: DO NOT SCALE DRAWING name date TITLE:

\section{WALL COLUMN} ASSEMBLY

BIEE 